\title{
PUBLICATIONS
}

$A 11102483636$

NATL INST OF STANDARDS \& TECH R.I.C.

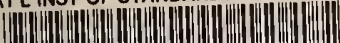

A11102483636

Domanskl, Plotr A/Modeling of a heat pum

QC100 U5753 NO.1218 V1986 C.1 NBS-PUB-C

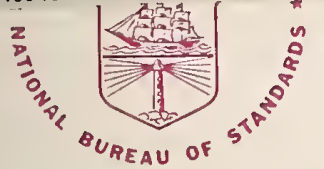

NBS TECHNICAL NO 1218

\section{Modeling of a Heat Pump Charged With a Non-Azeotropic Refrigerant Mixture}

\section{Piotr Domanski}

100

.45753

No. 1218

1986

c. 2 
he National Bureau of Standards' was established by an act of Congress on March 3, 1901. The Bureau's overall goal is to strengthen and advance the nation's science and technology and facilitate their effective application for public benefit. To this end, the Bureau conducts research and provides: (1) a basis for the nation's physical measurement system, (2) scientific and technological services for industry and government, (3) a technical basis for equity in trade, and (4) technical services to promote public safety. The Bureau's technical work is performed by the National Measurement Laboratory, the National Engineering Laboratory, the Institute for Computer Sciences and Technology, and the Institute for Materials Science and Engineering.

\section{The National Measurement Laboratory}

Provides the national system of physical and chemical measurement; coordinates the system with measurement systems of other nations and furnishes essential services leading to accurate and uniform physical and chemical measurement throughout the Nation's scientific community, industry, and commerce; provides advisory and research services to other Government agencies; conducts physical and chemical research; develops, produces, and distributes Standard Reference Materials; and provides calibration services. The Laboratory consists of the following centers:
- Basic Standards ${ }^{2}$

- Radiation Research

- Chemical Physics

- Analytical Chemistry

\section{The National Engineering Laboratory}

Provides technology and technical services to the public and private sectors to address national needs and to solve national problems; conducts research in engineering and applied science in support of these efforts; builds and maintains competence in the necessary disciplines required to carry out this research and technical service; develops engineering data and measurement capabilities; provides engineering measurement traceability services; develops test methods and proposes engineering standards and code changes; develops and proposes new engineering practices; and develops and improves mechanisms to transfer results of its research to the ultimate user. The Laboratory consists of the following centers:
- Applied Mathematics

- Electronics and Electrical Engineering ${ }^{2}$

- Manufacturing Engineering

- Building Technology

- Fire Research

- Chemical Engineering ${ }^{2}$

\section{The Institute for Computer Sciences and Technology}

Conducts research and provides scientific and technical services to aid Federal agencies in the selection, acquisition, application, and use of computer technology to improve effectiveness and economy in Government operations in accordance with Public Law 89-306 (40 U.S.C. 759), relevant Executive Orders, and other directives; carries out this mission by managing the Federal Information Processing Standards Program, developing Federal ADP standards guidelines, and managing Federal participation in ADP voluntary standardization activities; provides scientific and technological advisory services and assistance to Federal agencies; and provides the technical foundation for computer-related policies of the Federal Government. The Institute consists of the following centers:
- Programming Science and Technology

- Computer Systems Engineering

\section{The Institute for Materials Science and Engineering}

Conducts research and provides measurements, data, standards, reference materials, quantitative understanding and other technical information fundamental to the processing, structure, properties and performance of materials; addresses the scientific basis for new advanced materials technologies; plans research around cross-country scientific themes such as nondestructive evaluation and phase diagram development; oversees Bureau-wide technical programs in nuclear reactor radiation research and nondestructive evaluation; and broadly disseminates generic technical information resulting from its programs. The Institute consists of the following Divisions:
- Ceramics

- Fracture and Deformation ${ }^{3}$

- Polymers

- Metallurgy

- Reactor Radiation 


\section{Modeling of a Heat Pump Charged \\ With a Non-Azeotropic Refrigerant Mixture}

\section{Piotr Domanski}

National Bureau of Standards

Center for Building Technology

Building Equipment Division

Gaithersburg, Maryland 20899

January 1986

Sponsored by:

Electric Power Research Institute

Palo Alto, California 94303

U.S. DEPARTMENT OF COMMERCE, Malcolm Baldrige, Secretary 
National Bureau of Standards Technical Note 1218 Natl. Bur. Stand. (U.S.), Tech. Note 1218, 391 pages (Jan. 1986) CODEN: NBTNAE 
An analysis of the vapor compression cycle and the main components of an airto-air heat pump charged with a binary non-azeotropic mirture has been performed for steady-state operation. The general heat pump simulation model HPBI has been formulated which is based on independent, analytical models of system components and the logic linking them together. The logic of the program requires an iterative solution of refrigerant pressure and enthalpy balances, and refrigerant mirture and individual mirtare component mass inventories.

The modeling effort emphasis was on the local thermodynamic phenomena which were described by fundamental heat iransfer equations and oquation of state relationships among material properties. In the compressor model several refrigerant locations were identified and the processes taking place between these locations accounted for all significant heat and pressure losses. Evaporator and condenser models were developed on a tube-by-tube basis where performance of each coil tube is computed separately by considering the crossflow heat transfer with the external air stream and the appropriate heat and mass transfer relationships. A constant flow area expansion device model was formulated with the aid of Fanro flow theory. Equation of state for mirtures is described and equation constants for a R13B1/R152a mirture are given.

The developed heat pump model was validated by checking computer results against 1 aboratory tests data of one heat pump at two cooling and two heating rating points.

Program HPBI can be used to evaluate potentials of non-azeotropic mixtures vorking in a split residential heat pump. User's Manual and listing of the program is included in the report.

Key words: air conditioner; capillary tube: coil; compressor; condenser; expansion device; heat pump; modeling; mirture; non-azeotropic refrigerant: vapor modeling cycle 


\section{ACKNOWLEGEMENTS}

The anthor wishes to acknowledge the assistance received from Graham Morrison, Milliam Mulroy, Reinhard Radermacher, Howard Ross, and especially David Didion. who directed the Group's non-azeotropic mixture research.

Appreciation is due Preston McNal1, Victor Goldschmidt and Jesse Galloway for their comments on the report.

This work was funded by the Electric Power Research Institute, with Jim Ca1m serving as the project manager. 
ABSTRACT ..................................................

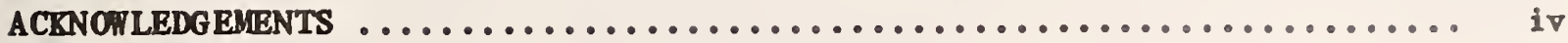

DISCLAIMER ..............................................

METRIC CONVERSION FACTORS .................................

LIST OF TABLES ........................................... ix

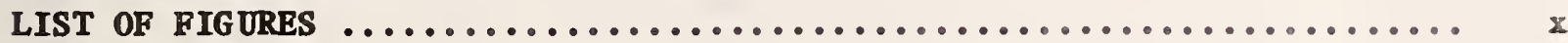

LIST OF SYMBOLS ..........................................

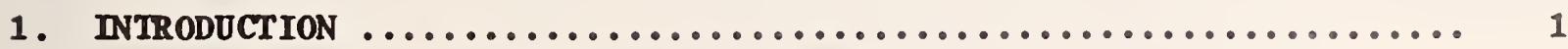

2. GUIDELINES FOR MODEL DEVELOPMENT .........................

3. EVALUATION OF THERMODYNAUIC AND TRANSPORT PROPERTIES OF A NON-AZEOTROPIC MIXTURE, R13B1/R152 \& .......................

3.1. Evalustion of Thermodynamic Properties .....................

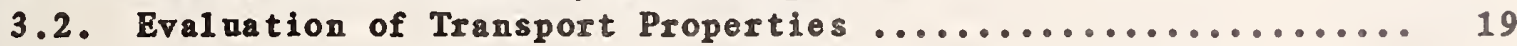

4. OPERATION OF A HEAT PUMP WITH A CONSTANT FLOT AREA EXPANSION

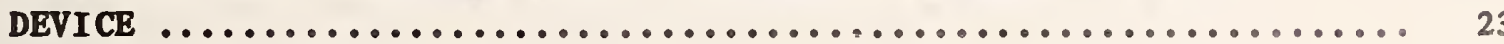

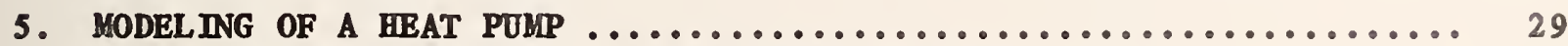

5.1. Logic of a Model of a Heat Pump Charged $\mathbf{w}$ th a

Non-Azeotropic Miztnre Refrigerant ................... 29

5.2. Modeling of a Reciprocating, Hermetic Compressor .......... 33

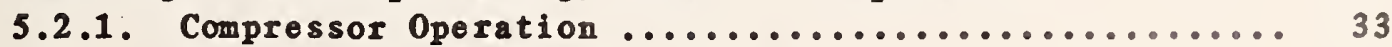

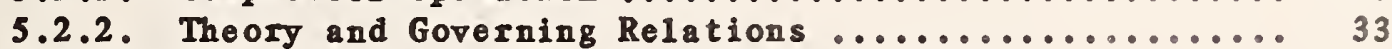

5.3. Hodeling of a Constant Flor Area Expansion Derice ..........47

5.3.1. Available Capillary Tube Performance Data .......... 47

5.3.2. Avaliable Short Tube Restrictor Performance Data ... 50

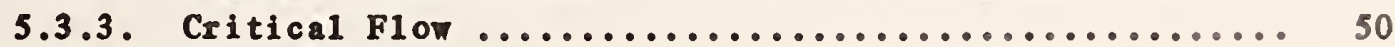

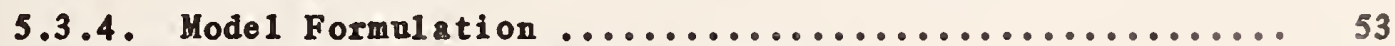

5.4. Modeling of an Evaporator and a Condenser ................56

5.4.1. Hodeling Methodology ........................ 56

5.4.2. Heat Transfer Rate for a Tube of a Cross-Fiow

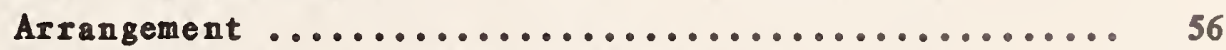

5.4.3. Refrigerant and Air Mass Flow Rates Associated

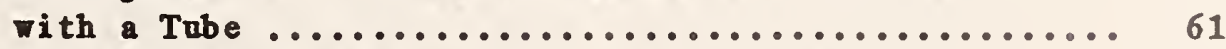

5.4.4. Overall Heat Transfer Coefficient for a Dry

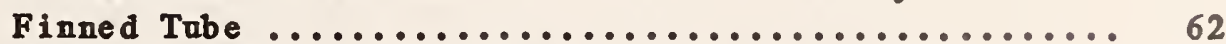

5.4.5. Forced Convection Heat Transfer Inside a Tube ....... 63

5.4.6. Forced Convection Heat Transfer at the Air-Side

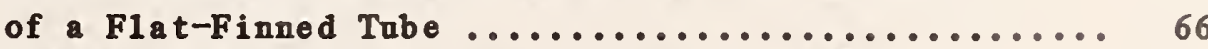

5.4.7. Overal1 Heat Transfer Coefficient for a Wet

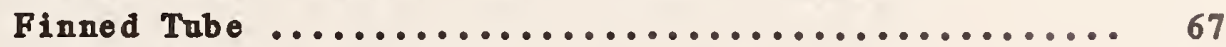

5.4.8. Pressure Drop in a Tube ......................... 74

5.5. Modeling of Additional Heat Pump Components .............. 77

5.6. Refrigerant Mass Inventory in a Heat Pump ................ 79

6. MODEL VERIFICATION ..................................... 
7. SUMMART AND CONCLUSIONS $\ldots \ldots \ldots \ldots \ldots \ldots \ldots \ldots \ldots \ldots \ldots \ldots \ldots \ldots \ldots$

8. REFERENCES ....................................... 91

APPENDIX A. Calculations of Properties of Moist Air .............. 95

APPENDIX B. Calculation of Water and Frost Properties .............. 97

APPENDIX C. Calculation of Critical Pressure for Tro-Phase

Fanno Flow of a Non-Azeotropic Mirture .............. 99

APPENDIX D. The Logic of the Main Program, BMAIN ................. 103

APPENDIX E. Compressor Simulation Subroutine, COMPRE ............. 107

APPENDIX F. Capillary Tube Simulation Subroutine, CAPIL ............ 109

APPENDIX G. Evaporator and Condenser Subroutines, EVAPHX and CONDHX .. 111

APPENDIX H. Jser's Manual ............................... 113

H1. General Information .......................... 113

H2. Input and Output Data Coding ................. 113

H2.1 Refrigerants Constants ................. 113

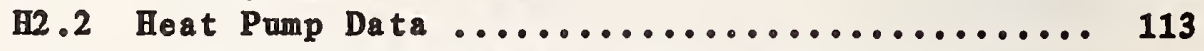

H2.3 Run Controlling Data, Indoor and Outdoor

Air Conditions, Refrigerant Parameters

and Output Data ....................... 114

APPENDIX I. Example of Run of the Program, HPBI ................. 131

APPENDIX $J$. Listing of the Program, HPBI .................... 215 


\section{DISCLAIMER}

In view of the presently accepted practice of the building industry in the United States and the structure of the computer software used in this project, common U.S. units of measurement have been used in this report. In recognition of the United States as a signatory to the General Conference of Weights and Measures, which gave official status to the SI system of units in 1960 , appropriate conversion factors have been provided in the table below. The reader interested in making further use of the coherent system of SI units is referred to: NBS SP330, 1972 Edition, 'The International System of Units,' or E380-72, ASTM Metric Practice Guide (American National Standard 2210.1). 


\begin{tabular}{|c|c|}
\hline Length & $\begin{array}{l}1 \text { inch }(\text { in })=25.4 \text { millimeters }(\mathrm{mm}) \\
1 \text { foot }(\mathrm{ft})=0.3048 \text { meter }(\mathrm{m})\end{array}$ \\
\hline Are a & $1 \mathrm{ft}^{2}=0.092903 \mathrm{~m}^{2}$ \\
\hline Volume & $1 \mathrm{ft}^{3}=0.028317 \mathrm{~m}^{3}$ \\
\hline Temperature & $F=9 / 5 C+32$ \\
\hline $\begin{array}{l}\text { Temperature } \\
\text { Interva } 1\end{array}$ & $1{ }^{\circ} \mathrm{F}=5 / 9^{\circ} \mathrm{C}$ or $\mathrm{K}$ \\
\hline Mas s & 1 pound $(1 \mathrm{~b})=0.453592 \mathrm{kilogram}(\mathrm{kg})$ \\
\hline $\begin{array}{l}\text { Mass Per Unit } \\
\text { Volume }\end{array}$ & $1 \mathrm{lb} / \mathrm{ft}^{3}=16.0185 \mathrm{~kg} / \mathrm{m}^{3}$ \\
\hline Energy & 1 Btu $=1.05506 \mathrm{kilojoules}(\mathrm{kJ})$ \\
\hline Specific Heat & $1 \mathrm{Btu} /\left[(1 \mathrm{~b})\left({ }^{\circ} \mathrm{F}\right)\right]=4.1868 \mathrm{~kJ} /[(\mathrm{kg})(\mathrm{K})]$ \\
\hline Ga11on & 1 ga11on $=0.0037854 \mathrm{~m}^{3}$ \\
\hline
\end{tabular}




\section{LIST OF TABLES}

Page

1. Equation of State Constants for Refrigerants $13 \mathrm{Bl}$ and 152 a ....... 11

2. Coofficients to be Osed in Equations (17), (18), and (19) ...... 18

3. Coefficients to be Used in Correlations for Fin Efficiency

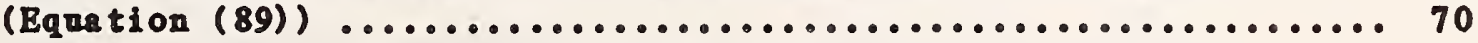

4. Laboratory Test and Computer Simalation Results in the Cooling Mode .....................................

5. Laboratory Test and Computer Simulation Results in the Heating Mode

B1. Water Property Evaluation Constants which are Osed in Equetion (B1)

H1. Refrigerant Property Single Precision Functions and Subroutines

H2. Refrigerant Property Double Precision Functions and Subroutines

H3. Functions and Subroutines for Heat Pump Component Simulation, Heat Transfer and Fluid Mechanic Calculations

44. Data File Containing Constants for Evaluntion of Thermodynamic Properties of R13B1/R152a Mirture ...................... 123

H5. Heat Punp Input Data Code to Program HPBI .................... 124

H6. Example of a Heat Pump Data File .........................129 
1. Oversimplified thermodynamic cycle of a heat pump ............. 2

2. Schematic of a heat pump $\ldots \ldots \ldots \ldots \ldots \ldots \ldots \ldots \ldots \ldots \ldots \ldots \ldots \ldots \ldots \ldots \ldots \ldots \ldots$

3. Thermodynamic cycle realized by a heat pump ................. 5

4. Flow chart for calculating the saturation pressure of single component refrigerant ............................... 12

5. F1ow chart for calculating the bubble point temperature of a non-azeotropic binary mirture..........................

6. Temperature-composition diagram for a non-azeotropic binary

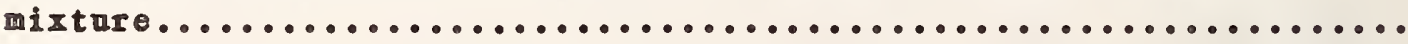

7. Simplified thermodynamic cycle realized by a heat pump charged with refrigerant 22 in the cooling mode at different

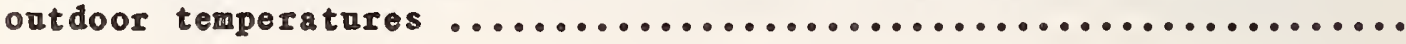

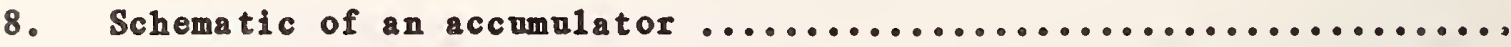

9. Overall logic of a program simulating a heat pump employing a constant flow area expansion device and charged with a non-azeotropic binary rerrigerant mizture $\ldots \ldots \ldots \ldots \ldots \ldots \ldots \ldots \ldots \ldots$

10. Schematic of a hermetic compressor $\ldots \ldots \ldots \ldots \ldots \ldots \ldots \ldots \ldots \ldots \ldots \ldots$

11. Typical speed (RPM) versus load curve for a permanent split-capacator two pole electric motor [15] .................

12. Simplified indicator diagram for a reciprocating compressor .......

13. Typical efficiency versus load curve for a permanent split-capacitor two pole electric motor [15] ..................

14. Pressure and temperature distribution along typical capillary tube $[23] \ldots \ldots \ldots \ldots \ldots \ldots \ldots \ldots \ldots \ldots \ldots \ldots \ldots \ldots \ldots \ldots \ldots \ldots \ldots \ldots \ldots \ldots \ldots \ldots$

15. Mass balance for an element of fluid in one-dimensional steady

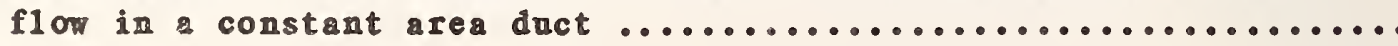

16. Momentum balance for an element of fluid in one-dimensional flow in a horizontal, constant area dact .....................

17. Energy balance for an element of fluid in one-dimensional, adiabatic, steady flow in a horizontal, constant area duct .......

18. Fanno Iine $\ldots \ldots \ldots \ldots \ldots \ldots \ldots \ldots \ldots \ldots \ldots \ldots \ldots \ldots \ldots \ldots \ldots \ldots \ldots \ldots \ldots \ldots$ 
19. Schematic of a heat pump heat exchanger ................... 57

20. Example of coil circuitry ........................... 58

21. Approximation method for treating a rectangular-plate fin of uniform thickness in terms of a flat circular-plate fin of

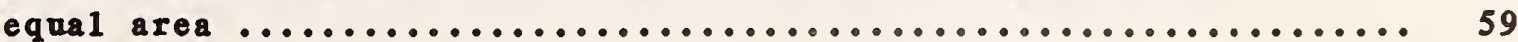

22. Cross section of a flat-finned tube indicating parameters which affect the air side heat transfer coefficient ................ 68

23. Efficiency for a circular-p1ate fin of uniform thickness ........ 69

24. Refrigerant phase in heat pump components ................. 80

C1. Logic to evaluate the quality of the Fanno flow of a given pressure for a non-azeotropic mixture ..................... 101

D1. Overall logic of program HPBI .......................... 105

F1. Logic of a constant flow area expansion device simulation program, CAPIL .................................. 110

G1. F10 chart for coll performance simulation programs ............ 112 


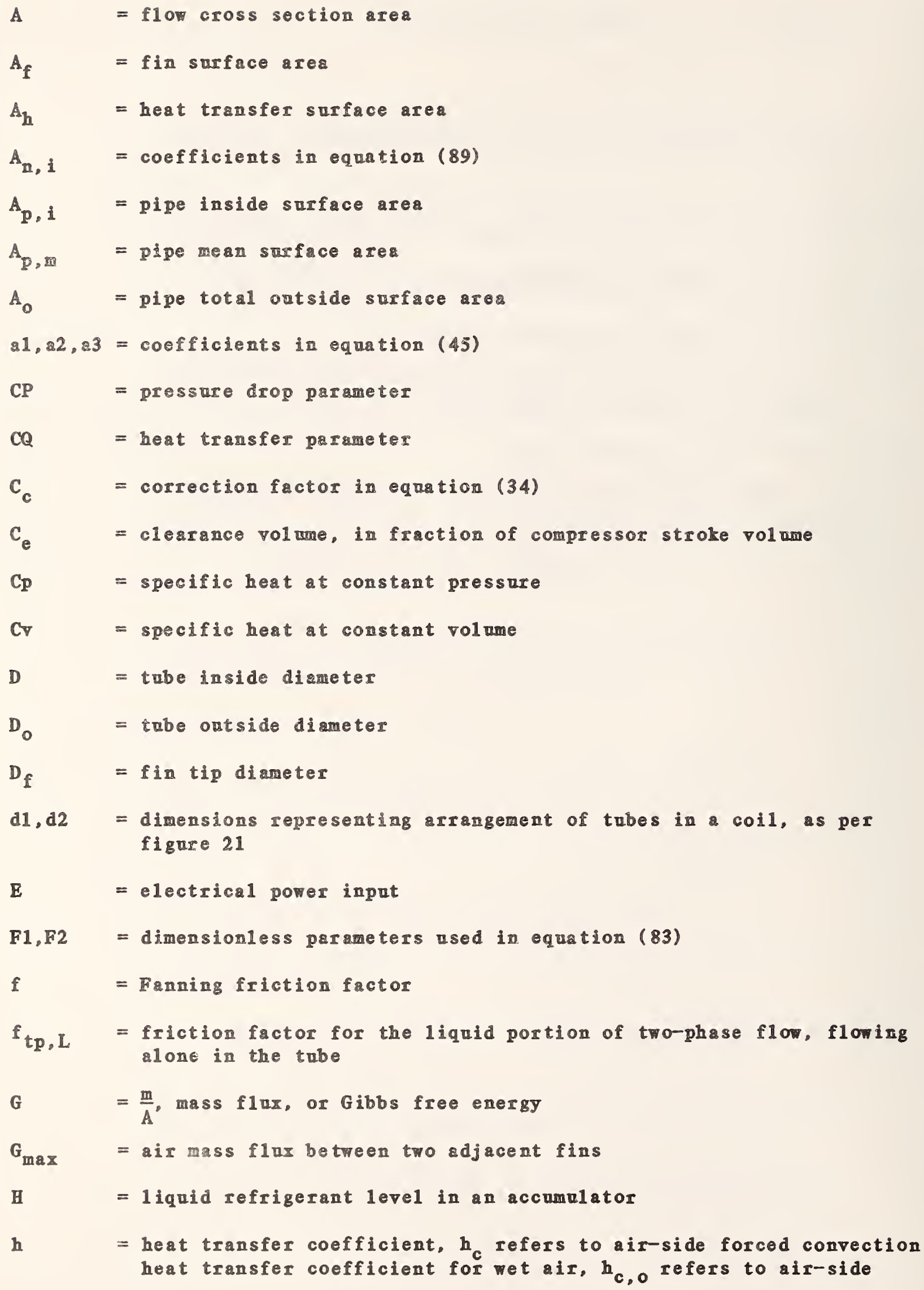


forced convection heat transfer coefficient for dry air, $h_{i}$ refers to inside tube convection heat transfer coefficient

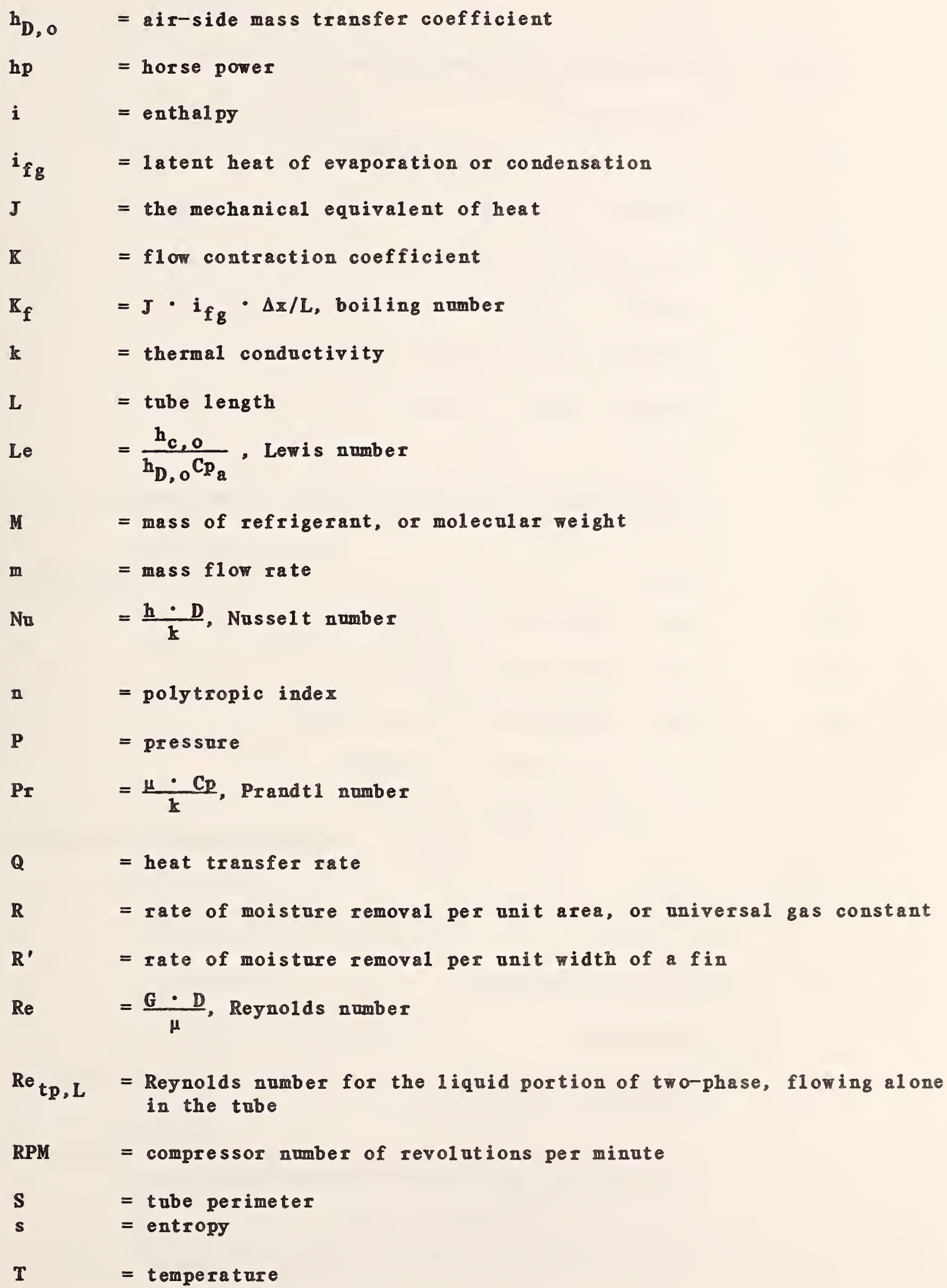




$$
\begin{aligned}
& T_{f, b} \quad=\text { fin base temperatare } \\
& T_{f, m} \quad=\text { mean } f \text { in temperature } \\
& T_{I, g}=\text { refrigerant saturation temperature } \\
& \text { t }=\text { fin thickness } \\
& \text { U = overal1 heat transfer coefficient } \\
& \mathrm{V} \quad=\text { velocity } \\
& \text { Vs = compressor swept volume } \\
& \forall \quad=\text { vol ume } \\
& \checkmark \quad=\text { specific volume or molar volume } \\
& W_{c} \quad=\text { mechanical power available for compession process } \\
& \text { We = wechanical power input } \\
& w_{a}=\text { humidity ratio of } a i r, w_{a, i} \text { refers to tube row. inlet, } w_{a, 0} \text { refers } \\
& W_{W} \quad \text { = humidity ratio of saturated ais at temperature of wetted water film } \\
& \text { XL = molar composition of liquid phase } \\
& \text { XM = molar composition of mixtere } \\
& \text { XV = molar composition of vapor phase } \\
& \text { XW = weight composition of mizture } \\
& z_{t t} \quad=\left(\frac{1-\Sigma}{z}\right)^{0.9}\left(\frac{\nabla_{L}}{v_{V}}\right)^{0.5}\left(\frac{\mu_{L}}{\mu_{L}}\right)^{0.1} \text {, Lockhart-Martine11i parameter } \\
& =\frac{m_{V}}{m_{V}+m_{L}} \text {, quality } \\
& x_{p} \quad=\text { pipe wal1 thickness } \\
& \text { y } \quad \text { fin height } \\
& \mathrm{Z}_{\mathrm{tp}} \quad=\text { fraction of the tube in the two-phase region for the tube with } \\
& \text { 1iquid and two-phase flow } \\
& \mathrm{Z}_{\mathrm{V}} \quad \text { = fraction of the tube in the superheated vapor region for the tube } \\
& \text { with tro-phase and superheated vapor flow } \\
& \text { z = distance between adjacent fins } \\
& \text { a } \quad \text { = void fraction }
\end{aligned}
$$




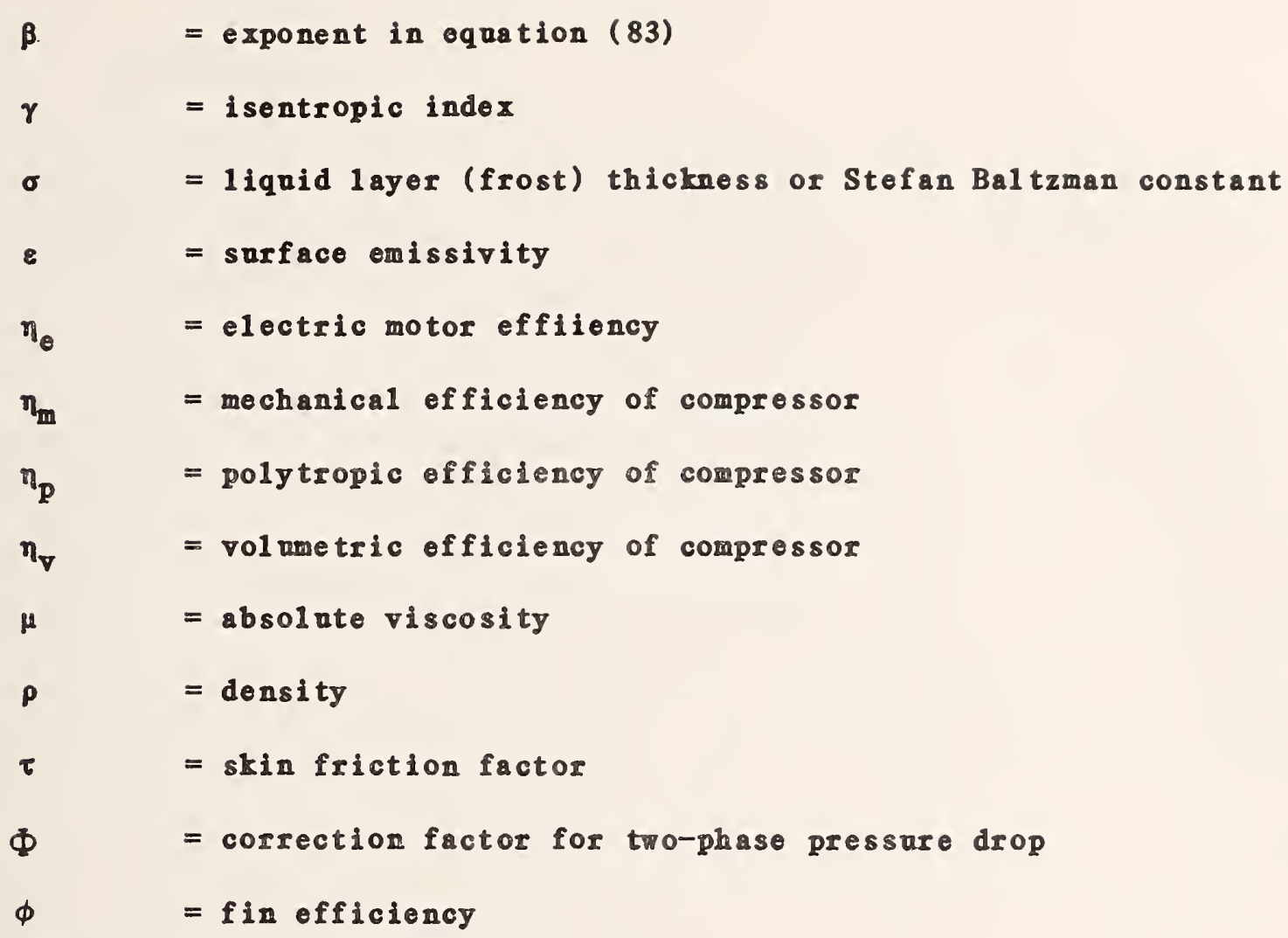

e exit

f $=$ frost or fin

1 inlet

L $=1$ iquid

m = mean valne

P = constant pressure process

I $=$ refrigerant

s = constant entropy process

t = total value

$\mathrm{V}=$ vapor

$\nabla \quad=$ constant volume process

w $\quad$ water

1 to 13 = refrigerant key locations in a heat punp, as per figure 4 , unless otherwise explained in the text 



\section{INTRODUCTION}

Among equipment providing thermal comfort for indoor spaces, the heat pump has gained in recent years substantial popularity for residential applications. The heat pump is unique since it is the only device, which can provide both heating and cooling. Heat pumps have become very competitive economically and are considered to be a very good investment by homeowners.

A heat pump works on the vapor compression cycle principle. The most important heat pump components are two heat exchangers, a compressor and an expansion device. The heat pump thermodynamic cycle can be explained by aralysing the processes that the refrigerant undergoes in these four components. The most convenient diagram for such an explanation and performance analysis is that of a pressure vs. enthalpy coordinate system, as shown in figure 1. The compressor receives low pressure and temperature refrigerant at state 1 and compresses it to a high pressure. This compression process is associated with an increase of refrigerant temperature. At state 2 , the high pressure and high temperature vapor enters the condenser. The refrigerant passing through the condenser rejects heat to the high temperature reservoir and changes, usua11y to a subcooled 1 iquid at state 3 . Then, the refrigerant flows through the expansion device undergoing a drop in pressure and temperature. Finally, the 1 ow pressure, 1 ow temperature, and low quality refrigerant at state 4 enters the evaporator, where it picks up heat from the 1 ow temperature reservoir, reaching a superheated (or high quality) vapor state 1 at the evaporator exit. In the explanation above, the low and high temperature reservoirs are the indoor and the outdoor environment, when the heat pump is operating in the cooling mode.

Besides the compressor, two heat exchangers, and the expansion device, there are, for practical reasons, many other components in an actual heat pump system. For modeling consideration, the most important are: tubes connecting basic elements, 4-way valve enabling refrigerant flow reversal in the unit to operate in the heating or cooling mode, and an accumalator (not used in all systems), which acts as a protective device for the compressor by storing excess refrigerant during part lord operation and preventing 1 iquid refrigerant from entering the compressor.

There are many concepts and designs of heat pump components, however, certain types are predominant. A reciprocating hermetic compressor is usually used for vapor compression in srall systems. Heat exchangers are usually in the form of staggered tubes with closely packed vavy fins (spine-fins or bristlefins are sometimes used). There are basically two types of expansion devices in application today. Constant flow area restrictors prevail in small capacity units such as household refrigerators, window-type air conditioners, and central residential air conditioners and heat pumps. Larger, more expensive residential units and commercial units are usualy equipped with a variable flow area device called a thermostatic expansion valve (TXV).

Al1 the above mentioned components male up an actual vapor compression system. Configuration of such a system and the thermodynamic cycle are illustrated in figure 2 and figure 3 , respectively. Refrigerant states in figure 3 correspond to particular locations in the system marked in figure 2 , which are: 

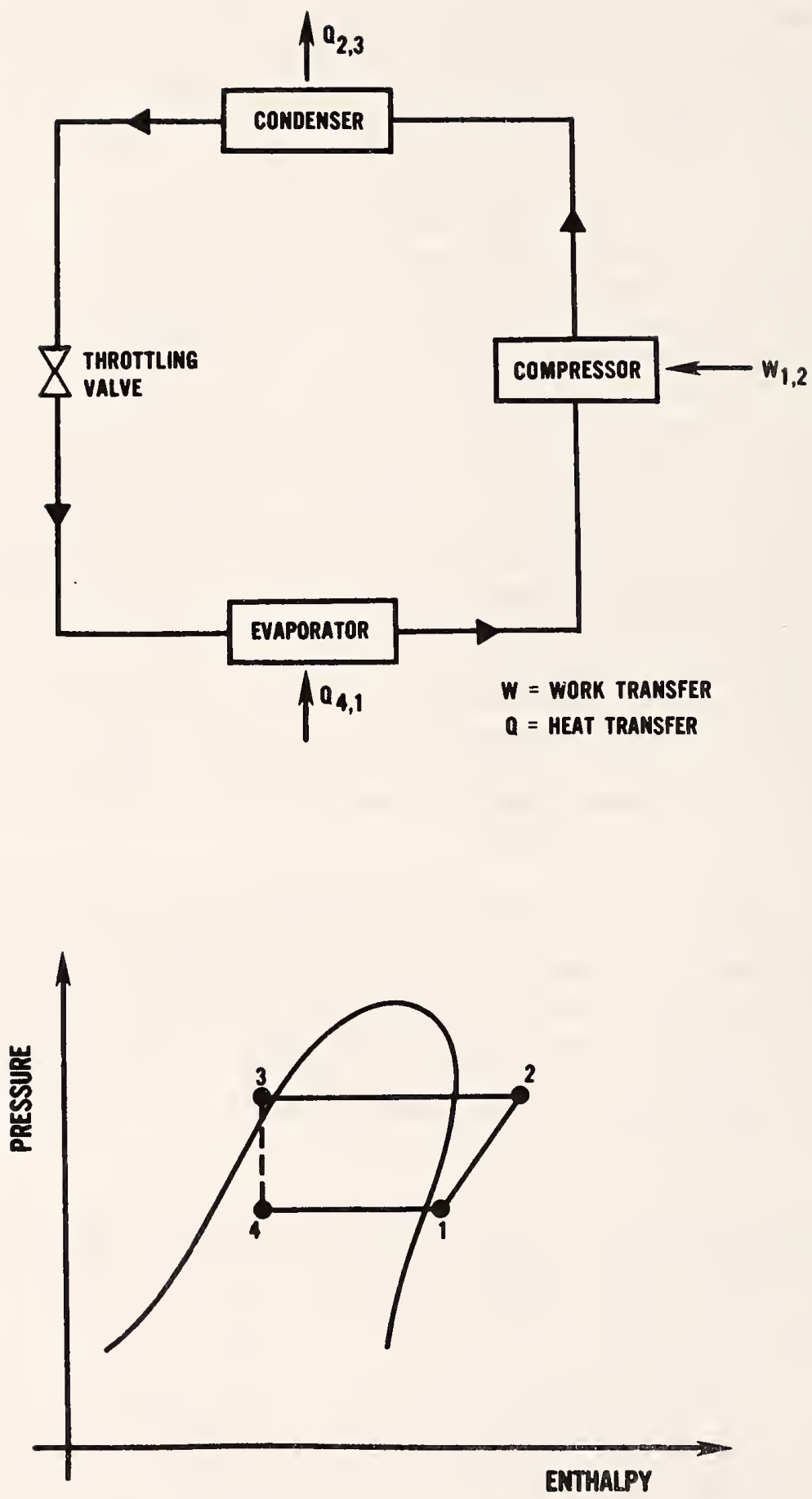

Figure 1. Oversimplified thermodynamic cycle of a heat pump. 


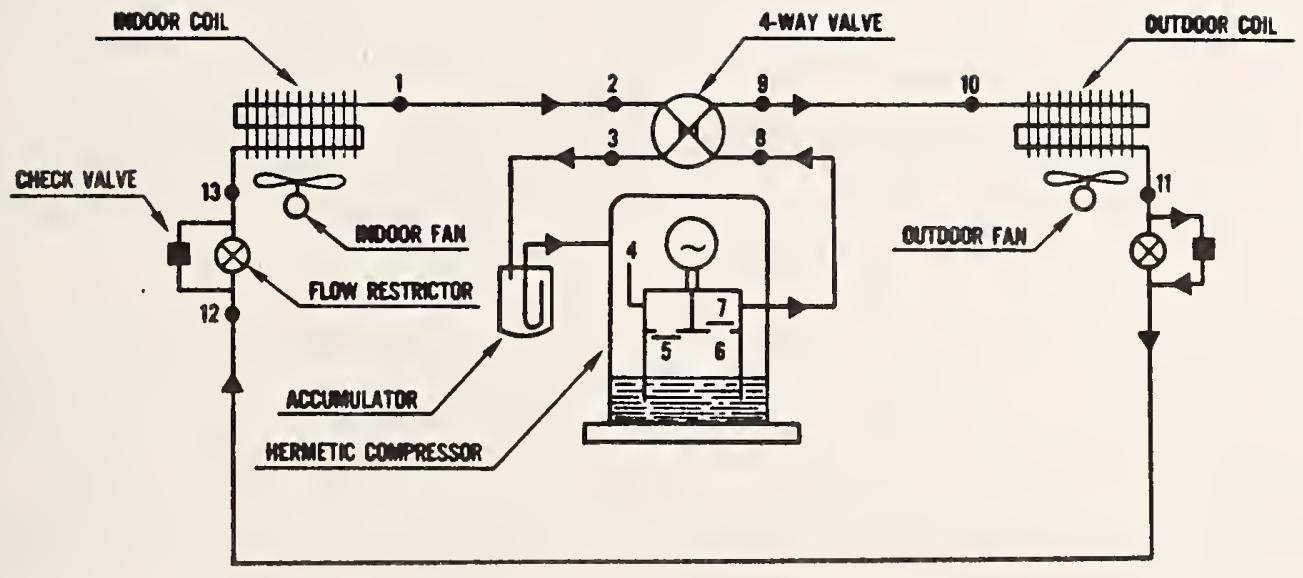

Note: 1. Refrigerant flow direction is marked for cooling operation.

2. Numbers 5 and 6 situated in the compressor correspond to refrigerant state before and after the compression process.

Figure 2. Schematic of a heat pump. 


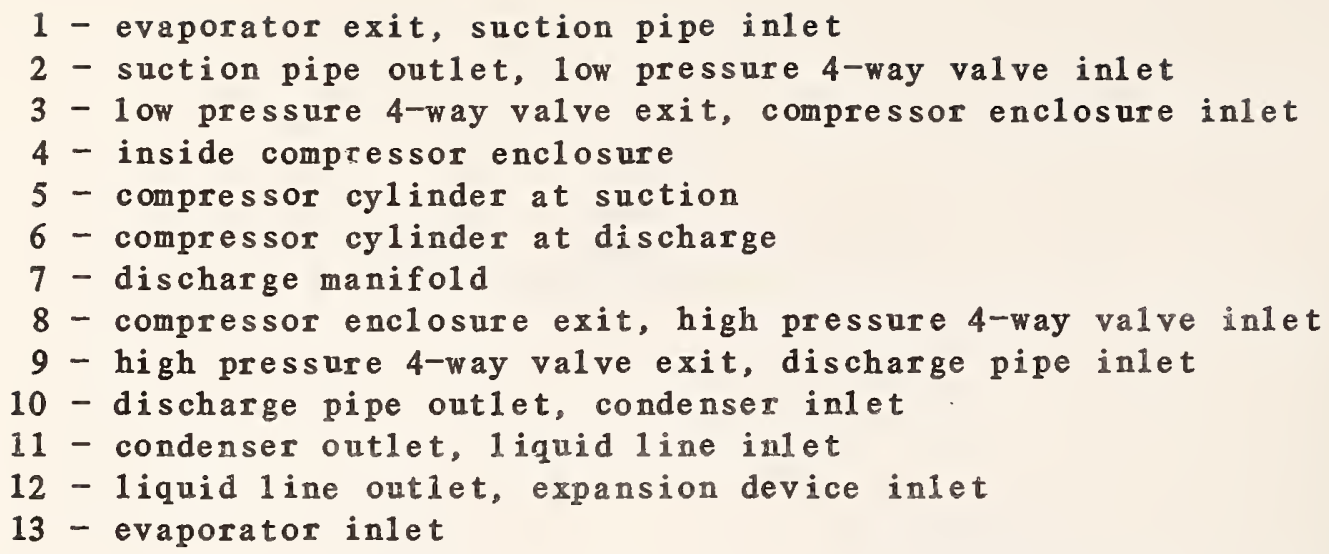

In the heat pumping process refrigerant is the carrier of heat from a 1 ow temperature reservoir to a high temperature reservoir. There are many kinds of refrigerants of different properties available and they are being used depending on the application. For residential air-to-air heat pumps, refrigerant 22 has been generally accepted as the most advantageous. However, in recent years interest has intensified in non-azeotropic binary mixtures as potential working fluids in heat pumps. Gliding evaporation/condensation temperatures and the possibility of heat pump capacity control via mixture composition shift are often cited as factors giving mixtures a theoretical performance edge over sing1e component refrigerants.

The objective of this study is the development of a mathematical model of an air-to-air heat pump working with a non-azeotropic mixture as the refrigerant in order to be able to investigate performance potentials of mixtures. The type of heat pump considered here is the one most comonly commercially available with a reciprocating hermetic compressor, fin-tube heat exchangers and a constant flow area expansion device. The mixture considered in this study consists of refrigerants $13 \mathrm{~B} 1$ and $152 \mathrm{a}$. 


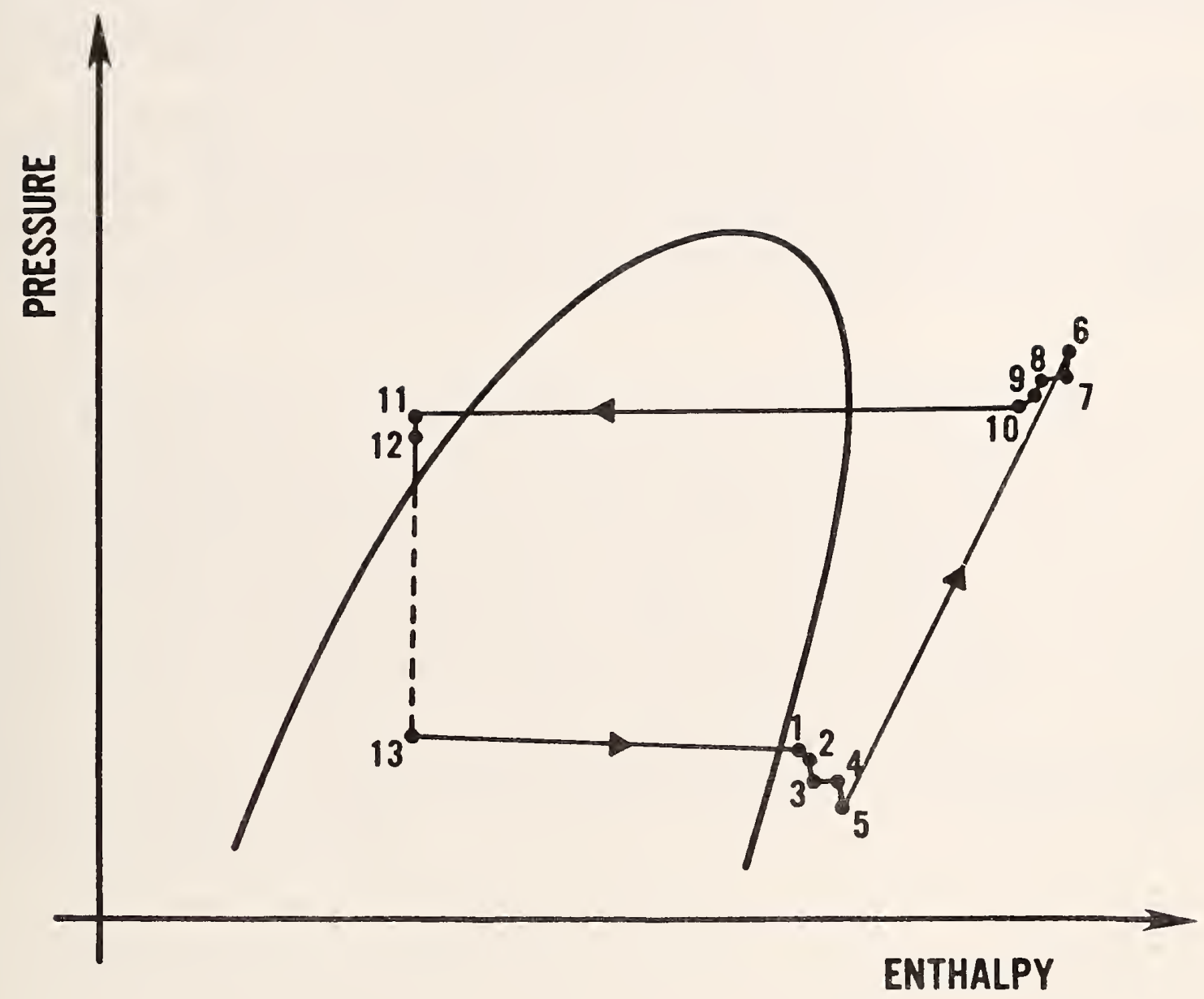

Figure 3. The rmodynamic cycle realized by a heat pump (for number location refer to figure 2). 

Before beginning the modeling effort, general guidelines for model development have to be established. These guidelines should evolve from future model applications as they are broadly perceived today. It is understood that the model is planned to be used for parametric studies by different users, applied to different hardware and possibly charged with different non-azeotropic refrigerants. The model should help prospective users to evaluate potential benefits of non-azeotropic mixtures, as applied to heat pumps, resulting from changing refrigerant temperature during evaporation/condensation and change of mixture composition which influences heat pump capacity.

It has been stated already that a heat pump equipped with a reciprocating compressor, compact staggered tube and flat fin heat exchangers, and a constant flow area expansion device will be modeled. This choice has been made since, in addition to the fact that components of this design type are most commonly encountered, results of initial research indicated a possibility of inherent to the system capacity modnlation in systems employing a capillary tube [1].

The model should be a first principle one. Regression analysis curve fits should be avoided so the program could be applied to simulate systems of different characteristics, size and performance level. Modular structure of the program should allow for later model upgrades on the local basis. Since heat exchangers and changing refrigerant mixture temperatures during change of phase are going to be investigated, a tube-by-tube approach to modeling of an evaporator and condenser vas adapted. Finally, an appropriate equation of state is desirable that could be universally used for accurate predictions of thermodyamic properties for a variety of possible non-azeotropic miztures.

During the past, a heat pump modeling effort has been underway at NBS [2,3]. The latest mode1 [3] fulfils the basic postulates of the guidelines and is adopted here for expansion to the simulation model of a heat prmp charged $w$ ith a non-azeotropic mixture. 


\section{EVALUATION OF THERMODYNAMIC AND TRANSPORT PROPERTIES OF A NONAZEOTROPIC MIXTURE, R13B1/R152a}

The refrigerant mixture, R13B1/R152a, discussed in this chapter showed promising performance in experiments performed by Cooper [1]. The choice of this mixture for development of this heat pump model does not mean this mixture to be optimum and precludes other mixtures to be more suitable for heat pump application.

\subsection{Evaluation of Thermodynamic Properties}

Evaluation of thermodynamic properties for a non-azeotropic mixture represents an additional complication as compared to single component refrigerants. The employed equation of state has to be able to provide predictions for a broad range of compositions that the circulating mixture may have during heat pump operation. This range of possible compositions is even further expanded by the fact that in the two-phase region, saturated liquid and saturated vapor in equilibrium have different compositions.

Connon and Drew [4] presented application of Redlich-Kwong-Soave (R-K-S) equation to generate R13B1/R152a mixture thermodynamic data. The R-K-S equation successfully generated all vapor thermodynamic information, however, prediction of liquid density of the mixture was based on densities of pure components and application of a mixing rule, since the R-K-S equation did not provide the desired accuracy.

It has been shown by Morrison et al. [5] that it is possible to describe both the 1 iquid and the vapor properties of pure-refrigerants as well as refrigerant mixtures with a single equation of state. They have shown that this approach has a significant advantage over the traditional methods, that of using a vapor equation of state and a library of liquid properties. This advantage is that it will accurately predict property values in the region near or above the critical point of either of the binary components [6]. Because of this advantage, Morrison's equation was adopted in this modeling effort. The basic features of this equation are given below, though for full review, the source report is recommended [5]. The equation of state has the following form:

$$
\frac{P v}{R T}=\frac{1+y+y^{2}-y^{3}}{(1-y)^{3}}-\frac{a}{R T(v+b)}
$$

where $\quad y=\frac{b}{4 v}$

For single component refrigerants, parameters a and $b$ can be determined by second degree polynomials:

$$
\begin{aligned}
& a=a_{0}+a_{1} T+a_{2} T^{2} \\
& b=b_{0}+b_{1} T+b_{2} T^{2}
\end{aligned}
$$


are $a_{0}, a_{1}, a_{2}, b_{0}, b_{1}$, and $b_{2}$ are constants which are based on empirical . ta for a given refrigerant. For mixtures, these parameters may be etermined by:

$$
\begin{aligned}
& a=w^{2} I^{a} I+{ }^{2}{ }^{w} I^{w} I I^{a} I, I I+{ }^{w} I I^{2} a I \\
& b=w^{2} I^{b} I+2{ }^{2} I^{w} I I^{b} I, I I+{ }^{w} I I^{2} b I I
\end{aligned}
$$

Parameters $a_{I},{ }^{a} I I, b_{I}$, and $b_{I I}$ are for pure refrigerants and are obtained by equations $(2)$ and (3).

Remaining parameters are:

$$
\begin{aligned}
& { }^{a_{I, I I}}=\left(1-f_{I, I I}\right)\left(a_{I}{ }^{a} I\right)^{0.5} \\
& b_{I, I I}=\left[\left(b_{I} 1 / 3+b_{I I} 1 / 3\right) / 2\right]^{3} \\
& f_{I, I I}=d-c T \quad \text { (6c) }
\end{aligned}
$$

(c and d constants are obtained from mixture measurements to allow for the interactions of the different molecular species.)

$$
\begin{aligned}
{ }^{w}, w_{I I}= & \text { molar composition of the mixture, fraction of } I \text { and } I I \\
& \text { components, respectively. }
\end{aligned}
$$

Numerical values of the above explained constants are given in Table 1 for refrigerants $R 13 B 1$ and $R 152 a$.

Having defined the mixture thermodynamic state, by specifying temperature, pressure and mixture composition, in order to evaluate other state thermodynamic properties it is essential to determine if the mixture is 1 iquid, vapor, or two-phase. In the latter case, evaluation of the compositions of 1 iquid and vapor in equilibrium is also required.

The starting point to this calculation procedure is pure component analysis to determine saturation pressure for a given temperature. Since equation (1) al so contains molar volume, an iterative process is mandatory for both the 1 iquid and vapor phases. The criteria for verifying that the saturation pressure and molar volume guesses are correct is the equality of the Gibbs free energy and pressure of the two phases. The logic of this procedure is illustrated in the flow diagram in figure 4, and Gibbs free energy is calculated by the equation:

$$
\begin{aligned}
& G=G^{p g}\left(p^{*}, T\right)+R T \ln \frac{R T}{p^{* v}}+(p v-R T)-\frac{a}{b} \ln \frac{v+b}{v} \\
& +\frac{4 R T y}{v-y}+\frac{R T y}{(v-y)^{2}}
\end{aligned}
$$$$
\text { where } G^{p g}=\text { perfect gas Gibbs free energy }
$$$$
p^{*}=\text { equilibrium vapor pressure of the pure } 1 \text { iquid at }
$$$$
\text { temperature } T
$$ 
Table 1. Equation of State Constants for Refrigerants R13B1 and R152a

\begin{tabular}{|c|c|c|}
\hline & R13 B1 & R152a \\
\hline $\begin{array}{l}a_{0} \\
a_{1} \\
a_{2} \\
b_{0} \\
b_{1} \\
b_{2}\end{array}$ & $\begin{array}{l}25.4145 \\
-0.063368 \\
4.140051 \mathrm{E}-5 \\
0.1353977 \\
-1.50409 \mathrm{E}-4 \\
-1.354434 \mathrm{E}-7\end{array}$ & $\begin{array}{l}27.39273 \\
-0.059421169 \\
3.3176956 \mathrm{E}-5 \\
0.1239878 \\
-1.445514 \mathrm{E}-4 \\
-1.9022: 81 \quad \mathrm{E}-8\end{array}$ \\
\hline c & \multicolumn{2}{|c|}{$\begin{array}{c}-2.241 \mathrm{E}-4 \\
0.1466\end{array}$} \\
\hline
\end{tabular}




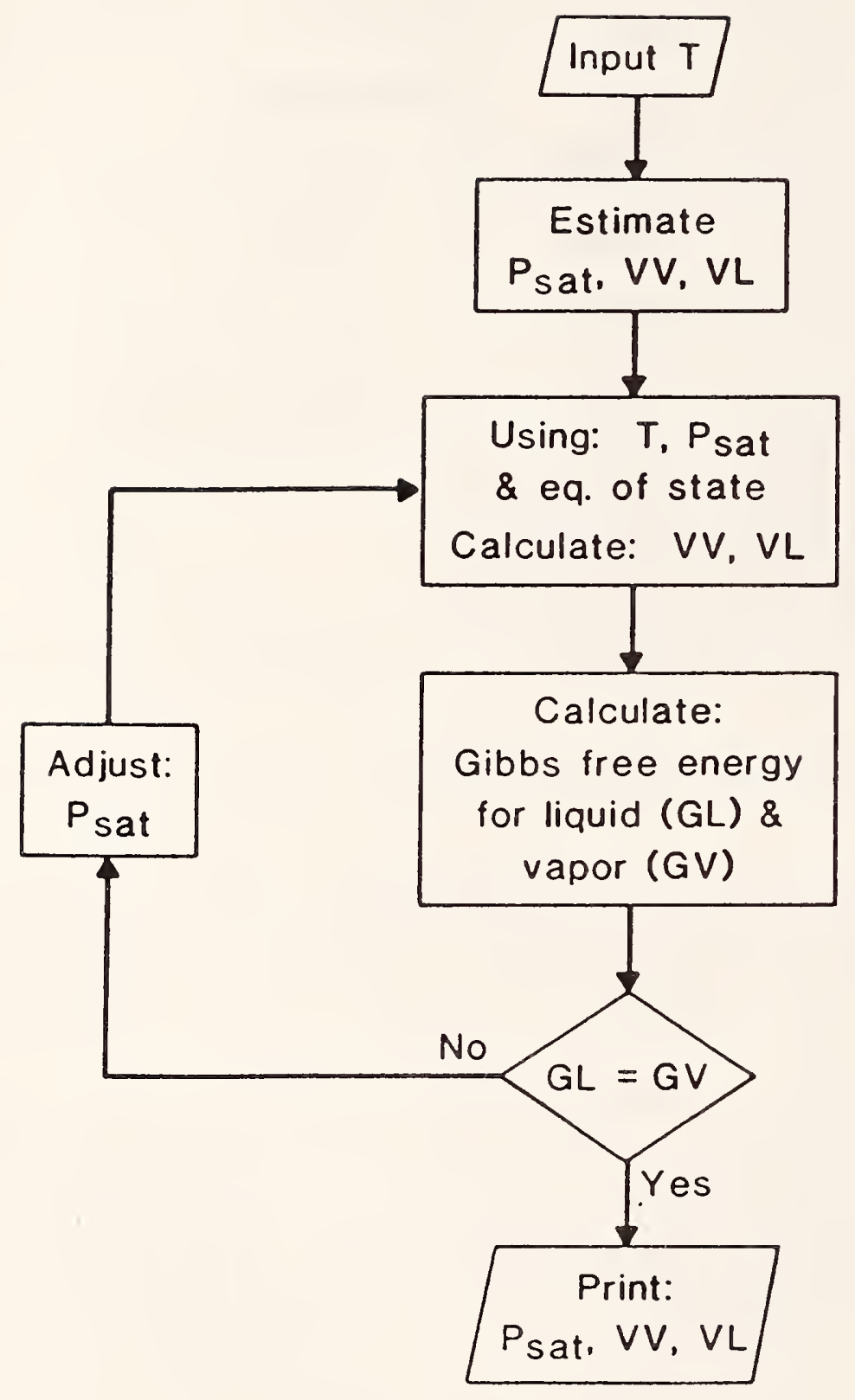

Figure 4. Flow chart for calculating the saturation pressure of single component refrigerant 
The evaluation of compositions of the 1 iquid and vapor phases in equilibrium follows the rule that phase equilibrium in mixtures is defined by equality of pressure, temperature and the chemical potential between phases of each of the species in the mixture. Determination of the equilibrium composition is a rather lengthy procedure and it is not practical to use a full algorithm in a large vapor compression cycle simulation program where property values are requested on the order of hundred of thousands of times. For this reason, equation of state was used to generate sufficient data to create a polynomial spline where saturated 1 iquid and saturated vapor compositions are functions of temperature, pressure and saturation pressures of pure components at mixture temperature. Generation of polynomial splines is discussed in [7] and [8]. The following equations were obtained for R13B1/R152a mixture:

$$
\begin{aligned}
& \mathbf{R P}=\frac{\mathbf{P} 1-\mathbf{P}}{\mathbf{P} 1-\mathbf{P} 2} \\
& \mathrm{~S} 1=12.58741-0.06465226 * \mathrm{TK} 9.56391 * 10^{-5} * \mathrm{TK}^{2} \\
& \mathrm{~S} 2=-48.9799-0.289414 * \mathrm{TK}-4.504945 * 10^{-4} * \mathrm{TK}^{2} \\
& \mathrm{~S} 3=47.7509-0.273993 * \mathrm{TK}+4.14487 * 10^{-4} * \mathrm{TK}^{2} \\
& \mathrm{~S}=\mathrm{S} 1+\mathrm{S} 2 * \mathrm{RP}+\mathrm{S} 3 * \mathrm{RP}^{2} \\
& \mathrm{Z} 1=-0.34065414-3.337532 * 10^{-3} * \mathrm{TK}+9.66115 * 10^{-6} * \mathrm{TK}^{2} \\
& \mathrm{Z} 2=-10.3139754+0.0653856 * \mathrm{TK}-9.9899162 * 10^{-5} * \mathrm{TK}^{2} \\
& \mathrm{Z3}=10.035917-0.05527259 * \mathrm{TK}+7.6821554 * 10^{-5} * \mathrm{TK}^{2} \\
& \mathrm{Z}=1+\mathrm{Z} 1 *(\mathrm{RP}-1)+\mathrm{Z} 2 *\left(\mathrm{RP}^{2}-1\right)+\mathrm{Z} 3 *\left(\mathrm{RP}^{3}-1\right) \\
& \mathrm{XL}=\frac{(1+S) * \mathrm{RP}}{(1+\mathrm{S} * \mathrm{RP})} \\
& \mathbf{X V}=\mathbf{X L} * \mathbf{Z} \\
& \text { where } \quad \text { TK = mixture temperature (K) } \\
& \mathbf{P}=\text { mixture pressure (std atm) } \\
& \text { P1 = saturation pressure of pure R13B1 at temperature TK (std } \\
& \text { a tm) } \\
& \text { P2 = saturation pressure of pure R152a at temperature TK (std } \\
& \text { a tm) } \\
& X L=\text { molar composition of } 1 \text { iquid phase (fraction of R152a) } \\
& \mathrm{XV}=\text { molar composition of vapor phase (fraction R152a) }
\end{aligned}
$$

Knowing saturated 1 iquid and vapor compositions and overall mixture composition, evaluation of mixture quality, $X Q$, is readily available:

$$
X Q=\frac{X L-X M}{X L-X V}
$$

where $\quad X M=$ mixture molar composition (fraction of R152a) 
Phase equilibrium compositions calculated by the spline agree to the third decimal point with compositions obtained using the equation of state.

Equation of state does not provide us directly with information about the bubble point or dew point. However, the bubble point for a mixture of given composition and pressure can be found by iteration using the criteria that, at bubble point the liquid composition (XL) is equal to a known mixture composition (XM). The solution logic is illustrated in the flow diagram of Figure 5 , where the parameters are graphed in the phase diagram of Figure 6 . Similar logic is used for calculating the dew point temperature; in this case, searching for the unique temperature at which the saturated vapor composition equals to composition of the mixture.

Evaluation of molar enthalpy is straight forward:

$$
\begin{aligned}
& h=h^{p g}+\frac{a^{\prime} b T K-a b^{\prime} T K-a b}{b^{2}} 1 n \frac{v+b}{v}+\frac{a b^{\prime} T-a b}{b(v+b)} \\
& +\frac{8 R T V(8 v-b)}{(4 v-b)^{3}}\left(b-b^{\prime} T\right)
\end{aligned}
$$

where prime denotes a temperature derivative.

The molar enthalpy of a perfect gas of a mixture, $h^{p g}$, is the integral of a linear weighting of the heat capacities of the component perfect gases.

$$
h^{p g}=\int_{T_{\text {ref }}}^{T} \quad C p d t=\int_{-40 F}^{T}\left(w_{I} C_{I}+{ }^{T I}{ }_{I I} p_{I I}\right) d T
$$

where $\mathrm{Cp}_{\mathrm{I}}$ and $\mathrm{Cp}_{\mathrm{II}}$ are perfect gas heat capacities of the respective components, functions of temperature, whose coefficients are determined empirical1y.

$$
\begin{aligned}
& \mathrm{C}_{\mathrm{p}_{I}}=\mathrm{C}_{\mathrm{I}}(1)+\mathrm{C}_{\mathrm{I}}(2) \cdot \mathrm{T}+\mathrm{C}_{\mathrm{I}}(3) \mathrm{T}^{2} \\
& \mathrm{Cp}_{\mathrm{II}}=\mathrm{C}_{I I}(1)+\mathrm{C}_{I I}(2) \cdot \mathrm{T}+\mathrm{C}_{I I}(3) \cdot \mathrm{T}^{2}
\end{aligned}
$$

Specific entropy can be calculated by the following expression:

$$
\begin{aligned}
& S_{m}=w_{I} \int_{-40}^{T} \frac{C v_{I}}{T} d T+R 1 n \frac{v}{{ }^{w} \cdot v_{I}^{*}}-\Delta S_{I}^{p g}\left(v_{I}^{*},-40^{\circ} C\right) \\
& +w_{I I} \int_{40}^{T} \frac{C_{v_{I I}}}{T} d T+R 1 n \frac{v}{{ }^{I I} \cdot v_{I I}^{*}}-\Delta S_{I I}^{p g}\left(v_{I I}^{*},-40^{\circ} C\right) \\
& +\Delta S_{m}^{p g}(v, T)
\end{aligned}
$$




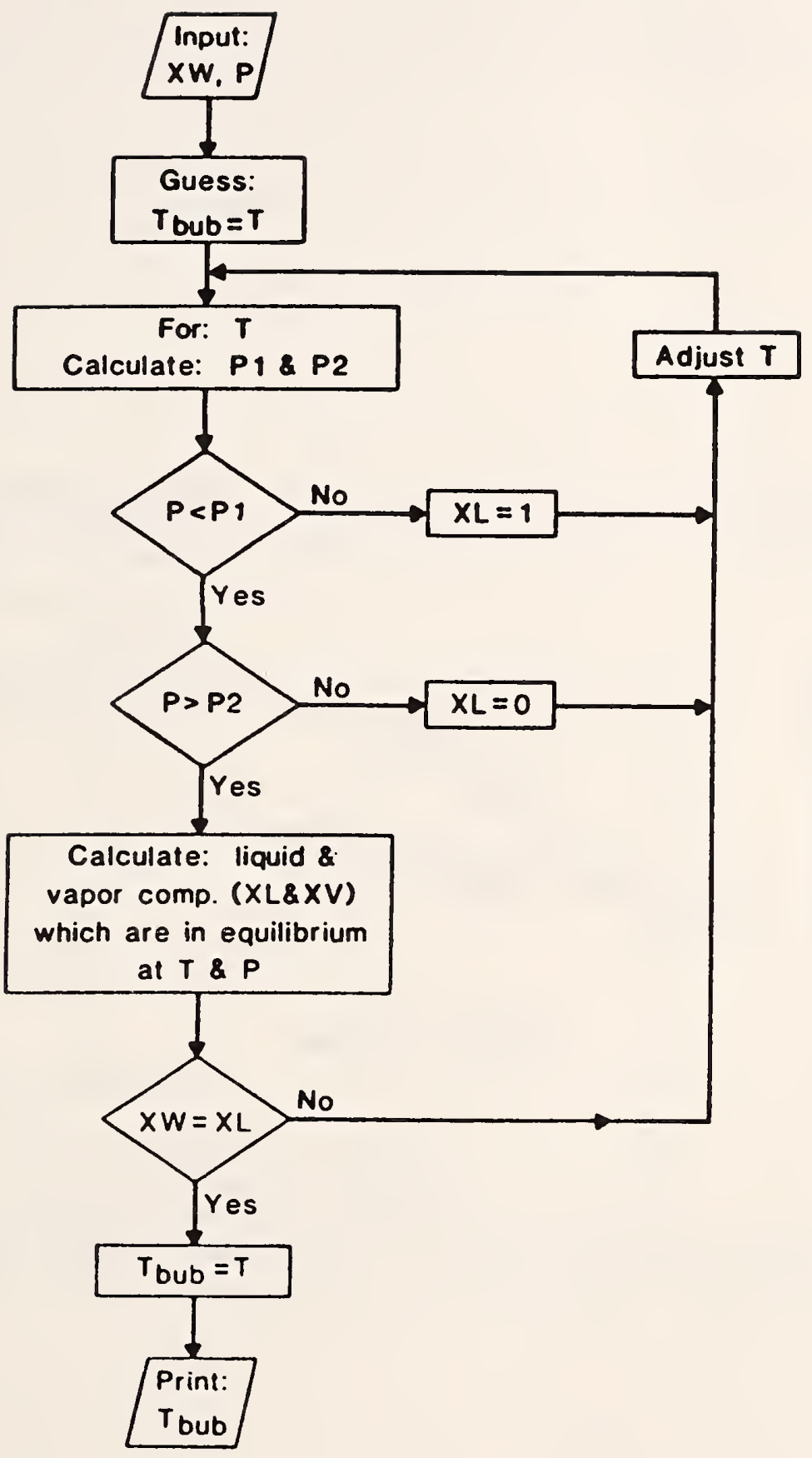

Figure 5. Flow chart for calculating the bubble point temperature of a nonazeotropic binary mixture. 


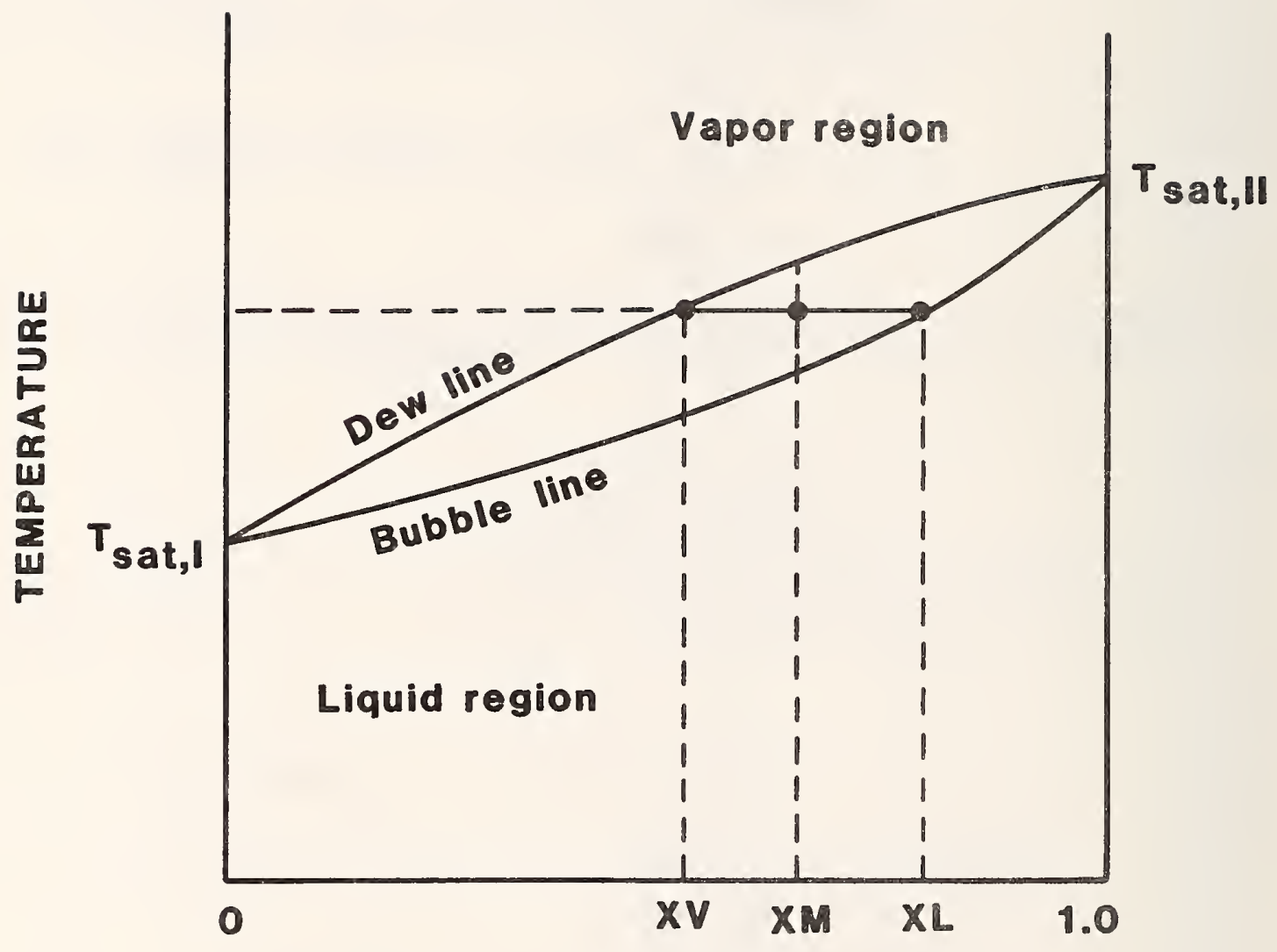

Figure 6. Temperature-composition diagram for a non-azeotropic binary mixture 
where:

$v_{I}^{*}$ and $v_{I I}^{*}$ are reference state $\left(-40^{\circ} \mathrm{C}\right)$ molar volumes of 1 iquid for each of the pure components, $\Delta S S^{P}$ and $\Delta S$ P $P$ are the differences of entropy between the perfect and the real gas having a molar volume of saturated pure component 1 iquid $I$ and $I I$, respectively, at the reference temperature $\left(-40^{\circ} \mathrm{C}\right), \Delta \mathrm{S}_{\mathrm{m}}^{\mathrm{pg}}$ is the difference of entropy between perfect and real mixture of the actual molar volume $v$ and at the actual mixture temperature $T$.

In either case, $\Delta S^{P B}$ can be evaluated by the equation (16) with $v$ put to $v^{*}$ or $v^{*}$, if pure components are considered.

$$
\begin{aligned}
& \Delta S^{p g}=\frac{a^{\prime} b-a b^{\prime}}{b^{\prime}} \ln \frac{v+b}{v}+\frac{a b^{\prime}}{b(v+b)}-\frac{R b(16 v-3 b)}{(4 v-b)^{2}} \\
& -\frac{8 R \operatorname{Rvv}(8 v-b)}{(4 v-b)^{3}}
\end{aligned}
$$

Application of the equation of state requires estimated values of the following pure component properties at saturation as function of temperature; pressure, molar volume of vapor, and molar volume of liquid. Correlations to provide these guesses are given below:

$$
\begin{aligned}
& P=\exp \sum_{I=1}^{3} a(I) \cdot T^{1-I} \\
& v_{V}=\sum_{I=1}^{3} b(I) \cdot T^{I} / P \\
& v_{L}=\sum_{I=1}^{3} c(I) \cdot T^{I-1}
\end{aligned}
$$

where $\quad P=$ saturation pressure

$\mathrm{T}=\mathrm{temperature}$

$v_{V}=$ molar volume of saturated vapor

$v_{L}=$ molar volume of saturated 1 iquid

The equation coefficients $a(I), b(I), c(I)$ for refrigerants $13 B 1$ and $152 a$ are given in Table 2 along with applicable units of refrigerant state property parameters. 
Table 2. Coefficients to be Used in Equations (17), (18) and (19)

\begin{tabular}{c|c|c} 
Coefficient & $R 13 \mathrm{~B} 1$ & $\mathrm{R} 152 \mathrm{a}$ \\
\hline $\mathrm{a}(1)$ & $1.00522804 \mathrm{E}+1$ & $1.06410518 \mathrm{E}+1$ \\
$\mathrm{a}(2)$ & $-2.2045632 \mathrm{E}+3$ & $-2.6428994 \mathrm{E}+3$ \\
$\mathrm{a}(3)$ & $9.6365313 \mathrm{E}+3$ & $4.6087585 \mathrm{E}+2$ \\
$\mathrm{~b}(1)$ & $-5.05060051 \mathrm{E}-2$ & $2.18192958 \mathrm{E}-2$ \\
$\mathrm{~b}(2)$ & $1.11455764 \mathrm{E}-3$ & $5.416820778 \mathrm{E}-4$ \\
$\mathrm{~b}(3)$ & $-2.56392871 \mathrm{E}-6$ & $-1.24731336 \mathrm{E}-6$ \\
$\mathrm{c}(1)$ & $2.749422 \mathrm{E}-1$ & $1.023688715 \mathrm{E}-1$ \\
$\mathrm{c}(2)$ & $1.702569 \mathrm{E}-3$ & $-4.0752759 \mathrm{E}-4$ \\
$\mathrm{c}(3)$ & $3.71008313 \mathrm{E}-6$ & $1.0409447 \mathrm{E}-6$
\end{tabular}

Applicable units of the state paraneter in equations (17), (18) and (19) are

$$
\begin{gathered}
\text { pressure - std. atm } \\
\text { temperature }-{ }^{\circ} \mathrm{K} \\
\text { molar volume }-\mathrm{L} / \mathrm{mol}
\end{gathered}
$$




\subsection{Evaluation of Transport Properties}

In addition to the thermodynamic state equation, algorithms have to be used to calculate thermal conductivity and absolute viscosity of the 1 iquid and vapor. These algorithms consist of curve-fitted correlations for the prediction of properties of pure components, and of some kind of a mixing rule. Reid, Prausnitz and Sherwood [9] gave a comprehensive review of miring rules that could be used depending on mixture component characteristics and component property data availability. Following their recommendation, appropriate correlations were selected and are listed below along with the correlations used for evaluation of the transport properties of pure components. Correlations for property calculations are given here as presented in the referenced documents. However, for use in the miring rule they were converted to common dimensions.

\section{Liquid Thermal Conductivity, k}

(The Filippov Equation is used. The error should not exceed 4\% [9].)

$$
k=k 1+(k 2-k 1) * X W *(0.72 * X W+0.28)
$$

where: $k 1, k 2=$ thermal conductivity of the pure components of $R 13 B 1$ and R152a, respectively.

$$
\begin{array}{lll}
\mathrm{k} 1=0.35-1.5 * 10^{-4} \cdot \mathrm{TF} & & (\mathrm{Btu} / \mathrm{h} \mathrm{ft} \mathrm{F)} \mathrm{[8]} \\
\mathbf{k} 2=0.11650-0.000497 \cdot \mathrm{TC} & (\mathrm{w} / \mathrm{m} \cdot \mathrm{K})[8] \\
\mathrm{TC}=\text { temperature } & \text { (C) } \\
\mathrm{TF}=\text { temperature } & \text { (F) } \\
\mathrm{XW}=\text { weight composition } & & \text { (fraction of R152a) }
\end{array}
$$

\section{Vapor Thermal Conductivity, k}

(The Wassiljewa Equation is used with the Lindsay and Bromley Modification. The error rarely exceeds 5\% [9].)

$$
k=\frac{(1-X M) \cdot k 1}{(1-X M) \cdot A_{11}+X M \cdot A_{12}}+\frac{X M \cdot k 2}{(1-X M) \cdot A_{21}+X M \cdot A_{22}}
$$

where: $k 1, k 2$ = thermal conductivity of the pure components of $R 13 B 1$ and R152a, respectively.

$$
\begin{aligned}
\mathrm{k} 1 & =8.2982 * 10^{-3}-5.1971 * 10^{-5} \cdot \mathrm{TK}+1.8413 * 10^{-7} \mathrm{TK}^{2} \\
\mathrm{k} 2 & \left.=-8.357 * 10^{-3}\right]+[8] \\
{[\mathrm{w} / \mathrm{m} \cdot \mathrm{K}][9] } & {\left[9.3 * 10^{-5} \cdot \mathrm{TK}+4.257 * 10^{-8} * \mathrm{TK}^{2}\right.}
\end{aligned}
$$$$
A_{i j}=0.25\left[1+\left(\frac{\mu_{i}}{\mu_{j}}\left(\frac{M_{j}}{M_{i}}\right)^{0.75} \frac{T K+S_{i}}{T K+S_{j}}\right)^{0.5}\right]^{2} \frac{T K+S_{i j}}{T K+S_{i}}
$$

$\mu_{1}, \mu_{2}=$ absolute viscosity of the pure components, R13B1 and R152a, respectively.

$M_{1}, M_{2}=$ molecular weight of the pure components, R13B1 and R152a, respectively.

TK = absolute temperature $(K)$ 


$$
\begin{aligned}
& \mathrm{S}_{\mathrm{i}}=1.5 \cdot \mathrm{T}_{\mathrm{b}} \\
& \mathrm{T}_{\mathrm{bi}}=\text { normal boiling point of ' } i \text { ' component (K) } \\
& s_{i j}=0.73 \cdot\left(s_{i} \cdot s_{2}\right)^{0.5} \\
& \text { XM = composition (mole fraction of R152a) }
\end{aligned}
$$

\section{Liguid Absolute Viscositz, 토}

(The Lobe correlation is used. The prediction error should be less than $15 \%$, [9].)

$$
\begin{aligned}
& \mu=\frac{1}{\nabla_{m}}\left(\phi_{1} \cdot \gamma_{1} e^{\phi_{2} a_{2}^{*}}+\phi_{2} \gamma_{2} e^{\phi_{1} a_{1}^{*}}\right) \\
& v_{\text {m }}=\text { mixture specific volume } \\
& D_{1}, D_{2}=\text { volume fraction of the pure components, R13B1 and R152a, } \\
& \text { respectively. } \\
& \gamma_{1}, \gamma_{2}=\text { kinematic viscosity of the pure components, R13B1 and R152a, } \\
& \text { respectively. (obtained, as shown below, through evaluation of } \\
& \text { the absolute yiscosities, } \left.\mu_{1} \text { and } \mu_{2}\right) \\
& \begin{array}{l}
\mu_{1}=\left(\mathrm{e}^{-4.22529+710.843 / \mathrm{TK}}\right)=10^{-3} \mu_{2}\left(\mathrm{~N} \cdot \mathrm{s} / \mathrm{m}^{2}\right)[10] \\
\mu_{2}=\left(\mathrm{e}^{-4.28224}+753.013 / \mathrm{TK}\right) \cdot 10^{-3} \quad\left(\mathrm{~N} \cdot \mathrm{s} / \mathrm{m}^{2}\right)[10]
\end{array} \\
& a_{1}^{*}=1.7 \ln \frac{\gamma_{2}}{\gamma_{1}} \\
& a_{2}^{*}=0.27 \ln \frac{\gamma_{2}}{\gamma_{1}}+\left(1.3 * 1 \mathrm{n} \frac{\gamma_{2}}{\gamma_{1}}\right) \\
& \text { TK = temperature (K) }
\end{aligned}
$$

\section{Vapor Absolute Viscosity, $\mu$}

(The equation derived from the vigorous kinetic theory of Chapman-Ensbog is used. The error seldom exceeds 4\% [9].)

$$
\mu=\frac{(1-X M) \mu_{1}}{(1-X M)+X M \cdot D_{12}}+\frac{X M \mu_{2}}{X M+(1-X M) D_{21}}
$$

where

$$
D_{12}=\frac{\left[1+\left(\frac{\mu_{1}}{\mu_{2}}\right)^{0.5}\left(\frac{M_{2}}{M_{1}}\right)^{0.25}\right]^{2}}{\left[8\left[1+\frac{M_{1}}{M_{2}}\right]\right]^{0.5}}
$$




$$
D_{21}=D_{12} \cdot \frac{\mu_{2}}{\mu_{1}} \frac{M_{1}}{M_{2}}
$$

$\mathrm{XM}=$ composition (mole fraction of R152a)

$\mu_{1}, \mu_{2}=$ absolute viscosity of the pure components, R13B1 and R152a, respectively.

$$
\begin{aligned}
\mu_{1}= & 0.67329 * 10^{-3}+7.60593+10^{-6} * \mathrm{TK}-2.81108 * 10^{-8} \mathrm{TK}^{2}+ \\
& 3.47410 * 10^{-11} \mathrm{TK}^{3} \quad\left(\mathrm{~N} \cdot \mathrm{s} / \mathrm{m}^{2}\right)[9] \\
\mu_{2}= & 3.205 * 10^{-5} * \frac{\mathrm{k}_{2} \mathrm{M}_{2}}{\mathrm{FE}} \quad\left(\mathrm{N} \cdot \mathrm{s} / \mathrm{m}^{2}\right)[11]
\end{aligned}
$$

$k 2$ = thermal conductivity of pure R152a vapor ( $/ \mathrm{m} \cdot \mathrm{K})$

$M_{1}, M_{2}=$ molecular weight of the pure components, R13B1 and $R 152 a$, respectively.

$$
\mathrm{FE}=0.115+0.354 \frac{\mathrm{Cp}_{\mathrm{PI}}}{\mathrm{R}}
$$

$\mathrm{Cp}_{\text {II }}=$ specific heat at constant pressure of R152a vapor.

$\mathrm{CP}_{\mathrm{II}}=-7.33704+0.093438 \mathrm{TK}-3.61094 \cdot 10^{-4} \mathrm{TK}^{2}+4.80449 \cdot 10^{-7} \cdot \mathrm{TK}^{3}$

$$
\left(\frac{\mathbf{k J}}{\mathrm{kg} \mathrm{K}}\right)
$$




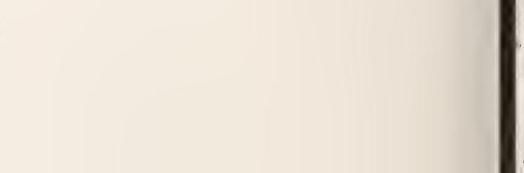




\section{OPERATION OF A HEAT PUMP WITH A CONSTANT FLOW AREA EXPANSION DEVICE}

In a heat pump with a capillary tube, vapor superheat at the compressor inlet will vary with changing operating conditions. As a matter of fact, no single refrigerant parameter stays constant when indoor or outdoor air conditions are altered and the system seeks equilibrium under new conditions. To understand the phenomena that cause refrigerant state changes, consider a heat pump working in the cooling mode at constant indoor air conditions subjected to a step increase of the outdoor temperature. For the purpose of the analysis, it is assumed that the overall heat transfer coefficient for both heat exchangers does not vary. It is also assumed, as a first approximation, that the latent heat of evaporation, $i_{f}$, is constant under the conditions discussed.

A step increase in the outdoor air temperature first affects the performance of the condenser. Since the condenser environmental temperature increases, the mean temperature difference between the air and the refrigerant decreases. Less heat is rejected by the refrigerant to the air and a smaller enthalpy change is realized in the coil. Consecutively, higher enthalpy, less subcooled 1 iquid refrigerant enters the capillary tube. The capillary tube is sensitive to the amount of subcooling and with less subcooling the mass flow rate through the capillary tube decreases. Since the compressor capacity stays unchanged, pressure builds up in the condenser. Thus point 11 on the p - h diagram (figure 3 ) moves in the direction of higher enthalpy and higher pressure.

The environment of the evaporator was not altered. However, refrigerant parameters in the evaporator change in response to the change of refrigerant state at location 11 as well as to the change in refrigerant mass flow rate. For pressure drop considerations, as a first spproximation, the 1 iquid 1 ine, the capillary tube, and the evaporator are viewed as one tube, in which a given flow experiences a certain pressure drop. As pressure of the refrigerant at location 11 increases, it pulls up the refrigerant pressure in the evaporator. Conflicting phenomena affect the change of refrigerant enthalpy at point 1. Increased enthalpy at point 11 and reduced refrigerant mass flow rate work against the impact of the smaller temperature difference between the air and the refrigerant causing refrigerant enthalpy to slightly decrease. The significance of the change of the refrigerant state at location 1 is a move towards smaller vapor specific volume. Since the compressor, as a first approximation, is a constant intake volume pump, increase in gas density results in a higher mass flow rate.

The indicated phenomena will balance themselves further until the refrigerant in the key locations of the system acquires the thermodynamic states that satisfy simultaneously the equilibrium of all the heat pump components. Since under steady-state operation, the refrigerant mass flow rate through the compressor and the capillary tube have to match, the pressure in the condenser will rise to an appropriate leve1. Again, higher pressure in the condenser implies some increase of pressure (and saturation temperature) in the evaporator. The smaller temperature difference between the indoor air and the refrigerant, and higher refrigerant mass flow rate result in a smaller refrigerant enthalpy increase in the evaporator. The refrigerant state at point 1 with respect to point 11 is determined by this enthalpy change and the appropriate pressure drop. Finally, when steady-state conditions are reached the following changes in refrigerant thermodynamic states can be noted: 
higher refrigerant pressure in the condenser, higher refrigerant pressure in the evaporator, less superheat at the evaporator exit, and less refrigerant 1 iquid subcooling at the condenser exit. The change of saturation temperature in the condenser corresponds approximately to the change in the outdoor air temperature. The change of pressure in the evaporator is a fraction (approximately 15 percent) of the pressure change in the condenser. Though the refrigerant is circulated in the system at a higher mass flow rate, the capacity of the heat pump is decreased due to the smaller refrigerant enthalpy change in the evaporator. The efficiency also drops since, in addition to a smaller cooling effect, the energy input to the compression process increases due to the higher refrigerant mas flow rate and the higher compression rate. A decrease of the outdoor air temperature would result in the opposite trends.

The change of refrigerant parameters is illustrated in figure 7, where results of three tests of a heat pump charged with refrigerant 22 are plotted on the pressure-enthalpy diagram (the plot is based on measured condenser and evaporator pressures and temperatures only). In these tests, the indoor air conditions were held constant while the outdoor air dry bulb temperature was changed producing modifications of the thermodynamic cycle.

For the heating mode operation. refrigerant flow is reversed by the action of the four-way valve. The flow direction of the refrigerant in the system is the opposite to that in the cooling mode, with the exception of the compressor. The indoor coil becomes the condenser while the outdoor coil serves as the evaporator. It is worthwile to note the effect this change has on the system. Since pressures in heat pump heat exchangers are functions of environment temperatures, the pressure in the evaporator is now much 1 ower than during cooling operation. Consequenty, the density of the refrigerant at the evaporator exit is smaller and less refrigeant is being pumped by the compressor. Al so note that an indoor coil is usually much smaller than an outdoor coil. The need for dehumidification is the primary reason. The indoor coil working as a condenser cannot condense and hold as much refrigerant as the outdoor coil during cooling operation. In heat pumps equipped with a capillary tube the excess of 1 iquid refrigerant is stored in an accumulator.

A schematic of an accumulator is given in figure 8. The accumulator is a closed container with two tubes. The longer bent tube has a small diameter hole on the bottom side, and is connected to the compressor. The other connects the tank with the evaporator exit.

If superheated vapor leaves the evaporator, only vapor is contained in the accumulator and no special function is fulfilled by the accumulator. If wet vapor enters the accumulator, 1 iquid droplets accumulate on the bottom of the tank, while saturated vapor enters the tube leading towards the compressor. Some 1 iquid refrigerant enters the vapor stream through the hole in the tube, driven by the static pressure difference in the liquid-vapor stream interface. It should be noted that during steady-state operation with 1 iquid in the accumulator, qualities of the vapor entering and leaving the accumulator must be equa1.

In a heat pump charged with a non-azeotropic mixture, incomplete evaporation in the evaporator and collection of 1 iquid refrigerant in the accumulator affect the actual mixture circulating composition. As shown on the temperature-composition diagram (figure 6) the liquid and vapor phases in 


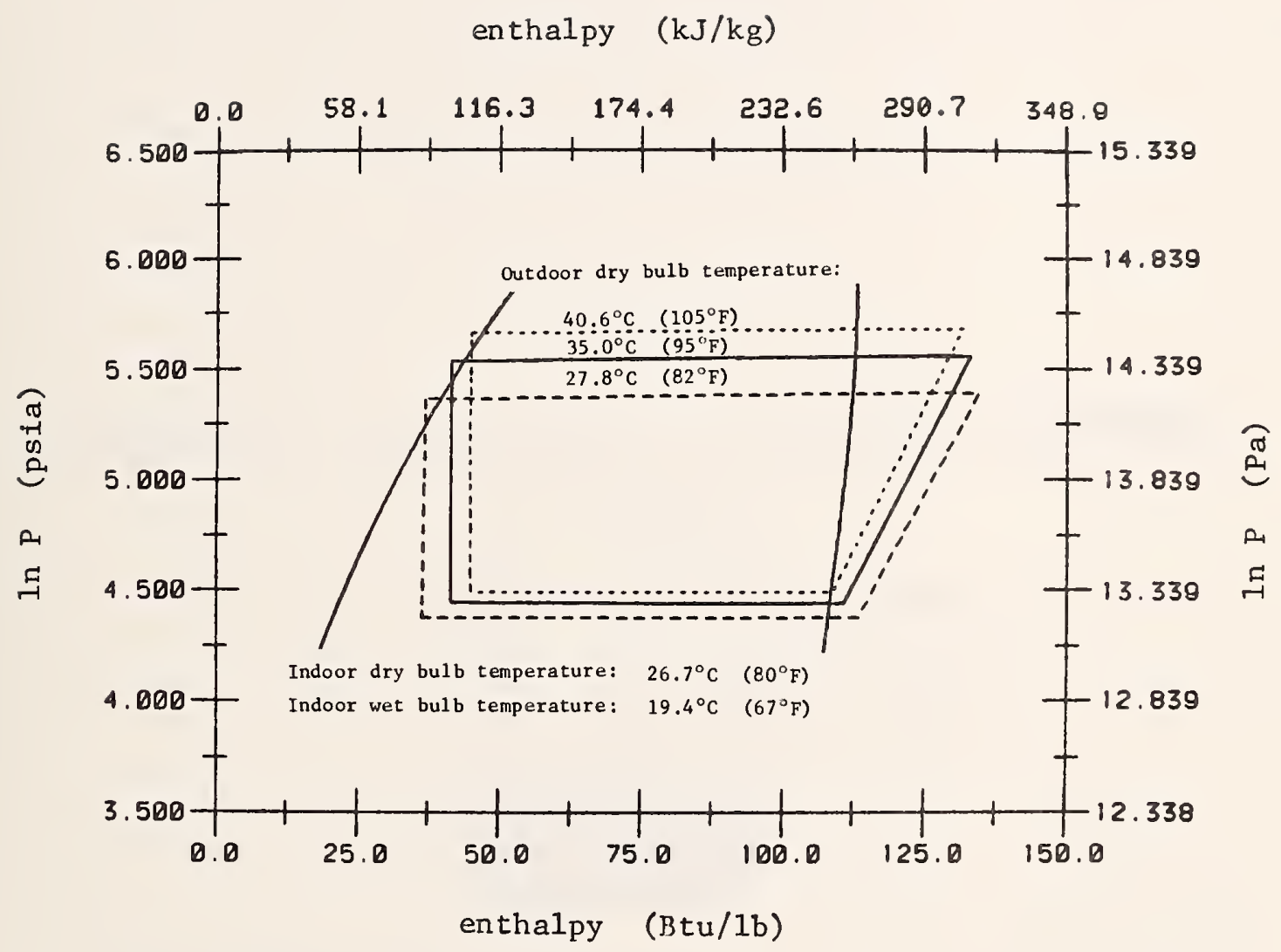

Figure 7. Simplified thermodynamic cycles realized by a heat pump charged with refrigerant 22 in the cooling mode at different outdoor temperatures. 


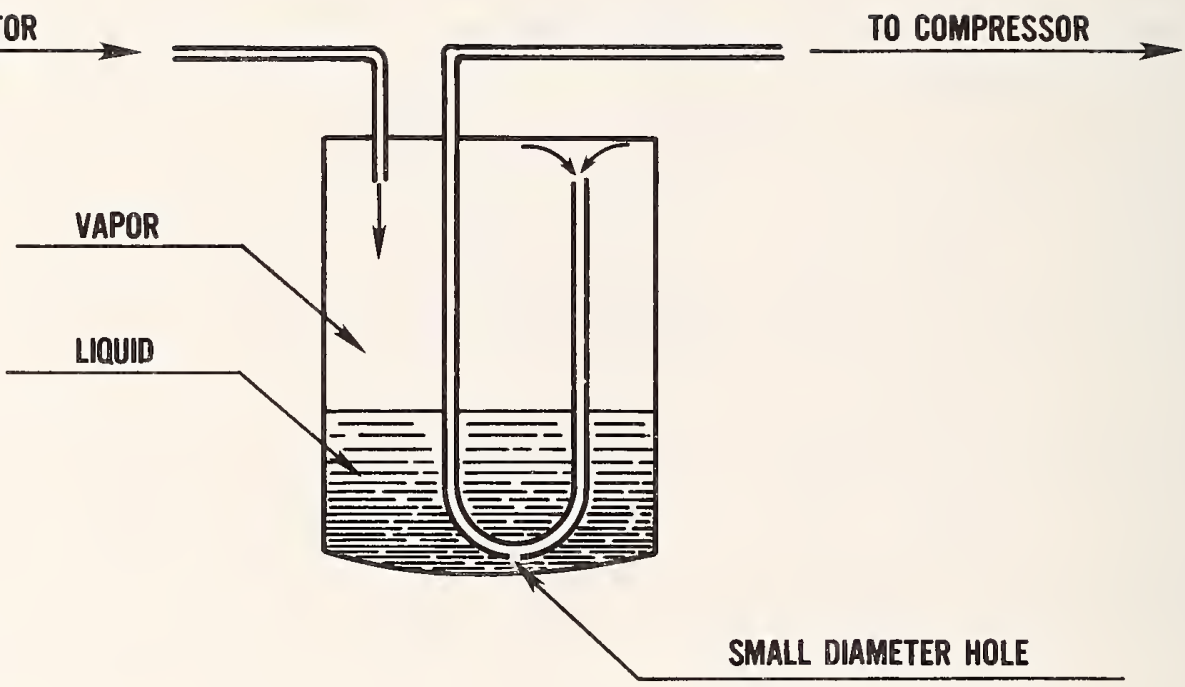

Figure 8. Schematic of an accumator. 
equilibrium at a given temperature and pressure have different compositions, the 1 iquid being richer in less volatile component. Consequently, with the first liquid drop stored in the accumulator, circulating mizture composition becomes richer in the lower boiling point refrigerant. This composition shift increases with the increase of accumulated Iiquid. 


\section{MODELING OF A HEAT PUMP}

\subsection{The Logic of a Model of a Heat Pump Charged with a Non-Azeotropic Refrigerant Mixture}

During heat pump operation, refrigerant parameters at any location are established at a level which satisfies the respective components of the system. There is a one-to-one relationship between working meding parameters and the operating conditions, i.e., for given outdoor and indoor air conditions, there is just one set of refrigerant parameters at any location within the system which satisfy steady state operation. This mique set of refrigerant parameters has to be determined by the heat pump simulation program in an iterative process.

In order to set up a heat pump iteration process (Fig. 9), balances taking place curing steady state operation have to be recognized. From the fact that a thermodynamic cycle is a closed loop, an analysis of a heat pump cycle on a pressure-enthalpy diagram allows the statement of two balances:

- Enthal py Balance

The net refrigerant enthalpy change in all components of the system has to equal zero.

- Pressure Balance

This balance implies that the increase of refrigerant pressure during the compression process has to be equal to the total pressure drop during the other processes forming the cycle. As pressure drop and mass flow rate are strictly related, this balance may be restated that pressure drop through each component has to be such that the mass flow rate in each component is the same.

The enthalpy balance and pressure balance can be supplemented by two additional balances resulting from the 1 aw of mass conservation, as expressed by the following equations:

$$
\begin{aligned}
& \frac{D}{D t} \int_{\Psi} \rho_{\mathrm{m}} \mathrm{d} q=0 \\
& \frac{D}{D t} \int_{\forall} \rho_{\mathrm{i}} \mathrm{dq}=0
\end{aligned}
$$

where

$$
\begin{aligned}
& \rho_{m}=\text { mean density of the mixture } \\
& \rho_{\frac{1}{1}}=\text { mean density of ' } i \text { ' compone } \\
& \frac{1}{\psi}=\text { system internal volume }
\end{aligned}
$$




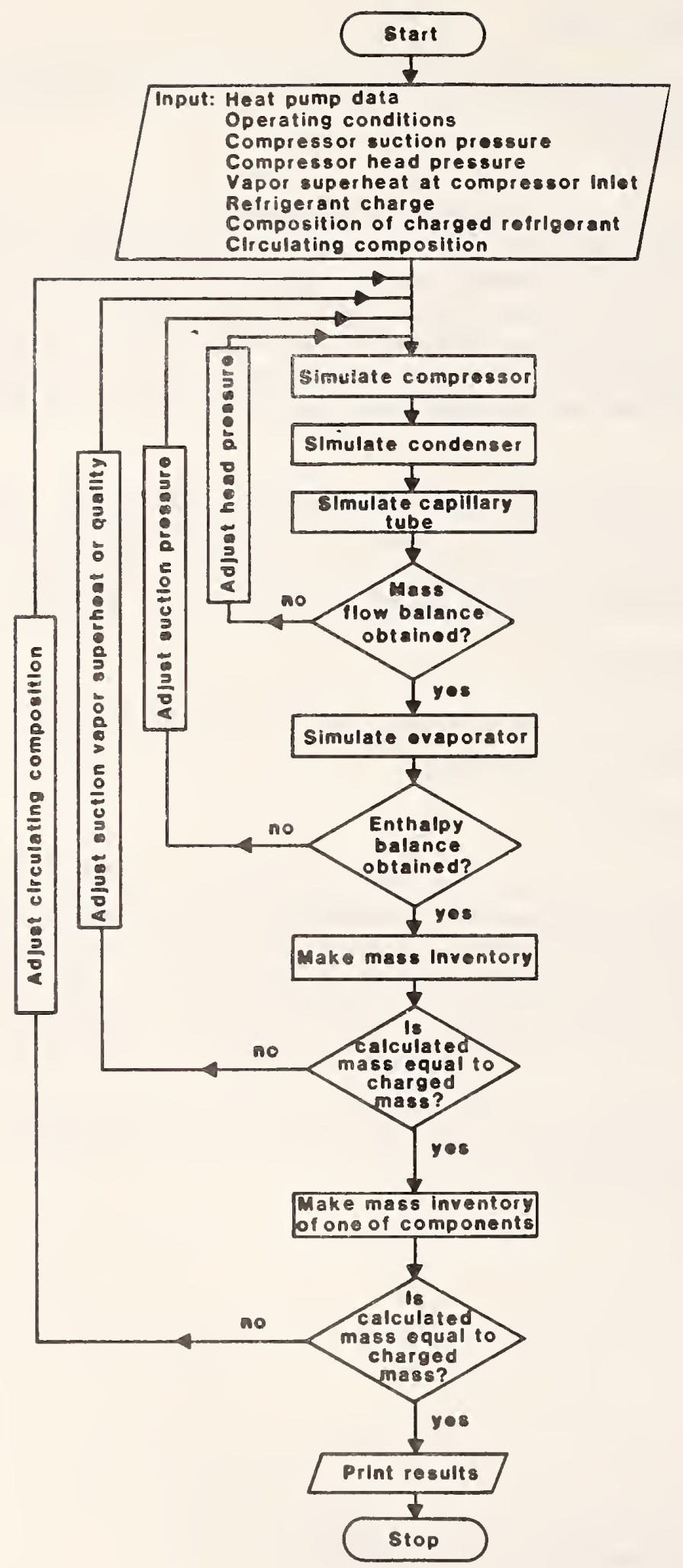

Figure 9. Overall logic of a program simulating performance of a heat pump employing a constant flow area expansion device and charged with a nox-azeotropic binary refrigerant mirture. 
Equation (24) implies, that the amount of working medium in the system is the same at all times, for any operating conditions. Equation (25) states the same conservation principle for each of the mixture components. Though for a binary mixture the 1 ast equation provides us with two balances, we can actually use only one of them with conjunction with equation (24).

It should be noted that the first three balances are sufficient to set up the logic of a simulation program of a heat pump charged with a single component refrigerant. These three balances were utilized in [3] allowing for iteration of the refrigerant thermodynamic states at key system locations without imposed restrictions on the refrigerant state anywhere in the system. In a heat pump charged with a non-azeotropic binary mixture, the circulating composition may change if some 1 iquid refrigerant is collected in the accumulator. The actual composition of the circulating mixture has to be evaluated during simulation. Since this change of composition represents an additional degree of freedom, an additional equation (equation (25)) is used in the solution logic.

The logic of the program is based on these four balances. It provides means for calculation of vapor superheat (quality) at the compressor can inlet and allows for heat pump simulation at the imposed operating conditions. Explanation of the logic, for sake of clarity, is done considering only four main components of a heat pump, i.e., a compressor, a condenser, a capillary tube, and an evaporator.

The required input consists of outdoor and indoor conditions, heat pamp data and composition of the charged and circulating mixture. The simulation process begins with estimated refrigerant pressure and vapor superheat at the compressor inlet, and the compressor discharge pressure. Using these data, the compressor performance is simulated yielding the refrigerant mass flow rate. Next, the condenser and the capillary tube are simulated and a mass flow balance is sought by comparing the refrigerant mass flow rates through the compressor and capillary tube. If the compared mass flows are not equal, simulation of the compresor, the condenser, and the capillary tube is redone with an unchanged refrigerant state at the compressor can inlet and a modified estimate of compressor discharge pressure. Increasing this pressure reduces refrigerant mass flow rate through the compressor. Then the condenser works with this smaller mass flow rate and at a higher saturation temperature. Consequently, refrigerant reaching the expansion device inlet is at a higher pressure and has more subcooling. Both factors promote greater mass flow rate through the capillary tube. Thus, increase in the discharge pressure has a clear and opposite impact on mass flow rates through the compressor and through the capillary tube and the appropriate discharge pressure can be found for which mass flow balance exists.

Once mass flow balance is reached, simulation of the evaporator is performed with the known refrigerant state at the evaporator exit and the refrigerant mass flow rate. Since the thermodynamic process in a capillary tube may be viewed as adiabatic, refrigerant enthalpy at the evaporator inlet should be equal to the enthalpy at the condenser outlet. If these enthalpies are not equal (energy balance is not reached), a new calculation starts from the beginning with a modified estimate of the refrigerant pressure at the compressor can inlet. From the condenser operation point of view, a change in compressor suction pressure induces a change in the refrigerant mass flow rate 
and a modification of the condenser saturation temperature resulting from the system flow balance search. These two changes have opposite effects on refrigerant enthalpy at the condenser exit, leaving it only slightly altered. On the other hand, the same change of compressor suction pressure has a strong effect on the refrigerant enthalpy at the evaporator inlet due to a change in the evaporator saturation temperature and refrigerant mass flow rate, both working to change the enthalpy in the same direction. Thus an appropriate suction pressure at the compressor inlet can always be found when an energy balance exists.

Once energy and pressure balances are established, two out of four refrigerant parameters estimated at the outset are determined. However, these two parameters, compressor suction and discharge pressures, were obtained for imposed refrigerant superheat (quality) at compressor can inlet and assuned circulating composition which still have to be verified. For refrigerant superheat (quality) verification, the refrigerant mass inventory is made. It is based on refrigerant states in the system which are found to satisfy energy and pressure balances with assumed vapor superheat at the compressor can inlet. The amount of refrigerant obtained from mass inventory calculations is compared to the actual refrigerant mass input (known design parameter). If the amount of refrigerant calculated is smaller than refrigerant input into the heat pump, the superheat (quality) estimate has to be decreased and all calculations have to be repeated from the outset.

Once, in addition to satisfied pressure and energy balances, the refrigerant superheat (quality) at the compressor can inlet is verified by means of mixture mass inventory, the ultimate verification of iterated refrigerant states has to be done by checking if the circulating mixture composition for which al1 results were obtained is in fact the actual circulation composition. This is done by performing a mass inventory of one of the mixture components. It should be mentioned that a composition shift of circulating mixture can occur only as a result of accumulation somewhere in the system of some of the mixture of composition different from the original, i.e., accumulation of 1 iquid in the accumulator. If such accumulation does not take place (refrigerant entering the compressor is a superheated vapor) the circulation composition equals the original composition of the charged refrigerant. If refrigerant entering the compressor has quality less than 1, the program has to proceed with mass inventory for one mixture component. This mass inventory is based on refrigerant states in the system which are found to satisfy enthalpy and pressure balances, and for which the total mass of refrigerant in the system is conserved. If the amount of calculated mixture component is not equal to the original amount charged into the system, the estimate of composition of the circulating refrigerant has to be adjusted and all calculations have to be repeated from the outset. The solution logic described here is presented graphically in figure 9. The actual implementation of thi in the main program of the model is presented in Appendix D. 


\subsection{Modeling of a Reciprocating, Hermetic Compressor}

\subsubsection{Compressor Operation}

The compressor is mechanically the most complex component of a heat pump. A reciprocating, hermetic compressor is most commonly used in heat pump systems. A schematic of this type of compressor is shown in figure 10. The compressor consists of a shell containing an electric motor, a cylinder/piston assembly with valves and manifolds and tubes. The electric motor is coupled to the compressor eccentric. Lubricating oil is collected on the bottom of the can and is in free contact with the refrigerant.

Flow of the refrigerant is from location 3 to location 8 , as marked in figure 10. Low pressure refrigerant enters the compressor can and is directed towards the electric motor to cool motor windings. The enthalpy of the refrigerant changes due to this and other heat transfer with other surrounding surfaces, namely, the compressor can, manifolds, the discharge tube and the cylinder body. Then the refrigerant at state 4 undergoes the process in the cylinder, which in case of positive displacement compressors consists of: expansion through the suction valve and mixing the residual gas, compression, expansion through the discharge valve, and re-expansion of residual gas. The compressed, high temperature and high pressure refrigerant vapor leaves the compressor through the discharge manifold and discharge line after giving up some heat to the 1 ow pressure refrigerant at state 4 .

The numbers marked in figure 10 identify key compressor locations and the corresponding refrigerant states. Even during steady state operation refrigerant parameters in the compressor can are not steady, since pressure changes in a series of rapid pulses. This pulsation is initiated by the periodic compression and suction processes and valve behavior. Because a certain pressure difference is needed for valve opening, the pressure in the cylinder at the end of compression is considerably above the discharge manifold pressure. Similarly, pressure during intake falls below suction manifold pressure. Particularly strong pressure peaks are observed in case of spring equipped valves. These pressure differences vary from compressor to compressor because of valve and manifold design, but also vary for the same compressor with compressor speed, compression pressure ratio, refrigerant, and mass flow rate.

Since processes taking place in a compressor are dymamic in nature, the most correct way of modeling a compressor would require dynamic simulation of valve motion, cylinder-manifold pressure interaction, and cylinder heat tronsfer. Such models ezist and are described in [12], [13], and [14]. However, these models usually require some very detailed experimental data or design information, not necessarily readily available for the prospective user of a heat pump simulation program. That is why a relatively simplot approach to corpressor modeling was taken in this study.

\subsubsection{Theory and Governing Relations}

Several assunptions are made for model formulation. The basic assumption is that the highly dynamic process in the compressor results in steady gas parameters throughout the can. The pressure and temperature in the cylinder and in suction and discharge manifolds are assumed to have constant values. 


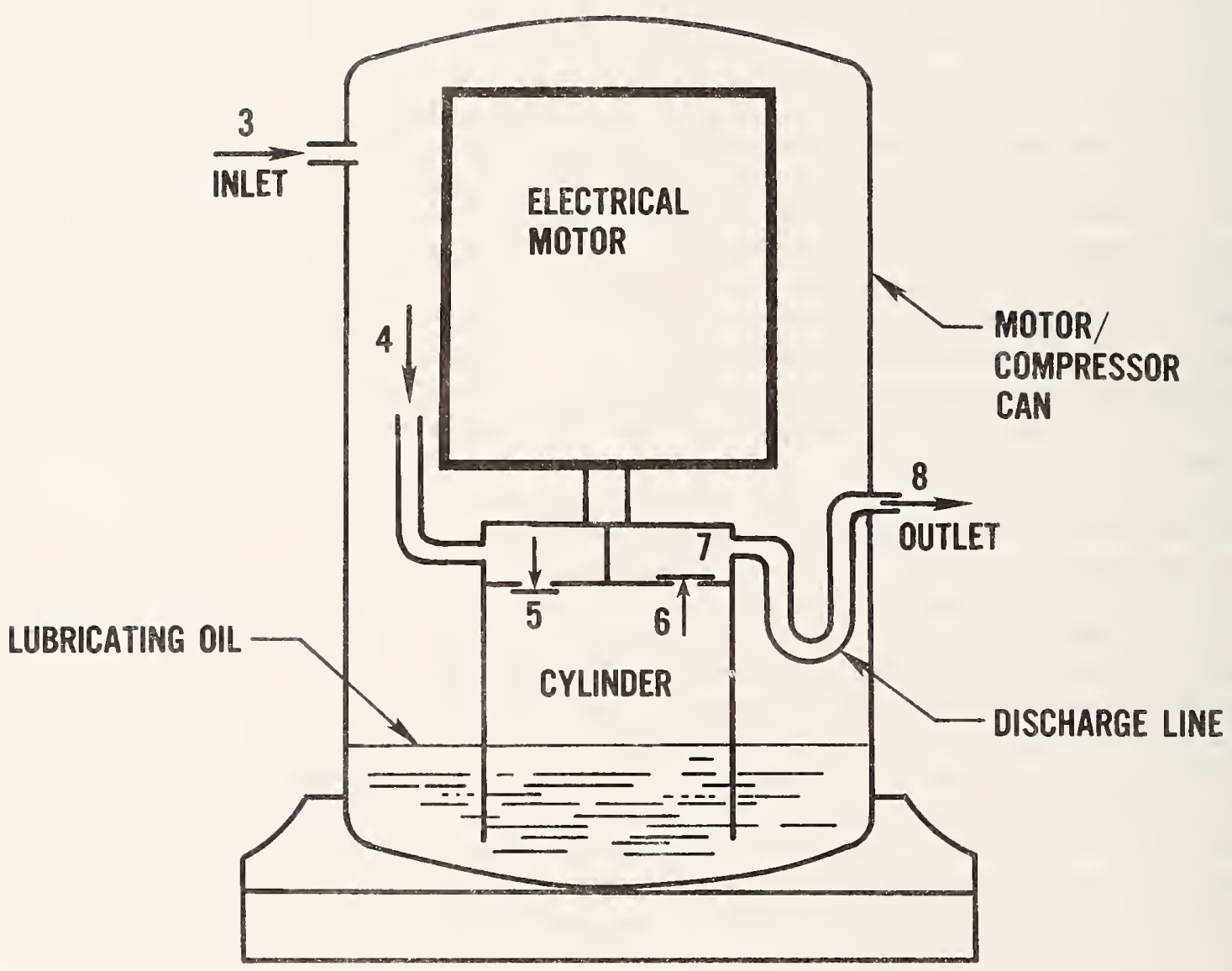

Note: Numbers 5 and 6 situated in the compressor correspond to the refrigerant state before and after the compression process.

Figure 10. Schematic of a hermetic compressor. 
The refrigerant is considered to have uniform thermodynamic properties throughout the space assigned to the particular location. Refrigerant flow through the compressor is assumed to be one-dimensional, so one-dimensional steady flow equations can be used. Though the model does not simulate valve dynamic behavior, it allows for valve representation by a steady difference between manifold and cylinder pressures.

In order to derive equations governing compressor balances, the thermodynamic irreversibilities taking place in the hermetically sealed compressor have to be identified. These losses can be put into four categories:

1. Those associated with incomplete conversion of electric energy into a mechanical energy available for vapor compression.

2. Those associated with the non-isentropic conversion process inside the cylinder.

3. Those due to heat transfer at the different locations.

4. Those due to pressure drop at the different 1ocations.

A11 of these losses contribute to the overall compressor efficiency and have to be considered in the modeling effort.

\section{Conversion of Electrica 1 to Mechanical Energy}

Electric energy is supplied to an electric motor to be converted into mechanical energy. This conversion has losses due to windage, friction, winding resistance, and hysteresis, which are accounted for by a motor efficiency, $\eta_{e}$. By definition

$$
\eta_{e}=\frac{W_{e}}{E}
$$

where $\quad E=$ electric power input

$W_{e}=$ mechanical power output

Electric motor efficiency, $\eta_{e}$, depends on 1 oad and is customarily given as a function of the fraction of actual mechanical power output to the maximum power output. In heat pumps with power requirements below $5 \mathrm{hp}$, single phase electric motors are usually used. They are permanent split-capacitor or capacitor-start capacitor-run types. A typical efficiency versus $10 a d$ curve for a permanent split-capacitor 2 pole electric motor is shown in figure 11 [15].

The electric motor is coupled to the compressor eccentric. Moving compressor parts experience friction and a power loss accounted for by the compressor mechanical efficiency, $\eta_{m}$. By definition

$$
\eta_{m}=\frac{w_{c}}{w_{e}}
$$




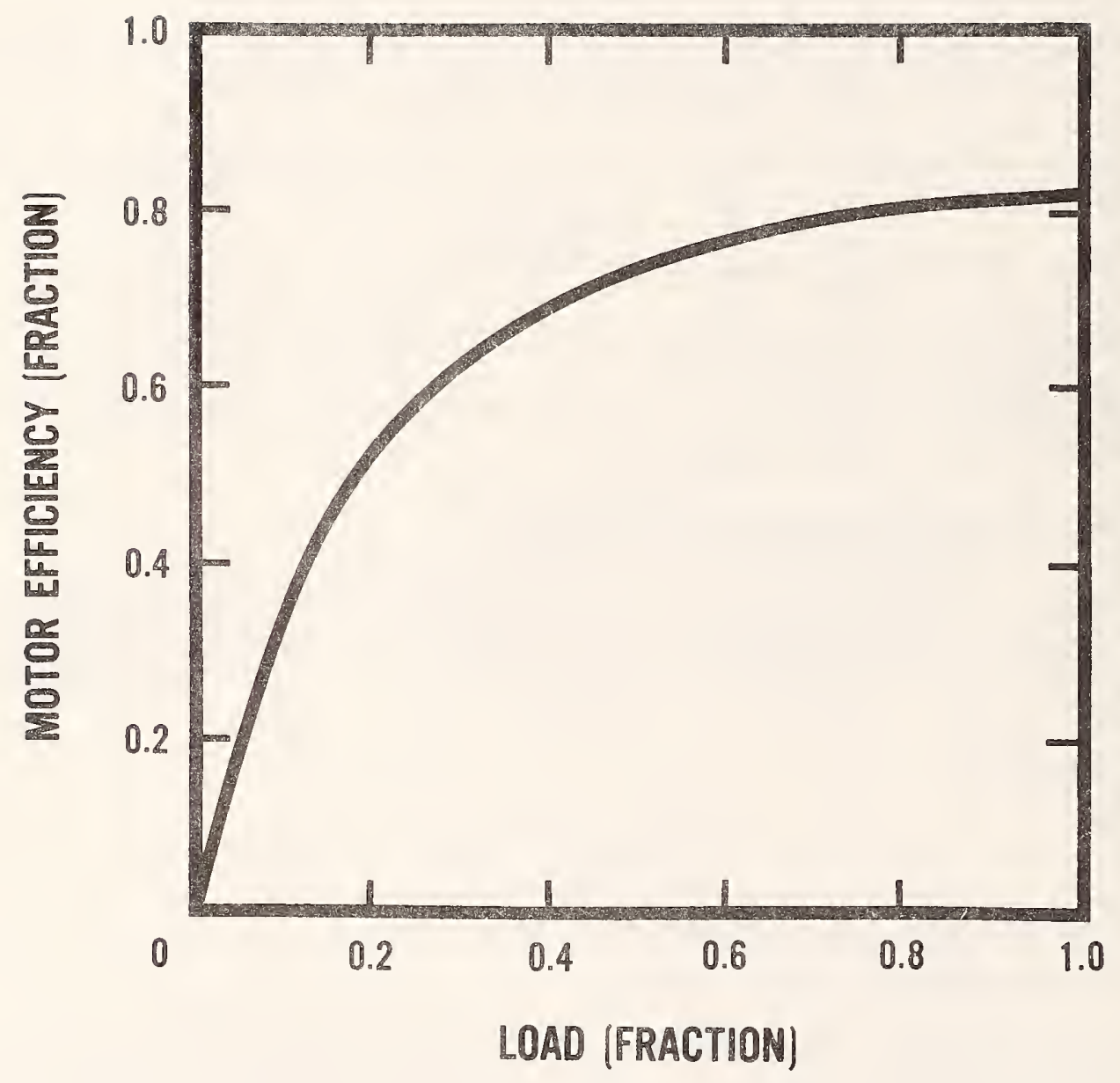

Figure 11. Typical speed (RPM) versus load curve for a permanent splitcapacitor 2 pole electrical motor [15]. 
where

$W_{c}=$ mechanical power available for compression

A common value of compressor mechanical efficiency falls in range of 0.95 to 0.98 .

\section{Compression Process}

A simplified indicator diagram is shown in figure 12. It shows idealized constant suction and discharge pressures in a cylinder. In modeling the cylinder process, both compression and re-expansion processes are assumed to be polytropic with the same polytropic index, $n$, following the equation [16]:

$$
\mathbf{P} \cdot \mathbf{v}^{\mathrm{n}}=\text { const }
$$

where

$$
\begin{aligned}
& \mathbf{n}=\text { polytropic index } \\
& \mathbf{P}=\text { pressure } \\
& \mathbf{v}=\text { refrigerant vapor specific volume }
\end{aligned}
$$

Since constant pressure and temperature are assumed during the discharge process, this implies no change in specific volume between points $C$ and $D$ (figure 12); consequently, the compression and re-expansion polytropic curves will coincide. This further means, that the net work required for compression of the residual gas is zero.

The refrigerant enthalpy increase during polytropic compression, $i_{6}-i_{5}$ (refer to figure 3), can be evaluated by the equation derived from the expressions for isentropic and polytropic work of compression at the same compression ratio. Equating these expressions results in the equation:

$$
i_{6}-i_{5}=\left(i_{6 s}-i_{5}\right) \frac{\frac{n}{n-1}}{\left.\frac{\gamma-1}{\gamma-P_{5}}\right)^{\frac{n-1}{n}}-1} \frac{\left(\frac{P_{6}}{P_{5}}\right)^{\gamma-1}-1}{P^{\gamma}}
$$

where $\quad i_{5}=$ refrigerant enthalpy before compression

$i_{6}^{5}=$ refrigerant enthalpy after coipression

${ }^{i} 6 s=$ refrigerant enthalpy after isentropic compression, rofined by pressure $p_{6}$ and entropy $s_{5}$

$n=$ polytropic indez

$\mathbb{P}_{5}=$ suction pressure

$\mathbf{P}_{6}=$ discharge pressure

$\gamma=$ isentropic index

The isentropic index, $\gamma$, and the polytropic index, $x$, are related by the polytropic efficiency of the compressor:

$$
\eta_{p}=\frac{\frac{y-1}{x-1}}{\frac{n}{n}}
$$




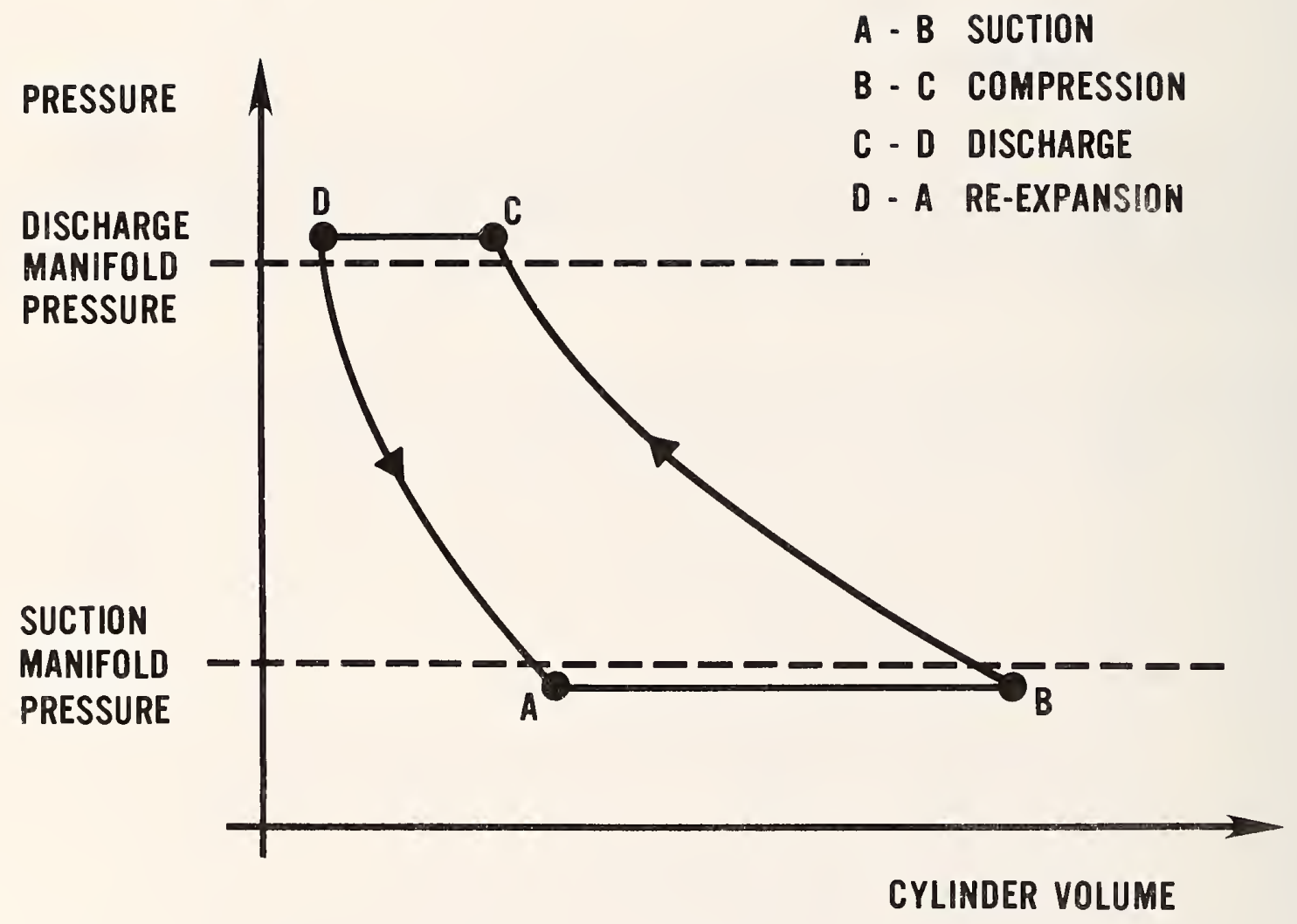

Figure 12. Simplified indicator diagram for a reciprocating compressor. 
The isentropic index, $\gamma$, equals the ratio of specific heats, as in the following formula:

$$
\gamma=\frac{C p}{C v}
$$

where

$$
\begin{aligned}
& \mathrm{Cp}=\text { specific heat at constant pressure } \\
& \mathrm{Cv}=\text { specific heat at constant volume }
\end{aligned}
$$

The specific heats of ideal gases are constant, thus the isentropic inder of an ideal gas is al so constant. For real gases such as refrigerant vapors, specific heats vary along the compression path. To accommodate this fact, the isentropic index can be evaluated by taking the average of the respective specific heat ratios at points 5 and $6 s$.

The refrigerant enthalpy increase during polytropic compression could also be calculated using the isentropic efficiency and enthalpy increase during isentropic compression at the same compression ratio. However, isentropic efficiency is sensitive to the compression ratio while polytropic efficiency is more consistent from one application to another and provides a more consistent representation of average compressor performance [17]. The imperfection of using isentropic efficiency may be traced to the general thermodynamic relation:

$$
\left(\frac{\partial i}{\partial s}\right)_{P}=T
$$

where

$$
\begin{aligned}
& \mathbf{i}=\text { enthalpy } \\
& \mathbf{P}=\text { pressure } \\
& \mathbf{s}=\text { entropy } \\
& \mathbf{T}=\text { temperature }
\end{aligned}
$$

which requires pressure 1 ines to diverge on a Mollier chart.

The refrigerant mass flow rate pumped by a compressor can be calculated by the following formula:

$$
m_{I}=\frac{60 \cdot R P M \cdot V s}{v_{5}} \eta_{v}
$$

where

$$
\begin{aligned}
m_{r} & =\text { refrigerant mass flow rate } \\
R^{r} & =\text { compressor speed (revolutions per minute) } \\
V s & =\text { compressor displacement per revolution } \\
v_{5} & =\text { refrigerant specific volume in the cylinder before compression } \\
\eta_{v} & =\text { volumetric efficiency }
\end{aligned}
$$

The RPM of a compressor is equal to that of an electric motor and is a function of load on the motor. A typical speed (RPM) versus load curve for a permanent split-capacitor 2-pole electric motor is shown in figure 13. 


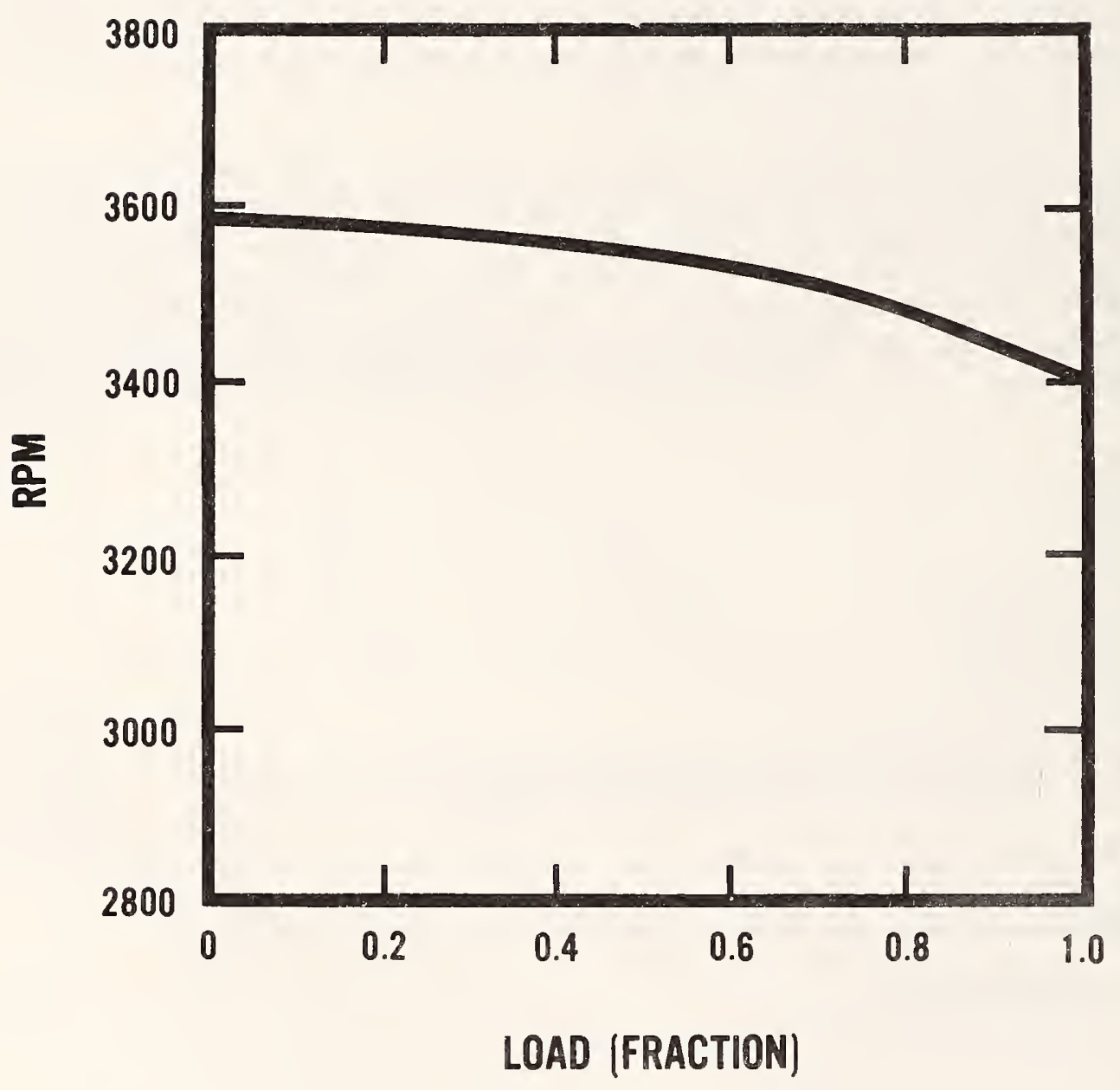

Figure 13. Typical efficiency versus load curve for a permanent splitcapacitor 2 pole electric motor [15]. 
With assumptions stated so far for the compression and re-expansion processes, the following formula for volumetric efficiency of a compressor can be derived [16]:

$$
\eta_{v}=c_{c}\left[1-c_{e}\left[\left(\frac{P_{6}}{P_{5}}\right)^{\frac{1}{n}}-1\right]\right]
$$

where

$$
\begin{aligned}
& \mathrm{C}_{c}=\text { correction factor for leakage from the piston, valves and for } \\
& \text { the throttling effect, assumed to be } 0.96, \text { [18] } \\
& \mathrm{C}_{e}=\text { clearance volume, fraction of displacement }
\end{aligned}
$$

\section{Heat Transfer Re1ations}

Electrical energy supplied to an electric motor is in part transferred to the refrigerant and in part is dissipated to the comprussor enviromment. This compressor heat balance can be expressed by the following equation:

$$
E+m_{r}\left(i_{3}-i_{8}\right)-Q_{C, A}=0
$$

where

$$
\begin{aligned}
E & =\text { electric energy input } \\
i_{3}, i_{8}= & \text { refrigerant enthalpy at respective locations (refer to } \\
& \text { figure } 10 \text { ) } \\
m_{T}= & \text { refrigerant mass flow rate } \\
Q_{C, A}= & \text { rate of heat rejected to ambient air }
\end{aligned}
$$

Reference is made to figure 10 , where key locations of refrigerant in the hermetic compressor are marked. In order to solve the compressor heat balance represented by equation (35), the following heat transfer losses are considered in the compressor model:

1. Heat transfer between the compressor can and ambient air, $Q_{C}, A$

2. Heat transfer between the refrigerant in the compressor can and the compressor can, $Q_{4, C}$

3. Heat transfer between the inlet refrigerant and the suction manifold and valve, $Q_{4,5}$

4. Heat transfer between the discharge refrigerant and the discharge valve and manifold, $Q_{6,7}$

5. Heat transfer between the refrigerant in the compressor can and the refrigerant in the discharge 1 ine, $Q_{7,8}$

The heat transfer between the compressor can and ambient air (item 1) is governed by free convection and radiation. Respetive heat transfer coefficients, $h_{c}$ and $h_{r}$, are calculated by the following equations [19]:

$$
\mathrm{h}_{\mathrm{c}}=0.18\left(\mathrm{~T}_{\mathrm{c}}-\mathrm{T}_{\mathrm{a}}\right)^{0.33} \quad\left(\mathrm{Btu} /\left(\mathrm{h} \cdot \mathrm{ft}^{2} \cdot \mathrm{F}\right)\right)
$$




$$
h_{r}=\sigma \cdot \varepsilon \cdot \frac{\left(T_{c}+459.67\right)^{4}-\left(T_{a}+459.67\right)^{4}}{T_{c}-T_{a}}\left(B t u /\left(h \cdot f t^{2} \cdot F\right)\right)
$$

where $\quad T_{\mathrm{a}}=$ ambient air temperature (F)

$\mathrm{T}_{\mathrm{c}}^{\mathrm{a}}=$ compressor she11 temperature (F)

$$
\begin{aligned}
& \sigma=0.1714 \times 10^{-4} \frac{\mathrm{Btu}}{\mathrm{h} \cdot \mathrm{ft} \cdot \mathrm{R}^{4}}=\text { Stefan-Boltzman constant } \\
& \varepsilon=\text { surface emissivity }(\varepsilon=0.9 \text { is used })
\end{aligned}
$$

The heat transfer rate between the compressor shell and the ambient air, $Q_{C}, A$ can be calculated by the equation:

$$
Q_{C, A}=Q_{C, A} \cdot\left(h_{c}+h_{r}\right) \cdot\left(T_{c}-T_{a}\right)
$$

where

$$
C Q_{C, A}=\text { heat transfer parameter }
$$

The heat transfer inside the compressor shell between the shell and refrigerant vapor (item 2) is governed by forced convection, for which the non-dimensional heat transfer parametric expression in terms of Nusselt, Reynolds, and Prandt1 numbers is in the form [20]:

$$
\mathrm{Nu} \propto \mathrm{Re}^{0.8} \cdot \mathrm{Pr}^{0.333}
$$

where $\quad \mathrm{Na}=\frac{\underline{h} \cdot \mathrm{L}}{\mathrm{k}}=$ Nosse1t Number

$$
\begin{aligned}
\operatorname{Pr} & =\frac{\mu \cdot C p}{k}=\operatorname{Prandt1} \text { Number } \\
\operatorname{Re} & =\frac{G \cdot L}{\mu}=\text { Reynolds Number } \\
G & =\frac{m_{r}}{A}=\text { refrigerant mass flux } \\
L & =\text { characteristic } 1 \text { ength } \\
\mathbf{K} & =\text { refrigerant thermal conductivity } \\
\mu & =\text { refrigerant dynamic viscosity }
\end{aligned}
$$

Using equation (39) the forced convection heat transfer coefficient, $h$, can be expressed as:

$$
\mathrm{h}=\mathrm{C} \cdot \mathrm{m}_{\mathrm{r}}^{0.8} \cdot \mathrm{k}^{0.666} \cdot \mathrm{Cp}^{0.333} \cdot \mu^{-0.467}
$$

where $\quad C=$ constant of proportionality, a function of wetted surface ge ome try 
Combining equations (36) and (40) yields the following expression for heat transfer rate between the compressor can and refrigerant:

$$
Q_{4, C}=C \cdot A_{h} \cdot m_{r}^{0.8} \cdot k_{4}^{0.667} \cdot C_{p_{4}}{ }^{0.333} \cdot \mu_{4}{ }^{-0.467}\left(T_{4}-T_{c}\right)
$$

or

$$
Q_{4, C}=C Q_{4, C} \cdot m_{r}^{0.8} \cdot k_{4}^{0.667} \cdot C_{p_{4}}{ }^{0.333} \cdot \mu 4^{-0.467}\left(T_{4}-T_{c}\right)
$$

where $\quad \mathrm{CQ}_{4, C}=$ heat transfer parameter

Obviously, heat transfer rates $Q_{C, A}$ and $Q_{4, C}$ are equal. Derivations of equations for the heat transfer rates between the inlet refrigerant and the suction manifold and $v a 1 v e, Q_{4,5}$, and between the discharge refrigerant manifold valve, $Q_{6,7}$, are similar to the derivation just performed for $Q_{4}, C$ since in these two cases heat transfer is al so by forced convection. Resulting expressions for these heat transfer rates are:

$$
\begin{aligned}
& Q_{4,5}=C_{4,5} \cdot m_{r}{ }^{0.8} \cdot k_{4,5} 0.666 \cdot C_{p_{4,5}}{ }^{0.333} \cdot \mu_{4,5}{ }^{-0.467} \\
& \left(T_{6}-T_{4}\right) \\
& Q_{6,7}=\mathrm{CQ}_{6,7} \cdot \mathrm{m}_{\mathbf{r}}{ }^{0.8} \cdot \mathrm{k}_{6,7}{ }^{0.667} \cdot \mathrm{Cp}_{4,5}{ }^{0.333} \cdot \mu_{6,7}-0.467 \\
& \left(T_{7}-T_{4}\right)
\end{aligned}
$$

The heat transfer between the refrigerant in the compressor can and the refrigerant in the discharge line (item 5) is modeled as forced convection heat transfer between the fluids separated by a barrier non-resistant to heat flow. Assuming that the temperature of refrigerant in the shell does not change (as a result of other heat transfers in the can and miring) and applying the logarithmic mean temperature difference, the following expression for heat transfer rate $Q_{7.8}$ can be derived [2]:

$$
Q_{7,8}=\mathrm{CQ}_{7,8} \cdot \mathrm{m}_{\mathrm{T}}^{0.8} \frac{\mathrm{a}}{\mathrm{a}+\mathrm{a3}}\left(\mathrm{T}_{7}-\mathrm{T}_{8}\right) / 1 \mathrm{n} \frac{\mathrm{T}_{7}-\mathrm{T}_{4}}{\mathrm{~T}_{8}-\mathrm{T}_{4}}
$$

where

$$
\begin{aligned}
& \mathrm{a}_{1}=\left(\mathrm{Cp}_{4} \cdot \mathrm{Cp}_{7,8}\right)^{0.333}\left(\mathrm{k}_{4} \cdot \mathrm{k}_{7,8}\right)^{0.667} \\
& \mathrm{a}_{2}=\mu_{4}{ }^{0.467} \cdot \mathrm{Cp}_{7,8}{ }^{0.333} \cdot \mathrm{k}_{7,8}{ }^{0.667} \\
& \mathrm{a}_{3}=\mu_{7,8}{ }^{0.467} \cdot \mathrm{Cp}_{4}{ }^{0.333} \cdot \mathrm{k}_{4}{ }^{0.667}
\end{aligned}
$$

Subscripts 3 through 8 refer to refrigerant key locations in the compressor in figure 10. If the subscripts are separated by a comma, the average value is impl ied.

The heat transfer to/from the flowing refrigerant changes the refrigerant enthalpy according to the equation:

$$
\mathbf{Q}=\mathbf{m}_{\mathbf{r}} \cdot \Delta \mathbf{i}
$$

where

$$
\Delta i=\text { refrigerant enthalpy change }
$$


Combining equations (43), (44), (45), and (46) yields the following expressions for the refrigerant enthalpy change during flow between respective locations of the compressor:

$$
\begin{aligned}
& i_{5}-i_{4}=C Q_{4,5} \cdot k_{4,5} 0.667 \cdot C_{p_{4,5}}{ }^{0.333}\left(\mathrm{~T}_{6}-\mathrm{T}_{4}\right) / \\
& \left(\mathrm{m}_{\mathrm{r}} 0.2 \cdot \mu_{4,5} 5^{0.467}\right) \\
& \mathrm{i}_{6}-\mathrm{i}_{7}=\mathrm{CQ}_{6,7} \cdot \mathrm{k}_{6,7} 0.667 \cdot \mathrm{Cp}_{6,7} 0.333\left(\mathrm{~T}_{7}-\mathrm{T}_{4}\right) / \\
& \left(\mathrm{m}_{\mathrm{r}} 0.2 \cdot \mu_{6,7}{ }^{0.466)}\right. \\
& \mathrm{i}_{7}-\mathrm{i}_{8}=\mathrm{CQ}_{6,7} \frac{\mathrm{a} 1}{\mathrm{a} 2+\mathrm{a3}}\left(\mathrm{T}_{7}-\mathrm{T}_{8}\right) /\left(\mathrm{m}_{\mathrm{T}} 0.2 \cdot \ln \frac{\mathrm{T}_{7}-\mathrm{T}_{4}}{\mathrm{~T}_{8}-\mathrm{T}_{4}}\right.
\end{aligned}
$$

where a1, a2, a3 are as in equation (45)

The derived heat transfer relations contain heat transer parameters $\mathrm{CQ}_{\mathrm{C}} \mathrm{A}$ $\mathrm{CQ}_{4,5}, \mathrm{CQ}_{6,7}$, and $\mathrm{CQ}_{7,8}$. These parameters are primarily functions of heat transfer surface geometry and have to be found empirically. If required laboratory test data for a given compressor are not available, typical compressor test data can be used. A large number of experimental compressor measurements have been published. The summary of these data and a 1 ist of references can be found in [21].

\section{Pressure Drop Re1ations}

Total pressure drop $\Delta \mathrm{P}_{\text {tot }}$ experienced by a flowing fluid results from pressure drops due to friction, momentum change, and gravity, i.e.,

$$
\Delta \mathrm{P}_{\text {tot }}=\Delta \mathrm{P}_{\text {friction }}+\Delta \mathrm{P}_{\text {acce } 1}+\Delta \mathrm{P}_{\text {gravity }}
$$

Pressure drop due to gravity in the hermetic compressor may be disregarded based on an order of magnitude analysis. On the same grounds, pressure drop of the flowing refrigerant between certain compressor locations may be attributed to either dynamic effect or viscous effect.

Pressure drop due to the dynamic effect, $\Delta \mathbf{P}_{\text {accel }}$, is proportional to velocity head, i.e.,

$$
\Delta \mathrm{P}_{\mathrm{acce} 1} \propto \rho \cdot \mathrm{V}^{2}
$$

which can be written, using the continuity equation:

$$
\Delta \mathrm{P}_{\mathrm{acce} 1}=\mathrm{CP} \cdot \mathrm{m}_{\mathrm{r}}{ }^{2 / \rho}
$$

where $\quad C P=$ pressure drop parameter

$$
\begin{aligned}
m_{r} & =\text { refrigerant mass flow rate } \\
\vec{V} & =\text { refrigerant velocity } \\
\rho & =\text { refrigerant density }
\end{aligned}
$$


The relation for the pressure drop due to the viscous effect, $P_{\text {friction, can }}$ be derived from the classical Fanning equation for pressure drop in a tube:

$$
\Delta \mathrm{P}_{\text {friction }}=2 \mathrm{f} \cdot \rho \cdot \mathrm{V}^{2} \cdot \mathrm{L} / \mathrm{D}
$$

where

$$
\begin{aligned}
& \mathbf{f}=\text { friction factor } \\
& D=\text { tube diameter } \\
& \mathbf{L}=\text { tube length }
\end{aligned}
$$

The friction factor, $f$, in the equation above is approximately proportional to the Reynolds number to the -0.2 power for the Reynolds number greater than 2000 [22]. Considering this and applying the equation of continuity, equation (53) becomes:

$$
\Delta \mathrm{P}_{\text {friction }}=\mathrm{CP} \cdot \mu^{0.2} \cdot \mathrm{m}_{\mathrm{r}} 0.8 / \rho
$$

The pressure drop of the refrigerant in the compressor can is modeled by evaluating individual pressure drops between refrigerant key locations indicated in figure 10. Based on equations (52) and (54), and attributing pressure drop between particular compressor locations either to the viscous or friction effect, the following pressure drop relations are proposed:

$$
\begin{aligned}
& \mathbf{P}_{3}-\mathbf{P}_{4}=\mathrm{CP}_{3,4} \cdot \mathrm{m}_{\mathrm{I}}^{2} / \rho_{3,4} \\
& \mathbf{P}_{4}-\mathbf{P}_{5}=\mathrm{CP}_{4,5} \cdot \mathrm{m}_{\mathbf{r}}^{2} / \mathrm{p}_{4,5} \\
& P_{6}-P_{7}=C_{6,7} \cdot m_{r}^{2} / P_{6,7} \\
& \mathrm{P}_{7}-\mathrm{P}_{8}=\mathrm{CP}_{7,8} \cdot \mathrm{m}_{\mathrm{T}}{ }^{1.8} \cdot \mu_{7,8} 0.2 / \mathrm{p}_{7,8} \text { for the discharge } 1 \text { ine }
\end{aligned}
$$

Pressure drop parameters $\mathrm{CP}_{3,4}, \mathrm{CP}_{4,5}, \mathrm{CP}_{6,7}$, and $\mathrm{CP}_{7}, 8$ have to be found experimentally for a given compressor or cán be calculated using test data for a similar compressor. For sources of such data, refer to [21].

Equations derived in this section are used in a compressor subroutine, COMPRE, described in Appendix E. These equations make it possible to carry out an energy balance of a hermetic compressor using an iterative process for electrical energy input, for heat lost to the ambient air, and for refrigerant parameters in compressor key locations. The variety of designs of refrigerant flow passages in the compressor caused the modeling of pressure drop and heat transfer to be done in an approximate manner in this general compressor model. In spite of several assumptions that were made to simplify the modeling process and reduce computing time, the model still retains sufficient details of the underlying physical principles to allow designers to determine which specific changes in the compressor design will lead to increased efficiency of the compressor and the heat pump system. 


$$
\bullet
$$




\subsection{Mode1 ing of a Constant Flow Area Expansion Device}

A constant flow area expansion device, as used in heating/air conditioning systems, is commonly called a capillary tube or a refrigerant flow restrictor. Usually it is a small bore tube of 1 ength as short as one-half inch up to a few feet, connecting the outlet of the condenser (or a liquid line) to the inlet of the evaporator. The main task of the constant flow area expansion device is to maintain the minimum pressure at the condenser at which all the flowing refrigerant can condense. Many researchers have investigated flow of a single component refrigerant through a capillary tube and a bibliography on the subject can be found in [15].

There is no experimental data known to the authors which would refer to the flow of a non-azeotropic mixture through a capillary tube or orifice. Because of 1 ack of data, single component refrigerant flow experience has been extrapolated to the non-azeotropic binary situation and the model developed accordingly.

\subsubsection{Available Capillary Tube Performance Data}

The capillary tube is a traditionally accepted name for a constant flow area expansion device used in heat pump systems. This name is inadequate and misleading since for tube diameters in the neighborhood of $1 / 16$ of an inch, capillary forces are negligible. The pressure drop consists primarily of:

- the loss due to sudden contraction at the entrance
- the loss due to flow in the tube
- the loss due to sudden enlargement at the exit to the evaporator

The flow of refrigerant through a constant bore tube is more complex than the geometric simplicity of the device would first indicate. The pressure and temperature distribution along a typical capillary tube is shown in figure 14. Bolstad and Jordan's description of the flow is as follows:

At the entrance to the tube, section 0-1, there is a slight pressure drop which was usually unreadable on the gages. From point 1 to point 2 the pressure drop is 1 inear. In the portion of the tube $0-1-2$ the refrigerant is entirely in the 1 iquid state and at point 2 the first bubble of vapor forms. From point 2 to the end of the tube the pressure drop is not 1 inear, the pressure drop per unit length increasing as the end of the tube is approached. For this portion of the tube, both the saturated 1 iquid and saturated vapor phases are present, the percent and volume of vapor increasing in the direction of flow...

With a saturation temperature scale corresponding to the pressure scale superimposed along the vertical axis, it is possible to plot the observed temperatures in a more meaningful way than if a uniform temperature scale were used. The temperature is constant for the first portion of the tube 0-1-2. At point 2, the pressure has dropped to the saturation pressure corresponding to this temperature. Further pressure drop beyond point 2 is accompained by a corresponding drop in temperatere, the 


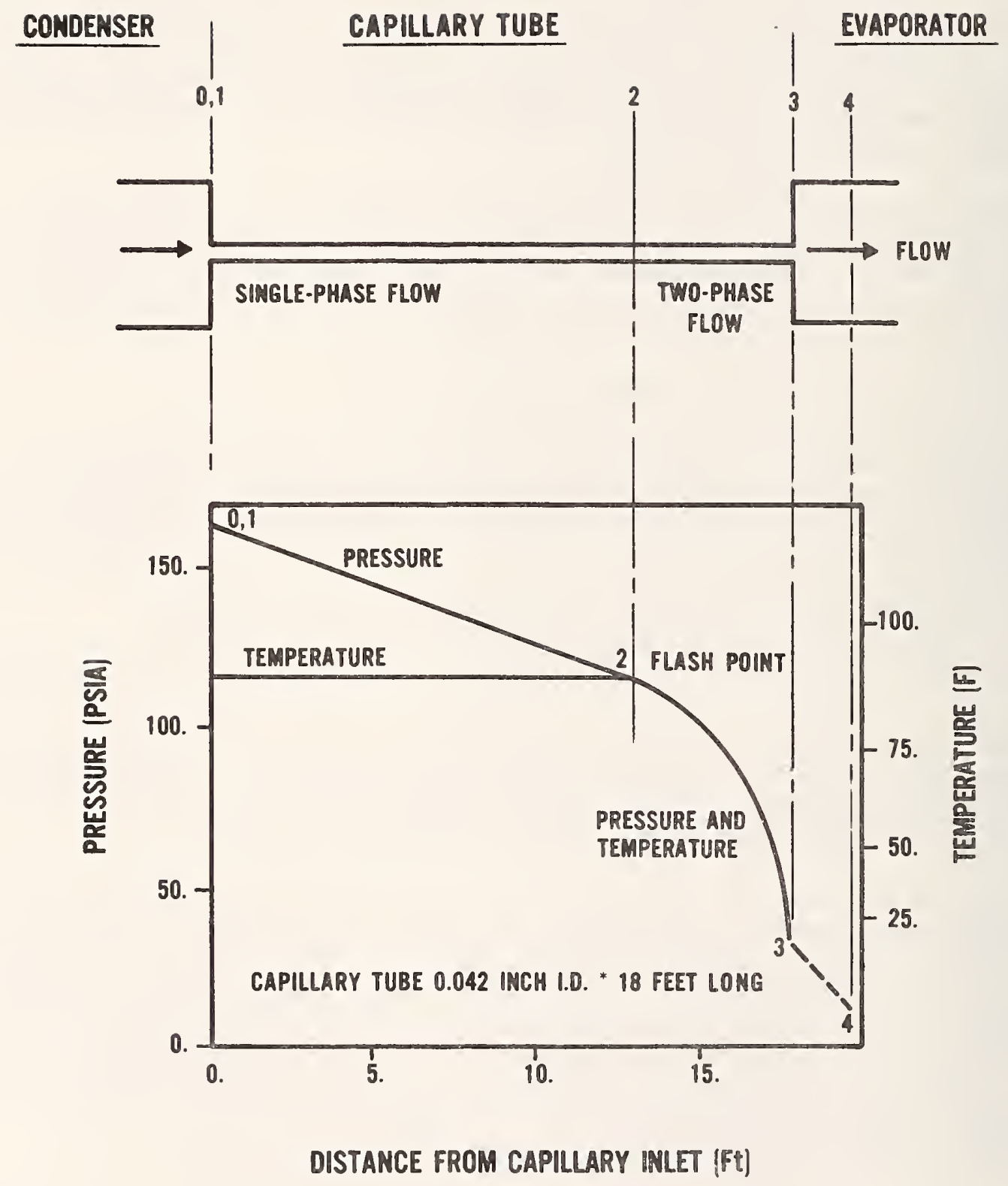

Figure 14. Pressure and temperature distribution along typical capillary tube [23]. 
temperature being saturation temperature corresponding to the pressure. As a consequence, the pressure and temperature lines coincide from point 2 to the end of the tube [23].

The point of the tube where the first bubble forms is called a bubble point or a flash point. The pressure at the point is called the flash pressure. Bolstad also presented an analytical method of solution for adiabatic flow through the capillary based on the Fanno flow theory.

Mikol [24] performed a capillary tube investigation from which his conclusions can be summarized as follows:

- Fluid flow through small bore tubes conforms to continuous flow as established for large bore tubes and pipes.

- The friction factor correlation of Moody and any others consistent with Moody's correlation [25] is applicable to single-phase flow in small bore tubes.

- The phenomena of metastability, persistance of the liquid state at pressures less than the saturation pressure corresponding to its local temperature, has been found to occur.

- The phenomenon of choked flow in two-phase flow occurs in the same way and for the same reasons as in the case of gaseous flow. Sonic velocity occurs at the tube exit.

One of Mikol's findings, existence of superheated liquid in a small portion of a tube, was not observed by Bolstad and Jordan [23]. However, it was reported by Cooper et a1. [26] and Rezk [27]. Investigators have found that delayed evaporation is affected by initial disturbances and flow agitation, but there is not enough data in the current 1 iterature to assess all the factors promoting or eliminating this phenomena and making it possible to consider metastability in a capillary tube model at this time.

The pressure and temperature distributions along a capillary tube as shown in figure 14 occur at design operating conditions of a 1 ong (a few feet) capil1ary. Part of the capillary is filled with flowing liquid, while two-phase flow exists in the other part. However, there are al so other possible modes of operation, i.e., with only two-phase flow in the capillary (the case of incomplete condensation in the condenser) or with only liquid flow (the case of short restrictor). All these cases are observed in practice and have to be simulated by a general model of the constant flow area expansion device.

Based on the experimental evidence and the theory of 1 arge tube fluid mechanics, the following assumptions were used for model formulation:

1. The capillary tube is straight, horizontal, and has a constant inner diameter.

2. F1ow in the capillary is one-dimensional and homogeneous.

3. F1 ow in the capillary is adiabatic. 
4. F1ow resistance in the capillary tabe can be subdivided into

a. Resistance due to the entrance effect

b. resistance due to flow in a tube which consists, in the general case, of single-phase 1 iquid flow resistance from the entrance to the flash point, and two-phase mirture flow resistance in the rest of the tube. The existence of the delayed evaporation phenomena is neglected. Resistance due to the exit effect is neglected as meaningless for a choked flow and insignificant for a non-choked f10w [23].

5. Choked flow phenomenon for tro-phase flow of a non-azeotropic mirture is governed by the same laws as for single-phase flow of a single component fluid and can be modeled accordingly.

\subsubsection{Available Short Tube Restrictor Performance Data}

An experimental study on the flow of refrigerant 12 through short tubes was performed by Pasqua [28]. From his visual observations he found that the refrigerant flowed through the restrictor in the form of a metastable 1iquid core surrounded by a vapor annulus. Based on his experiment, Pasqua also determined flow characteristics of subcooled and saturated liquid through a short tube restrictor. A study of short tube restrictors applied to refrigerant 22 was performed by Mei [29]. He tested five restrictors of a length/diameter rations from 7.5 to 11.9. He confirmed occurrence of firststage choking but reported that second-stage choking did not take place at conditions obtainable in his test facility. Mei provided two correlations for evaluation of refrigerant mass flow rate which, however, are limited to the tested refrigerant 22 .

As experimentally obtained information available in the literature is not sufficient for a development of a general simulation model of a short restrictor applicable to different refrigerants, such models have to be developed using fundamental equilibrium fluid mechanics. Using this approach, the analysis and basic assumptions made in the previous section regarding a capillary tube would apply to a short tube.

\subsubsection{Critical F1ow}

Refrigerant mass flow rate through a given flor restrictor 111 depend on the inlet refrigerant state and on the pressure that will be established at the tube outlet. This pressure may equal the evaporator pressure or may have a higher value if the flow is choked. Since pressure at the tube exit is one of the parameters affecting the flow, it has to be known for accurate refrigerant mass flow rate prediction.

The assumption that the flow in the flow restrictor is one-dimensional and homogeneous enables the tro-phase flow in the tube to be treated as single-phase flow with uniform properties at any cross-section of the flow and allors use of the single-phase, one-dimensional form of the governing equations as presented in figure 15, 16 and 17. 


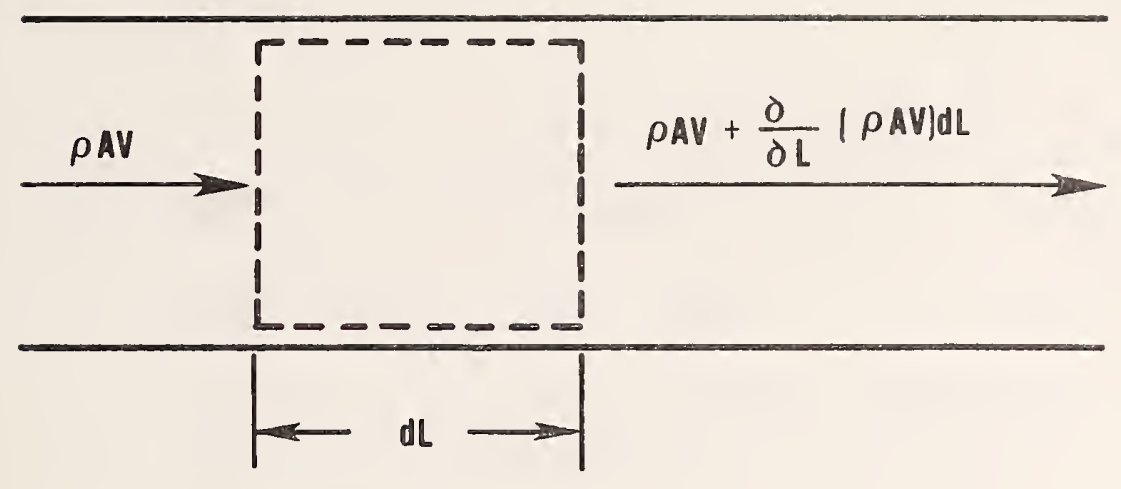

CONTINUITY EQUATION: $d(\rho V)=0$

Figure 15. Mass balance for an element of fluid ia ore-dimensional steady flow in a constant area duct.

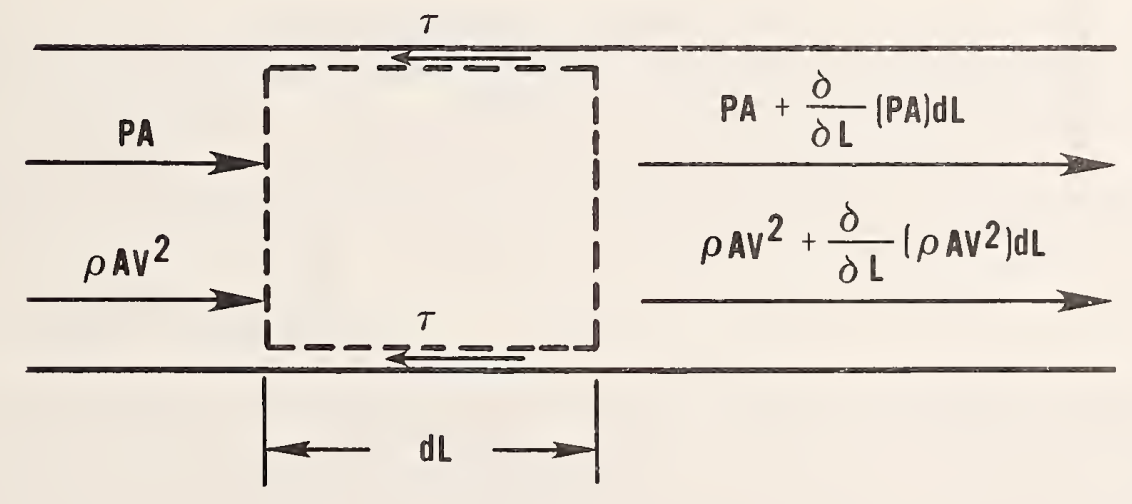

MOMENTUM EQUATION: AdP $+\rho A V d V+\tau S d L=0$

Figure 16. Momentum balance for an element of fluid in one-dimensional steady flow in a horizontal, constant area duct. 


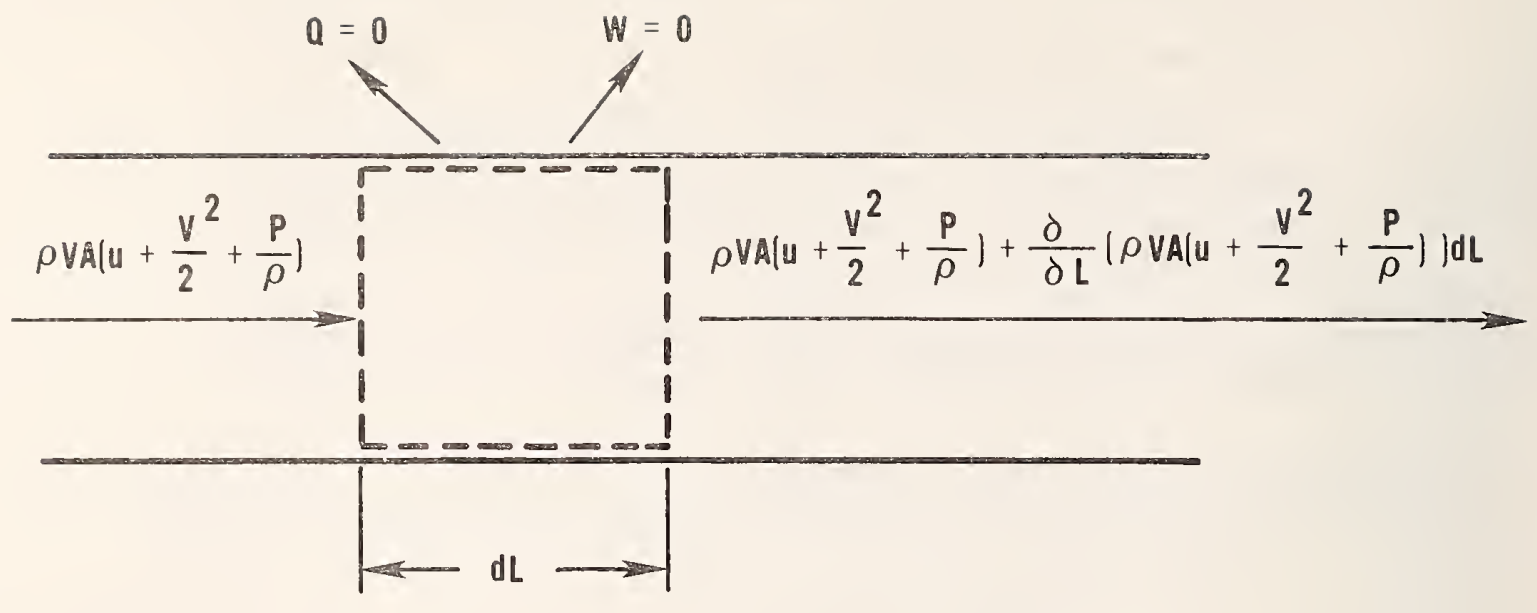

ENERGY EQUATION: $d\left(u+\frac{v^{2}}{2}+\frac{P}{\rho}\right)=d\left(i+\frac{v^{2}}{2}\right)=0 \quad i+\frac{v^{2}}{2}=i_{0}$

Figure 17. Energy balance for an element of fluid in one-dimensional adiabatic, steady flow in a horizontal, constant area duct.

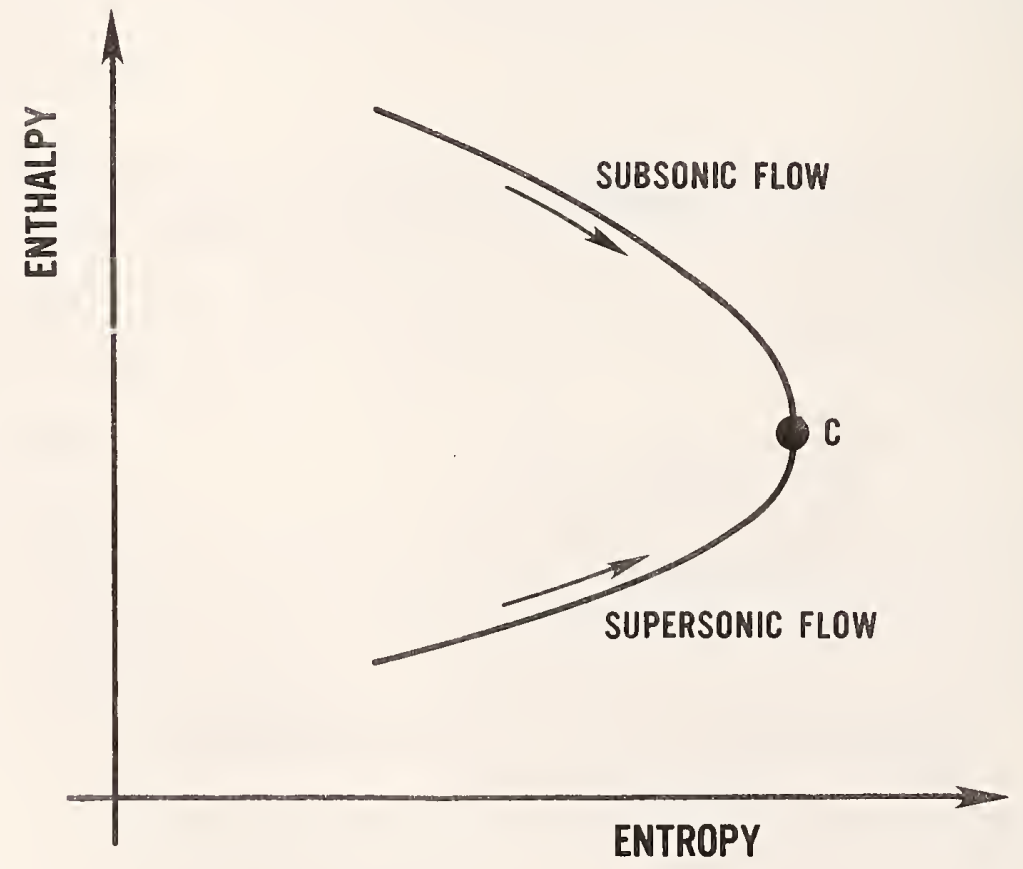

Figure 18. Fanno 1 ine. 
Adiabatic flow through a capillary tube is classical example of so called Fanno flow: adiabatic flow with friction in a constant area duct. The energy equation for such flow has the form:

$$
d i+V \cdot d V=0 \quad \text { or } \quad i_{0}=i+V^{2} / 2=\text { const }
$$

which, when combined with the equation of continuity yields:

$$
i_{0}=i+{\frac{m_{I}}{A}}^{2} \cdot \nabla^{2} / 2=i+G^{2} \cdot v^{2} / 2
$$

where

$$
\begin{aligned}
\mathbf{A} & =\text { tube cross-sectional area } \\
\mathbf{G} & =\frac{m_{\mathbf{I}}}{\mathbf{A}}, \text { refrigerant mass flax } \\
\mathbf{I} & =\text { refrigerant enthalpy } \\
i_{0} & =\text { refrigerant stagnation enthalpy } \\
\mathbf{m}_{\mathbf{r}} & =\text { refrigerant mass flow rate } \\
\mathbf{V} & =\text { flow velocity } \\
\nabla & =\text { refrigerant specific volume }
\end{aligned}
$$

Graphical representation of equation (60) on the enthalpy-entropy diagram, as shown in figure 18, is called a Fanno line. Fanno flow, as an irreversible adiabatic process, can sequentially exist only in the direction of increasing entropy. The upper branch of the Fanno line corresponds to subsonic, accelerating flow while the 1 ower branch is the supersonic, decelerating flow. Both flows tend towards point $C$ where the sonic velocity is reached.

The Fanno 1 ine implies that fluid cannot reach the velocity of sound inside a constant area duct, because, if this happened, further flow in tho duct would have to be associated $\mathbf{w i t h}$ a decrease of entropy (figure 18), and that would be in violation of the Second Law of Thermodynamics. Thus, the only location of a tube at which sonic velocity can be reached is the tube exit, and choking will never occur inside the capillary regardless of external outlet pressure.

\subsubsection{Mode1 Formulation}

Refrigerant flow from the 1 iquid 1 ine into the capillary tube experiences a pressure drop due to sudden contraction. This pressure drop consists of an acceleration loss and entrance friction loss and is usually expressed by a decrease in the Bernoulli head and a contraction coefficient referred to tho kinetic energy of the flow in the section of smaller flow area:

$$
\frac{P_{0}-P_{1}}{P_{0,1}}+\frac{V_{0}^{2}-V_{1}^{2}}{2}=K \frac{V_{1}^{2}}{2}
$$

Subscripts in eq. (61) refer to sections shown in figure 14. Combining with the equation of continuity, the above can be rearranged to: 


$$
P_{0}-P_{1}=\Delta P=(1+K) \rho_{0,1} \cdot \frac{V_{1}^{2}}{2}
$$

The contraction coefficient, $K$, given in the 1 iterature is strict $1 y$ empirical and is represented as a function of the contraction area ratio. Several sources are in disagreement about its value. The value of $K=0.15$, used here is from a derivation based on Kays' general formula [30]. It was calculated for a normal range of contraction area ratios for capillary tubes with slightly beveled entrances.

The equation of motion for steady flow in a constant cross-section area pipe has the following form:

$$
\rho \cdot A \cdot V \cdot d V+A \cdot d P+\tau \cdot S \cdot d L=0
$$

The skin friction coefficient, $\tau$, can be expressed in terms of the friction coefficient, $f$, and the velocity head:

$$
\tau=\frac{1}{2} f \cdot \rho \cdot v^{2}
$$

The flow velocity term, $V$, can be eliminated by means of the equation of continuity:

$$
d(V \cdot \rho)=0
$$

Substituting and rearranging, the equation of motion assumes the following form:

$$
\left(\frac{A}{m_{r}}\right)^{2} \cdot \int_{P_{i}}^{P_{i}+1} \rho \cdot d P+\frac{2}{D} \int_{L_{i}}^{L_{i}+1} f \cdot d L+1 n \frac{\rho_{i}}{\rho_{i}+1}=0
$$

As discussed before, flow in a capiliary tube, in the general case, can be subdivided into two parts separated by a flash point: the liquid flow part and the two-phase mixture part. The same equation will be applicable for both flows though in the case of 1 iquid flow, it can be simplified on grounds of incompressibility. In fact, it reduces to the Fanning pressure drop formula in the following form:

$$
\Delta P=\frac{2 f \cdot G^{2} \cdot L}{\rho-D}
$$

where the friction factor, $f$, can be evaluated by the empirical formula:

$$
f=0.046 \cdot \mathrm{Re}^{-0.2}
$$

for the Reynolds number, Re, greater than 2000 [22].

For the two-phase mixture flow, equation (66) has to be solved in its full form. This was done by Whitese1 [31,32] for refrigerants 12 and 22 , but with significant oversimplified approsimations for the refrigerant properties. In 
solving equation (42), difficulty arises with evaluating the first term because it depends directly on the pressure-density relation along the path of flow. However, the relation can be obtained by considering the adiabatic flow case. The specific volume at a given pressure can be expressed in terms of the property values for saturated liquid and vapor and in terms of quality:

$$
v=v_{L}+\mathbf{z}\left(\nabla_{V}-\nabla_{L}\right)
$$

where $\quad \nabla=$ specific volume

$\mathbf{z}=$ quality

Subscripts $L$ and $V$ are for 1 iquid and vapor, respectively

The quality of the flow in the Fanno path can be found as explained in Appendix C. Thus integration of refrigerant density over a given pressure interval can be done numerical1y. Still another problem is faced in evaluating the second term of equation (66), which includes a two-phase friction factor as a function of tube length. Erth [33] made an effort to correlate two-phase average friction factor in a capillary tube for refrigerant 12 and refrigerant 22. His regression analysis, based on four sets of data from four different experiments, yielded the following correlation for a two phase flow mean friction factor, $f_{m}$, as a function of the inlet conditions only:

$$
f_{m}=\frac{0.775}{\operatorname{Re}^{0.5}} \exp \left[\left(1-x_{i}^{0.25}\right) / 2.4\right]
$$

where $\quad z_{i}=$ quality of refrigerant entering capillary tabe

$$
\operatorname{Re}=\frac{G \cdot D}{\mu_{L}+\mathbf{z}_{\mathbf{i}}\left(\mu_{V}-\mu_{L}\right)}
$$

Using this mean friction factor, $f_{m}$, equation (66) may be written for the twophase portion of the flow in the following form:

$$
m_{r}=A\left[\frac{-\int_{\rho d P}^{P_{3}}}{\frac{P_{2}}{D} f_{m} \cdot\left(L_{3}-L_{2}\right)+1 n\left(\rho_{2} / \rho_{3}\right)}\right]^{0.5}
$$

where the numbers used as subscripts denote location consistent $w$ ith figure 14.

A subroutine, CAPIL, modeling a constant flow area expansion device is based on the equations presented above. These equations have to be solved in a highly iterative process since choking pressure, friction factor, fraction of capillary tube length with 1 iquid and two-phase flow, and the velocity head used to correct enthalpy are functions of refrigerant mass flow rate which has to be determined. Additional information about the subroutine CAPIL is given in Appendix F. 


\subsection{Mode1 ing of an Evaporator and a Condenser}

\subsubsection{Modeling Me thodology}

There are two heat exchangers in a heat pump: an indoor coil and an outdoor coil. Both coils are made in a similar way and both serve as an evaporator or condenser depending on the heat pump operation mode. A schematic of a typical heat pump heat exchanger is show $n$ in figure 19. It consists of a set of finned tubes connected in a specifically designed circuit configuration. The refrigerant flows through the tubing while air flows over the outside of the coil. Various schemes of circuiting the tubes together can be used. An example of the coil circuitry is illustrated in figure 20 .

The tube-by-tube modeling technique is applied here to model the coil. This technique depends on imaginary isolation of one tube with appropriate fin surfaces from the coil assembly and calculating the performance independently. The heat transfer to and from a tube is calculated with the aid of the heat exchanger cross-flow theory. Input for calculations consists of finned tube design data, refrigerant and air mass flow rates, and inlet refrigerant and air thermodynamics states. These are uniquely evaluated by the model for each particular tube. Performance calculations are conducted for each tube independently in proper sequence and their summation results in total coil capacity.

In order to perform heat transfer calculations, four surfaces associated with the tubes must be defined. Following Carrier and Anderson [34], it was assumed that the fin area served by each tube is equivalent in performance to a circular-plate fin of equal area. Thus a single tube is considered with a circular fin of diameter, $D_{t}$, as shown in figure 21 .

\subsubsection{Heat Transfer Rate for a Tube in a Cross-F1ow Arrangement}

Usually a heat pump coil employs some form of cross-flow arrangement. If a separate tube is considered, the problem is one of pure cross flow. Fortunately, this kind of arrangement has received much attention in theoretical investigations. According to the general heat transfer equation:

$$
Q=\mathrm{U} \cdot \mathrm{A}_{\mathrm{h}} \cdot \Delta \mathrm{T}
$$

where

$$
\begin{aligned}
A_{\mathrm{h}} & =\text { heat transfer surface area } \\
\bar{U} & =\text { overall coefficient of heat transfer } \\
\Delta \mathrm{T} & =\text { temperature difference }
\end{aligned}
$$

In the case of a pure cross-flow arrangement with changing temperatures of both fluids during heat exchange, the following equation for mean temperature difference between fluids applies [16]:

$$
\Delta \mathrm{T}_{\mathrm{m}}=\frac{\mathrm{t}_{2}-\mathrm{t}_{1}}{\frac{\mathrm{T}_{1}-\mathrm{T}_{2}}{\mathrm{t}_{2}-\mathrm{t}_{1}}}
$$




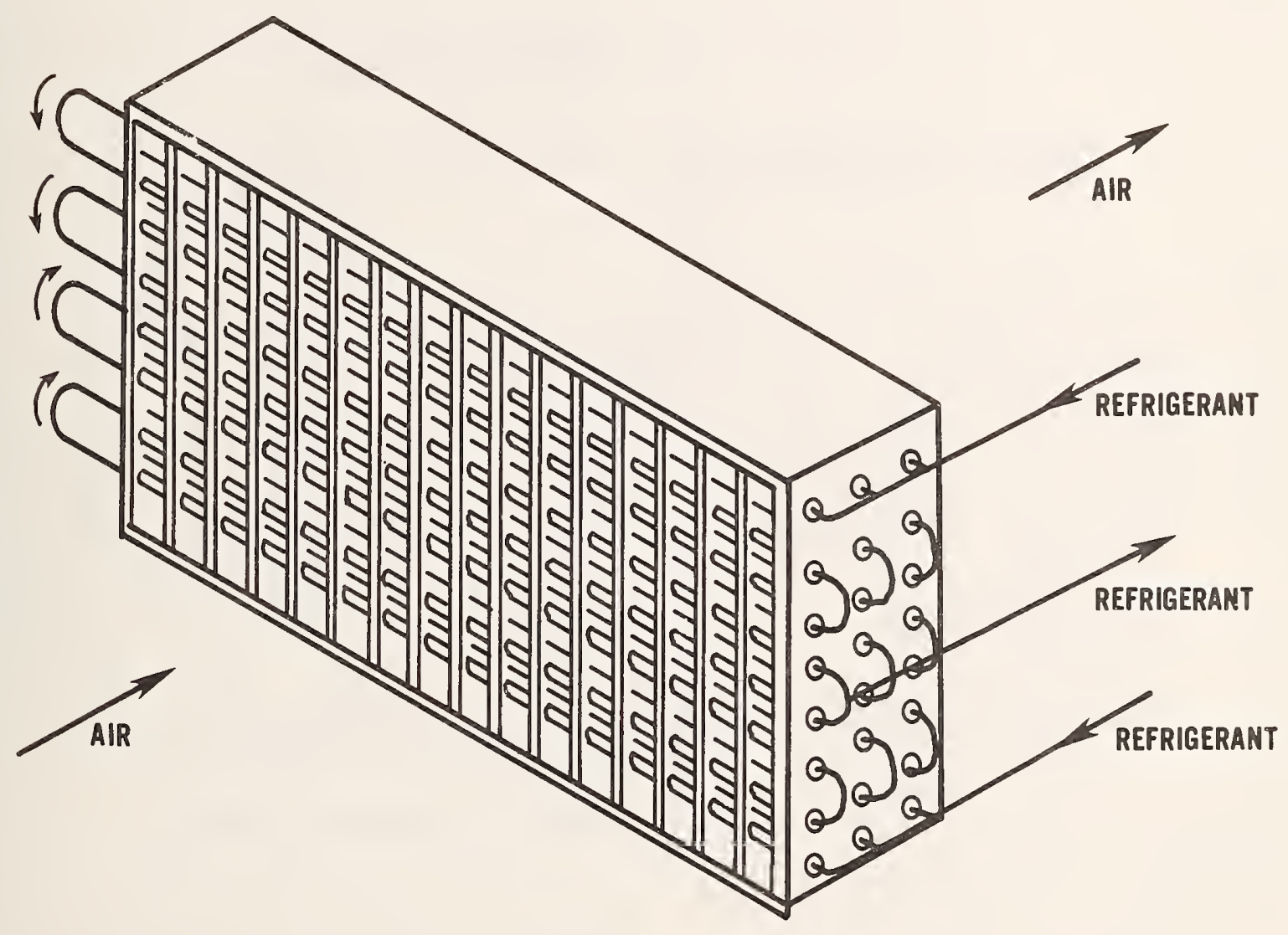

Figure 19. Schematic of a heat pump heat exchanger. 


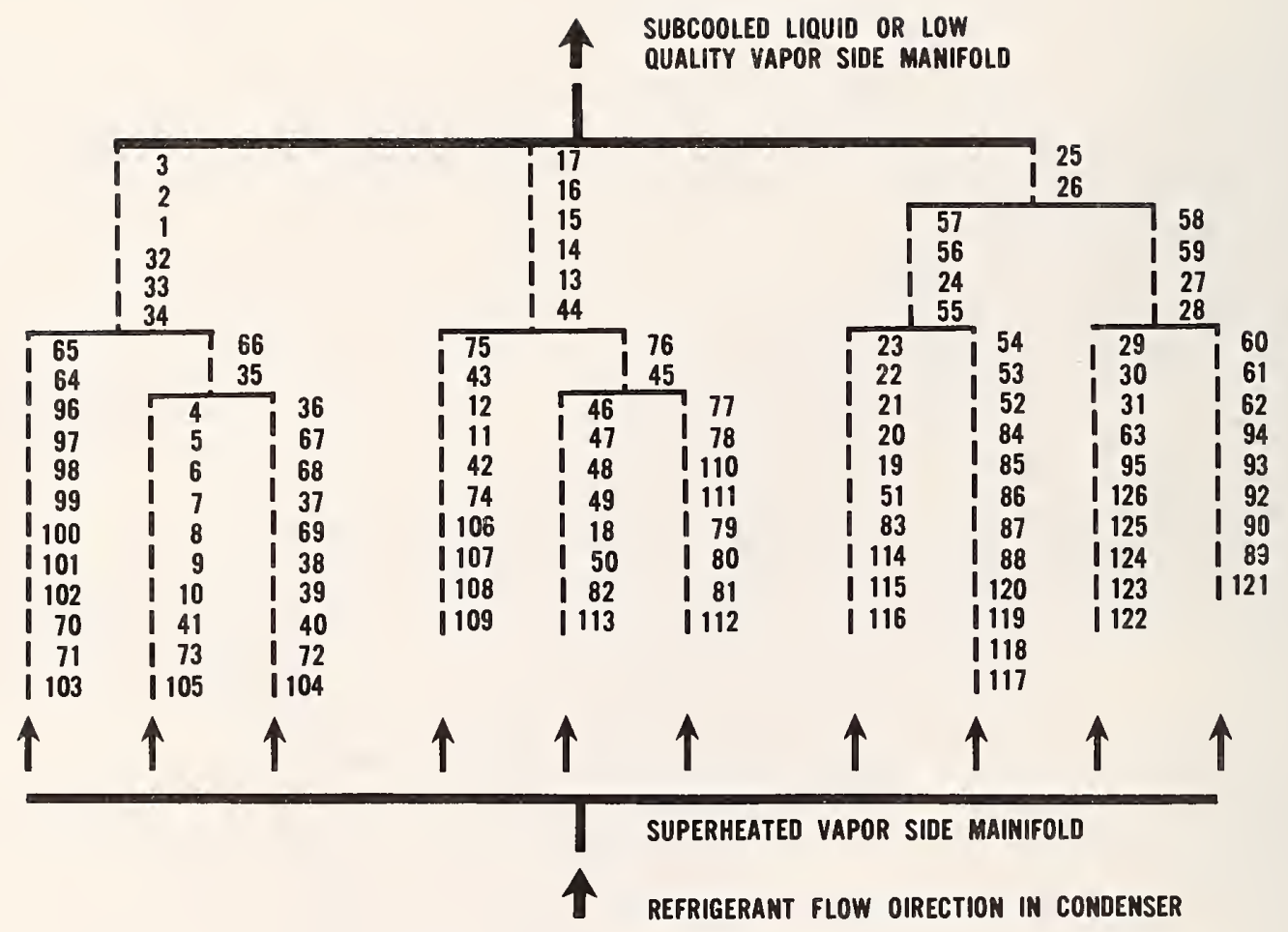

Numbers in the figure represent a location of the particular tube counting them left to right in each depth row starting with the row facing the incoming air.

Figure 20. Example of coil circuitry. 


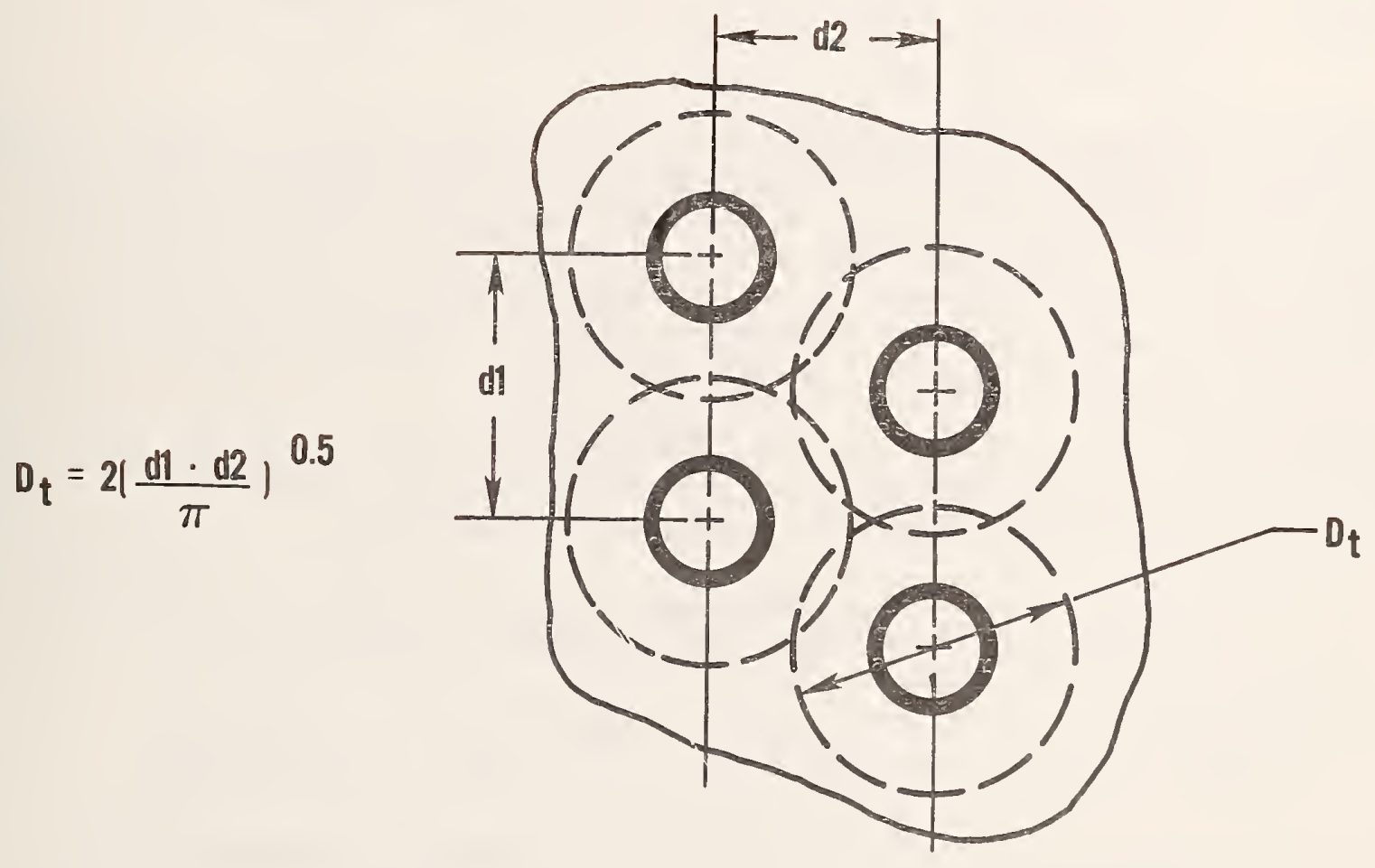

Figure 21. Approximation method for treating a rectangular-plate fin of uniform thickness in terms of a flat circularplate fin of equal area. 
where

$$
\begin{aligned}
T= & \text { temperature of one fluid } \\
t= & \text { temperature of another fluid } \\
\Delta T_{m}= & \text { mean temperature difference } \\
& \text { subscripts } 1 \text { and } 2 \text { refer to tube inlet (upstream) and outlet } \\
& \text { (downstream) conditions, respectively. }
\end{aligned}
$$

The heat exchanged with the fluid can be calculated by the equation:

$$
Q=m \cdot\left(i_{2}-i_{1}\right)
$$

or

$$
Q=\mathrm{m} \cdot \mathbb{C P}_{\mathrm{p}} \cdot\left(\mathrm{T}_{2}-\mathrm{T}_{1}\right)
$$

where $\quad C_{p}=$ average specific heat of f1uid at constant pressure

$i=$ enthalpy

$\mathrm{m}=$ mass flow rate

Looking at any vapor compression cycle $\mathrm{P}-\mathrm{h}$ or $\mathrm{T}$-s diagram, it can be realized that both single-phase and two-phase refrigerant flow usually exists in a given heat exchanger. Also, both flow patterms can actualiy exist in one tube. That means that the rate of change of temperature of refrigerant flowing in the tube will not be uniform over tube length. Not only the mean temperature between fluids is affected by the flow pattern inside the tube but al so the refrigerant pressure drop.

Equations presented below, derived from equations (73), (74), and (75), a110w for detailed consideration of these problems. The heat transfer rate for each mentioned flow condition can be calculated as follows:

- single-phase or two-phase flow only, refrigerant is superheared, subcooled or in two-phase at both inlet and outlet

$Q=C p r \cdot m_{I}\left(T_{I, i}-T_{a, i}\right)\left(1-\exp \left(-\frac{C_{p_{a}} \cdot m_{a}}{C_{p_{I}} \cdot m_{I}}\left(1-\exp \left(\frac{-\mathbb{U} \cdot A_{0}}{C_{p_{a}} \cdot m_{a}}\right)\right)\right)\right)(76)$

- superheated vapor at tube inlet, two-phase at the tube outlet

$$
\begin{aligned}
& Q=m_{I}\left(i_{T, i}-i_{T, V}\right)+C_{P_{I}} \cdot m_{T} \cdot\left(T_{T, V}-T_{Q, i}\right)
\end{aligned}
$$

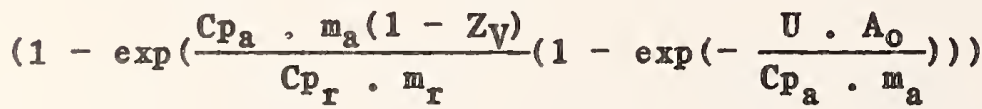

where $\mathrm{Z}_{\mathrm{V}}=$ fraction of the tube length in the superheated region which can be calculated by the equation: 


$$
\mathrm{Z}_{\mathrm{V}}=\frac{-\mathrm{Cp}_{\mathrm{r}} \cdot \mathrm{m}_{\mathrm{r}} \cdot \ln \left(1-\frac{\mathrm{i}_{\mathrm{r}, \mathrm{i}}-\mathrm{i}_{\mathrm{r}, \mathrm{V}}}{\left.\mathrm{C}_{p_{\mathrm{r}}\left(\mathrm{T}_{\mathrm{r}, \mathrm{i}}-\mathrm{T}_{\mathrm{a}, \mathrm{i}}\right)}\right)}\right.}{\mathrm{Cp}_{\mathrm{a}} \cdot \mathrm{m}_{\mathrm{a}}\left(1-\exp \left(-\frac{\mathrm{U} \cdot \mathrm{A}_{\mathrm{O}}}{\left.\mathrm{Cp}_{\mathrm{a}} \cdot \mathrm{m}_{\mathrm{a}}\right)}\right.\right.}
$$

- two-phase at tube inlet, subcooled liquid at the tube outlet

$$
\begin{aligned}
& Q=C p_{I} \cdot m_{r}\left(T_{T, L}-T_{a, i}\right)\left(1-\exp \left(-\frac{C p_{a} \cdot m_{Q}\left(1-Z_{t p}\right)}{C_{p_{I}} \cdot m_{T}}\right.\right. \\
& \left.\left(1-\exp \left(-\frac{U \cdot A_{0}}{C p_{a} \cdot m_{a}}\right)\right)\right)+m_{I}\left(i_{I, i}-i_{I, L}\right)
\end{aligned}
$$

where $\quad z_{t p}=$ fraction of the tube length in the two-phase region which can be calculated by the equation:

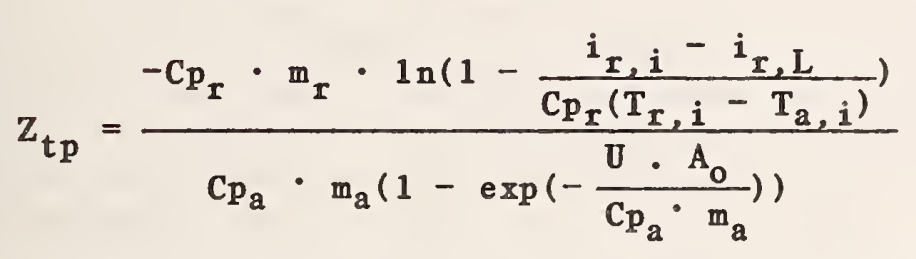

In equations (76) through (79) the following nomenclature was used:

$A_{0}=$ total exterior surface area associated with the tube wetted by air

$\mathrm{Cp}_{\mathrm{a}}=$ air specific heat at constant pressure

$\mathrm{Cp}_{\mathrm{r}}=$ refrigerant specific heat at constant pressure (in the two-phase region the specific heat is assumed to be a ratio of enthalpy change to temperature change, i.e., $C_{p_{r}}=\Delta i_{r} / \Delta T_{r}$ )

$i_{r, i}=$ refrigerant enthalpy at the tube inlet

$i_{r, L}^{r, i}=$ enthalpy of refrigerant saturated 1 iquid

$i_{r, V}=$ enthalpy of refrigerant saturated vapor

$\dot{m}_{\mathrm{a}}=$ air mass $\mathrm{fl}$ ow rate associated with the tube

$\mathrm{m}_{\mathrm{r}}=$ refrigerant mass flow rate in the tube

$T_{a, i}=$ air temperature upstream of the tube

$T_{r, i}^{a, i}=$ refrigerant temperature at tube inlet

$T_{r, L}=$ refrigerant bubble-point temperature

, $\vec{U}=$ overall tube heat transfer coefficient

5.4.3 Refrigerant and Air Mass Flow Rates Associated with a Tube

\section{Refrigerant Mass Flow Rate}

During flow through a heat pump heat exchanger, the refrigerant undergoes a change of phase in the course of evaporation or condensation. The change of phase is associated with a dramatic change of density which affects the 
velocity and pressure drop of the working fluid. In order to prevent a high pressure drop, tubes in some heat pump coils are connected to form branched circuits. An example of such coil circuitry is shown in figure 20 .

Refrigerant flow direction marked in figure 20 is for the coil working as a condenser. Superheated vapor enters the vapor side manifold and is distributed into 10 tubes. On its flow path, refrigerant merges several times and finally merges in the 1 iquid manifold to enter the 1 iquid 1 ine. For the coil operating as an evaporator, the direction of flow is opposite to that marked in the figure. Low quality refrigerant enters the coil and the flow is subdivided into three circuits. On its way through the coil the refrigerant evaporates and splits several times on its way towards the exit where it is finally collected into one larger diameter vapor 1 ine. The mass flow rates through the particular circuits of the coil are self adjusting so the pressure at merging (splitting) tubes is the same.

To perform a simulation of a coil by the tube-by-tube method, refrigerant mass flow rate for each tube has to be known. Since total refrigerant mass flow rate supplied to the coil is known, the problem reduces to the determination of refrigerant distribution. This could be determined by the model itself, however, at expense of going through iterative calcuations. Another approach was tried to determine refrigerant flow distribution. Since most of the coil total pressure drop may be expected to result from superheat vapor and twophase flow, it was assumed that the refrigerant flow is uniformly distributed among tubes connected to the vapor side manifold and that mass flow rates in other tubes may be found by following the refrigerant path with direction of flow as marked in figure 20. The resulting distribution was checked by examining calculated refrigerant (R22) pressures at the ends of circuit branches. These pressures should be equal if the assumed distribution is correct. The maximum pressure discrepancy found was equal to 0.3 psi which represented less than a $0.2^{\circ} \mathrm{F}$ variation in the saturation temperature of refrigerant 22 between merging tubes. This was considered satisfactory and the method was adopted for determining refrigerant ass flow rate distribution in a coil.

\section{Air Mass Flow Rate}

Air mass flow rate was assumed to be distributed uniformly over the whole coil face regardless of the coil and fan respective locations, so each tube in particular depth row was associated with the same air mass flow.

The temperature of the inlet air for a given tube was assumed to be equal to the temperature of air exiting from the upstream tube and not to be affected by mixing with air leaving neighboring tubes.

\subsubsection{Overal1 Heat Transfer Coefficient for a Dry Finned Tube}

Dry finned tube analysis is applicable to a condenser and also to an evaporator if no dehumidification takes place. The overal1 heat transfer coefficient, $U$, for a dry finned tube can be derived by summing the individual resistances between the refrigerant and the air, [16]: 


$$
U=\left[\frac{A_{0}}{A_{p, i} h_{i}}++\frac{A_{0} p_{p}}{A_{p, m^{k}}}+\frac{1}{h_{c, 0}\left(1-\frac{A_{f}}{A_{0}}(1-\phi)\right)}\right]^{-1}
$$

where $\quad A_{f}=$ fin surface area

$A_{0}=$ total exterior surface area exposed to air

$A_{p, i}=$ pipe inside surface area

$A_{p, m}^{p, i}=$ pipe mean surface area

$h_{c, o}^{p}=$ convection heat transfer coefficient at the exterior surface

$h_{i}=$ inside tube convection heat transfer coefficient

$k_{p}=$ thermal conductivity of pipe material

$x_{p}=$ thickness of pipe wall

$\phi=\frac{T_{f, m}-T_{a}}{T_{f, b}-T_{a}}$, fin efficiency

$T_{a}=$ air temperature

$T_{f, b}=f$ in base temperature

$T_{f, m}=$ mean fin temperature

The second term of equation (81) can be evaluated if the heat exchanger material and geometry are known. Terms 1 and 3 , which refer to the inside and outside convection resistance respectively, required considerably more analysis to establish the proper algorithm for determining the heat transfer coefficient.

\subsubsection{Forced Convection Heat Transfer Inside a Tube}

Analyzing the problem for both an evaporator and a condenser, the following modes of forced convection are encountered:

- single-phase forced convection

- two-phase forced convection with condensation

- two-phase forced convection with evaporation

The physics of these phenomena are very much different and forced convection in each mode have to be considered separately.

\section{Single-Phase Forced Convection}

Single-phase forced convection takes place in a condenser, at the entrance section where the superheated vapor is being cooled, and at the exit section where a subcooled liquid is being cooled. It is also applicable in the evaporator, as the superheated vapor passes through the exit tubes. The nondimensional heat transfer parameter describing this phenomena, Nusselt number, is related to the non-dimensional Reynolds and Prandtl numbers in the following form [19]:

$$
\mathrm{Nu}=0.023 \cdot \operatorname{Re}^{0.8} \cdot \operatorname{Pr}^{\mathrm{a}}
$$


where $\quad \mathrm{Nu}=\frac{h \cdot \mathrm{D}}{\mathrm{k}}=$ Nusselt Number

$$
\begin{aligned}
\operatorname{Pr} & =\frac{\mu \cdot C_{p}}{k}=\operatorname{Prandt1} \text { Number } \\
\operatorname{Re} & =\frac{G \cdot D}{\mu}=\text { Reynolds Number } \\
a & =0.3 \text { for cooling: } 0.4 \text { for heating } \\
C_{p} & =\text { specific heat at constant pressure } \\
D & =\text { inside diameter of a tube } \\
G & =\text { refrigerant mass flux } \\
h & =\text { convection heat transfer coefficient } \\
\mathbf{k} & =\text { refrigerant thermal conductivity } \\
\mu & =\text { refrigerant absolute viscosity }
\end{aligned}
$$

\section{Two-Phase Forced Convection with Condensation}

The predominant flow pattern during condensation in a heat pump condenser is annular flow with 1 iquid refrigerant flowing on the pipe wall and vapor refrigerant flowing in the core. To the author's knowledge there are no data or correlations available in the literature on the forced convection condensation heat transfer coefficient of non-azeotropic mixtures flowing inside a tube. The only option left here is to use one of the correlations developed for condensing heat transfer coefficient for single component refrigerant. The the correlation proposed by Traviss, Baron and Rohsenow [35] was chosen as the most theoretically derived, thus having most chances to provide reasonable predictions for other than the tested refrigerants 12 and 22 . The correlation proposed in [35] and adopted in this modeling effort without modification is expected to overpredict condensing heat transfer coefficient for binary mixtures.

The theoretical background for the Traviss et al. correlation is as follows: the von Karman universal velocity distribution in the condensate film was assumed (1ike on a flat plate), pressure was calculated using the Lockhart-Matinelli method [22], and the momentum and heat transfer analogy was applied. The proposed correlation has the following form:

$$
\mathrm{Nu}=\frac{\operatorname{Re}_{\mathrm{L}}{ }^{0.9} \cdot \mathrm{Pr}_{\mathrm{L}} \cdot \mathrm{F} 1^{\beta}}{\mathrm{F} 2}
$$

$$
\text { where } \quad \begin{aligned}
\mathrm{Nu} & =\frac{\mathrm{h} \cdot \mathrm{D}}{\mathrm{k}_{\mathrm{L}}} \\
\mathrm{h} & =\text { condensation heat transfer coefficient } \\
\mathrm{D} & =\text { tube inside diameter } \\
\mathrm{k}_{\mathrm{L}} & =\text { thermal conductivity of liquid refrigerant }
\end{aligned}
$$




$$
\begin{aligned}
& \mathrm{Re}_{\mathrm{L}}=\frac{G(1-\Sigma) D}{\mu_{L}} \\
& G=\text { refrigerant mass flux } \\
& \mathrm{z}=\mathrm{qu} \text {. } 1 \mathrm{ity} \\
& \mu_{L}=1 \text { iquid refrigerant absolute viscosity } \\
& \operatorname{Pr}_{\mathrm{L}}=\frac{\mu_{\mathrm{L}} \cdot \mathrm{CP}_{\mathrm{L}}}{\mathrm{k}_{\mathrm{L}}} \\
& \beta=1 \text { for } F 1 \leq 1, \beta=1.15 \text { for } F 1>1
\end{aligned}
$$

F1 and F2 in equation (83) are dimensionless parameters expressed as follows:

$$
\begin{aligned}
F 1= & 0.15\left(X_{t t}{ }^{-1}+2.85 \mathrm{X}_{t t} 0.524\right) \\
F 2= & 0.707 \cdot \operatorname{Pr}_{\mathrm{L}} \cdot \operatorname{Re}_{\mathrm{L}} 0.5 \quad \text { for } \operatorname{Re}_{\mathrm{L}}<50 \\
\mathrm{~F} 2= & 5 \cdot \mathrm{Pr}_{\mathrm{L}}+5 \cdot \ln \left(1+\operatorname{Pr}_{\mathrm{L}}\left(0.09636 \cdot \operatorname{Re}_{\mathrm{L}} 0.585-1\right)\right) \text { for } 50<\operatorname{Re}_{\mathrm{L}}<1125 \\
\mathrm{~F} 2= & 5 \cdot \mathrm{Pr}_{\mathrm{L}}+5 \cdot 1 \mathrm{n}\left(1+\mathrm{Pr}_{\mathrm{L}}\right)+2.5 \cdot 1 \mathrm{n}\left(0.00313 \cdot \operatorname{Re}_{\mathrm{L}} 0.812\right) \\
& \text { for } \operatorname{Re}_{\mathrm{L}}<1125
\end{aligned}
$$

Parameter, $\mathbb{X}_{t t}$, formulated by Lockhart-Martine11i [22] with the assumption of no radial pressure gradient and a smooth pipe, has the following form:

$$
z_{t t}=\left(\frac{1-z}{x}\right)^{0.9}\left(\frac{v_{L}}{\nabla_{V}}\right)^{0.5}\left(\frac{\mu_{L}}{\mu_{V}}\right)^{0.1}
$$

Parameter $\mathbb{X}_{t t}$ is inversely proportional to flow quality and refers to turbulent vapor and turbulent liquid flow. Physically it is equal to the square root of the ratio of the frictional pressure drop of the liquid phase to the frictional pressure drop of the vapor phase if each of these phases was flowing alone in the tube, i.e.,:

$$
x_{t t}=\left[\frac{\left(\frac{d P}{d L}\right)_{L}}{\left(\frac{d P}{d L}\right)_{V}}\right]^{0.5}
$$

Equation (83) is applicable where conditions for annuiar condensation in a tube exists. Such conditions may be assumed to exist for flow qualities ranging from 0.1 to 0.9 . At qualities larger than 0.9 , tra whole tube inner surface is not covered by a liquid film and part of the heat transfer is just that of single-phase convection. At qualities less than 0.1, flow was observed to be in the slug regime [35]. It is assumed, that in the quality range 0.0 to 0.1 and 0.9 to 1.0 , the heat transfer coefficient changes linearly from a two-phase flow value to a single-phase flow value and is calculated using 1 inear interpolation between values obtained from equations (82) and (83). 


\section{Two-Phase Forced Convection with Evaporation}

Refrigerant enters an evaporator from an expansion device at a quality of about 20 percent and forms an annular flow instantly. The quality increases with the proceeding flow and the annular flow pattern is maintained until the quality reaches about 0.90 , at which point refrigerant vapor has enough kinetic energy to gradually destroy the liquid layer and patches of dry wall appear.

Many experiments were performed and correlations published for calculating the forced convection evaporative heat transfer coefficient for R12 and R22, however, no data are available in the literature on non-azeotropic mixtures. Simultaneous to the development of this model, evaporative heat transfer coefficient measurements were performed at NBS on mixtures of R13B1/R152a at a variety of compositions. The results of preliminary tests with heat balances within $10 \%$ were correlated for use in this model in the following form:

$$
\mathrm{h}_{\mathrm{ev}, \mathrm{m}}=\mathrm{h} * 3.22 \mathrm{x}_{\mathrm{tt}}-0.3
$$

where $h_{e v, m}=$ evaporative heat transfer coefficient of the refrigerant mixture

$h=$ forced convection heat transfer coefficient of the liquid film calculated by equation (82) for 1 iquid flow rate equal to twophase refrigerant flow rate in the tube.

$\mathrm{X}_{\mathrm{t} t}=$ Lockhart-Martine11i parameter, equation (84)

It is important to note that al1 1 iquid and vapor properties used in $\mathbb{X}_{t}$ parameter have to be evaluated for 1 iquid and vapor phases being in equilibrium based on mixture temperature, pressure and composition of mixture in the tube.

Predictions of the above correlation agree within accuracy of $\pm 10 \%$ for 50 percent of experimental data, $\pm 20 \%$ for $89 \%$ of data, and within $\pm 31 \%$ for all data. This correlation is applicable for annual flow at qualities from $10 \%$ to $90 \%$.

\subsubsection{Forced Convection Heat Transfer at the Air-side of a F1at-Finned Tube}

In order to evaluate the forced convection heat transfer outside a flat-finned tube (term 4 of equation (81)), the total exterior surface area, $A_{0}$, the fin area, $A_{f}$, the air-side heat transfer coefficient, $h_{c, 0}$, and fin efficiency, , have to be known.

From the number of air-side heat transfer correlations available in the literature, the one proposed by Briggs and Young [36] is most applicable here. This correlation was developed after extensive tests or 18 tube banks of different fin geometry. A regression analysis of the test data for the air Reynolds number raxge from 1000 to 20000 yielded the following equation:

$$
\mathrm{Nu}=\frac{\mathrm{h}_{\mathrm{c}, \mathrm{O}} \cdot \mathrm{D}_{\mathrm{o}}}{\mathrm{K}_{\mathrm{a}}}=0.134 \cdot \operatorname{Re}_{\mathrm{a}}{ }^{0.681} \cdot \operatorname{Pr}_{\mathrm{a}}{ }^{0.333} \cdot\left(\frac{\mathrm{z}}{\mathrm{y}}\right)^{0.2} \cdot\left(\frac{\mathrm{z}}{\mathrm{t}}\right)^{0.1134}
$$

where

$$
\begin{aligned}
D_{0} & =\text { outside tube dismeter } \\
h_{c, 0} & =\text { air-side mean convective heat transfer coefficient for ary air }
\end{aligned}
$$




$$
\begin{aligned}
\mathbf{k}_{a} & =\text { air thermal conductivity } \\
\operatorname{Pr}_{a} & =\frac{\mu_{a} \cdot C p_{a}}{k_{a}}, \text { Prandt1 number } \\
\operatorname{Re}_{a} & =\frac{G_{\max } \cdot D_{0}}{\mu_{a}}, \text { Reynolds number } \\
G_{\max } & =a i r \text { mass flux at minimum cross section } \\
t & =\text { fin thickness } \\
y & =\text { fin height } \\
z & =\text { distance between adjacent fins }
\end{aligned}
$$

The geometric parameters affecting the heat transfer are illustrated in figure 22. The accuracy of equation (87) was further verified by Jones and Russe11 [37].

The addition of fins to the tubes greatly increases the outer heat transfer area but at the expense of decreasing the mean temperature difference between the surface and the air stream. The parameter, fin efficiency, $\phi$, is used to rate the thermal effectiveness of a $\mathrm{fin}$. As mentioned in Section 5.4.1, it is assumed in this study for heat transfer analysis, that each tube is served by a circular-plate fin of equilivalent surface area, as in figure 21. Gardner [38] solved the differential equation for describing the temperature distribution in a circular $f$ in and presented fin efficiency curves in terms of two parameters.

$$
D_{0} / D_{t} \text { and } y\left[\frac{2 \cdot h_{c}}{k_{f} \cdot t}\right]^{0.5}
$$

The theoretical results are correlated well by the following equation (see al so figure 23) [2]:

$$
\phi=\sum_{i=1}^{8}\left(A_{1, i}+A_{2, i} \frac{D_{0}}{D_{t}}+A_{3, i}{\frac{D_{0}}{D_{t}}}^{2}\right)\left(y\left(\frac{2 \cdot h_{c}}{k_{f} \cdot t}\right)^{0.5}\right)^{i-1}
$$

where $\quad h_{c}=$ air-side convective heat transfer coefficient

$\mathbf{k}_{\mathrm{f}}=\mathrm{fin}$ material thermal conductivity

The geometric parameters are indicated in figure 22. The coefficients, $A_{n, i}$ are given in Tab1e 3 .

\subsubsection{Overal1 Heat Transfer Coefficient for a Wet Finned Tube}

Wet finned tube analysis is applicable to an evaporator when its temperature is below the dew point temperature of ambient air. In such a case, moisture is being removed from an air stream and is transferred to the evaporator external surface. If the evaporator temperature is above $32^{\circ} \mathrm{F}$, a water film flows dow the fin under force of gravity. If the exterior evaporator temperature is below $32^{\circ} \mathrm{F}$, frost is accumulated. 


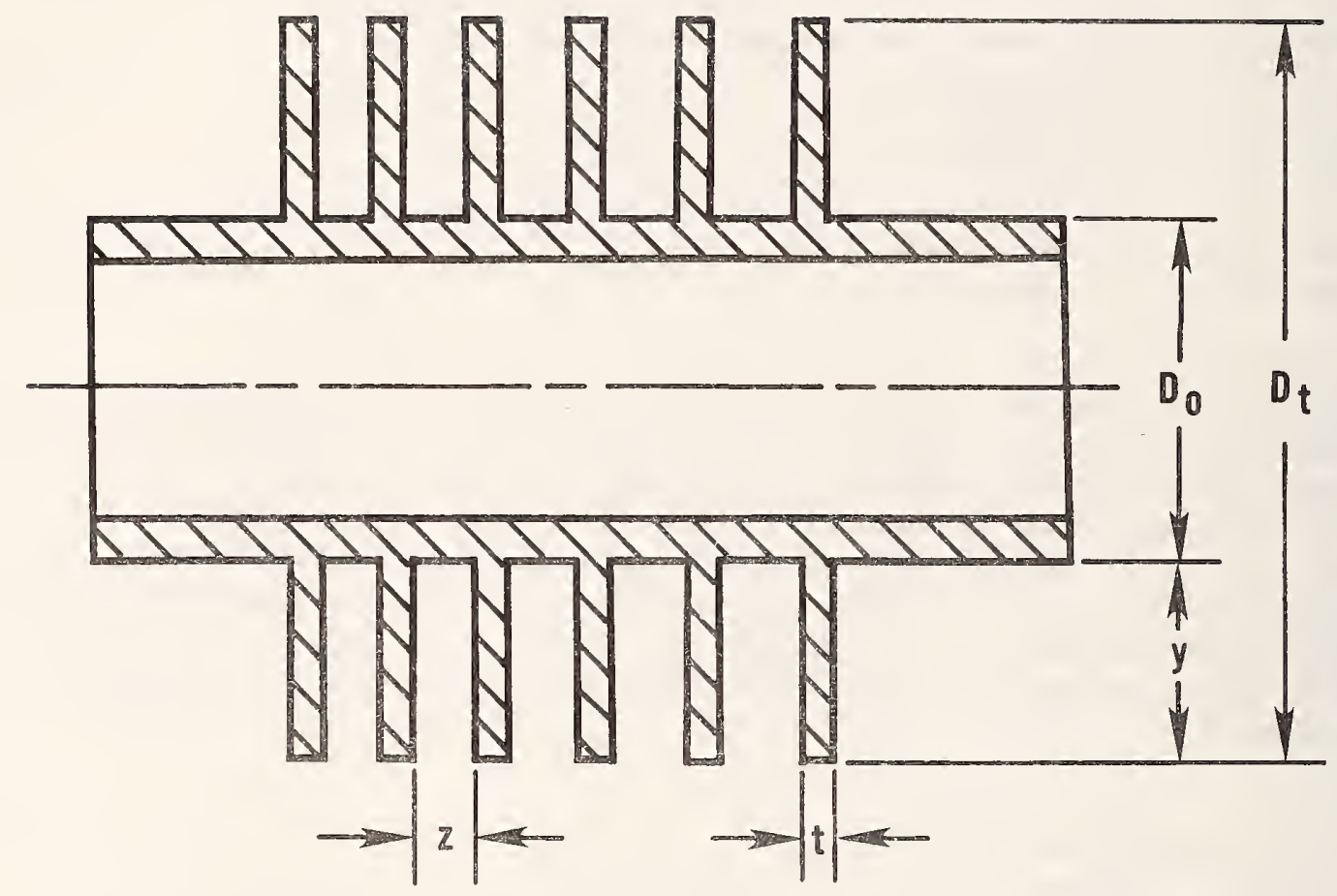

$D_{0}=$ TUBE OUTSIDE DIAMETER

$D_{\mathrm{t}}=$ FIN TIP DIAMETER

$y=$ FIN HEIGHT

$t=$ FIN THICKMESS

$z$ = DISTANCE BETWEEN ADJACENT FINS

Figure 22. Cross section of a flat-finned tube indicating parameters which affect the air side heat transfer coefficient. 


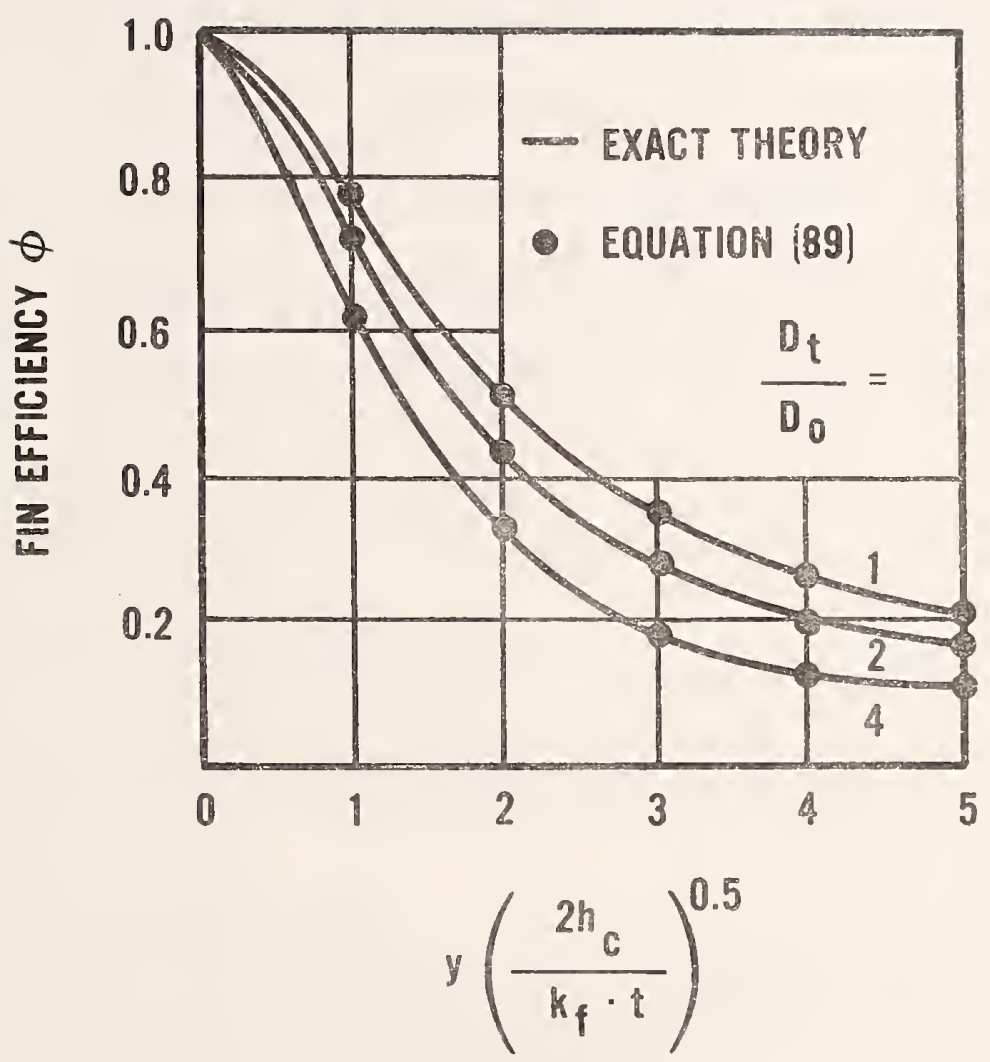

Figure 23. Efficiency for a circularplate fin of uniform thickness. Comparison of exact theory results with those obtained by equation (89). 
Tab1e 3. Coefficients to be Used in Correlation for Fin Efficiency (Equation (89)).

\begin{tabular}{l|l|l|l}
$i$ & $A_{1, i}$ & $A_{2, i}$ & \multicolumn{1}{|c}{$A_{3, i}$} \\
\hline 1 & 1.0 & 0.0 & 0.0 \\
2 & $-0.22920 \mathrm{E}-01$ & $-0.13755 \mathrm{E}+00$ & $0.20130 \mathrm{E}-01$ \\
3 & $0.16106 \mathrm{E}+00$ & $0.81890 \mathrm{E}-01$ & $-0.11440 \mathrm{E}-01$ \\
4 & $-0.64975 \mathrm{E}+00$ & $-0.55500 \mathrm{E}-01$ & $-0.28753 \mathrm{E}-01$ \\
5 & $0.53491 \mathrm{E}+00$ & $0.18040 \mathrm{E}-01$ & $0.42477 \mathrm{E}-01$ \\
6 & $-0.19286 \mathrm{E}+00$ & $0.36494 \mathrm{E}-03$ & $-0.20335 \mathrm{E}-01$ \\
7 & $0.32564 \mathrm{E}-01$ & $-0.10660 \mathrm{E}-02$ & $0.40947 \mathrm{E}-02$ \\
8 & $-0.20972 \mathrm{E}-02$ & $0.12410 \mathrm{E}-03$ & $-0.29673 \mathrm{E}-03$
\end{tabular}


Considering the heat transfer from the refrigerant to the air, one can realize that the dehumidification process will alter the heat transfer situation on the external surface of the finned tube, while other processes in the tube and refrigerant stay unaffected and are governed by relations already proposed in previous sections. Thus only processes connected with dehumidification need to be discussed.

The heat transfer rate between the air stream and the water surface is described by the following equation:

$$
d Q=\left(h_{c, o}\left(T_{a}-T_{w}\right)+h_{D, o}\left(w_{a}-w_{w}\right) i_{f g, w}\right) d A_{0}
$$

The first term accounts for sensible heat transfer and the second term accouts for latent heat transfer. For air at atmospheric pressure the Lewis number,

$$
\text { Le }=\frac{h_{C, 0}}{h_{D, 0} C_{p_{a}}} \text {, }
$$

is close to 1 [16]. Therefore, equation (90) assumes the following form for a tube with flat fins:

$$
d Q=h_{c}\left(1-\frac{A_{f}}{A_{0}}(1-\phi)\right)\left(T_{a}-T_{w}\right) d A_{0}
$$

where $\quad h_{c}=h_{c, 0}\left(1+\frac{i_{f g, w}\left(w_{a}-w_{w}\right)}{C_{p_{a}}\left(T_{a}-T_{w}\right)}\right)$

Symbols used in equations (90), (91) and (92) denote:

$$
\begin{aligned}
& A_{f}=\text { fin area } \\
& A_{0}=\text { total external area } \\
& \mathrm{Cp}_{\mathrm{a}}=\text { specific heat of air } \\
& h_{c}=a i r-s i d e \text { forced convection heat transfer for wet air } \\
& h_{c, 0}=a i r-s i d e \text { forced convection heat transfer coefficient for dry air } \\
& \mathrm{h}_{\mathrm{D}, 0}=\text { air-side mass transfer coefficient } \\
& i_{f g, w}=1 \text { latent heat of condensation for water (frost sublimation) } \\
& \begin{aligned}
\mathrm{T}_{\mathrm{a}}^{\mathrm{W}} & =\text { temperature of } 1 \text { iquid water (frost) } \\
\mathbf{Q} & =\text { heat transfer rate }
\end{aligned} \\
& w_{a}=\text { humidity ratio of air } \\
& w_{w}^{a}=\text { humidity ratio of saturated air at } T_{w} \text { temperature } \\
& \phi=\frac{T_{W, m}-T_{a}}{T_{w, b}-T_{a}} \text {, fin efficiency } \\
& T_{w, m}=\text { mean temperature of water film (frost) } \\
& T_{w, b}=\text { temperature of water film (frost) at fin base }
\end{aligned}
$$


The one-dimensional heat conduction across the condensate (frost) film can be expressed by the equation:

$$
\mathrm{dQ}=\mathrm{h}_{\mathrm{L}} \cdot \Delta \mathrm{T}_{\mathrm{L}} \cdot \mathrm{dA_{0 }}
$$

where $\quad h_{L}=\frac{k_{W}}{\delta}$, heat transfer coefficient for the condensate (frost) film

$$
\begin{aligned}
k_{W}= & \text { thermal conductivity of water (frost) } \\
= & \text { thickness of condensate (frost) film (for evaluation of water } \\
& \text { and frost properties see Appendir B) } \\
\Delta \mathrm{T}_{\mathrm{L}}= & \text { temperature difference across the condensate (frost) film }
\end{aligned}
$$

Using equations (92) and (93) and ceferring to equation (81), the following relation for overall heat transfer coefficient for a wet finned tube can be derived.

$$
\delta=\left[\frac{A_{0}}{h_{i} A_{D, i}}+\frac{A_{0}{ }_{p}}{A_{p, m} k_{p}}+\frac{1}{h_{L}}+\frac{1}{h_{c, 0}\left(1+\frac{i_{f, D}\left(w_{Q}-w_{W}\right)}{C_{p_{Q}}\left(T_{Q}-T_{W}\right)}\right)\left(1-\frac{A_{f}}{A_{0}}(1-\phi)\right)}\right]^{-1}
$$

where symbols used are defined as in equations (81) and (92).

In the above formulation of the overall heat transfer coefficient, it is assumed that the temperature difference across the 1 iquid film (frost) is uniform. The calculated value for wet fin efficiency is affected by the change of the airside heat transfer coefficient caused by the air passage geometry alteration by liquid (frost) accumulation, and by the released latent heat of condensation (sublimation). The effect of water (frost) conductance on fin efficiency is neglected. In summary, the heat transfer phenomena that occurs diring dehumidification on the air-side may be itemized as foll ow : :

(1) the lager of wet (frost) offers additional heat flow resistance

(2) the airomide hoat transfer Iosistance is decreased due to effect of condensation.

(3) the airside heat transfer coefficient, $b_{c}$, has an increased value since it is sensitive to external surface geometry and the Reynolds number (

(4) Iin efficiency decreases as $h_{c}$ is increased (see figure 23).

(5) the cross sectionel area of the air flow passage between the fins has decreased, decreasing the flow rate.

In order to evaluate water (frost) layer thickness, consider the mass transfer equation:

$$
m_{a, d} \cdot d w_{a}=-h_{D, 0}\left(w_{Q}-w_{w}\right) d A_{0}
$$


For the Lewis number equal to 1 equation (95) assumes the following form:

$$
m_{a, d} \cdot d w_{a}=-\frac{h_{c, o}}{C p_{a}}\left(w_{a}-w_{w}\right) \cdot d A_{o}
$$

The change in the air humidity ratio can be calculated by integrating equation (96), which yields:

$$
w_{a, e}=w_{a, i}-\left(w_{a, i}-w_{w}\right)\left(1-\exp \frac{-h_{c, 0} \cdot A_{0}}{c_{p_{a}} \cdot m_{a, d}}\right)
$$

The rate of moisture removal per unit area, $R$, can now be calculated:

$$
R=m_{a, d}\left(w_{a, i}-w_{a, e}\right) / A_{0}
$$

where

$$
\begin{aligned}
& m_{a, d}=\text { mass flow rate of dry air } \\
& w_{a, e}=\text { humidity ratio of air at tube row exit } \\
& w_{a, i}=\text { humidity ratio of air at tube row inlet }
\end{aligned}
$$

If the evaporator temperature is below the freezing point, moisture removed from the air stream accumulates on the evaporator external surface in the form of frost. Its thickness, $\delta_{f}$, can be evaluated by integrating the rate of moisture removal with respect to time, i.e.,:

$$
\delta_{f}=\int_{0}^{t} \frac{R}{\rho_{f}} d t
$$

where

$$
\begin{aligned}
t & =\text { time } \\
R & =\text { rate of moisture removal per unit area } \\
\delta_{f} & =\text { frost layer thickness } \\
\rho_{f} & =\text { frost density }
\end{aligned}
$$

In case of evaporator temperature above $32^{\circ} \mathrm{F}$, condensate flows dow on the fin. Assuming no air drag on the liquid layer, its local velocity is expressed by the closed solution of the Navier-Stokes equation for a viscous flow on a vertical wal1 [39]:

$$
V_{z}=\frac{\rho g \delta^{2}}{2 \mu}\left[1-\left(\frac{Z}{\delta}\right)^{2}\right]
$$

where

$$
\begin{aligned}
V_{z} & =10 c a l \text { liquid layer velocity } \\
p & =1 \text { iquid density } \\
g & =\text { gravitational acceleration } \\
y & =\text { distance from the wall } \\
\delta & =1 \text { iquid layer thickness } \\
\mu & =1 \text { iquid absolute viscosity }
\end{aligned}
$$

Applying to the liquid film the continuity equation: 


$$
m(z)=\rho \int_{0}^{\delta} v_{z} d y
$$

and assuming uniform condensation rate on the fin (i.e., $m(z)=m * z / h$, where: $m(z)=$ mass flow rate of condensate at elevation $z, m=$ water condensation rate by a fin of height $h$ ), the average condensate layer thickness can be obtained by integrating a local layer thickness over the fin height and dividing the obtained expression by the height. The resulting expression is:

$$
\delta_{f}=1.082\left[\frac{\mu_{\mathrm{w}} \cdot \mathrm{R}^{\prime}}{\mathrm{g} \cdot \rho_{\mathrm{w}}{ }^{2}}\right]^{1 / 3}
$$

where

$$
\begin{aligned}
g & =\text { gravitational acceleration } \\
\mathbf{R}^{\prime} & =\text { condensation rate per unit width of a fin } \\
\mu_{m} & =\text { water dynamic viscosity } \\
\rho_{w} & =\text { water density }
\end{aligned}
$$

\subsubsection{Pressure Drop in a Tube}

As expressed by equation (50), the total pressure drop experienced by a floring fluid results from pressure drops due to friction, momentum change, and gravity. In an actual heat pump heat exchanger, pressure drop due to gravity effect is very small and may be neglected. Only pressure drop due to friction and due to momentum change will be considered for the different f1ow patterns in a tube.

\section{Single-Phase F10}

Frictional pressure drop for a single-phase turbulent flow in a tube can be calculated by the Fanning equation with the Fanning friction factor, equations (67) and (68):

$$
\begin{aligned}
& \Delta P=\frac{2 f \cdot G^{2} \cdot L}{D \cdot \rho} \\
& f=0.046 \operatorname{Re}^{-0.2} \quad \text { (for } \operatorname{Re}>2000,[22] \text { ) }
\end{aligned}
$$

Pressure drop due to momentum change can be calculated by the following equation:

$$
\frac{d P}{d L}=-G^{2} \frac{d v}{d L}
$$

$$
\text { where } \quad \begin{aligned}
& G=\text { refrigerant mass flux } \\
& \text { L }=\text { tube length } \\
& \quad V=\text { refrigerant specific volume }
\end{aligned}
$$

\section{Tro-Phase Flor with Condensation}

The frictional pressure drop for two-phase flow with condensation can be calculated by the method proposed by Lockhart and Martine11i [22]. They 
performed a semi-empirical study of adiabatic two-phase flow with air and different 1 iquids including benzene, kerosene, water, and various oils in tubes varying in diameter from 0.586 to 1.017 inch. They related the pressure drop of two-phase flow to the pressure drop of the liquid portion of the flow flowing alone in the pipe, by a dimensionless paraneter $x_{t}$, i.e..:

$$
\frac{\Delta P_{t p}}{\Delta P_{L}}=f\left(x_{t t}\right)=\Phi \quad \text { or } \quad \Delta P_{t p}=\Delta P_{L} \cdot \Phi
$$

where $\Delta P_{L}=$ frictional pressure drop of the liquid portion of two-phase flow flowing alone in the tube

$\Delta \mathrm{P}_{\mathrm{t}} \mathrm{p}=$ frictional pressure drop of two-phase flow

$\Phi^{t p}=$ correction factor for two-phase pressure drop

$\mathrm{X}_{\mathrm{tt}}=$ as given by equation (84)

The pressure drop, $\Delta \mathrm{P}_{L^{\prime}}$ is calculated by the single-phase pressure drop relation with the 1 iquid Reynolds number and friction factor calculated as follows :

$$
\begin{aligned}
& \operatorname{Re}_{t p, L}=\frac{(1-x) G \cdot D}{\mu_{L}} \\
& f_{t p, L}=0.046 \cdot \operatorname{Re}_{t p, L}-0.2 \quad\left(\text { for } \operatorname{Re}_{t p, L}>2000\right)
\end{aligned}
$$

where $\quad f_{t p, L}=$ friction factor for the 1iquid portion of two-phase flow flowing alone in the pipe

$$
\begin{aligned}
\operatorname{Re}_{t p, L}= & \text { Reynolds number for the two-phase liquid portion flowing } \\
& \text { alone in the pipe } \\
x= & \text { quality } \\
\mu_{L}= & \text { liquid dynamic viscosity }
\end{aligned}
$$

A correction factor for two-phase pressure drop, $\Phi$, was correlated by the following equation:

$$
\Phi=\exp \left(\sum_{i=1}^{5} A_{i} \cdot x_{t t}-0.25 \cdot i\right)^{2}
$$

where

$$
\begin{aligned}
& A_{1}=-0.418956 \\
& A_{2}=1.47330 \\
& A_{3}=0.668583 \\
& A_{4}=-0.321168 \\
& A_{5}=0.0408167
\end{aligned}
$$

Combining equations (106), (107), (108), and (109), the two-phase pressure drop equation assumes the form:

$$
\Delta P_{t p}=2 f_{t p, L} \cdot G^{2}(1-x)^{2} L \cdot \Phi /\left(D \cdot \rho_{L}\right)
$$

The pressure drop due to momentum change for separated two-phase flow can be estimated by the following equation: 


$$
\frac{d p}{d L}=-G^{2} \frac{d}{d L}\left(\frac{v_{V} \cdot x^{2}}{\alpha}+\frac{v_{L}(1-x)^{2}}{(1-\alpha)}\right)
$$

where.

$$
\begin{aligned}
\mathbf{x} & =\text { quality } \\
\mathbf{v}_{\mathbb{L}} & =\text { specific volume of liquid } \\
\mathbf{v}_{V} & =\text { specific volume of vapor } \\
\alpha & =\text { void fraction }
\end{aligned}
$$

Void fraction, $a$, percent of tube filled with vapor, was show in by Lockhart and Martinelli to be a function of $x_{t t}$ under any flow conditions for separated flow with both phases turbulent. Wallis [40] correlated their results in the following form:

$$
\alpha=\left(1+x_{t t} 0.8\right)^{-0.378}
$$

This expression was found to correlate well with data presented in [22] for values of $X_{t t} \leq 10$. For $X_{t t}$ greater than 10 , another curve fitted formula is used:

$$
a=0.823-0.157 \cdot \ln \mathrm{x}_{\mathrm{t} t}
$$

\section{Two-Phase Flow With Evaporation}

The Lockhart-Martinel1i method for pressure drop calculation of two-phase flow is widely used for adiabatic and condensing flows of single component refrigerants. However, it does not yield accurate prediction for evaporative flow. Instead of the Lockhart-Martinelli correlation, Anderson, Rich and Geary [41] recommended a method proposed by Pierre [42]. In order to evaluate the accuracy of this correlation for the $\mathbb{R} 13 \mathbb{B} 1 / R 152$ a mixture, pressure drop predictions were compared with laboratory data of evaporator tests performed in NBS enviromental chambers. It was found that this correlation underpredicted pressure drop by about 40 percent in a consistent manner. Until pressure drop of non-azeotropic mixtures is fully investigated Pierre's correlation will be used for calculation of pressure drop with a correction factor of 1.4 .

The correlation of Pierre based on experiments with refrigerants 12 and 22 has the following form:

$$
\Delta P=\left(f \frac{L}{D}+\frac{\bar{\Delta} z}{x_{m}}\right) G^{2} \cdot v_{m}
$$

where

$$
\begin{aligned}
D & =\text { inner tube diameter } \\
D & =\text { tube length } \\
\hat{I} & =\text { friction factor (calculated by equation }(115)) \\
\mathrm{z}_{\mathrm{m}} & =\text { mean quality } \\
\Delta \mathrm{x} & =\text { qual } \mathrm{ity} \text { change } \\
\mathrm{v}_{\mathrm{m}} & =\mathrm{v}_{\mathbb{L}}+\mathrm{z}_{\mathrm{m}}\left(\mathrm{v}_{\mathrm{V}}-\mathrm{v}_{\mathbb{L}}\right), \text { mean specific volume }
\end{aligned}
$$

The friction factor to be used in equation (114) was correlated by Pierre from his experimental data by the following erpixical equation valid for $\operatorname{Re} / K_{f}>1$ : 


$$
f=0.0185\left(K_{f} / \operatorname{Re}\right)^{0.25}
$$

where

$$
\begin{aligned}
\mathbb{K}_{\mathrm{f}} & =\frac{J \cdot i_{f g} \cdot \Delta z}{L} \text {, boiling number } \\
R e & =\frac{G \cdot D}{\mu_{L}}, \text { Reynolds number } \\
J & =\text { mechanical equivalent of heat }
\end{aligned}
$$

The correlation proposed by Pierre is in the conventional format for the single pressure drop formula. The first term of equation (114) is for frictional pressure drop while the second is for pressure drop due to change of momentum. The formula for the friction factor contains the Reynolds number divided by the boiling number, making the friction factor sensitive to vapor generation rate at the vapor-1iquid interface.

\subsection{Modeling of Additiona1 Heat Pump Components}

In the previous sections, modeling of the main heat pump components has been discussed. The analysis included a compressor, a constant flow area expansion device, a condenser, and an evaporator. Additional components that have to be considered for a more accurate heat pump performance prediction by the model are: the vapor suction and discharge lines, the 1 iquid 1 ine, and the four-way valve. Their modeling is briefly explained below.

\section{Vapor Lines}

A suction 1 ine connects the evaporator with the compressor. A compressor and a condenser are connected by a discharge line. Usually, single-phase flow exists in the vapor lines in the form of either saturated or superheated vapor. Heat transfer rates between the refrigerant vapor flowing in the vapor lines and ambient air can be calculated by a general heat transfer equation for a circular duct ith insulation [19]. Single-phase forced convection is assumed inside the tube (equation (82)) and free convection is assumed outside the tube. The following equation is used for calculation of the free convection heat transfer coefficient for a horizontal tube [43]:

$$
h=0.27\left(\frac{\Delta T}{D_{0}}\right)^{0.25}
$$

for Grashof numbers from $10^{3}$ to $10^{9}$.

where $\Delta T=$ temperature difference between tube wall and air $D_{0}=$ tube outside diameter

Pressure drop in vapor lines can be calculated by the single-phase pressure drop equation (67). 


\section{Liquid Line}

A liquid 1 ine connects the evaporator and the condenser. This 1 ine is filled with subcooled liquid or 10w quality two-phase flow. The pressure drop can be calculated by equations (67) or (108), depending on the flow pattern. Heat $108 s$ from the liquid line to the amblent air is neglected.

\section{Four-Way Valve}

The main function of a four-way valve is to direct refrigerant flow from the compressor to the indoor or outdoor coll deponding on the mode of operation (heating or cooling). The side effects of flow through a four-way valve are changes in the refrigerant thermodynamic state due to the pressure drop and heat exchange. Assuming for simplicity an adiabatic exterior wal1, all heat lost by the discharge refrigerant is gained by the saction refrigerant. The heat transfer rate and refrigerant pressure drop in the valve can be ovaluated by formulas similar to equations (45) and (55), respectively. Hoat transfer and pressure drop parameters in these formulas have to be found using a subroutine VALPAR and a four-way valve (heat pump) one test data as explained in Appendis $E$.

\section{Accumnlator}

Simplified schematic of an accumulator is shown in Figure 8. It is assumed that refrigerant experiences no pressure drop while flowing through the accumulator, and the accumulator is adiabatic. With these assumptions the refrigerant state at the accumiator inlet is equal to the state the outlet during steady-state operation. The main purpose of the simulation of the accumalator is the calcalation of mass of refrigerant contained in it. If superheated vapor is entering the accumulator, the accumulator is filled with vapor and calculation is straight forward. If wet vapor is entering the accumulator, refrigerant 1 iquid is collected in it and the liquid level has to be evaluated. The liquid level is found by evaluating the hydrostatic pressure that along with the dyumic pressure exerted at the oil return hole would cause refrigerant 1 iquid to flow through the hole at the rate that would change saturated vapor in the accumulator tube into a wet vapor of the inlet vapor quality.

The basic equations used in the accumalator subroutine, FACCUM, are:

$$
\begin{aligned}
& \mathrm{m}(1-\mathrm{x})=\mathrm{A} \cdot \mathrm{K} 2 \cdot \Delta \mathrm{P} \cdot \rho_{\mathrm{L}} \\
& \Delta \mathrm{P}=\mathrm{H} \cdot \rho_{\mathrm{L}} \cdot \mathrm{g}+0.5\left(\frac{\mathrm{I} \cdot \mathrm{m}}{\mathrm{A}}\right)^{2} \nabla_{\mathrm{V}}
\end{aligned}
$$

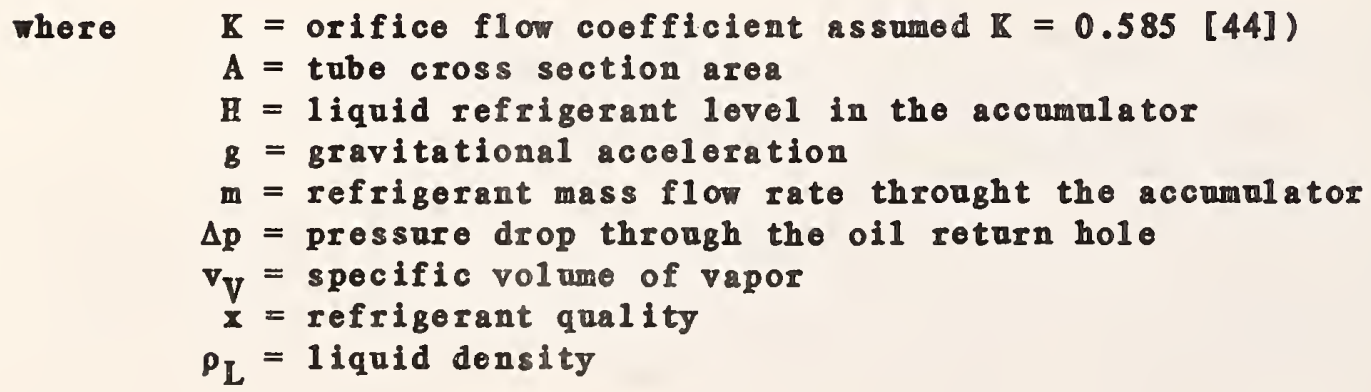


Equation (117) is the orifice equation applied to the oil return hole. In equation (118) the first term represents the hydrostatic pressure while the second term expresses the dynamic pressure exerted at the hole (velocity of the liquid is neglected).

Some accumolators have two holes in the accumalator suction 1 ine. In addition to the regular oil return hole at the bottom of the suction 1 ine, they have another hole located some distance (approzimately 1.5 inch) above. The accumalator subroutine is capable of simulating both types of accumators. In either case the subroutine is solving for the liquid level, $B$, using equations (117) and (118).

\subsection{Refrigerant Mass Inventory in a Heat Pump}

The mass, $M$, of a substance occupying a known volume, $\forall$, may be determined by:

$$
M=\int_{V} \rho \cdot d \Psi
$$

where

$$
p=10 c a l \text { density }
$$

In reality, the mass of refrigerant in the system cau be found by estimating the masses of the refrigerant in each system component and adding them up. For this purpose, equation (119) can be witten in the form:

$$
\begin{aligned}
& M=\sum M_{i} \\
& M_{i}=\forall_{i} \cdot \rho_{m, i}
\end{aligned}
$$

where

$$
\begin{aligned}
M_{i} & =\text { mass of refrigerant in particular component } i \\
\nabla_{i} & =\text { internal volume of component } i \\
\rho_{m, i} & =\text { mean fluid density in component } i
\end{aligned}
$$

In order to make mass inventory of individual mixture components, the following equation can be applied with the known mixture weight composition, $X$, of the considered refrigerant:

$$
M_{i, I}=M_{i} \cdot X T
$$

where $M_{i, I}=$ mass of refrigerant ' $I$ ' in heat pump component, ' $i$ '

The heat pump components taken into account in the mass inventory calculations are shown in figure 24. The refrigerant phase in each of the components as indicated in the figure, is based on the following considerations:

Discharge line - receives and is filled with superheated vapor from the compressor.

Condenser - receives superheated vapor from a discharge 1 ine. In the course of passage through the condenser tubes, vapor temperature is brought to the dew point temperature. Starting at this point, a thin condensed 1 iquid layer forms on the tube walls. Depending on the mass flux, this liquid film may bo swept and ontrained within the vapor as a mist forming a dispersed flow. With more condensed vapor, 


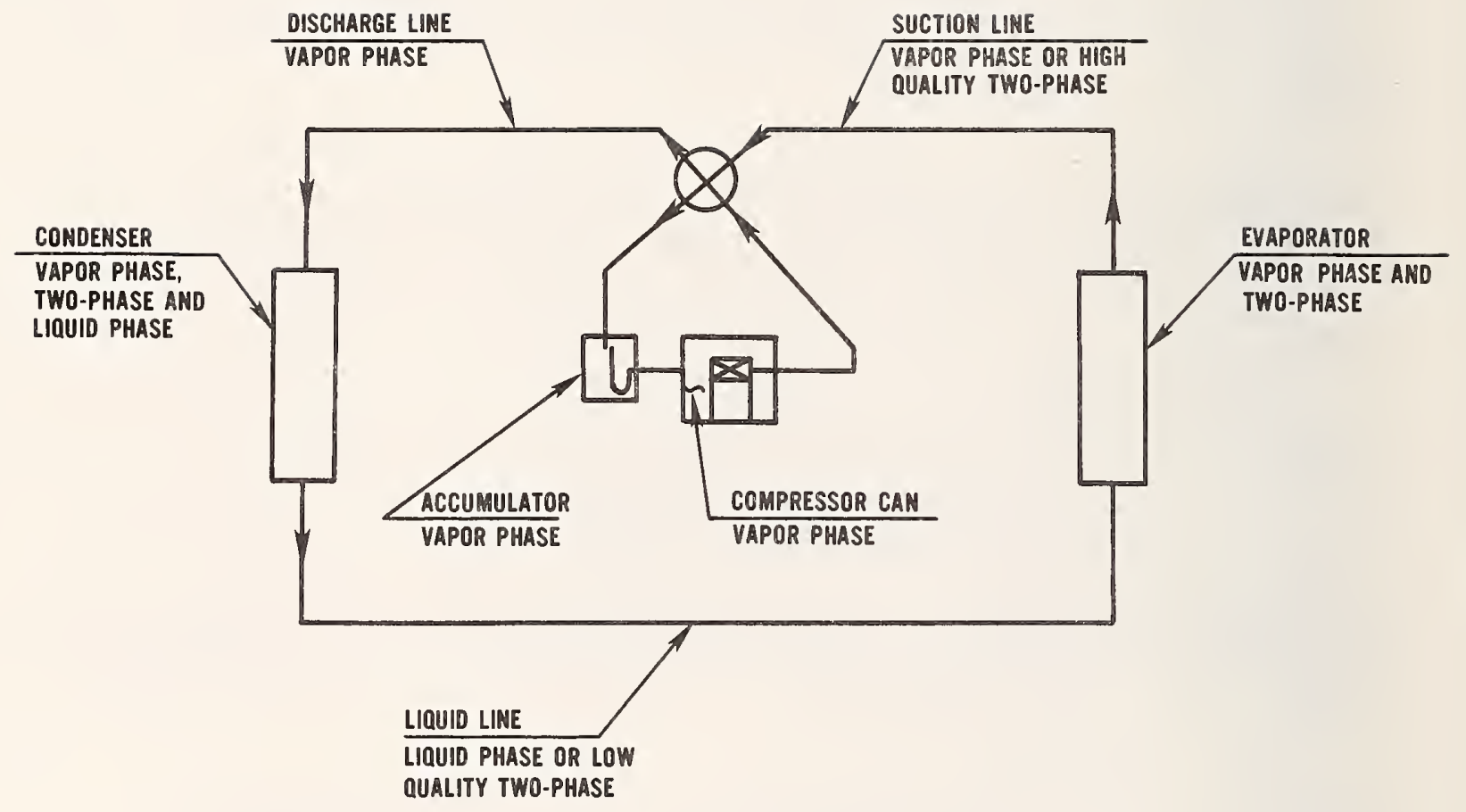

Figure 24. Refrigerant phase in the heat pump components. 
the velocity of vapor decreases, a permanent liquid film is formed on the wall, and the flow proceeds further as the annular or semiannular flow. The quality decreases and the flow slows down in the direction of flow. Gravity forces result in a stratifying effect and the 1 iquid flowing in the bottom of the tube periodically wets the upper tube wall (slug flow). If full condensation is reached, there will be subcooled liquid at the condenser exit.

Thus there are four two-phase flow patterns in the condenser, i.e., dispersed flow, annular flow, semi-annular flow, and slug flow as identified by $T r a v i s s$ and Rohsenow [45]. Soliman and Azer [46] identified nine flow regimes, however, five of them may be considered as transition regimes and can be put into the above basic four categories. The effect of return bends connecting condenser tubes was investigated in [47]. It was found that this effect is insignificant and that the annular flow pattern 'recovers' almost immediately in a tube after a return bend.

Liquid line - receives refrigerant from a condenser and delivers it to an expansion device. Entering the expansion device at the end of the liquid line, the flow experiences a sudden contraction. The entrance pressure drop depends upon the mass flow rate and the fluid density. This, along with the capillary tube sensitivity to the amount of subcooling, provides a flow controlling mechanism for the system. It is common practice to design a capillary tube so the refrigerant mass flow rate through it would balance with the mass flow rate through the compressor when a 1iquid seal exists at the capillary tube entrance. Temporary existence of vapor at the capillary tube would decrease the mass flow rate and build up the pressure. This would provide better conditions for condensation in the condenser and thus cause a return to a subcooled 1 iquid or very 1 ow quality two-phase flow in the 1 iquid 1 ine.

Expansion device - due to its small internal volume it may be disregarded for mass inventory purposes.

Evaporator - receives refrigerant from a capillary tube in the form of homogeneous flow of about 20 percent quality, at sonic or close to sonic velocity. This flow experiences a sudden deceleration and is assumed to form an annular or semi-annular flow very quickly having a void fraction about 0.8 [48]. The 1 iquid phase flows along the tube in the form of an annulus and the vapor phase flows in the core. This type of 1 ow prevails in most of the evaporator. With increasing quality, the velocity of the vapor increases and becomes eventually high enough to entrain small liquid drops and tear the liquid film apart. This results in a mist flow at qualities close to 1 . Finally, the flow may reach the evaporator exit in a superheated vapor state.

Suction line - receives refrigerant from the evaporator as a mist flow or superheated vapor flow and the same flow pattern exists at the exit.

Accumulator - receives the refrigerant from a suction line after some heat is added in the four-way valve. Superheated, saturated or wet vapor may be expected. With wet vapor entering, 1 iquid will be accunulated on the accumulator sump. 
Compressor can - receives refrigerant from the accumulator as a saturated or superheated vapor which is further superheated due to heat transfer from an electric motor, discharge 1 ine, and compressor cylinder body.

According to the above analysis, refrigerant in the compressor can, suction line, and discharge 1 ine is in (or close to) a homogeneous vapor state. In the 1 iquid 1 ine, homogeneous 1 iquid flow or two-phase flow exists but in a state so close to saturation that for simplicity of mass inventory no slip is assumed and the flow is considered to be homogeneous. The mass of refrigerant in these components can be then calculated straight forward in a similar manner since refrigerant densities in the components described above will be known as a result of the simulation program iteration process.

In order to calculate the mass of refrigerant in the accumulator, it is necessary to determine the portion of the internal volume filled with liquid. Once this is found, total refrigerant mass in the accumulator can be calculated based on refrigerant state parameters.

In the case of both coils, the refrigerant flow is partiy homogeneous, however, in most of the coil some type of annular flow prevails. For a separated flow regime, like an annular flow, density of the flowing fluid is determined from vapor and 1 iquid densities and the fractions of tube volume occupied by the 1 iquid and vapor phase:

$$
p_{\mathrm{m}}=\frac{\rho_{\mathrm{L}} \cdot \forall_{\mathrm{L}}+\rho_{\mathrm{V}} \cdot \forall_{\mathrm{V}}}{\forall_{\mathrm{t}}}=\rho_{\mathrm{L}}(1-\alpha)+\rho_{\mathrm{V}} \cdot a
$$

where

$$
\begin{aligned}
& M=\text { mass } \\
& \forall=\text { occupied vol ume } \\
& p=\text { density } \\
& \alpha=\forall_{V} / \Psi_{t}, \text { void fraction }
\end{aligned}
$$

subscripts refer to:

$$
\begin{aligned}
& \mathrm{L}=1 \text { iquid phase } \\
& \mathrm{m}=\text { mean value } \\
& \mathrm{t}=\text { total value } \\
& \mathrm{V}=\text { vapor phase }
\end{aligned}
$$

It should be pointed out that for a two-phase flow of a given quality, mean density depends not only on the thermodynamic parameters affecting densities of the liquid and vapor, but also on the ratio of mean velocities of the vapor and 1 iquid referred to often as the slip ratio. This can easily be noticed by eramining the equation for the void fractinn, which can be derived, considering mass flow rates of each phase:

$$
a=\frac{1}{1+\frac{1-x}{z} \frac{V_{V}}{V_{L}} \frac{\rho_{V}}{\rho_{L}}}
$$

where $\quad V=$ velocity 
Calculation of the void fraction has received much less attention than the calculation of heat transfer or pressure drop, although all three of these quantities are undoubtediy inter-related. The most of ten referenced method for void fraction calculation is that of Lockhart and Martine11i [22] based on their experimental void fraction data reported along with pressure drop results. They correlated void fraction with the dimensionless parameter, $X_{t t}$. Their experiment dealt with adiabatic flow, however, in the conclusions of their paper they suggested that their pressure drop correlation could be used for prediction of pressure drop during evaporation and condensation as well. On similar grounds, their void fraction data should be applicable beyond the adiabatic case.

There is another, earlier, method available for void fraction calculation by Martine11i and Nelson [49], derived with the same assumptions for water/steam evaporating flow. The method is similar to the Lockhart-Martinelli method so the Lockhart-Martinelli method was used in this study for calculation of the void fraction in both the evaporator and the condenser coils.

Correlations for void fraction based on [22] have already been given in section 5.4.8 (equations (112) and (113)).

$$
\begin{array}{ll}
\alpha=\left(1+x_{t t} 0.8\right)-0.378 & \text { for } x_{t t} \leq 10 \\
\alpha=0.823-0.157 \ln x_{t t} & x_{t t}>10
\end{array}
$$

The parameter $X_{t t}$, as defined by equation (84), is a function of flow quality, specific volume, and the viscosities of the liquid and vapor, and is not sensitive to the mass flux which in turn affects the slip ratio.

Experimental data of Staub and Zuber [48] for evaporating refrigerant 22 indicate that void fraction increases with increased mass flow rate. Comparison of their data with the void fraction predicted by the Martinelli-Nelson method showed a discrepancy which may lead to underestimation of the two-phase flow mean density by as much as 300 percent. (Note that a small difference in void fraction results in a large error in mean density prediction as density of the vapor and 1 iquid are vastly different.) The results of Staub and Zuber also indicate that approximate agreement with the Martinelli-Nelson could be obtained at high mass fluxes, more than five times higher than those observed in usual heat pump evaporators.

In discussing the accuracy of a refrigerant mass prediction method, it should be noted that it al so depends upon an accurate measurement of the internal volume of the system. Internal volume can be easy calculated for straight pipes. For valves, bends, etc., it can only be approximated.

Mass inventory of a heat pump system, as explained in Section 5.1, is intended to be used for iteration of refrigerant superheat at the compressor can inlet. The discussions above indicated that mass inventory calculations may have errors from two different sources. These sources are: inaccurate density prediction in the two-phase region, and inaccurate internal volume measurement. However, effect of these inaccuracies can be diminished since the program does not really need prediction of the absolute value of refrigerant mass in the system, but rather requires sensitivity in relative mass predictions in a heat pump with changes of operating conditions. This requirement 
should be satisfied by the Lockhart-Martinelli method and very precise knowledge of the internal volume should not be that important. Since the refrigerant vapor superheat at the compressor can inlet is known at the cooling design operating mode at conditions (usually: outdoor temperature $95^{\circ} \mathrm{F}$, indoor dry bulb temperature $80^{\circ} \mathrm{F}$, and wet bulb $67^{\circ} \mathrm{F}$ ) and does not have to be iterated for solution, refrigerant mass in the system can be calculated at this operating condition. This calculated mass of refrigerant may then be used as input data for refrigerant vapor superheat/quality iteration at other outdoor or indoor air conditions at which heat pump simulation results are required. 


\section{MODEL VERIFICATION}

A verification of the compter model has been done by comparison of computer predictions with perfornance data obtained from Iaboratory tests conducted in NBS environental chambers. In this sage of model development, laboratory data were available on one heat pum tested at two cooling and two heating standard rating operating conditions [50].

The sequence for runming laboratory tests was as follows:

1. DoE cooling test $A, T_{\text {ont }}=95^{\circ} \mathrm{F}$

indoor conditions: $80^{\circ} \mathrm{F} \mathrm{FB}, 67^{\circ} \mathrm{F}$.

refrigerant charge adjusted to obtain $10^{\circ}$ superheat at evaporator outlet

2. DoE cooling test $B, T_{\text {ont }}=82^{\circ} \mathrm{F}$

indoor conditions: $80^{\circ} \mathrm{F}, 67^{\circ} \mathrm{F}$ FB

3. DoE high temperature heating test

outdoor conditions: $47^{\circ} \mathrm{F} \mathrm{DB}, 43^{\circ} \mathrm{F}$ WB

indoor temperature: $70^{\circ} \mathrm{F}$

4. DoE 1 ow temperature heating test

outdoor conditions: $17^{\circ} \mathrm{F} \mathrm{DB}, 15^{\circ} \mathrm{F}$ WB

indoor temperature: $70^{\circ} \mathrm{F}$

The laboratory data of DoE cooling Test A were used first to obtain compressor and four-way $\nabla a 1 v e$ parameters as explained in Appendix $E$. Then corputer model runs were started with simulation of performance at DoE cooling test A conditions with the appropriate imposed superheat at compressor car inlet trat resulted in $10^{\circ} \mathrm{F}$ superheat at evaporator inlet as observed during laboratory test at this operating condition. Along with the performance results, refrigerant charge at the imposed superheat was obtained. This charge was then included in a heat pump data file to be used for iteration of superheat/ quality of vapor at compressor can inlet, and composition of the circulating refrigerant at other operating couditions.

The laboratory and simulation results are given in Table 3 for the cooling mode and in Table 4 for heating operation.

During cooling operation no liquid was collected in the accunulator and the composition of the mirture did not change. In the heating mode refrigerant 1 iquid was collected and a shift of composition of the circulating misture occured.

For both the cooling and heating modes, agreement between the resuls is very good, in fact, better than could be expected taking into account the number of simplifications used in the model, even the model is complez. It is not implied that this type of agreement can be obtained for every heat pump. It is believed that the accuracy of prediction stems partially from the effective cancellation of different simulation approximations, and this may not necessarily occur in simulation of every heat pump. However, the model 
proved to provide physically consistent predictions including evalustion of refrigerant superheat/quality at the compressor can inlet and the oirculating composition. Validation of this model with test data at other heat puns charged with different mixtures, when availablo, would further incroase confidence in the model.

Since two-phase heat transfer and pressure drop of non-azeotropic mixtures have not been fully presented in the 1 iterature and appropriate correlations are not available, the evaporative and condensation heat transfer coefficients and respective pressure drops are oalculated in the model by formulas that may provide approximate values of the parameters in question. To evaluate the impact of possible approximate prediction on the final results, a few simalation runs were performed in which the specific parameter calculated within the program was altered by a preset factor. Change of calculated system capacity and COP as compared to performance obtained with unaltered parameter describes sensitivity of the model on this particular parameter.

The following results were obtained. Increase of the evaporative heat transfer coefficient by $50 \%$ resulted in $2.5 \%$ increase of system capacity and $1.8 \%$ of system COP. Decrease of the evaporative heat transfer coefficient by $50 \%$ caused reduction in capacity and COP by $6.9 \%$ and $5.4 \%$, respectively. Increase in the condensation heat transfer coefficient by $50 \%$ increased capacity by $0.6 \%$ and $C O P$ by $1.1 \%$. The sane reduction in the condensation heat transfer coefficient caused reduction of system capacity by $2 \%$, and reduction of $\mathrm{COP}$ by $3.1 \%$.

Similar comparison was done for the evaporative and condensation pressure drops. Sensitivity of the final performance predictions was much smaller. In either considered case a change in pressure drop by $50 \%$ did not affect system capacity or $\mathrm{COP}$ by more than $1 \%$.

Comparison numbers given above were obtainea at indoor and outdoor air conditions specified for DoB cooling Test $A$. These findings should still apply for other operating conditions. Somewhat different results could be obtained simulating different systems. 


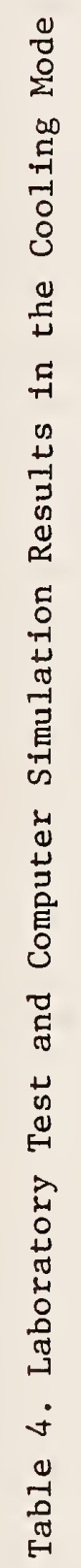

\begin{tabular}{|c|c|c|c|c|c|}
\hline 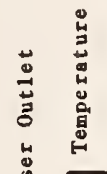 & 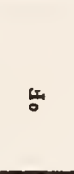 & $\stackrel{\bullet}{\dot{a}}$ & $\stackrel{\circ}{\doteq}$ & $\begin{array}{l}\dot{0} \\
\infty \\
\infty\end{array}$ & $\begin{array}{l}n \\
\dot{a} \\
\alpha\end{array}$ \\
\hline 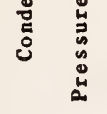 & $\vec{\Delta}$ & $\bar{a}$ & నี & Jे & I \\
\hline 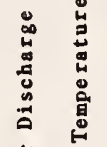 & .5 & $\stackrel{\sim}{\sim}$ & 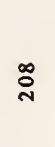 & $\cong$ & ळ \\
\hline 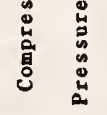 & $\stackrel{\vec{\Delta}}{\stackrel{\Delta}{\circ}}$ & $\stackrel{\text { Dे }}{\text { d }}$ & స్ & $\stackrel{m}{i}$ & $\tilde{\tilde{~}}$ \\
\hline 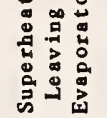 & 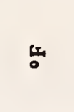 & $\hat{a}$ & $\hat{a}$ & $\stackrel{+}{9}$ & $\ddot{I}$ \\
\hline 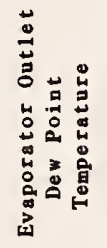 & $\frac{5 x}{0}$ & $\begin{array}{r}n \\
\dot{n}\end{array}$ & $\stackrel{\infty}{\stackrel{\infty}{*}}$ & $\begin{array}{l}\dot{0} \\
\dot{\sigma} \\
\dot{\sigma}\end{array}$ & $\stackrel{\infty}{\dot{q}}$ \\
\hline 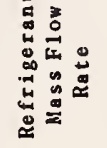 & $\stackrel{5}{\varrho}$ & ڤ్ & 怘 & $\tilde{n}$ & $\tilde{\tilde{n}}$ \\
\hline 獣 & 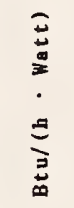 & $\stackrel{m}{\stackrel{m}{0}}$ & 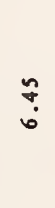 & $\stackrel{\overrightarrow{7}}{\circ}$ & $\stackrel{m}{\rightarrow}$ \\
\hline 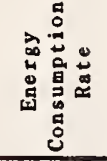 & $\begin{array}{l}\overrightarrow{\mathrm{a}} \\
\stackrel{\Delta}{*}\end{array}$ & 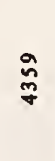 & ָี & $\stackrel{+}{+}$ & $\stackrel{\infty}{0}$ \\
\hline 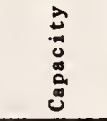 & 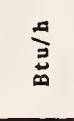 & 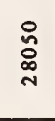 & \begin{tabular}{l}
$\stackrel{D}{\infty}$ \\
$\underset{\infty}{\infty}$ \\
\multirow{N}{*}{}
\end{tabular} & : & 莒 \\
\hline 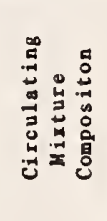 & 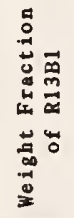 & $\tilde{n}$ & $\tilde{\varrho}$ & $\stackrel{n}{n}$ & $\tilde{n}$ \\
\hline \multicolumn{2}{|c|}{ 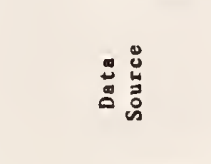 } & 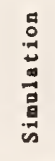 & 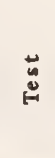 & 总 & 免 \\
\hline & เ & & & & \\
\hline
\end{tabular}




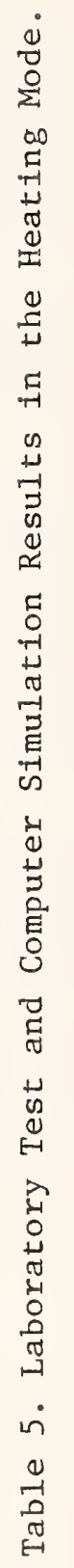

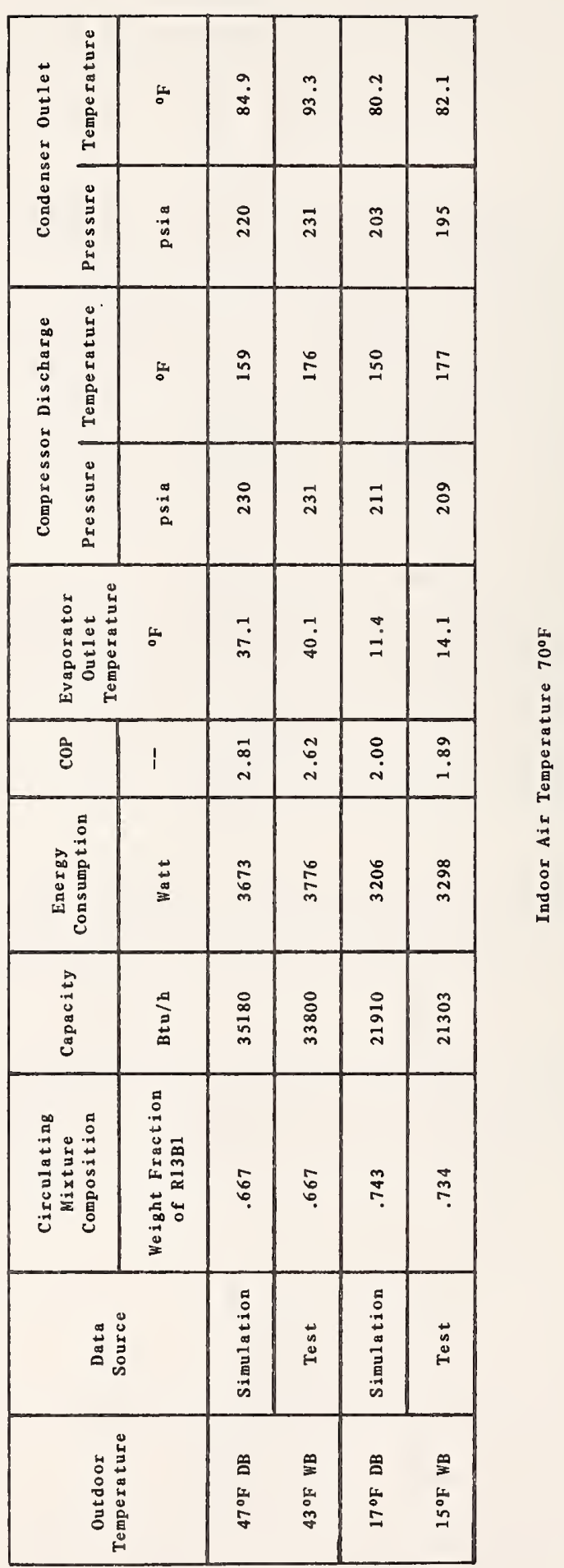




\section{SUMMARY AND CONCLUSIONS}

This report describes a model of a heat pump equipped with a constant flow area expansion device and operating with a non-azeotropic binary mirture refrigerant.

The model is able to simulate performance of a heat pump at imposed operating conditions without restriction on the refrigerant thermodynamic state at any point of the system. The simulation power includes simulation of the circulating mixture composition shift resulting from incomplete evaporation and accumulation of 1 iquid refrigerant in the accumulator.

The model has a modular structure; it consists of independent models of heat pump components linked together by appropriate logic iterating refrigerant thermodynamic states in thirteen key locations of a heat pomp thermodynamic cycle. Heat pump system component models were developed with emphasis on description of processes by fundamental heat transfer and pressure drop equations, and equation of state relationships among material properties. In the compressor model several refrigerant locations were identified and processes taking place between them accouted for all significant heat and pressure losses. Evaporator and condenser models vere developed on a tube-by-tube basis where performance of each coil tabe is computed separately by considering the cross-flow heat transfer with the external air-stream and the appropriate heat and mass transfer relationship. A constant flow area expansion device model was formulated with the aid of the Fanno flow theory.

The model utilizes an equation of state which is applicable for both liquid and vapor phases. Unlike the usual practice of using separate pressuresaturation temperature and liquid density-saturation temperature correlations with P-V-T relationship, one P-V-T relationship and physical equilibrium criteria are used in this project in establishing saturation state parameters and properties.

Since two-phase heat transfer and pressure drop of non-azeotropic mixtures have not been fully presented in the literature and appropriate correlations for their evaluation are not available, the evaporative and condensation heat transfer coefficients and respective pressure drops are calculated in the model by formulas either derived from a single component flow experiments or at best from limited experiments of one mixture only. There is certainly a need of research in these areas and this model should be updated when our knowledge about these phenomena is advanced.

The model was validated by comparing its performance predictions ith laboratory test results of one heat pump charged with R13B1/R152a mixture at two cooling and heating operating conditions. The data presented in this report, established our confidence in the model. The choice of R13B1/R152a for development and verification of this model does not mean recommendation of this mirture as an optimum and precludes other mirtures to be more suitable for heat pump application.

This report is intended for research and development engineers to provide a tool for evaluation of the potential of non-azeotropic binary mixtures in heat 
pumps. The User's Mannal of the developed program, HPBI, is included in the Appendix Section to enable readers to prepare input data for other heat pumps and to run the program even without detailed knowledge of the main section of this report. An example run and a listing of the program are also includsd.

HPBI program is applicable to the standard split residential system. It is, however, limited in the form presented here to the R13B1/R152a mixture due to a saturated refrigerant property spline which was incorporated in the program to decrease required computing time. Once a more efficient scheme is developed to use the equation of state directly, the spline will be removed along with present model 1 imitations.

The current version of the model can be used to evaluate performance of a heat pump charged with R13B1/R152a mixture at different operating conditions and different mixtare compositions. It is intended to be used, after slight modification, to predict the possible potentials of mixtures working in modified thermodynamic cycles. The computing time required by the program will vary with the size of the heat exchangers and the accuracy of estimated refrigerant parameters given as inputs. On the average, HPBI program run on Sperry 1100/82 computer requires 10 minutes of CPU time to converge $\mathbf{\text { ith }}$ iteration of superheat, and about 40 minutes to converge iterating refrigerant quality at the compressor can inlet and the circulating mixture composition. 


\section{REFERENCES}

1. Cooper, W.D., The Use of Mized Refrigerants in Air-to-Air Heat Pumps, ASHRAE Transaction, Vo1. 88, 1982.

2. Chi, J., A Computer Model HTPUMP for Simnlation of Heat Pump Steady-State Performance, National Burean of Standards, Internal Report, Mashington, D.C., 1979.

3. Domanski, P., and Didion, D., Computer Modeling of the Vapor Compression Cycle with Constant Flow Area Expansion Device, National Burean of Standards, Building Science Series 155, Washington, D.C., Mry 1983.

4. Cannon, H.A., and Drew D. W., Estimation and Application of Thermodynamic Properties for a Non-Azeotropic Refrigerant Mixture, IIR Meeting of Commissions B1, B2, E1 and E2, Essen, West Germany, September 1981.

5. Morrison, G., Francis, G., and Bland, T., An Equation of State for Refrigerant Mixtures, Report to EPRI, National Burean of Standards, March 1983.

6. Morrison, G., The Importance of Including the Liquid Phase in Equation of State for Non-Azeotropic Refrigerant Mixtures, ASHRAR Transactions, Vo1. 91. Part 1, 1985.

7. Baker, G.A, Gamme1, J.L., and W11s, J.G., Jouraal of Mathomatical Analysis Application, Vo1.2, p. 405, 1961.

8. Bakex, G.A., Advancos in Theoretieal Physies, Bxuecknor, K.A., od., Academic Press, New York, 1965.

9. Roid, R.C., Pransitz, J.M., and Shermood T.K., The Propertlos of Gases and Liquids, McGraw-H11 Book Company, New York, 1977.

10. Thermophysical Properties of Refrigerants, American Society of Heating, Refrigerating and Air Conditioning Engineors, Inc., Atlonta, Georgia, 1976.

11. Afshar, Ro, and Sazena, S.C., International Journal of Thermophysics, Vol. 1, 1980 .

12. Procoedings of the 1976 Purdue Compressor Technology Conference, Purdue Research Foundation, 1978.

13. Proceedings of the 1978 Purdue Compressor Technology Conference, Purdne Research Foundation, 1978.

14. Proceodings of the 1980 Purdue Compressor Technology Conferonce, Purdue Research Foundation, 1980.

15. American Society of Heating, Refrigerating and Air Conditioning Engineers, ASHRAE Guide and Data Book, Equipment Volume, New York, 1983. 
10. Threlke1d, J.L., Thermal Environmental Engineering, Prentice-Ha11, Inc., New Jersey, 1970.

17. Schultz, J.M. The Polytropic Efficiency of Centrifugal Compressors, ASME Transactions. Journal of Engineering for Power, Vo1. 84, January 1962.

18. Hirsch, S.R., On the Relation of Compressor Theory to Performance, ASHRAE Journa1, Ju1y 1973 .

19. American Society of Heating, Refrigeranting and Air Conditioning Engineers, Inc., ASHRAE Handbook, Fundamentals Volume, New York, 1985.

20. Colburn, A.P., A Method of Correlating Forced Convection Heat Transfer Data and a Comparison ith Fluid Friction, AIChE Transactions, Vo1.29, p. 174, 1930 .

21. Hiller, C.C. and Glickman, L.R., Improving Heat Pump Performance via Capacity Control - Analysis and Test, Report No. 24525-96, Heat Transfer Laboratory, Massachusetts Institute of Technology, Cambridge, Mas sachusetts, 1976.

22. Lockhart, R.W., and Martine11i, R.C., Chemical Engineering Progress, Vol. 45, pg. 39, 1949.

23. Bolstad, M.M. and Jordan, R.C. Theory and Use of the Capillary Tube Expansion Device, Refrigeration Engineering, Voi. 56, No.6, pg. 519, December 1948.

24. Miko1, E.P., Adiabatic Single and Two-Phase F1ow in Smal1 Bore Tubes, ASHRAE Journa1, Vol. 5, No. 11, November 1963.

25. Moody, L.F., Friction Factors for Pipe Flows, ASME Transactions, Vo1. 66 , No. 8, pg. 671, November 1944.

26. Cooper, L., Chu, C.K., and Brosken, W.R., Simple Selection Method for Capillaries Derived from Physical Flow Concitions, Refrigeration Engineering, Vo1.65, No. 7, pg. 37, July 1957.

27. Rezk, A.M.A., Investigation on F1ow of R12 Through Capillary Tubes, Paper presented on XV International Congress of Refrigeration, Venezia, September 23-29, 1979.

28. Pasqua, P.F., Metastable F1 ow of Freon-12, Refrigerating Engineering, Vo1. 61, No. 10, 1953.

29. Mei, V.C., Short-Tube Refrigerant F1 ow Restrictors, ASHRAE Transactions, Vo1. 88, Pt. 2, 1982 .

30. Kays, H.M., Loss Coefficient for Abrupt Changes in Flow Cross Section with Low Reynolds Number Flow in Single and Multiple-Tube Systems, ASME Transactions, Vo1. 72, No. 8, pg. 1067, November 1950.

31. Whitese1, H.A., Capillary Two-Phase F1ow, Refrigerating Engineering, Vol. 65, No. 4, pg. 49, Apri1 1957. 
32. Whitese1, H.A., Capil1ary Two-Phase F1ow, Part II, Refrigerating Engineering, Vo1. 65, No. 9, pg. 35, September 1957.

33. Erth, R.A., Two-Phase Flow in Refrigeration Capillary Tubes: Analysis and Prediction, Ph.D. Thesis, Purdue University, January 1970.

34. Carrier, W.H. and Anderson, S.W., The Resistance to Heat F1ow Through Finned Tubing, ASHVE Transactions, Vo1. 50, 1944.

35. Traviss, D.P., Baron, A.B., and Rohsenow, W.M., Forced-Convection Condensation Inside Tubes, Technical Report No. 72591-74, Massachusetts Institute of Technology, Cambridge. Massachusetts, 1971.

36. Briggs, D.E. and Young, E.H., Convection Heat Transfer and Pressure Drop of Air Flowing Across Triangnlar Pitch Banks of Finned Tubes, 5 th AICHE/ASME National Heat Transfer Conference, Houston, Tezas, 1962.

37. Jones, T.V. and Russe11, C.B.B., Heat Transfer Distribution on Annular Fins, AIAA-ASME Thermophysics Heat Transfer Conference, Palo Alto, California, May 1978, ASME Paper No. 78-HT-30.

38. Gardner, K.A., Efficiency of Extended Surface, ASME Transactions, Vo1. 67,1945 .

39. Bird, R.B., Steward, W.E., and Lightfoot, E.N., Transport Phenomena, John Wiley and Sons, Inc., 1980.

40. Wa11is, G.B., One-Dimensional Two-Phase F1ow, McGraw-Hil1, 1969.

41. Anderson, S.W., Rich, D.C., and Geary, D.F., Evaporation of Refrigerant 22 in a Horizontal $3 / 4$ - in. OD Tube, ASHRAE Transactions, Vo1.72, 1966 .

42. Pierre, B., F1ow Resistance with Boiling Refrigerants, ASHRAE Journal, September 1964.

43. McAdams, W.H., Heat Transmission, McGraw-Hi11, Inc., New York, New York, 1954.

44. Fox, R.W., McDonald, A.T., Introduction to Fluid Mechanics, J. Wiley and Sons, 1978 .

45. Traviss, D.P. and Rohsenow, W.M., F1ow Regimes in Horizontal Two-Phase F1ow with Condensation, ASHRAE Transactions, Vo1. 79, Part 2, pg. 31 , 1973.

46. Soliman, H.M. and Azer, N.Z., Flow Patterns During Condensation Inside a Horizontal Tube, ASHRAE Transactions, Vo1. 77, Part 1, pg. 210, 1971.

47. Traviss, D.P., and Rohsenow, W.M., The Influence of Return Bends on Downstream Pressure Drop and Condensation Heat Transfer in Tubes, Heat Transfer Laboratory, Massachusetts Institute of Technology, Cambridge, Mas sachusett s, 1971 . 
48. Staub, F.W. and Zuber, N., Void Fraction Profiles, Flow Mechanisms, and Heat Transfer Coefficients for Refrigerant 22 Evaporating in a Vertical Tube, ASHRAE Transactions, Vo1. 72, 1966.

49. Martine11i, R.C. and Ne1son, D.B., Prediction of Pressure Drop During Forced Circulation Boiling of Water, ASME Transactions, No. 6.

50. Federal Register, Part III, Test Procedures for Central Air Conditioners, Including Heat Pumps, Vo1. 44, No. 249, p. 76708, December 27, 1979.

51. Biguris, G. and Wenze1, L.A., Industrial Engineering Chemica1 Fundame ntals, Vo1. 9, No. 1, 1970 .

52. International Critical Tables, McGraw-Hill Book Co., pg. 216, New York, 1929. 


\section{APPENDIX A. CALCULATION OF PROPERTIES OF MOIST AIR}

For the selection of moist air property equations, it was assumed that air dry bulb temperature, $T$, relative humidity, $R H$, and pressure would be known, and the properties that would be the output were: humidity ratio, specific heat at constant pressure, gas constant, dynamic viscosity, and thermal conductivity. Presented here are the psychometric equations in their fundamental form. They are derived assuming moist air to be a mixture of two independent perfect gases, so that the perfect gas equation of state and Dalton's rule can be applied. The transport properties, dynamic viscosity, and thermal conductivity of air are assuned to be negligibly affected by the moisture content. Correlations for these two properties were based on dry air data.

Relations of this appendix were applied in air properties subroutine AIRPR.

The humidity ratio is determined by [16]:

$$
\mathrm{w}=0.622 \mathrm{PSAT} /(\mathrm{PATM}-\mathrm{RH} \cdot \mathrm{PSAT})
$$

where PATM = atmospheric pressure

$$
\begin{aligned}
\text { PSAT } & =\text { saturation pressure of water vapor at temperature } T \\
\text { W } & =\text { humidity ratio ( } 1 \mathrm{~b} \text { water/1b dry air) } \\
\text { RH } & =\text { relative humidity }(-)
\end{aligned}
$$

The saturated water vapor pressure, PSAT (psi), is calculated by the polynominal approximation [2]:

$$
\begin{aligned}
& \text { For } T>32^{\circ} \mathrm{F} \\
& \text { PSAT }=\exp \left(19.504-10.431 \mathrm{z}-0.2755 \mathrm{z}^{2}+0.03940 \mathrm{z}^{3}\right) \\
& \text { For } 32 \leq \mathrm{to}\left\langle 180^{\circ} \mathrm{F}\right. \\
& \text { PSAT }=\exp \left(13.4353-5.0988 \mathrm{z}-1.6896 \mathrm{z}^{2}+0.17829 \mathrm{z}^{3}\right) \\
& \text { For } \mathrm{T} \geq 180^{\circ} \mathrm{F} \\
& \text { PSAT }=\exp \left(16.8255-14.213 \mathrm{z}+7.5568 \mathrm{z}^{2}-4.01506 \mathrm{z}^{3}+0.17692 \mathrm{z}^{4}\right) \\
& \text { Where } \quad \mathrm{z}=\frac{1000}{460+\mathrm{T}}
\end{aligned}
$$

The specific heat at constant pressure is [16]:

$$
C_{p}=\left(C_{p_{d x y}}+0.444\right) /(1+w)
$$

where

$$
\begin{aligned}
\mathrm{Cp}= & \text { specific heat at constant pressure of moist air } \\
& (\text { Btu/(1b moist air } \mathrm{F})) \\
\mathrm{CP}_{\mathrm{dry}}= & \text { specific heat at constant pressure of dry air }(B t u /(1 \mathrm{~b} \cdot \mathrm{F})) \\
\mathrm{w} & \text { hmidity ratio (1b water/1b dry air) }
\end{aligned}
$$

The specific heat at constant pressure of dry air is approximated by the following polynominal [2]:

$$
\begin{gathered}
\mathrm{Cp}_{\mathrm{d} \text { ry }}=0.2478786-0.4204563 \cdot 10^{-4} \cdot \mathrm{TR}+0.567857 \cdot 10^{-7} \\
\cdot \mathrm{TR}^{2}-0.14936056 \mathrm{TR}^{3}
\end{gathered}
$$


where $\quad T R=T+460^{\circ} \mathrm{F}$ is the dry bulb air temperature on the Rankine scale

The gas constant is [16]:

$$
R=(53.34+85.76 \cdot w) /(1+w)
$$

where $\quad R=$ moist air gas constant $\frac{1 b f \cdot f t}{1 b \quad R}$

$$
w=\text { bumidity ratio (1b water/1b dry air) }
$$

The dynamic viscosity and thermal conductivity values are obtained from the respective equations [2]:

$$
\begin{aligned}
\mu= & 5.5029 \cdot 10^{-3}+8.7157 \cdot 10^{-5} \mathrm{TR}-2.9464 \cdot 10^{-8} \mathrm{TR}^{2} \\
& +6.25 \cdot 10^{-12} \mathrm{TR}^{3} \\
\mathrm{k}= & -2.853 \cdot 10^{-4}+3.268 \cdot 10^{-5} \mathrm{TR}-8.253 \cdot 10^{-9} \mathrm{TR}^{2} \\
& 1.239 \cdot 10^{-12} \mathrm{TR}^{3}
\end{aligned}
$$

where $\quad k=$ moist air thermal conductivity (Btu/(h $\cdot F \cdot f t)$ ) $T R=460+T=d r y$ bulb air temperature on the Rankine scale (R) 
APPENDIX B. CALCULATION OF VATER AND FROST PROPERTIES

The equations presented below are for calculation of the properties of water and frost deposited on heat pump evaporator outer surface. These properties, for specific conditions, are either solely a function of temperature or can be assumed to be constant. The equations below are used in the water and frost properties subroutine, WATPR.

The following fourth degree polynomial expression is used for calculating water density, conductivity, dynamic viscosity and latent heat of condersatio [2]:

$$
\text { PROP }=\sum_{I=1}^{5} A(I) \cdot T^{I-1}
$$

where

$$
\begin{aligned}
A(I) & =\text { five constants per calculated property } \\
\text { PROP } & =\text { calculated property } \\
T & =\text { water temperature (F) }
\end{aligned}
$$

Constants $A(I)$ for each property are given in Table B1. The specific heat of water at constant pressure is assuned to be independent of temperature and to have a constant value of $C_{p}=1 \frac{\mathrm{Bta}}{1 \mathrm{~b} \cdot \mathrm{F}}$

The density of frost, as proposed in [51], is calculated by the following equation:

$$
P_{f}=\exp \left(b_{1}+b_{2} \cdot(T P-T P)\right)
$$

where

$$
\begin{aligned}
& b_{1}=11.9521+0.02422 \mathrm{TPR}+35.5498 \mathrm{WA}-9.1742 \cdot 10^{-7} \mathrm{VA}+ \\
& 3.1138 \cdot 10^{-9} \mathrm{VA} \cdot \mathrm{TPR}-0.03838 \\
& \text { b2 }=13.1606-0.02133 \mathrm{TPR}-81.955 \mathrm{WA} / 32.018-\mathrm{TP} \\
& \text { TP }=\text { tube temperature (F) } \\
& T P R=T P+460 \text {. tube temperature (R) } \\
& T \text { F }=\text { water (frost) temperature (F) } \\
& \text { WA }=\text { air humidity ratio } \\
& V A=\text { air velocity (ft/sec) } \\
& P_{f}=\text { density of frost }\left(1 b / \mathrm{ft}^{3}\right)
\end{aligned}
$$

The frost conductivity, as proposed in [52], is calculated by the equation:

$$
k_{f}=0.012138+3.890910^{-3} \cdot p_{f}+5.140910^{-6} \cdot p_{f}
$$

where $\quad \mathrm{L}_{\mathrm{f}}=$ erost conductivity $(\mathrm{Btu} /(\mathrm{h} \cdot \mathrm{F} \cdot \mathrm{ft}))$

$$
P_{f}=\text { frost dexsity }\left(1 b / f t^{3}\right)
$$

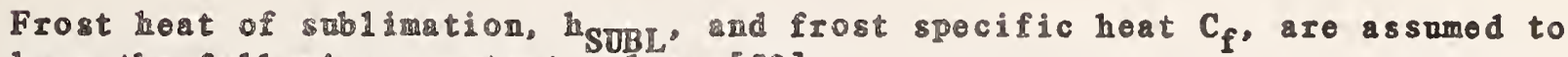
have the following constant values [52]:

$$
\begin{aligned}
\mathrm{h}_{\mathrm{SOBL}} & =1219.0 \mathrm{Btw} / 1 \mathrm{~b} \\
\mathrm{C}_{\mathrm{f}} & =0.46 \mathrm{Btu} /(1 \mathrm{~b} \cdot \mathrm{F})
\end{aligned}
$$


Tab1e B1. Water Property Evaluation Constants which are Used in Equation (B1)

\begin{tabular}{|c|c|c|c|c|}
\hline $\begin{array}{c}\text { Calculated } \\
\text { Property }\end{array}$ & $\begin{array}{c}\text { Density } \\
1 \mathrm{~b} / \mathrm{ft}^{3}\end{array}$ & $\begin{array}{c}\text { Dynamic } \\
\text { viscosity } \\
\mathrm{b} /(\mathrm{h} \cdot \mathrm{ft})\end{array}$ & $\begin{array}{c}\text { Thermal } \\
\text { conductivity } \\
\mathrm{Btu} / \mathrm{h} \cdot \mathrm{F} \cdot \mathrm{ft})\end{array}$ & $\begin{array}{c}\text { Latent heat } \\
\text { of } \begin{array}{c}\text { condensation } \\
\text { Btu/1b }\end{array}\end{array}$ \\
\hline $\mathrm{A}(\mathrm{I})$ & $0.11647 \mathrm{E}+03$ & $0.79422 \mathrm{E}+03$ & -0.27694 & $0.31514 \mathrm{E}+04$ \\
$\mathrm{~A}(1)$ & -0.40054 & $0.47589 \mathrm{E}+01$ & $0.45215 \mathrm{E}-03$ & $0.13714 \mathrm{E}+02$ \\
$\mathrm{~A}(3)$ & $0.10815 \mathrm{E}-02$ & $0.10622 \mathrm{E}-01$ & $0.49008 \mathrm{E}-05$ & $0.35945 \mathrm{E}-01$ \\
$\mathrm{~A}(4)$ & $0.12387 \mathrm{E}-05$ & $0.10416 \mathrm{E}-04$ & $0.88613 \mathrm{E}-08$ & $0.43525 \mathrm{E}-04$ \\
$\mathrm{~A}(5)$ & $0.49002 \mathrm{E}-09$ & $0.37690 \mathrm{E}-08$ & $0.41387 \mathrm{E}-11$ & $0.19695 \mathrm{E}-07$ \\
\hline
\end{tabular}




\section{APPENDIX C. CALCULATION OF CR ITICAL PRESSURE FOR TWO-PHASE FANNO FLOW OF A NON-AZEOTROPIC MIXTURE}

In this appendix, the procedure is explained for calculation of critical pressure of a flow if mass flow rate and stagnation enthalpy are given. The procedure focuses on the fact that Fanno flow assumes maximum entropy at the flow critical pressure. Hence, the point of maximum entropy on the Fanno line is being sought and once determined, the choking pressure is found.

The entropy of two-phase flow is calculated by the equation:

$$
s=s_{L}+x\left(s_{V}-s_{L}\right)
$$

where $\quad s=$ entropy

$x=$ quality

subscripts $L$ and $V$ refer to 1 iquid and vapor phase, respectively, being in equilibrium

The quality for Fanno flow can be found usino the energy equation:

$$
i_{0}=i+\frac{G^{2}}{2} \cdot v^{2}
$$

where

$$
\begin{aligned}
\mathbf{G} & =\text { mass flux } \\
\mathbf{i} & =\text { enthalpy } \\
\dot{i}_{0} & =\text { stagnation enthalpy } \\
\mathbf{v} & =\text { specific volume }
\end{aligned}
$$

Two-phase specific enthalpy and specific volume are:

$$
\begin{aligned}
& \mathbf{i}=\mathbf{i}_{\mathrm{L}}+\mathbf{x}\left(\mathbf{i}_{\mathrm{V}}-\mathbf{i}_{\mathrm{L}}\right) \\
& \mathbf{v}=\mathrm{v}_{\mathrm{L}}+\mathrm{x}\left(\mathrm{v}_{\mathrm{V}}-\mathrm{v}_{\mathrm{L}}\right)
\end{aligned}
$$

Substituting and rearranging, the following quadratic equation can be obtained [23]:

$$
x^{2}+x \cdot b+c=0
$$

where

$$
\begin{aligned}
& b=\frac{2\left(v_{V}-v_{L}\right) \cdot v_{L} \cdot G^{2}+i_{V}-i_{L}}{G^{2}\left(v_{V}-v_{L}\right)^{2}} \\
& c=\frac{2\left(i_{L}-i_{O}\right)}{G^{2}}+v_{L}^{2} \frac{1}{\left(v_{V}-v_{L}\right)^{2}}
\end{aligned}
$$

It should be noticed that saturated volumes of liquid and vapor and respective enthalpies are uniquely defined at a given pressure for a single component refrigerant. In such situation, refrigerant quality for a given Fanno flow can be calculated explicitly. When a non-azeotropic mixture is used, saturated liquid and vapor properties needed for evaluation of the quality are function of the quality thus an iterative procedure has to be used as shown 
in the figure $\mathrm{Cl}$. Once the mixture quality (thus also temperature) at the Fanno flow at a given pressure is known, entropies of the saturated 1 iquid and vapor are readily available and the flow entropy can be evaluated by equation $\mathrm{Cl}$.

The above described equations and procedures are applied in a double precision function, DFANNO. The Secant Method is used to provide the solution. 


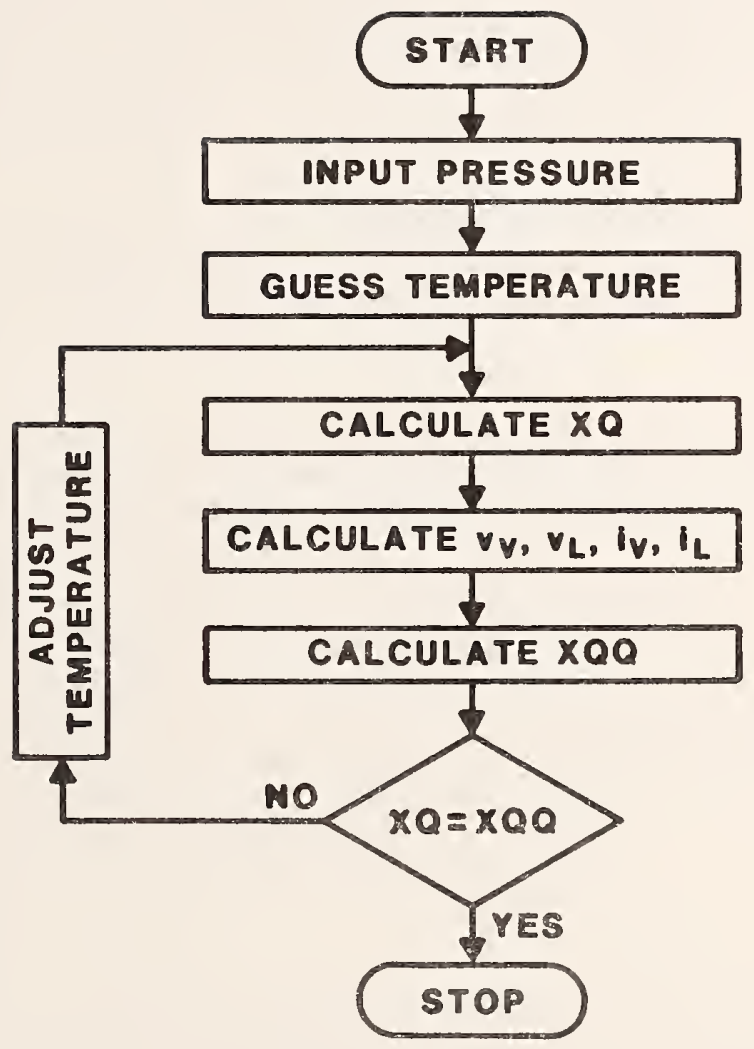

$$
\begin{aligned}
& X Q \text { - quality calculated by equation of state } \\
& X Q Q \text { - quality calculated by equation C5 }
\end{aligned}
$$

Figure C1. Logic to evaluate the quality of the Fanno flow at a given pressure for a non-azeotropic mixture. 

The principle of the logic for the heat pump model has been explained in section 5.1. It is based on four balances:

- enthalpy balance
- pressure balance
- mass balance of non-azeotropic mixture
- mass balance of one of the mixture components

The enthalpy balance and pressure (mass flow rate) balance are interdependent and have to be found in a simultaneous iteration process. Their solution provides refrigerant properties in the system which are required for mass inventory calculations. The balances are performed in the manner as show in figure D1, which presents the logic developed during this study and contained in the main program, BMAIN.

The objective of the logic is to iterate refrigerant states at key locations of a heat pump for given outdoor and indoor conditions. The addresses of the key locations used in the program BMAIN are consistent with those marked in figures 2 and D1:

1 - evaporator exit, suction tube inlet

2 - suction tube outlet, 1 ow pressure four-way valve inlet

3 - low pressure four-way valve exit, compressor can inlet

4 - inside compressor can

5 - compressor cylinder during suction stroke

6 - compressor cylinder during discharge

7 - discharge manif old

8 - compressor can exit, high pressure four-way valve inlet

9 - high pressure four-way valve exit, discharge tube inlet

10 - discharge tube outlet, condenser inlet

11 - condenser outlet, liquid 1 ine inlet

12 - 1 iquid out 1 et, expansion device outlet

13 - evaporator inlet

The thermodynamic processes shown in figure D1 are covered by five subroutines: COMPRE, CONDHX, BLINE, CAPIL, and EVAPHX. The iteration process starts with an estimated refrigerant pressure, superheat or quality at the compressor can inlet, and an estimated compressor discharge pressure (points 3 and 6): From these data, the compressor simulation subroutine COMPRE computes the refrigerant mass flow rate through the compressor and refrigerant parameters from point 1 to 10 . The condenser and 1 iquid 1 ine subroutines CONDHX and BLINE are called next to perform calculations which yield refrigerant states at point 11 and 12 . Subsequently, the expansion device simulation program, CAPIL computes refrigerant mas flow rate through the flow restrictor. At this point, the mass flow rate through the compressor, RMASS, and mass flow rate through the expansion device, XMASS, are compared and mass flow balance is sought. This is done by resuming calculations by COMPRE, CONDHX, BLINE, and CAPIL, holding constant refrigerant parameters at the compressor can inlet (point 3 ) and adjusting refrigerant saturation temperature at discharge (point 6) until an appropriate saturation temperature at point 6 is found at which RMASS and XMASS are equal. 
The evaporator performance simulation routine, EVAPHX, was chosen in this logic to be run after the mass flow balance between the compressor and expansion device has been found. (EVAPHX requires the most computing time of all the subroutines.) Evaporator simulation requires input of refrigerant mass flow rate and refrigerant state at the evaporator inlet. The refrigerant enthalpy at the evaporator inlet, H13, is equal to that at point 11, H11, since the thermodynamic processes in the 1 iquid 1 ine and flow restrictor are adiabatic. The refrigerant pressure at evaporator inlet, P13, is unknown and has to be guessed based on the known pressure at location 1 and the pressure drop across the evaporator (if known from the previous simulation of the evaporator). The output from EVAPHX is pressure and enthalpy at evaporator outlet, P1E and H1E, respectively. If the pressure P1E is not equal within imposed tolerance to $P 1$, pressure $P 13$ has to be adjusted and evaporator simulation repeated. After the condition of equal P1E and P1 pressures is satisfied, the refrigerant enthalpy at the evaporator outlet obtained from evaporator simulation, H1E, and enthalpy H1 are compared. If these enthalpies are not equal, the refrigerant saturation temperature at the compressor can inlet is adjusted and all calculations repeat.

Once an enthalpy balance and mass flow balance are established, two out of four refrigerant parameters estimated at the outset are temporarily obtained. To verify the third estimated parameter, i.e.. the refrigerant vapor superheat at the compressor can inlet, the refrigerant mass inventory in the system is used. Each time the mass inventory is performed, it provides a result based on refrigerant states in the system found after solving enthalpy and pressure balances with the assumed vapor superheat (quality) at points 3 and refrigerant mixture composition. If the amount of refrigerant obtained from the mass inventory calculations is less than the refrigerant in the system, the superheat (quality) estimate has to be decreased and all calculations have to be repeated from the beginning.

After the refrigerant mixture mass inventory agrees with mass of charged refrigerant, the mass inventory of one of the mixture components is made. If, based on this inventory, calculated circulating mixture composition is not equal (within imposed tolerance) to circulating composition estimated for use for simulation calculations, new estimate for the circulating composition is computed and all the calculations have to be repeated from the outset. If the equality between the estimated and calculated compositions is obtained, the iteration process is ended and the results are printed.

In the course of the computing process, the main program gathers information about the heat pump components' performance, updated at each iteration 1oop, and applies them to anticipate changes of some parameters caused by a change in some state property. This allows for the iteration process to converge faster with each iteration 1oop. The Secant Method is used in the solution of the two most internal loops and the external $100 \mathrm{p}$ of the main program. The Binary Search Method was applied to the mixture mass inventory 100 due to highly non-linear characteristic of the accumulator. 


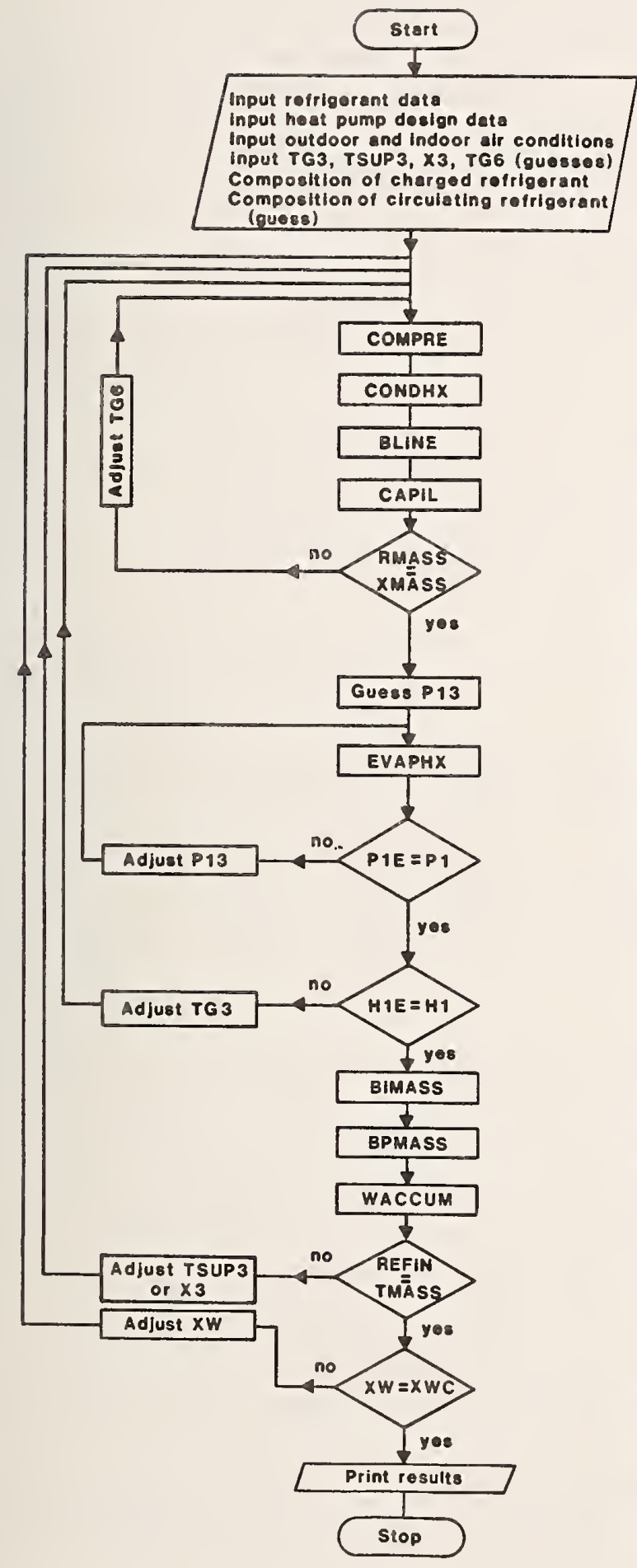

Symbols:

H1 - refrig. euthalpy at evaporator outlet calculated by COMPRE H1E - refrig. euthalpy at evaporator outlet caluclated by EVAPHX

H12 - refrig. enthalpy at poiut 12

H13 - refrig. euthalpy at point 13

Pl - refrig. pressure at evaporator outlet calculated by COMPRE

P1E - refrig. pressure at evaporator outlet calculated by EVAPBX

REFIN - mass of refrig. charged iuto a machiue

RMASS - refrig. mass flow rate through a compressor

TG3 - Iefrig. dew point temp. at point 3

TG6 - refrig. dew poiut temp. at point 6

TMASS - total mass of refrig. calculated by iuveutory program

TSUP3 - refrig. superheat at poiut 3

XMASS - refrig. mass flow rate throuth au expausiou device

$X W$ - circulatiug refrigeraut misture composition used for the calculatious

XWC - circulatiug refrigeraut mirture composition resultiug from the compouent refrigeraut mass inveutory

X3 - refrig. quality at point 3

BIMASS - calculates mass of refrigerant in a coil

Input: refrig. State at each coil tube eud

Output: mass of refrig, in a coil

BLINE - calculates pressure drop in a liquid liue

Input: RMASS and refrig. State at point 11

Output: refrig. state at poiut 12

BPMASS - Calculates mass of refrig. in a tube Input: refrig. state at tube ends

Output: mass of refrig. in a tube

CAPIL - calculates performace of au expausion device Input: refrig state at poiut 12 and pressure at point 13 Output: XMASS

COMPRE - calculates performace of a compressor with 4-way value ad tubiug conuectiug compresor with both coils

Input: refrig. State at point 3 aud pressure at point 6 Output: RNASS and refrig. state at poiuts 1 through 10

CONDHX - calculates performauce of a coudenser Iuput: RMASS aud refrig. state at point 10 Output: refrig. state at point 11

EVAPHX - calculates performance of a evaporator Input: RMASS aud refrig. State at poiut 13 Output: refrig. state at point 1

WACCUM - calculates mass of refrigerat in an accumulator Input: refrig. State iu au accumulator Output: mass of refrig. in a accumulator and mean refrig. composition

Figure D1. Overal1 logic of the program HPBI. 

Refer to figure 2 where the configuration of the main heat pump components is shown. It was decided to include in one subroutine called COMPRE all

refrigerant processes from point 1 to point 10. The refrigerant path covered by this subroutine consists of:

- flow through a suction tube 1-2 and discharge tube 9-10

- flow through a reversing valve on suction side $2-3$ and discharge side 8-9

- flow through a compressor 3-8

Input data to COMPRE is detailed in comment statements inserted in the program. Basically they consists of:

- connecting tubing design data

- compressor design and performance parameters

- four-way valve performance parameters

- refrigerant state at the compressor can inlet (point 3)

- refrigerant pressure after compressor (point 6)

The design input data are explained in Table H5. The compressor performance parameters include:

$\eta_{e}=$ electric motor efficiency versus $10 a d$

RPM = electric motor speed (RPM) versus load

$\eta_{m}=$ compressor mechanical efficiency

$\mathrm{C}_{\mathrm{e}}=$ compressor effective clearance

$\eta_{p}=$ compressor polytropic efficiency

CPE34, CPC45, CPC67, CPC78 = pressure drop parameters

CQC4C, CQCCOA, CQC45, CQC67, CQC78 = heat transfer parameters

One of the parameters, the compressor mechanical efficiency, $\eta_{m}$, is assumed here to be constant and equal to 0.96 . The rest of the parameters can be calculated by means of subroutine COMPAR using compressor test data. The efficiency, $\eta_{e}$, and RPM as functions of mechanical load are both part of typical electric motor characteristic for its class (see figures 11 and 13). Coefficients describing these curves are required as an input to COMPAR. In the course of calculations, new coefficients are computed which retain the shape of the characteristic curves, although changes in absolute values of efficiency, $\eta_{e}$, and RPM do occur according to test data supplied. The rest of the parameters (compressor effective clearance, compressor polytropic efficiency, five heat transfer parameters, and four pressure drop parameters) are calculated by COMPAR using relations presented in section 5.2.2.

Subroutine COMPAR is incorporated in the general program HPBI and is called by the main program, BMAIN. It is executed by running the HPBI program with appropriate run control data input as explained in Appendix $H$.

Four-way valve performance parameters are the pressure drop parameter and heat transfer parameter. These parameters are calculated by subroutine VALVPA using four-way valve test data. Similar to the compressor case, VALVPA is a part of the program HPBI and can be accessed by executing it with appropriate run control input data (refer to Appendix H). 
Organization of COMPRE may be followed by examining the program 1 isting with inserted comment statements. Based on a known refrigerant state at the can inlet (point 3), refrigerant parameters at the suction valve (point 5) are estimated and since the compression pressure is given, the compression process can be computed. Using the refrigerant mass flow rate from this computation and applying equations given in section 5.2.2, enthalpy and pressure balances are conducted. If balance is not reached, the estimate of the refrigerant state at the suction valve, point 5 , is adjusted. Calculations are repeated until balances are satisfied.

Once refrigerant states at compressor stations 3 to 8 and the mass flow rate are known, the properties for other refrigerant paths are computed. Simulation of the four-way valve and tubes are done by routines MVAL4 and PIPE, respectively.

The output from subroutine COMPRE is detailed in the program comment statements and consists of refrigerant thermodynamic properties for points 1 to 10 , refrigerant mass flow rate, compressor RPM, energy consumption rate, electric motor efficiency, compression efficiency, and volumetric efficiency. 
APPENDIX F. CONSTANT FLOW AREA EXPANSION DEVICE SUBROUTINE, CAPIL

The purpose of the capillary tube simulation subroutine is to calculate the refrigerant mass flow rate when the capillary dimensions, refrigerant inlet state, and pressure in the evaporator are given. Depending on the above input, there are several possible capillary tube operation modes. For example, the refrigerant can be either a single -or a two-phase state prior to the tube entrance. If there is a two-phase mixture at the inlet (a highly undesirable but possible condition under very low load operating conditions), two-phase flow will exist along the whole tube length.

For a subcooled liquid at the inlet, there are three flow alternatives:

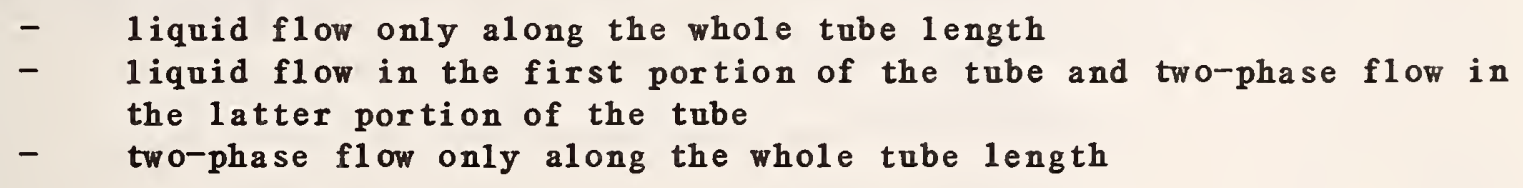

Each of the above flow situations can exist with or without choking condition at the tube exit. The capillary tube simulation routine, CAPIL can distinguish among all of the above operational modes. During the course of the calculations, the refrigerant critical pressure is found by routine CHOKE using a binary search iteration method. Then equations (62), (67), and (72) are used to compute the refrigerant mass flow rate. The density-pressure integral in equation (72) is evaluated by routine SIMP based on Simpson's rule. An iteration solution is required to obtain the final simulation result since choking pressure, friction factor, fractions of tube length with liquid and two-phase flow, and the velocity head used to correct enthalpy are functions of refrigerant mass flow rate which has to be found. The Secant liethod is used in the iteration for the final CAPIL solution. The logic of the routine CAPIL is presented in figure F1. The input and output data are 1 isted in comment statements in this routine. 


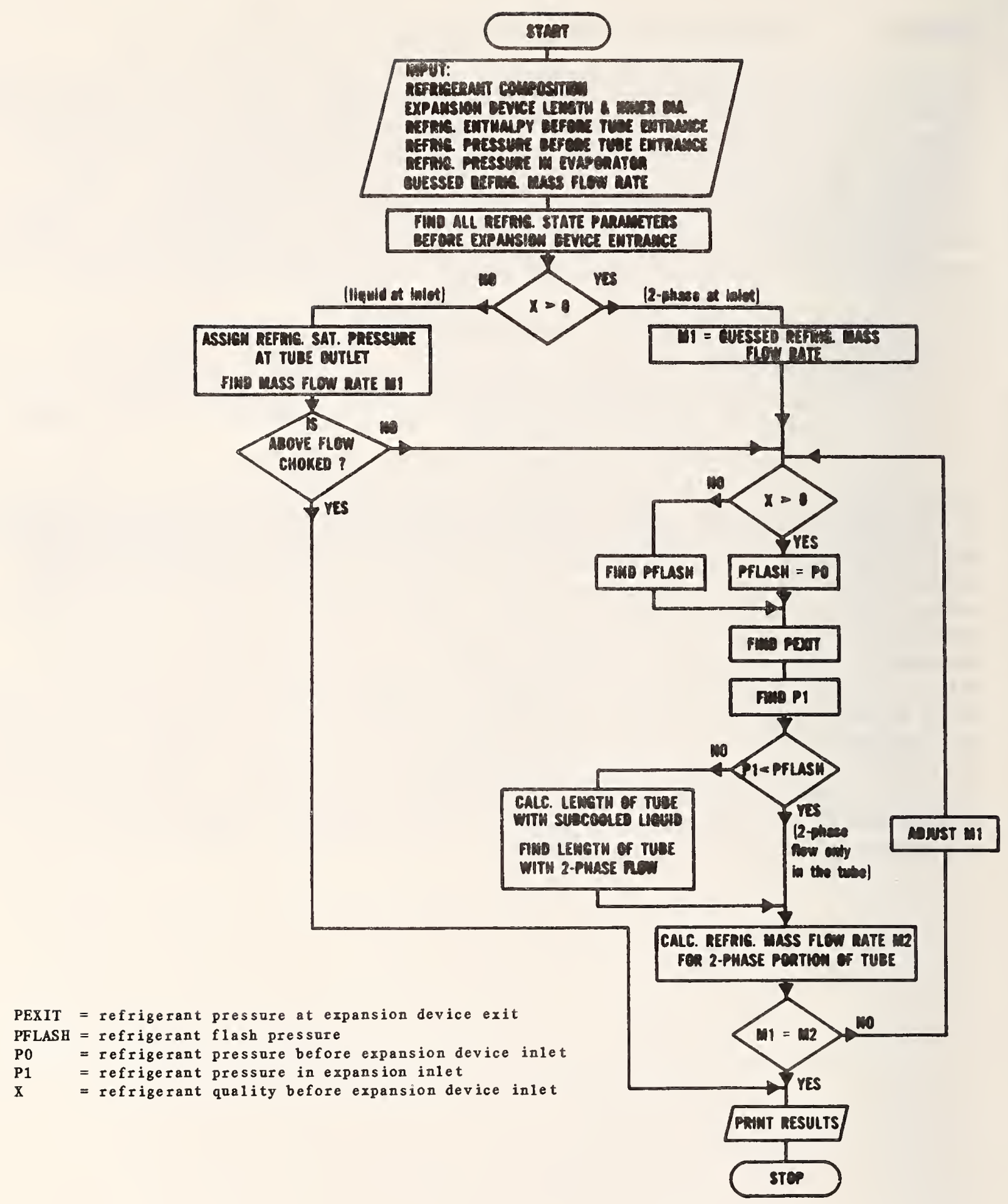

Figure F1. Logic of a constant flow area expansion device simulation program, CAPIL 
The tube-by-tube approach to coil simulation has been explained in section 5.4 where applicable heat, mass, and momentum transfer equations have also been presented. Though the evaporator and condenser simulation prograns require somewhat different heat transfer relations, their $\log$ ic is the same as shown in figure $\mathrm{Gl}$.

The input to coil simulation program are coil design data, refrigerant flor distribution sequence, and air and refrigerant inlet conditions. During the first bank of tubes' performance calculations, the air parameters apstream of each tube row are estimated. The program then computes the heat transer rate and pressure drop tube-by-tube from the inlet to the exit of the coil. Mean refrigerant properties in each tube are used to calculate heat transfer rates and pressure drops applying equations described in section 5.4. An iterative procedure is used for each tube since only refrigerant conditions at tube inlet are known when the calculations begin, and initially the average refrigerant properties in a tube have to be assumed to be those at the inlet conditions. Upstream air temperature and humidity ratio may not be accurately known at the time tube performance is being calculated, so a second iterative loop is used to update airside data after each loop. It should be realized that, while in the condenser case, update of air-side data means update of air temperature and humidity ratio; for the evaporator it al so includes 1 iquid (frost) layer thickness. Then refrigerant enthalpy values at the coil exit obtained from two consecutive loops are within the imposed tolerance, the calculations of coil performance are completed and results printed.

Both the evaporator coil simalation model EVAPHX and condenser coil simulation model CONDHX comply with the logic and equations previously described. Input and outpet data for these programs are detailed in comment statements at the beginning of each subroutine. Other inserted comment statements along with the flow chart (figure G1) facilitate the understanding of both program's organization. 


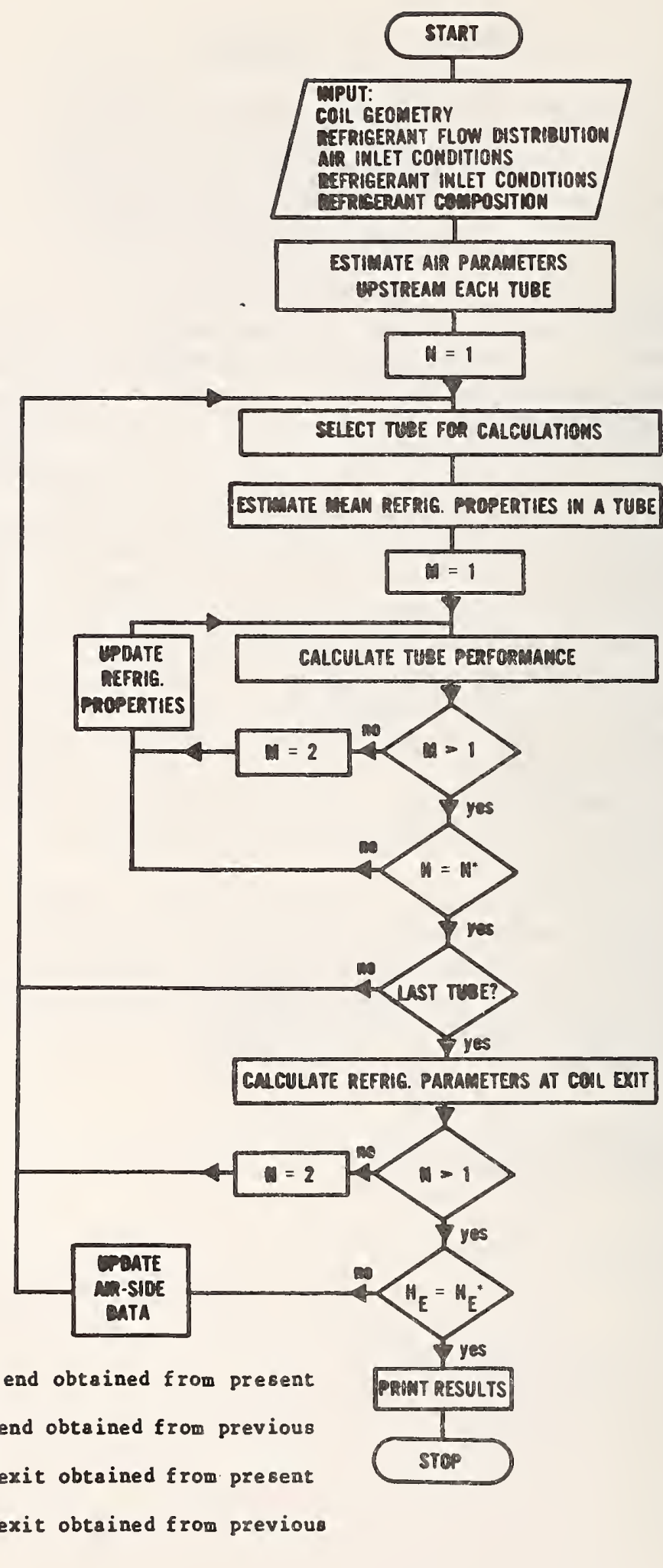

Figure G1. Flow chart for coil performance simulation programs. 


\section{H1. General Information}

The simulation program of a heat pump charged with a non-azeotropic binary mixture, HPBI, is written in Fortran 77 and makes use of standard Fortran mathematical functions. It is built around the main program, BMAIN, and 65 subprograms for heat pump component simulation, and heat transfer, fluid mechanics, and fluid property calculation. Relations used in the heat pump component simulation subprograms, f1uid mechanics, heat transfer and refrigerant property routines are described in the main text of this report. Equations used in moist air, water and frost property routines are described in Appendices $A$ and $B$.

Tab1es H1, H2, and H3 1ist, by categories, a11 programs used in the heat pump mode1. Short descriptions of applications are stated in the tables for each program. A 1 isting of all programs is given in Appendix J. A detailed description with specification of input and output parameters is given in the comment statements at the beginning of each program. Comment statements are also located within routines to facilitate understanding of the program organization. Organization of principal program modales is explained in Appendices $D, E, F$ and $G$.

\section{Hi2. Input and Output Data Coding}

There are five categories of input values for the HPBI runs, namely:

- refrigerant constants

- heat pump data

- run control1ing data

- indoor and outdoor air conditions

- refrigerant parameters

\section{H2.1 Refrigerant Constants}

Refrigerant constants for evaluation of thermodynamic properties are read into the program by subroutine BCONST from a disc file. File 7 is used in the program for input of these data. Table 14 presents a proper format of refrigerant data file. Comment statements inserted into subroutine BCONST help to identify specific constants.

Refrigerant constants for evaluation of transport properties are contained in function VISCON. Input of these data is not organized through reading of a data file since transport property constants may not have the same format for a11 mixtures. This may be due to different availability of data as well as different data possibly required by other kinds of mixing rules that could be more applicable for the new mixtures under consideration. Thus, if a new mixture is to be examined, function VISCON has to be redone from the source element.

\section{H2.2 Heat Pump Data}

Heat pump data are read into the program by the main program, BMAIN, from a disc file. File 8 is used in the program for input of heat pump data. Coding 
of these data is described in Table H5 which includes Fortran symbols with their explanation. An example of a heat pump data file is given in Table H6. Heat pump data include information about each modeled component, i.e., compressor, condenser, expansion device, evaporator, accumulator, four-way valve, vapor 1 ine and 1 iquid line. All components with the exception of the compressor and the four-way valve are described by design data only (dimensions, material properties, coil circuitry, etc.). The compressor and the four-way valve simulation also require performance parameters which are derived from test information using subroutines COMPAR and VALVPA, respectively, included in this report (see Appendix E). The subroutines are run by executing the HPBI program and providing appropriate run controlling data, as explained below in this appendix. Heat pump data also include the amount of refrigerant charge. This input is used in simulation runs in which iteration of refrigerant superheat (quality) at the compressor can inlet is required. For determining this input value refer to Chapter 5.6.

H2.3 Run Contro11ing Data, Indoor and outdoor Air Conditions, Refrigerant Parameters and Output Data

This category of input data have to be contained in a runstream file or are read from a terminal if simulation run is executed in the interactive mode. They are clearly requested by the program and responses have to be given in a Fortran free format.

The sequence of requests depends on the response to the first request given by the program to determine which one of three possible tasks the user wants the program to perform, i.e.,

1. evaluation of compressor parameters

2. evaluation of four-way valve parameters

3. simulation of heat pump performance

Evaluation of compressor parameters and evaluation of four-way valve parameters has to be done once to generate performance parameters of these components needed as input for simulation of performance of a heat pump system.

\section{Evaluation of Compressor Parameters}

The following is the sequence of program requests and explanation of reponses for evaluation of compressor parameters:

1. Reque st: COMPRESSOR PARAMETER (1), FOUR-WAY VALVE PARAMETERS (2)

Response: 1 OR HEAT PUMP PERFORMANCE (3)

2. Reque st: DETERMINE COMPRESSOR PERFORMANCE PARAMETERS ENTER: 0 FOR PARTIAL TEST DATA OR 1 FOR FULL TEST DATA =

Response: as explained in the request. Most 1 ikely user will not have detailed compressor test data. Assume then that response is 0 
3. Request: ENTER: ELEFUL, ELEIPT, RPMCP, SWPVOL, RMASS, TOA =

Response: ELEFUL = compressor motor energy input rate at max. rated 1 oad (kW)

ELEIPT = compressor motor energy input rate at test conditions (kW)

RPMCP = compressor number of revolution per minute at test ( $1 / \mathrm{min}$ ), enter 0 if not measured.

SWPVOL = total compressor displacement volume per revolution $\left(i^{3}\right)$

RMASS = refrigerant mass flow rate at test conditions $(1 \mathrm{~b} / \mathrm{h})$

TOA = ambient air temperature (F)

4. Request: WEIGHT COMPOSITION OF MIXTURE IN FRACTION OF MORE VOLATILE COMPONENT, XYT =

Response: weight composition (decimal fraction)

5. Request: ENTER: T3, P3 =

Response: $T 3$ = refrigerant temperature at compressor can inlet (F)

P3 = refrigerant pressure at compressor can inlet (psia)

6. Request: ENTER: T8, $P 8=$

Response: $\quad T 8=$ refrigerant temperature at compressor can inlet (F)

P8 = refrigerant pressure at compressor can inlet (psia)

At this point, the program will evaluate refrigerant state at key compressor locations and compressor parameters. Output symbols are explained in the comment statements in the beginning of subroutine COMPAR. Note, that evaluation of compressor parameters requires information on standard electric motor characteric (motor efficiency vs. load and RPM vs. load) that should be contained in the mass storage data file in 1 ines $2,3,4,5$ and 6 as explained in Table 115. This file should be assigned to number 8 for program execution.

\section{Evaluation of Four-Way Valve Parameters}

The following is the sequence of program requests and explanation of responses for evaluation of four-way valve parameters.

1. Request: COMPRESSOR PARAMETER (1), FOUR-WAY VALVE PARAMETRS (2) OR HEAT PUMP PERFORMANCE (3)

Response: 2

2. Request: EVALUATION OF FOUR-WAY VALVE PARAMETERS, ENTER: T2, P2, T3, $\mathrm{P3}=$

Response: $\quad T 2$ = refrigerant temperature at valve 1 ow pressure inlet (F)

P2 = refrigerant pressure at valve 1 ow pressure inlet (psia)

T3 = refrigerant temperature at valve 1 ow pressure outlet (F)

P3 = refrigerant pressure at valve 1 ow pressure out 1 et (psia) 
3. Request: ENTER: T8, P8, T9, P9=

Response: $T 8=$ refrigerant temperature at valve high pressure inlet (F)

P8 = refrigerant pressure at valve high pressure inlet (psia)

T9 = refrigerant temperature at valve high pressure outlet (F)

P9 = refrigerant pressure at valve high pressure outlet (psia)

4. Reque st: ENTER: RMASS, XW =

Response: RMASS = refrigerant mass flow rate at test conditions $(1 \mathrm{~b} / \mathrm{h})$

$X W=$ refrigerant weight composition (decimal fraction of the more volatile component)

At this point, the program will evaluate the four-way valve pressure drop parameter, CPD, and heat transfer parameter, CQ. (All output symbols are explained in the comment statements in the beginning of subroutine VALVPA.) Parameters CPD and $C Q$ are to be included in heat pump data file in 1 ine 10 as exp1ained in Tab1e H5.

\section{Simulation of Heat Pump Performance}

Simulation of heat pump performance is the ultimate purpose of the program HPBI. Once the heat pump data file is completed with the compressor and fourway valve performance parameters, simulation runs of heat pump performance can be conducted for a full range of operating conditions in the heating and cooling mode.

The following is the sequence of program requests and explanation of responses for the heat pump performance simulation run:

1. Request: COMPRESSOR PARAMETERS (1), FOUR-WAY VALVE PARAMETERS (2) OR HEAT PUMP PERFORMANCE (3)

Response: 3

2. Request: ANSWER 1 FOR YES OR 0 FOR NO

DO YOU WANT ANY INPUT DATA PRINTED? LPF =

Response: 0 for no input data print

or 1 for input data printout (request for specification of desired data will follow)

3. Request: OUTDOOR AND INDOOR AIR CONDITIONS, POA, TOA, RHOA, PRA, TRA, RHRA = ?

Response: $\quad P O A=$ outside air pressure (psia)

TOA = outside air temperature (F)

RHOA = outside air relative humidity (decimal fraction)

PRA = indoor air pressure (psia)

TRA = indoor air temperature (F)

RHRA = indoor air relative humidity (decimal fraction)

4. Request: NSYS $=1$ FOR HEATING, NSYS $=2$ FOR COOLING, NSYS $=$ ?

Response: as explained in the request 
5. Reque st: IS ITERATION OF SUPERHEAT/QUALITY REQUESTED?

ITER = 0 FOR NO, ITER = 1 FOR YES, ITER = ?

Response: as explained in the request

6. Request: IS ITERATION OF CIRCULATING COMPOSITION REQUESTED?

ITERXW = 0 FOR NO, ITERXW = 1 FOR YES, ITERXW?

Response: as explained in the request

7. Request: COMPOSITION OF CHARGED REFRIGERANT =

(WEIGHT FRACTION OF MORE VOLATILE COMPONENT)

Response: as explained in the request (decimal fraction)

8. Request: COMPOSITION OF CIRCULATING REFRIGERANT =

(WE IGHT FRACTION OF MORE VOLATILE COMPONENT)

Response: as explained in the request (decimal fraction)

9. Request: REFRIGERANT STATE ESTIMATES: TG3, X3, TSUP3, TG6

Response: TG3 = dew point temperature of refrigerant at the compressor can inlet (F)

X3 = refrigerant quality at the compressor can inlet (decimal fraction)

TSUP3 = refrigerant superheat at the compressor can inlet (F)

TG6 = dew point temperature of refrigerant vapor in compressor cylinder after compression

(a11 above values are estimates, subject to iteration)

At this point, the program starts to iterate the refrigerant thermodynamic states at heat pump key locations following the logic explained in Appendix $D$. Once a solution is obtained the results are printed in the format shown in a printout of an example run (Appendix I).

The output data consist of refrigerant thermodynamic states at 13 key heat pump locations, identified on figure 4, along with results describing the heat pump performance. Symbols used for output data have the following meaning:

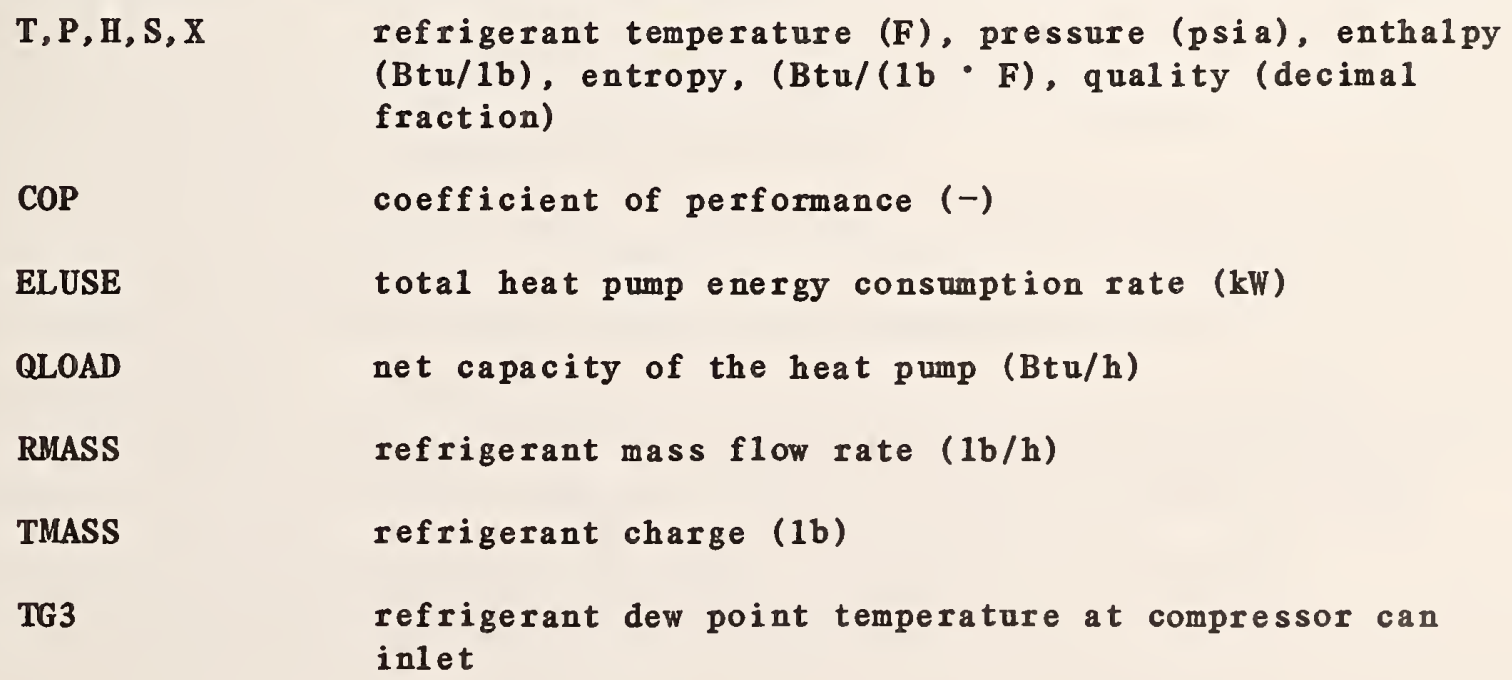



valve (F)

TSUP3

refrigerant superheat at compressor can inlet (F)

The last three output data TG3, TG6, and TSUP3 are final values iterated from TG3, TG6, and TSUP3 supplied as estimated input values.

It should be noted that the program prints the above results every time the enthalpy loop and pressure loop are closed, and proceeds with further calculations until the mass inventories of the mixture and one of mixture components are satisfied. The final results in the printout are the requested heat pump performance data at the imposed operating conditions. 
Tab1e H1. Refrigerant Property Sing1e Precision Functions and Subroutines

\begin{tabular}{|c|c|}
\hline NAME & SE \\
\hline BUBPRE & $\begin{array}{l}\text { Calculate bubble point pressure from a given composition and } \\
\text { temperature. }\end{array}$ \\
\hline BUBTEM & $\begin{array}{l}\text { Calculate bubble point temperature from a given composition and } \\
\text { pressure. }\end{array}$ \\
\hline DEWPRE & $\begin{array}{l}\text { Calculate dew point pressure from a given composition and } \\
\text { temperature. }\end{array}$ \\
\hline DEWTEM & $\begin{array}{l}\text { Calculate dew point temperature from a given composition and } \\
\text { pressure. }\end{array}$ \\
\hline EBUBPR & $\begin{array}{l}\text { Estimate bubble point pressure from a given composition } \\
\text { and temperature. }\end{array}$ \\
\hline EBUBTE & $\begin{array}{l}\text { Estimate bubble point temperature from a given composition } \\
\text { and pressure. }\end{array}$ \\
\hline ENTROP & $\begin{array}{l}\text { Calculate specific entropy of refrigerant being in a single } \\
\text { phase from given composition, temperature and specific volume. }\end{array}$ \\
\hline ENTR02 & $\begin{array}{l}\text { Calculate specific entropy from given composition, temperature } \\
\text { and pressure. }\end{array}$ \\
\hline EQPAR & Calculate parameters for the equation of state. \\
\hline ESVOL, & $\begin{array}{l}\text { Estimate specific volume of liquid or vapor from given } \\
\text { composition, temperature and pressure }\end{array}$ \\
\hline FGIBBS & Calculate Gibbs free energy \\
\hline HCVCP & $\begin{array}{l}\text { Calculate enthalpy, specific heat at constant volume and } \\
\text { specific heat at constant pressure from given composition, } \\
\text { temperature, and specific volume. }\end{array}$ \\
\hline HPAR & $\begin{array}{l}\text { Calculate parameters for calculation of specific enthalpy, } \\
\text { specific heat at constant volume, specific heat at constant } \\
\text { pressure and specific entropy. }\end{array}$ \\
\hline HPIN & $\begin{array}{l}\text { Calculate temperature from given composition, enthalpy and } \\
\text { pressure. }\end{array}$ \\
\hline HPPROP & $\begin{array}{l}\text { Calculate thermodynamic and transport properties from given } \\
\text { composition, pressure and enthalpy. }\end{array}$ \\
\hline PXQIN & $\begin{array}{l}\text { Calculate temperature of two-phase refrigerant from given } \\
\text { composition, pressure and quality. }\end{array}$ \\
\hline PXQIN2 & $\begin{array}{l}\text { Calculate temperature and enthalpy of two-phase refrigerant } \\
\text { from given composition, pressure and quality. }\end{array}$ \\
\hline QLITY & $\begin{array}{l}\text { Calculate quality of R13B1/R152a mixture from given } \\
\text { composition, temperature and pressure. }\end{array}$ \\
\hline SATCOM & $\begin{array}{l}\text { Calculate pressure, specific volume of } 1 \text { iquid and specific } \\
\text { volume of vapor of mixture pure components at saturation from } \\
\text { given temperature. }\end{array}$ \\
\hline SPIN & $\begin{array}{l}\text { Calculate temperature from given composition, entropy, and } \\
\text { pressure. }\end{array}$ \\
\hline SATLIB & $\begin{array}{l}\text { Estimate saturation pressure, specific volume of } 1 \text { iquid and } \\
\text { specific volume of vapor at given temperature for pure components. }\end{array}$ \\
\hline TPPROP & $\begin{array}{l}\text { Calculate specific enthalpy, specific volume and quality from } \\
\text { given composition, temperature and pressure. }\end{array}$ \\
\hline TXQIN & $\begin{array}{l}\text { Calculate pressure of two phase refrigerant from given } \\
\text { composition, temperature, and quality. }\end{array}$ \\
\hline
\end{tabular}




\begin{tabular}{l|l} 
TXQIN2 & $\begin{array}{l}\text { Calculate pressure and specific enthalpy of two-phase } \\
\text { refrigerant from given composition, temperature and quality. } \\
\text { VISCON }\end{array}$ \\
volite absolute viscosity and thermal conductivity of 1 iquid \\
or vapor and specific heat of saturated liquid of R13B1/R152a \\
mixture from given composition and temperature. \\
Calculate specific volume from given composition, temperature \\
and pressure.
\end{tabular}


Table H2. Refrigerant Property Double Precision Function and Subroutines

\begin{tabular}{l|l} 
NAME & PURPOSE \\
\hline DBUBTE & as BUBTEM (Table H1) \\
DDEWTE & as DEWTEM (Table H1) \\
DENTRO & as ENTROP (Table H1) \\
DH & $\begin{array}{l}\text { Calculate specific enthalpy from given composition, temperature } \\
\text { and specific volume. }\end{array}$ \\
DQLITY & $\begin{array}{l}\text { as QLITI (Table H1) } \\
\text { as VOLIT1 (Table H1) }\end{array}$
\end{tabular}


Table H3. Functions and Subroutines for Heat Pump Component Simulation, Heat Transfer and Fluid Mechanics Calculations

\begin{tabular}{|c|c|}
\hline NAME & PURPOSE \\
\hline AIRHT & Calculate finned tube air-side heat transfer coefficient. \\
\hline AIRPR & Calculate air properties. \\
\hline BCONST & Read refrigerant constants for equation of state. \\
\hline BIMASS & Calculate mass of refrigerant in a coil. \\
\hline BLINE & Calculate frictional pressure drop in a liquid line. \\
\hline BMAIN & Main program, solution logic contained. \\
\hline BPMASS & Calculate mass of refrigerant in a tube. \\
\hline BSIMP & Integrate numerically using Simpson's $1 / 3$ Rule. \\
\hline CAPIL & Simulate performance of a constant flow area expansion device. \\
\hline CHOKE & $\begin{array}{l}\text { Calculate the thermodynamic critical pressure for a non- } \\
\text { azeotropic mixture in two-phase Fanno flow. }\end{array}$ \\
\hline COMPAR & Evaluate compressor parameters. \\
\hline COMPRE & Simulate compressor performance. \\
\hline CONDHX & Simulate condenser performance. \\
\hline DDENFA & $\begin{array}{l}\text { Calculate refrigerant density for two-phase Fanno flow (double } \\
\text { precision). }\end{array}$ \\
\hline DFANNO & $\begin{array}{l}\text { Calculate refrigerant entropy for two-phase Fanno flow (double } \\
\text { precision). }\end{array}$ \\
\hline DPDYN1 & $\begin{array}{l}\text { Calculate dynamic pressure drop for a single-phase flow in a } \\
\text { tube. }\end{array}$ \\
\hline DPDYN2 & Calculate dynamic pressure drop for a two-phase flow in a tube. \\
\hline EVAPHX & Simulate evaporator performance. \\
\hline EVDP & Calculate frictional evaporation pressure drop. \\
\hline FINEFF & Calculate fin efficiency. \\
\hline FEELIQ & $\begin{array}{l}\text { Calculate Lockhart-Martinelli correction factor for two-phase } \\
\text { pressure drop. }\end{array}$ \\
\hline HTCCON & Calculate condensation heat transfer coefficient. \\
\hline ITCEV & Calculate evaporation heat transfer coefficient. \\
\hline HXCODE & Determine refrigerant and air flow distribution in a tube. \\
\hline MVAL4 & Simulate four-way valve performance. \\
\hline OVLHTC & $\begin{array}{l}\text { Calculate overall heat transfer coefficient for a dry finned } \\
\text { tube. }\end{array}$ \\
\hline OVLWET & $\begin{array}{l}\text { Calculate overall heat transfer coefficient for a wet finned } \\
\text { tube. }\end{array}$ \\
\hline PFLASH & Calculate flashing pressure and temperature for a Fanno flow. \\
\hline PIPE & Simulate flow through a tube. \\
\hline SPHDP1 & Calculate frictional single-phase pressure drop in a tube. \\
\hline SPHTC & Calculate single-phase heat tranfer coefficient in a tube. \\
\hline VALVPA & Calculate four-way valve parameters. \\
\hline WACCUM & Calculate mass of refrigerant in accumulator. \\
\hline WATPR & Calculate water properties. \\
\hline
\end{tabular}


Table H4. Data File Containing Constants for Evaluation of Thermodynamic Properties of R13B1/R152a Mixture

$25.4145,-0.063368,4.140051 E-05$

$0.1353977,-1.50409 \mathrm{E}-04,-1.354434 \mathrm{E}-07$

$27.392729669,-0.0594211671,3.317695607 E-5$

$0.1239878,-1.445514007 E-4,-1.90223806 E-8$

$0.1466,-2.241 E-04$

$148.93,66.05$

$340.15,386.65$

$10.0522804,-2204.5632,9636.5313$

$-0.05060051,1.1455764 \mathrm{E}-03,-2.56392871 \mathrm{E}-06$

$0.2749422,-1.702569 E-03,3.71008313 E-06$

$10.6410518,-2642.8994,460.87585$

$0.0218192958,5.416820778 E-04,-1.24731336 E-06$

$0.1023688715,-4.0752759 E-04,1.0409447 E-06$ 
Table H5. Heat Pump Input Data Code to Program HPBI

All input data are in FORTRAN free field input format with data values on the same 1 ine separated by commas.

Line 1: ATITLE

ATITLE $=$ title, maximum 80 characters

Line 2: $\quad \operatorname{EMETA}(I), I=1,5$

EMETA(I) = compressor motor effiiency in fraction at fraction of full load specified by EMOPT(I), $I=1,5(-)$

Line 3: $\quad \operatorname{EMETA}(I), I=6,11$

EMETA(I) = compressor motor efficiency in fraction at fraction of

Line 4: $\quad \operatorname{EMOPT}(I), I=1,5$

ful1 load specified by $\operatorname{EMOPT}(I), I=6,11(-)$

Line 5: $\quad \operatorname{EMOPT}(I), I=6,11$

EMOPT $(I)=$ compressor motor full load fraction (decimal fraction)

Line 6: $\quad \operatorname{EMRPM}(I), I=1,6$

Line 7: ELEFUL, SW PVOL, ETAPLY, CLREFF

ELEFUL = compressor motor energy input rate at max. rated $\frac{1}{3}$ oad (kW)

SWPVOL = compressor displacement volume per revolution (in ${ }^{3}$ )

ETALPY = compressor polytropic efficiency (-)

CLREFF = compressor clearance volume as fraction of displacement vol une (-)

Line 8: $\quad$ CPC34, CPC45, CPC67, CPC78

CPC34 = pressure drop parameter at compressor can inlet $\left(\left(1 \mathrm{bf} \cdot \mathrm{h}^{2}\right) /\left(1 \mathrm{~b} \cdot \mathrm{in} \mathrm{n}^{2} \cdot \mathrm{ft}^{3}\right)\right)$

CPC45 = pressure drop parameter at compressor suction valve $\left(\left(1 \mathrm{bf} \cdot \mathrm{h}^{2}\right) /\left(1 \mathrm{~b} \cdot \mathrm{in} \mathrm{n}^{2} \cdot \mathrm{ft}^{3}\right)\right)$

CPC67 = pressure drop parameter at compressor discharge valve $\left(\left(1 b f \cdot h^{2}\right) /\left(1 b \cdot i n^{2} \cdot f^{3}\right)\right)$

CPC78 = pressure drop parameter at compressor can exit

$\left(\left(1 b f \cdot h^{2} .2\right) /\left(1 b \cdot i n^{2} \cdot f t^{2.8}\right)\right)$

Line 9: CQC4C, CQCCOA, CQC45, CQC67, CQC78

CQC4C = parameter for compressor can wall - refrigerant vapor heat transfer $\left(\mathrm{ft}^{0.2}\right)$

CQCCOA = parameter for compressor can - ambient air heat transfer $\left(\mathrm{Btu} / \mathrm{h} \cdot \mathrm{F}^{1.333}\right)$

CQC45 = suction valve heat transfer parameter $\left(\mathrm{ft}^{0.2}\right)$

CQC67 = discharge valve heat transfer parameter $\left(\mathrm{ft}^{0.2}\right)$

CQC78 = heat transfer parameter at can exit $\left(\mathrm{ft}^{0.2}\right)$

Line 10: CQ, CPDR, VCAN, REFIN

$C Q=$ parameter for 4-way valve heat transfer $\left(\mathrm{ft}^{0.2}\right)$

CPDR = pressure drop parameter for a 4-way valve $\left(1 b f^{2} h^{2} /\left(1 b * i^{2} * f t^{3}\right)\right.$

VCAN = volume of compressor can filled by liquid $\left(\mathrm{ft}^{3}\right)$

REFIN = refrigerant charge (1b)

Line 11: AHGT, DACC, DHOLE(1), DHOLE(2), DTUBE, HDIS

AHGT = distance between accumulator top and oil return hole (ft)

DACC = inner diameter of accumulator ( $f t$ )

$\operatorname{DHOLE}(1)=$ diameter of oil return hole ( $\mathrm{ft}$ )

DHOLE(2) = diameter of upper hole in accumulator tube ( $f t$ ) 
DTUBE = diameter of accumulator tube ( $f t)$

HDIS = vertical distance between holes in accumulator tube ( $f t$ )

Line 12: $\operatorname{NDEP}(1), \operatorname{NROW}(1)$

$\operatorname{NDEP}(1)=$ number of indoor coil tube depth rows (-)

NROW(1) = number of tubes per indoor coil depth row (-)

Line 13: $\mathrm{DI}(1), \mathrm{DO}(1), \operatorname{Dr}(1), \operatorname{RPCH}(1), \operatorname{DPCH}(1), \operatorname{WIDTH}(1)$

DI $(1)=$ inner diameter of indoor coil tubes (in)

DO(1) = outer diameter of indoor coil tubes (in)

DT $(1)=$ indoor coil fin tip diameter (in) (refer to figure 21)

RPCH(1) = pitch between tubes of the same depth row in indoor coil (in)

DPCH(1) = pitch for indoor coil tube depth rows (in)

WIDTP9H(1) = indoor coil width (equal tube length) (in)

Line 14: FPCH (1), FTK(1), FMK (1), TMK(1), AMAS(1)

$\operatorname{FPCH}(1)=$ indoor coil fin pitch (in)

FTK(1) = indoor coil fin thickness (in)

FMK $(1)=$ indoor coil fin material thermal conductivity $(\mathrm{Btu} /(\mathrm{ft} \cdot \mathrm{h} \cdot \mathrm{F}))$

TMK(1) = indoor coil tube material thermal conductivity $(B t u / f t \cdot h \cdot F))$

$\operatorname{AMAS}(1)=$ air mass flow rate through indoor coil $(1 \mathrm{~b} / \mathrm{h})$

Line 15: $\operatorname{CONST}(1), \operatorname{CPOH}(1), \operatorname{ANGLE}(1)$

CONST(1) = constant for air side heat transfer correlation for indoor coil equal to $0.134(-)$

CPOW(1) = constant for air side heat transfer correlation for indoor coil equal to $0.681(-)$

ANGLE(1) = angle between indoor coil face and air streamlines (rad)

Line 16: EIDFAN

EIDFAN $=$ indoor fan energy input rate (kW)

Line 17: NREPTI

NREPTI = number of repeating sections in indoor coil (-)

Line 18: $\operatorname{NTUBE}(1, I) \quad I=1,5$

$\operatorname{NTUBE}(1,1)=$ number of tubes in first row in each section of indoor $\operatorname{coil}(-)$

$\operatorname{NTUBE}(1,2)=$ number of tubes in second row in each section of indoor coil (-)

$\operatorname{NTUBE}(1,3)=$ number of tubes in third row in each section of indoor $\operatorname{coil}(-)$

$\operatorname{NTUBE}(1,4)=$ number of tubes in fourth row in each section of indoor coil (-)

$\operatorname{NTUBE}(1,5)=$ number of tubes in $f$ ifth row in each section of indoor coil (-)

Line 19: $\operatorname{IFROM}(1, I), I=1,10$

$\operatorname{IFROM}(1,1)=$ number of tube of indoor coil from which tube 1 receives refrigerant when indoor coil works as evaporator (-)

$\operatorname{IFROM}(1,2)=$ number of tube of indoor coil from which tube 2 receives refrigerant when indoor coil works as

$\operatorname{IFROM}(1,3)=$ evaporator $(-)$

$\operatorname{IFROM}(1,9)=$

$\operatorname{IFROM}(1,10)=$ number of tube of indoor coil from which tube 10 receives refrigerant when indoor coil works as evaporator (-) 
Line 20: $\quad \operatorname{IFROM}(1, I), I=11,20$

$\operatorname{IFROM}(1, I)=$ number of tube of indoor coil from which tube $I$ receives refrigerant when indoor coil works as evaporator (-)

Line 21: $\operatorname{IFROM}(1, I), I=21,30$

Line 22: $\quad \operatorname{IFROM}(1, I), I=31,40$

Line 23: $\quad \operatorname{IFROM}(1, I), \quad I=41,50$

Line 24: IFROM( $1, I), I=51,60$

Line 25: $\quad \operatorname{IFROM}(1, I), I=61,70$

Line 26: $\quad \operatorname{IFROM}(1, I), I=71,80$

Line 27: $\quad \operatorname{IFROM}(1, I), I=81,90$

Line 28: $\quad \operatorname{IFROM}(1, I), \quad I=91,100$

Line 29: $\quad \operatorname{IFROM}(1, I), I=101,110$

Line 30: $\quad \operatorname{IFROM}(1, I), I=111,120$

Line 31: $\quad \operatorname{IFROM}(1, I), I=121,130$

$\operatorname{IFROM}(1, I)=$ number of tube of indoor coil from which tube I receives

Line 32: $\operatorname{NDEP}(2), \operatorname{NROW}(2)$ refrigerant when indoor coil works as evaporator $(-)$

$\operatorname{NDEP}(2)=$ number of outdoor coil tube row depths $(-)$

NROW(2) = number of tubes per outdoor coil depth row (-)

Line 33: $\mathrm{DI}(2), \operatorname{DO}(2), \operatorname{DT}(2), \operatorname{RPCH}(2), \operatorname{DPCH}(2)$, WIDTH (2)

DI(2) = inner diameter of outdoor coil tubes (in)

DO(2) = outer diameter of outdoor coil tubes (in)

DT(2) = outdoor coil fin tip diameter (in) (refer to figure 21)

RPCH(2) = pitch between tubes of the same depth in outdoor coil (in)

$\operatorname{DPCH}(2)=$ pitch between tube depth rows for outdoor coil (in)

WIDTH(2) = outdoor coil width (equal tube length) (in)

Line 34: FPCH (2), FTK(2), FMK (2), TMK (2), AMAS (2)

FPCH(2) = outdoor coil fin pitch (in)

FTK (2) = outdoor coil fin thickness (in)

FMR $(2)=$ outdoor coil fin material thermal conductivity $(B t u /(f t \cdot h \cdot F))$

TMK $(2)=$ outdoor coil tube material thermal conductivity $(B t u /(f t \cdot h \cdot F))$

Line 35: $\operatorname{CONST}(2), \operatorname{CPOW}(2), \operatorname{ANGLE}(2)$

AMAS $(2)=a i r$ mass flow rate through outdoor coil $(1 \mathrm{~b} / \mathrm{h})$

CONST $(2)=$ constant for air side heat transfer correlation for outdoor coil equal to $0.134(-)$

CPOW(2) = constant for air side heat transfer correlation for outdoor coil equal to $0.681(-)$

$\operatorname{ANGLE}(2)=$ angle between outdoor coil face and air streamlines

Line 36: EIDFAN (rad)

Line 37: NREPTO

NREPTO = number of repeating sections in outdoor coil (-)

Line 38: $\quad \operatorname{NTUBE}(2, I), \quad I=1,5$

$\operatorname{NTUBE}(2,1)=$ number of tubes in first row in each section of outdoor coil (-)

$\operatorname{NTUBE}(2,2)=$ number of tubes in second row in each section of outdoor coil (-)

$\operatorname{NTUBE}(2,3)=$ number of tubes in third row in each section of outdoor coil (-) 
Line 39: $\operatorname{IFROM}(2, I), I=1,10$

$\operatorname{NTUBE}(2,4)=$ number of tubes in fourth row in each section of outdoor coil (-)

$\operatorname{NTUBE}(2,5)=$ number of tubes in $f$ if th row in each section of outdoor coil ( - )

$\operatorname{IFROM}(2,1)=$ number of tube of outdoor coil from which tube 1 receives refrigerant when outdoor coil works as evaporator (-)

$\operatorname{IFROM}(2,2)=$ number of tube of outdoor coil from which tube 2 receives refrigerant when outdoor coil works as

$\operatorname{IFROM}(2,3)=$ evaporator (-)

Line 40: $\quad \operatorname{IFROM}(2, I), \quad I=11,20$

$\operatorname{IFROM}(2, I)=$ number of tube of outdoor coil from which tube I receives refrigerant when outdoor coil works as evaporator $(-)$

Line 41: $\quad \operatorname{IFROM}(2, I), I=21,30$

Line 42: $\quad \operatorname{IFROM}(2, I), \quad I=31,40$

Line 43: $\quad \operatorname{IFROM}(2, I), I=41,50$

Line 44: IFROM(2,I), I $=51,60$

Line 45: $\operatorname{IFROM}(2, I), I=61,70$

Line 46: IFROM(2,I), I $=71,80$

Line 47: $\quad \operatorname{IFROM}(2, I), I=81,90$

Line 48: $\operatorname{IFROM}(2, I), I=91,100$

Line 49: $\quad \operatorname{IFROM}(2, I), \quad I=101,110$

Line 50: $\operatorname{MROM}(2, I), I=111,120$

Line 51: $\quad \operatorname{IFROM}(2, I), I=121,130$

$\operatorname{IFROM}(2, I),=$ number of tube of outdoor coil from which tube I receives refrigerant when outdoor coil works as evaporator $(-)$

Line 52: CAPID1, CAPL1, NCPL1, CAPID2, CAPL2, NCPL2

CAPID1 = inner diameter of cooling operation expansion device (in)

CAPL1 = length of cooling operation expansion device (in)

NCPL1 = number of cooling operation expansion devices ( - )

CAPID2 = inner diameter of heating operation expansion device (in)

CAPL2 = length of heating operation expansion device (in)

NCPL2 = number of heating operation expansion devices (-)

Line 53: $\mathrm{YL}, \mathrm{YD}, \mathrm{YK} 1, \mathrm{YD} 1, \mathrm{YK} 2, \mathrm{YD} 2$

$\mathrm{YL}=$ length of compressor-outdoor coil tubing (in)

YD, inner diameter of compressor-outdoor coil tubing (in)

YK1 = thermal conductivity of compressor-outdoor coil tubing material (Btu/(ft $\cdot h \cdot F))$

YD1 = outer diameter of compressor-outdoor coil tubing (in)

YK2 = thermal conductivity of compressor-outdoor coil tubing insulation (Btu/( $\mathrm{ft} \cdot \mathrm{h} \cdot \mathrm{F}))$

YD2 = outer diameter of compressor-outdoor coil tubing insulation $(\mathrm{Btu} /(\mathrm{ft} \cdot \mathrm{h} \cdot \mathrm{F}))$ 
Line 54: RL, RD, RK1, RD1, RK2, RD2

$R L=1$ ength of compressor-indoor coil tubing (in)

$R D=$ inner diameter of compressor-indoor coil tubing (in)

RK1 = thermal conductivity of compressor-indoor coil tubing material (Btu/(ft・h $\cdot \mathrm{F}))$

RD1 = outdoor diameter of compressor-indoor coil tubing (in)

RK2 = thermal conductivity of compressor-indoor coil tubing insulation (Btu/(ft $\cdot h \cdot F)$ )

RD2 = outer diameter of compressor-outdoor coil tubing insulation $(B t u /(f t \cdot h \cdot F))$

Line 55: RYL, RYD

RYL = 1 iquid 1 ine length (in)

RYD = 1 iquid line diameter (in)

Note on coil input data:

The coil depth is in the direction perpendicular to a coil surface facing the incoming air. Lines 19-31 and 39-51: tubes are numbered consecutively from the first tube in the first row (facing incoming air) to the last tube in the 1ast row. Enter 0 (zero), if considered tube receives refrigerant from coil inlet port; enter 999, if the tube is nonexistent. 
Table H6. Example of a Heat Pump Data File

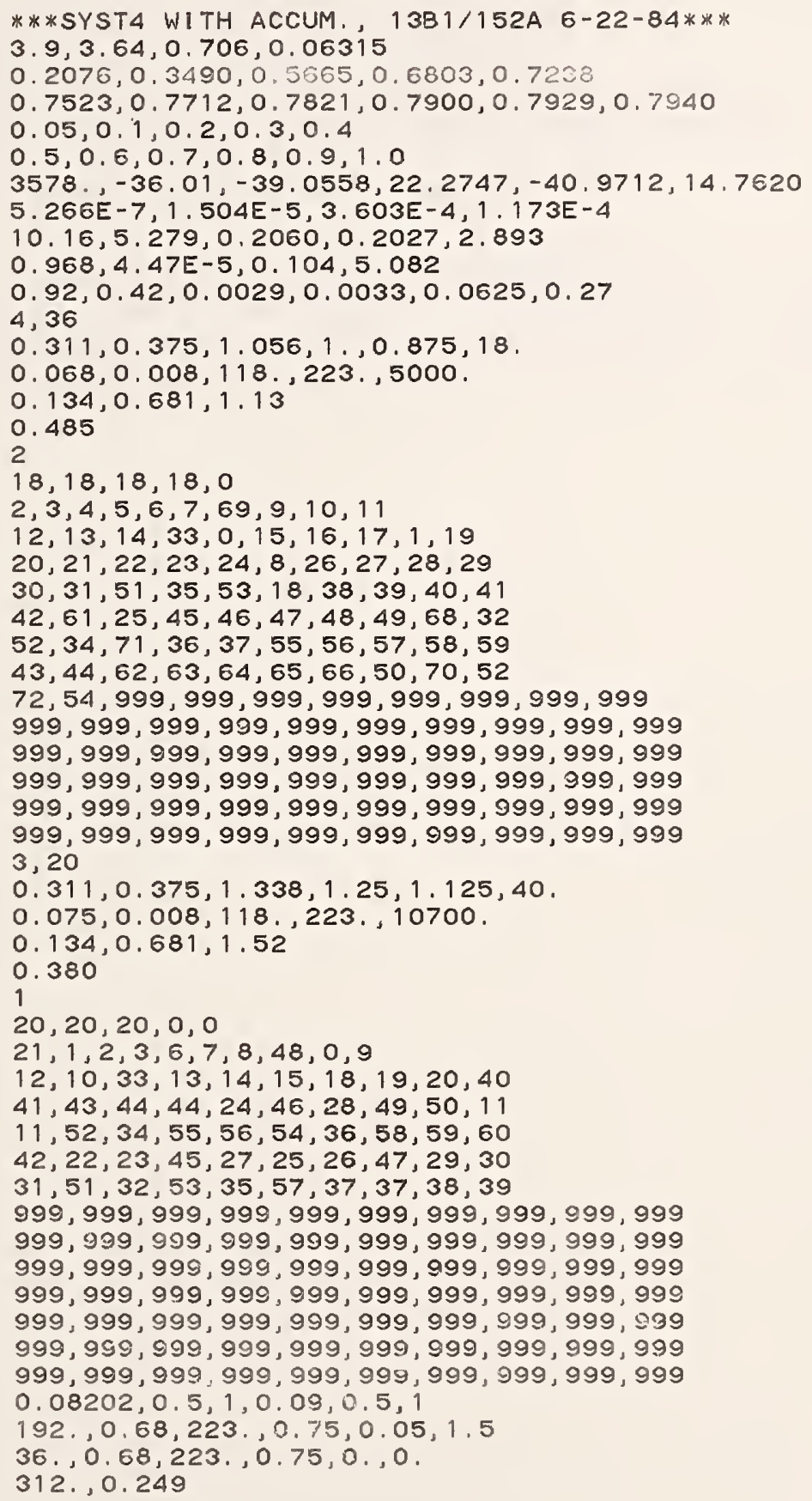



APPENDIX I. EXAMPLE OF RUN OF THE PROGRAM HPBI

The following is a computer printout for a HPBI run in which performance of a heat pump in the heating mode was simulated. Input for this run was as foll ows :

- refrigerant data - as shown in Table H4

- heat pump data - as shown in Table H6

- run controlling data, operating conditions, estimated refrigerant parameters - as shown on the printout ( 1 ines 4-36).

The results of this run are included in Table 5.

The solution was iterated in two loops iterating composition of the circulating refrigerant ( 1 ines $39-2592$ and 2593-4762). The second $100 p$ required eight internal loops in which the total refrigerant mass conservation was sought (TMASS $=$ REFIN). The run required 33 minutes of CPU on a Sperry $1100 / 82$ computer. 


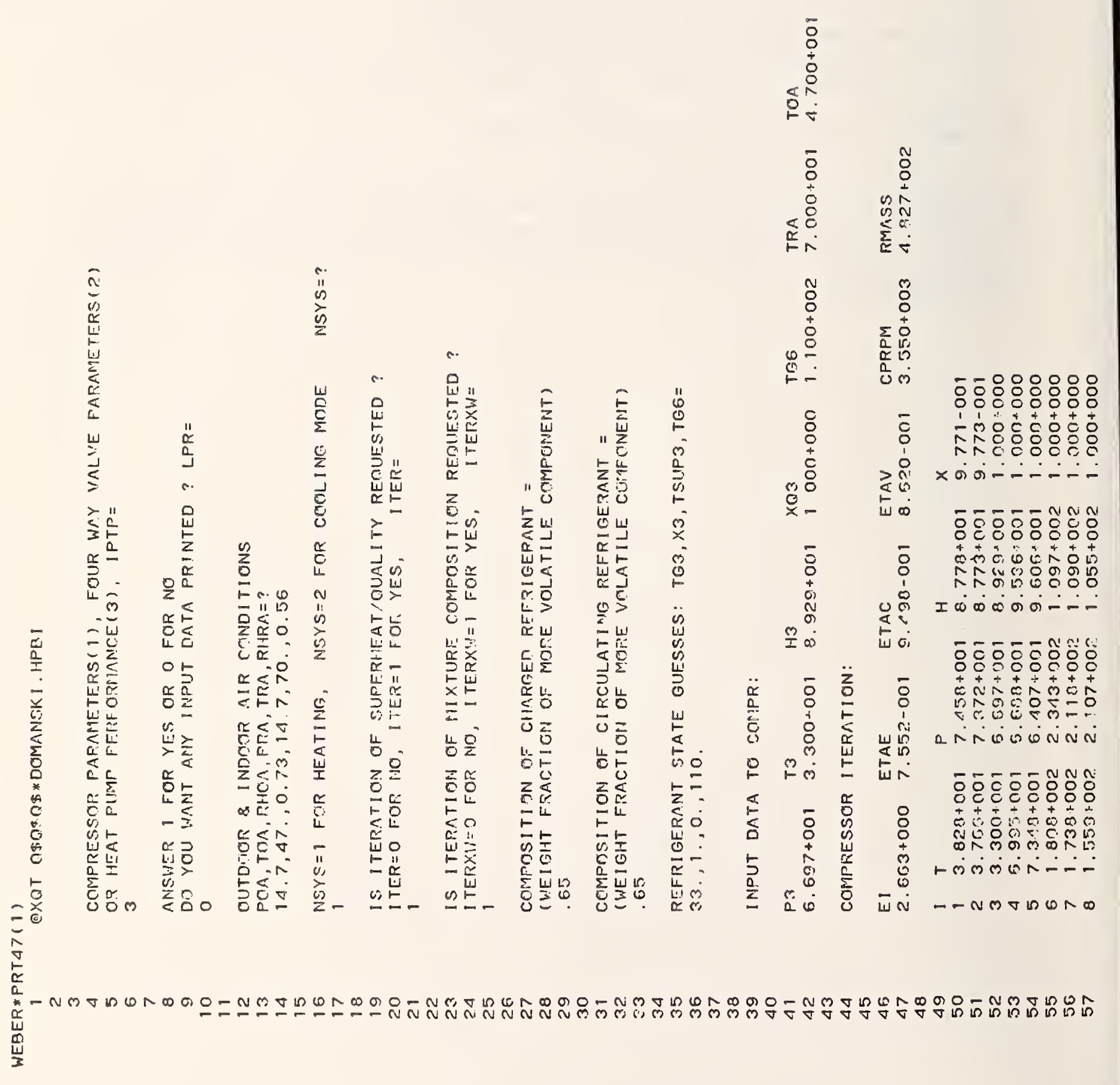




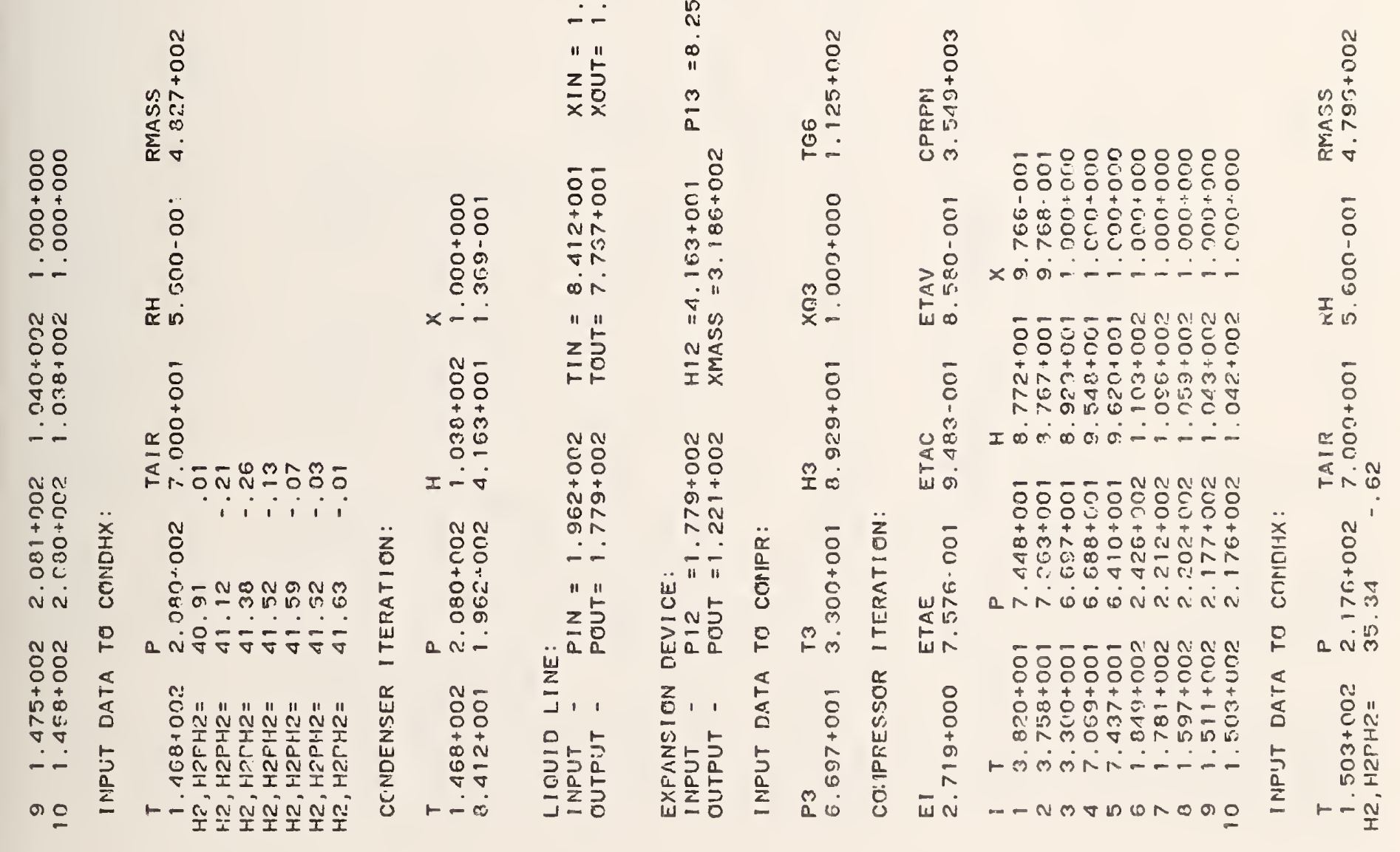

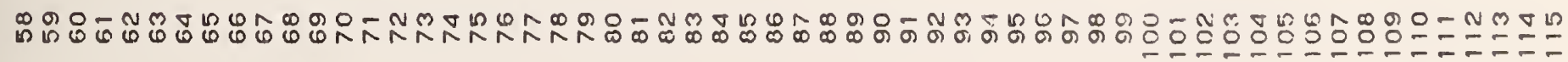




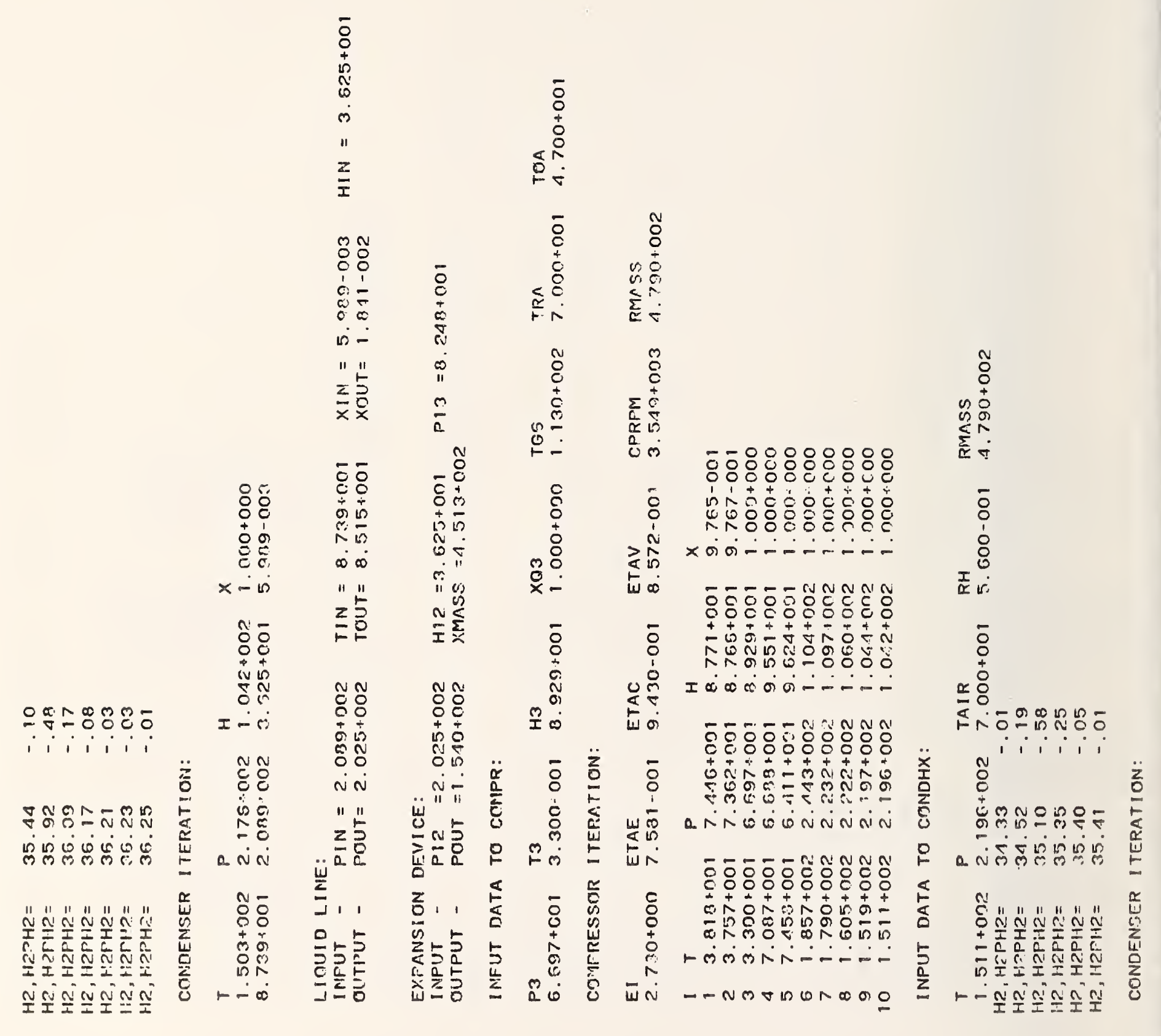

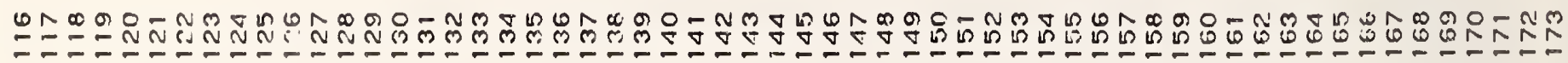




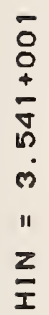

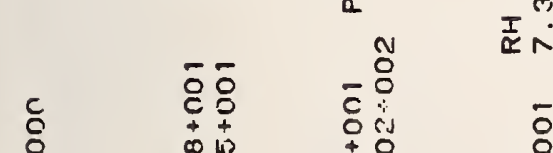

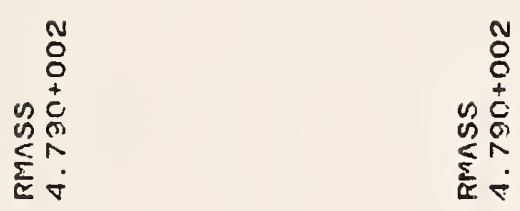

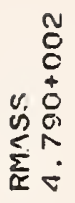

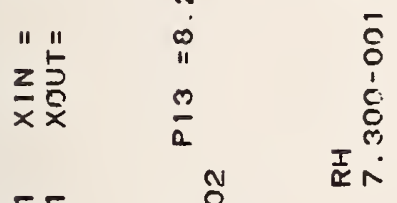

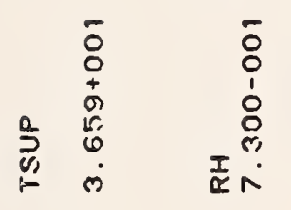

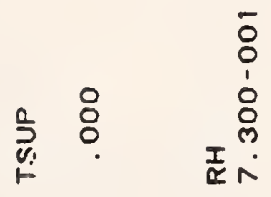

$\underset{\substack{0 \\ 0}}{\stackrel{0}{0}} \stackrel{8}{0}$
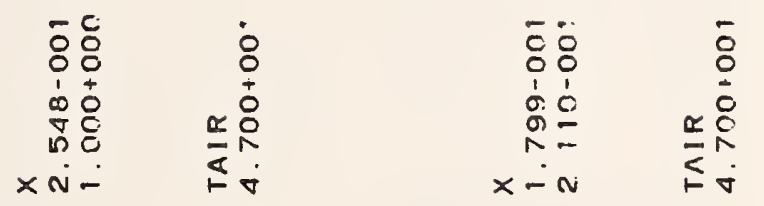

후음

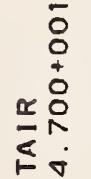

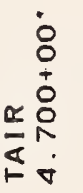

宓芯 $\times$ ते

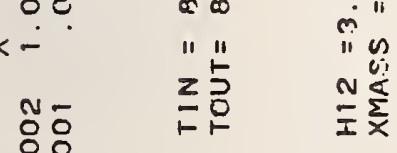

$\stackrel{+}{\mathrm{i}} \frac{+}{\mathrm{i}}$

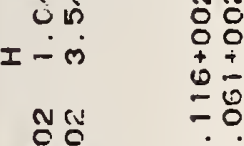

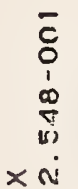

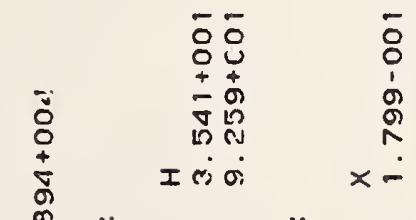

휴 ह

둥

ถึ

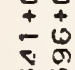

Imं $\quad$ त

Irin

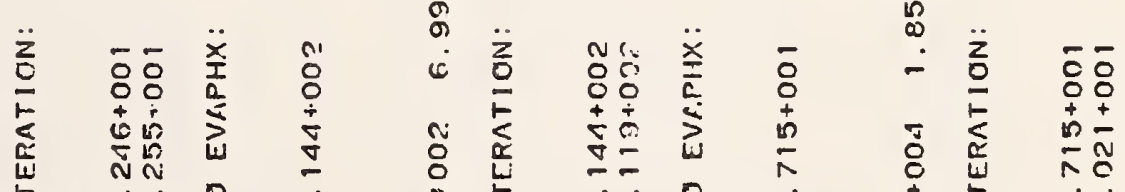

ஸ்

กั่

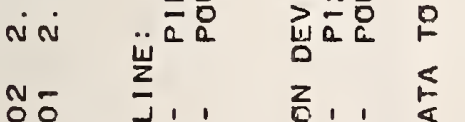

a.

a. कंष 0 -

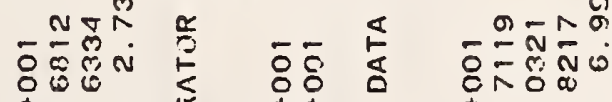

ํㅜㄹ

लक्ष कै

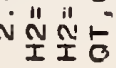

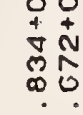

a. $0^{\circ}$

a.

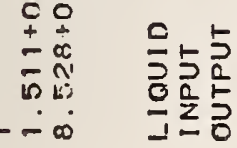

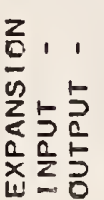

5
5
0
0

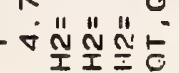




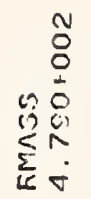

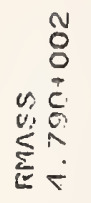

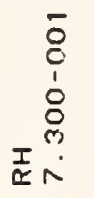

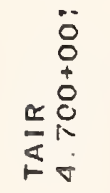
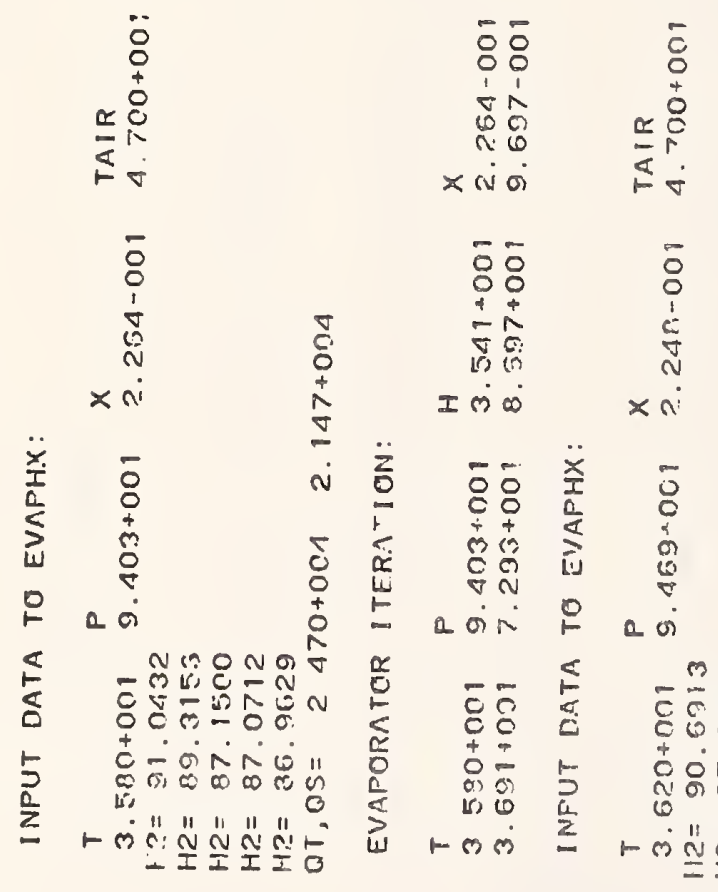

I $\operatorname{li}^{\circ} \times \mathrm{n}^{\circ}$

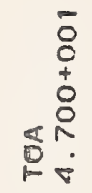
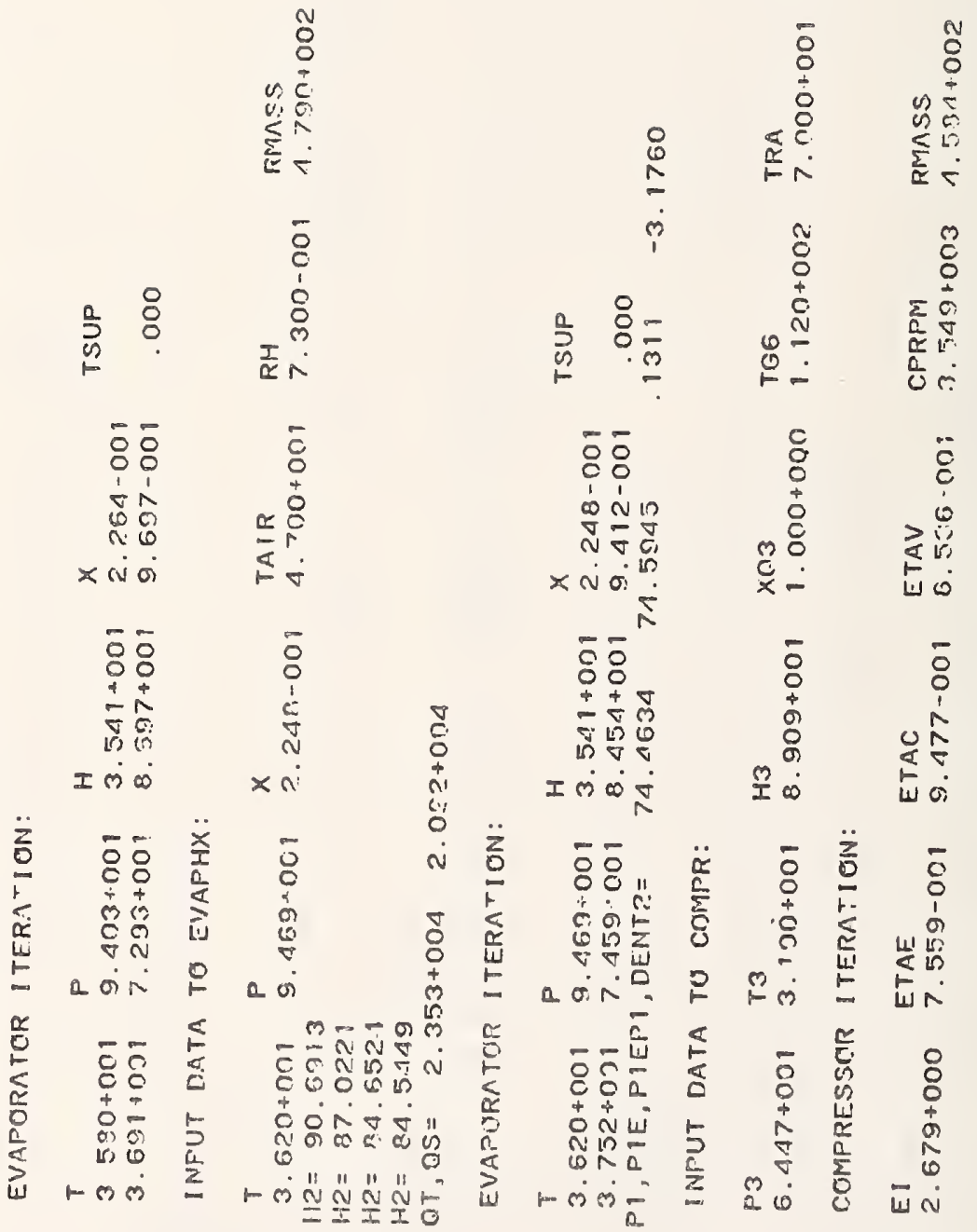

$\ddot{c}$

흐융융유 $1,+4+0 c 0$

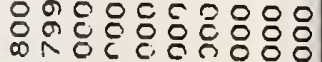
这说:

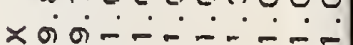

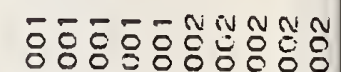
-i. $\begin{array}{llll}7 & 0 \\ 0 & 0 & 0\end{array}$ เด I लिख

Wo I 0 ic $000 \div-\therefore$

-

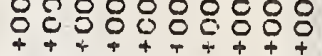

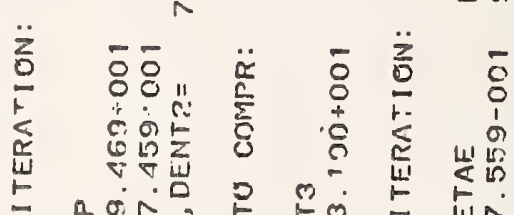
-

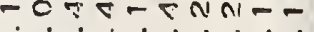
añ⿻上丨तกN

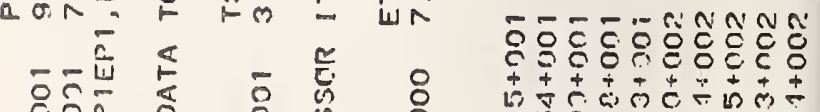

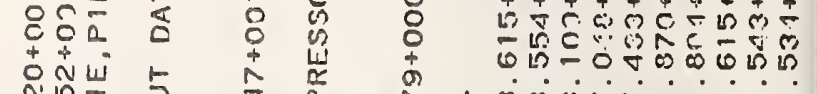

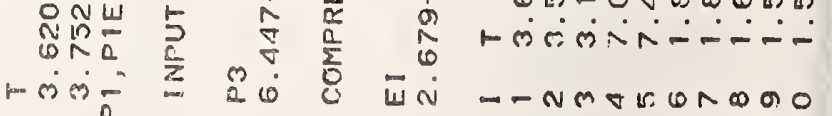

$\vec{\omega} \dot{\sim}$ - nmosingon

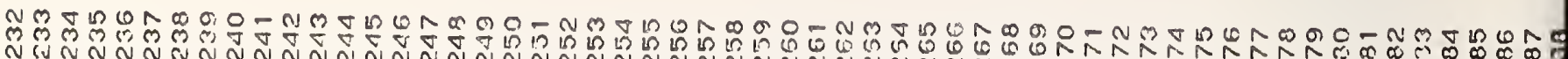

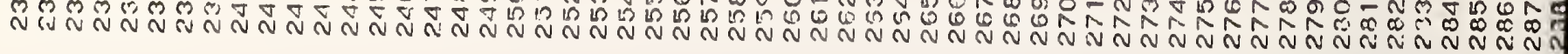


\begin{tabular}{l}
0 \\
0 \\
+ \\
0 \\
0 \\
0 \\
11 \\
$z$ \\
1 \\
\hline 1
\end{tabular}

¿๕

竞

0
$\vdots$
$\vdots$
$\vdots$
0

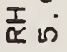

$\bar{\delta}$
$\stackrel{+}{ \pm}$
$\stackrel{8}{0}$

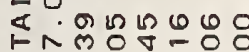

i i i i i

ํํㅇ

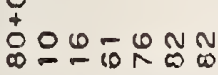

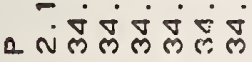

ก๊

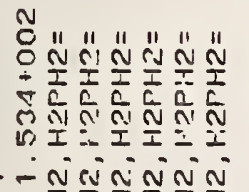

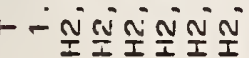

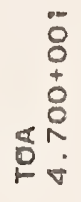

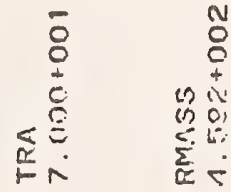

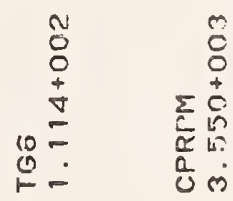

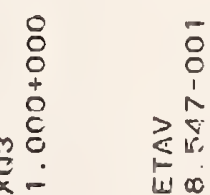

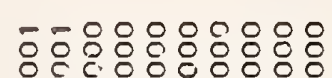

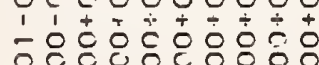
\& ×ง

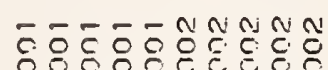

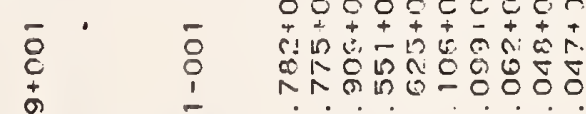

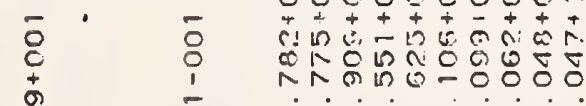
I $\dot{\omega} \dot{\infty} \dot{\sigma} \sigma \ldots$

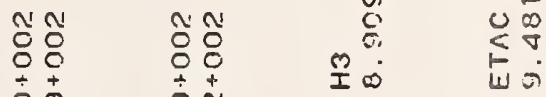

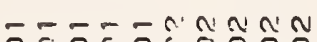

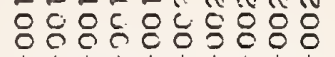

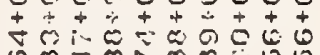

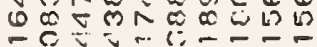

- ríogoninina

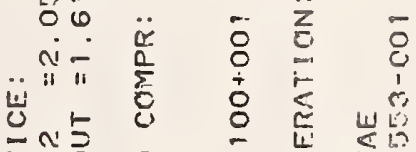

"z⿺

- - - - -

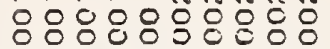

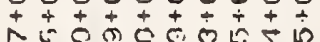

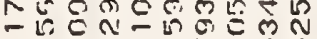

un $-0 \nabla \infty \pi 0$ ถ

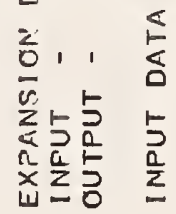

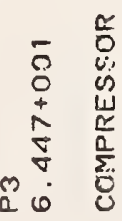

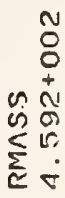

8

1
0
-8

它

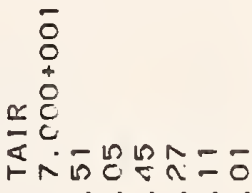

ธิ

$\overline{0}$

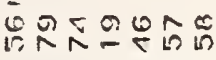

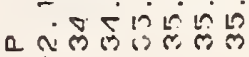

c.

○.

$\therefore$ a

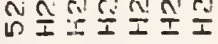

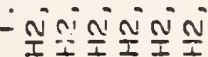

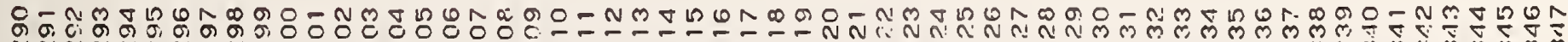
N 


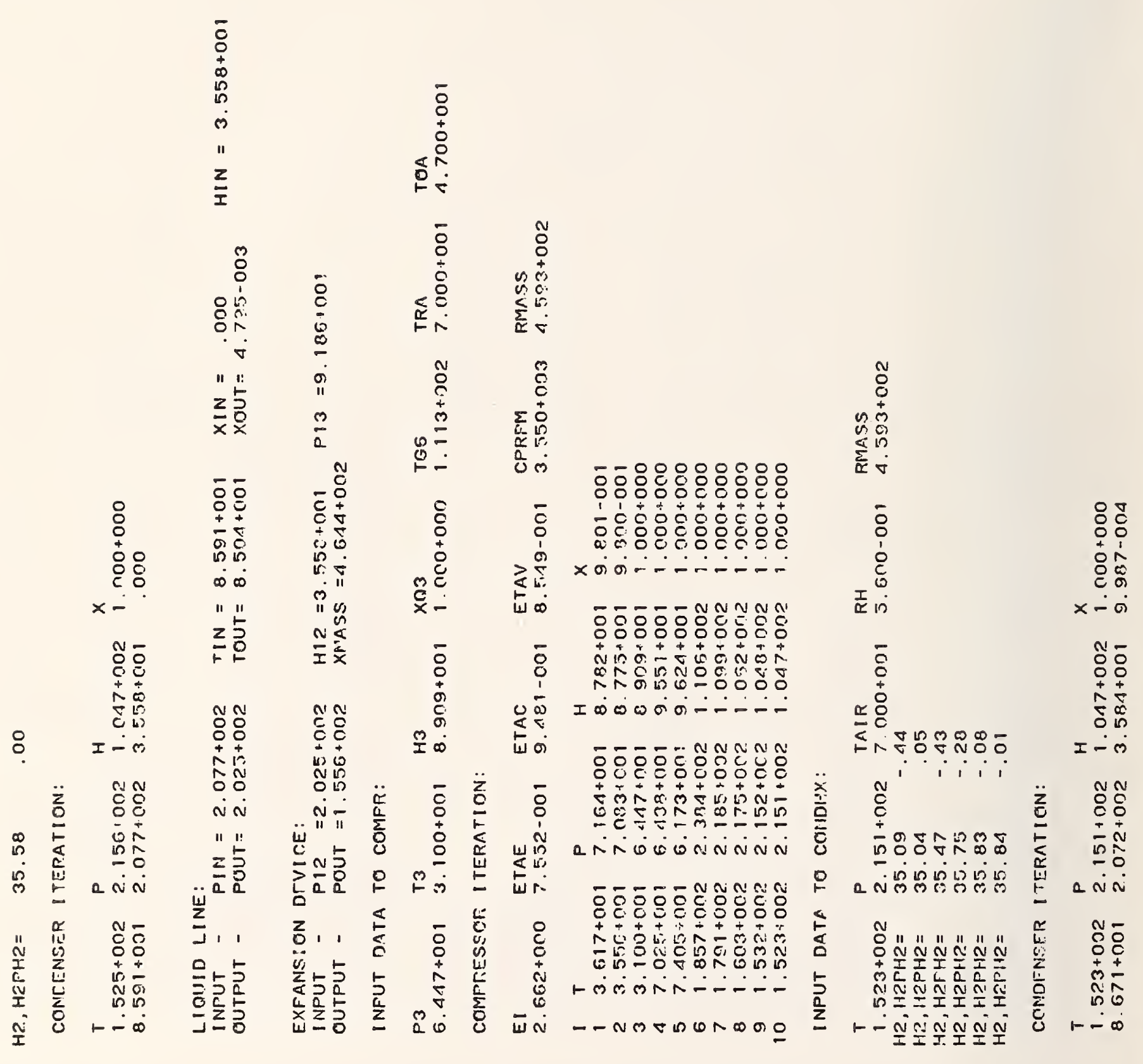

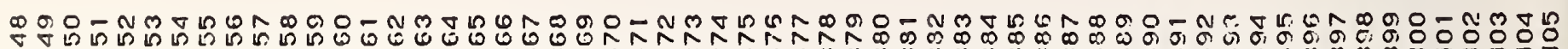

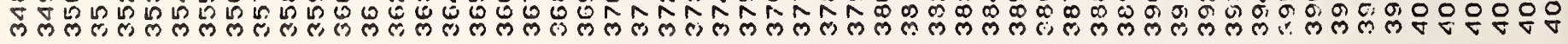


5
0
+
5
0
0
r
11
$z$
$z$
1

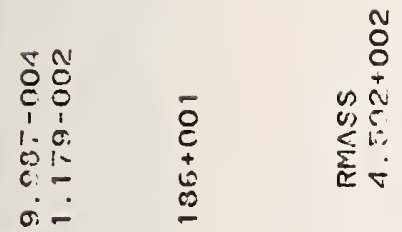<smiles>O[C@H]1O[C@@H]2C=C[C@H]1O2</smiles><smiles>O[C@H]1CC2CCC(C2)[C@H]1O</smiles>

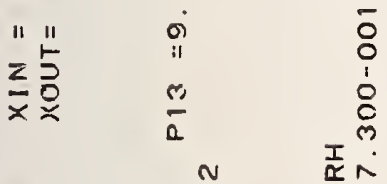

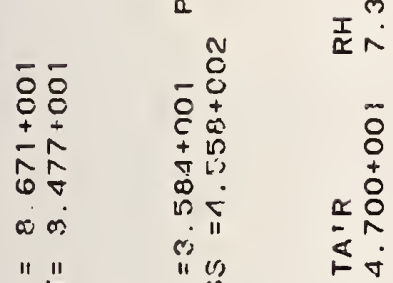

"

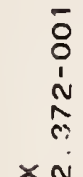

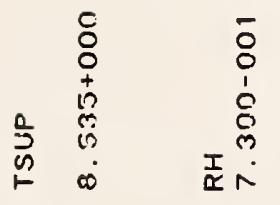

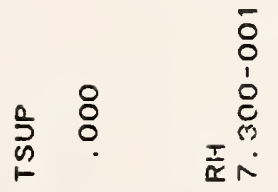

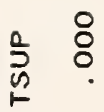

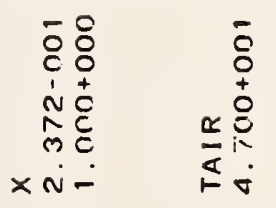

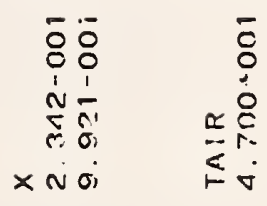

응요

동

$\times$ त

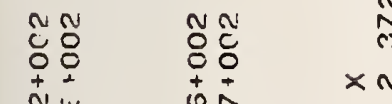

훙

웅

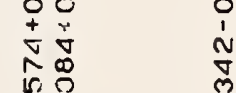

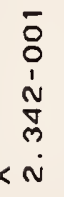

ô

I लें

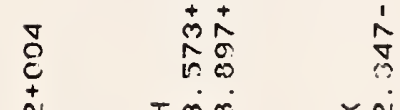

¿

58

$+$

${ }^{3} \stackrel{\infty}{5}$

Iक $x \quad \times N$

I लं०

ni

" II

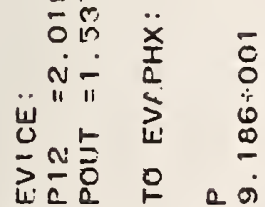

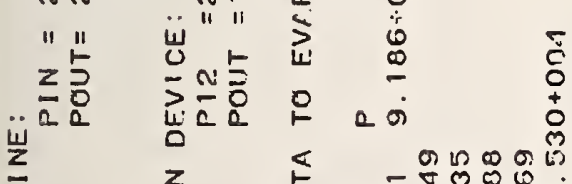

J

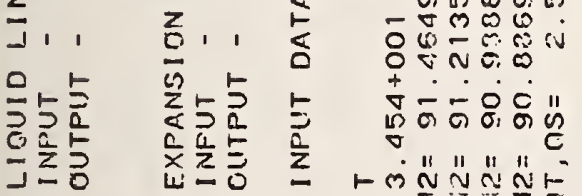
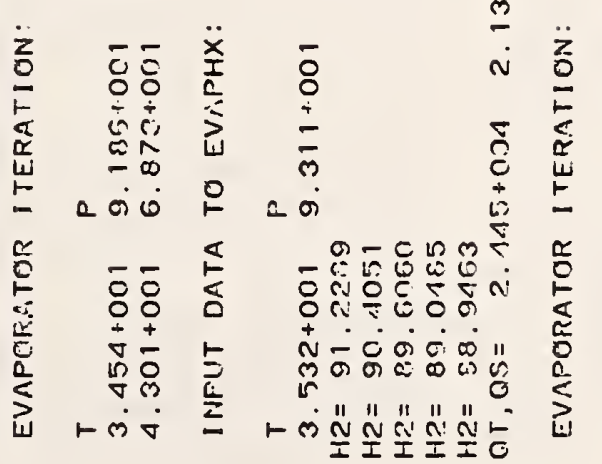

둉

$\sum_{\substack{x \\ \frac{x}{5}}}^{\ddot{x}}$

a ar

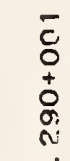

a 0

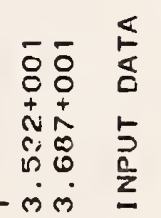

ODON-

on

के

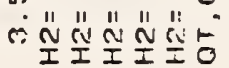

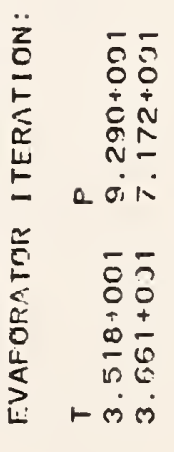

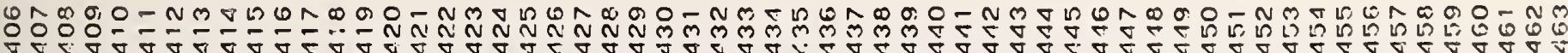




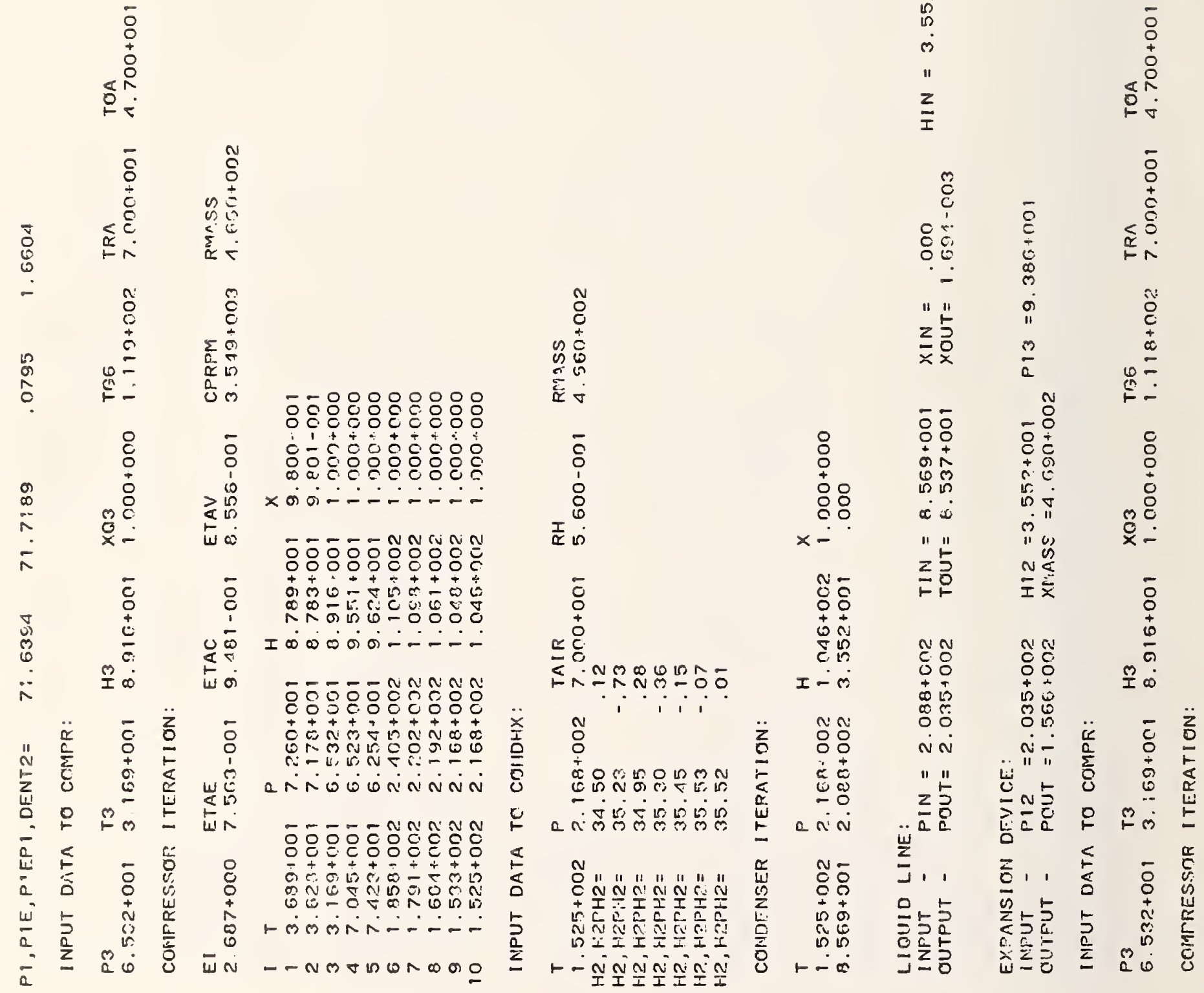

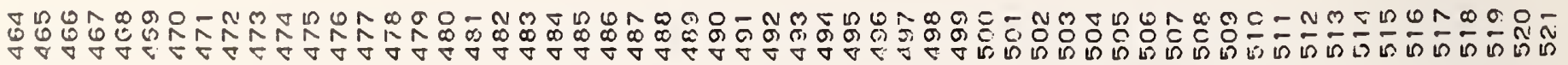




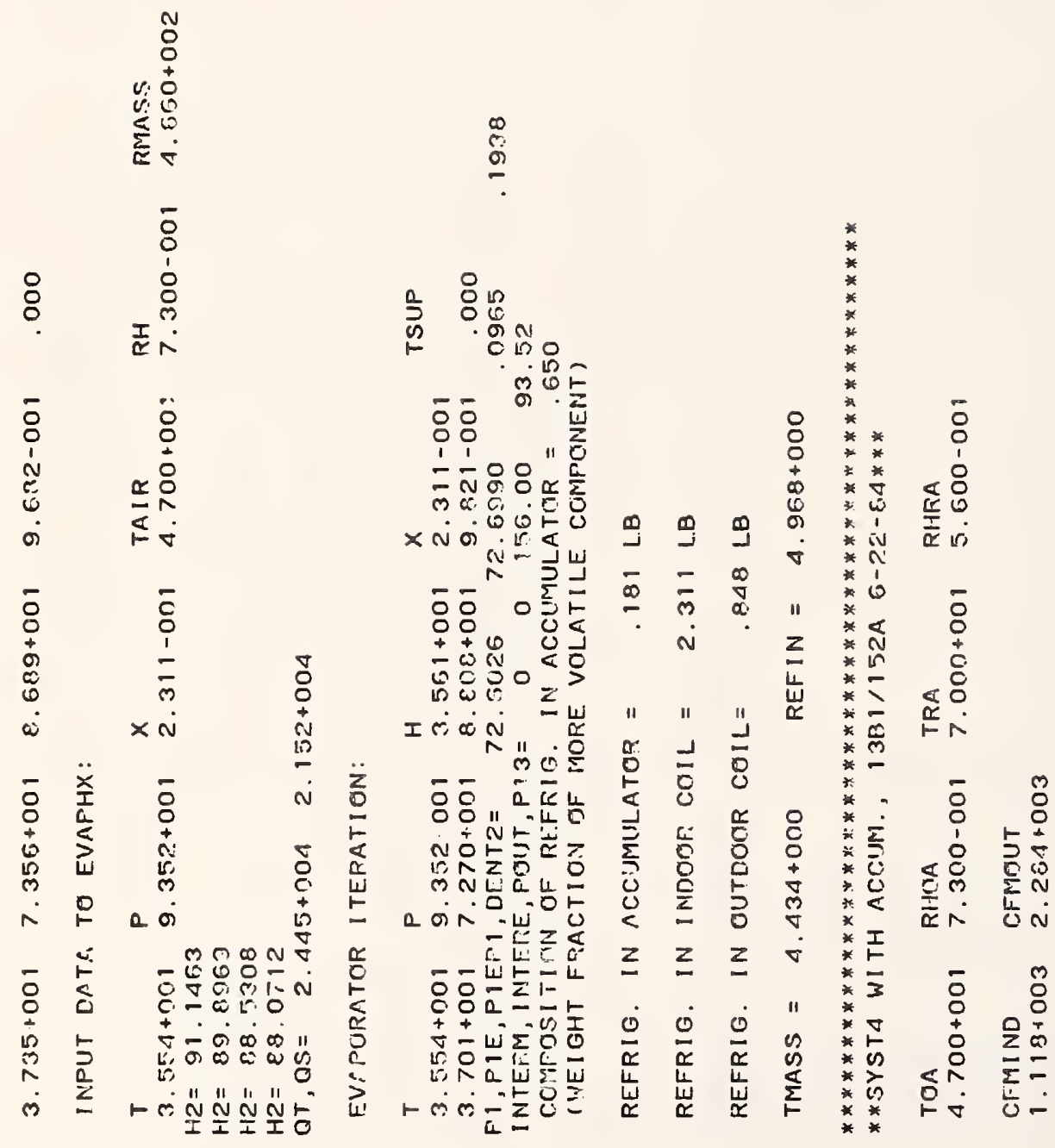

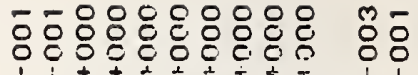

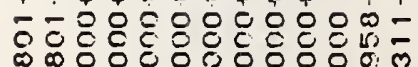

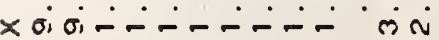

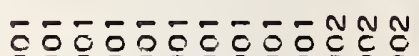
8.88 .88808808 - - in ON o o m in in n N

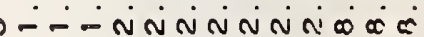

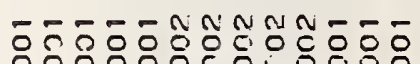
每 -

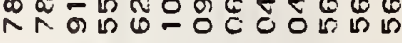
I $\infty 000$ O

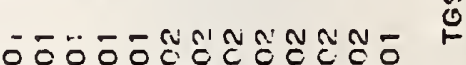

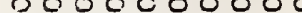

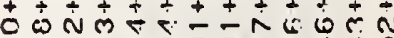
ต

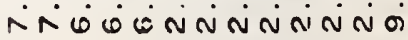

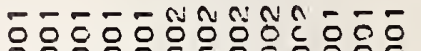
$\frac{3}{2}$ 800800080080

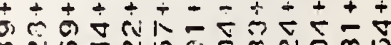

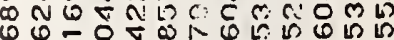

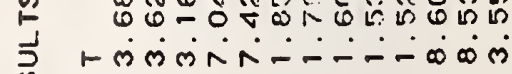
$\stackrel{M}{0}$

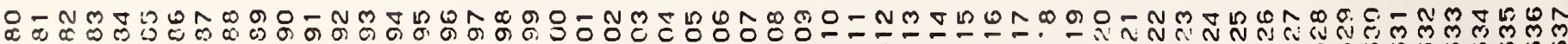
正 
$\begin{array}{r}-5 \\ 0 \\ 0 \\ 0 \\ 0 \\ 0 \\ \hline\end{array}$

5
0
+5
0
0
0
11
11
$z$
$I$

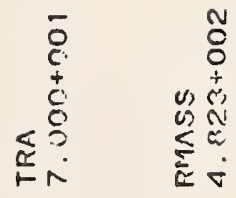

용응

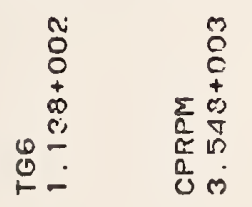

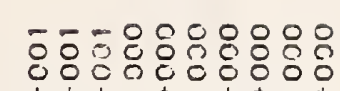

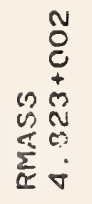

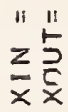

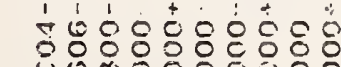

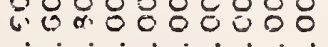

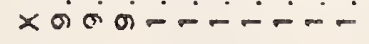

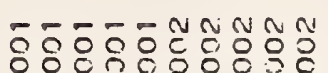

o to

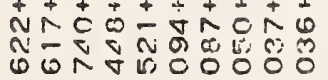

I $\cos 0$ oi $\sigma:-\ldots$

- -

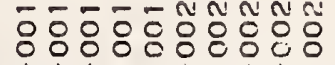

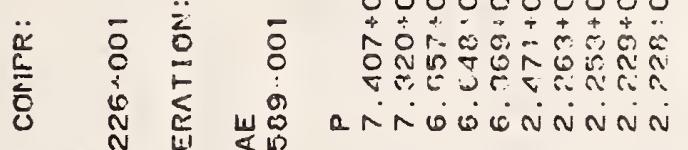

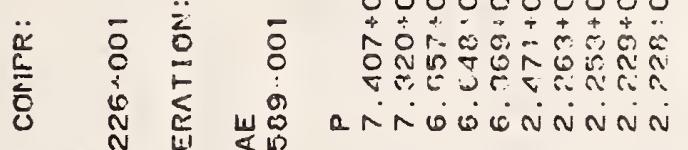

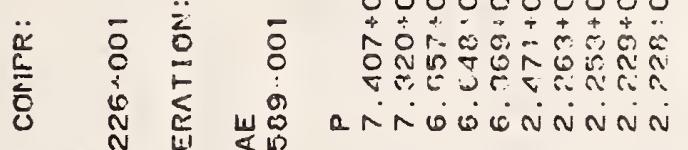

:

อํํำํํำำ

占

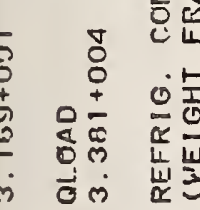

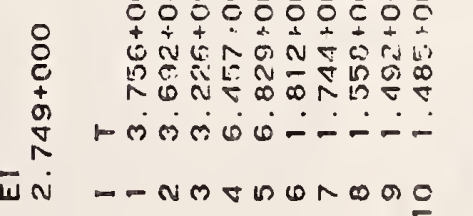

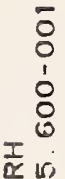

¿

cc c

¿ே용

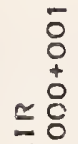

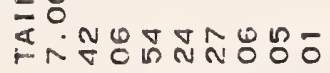

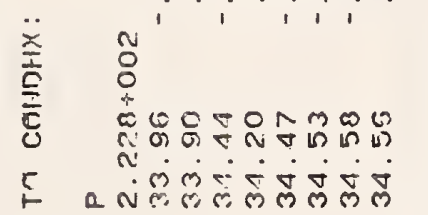

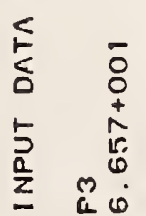

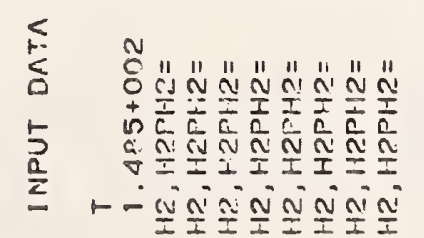

a $\sim$ i

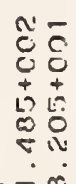

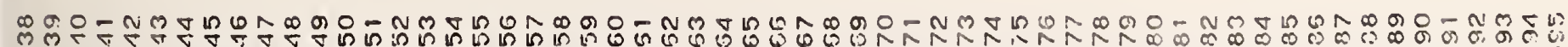

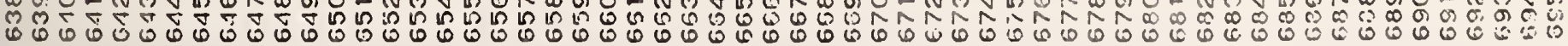




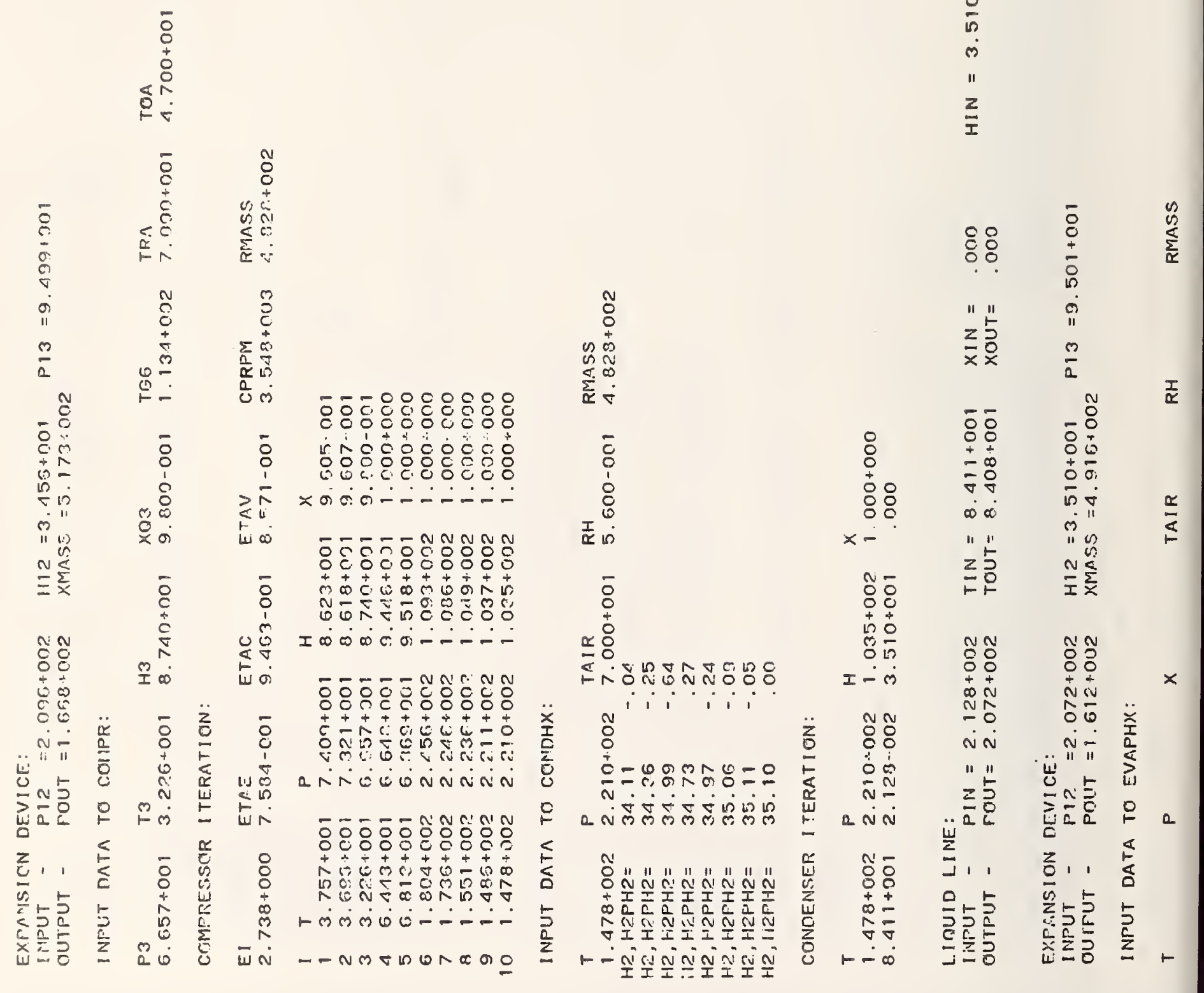

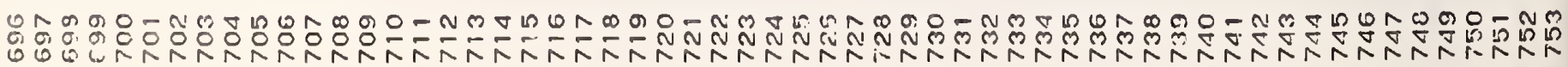




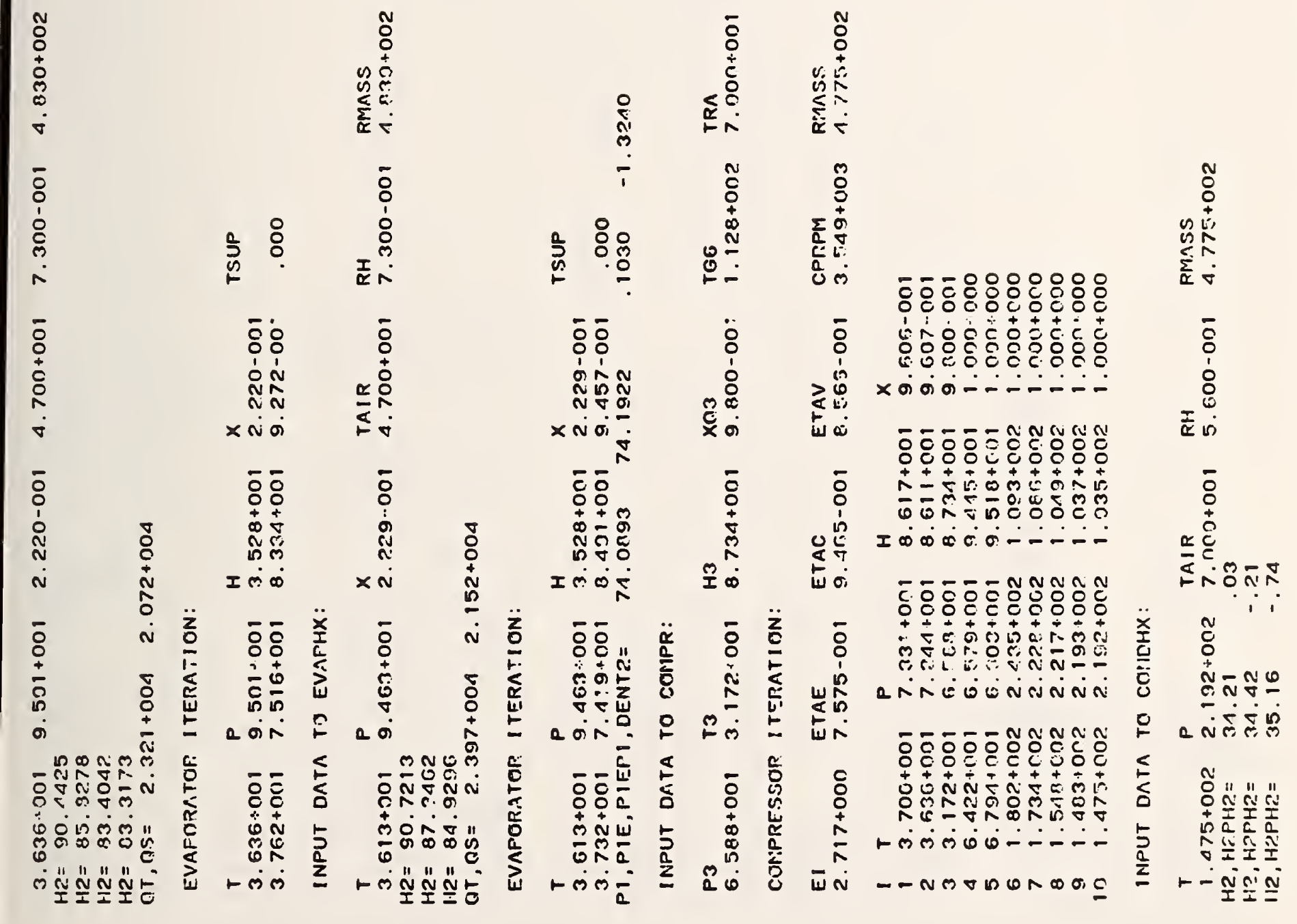

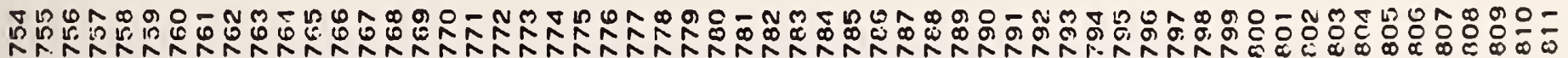




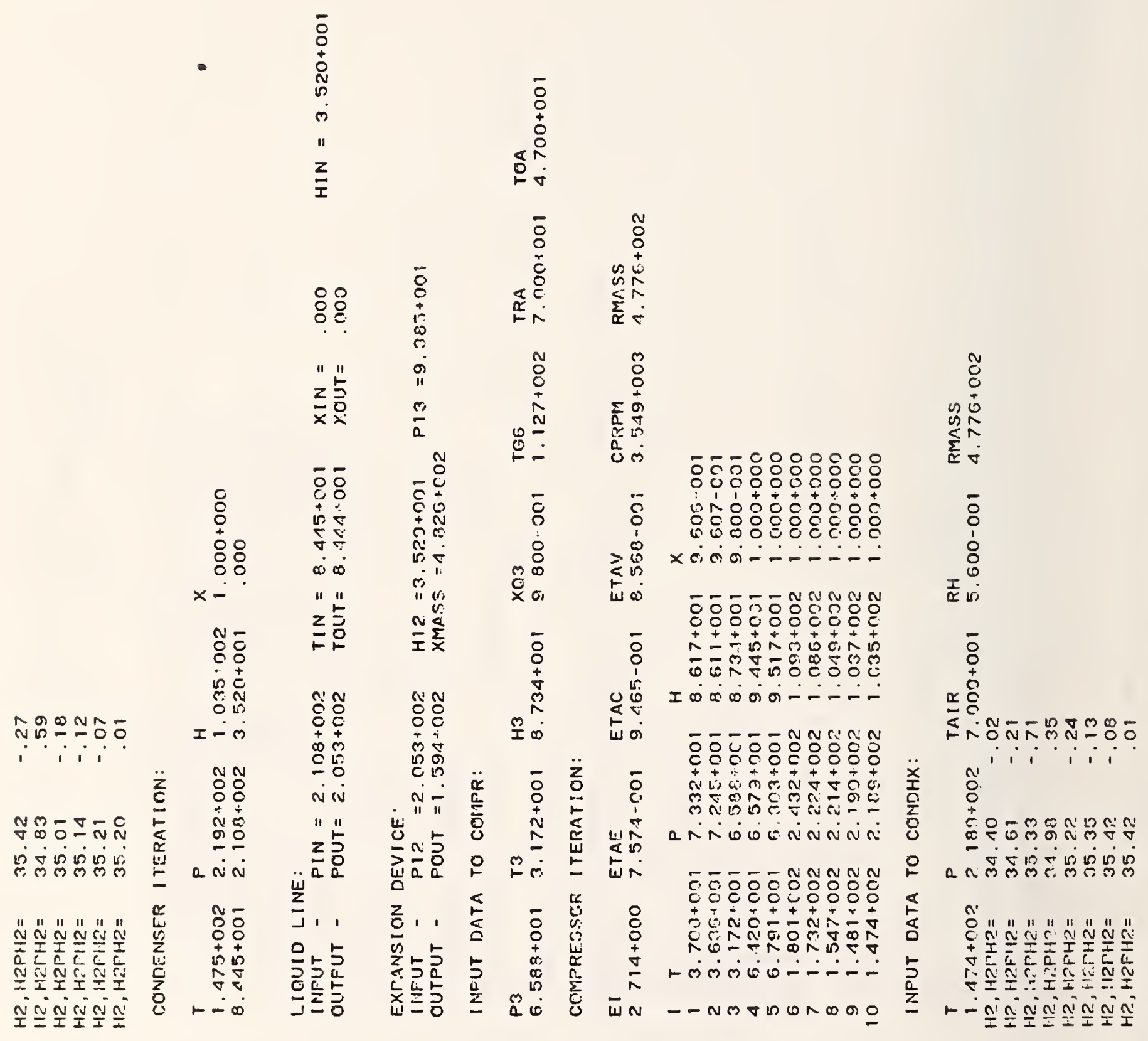

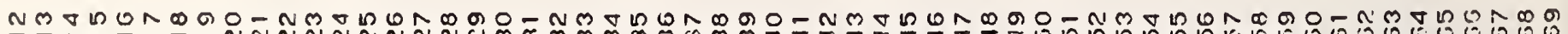

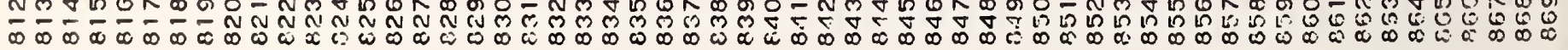




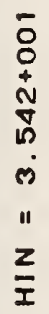

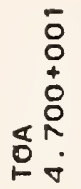

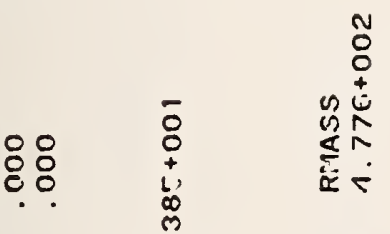

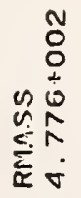

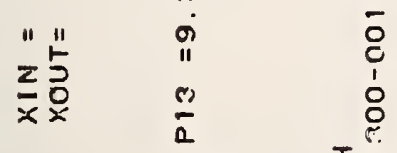

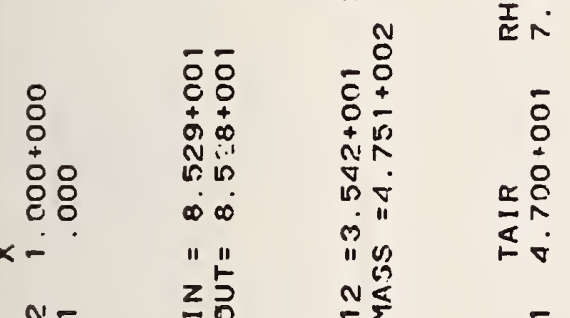

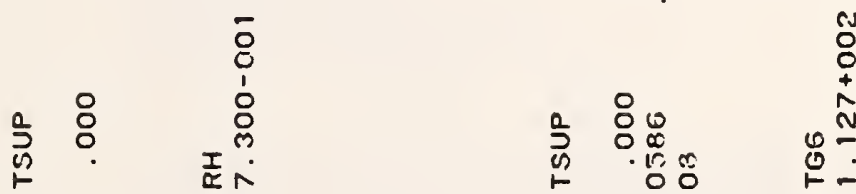

$\frac{m}{\nabla}$

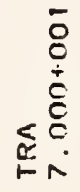

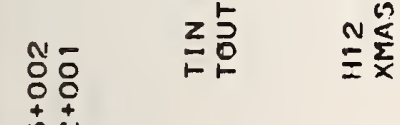

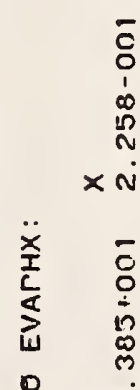

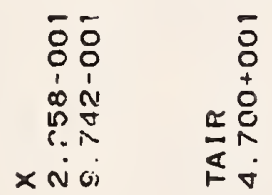

용

$1 \dot{0}$

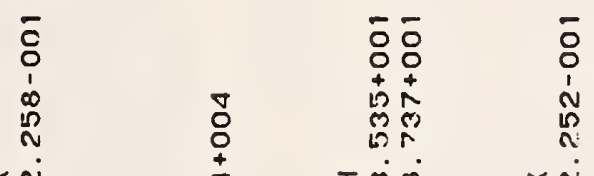

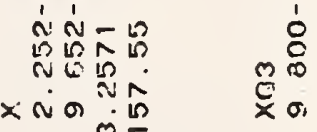

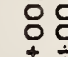

I $\dot{\infty} \dot{\infty}$

$\times 0 i$

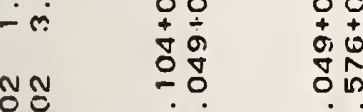

$\overline{0} \quad \bar{n}$

i

i-

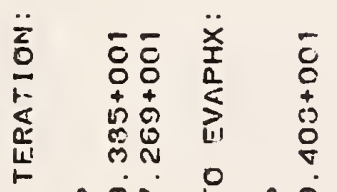

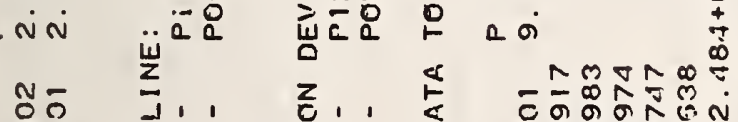

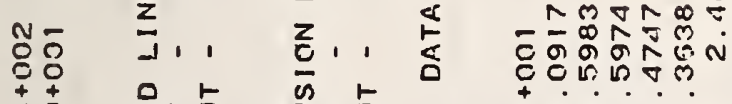

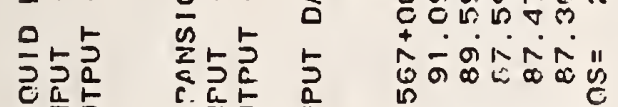

$\underset{\sim}{\infty}$ is

$\checkmark s$

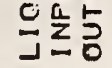

学起起

ल"

Е adi

ह̄ं

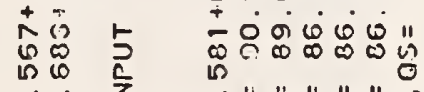

- लि

?"11 11 " 11 .

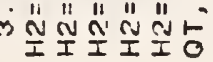

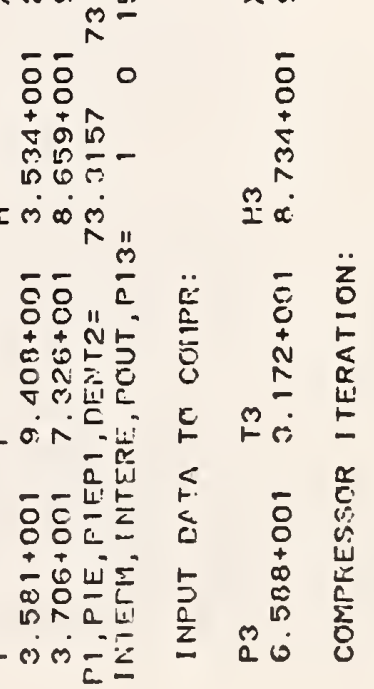

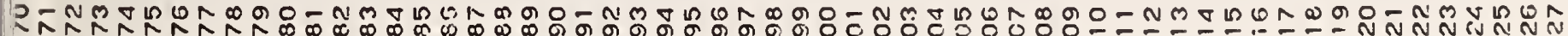

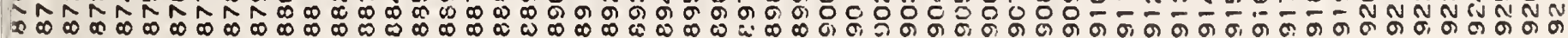




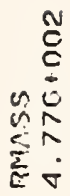

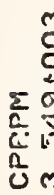

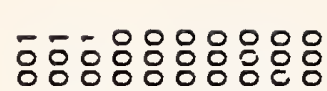

은

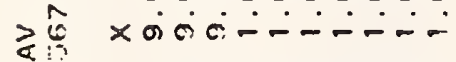

w

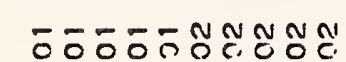

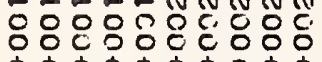

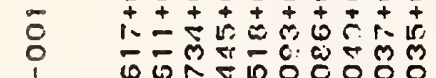

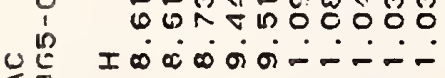

\&

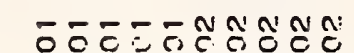

820080800

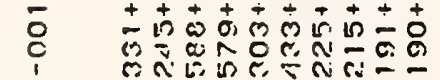

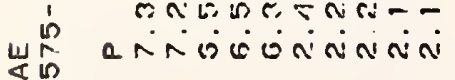

แn

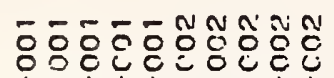

을

1 -लिंक्ष०-:-

स̄ - NMOUONDOO

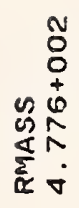

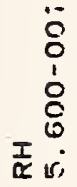

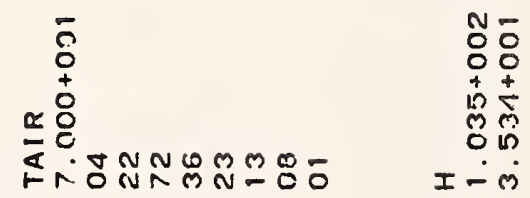

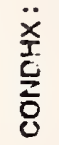

- -

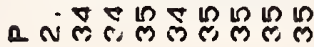

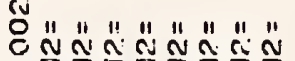

ำ

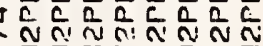

-

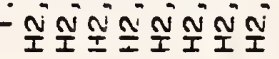

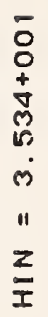

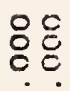

11
25
$x$
$x$
$x$

응

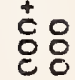

$x-$

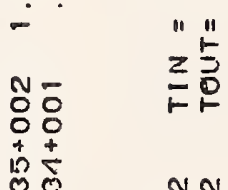

ํํำ

든

वू.

$1+$

음

ani

주를

崩

毃更,

$+\frac{1}{2}$

ก

$\rightarrow$ is:

돈

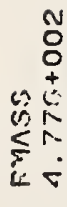

웅

응

$\frac{T}{\alpha} n$

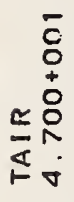

$\overline{8}$
$\vdots$
$\vdots$
N.
N

$\times n i$

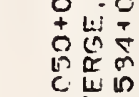

.. त $\sum_{i}$ -

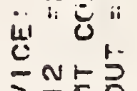

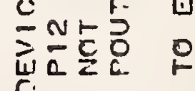

z, 岂, 造

a $\dot{0}$

Tำm

-

OE. T. Th

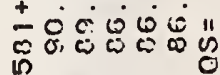
in

ตั

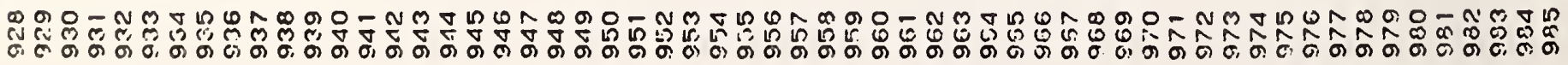




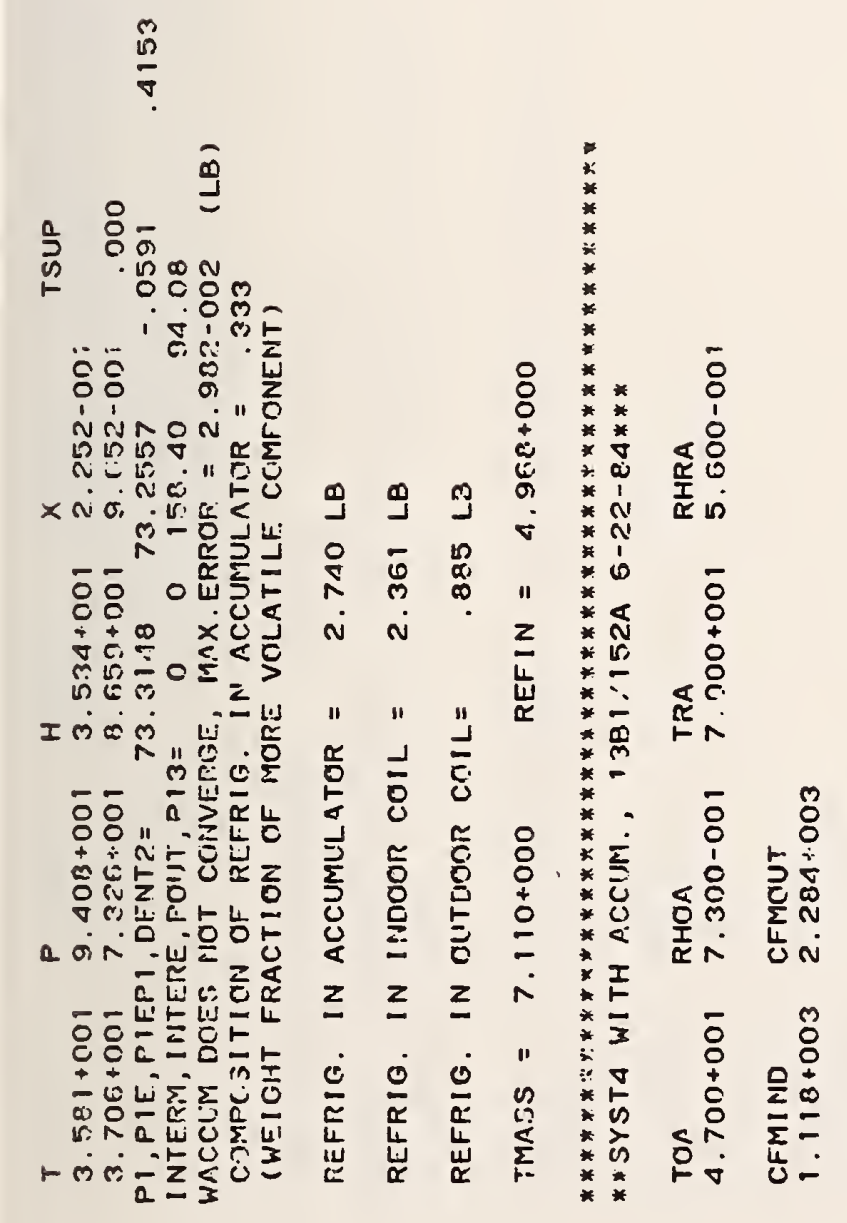

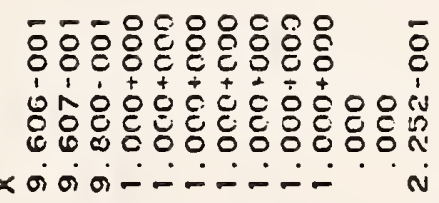

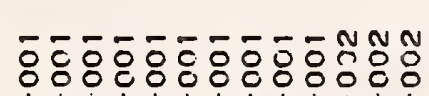

1 íc \begin{tabular}{c}
1 \\
\hline
\end{tabular}

o) $\infty 心 \tilde{N}$

000.064

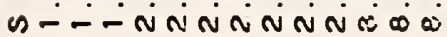

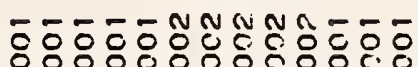

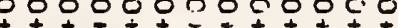

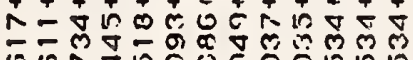
क

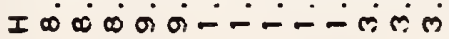

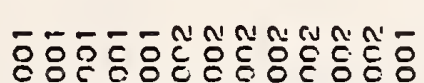

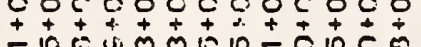

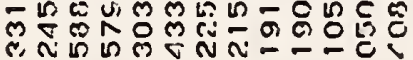

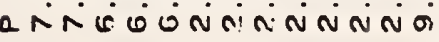

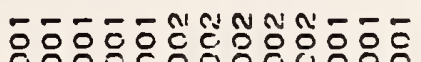
$7+9$
0

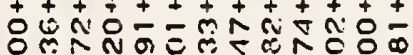
今 虫

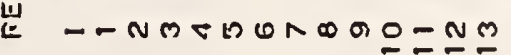

究:

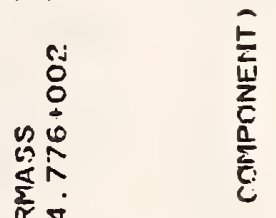

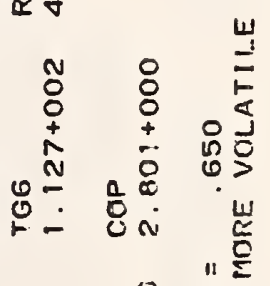

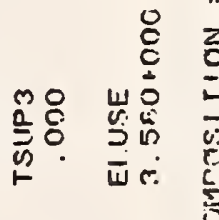

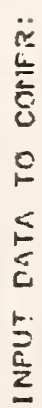

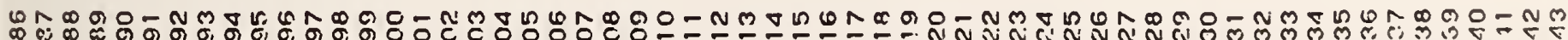

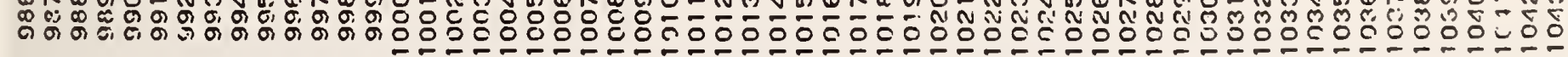


0
0
$\vdots$
0
0
0
0

5
0
+
0
1
50
0
11
2
2
1

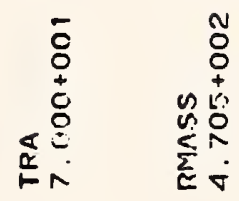

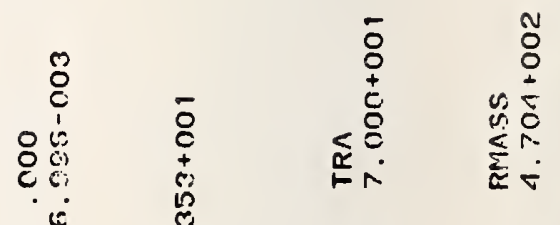

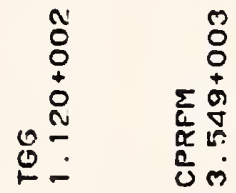

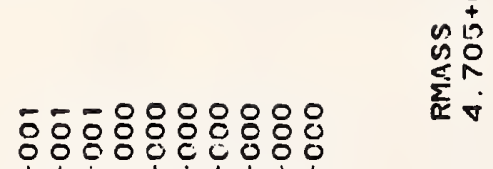

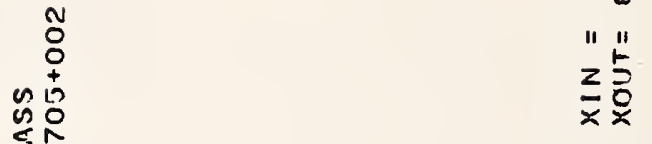

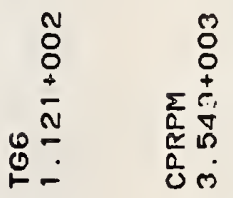

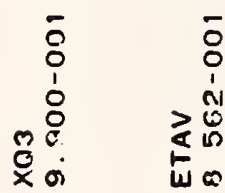

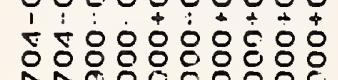
$\times a 0^{2}=a^{\circ}$

-อะรอ

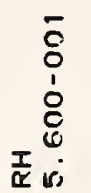

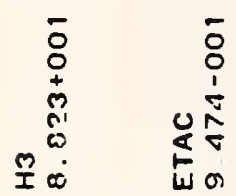

유융융유

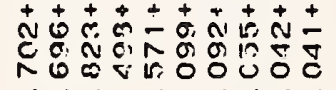

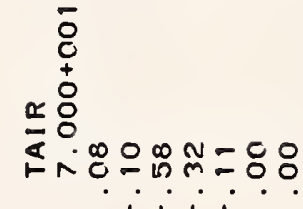

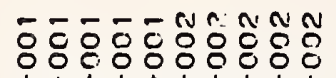

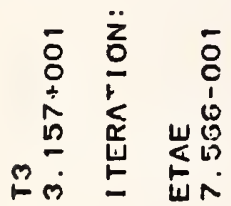

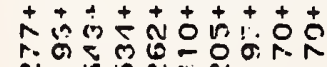

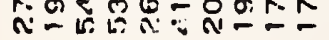

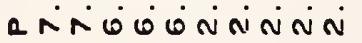

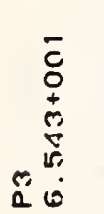

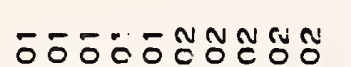

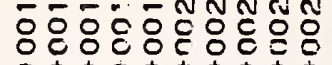

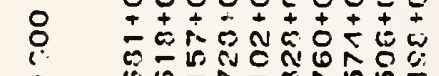

व

Wั

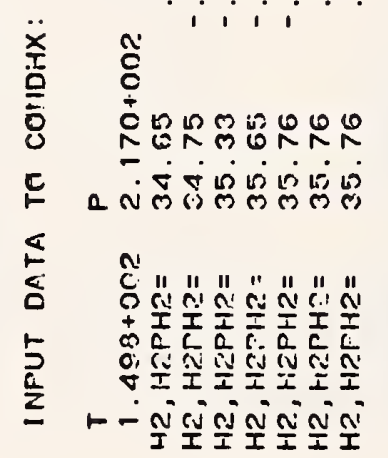

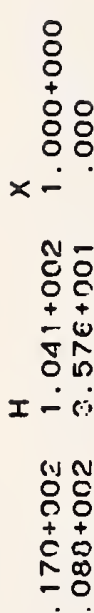

a ini

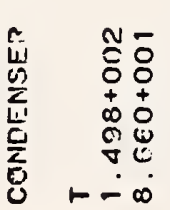

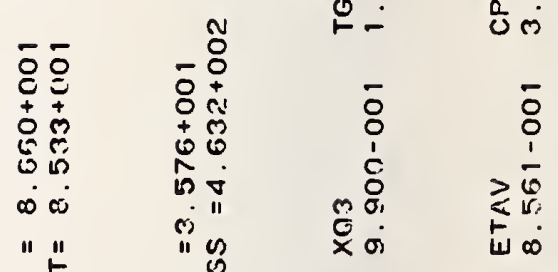

늘

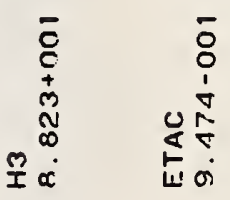

要品

ํำ

S.:

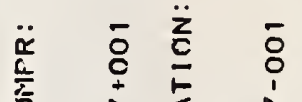

茪"

in

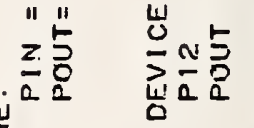

峞芯

$\ddot{w}^{2}$

0 웅

wn

了。

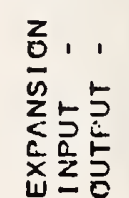

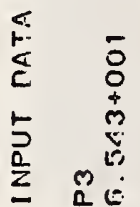

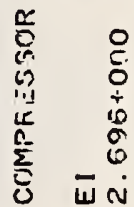

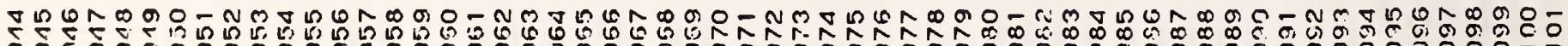

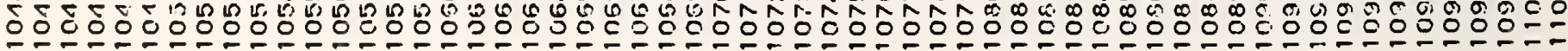




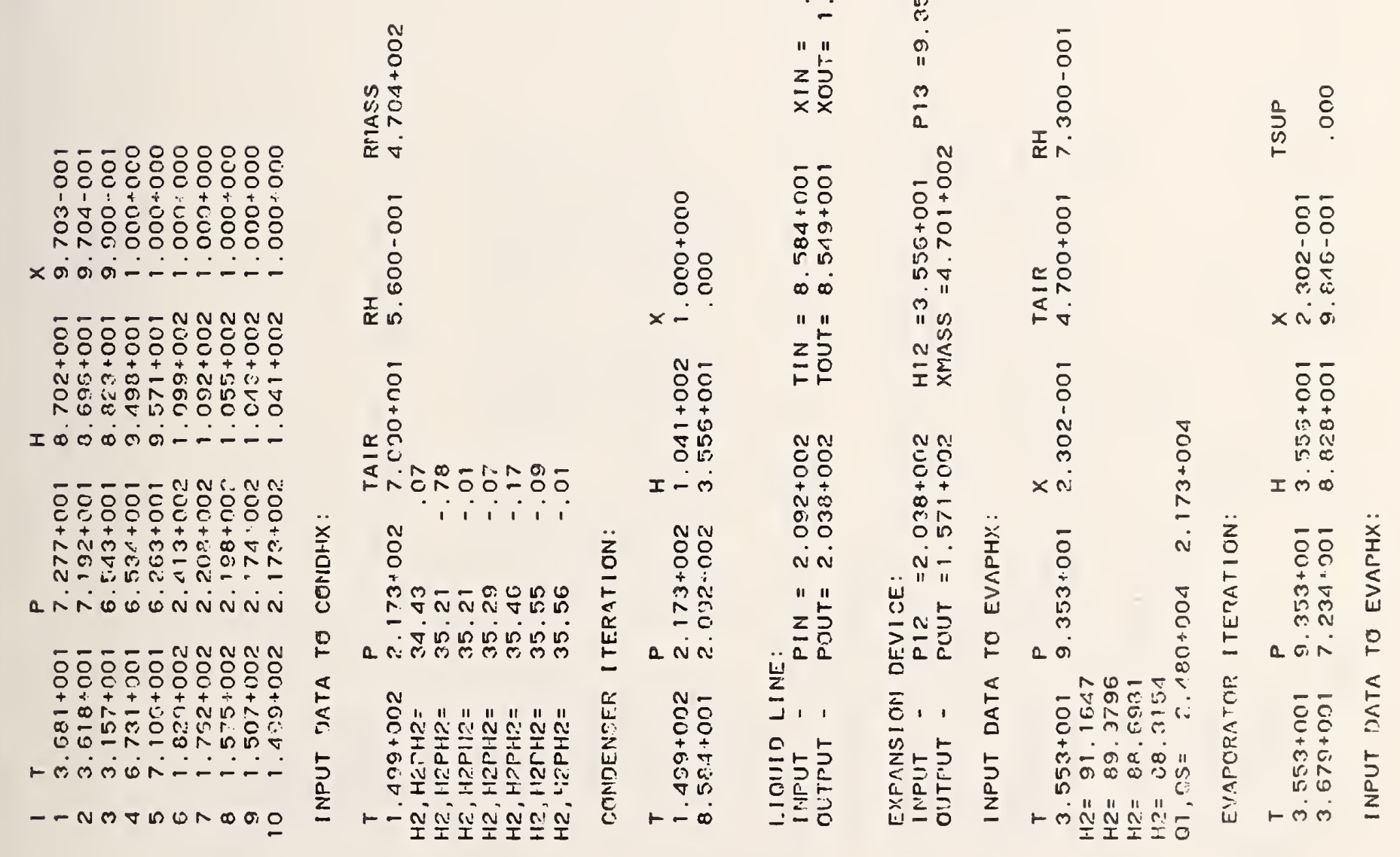

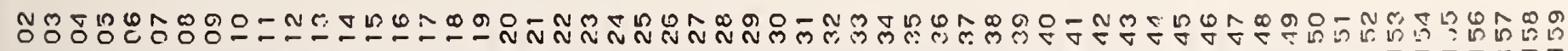

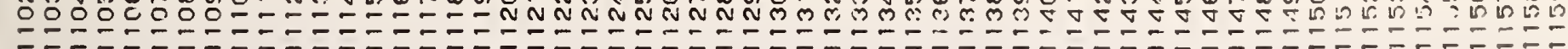


$\sum_{\substack{0 \\ \hdashline}}^{\substack{n \\ 0}}$

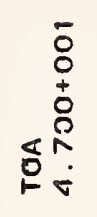

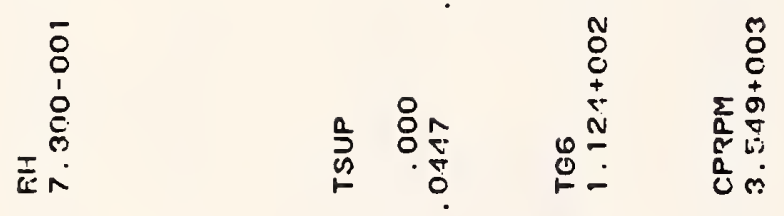

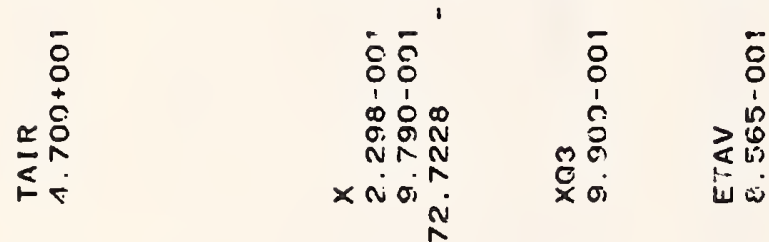

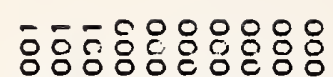

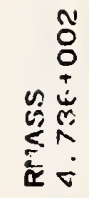

$\begin{array}{ll}0 \\ 1 \\ 1 & 1 \\ 0 & 1\end{array}$

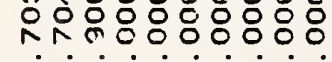

×

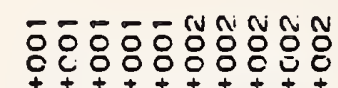

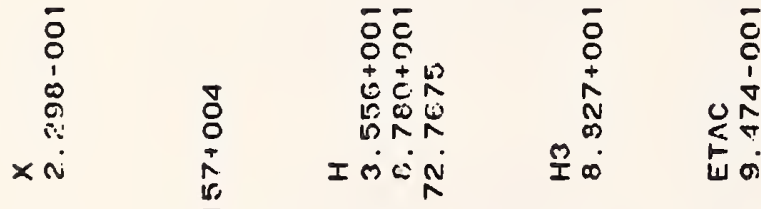

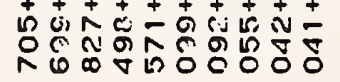

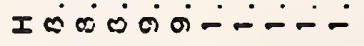

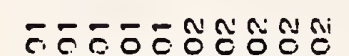

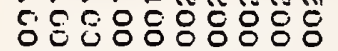

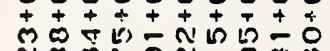

Ne

inio

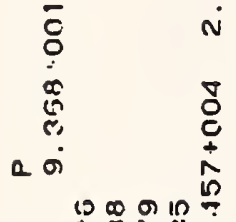

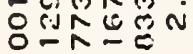

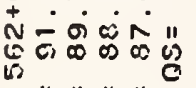

-

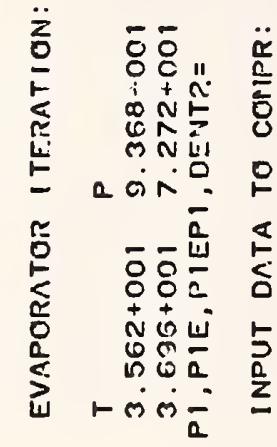

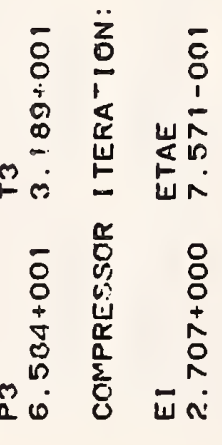

-

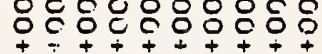

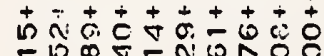

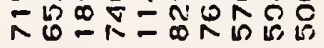

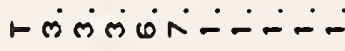

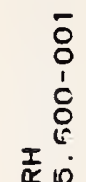

8
8
$\vdots \circ$
$\circ 8$

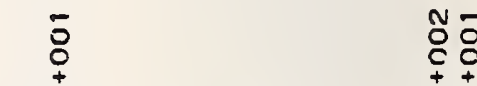

ㄷㅇㅇ

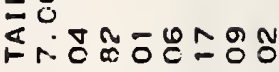

逢

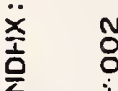

O̊

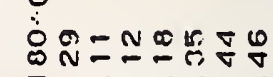

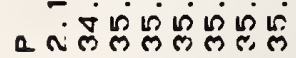

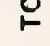

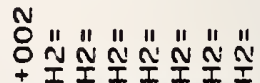

0
0
0
$T$

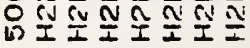

-

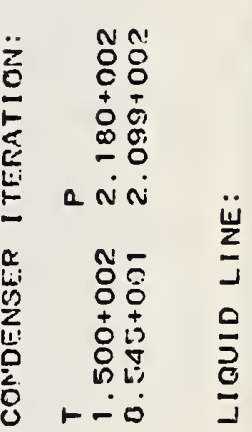

O- ผ

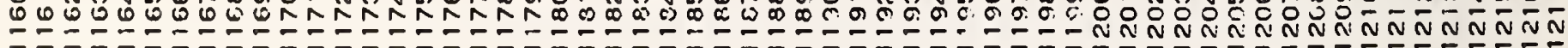




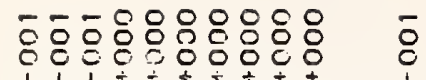

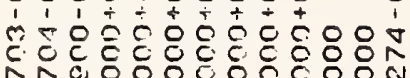

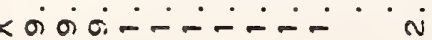

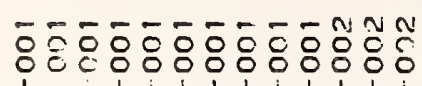

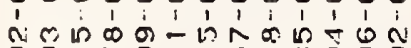

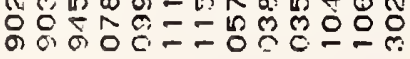
$\omega-\therefore-N N N N N N \dot{N} \dot{N}$

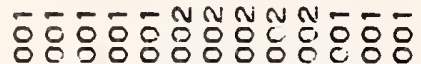

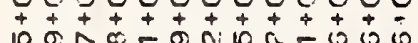
ñ.

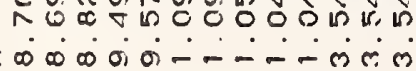

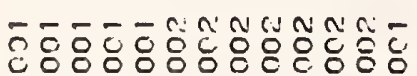

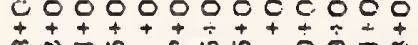

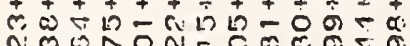

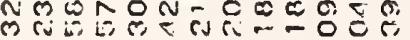
- NNCDONNN

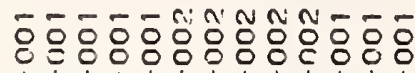

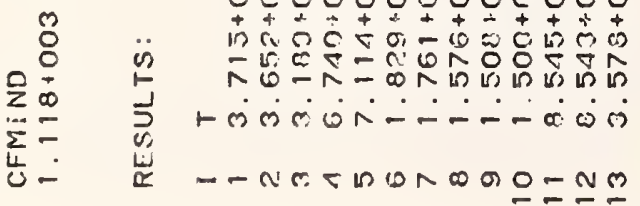

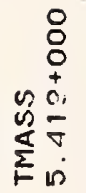

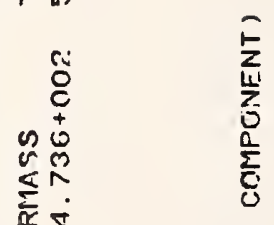

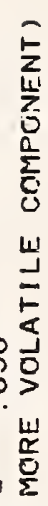

¿

象员 药望

- $\quad$ 选选

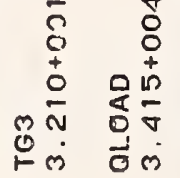

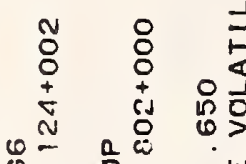

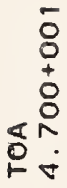

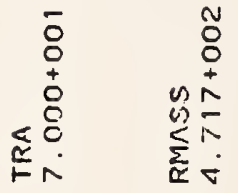

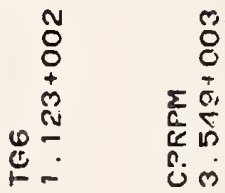

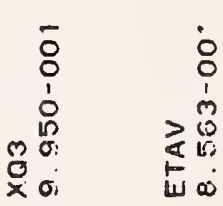

훙ㅇㅇㅇㅇㅇㅇㅇㅛ i.

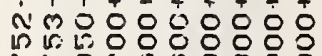
人र⿵冂卄

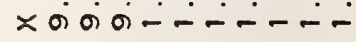

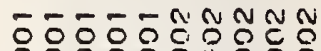
80000 U0.0

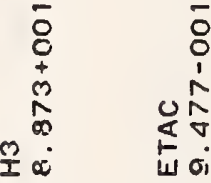

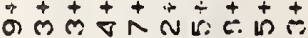

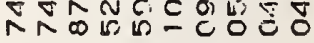
$I \infty \infty \phi i \dot{0} 0 \ldots$

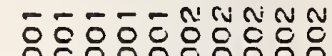

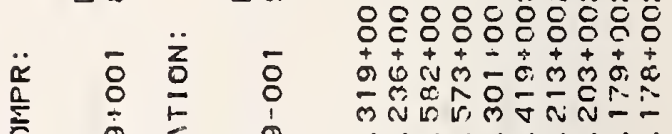
añoboniñón 


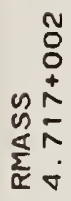

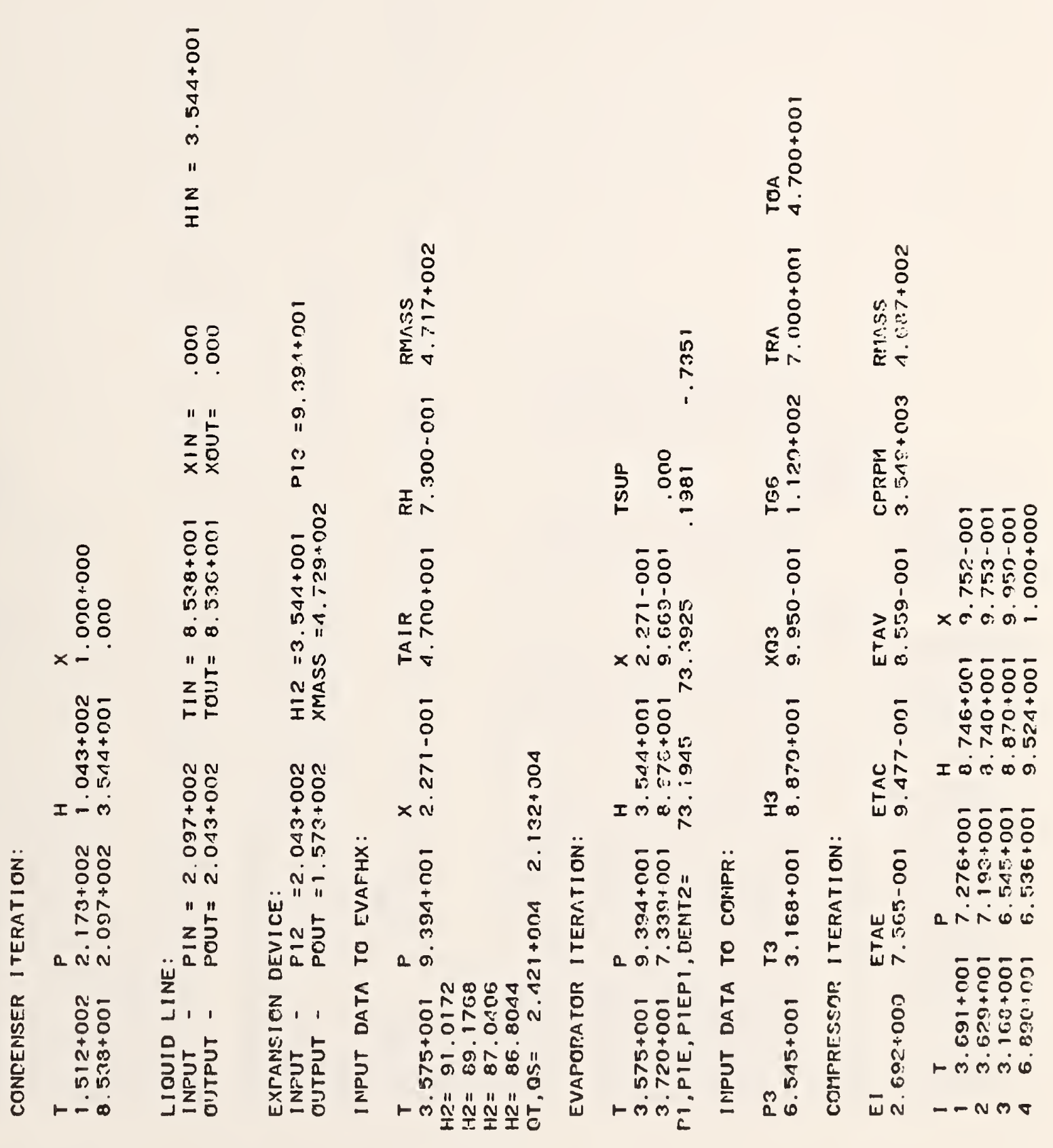

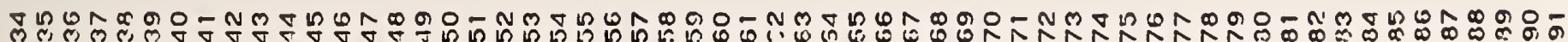

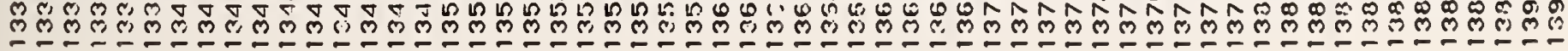




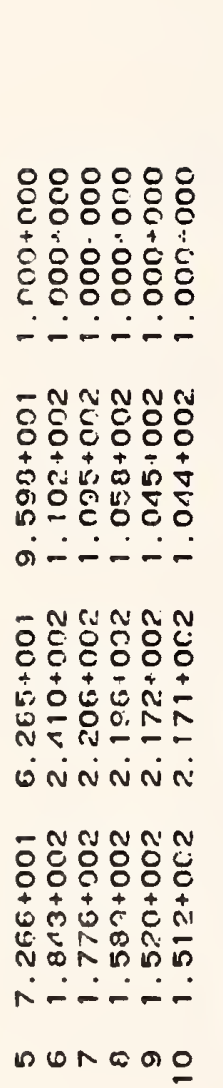

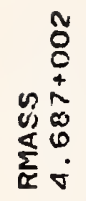

5
0
+
0
0
0
11
$z$
2

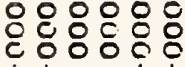

รุด

.....

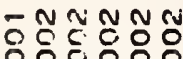

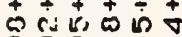

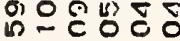

ตับ

อรำำ

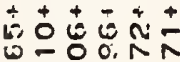

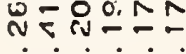

DnNNNA

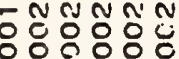

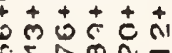

N

UUNAOO

8
8
0
8
0
1
10

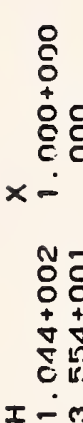

ํำำำำ.

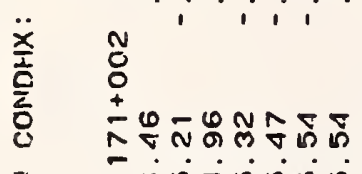

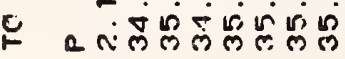

$\S$

웅

$\simeq \stackrel{\dot{8}}{\circ}$

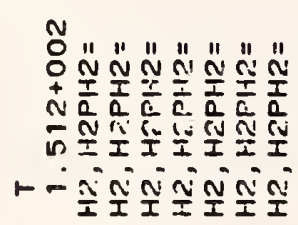

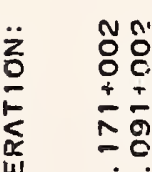

a तंN

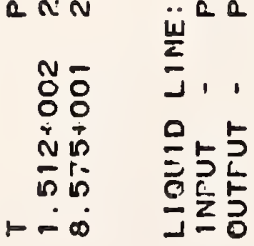

nin

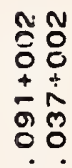

z芺 㟧

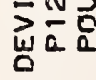

否, 1

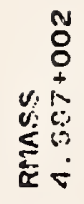

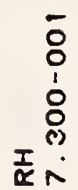

$\sum_{\substack{0 \\ \leftrightarrow}}^{0}$

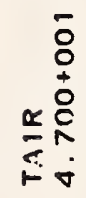

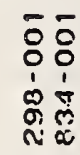

$x$ No

$\overline{8}$
1
0
$\stackrel{2}{2}$
$\times \stackrel{\text { n }}{0}$

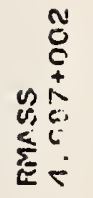

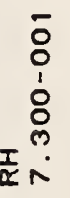

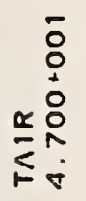

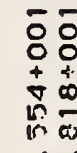

I लंख

$\begin{array}{ll}\ddot{x} & \\ \frac{1}{c} & 0 \\ \frac{1}{2} & \frac{1}{10} \\ 0 & 0\end{array}$

< a

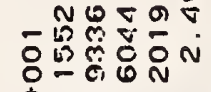

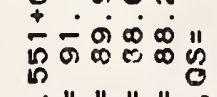

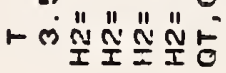

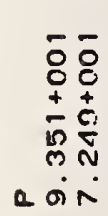

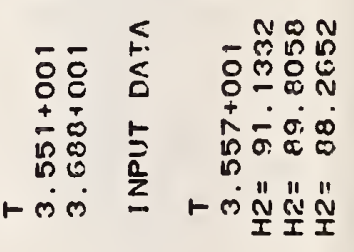

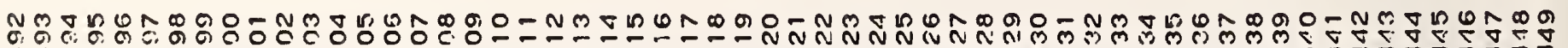

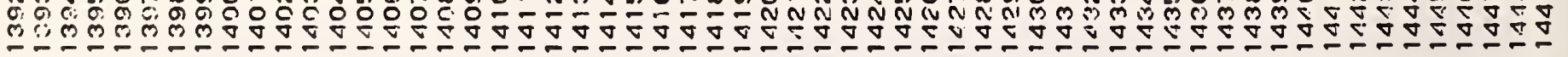




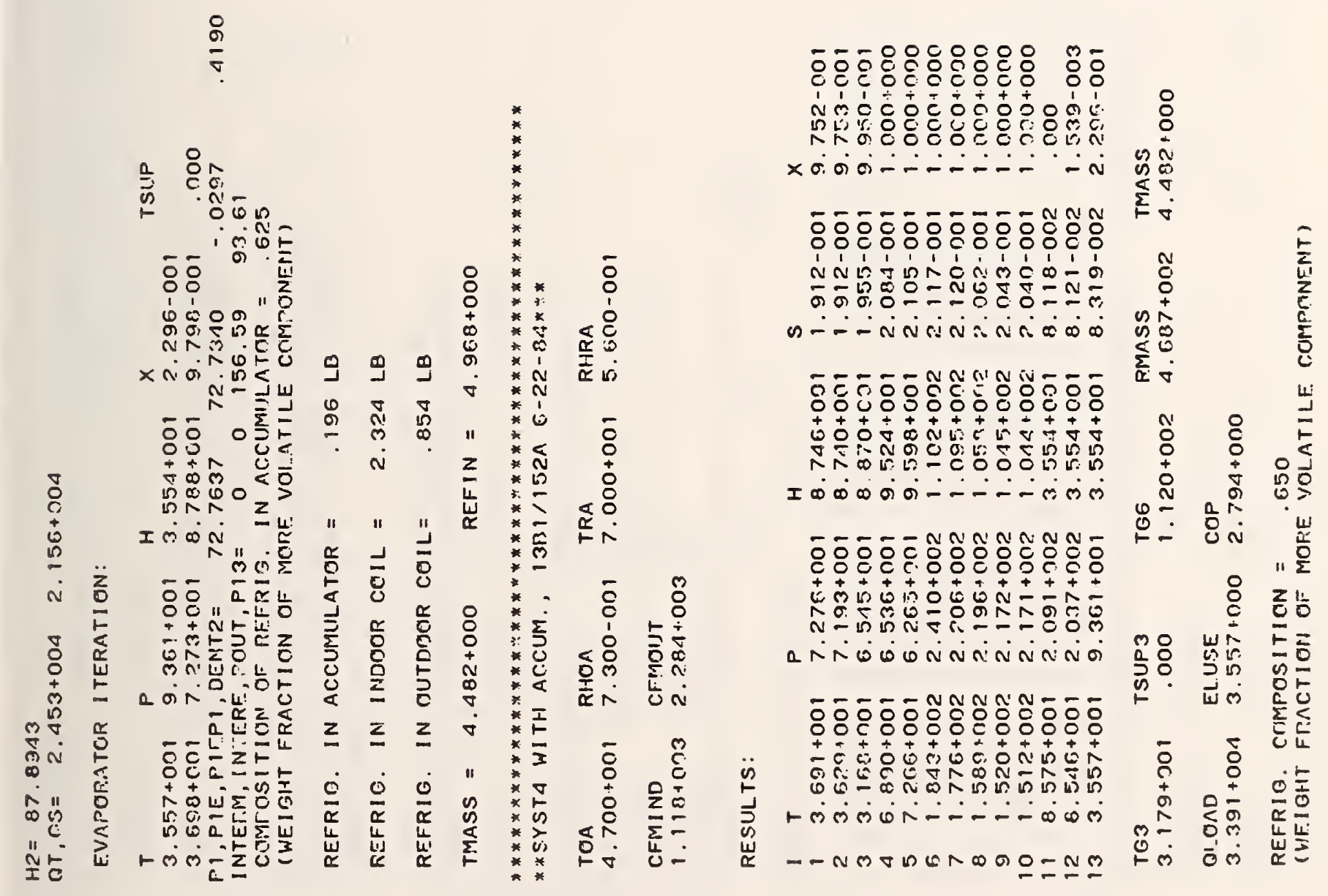

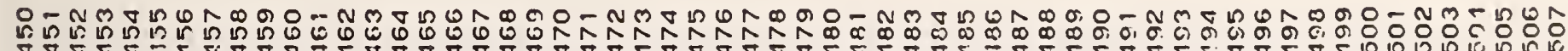

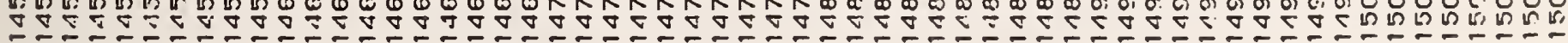




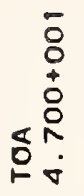

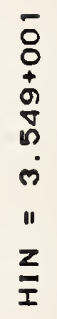

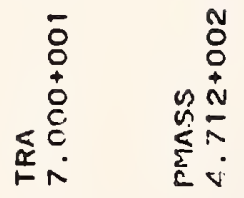

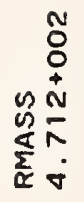

$\because$

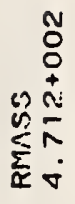

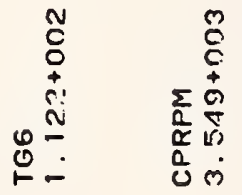

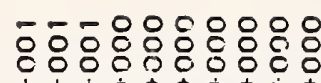

z"

$\begin{array}{r}\overline{8} \\ \vdots \\ \vdots \\ 8 \\ 0 \\ \hdashline\end{array}$

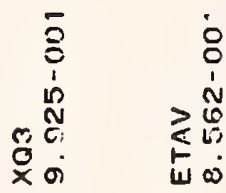

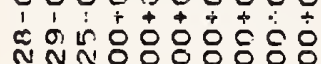

hN

$\times$ oino $\therefore-\cdots-1$

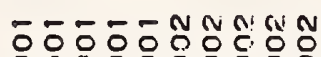

8008808280

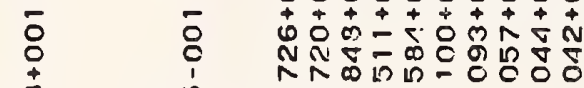

造足

I $\infty \dot{\infty} \infty \dot{0} 0 \div-\cdots$

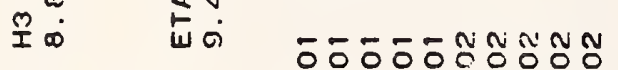

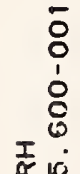

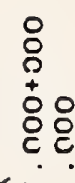

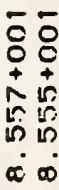

$x-$

훙

$\simeq 8$

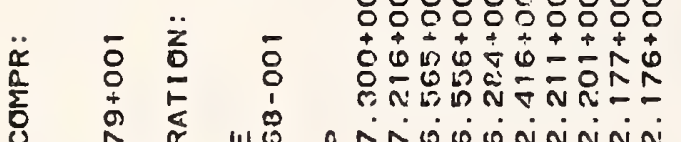

aniogonanina

욤ㄷㄴ

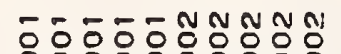

$\leqslant-\frac{L}{6}$

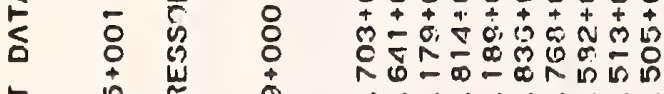

5 总

ํํำกำกำ

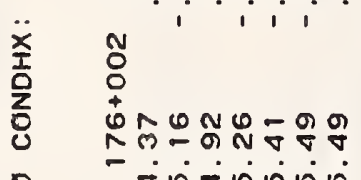

-

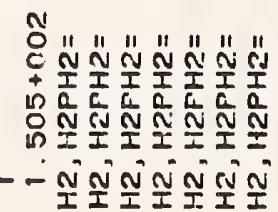

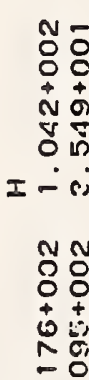

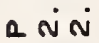

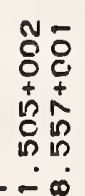

0
8
+
0
0
0
0
11
$m$
0
0

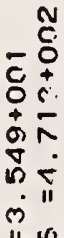

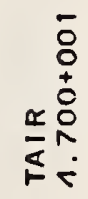

I $\sum_{X}$

$\bar{c}$
$\grave{d}$
$\stackrel{N}{N}$
$\times \stackrel{N}{N}$

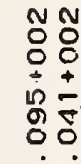

용ำ

$\overline{0}$ in

i⿱⺌兀)

$\bar{\delta}$
0
+
1
0
0
0

ż⿺辶一

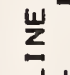

吉, 它

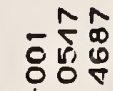

노

눙

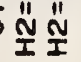

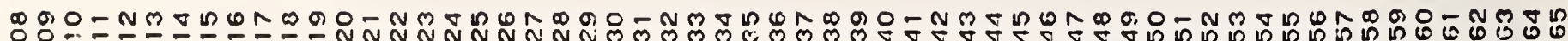

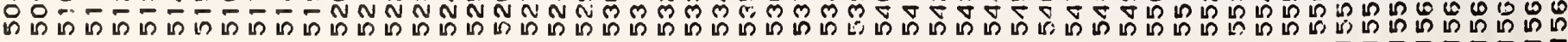




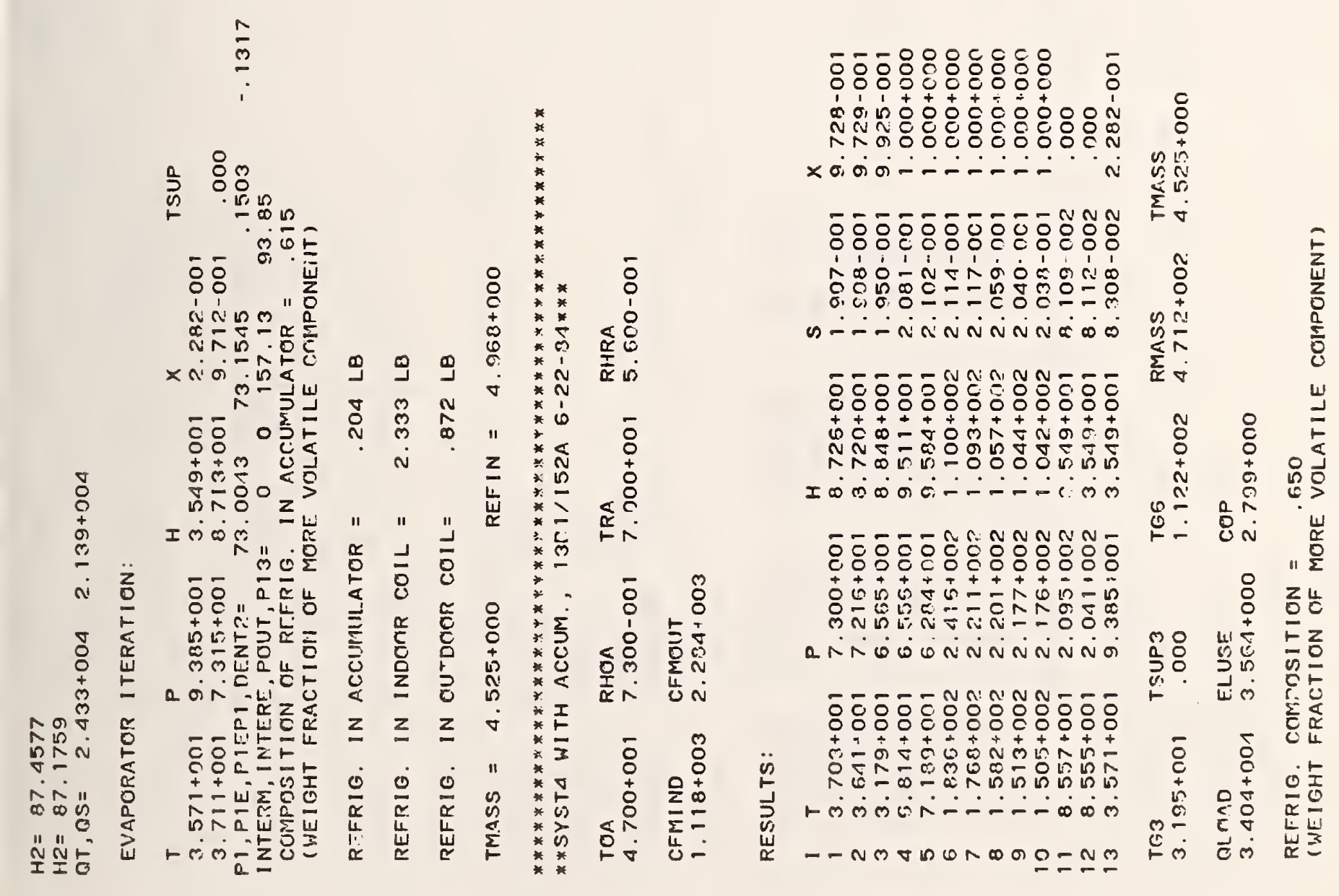

BNDO O-NG

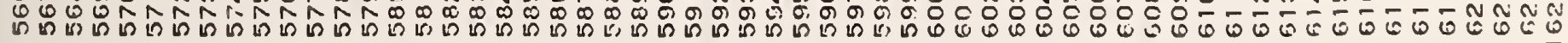




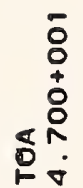

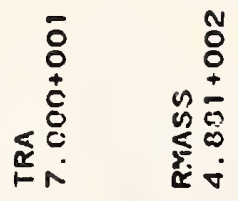

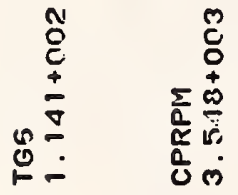

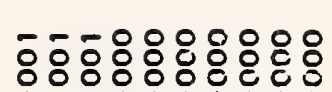

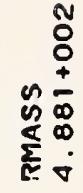

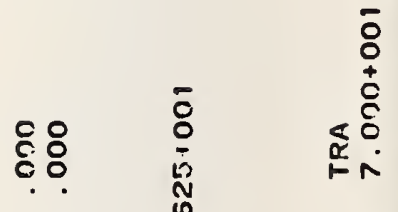

0
8
0
0
0
0
0
1

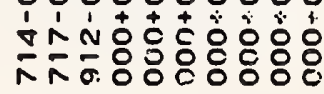

กั่

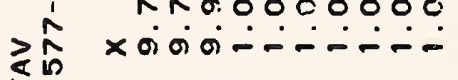

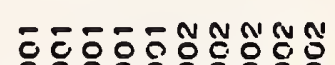

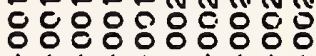

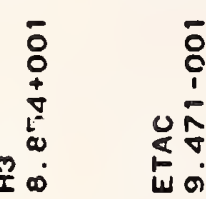

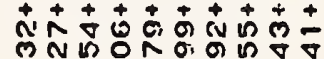

กำ

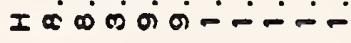

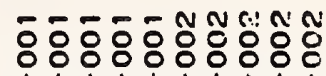

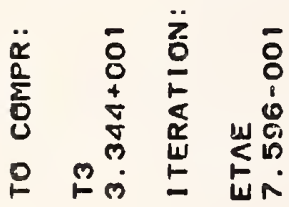

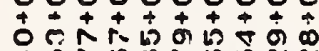

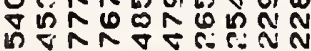

antiogensinas

< 휴유ํํํํำ

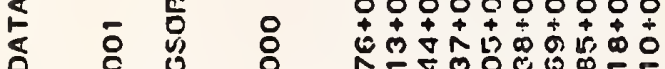

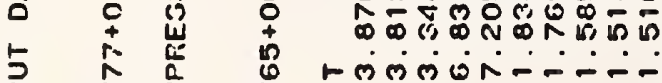

吉

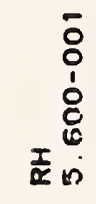

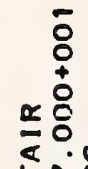

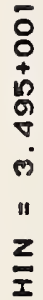

2

"z돔

¿ั

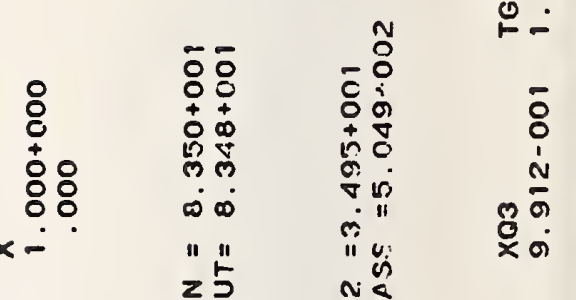

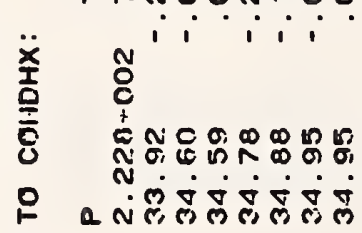

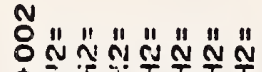

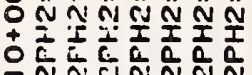

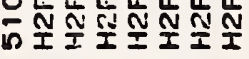

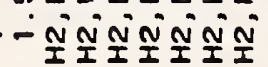

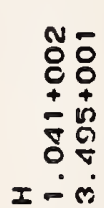

I这

@.

苂

ํํㅇㅇํㅇ

FF

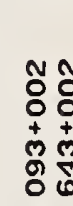

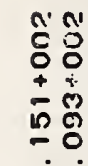

ผั่

लें

点

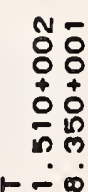

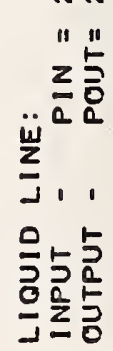

ii

峞"

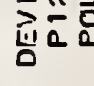

告,

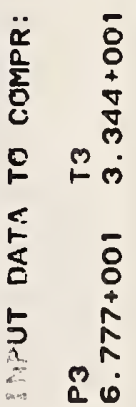

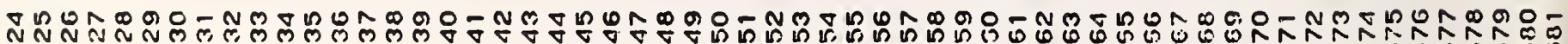

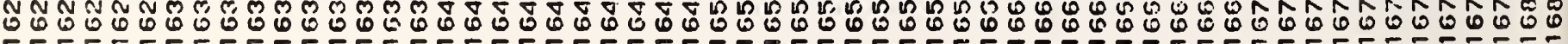




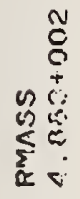

$\overline{8}$
0
$\vdots$
0
0
in
in
11
$z$
$z$

管

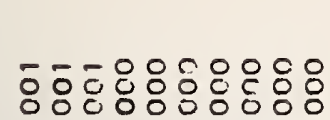

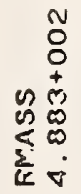

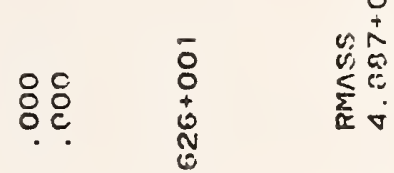

ñ

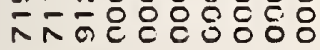

×oino:- …

-

$\frac{z}{x} \bar{z}$

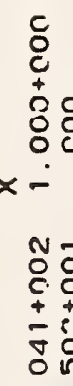

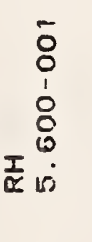

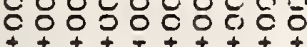

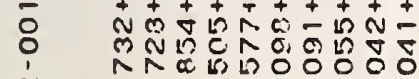

UN I $\frac{N}{N} \cos \theta 0-\cdots \cdot$

出

-

0.808080 .8

z

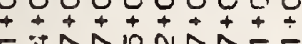

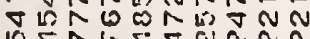

b.

mo añóóññNiñ

s?

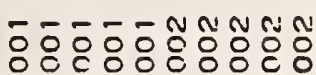

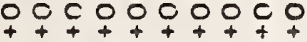

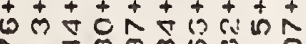

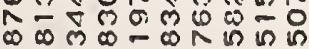

मलंलिएं-.-:

$\bar{\omega} \dot{\sim}$

$\begin{array}{r}\overline{8} \\ 0 \\ \vdots \\ \hdashline \\ \hline\end{array}$

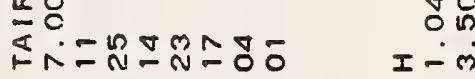

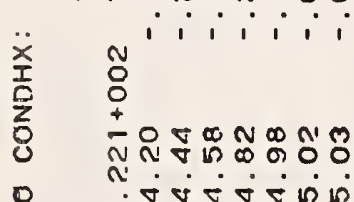

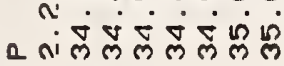
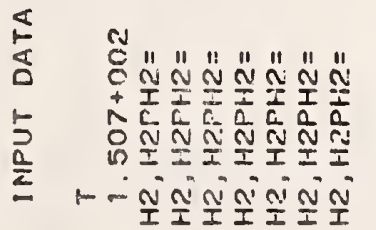

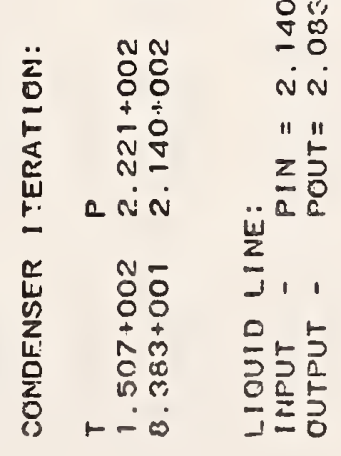

$\begin{array}{r}\overline{8} \\ \vdots \\ \vdots \\ \hline \\ \hline\end{array}$

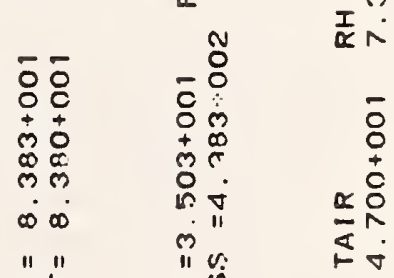

$\sum_{\substack{5 \\ 5}}^{0}$

8

$\underline{\hat{n}}$

$\times \sim$

$\overline{0}$
0
1
0
0

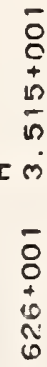

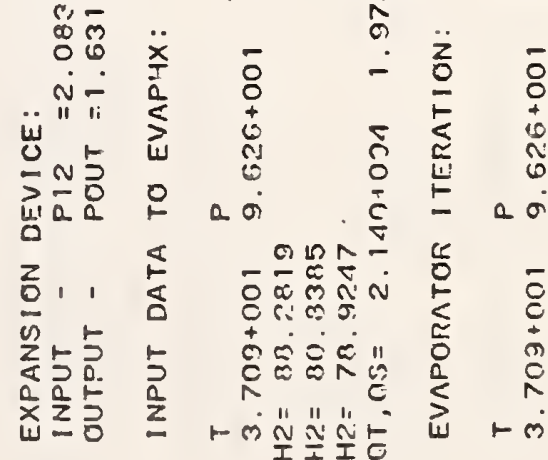

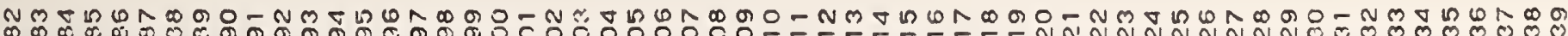

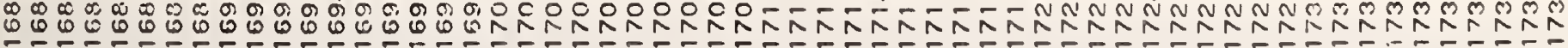




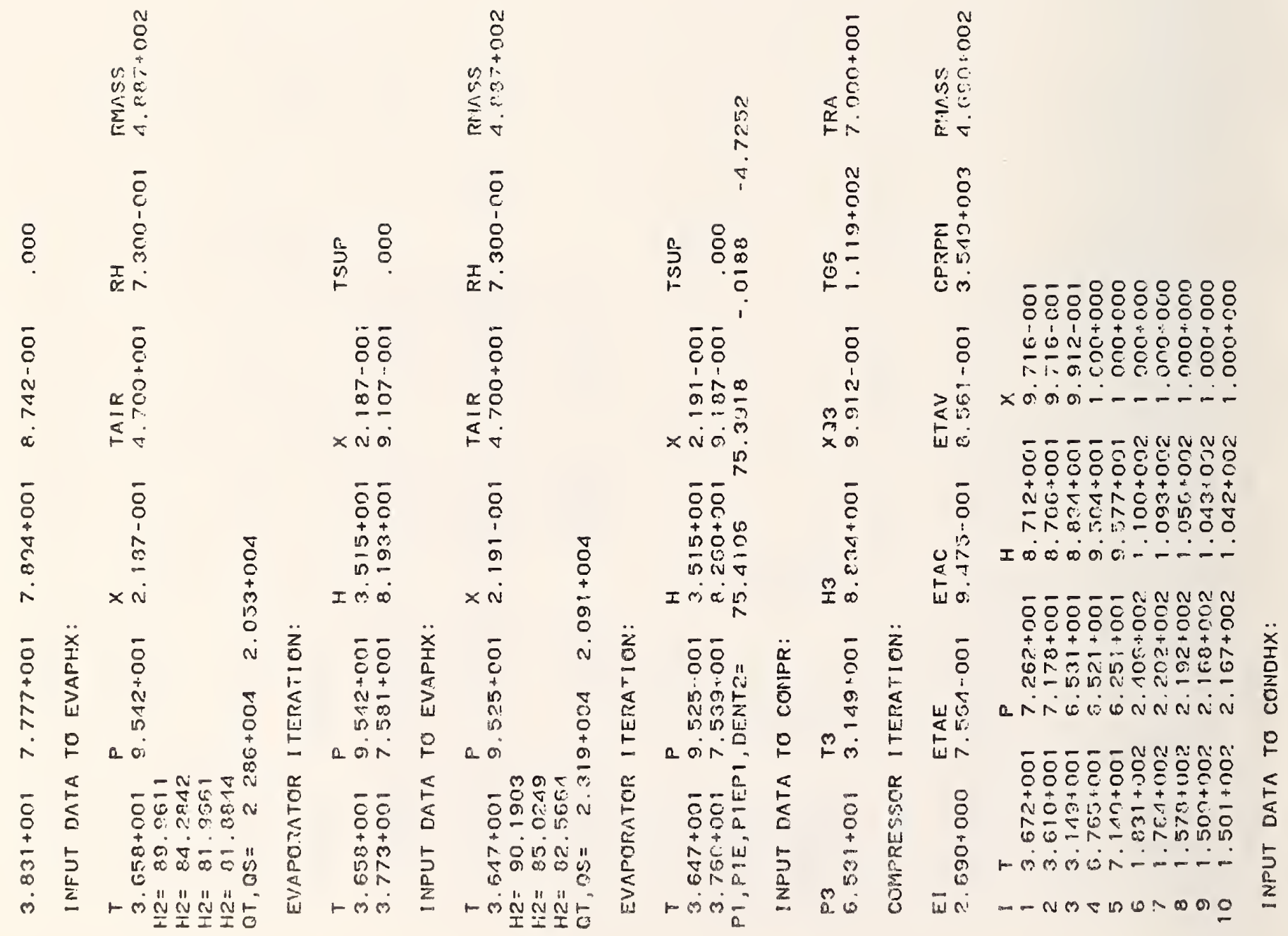

O-NM П tivis 
5
8
5
5
0
0
$1 "$
$z$
$\frac{2}{2}$

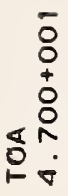

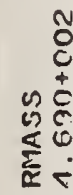

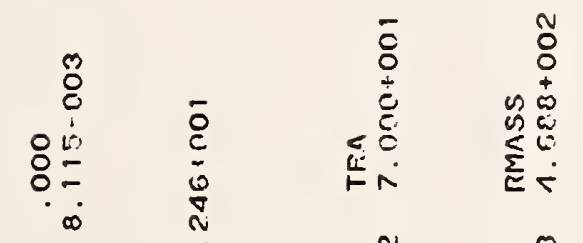

" 11 व

름

¿

$\overline{8} \overline{0}$

$\frac{m}{a}$

象:

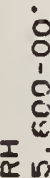

$\overline{8}$

$\simeq \stackrel{+}{0}$

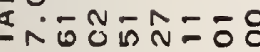

융응

줄

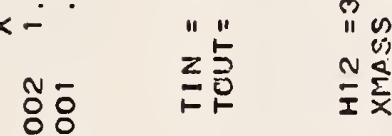

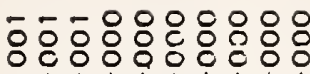

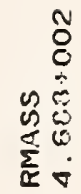

$\tilde{\check{o}}$

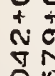

पु

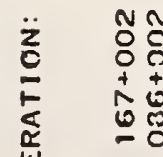

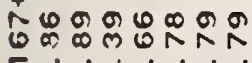

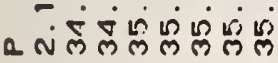

ถั

a พंख

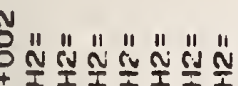

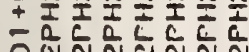

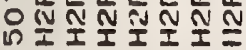

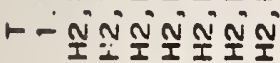

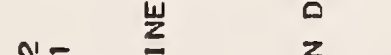

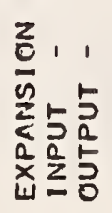

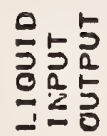

0
0.5

65

कि

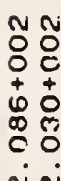

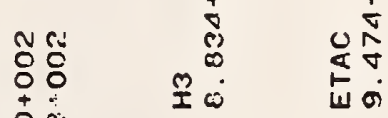

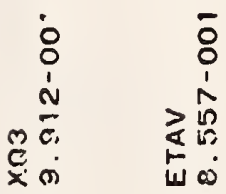

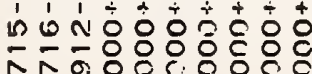

×नंब-:- :-

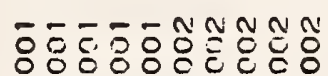

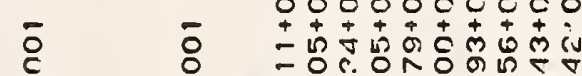

人⿻一𠃋火

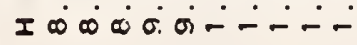

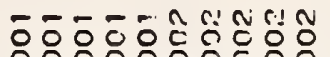

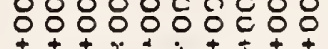

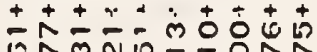

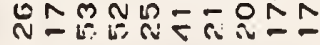
bñobiññ

-

응영응ㅇㅇㅇㅇㅇㅇㅇㅇ

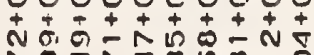

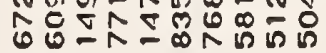

लिंक्षि-‥-
-

แิ
$-N m \nabla \operatorname{ran}$

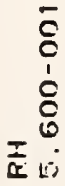

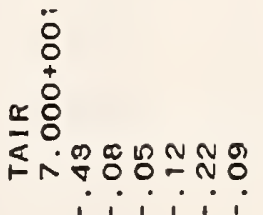

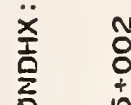

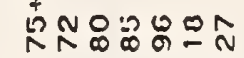

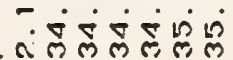

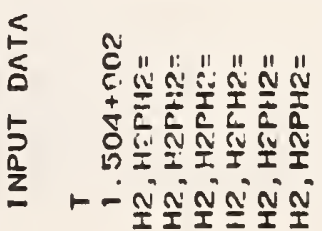

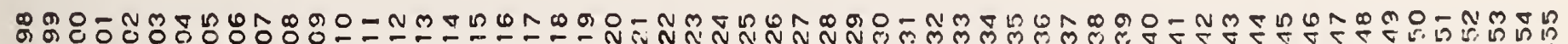

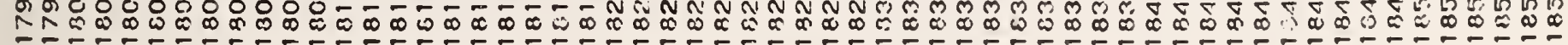




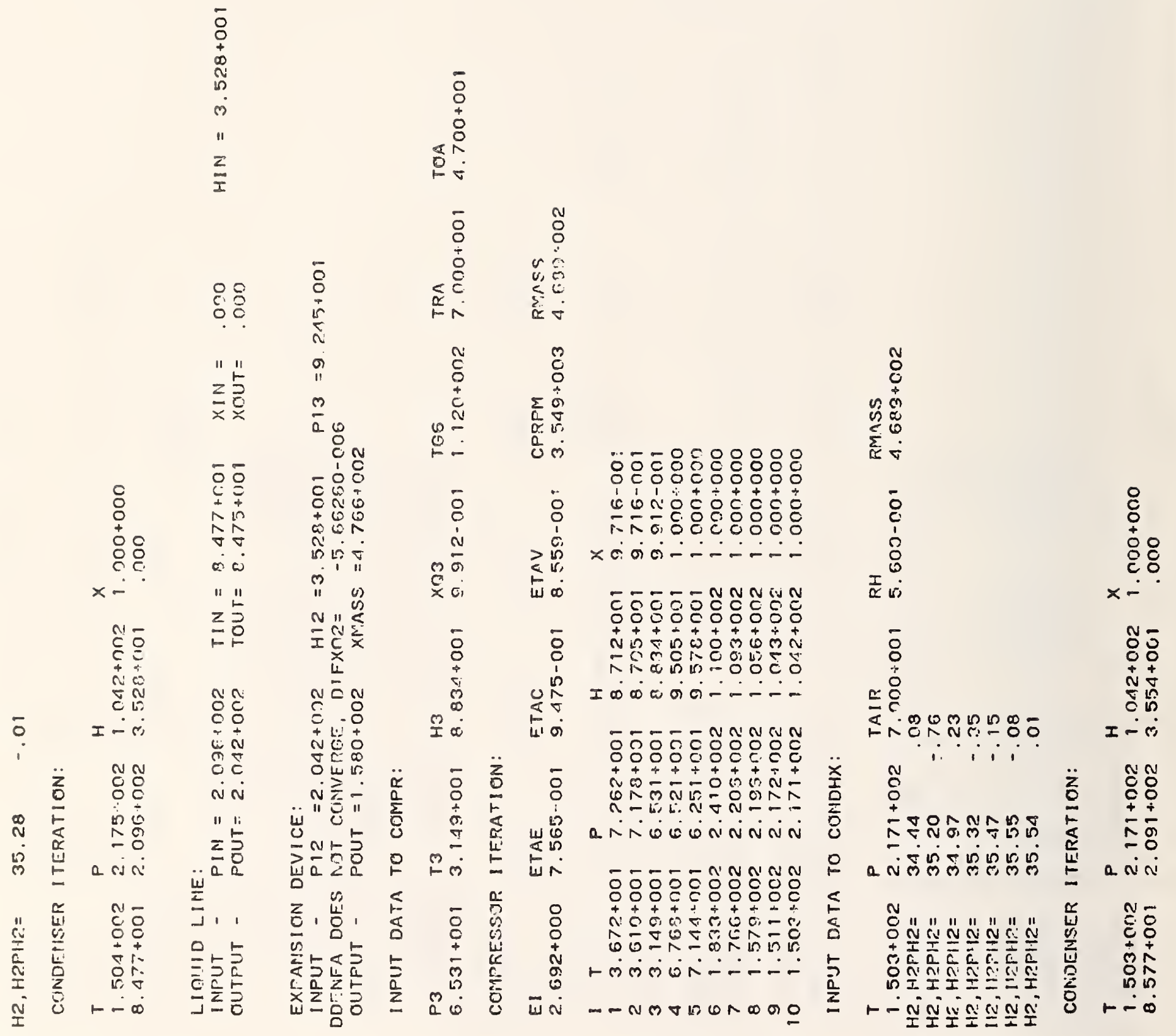

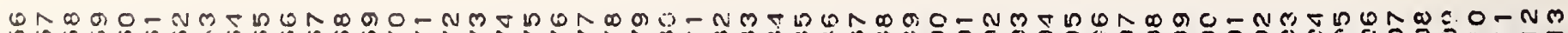

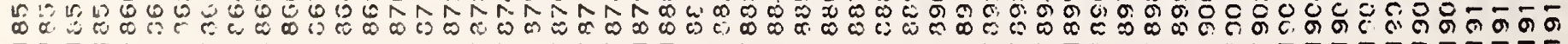


5
8
+
4
0
0
n'
11
$z$
$z$

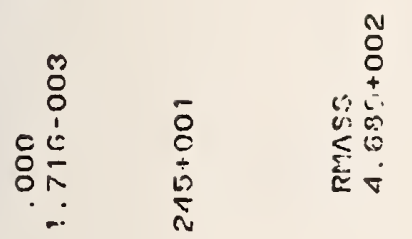

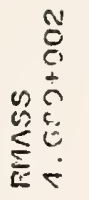

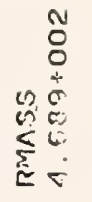

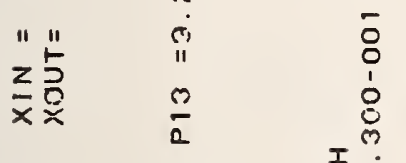

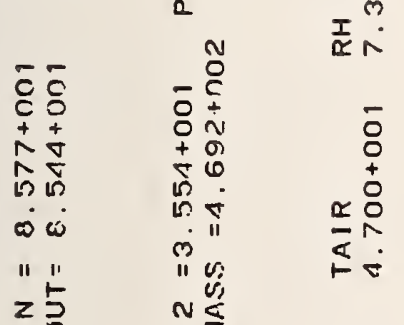

$\begin{array}{lll}8 & \overline{0} & \bar{c}\end{array}$

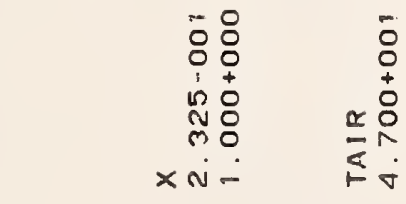

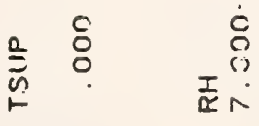

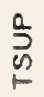

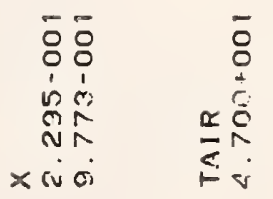

$\overline{0}$
$\dot{0}$
$\dot{0}$
$\stackrel{2}{\times}$
$\times \dot{v}$

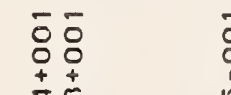

됴

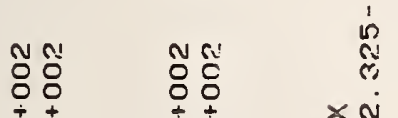

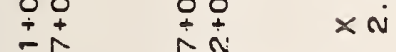

नूँ

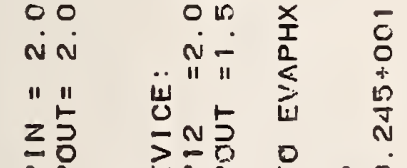

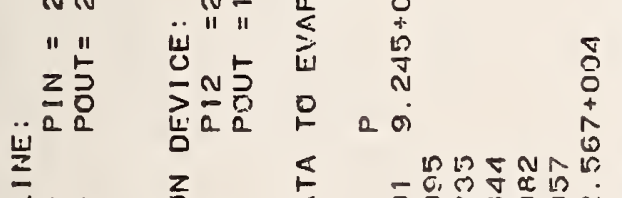

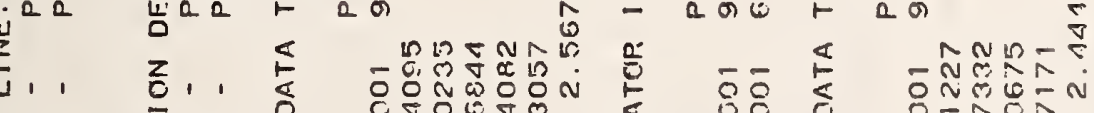

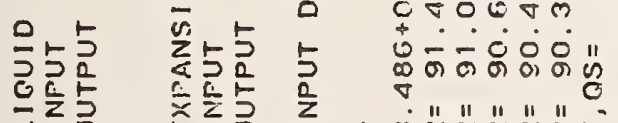

는

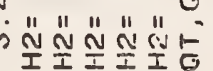
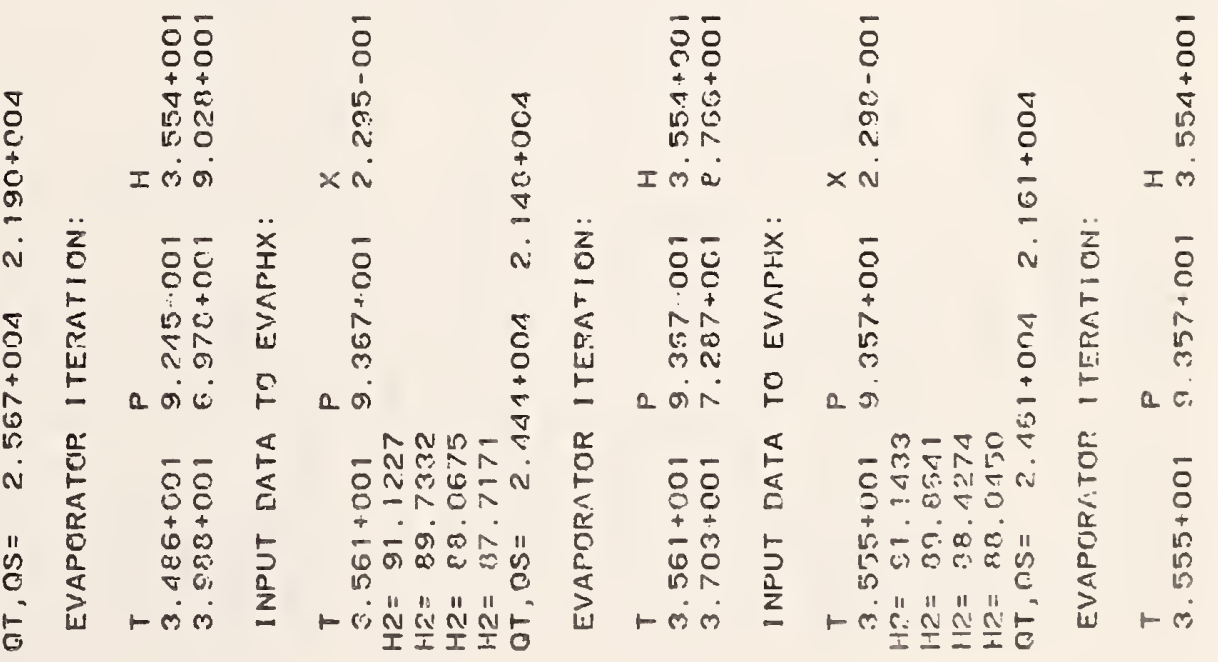

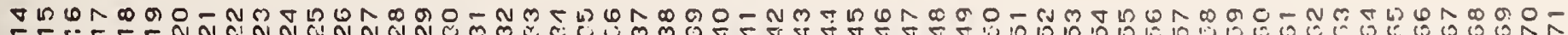

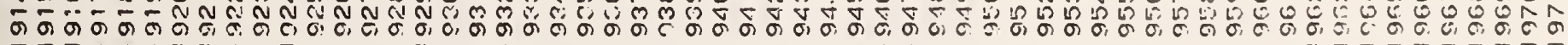



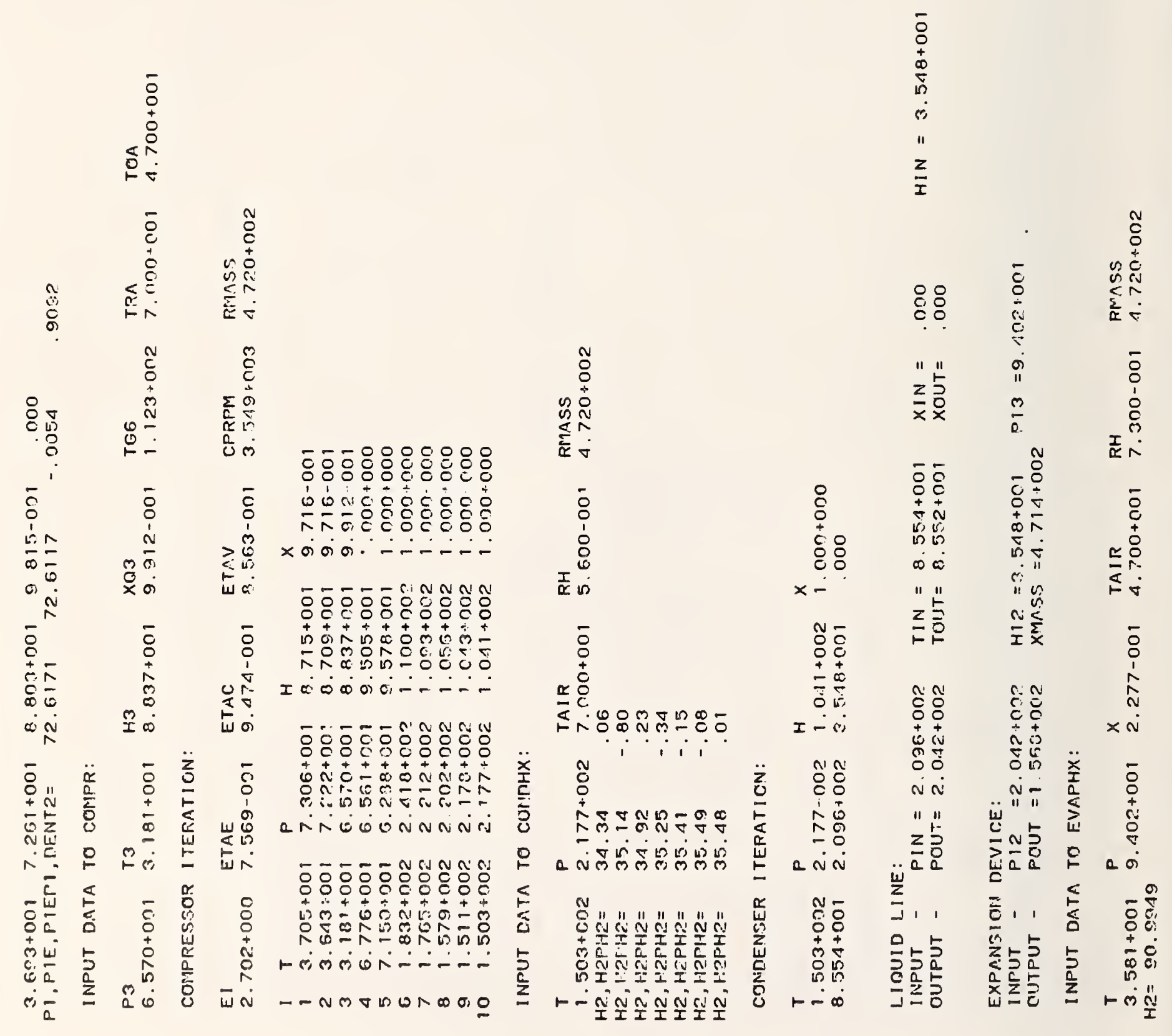

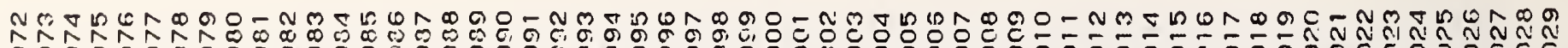

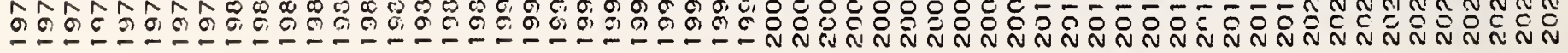




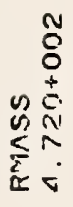

$\stackrel{n}{\circ}$

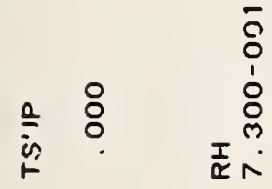

点

둥ㅇㅇㅇ

ón

ススの次

×0ं0:-

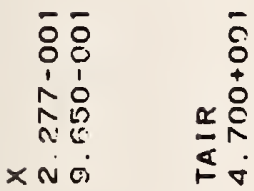

ㄷํㅇ

- in

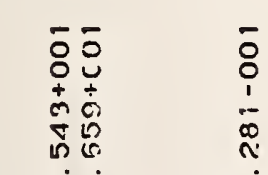

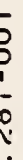

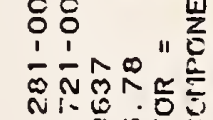

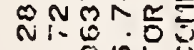

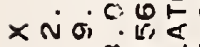

م-ग

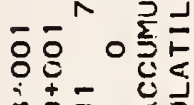

i商它o

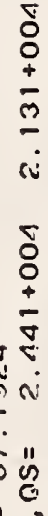

I लिक $x$

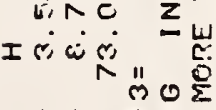

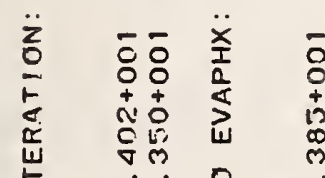

acin

a $a$

$2 \pi)^{2}$

लैख़

कiष

"1 " "

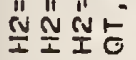
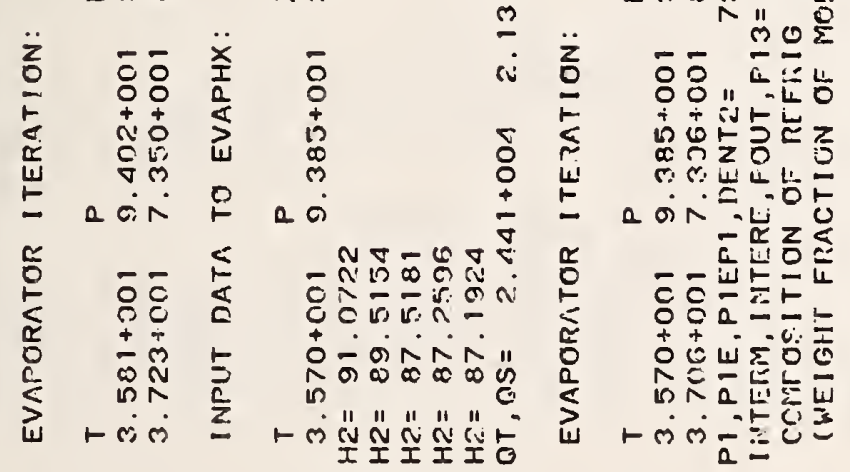

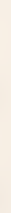

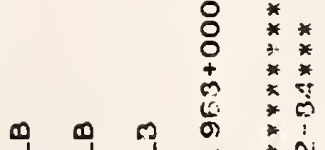

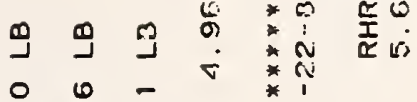

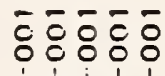

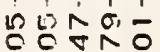

करेट-

$n-\cdots n$

휴웡융

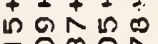

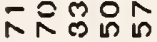

Iक्षंक

흥ํㅇㅇㅇㅇ

$++\cdots+$

ON

añó

วิ

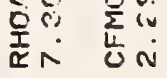

흐읋홍

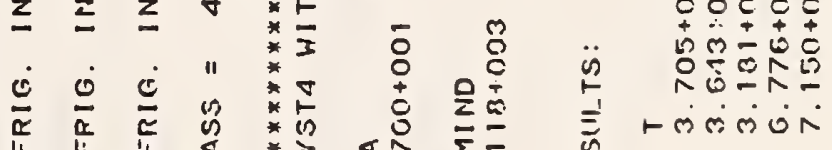

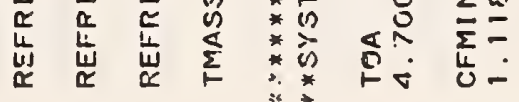

崖

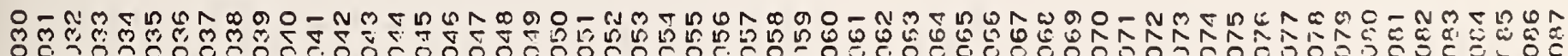

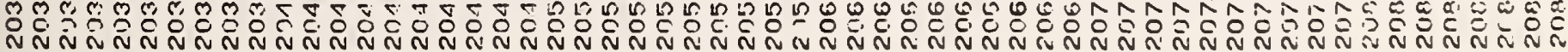




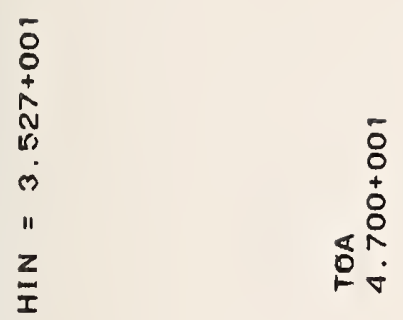

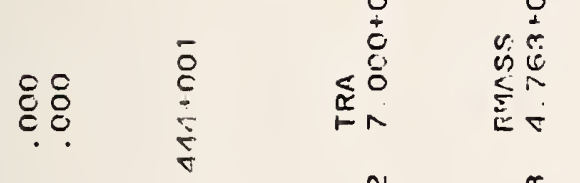

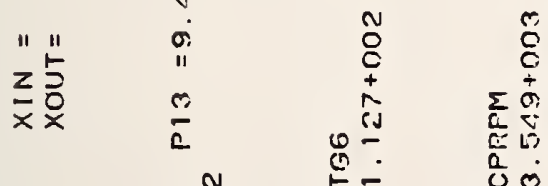

○

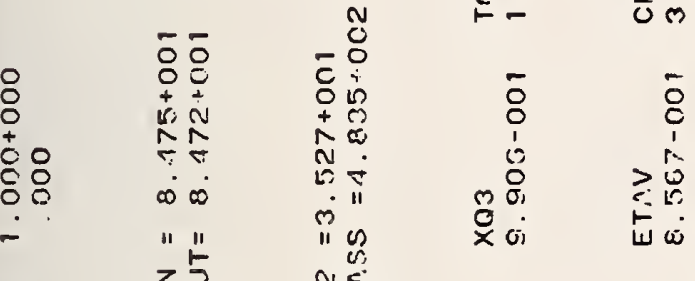

흥ㅎㅇㅇㅇㅇㅇㅇㅇㅇㅇㅇ응

1.1

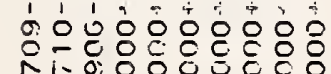

×ण

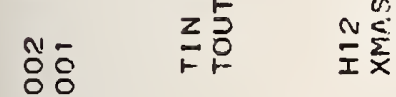

-

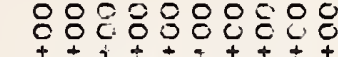

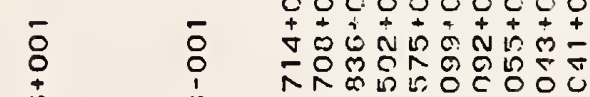

I $\sin \theta \sigma^{\circ}-\therefore-$

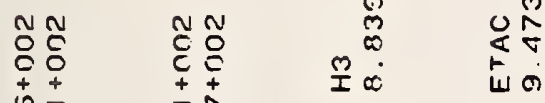

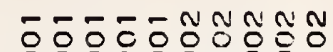

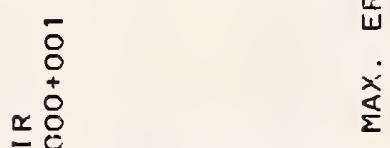

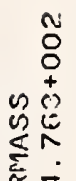

$\sum_{\substack{c \\ \vdots}}^{2}$

व

告

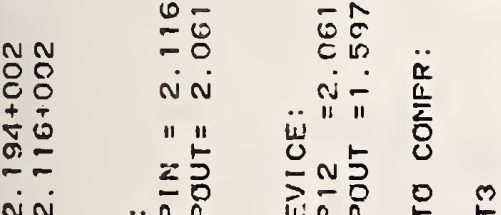

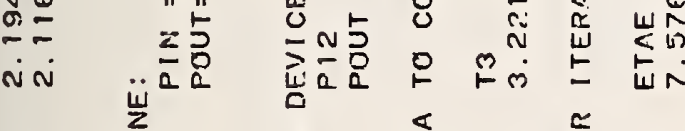

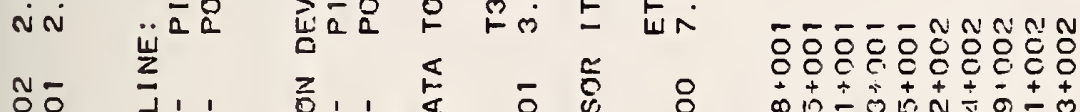

8080808000

$0 \pm 0$
0

0 wa

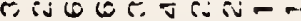

añouninan

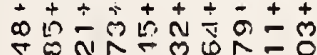

NEN N

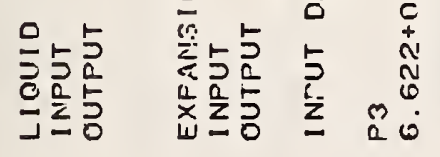

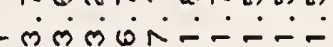

$\frac{1}{n}$

$\bar{\omega} \mathrm{N}$
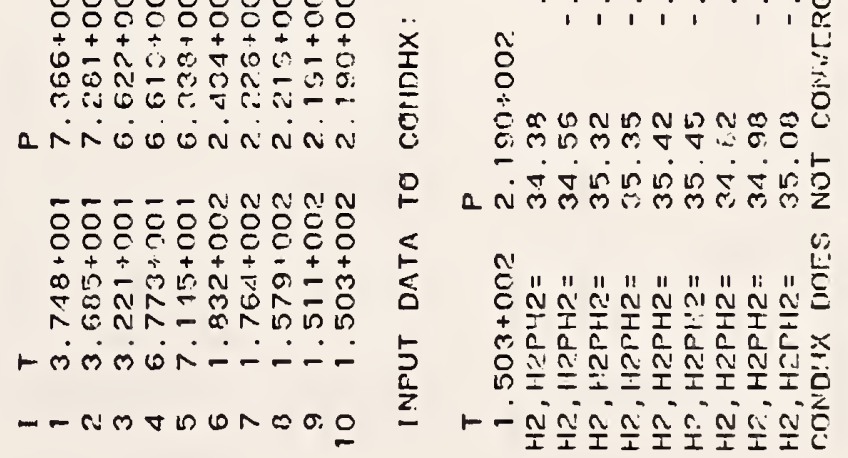

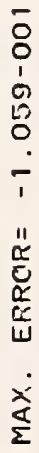

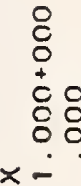

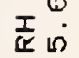

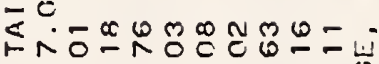

a-

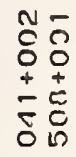

I-

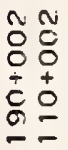

a ก

든

co

कㅇ

เ: ?

$1-\infty$

$\underset{z}{u}$

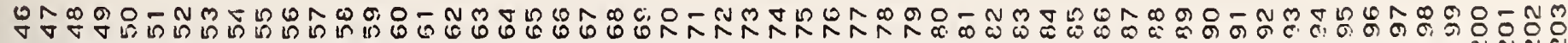

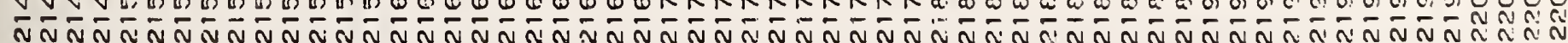


$\overline{8}$
+
+
0
0
0
0
$1 "$
$z$
$z$
$z$

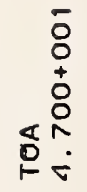

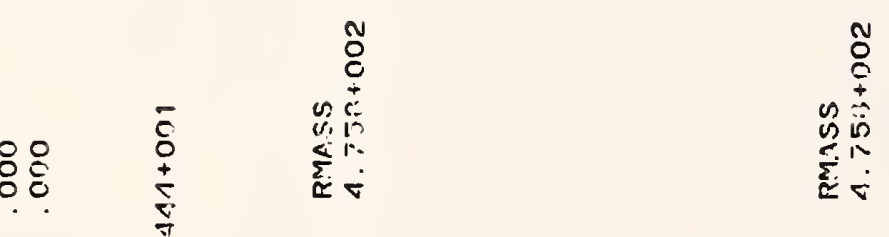

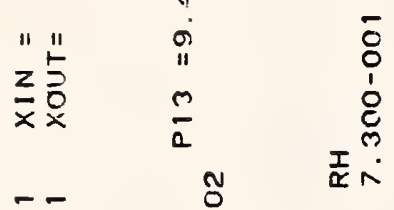

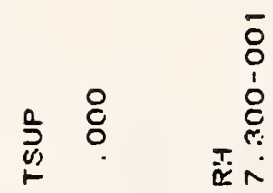

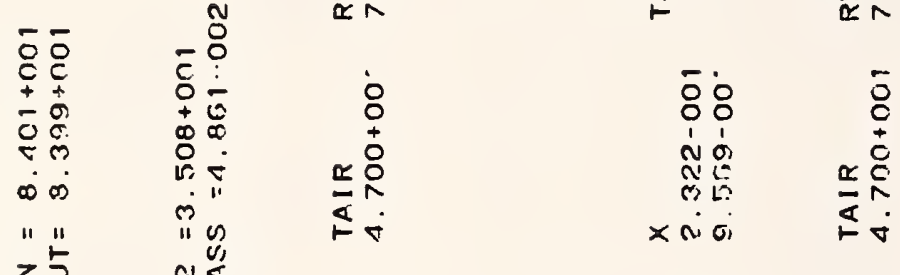

z"

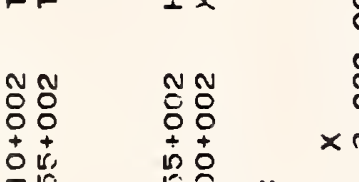

$\begin{array}{rl}0 & 0 \\ 0 & 0 \\ 0 & 0\end{array}$

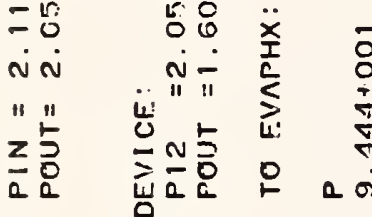

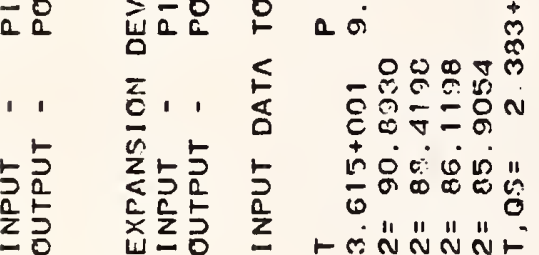

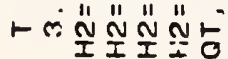

\begin{tabular}{l}
$\overline{8}$ \\
8 \\
\hline \\
\hdashline \\
0 \\
0
\end{tabular}

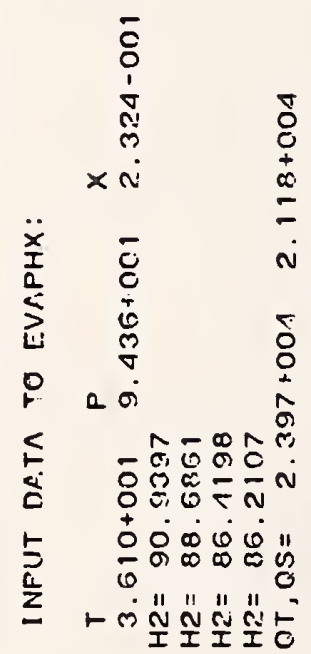

\section{ㅎㅇ}

싱요

ल

$\times a$

$-e^{2}$

ह̄:

$\pm+\frac{0}{0} 00$

要造

I $\dot{0} \dot{\sim}$

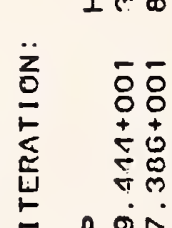

- an

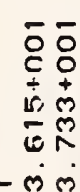
Q oin:

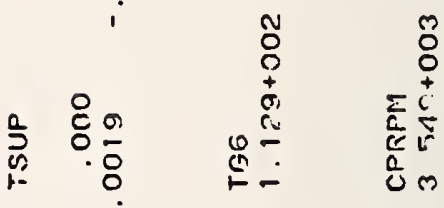

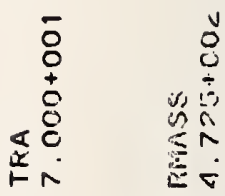

둥흉ㅇㅇ융

ब่́

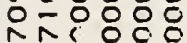

×oio:-

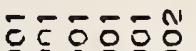

tit

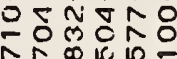

Uत I

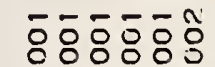

$+4+7+$

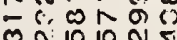

ํ. añogon

두응융

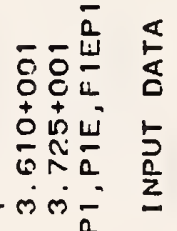

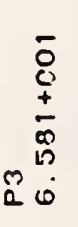

win

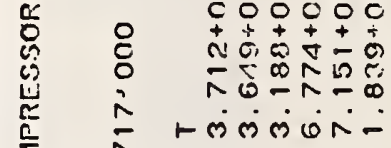

चัN-nMrno

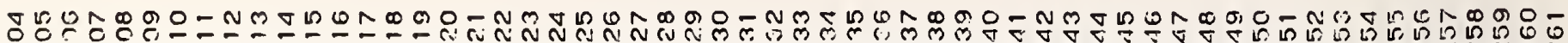

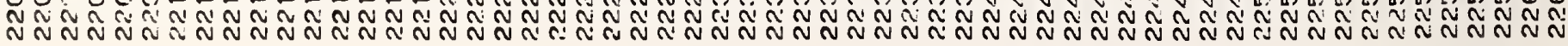




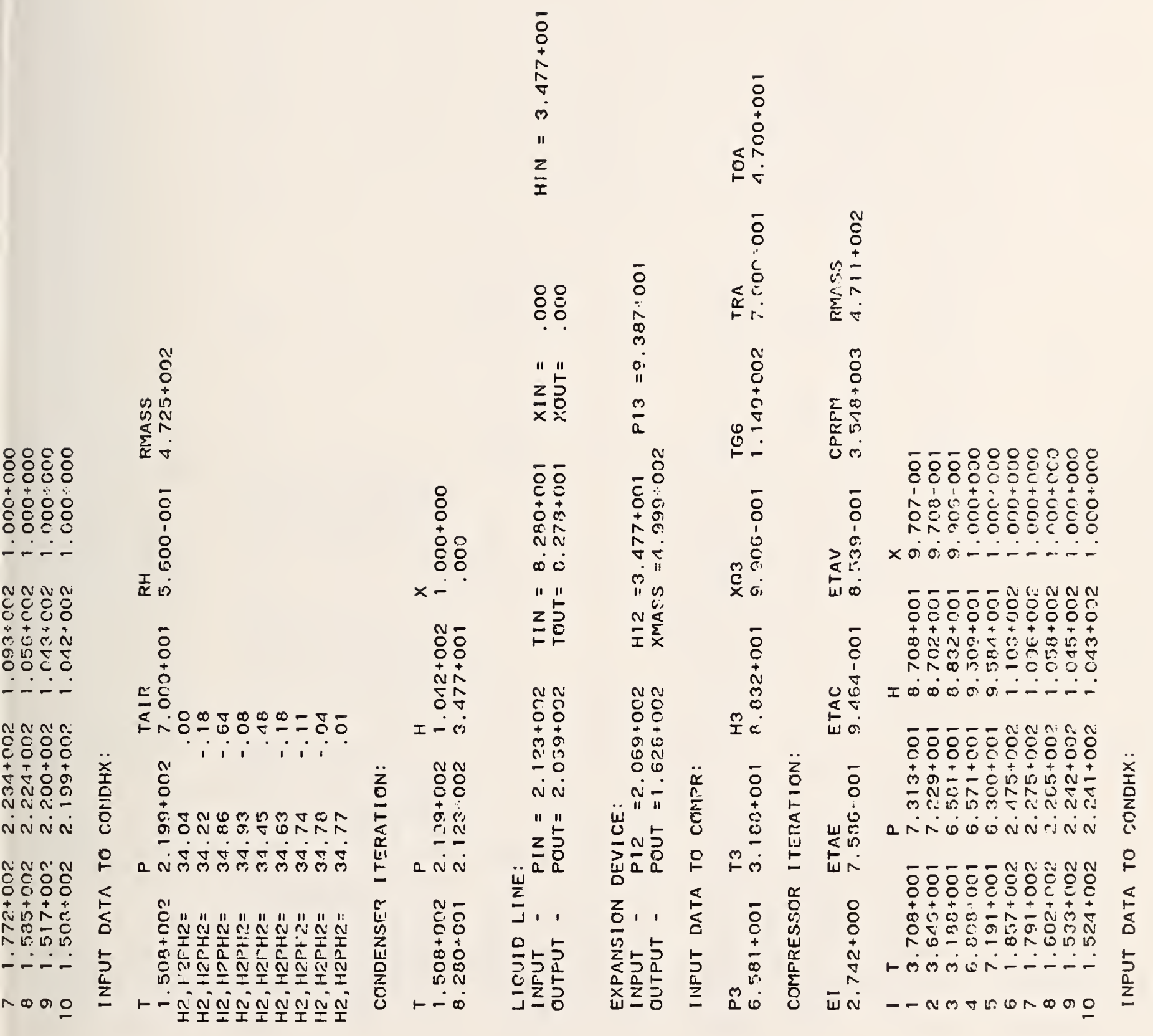

母M N NลN 


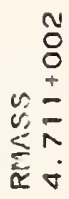

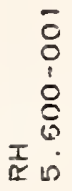

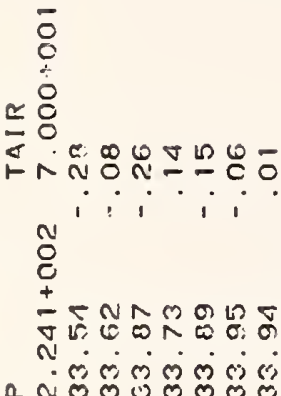

OัN"

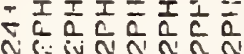

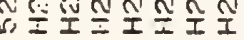

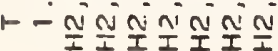

$\overline{8}$
0
+
0
0
$\dot{8}$
$1 "$
$z$
$z$

¿̊

" "

를

8

c:

$x-$

을

竞岁

I-ल

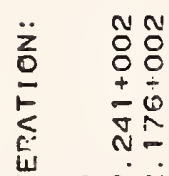

a Nं

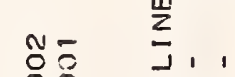

88

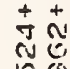

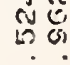

$\begin{array}{r}5 \\ 0 \\ + \\ 0 \\ 8 \\ 0 \\ 0 \\ \hline\end{array}$

$\overline{8}$
0
0
0
0
$\sigma$
11
$\frac{m}{2}$

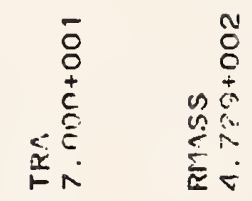

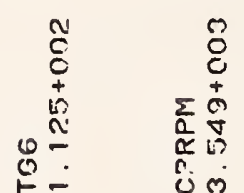

훋ㅇㄴㅇㅠ:8: 1.504060\%

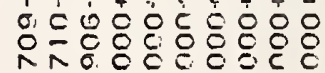
×नंब

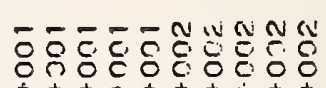

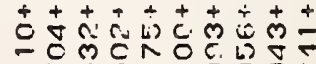

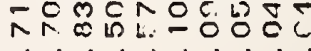

I के $\infty 0^{\circ} 0$

-

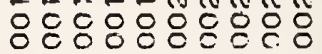
t t+t t+t t+ क

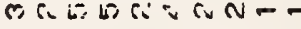

arnogontina

苅"

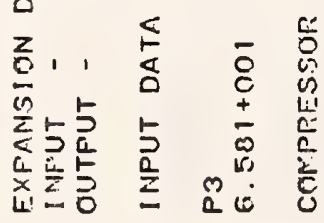

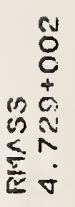

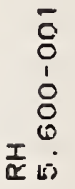

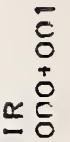

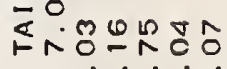

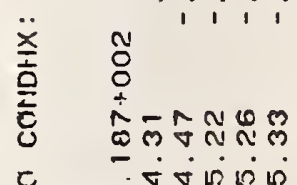

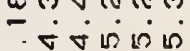
ट व तेल लूलूल

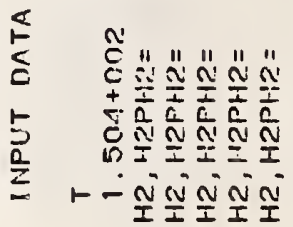

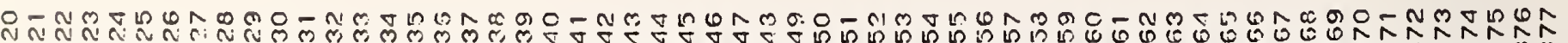

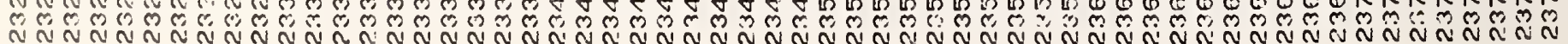


\begin{tabular}{l}
$\overline{8}$ \\
+ \\
0 \\
0 \\
0 \\
0 \\
11 \\
$z$ \\
$z$ \\
\hline
\end{tabular}

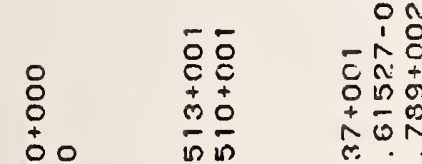

용으

$x-$

용

잉

定商

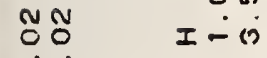

लू

$\frac{N 1}{T}$

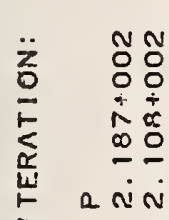

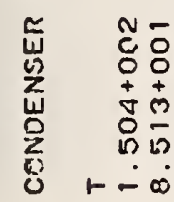

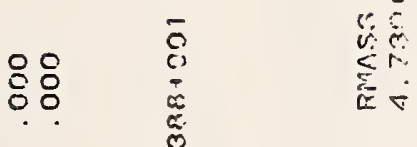

" "

䜤高

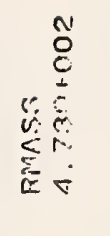

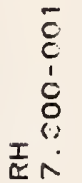

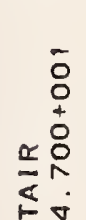

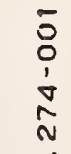

$\times \dot{N}$

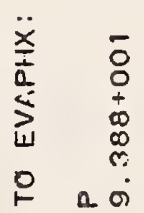

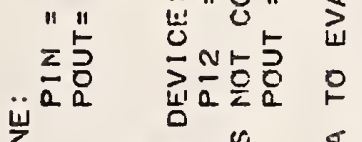

z

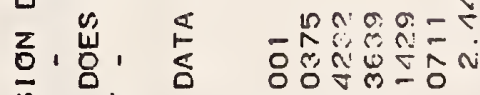

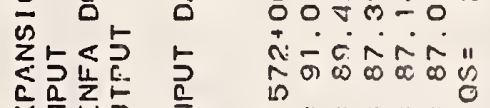

出之捗

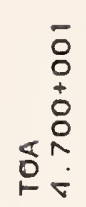

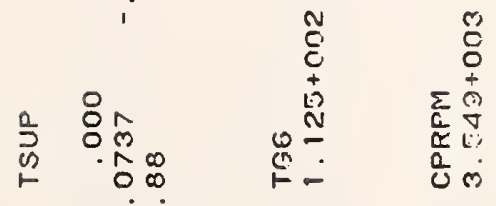

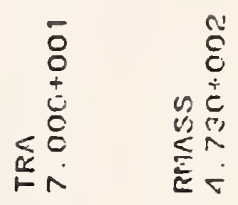

응

11

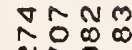

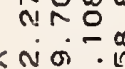

$x \cos 2 \pi$

융응

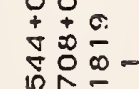

Iलिंल

एर्ष

के

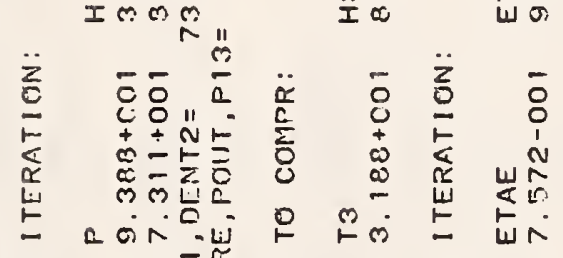

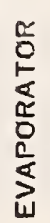

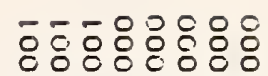

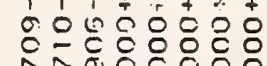
$\times 000$

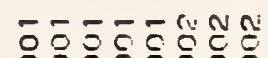
००ดธรถั

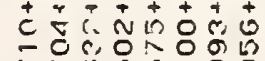

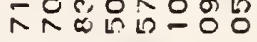

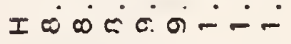

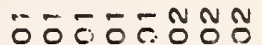
80.06080

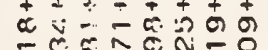
-

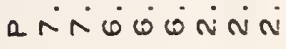

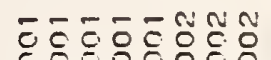

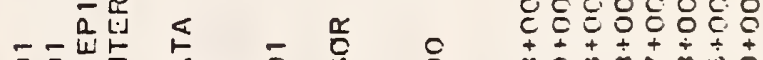

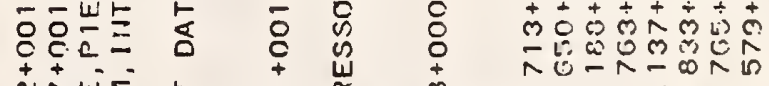

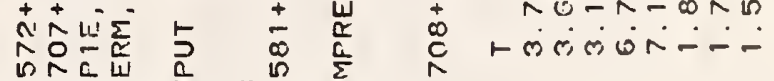

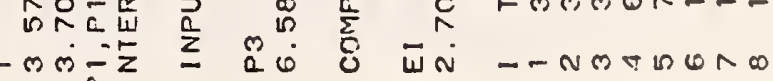

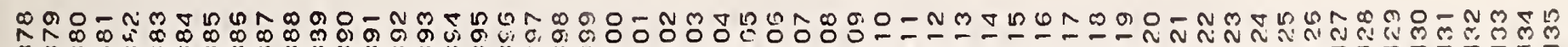
N 


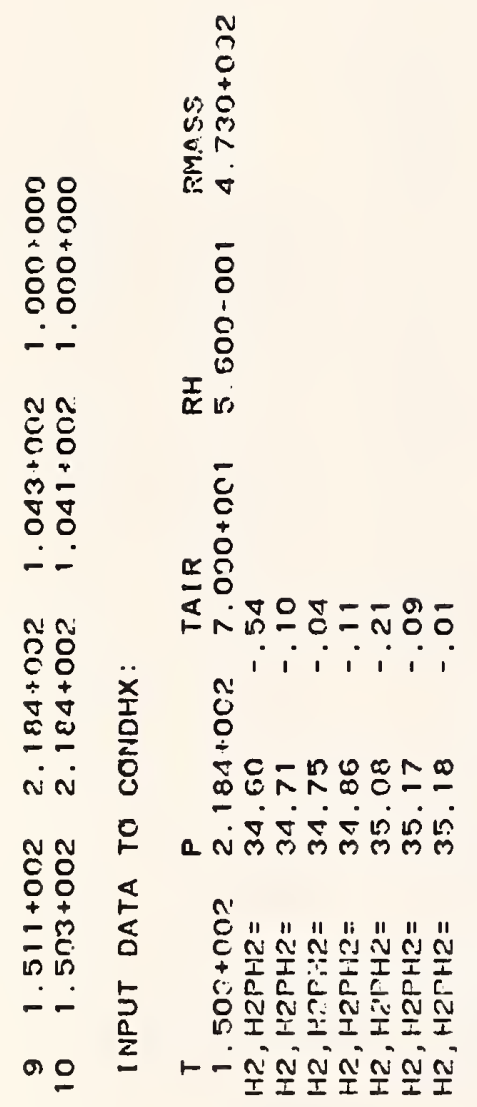

5
0
+
0
$\vdots$
nं
11
$z$
I

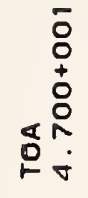

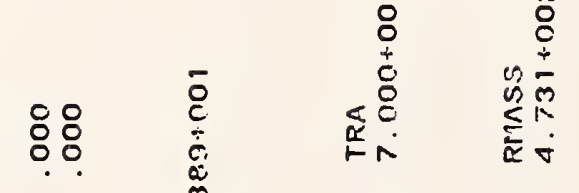

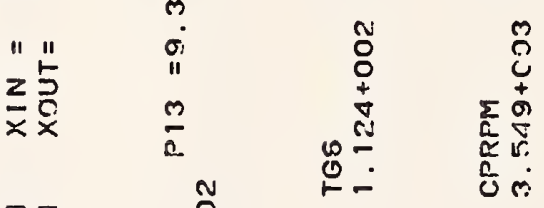

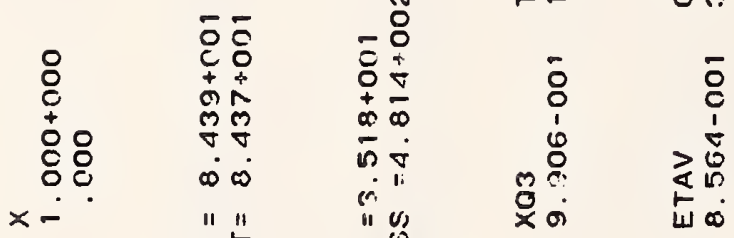

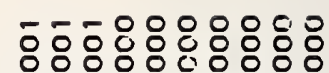

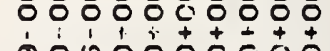

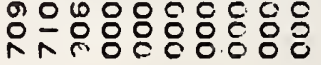

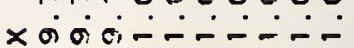

" 255 "W

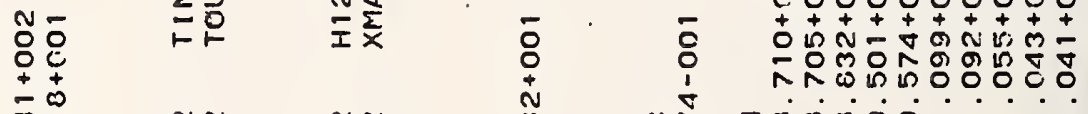

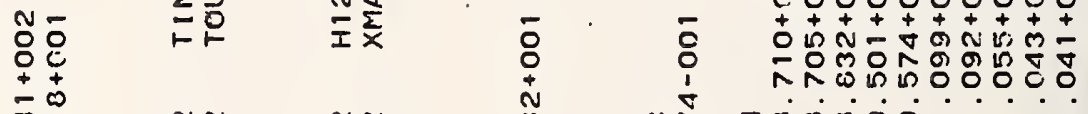

W

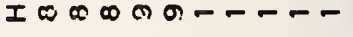

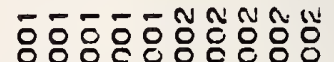

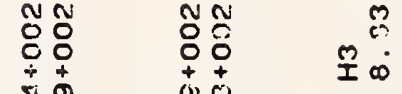

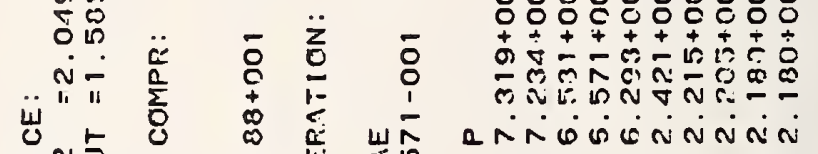

- is

No

in

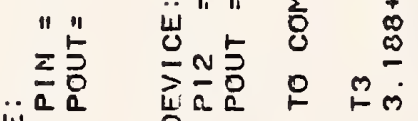

岩合

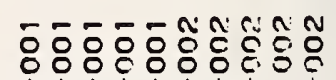

กิธ ฮั

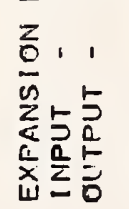

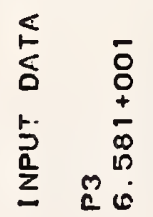

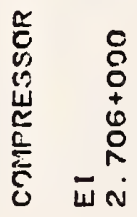

$+7++t+7$

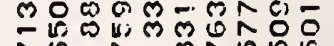

人

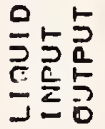

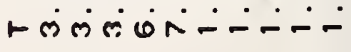

- nM

$\sum_{\substack{0 \\ 0}}^{\substack{0 \\ 0}}$

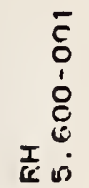
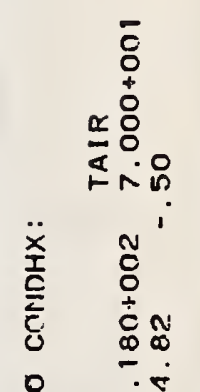

वतले

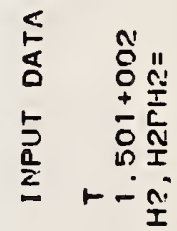

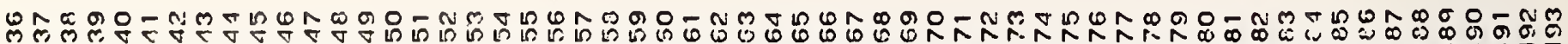

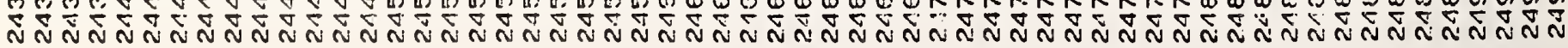




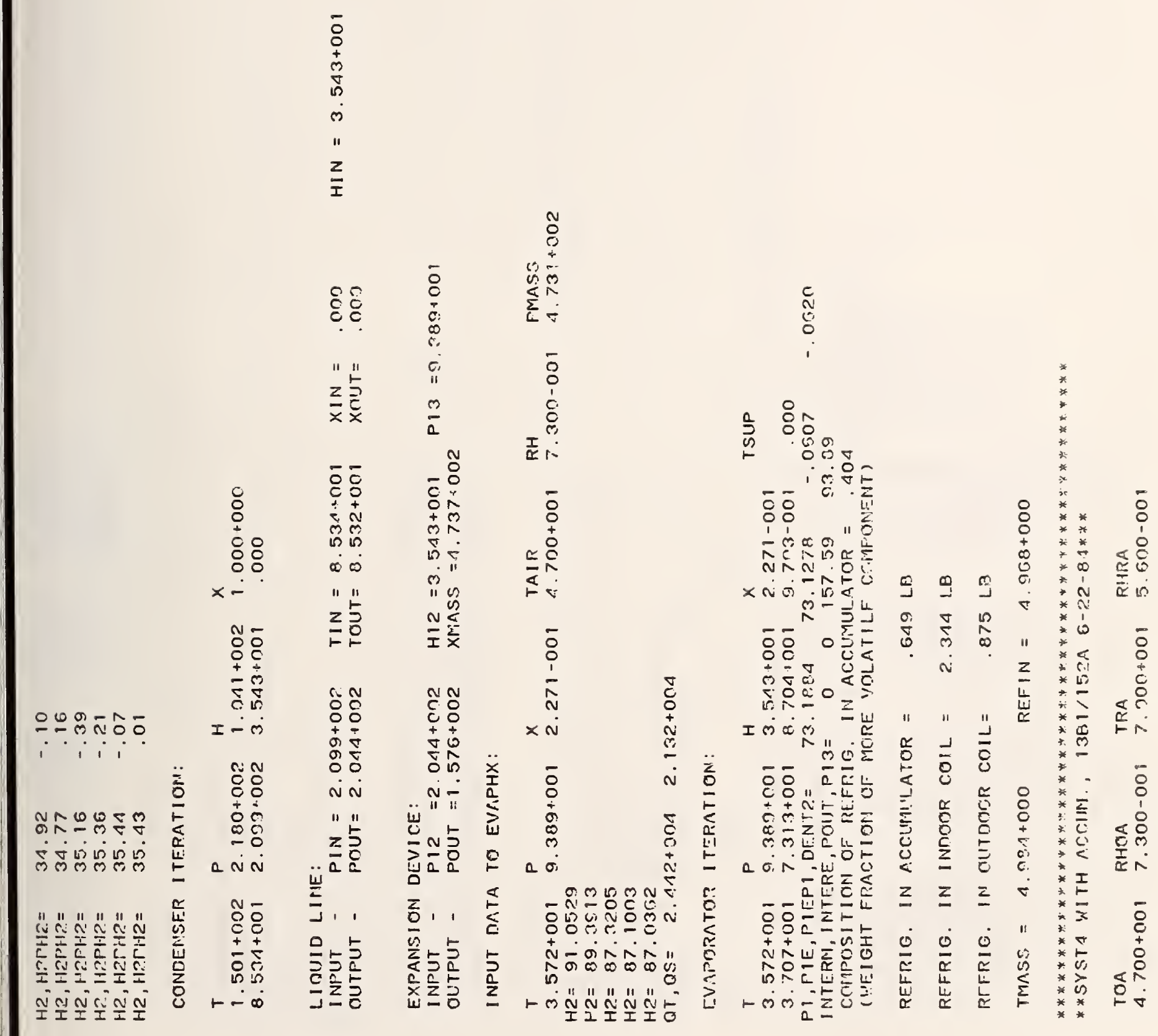

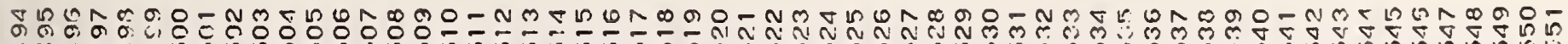

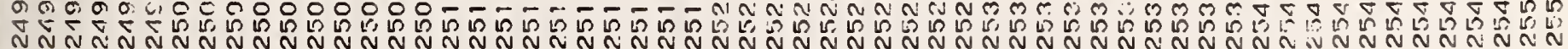




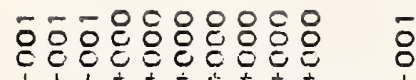

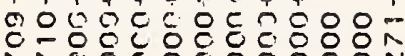
个रำ

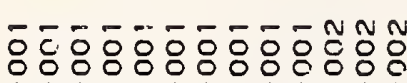

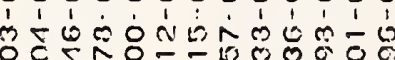

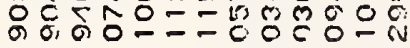

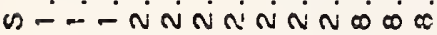

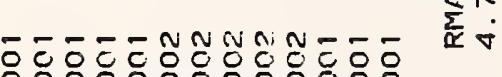

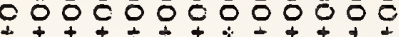
ㄴํำ

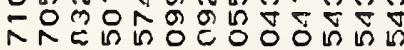

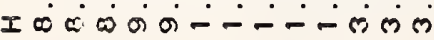

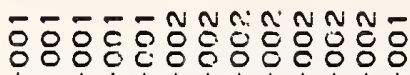

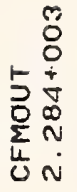

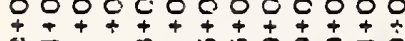

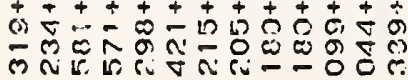

a NN

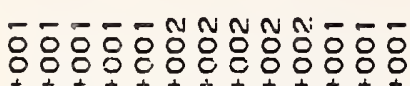

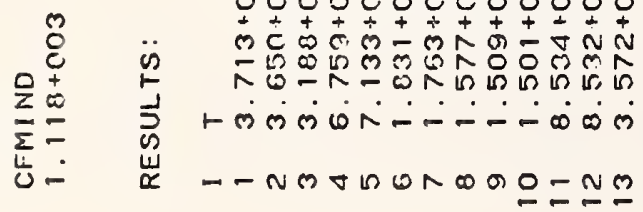

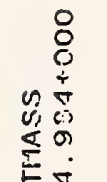

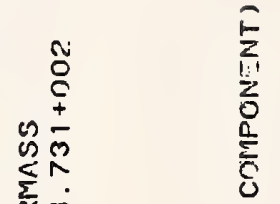

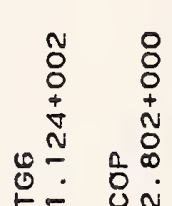

r.

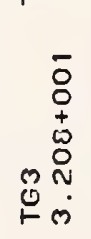

11

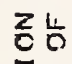

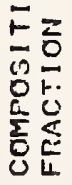

要

莣崖

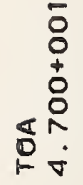

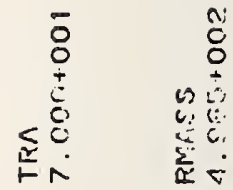

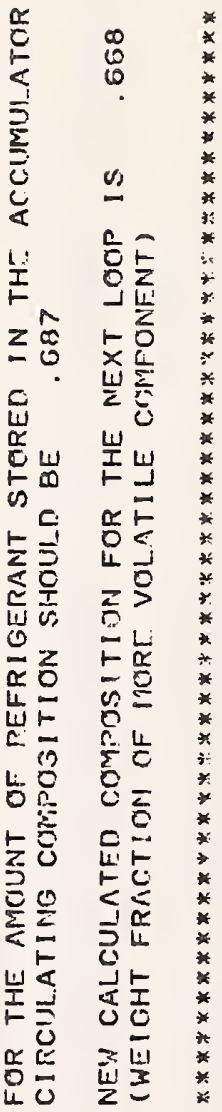

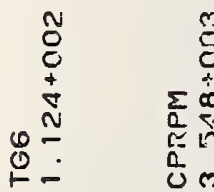

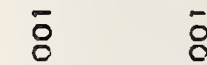

芯

$\geq \hat{n}$
¿ृं

$\overline{0} \overline{0} 0: 0$

잉



रREO

×ंकंज:-

등ㅎㅁ용

$+7++7$ $\checkmark \begin{gathered}+ \\ \sim\end{gathered}$

15. $156 \mathrm{sj}$

I $\infty \infty \infty_{0}^{\circ} \sigma^{\circ}$

흐응응

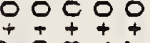

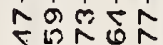

B.

añiिio

드응

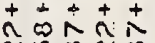

N

मलंलिं

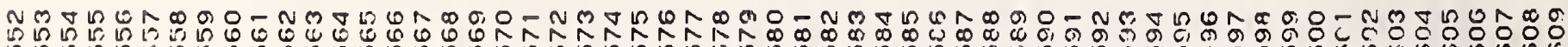
N 


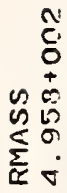

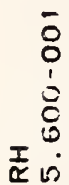

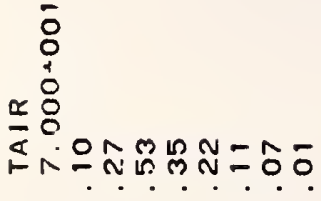

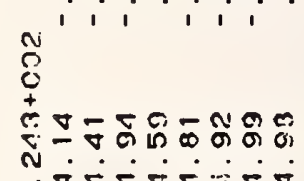

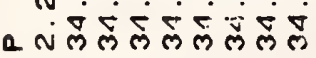

๊

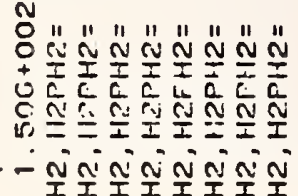

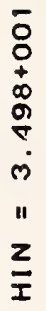

๕๕

"

중

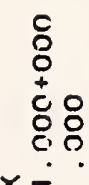

ํํ음

ㅇํㅇ

家实

I- लं

$\underset{\frac{z}{0}}{\frac{z}{5}}$

กN

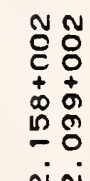

80

离葛

空

a $\sin$

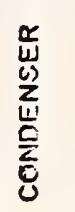

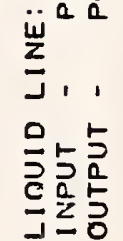
菓骂
5
0
+
0
0
0

$\overline{8}$
$\frac{5}{6}$

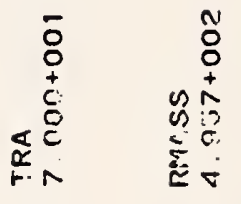

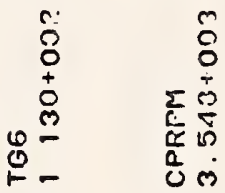

$z$
$\frac{2}{0} 1$
$\frac{0}{2} 55$
$<50$
$\frac{0}{x} \frac{1}{2} 5$
$w=0$

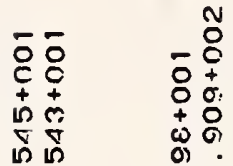

में

"

लำ

" 2 "

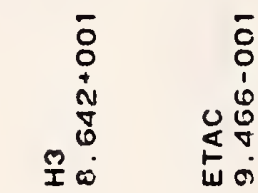

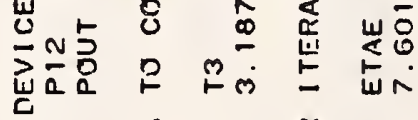

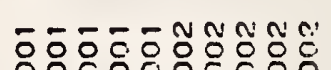

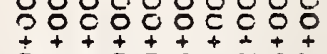

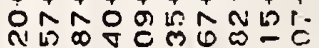

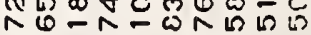

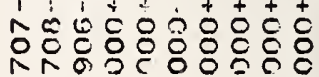
xoro:-..-

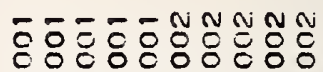

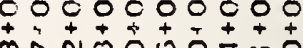
กี่

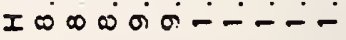

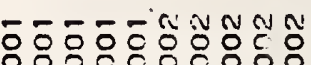

titition

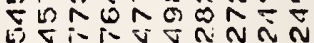

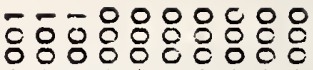

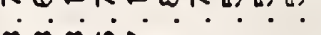

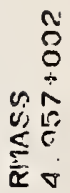

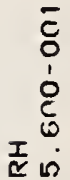

$\frac{\pi}{8}$

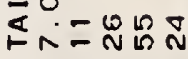
a 1

잉

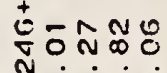
द तलेंगे

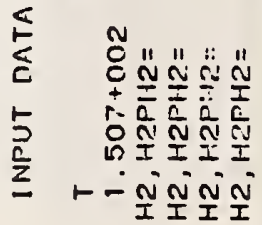

DOO

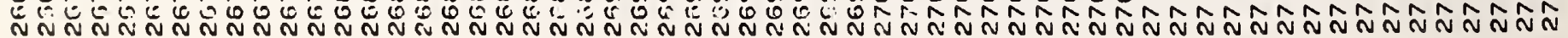




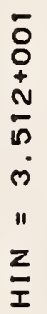

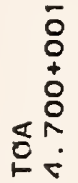

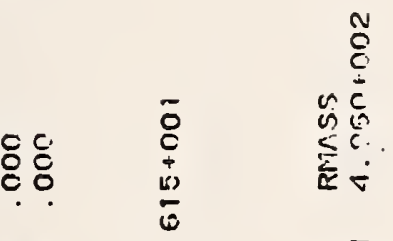

告

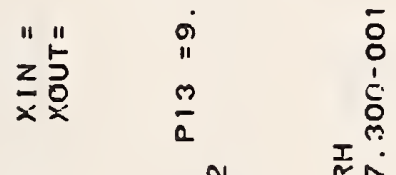

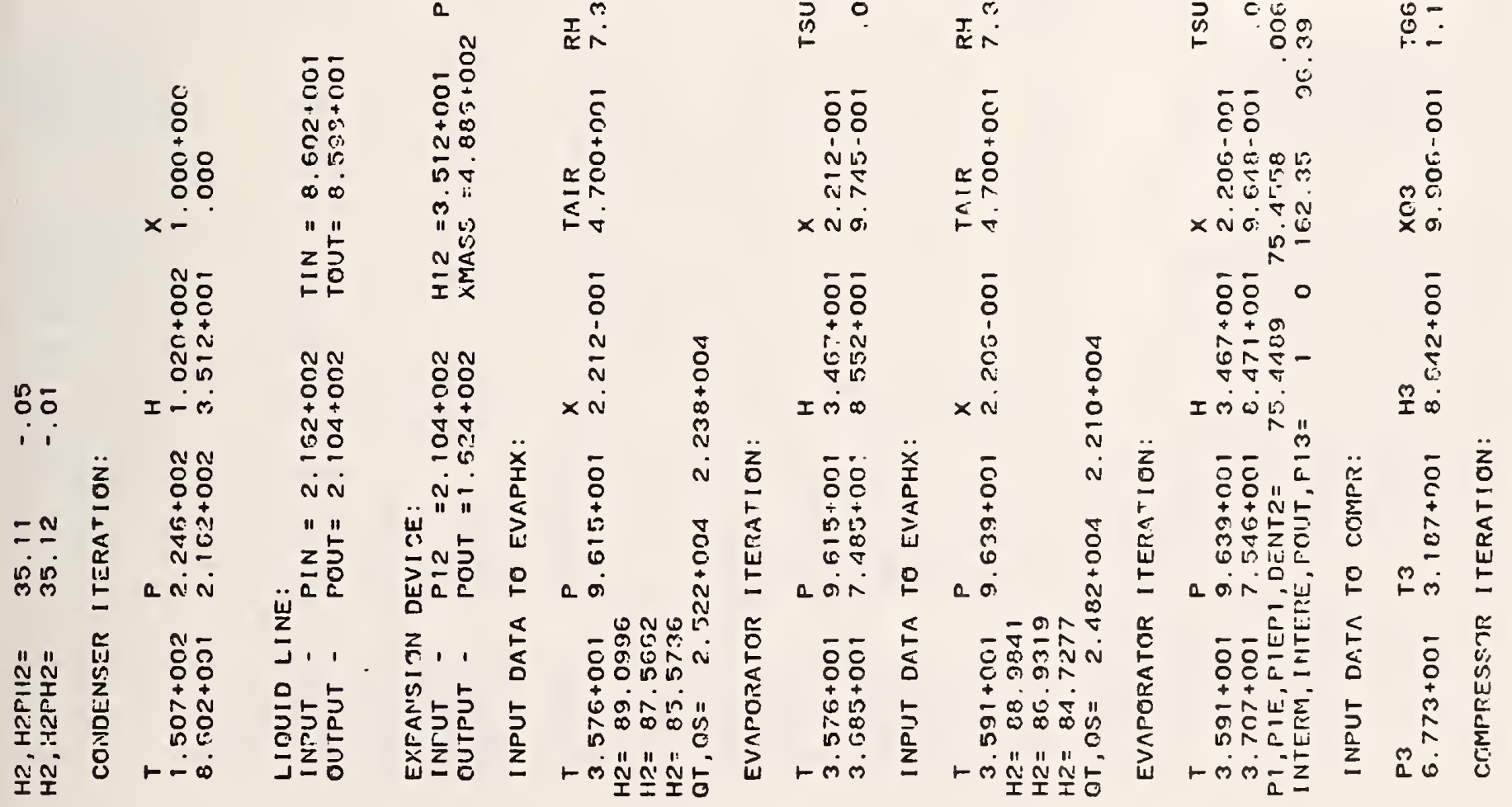

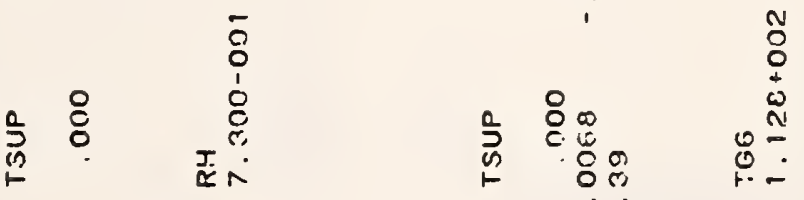

N

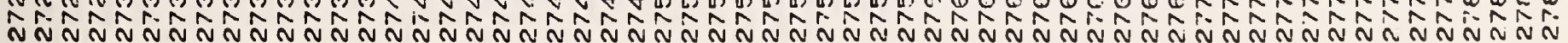




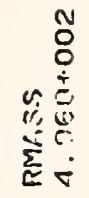

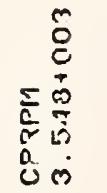

훙형웅영영연

ó

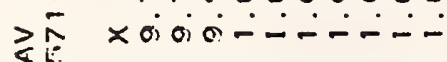

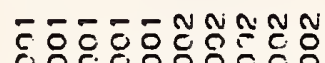

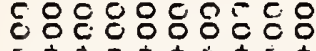

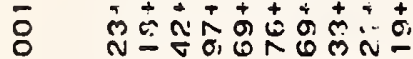

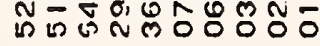

I $\infty 0_{000}^{\circ} 0$

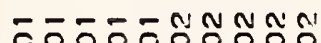

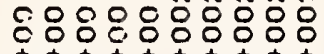

-

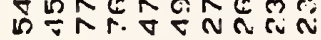

añoogninin

造它。

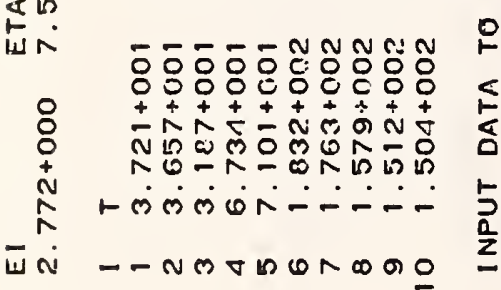

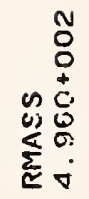

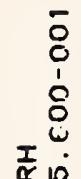

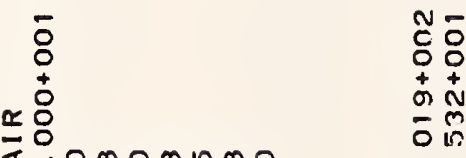

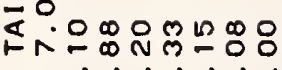

O

airitio

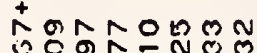

त.

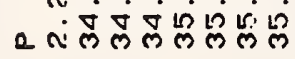

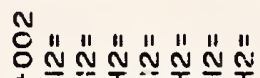

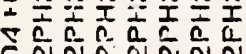

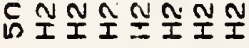

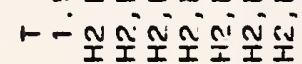

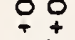

它去 $\overline{8}$
0
$N$
0
0
0
i
11
$z$
$z$

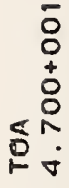

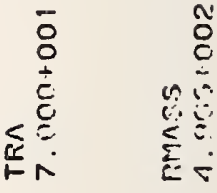

11:

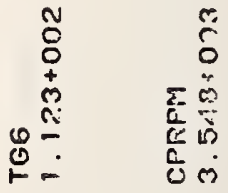

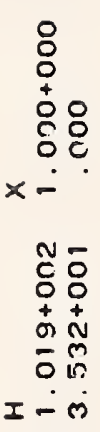

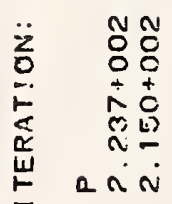

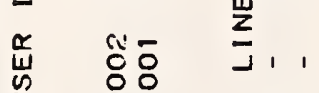

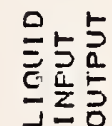

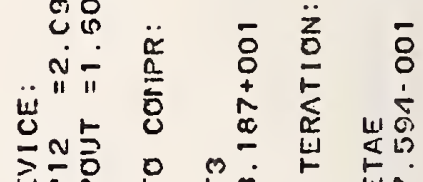

등ㅇㅇㅇ

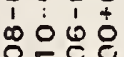

중요 रणिं०-

동

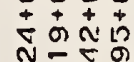

ถิธีอ

I बिल

등ㅎㅇㅇㅠ

2
$\infty$
$\infty$

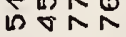

arió

嵓员

竞,

둥응

$+7+$

Nis

AC-N

मलंलिं

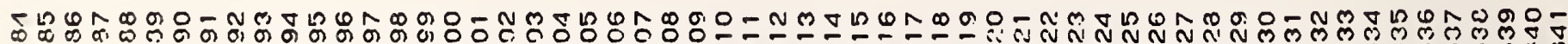

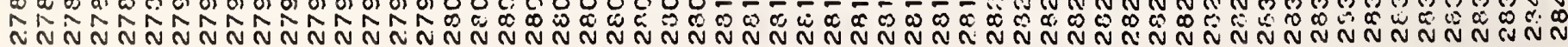



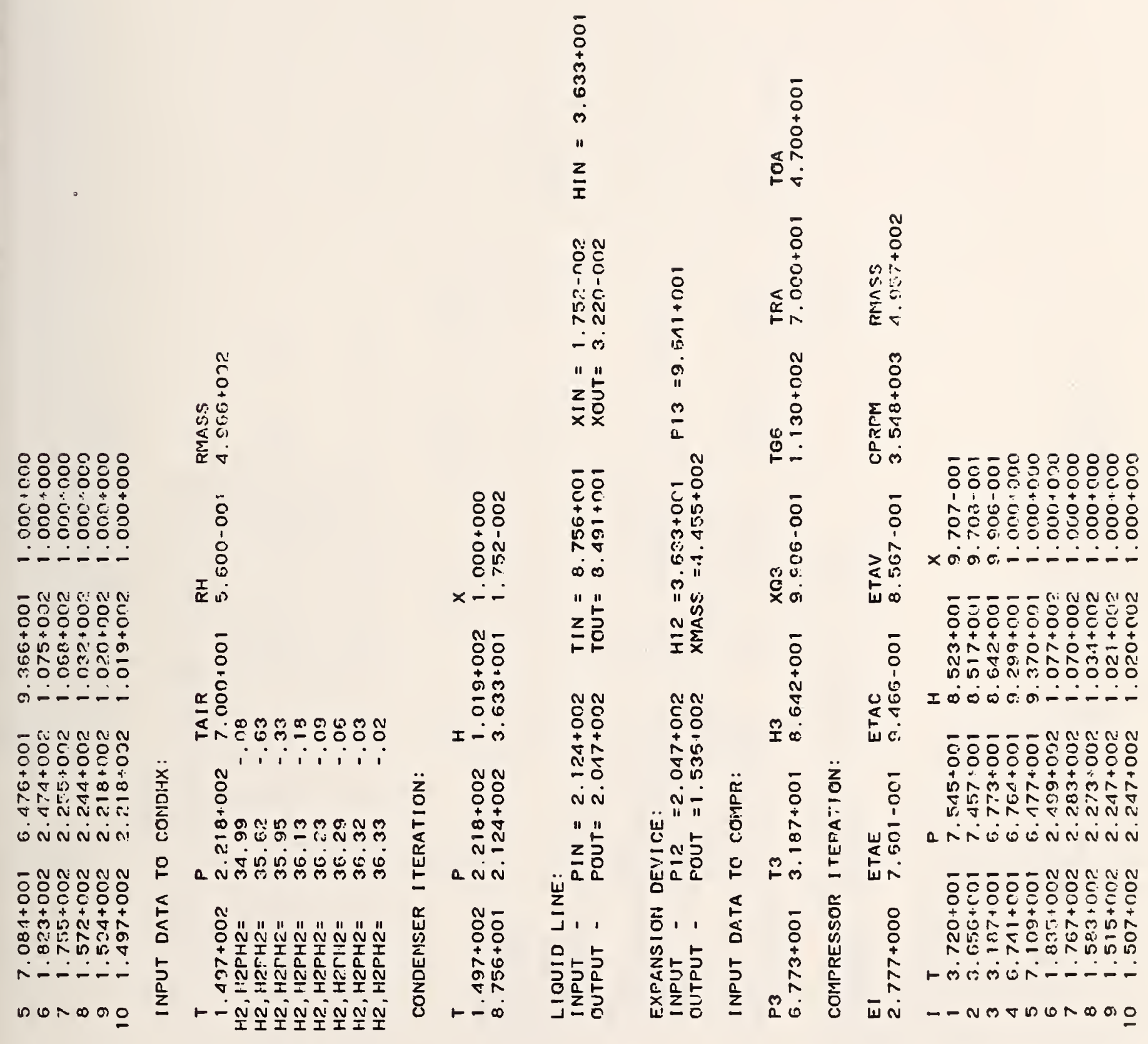

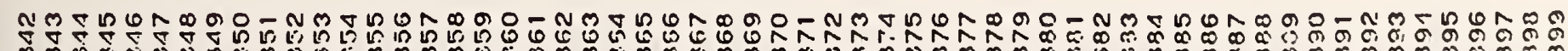

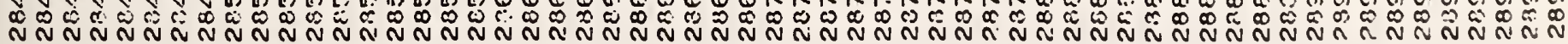




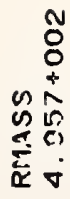

$\overline{8}$
+
8
8
6
8
11
$z$
$z$

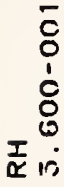

$£ \AA$

$\overline{8}$
+
$c$
0
0
0

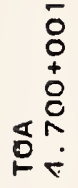

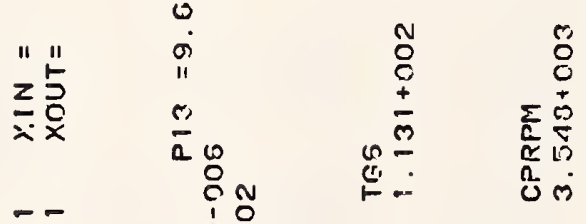

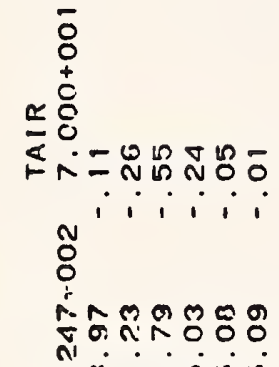

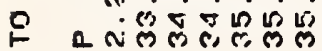

迠

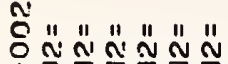

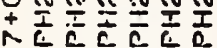

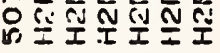

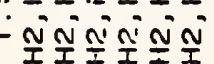

8
8
8
80
80

-

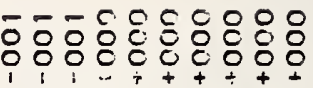

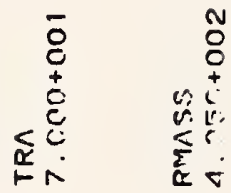

0
0
0

踏

iा

กั๊

"

$+2$

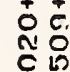

nก ñก

I- लं

잉

兽点

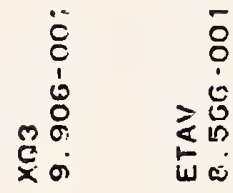

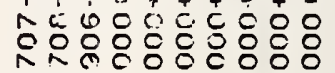

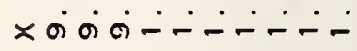

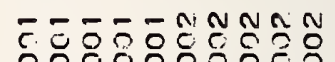

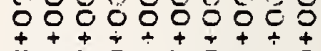

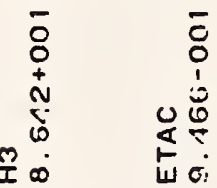

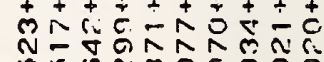

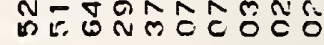

I $\infty 0_{\infty} \infty \sigma^{\circ}-\ldots$

+1: M足

- - - - -

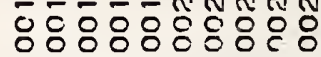

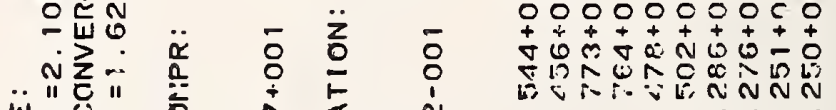

$\sum^{+} \sum^{+}$nin

añogeñNa

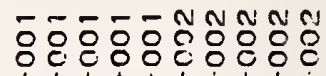

a लंN

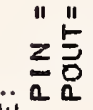

岁"

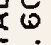

㟧丘是吕

응ำ

岁

吉, 岀,

占

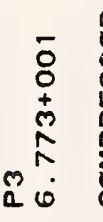

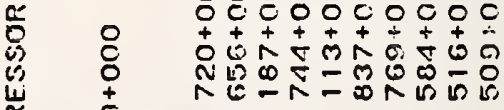

은?

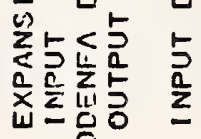

मलंखित-‥

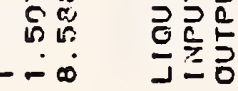

în

- NM

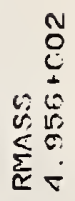

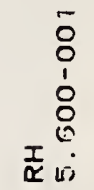

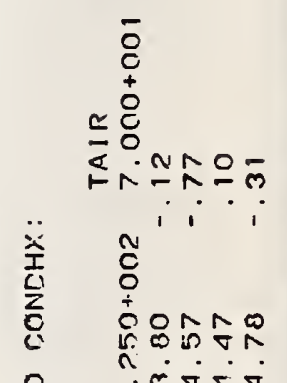

$\stackrel{0}{r}$

a तल लें ले

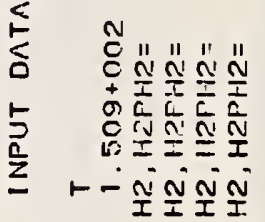

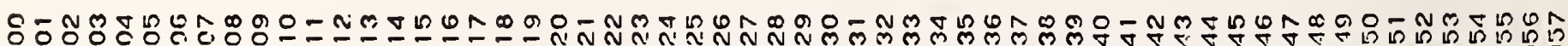
NลN N N N N 
$\overline{8}$
8
+
5
0
$\dot{0}$
11
$z$
$z$

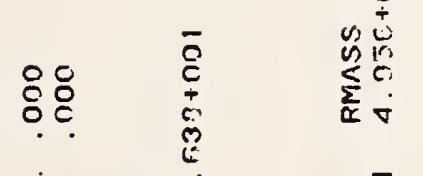

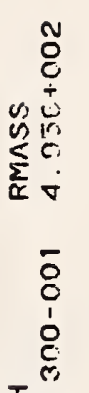

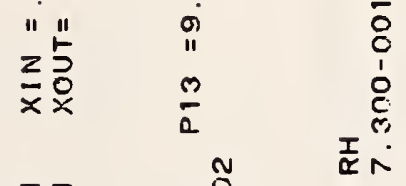

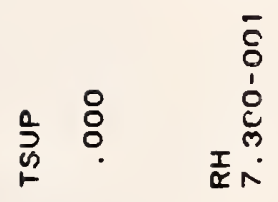

$\bar{a}$
0
$\vdots$
$i$

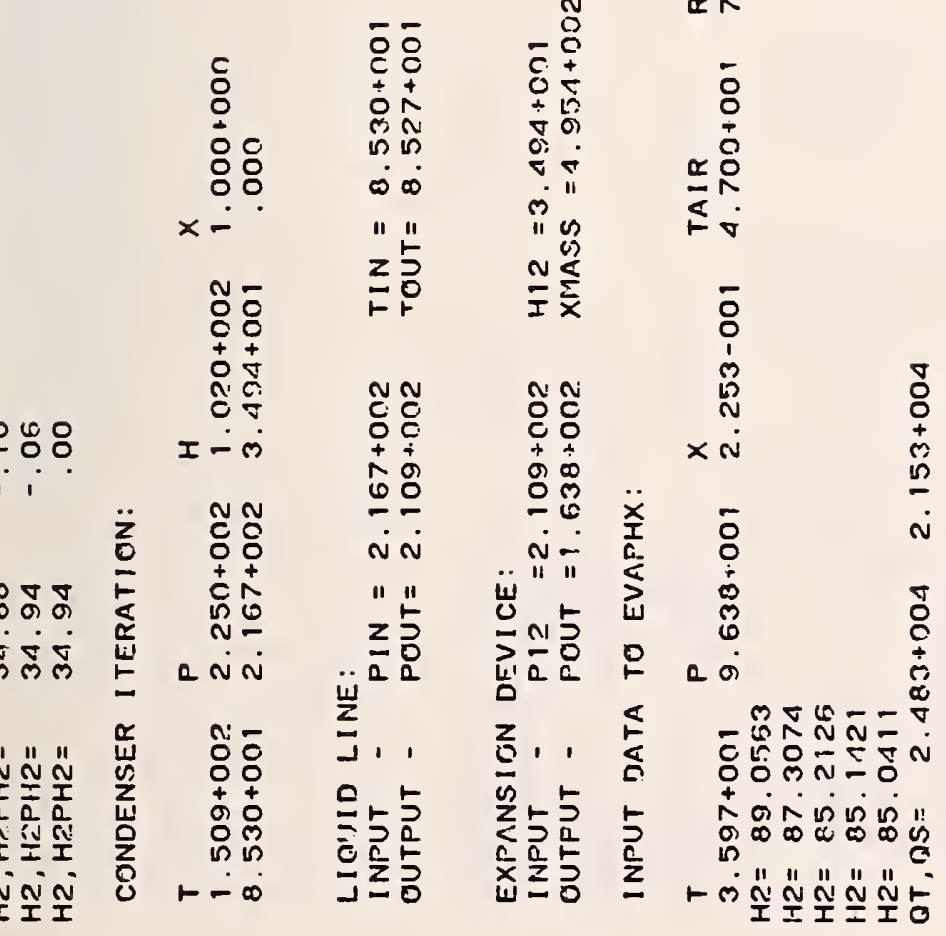

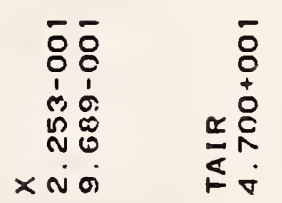

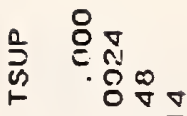

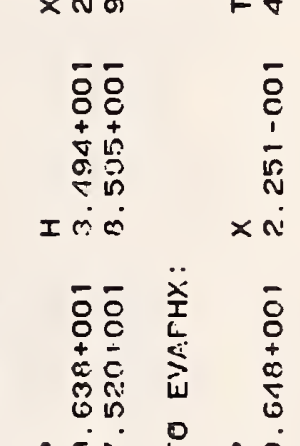

$\bar{\circ} \overline{0}$ i क्ष

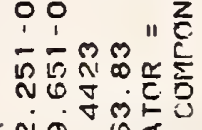
مْ 용 ०

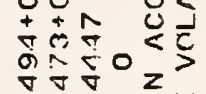
I

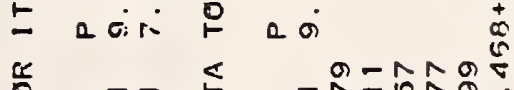

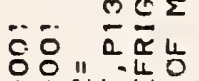

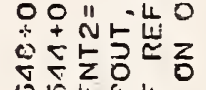
ôे⿺乚一匕 a on $-\frac{1}{2} z$

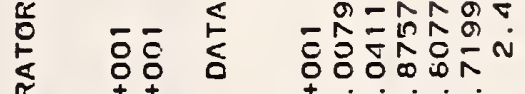

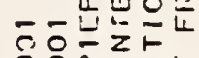
ㅇ으는

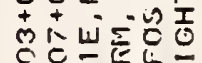
c.

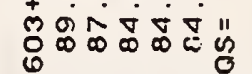

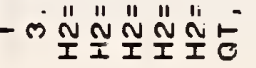
मिं

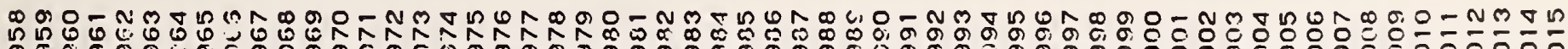

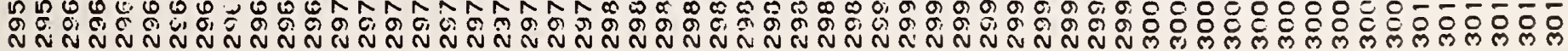




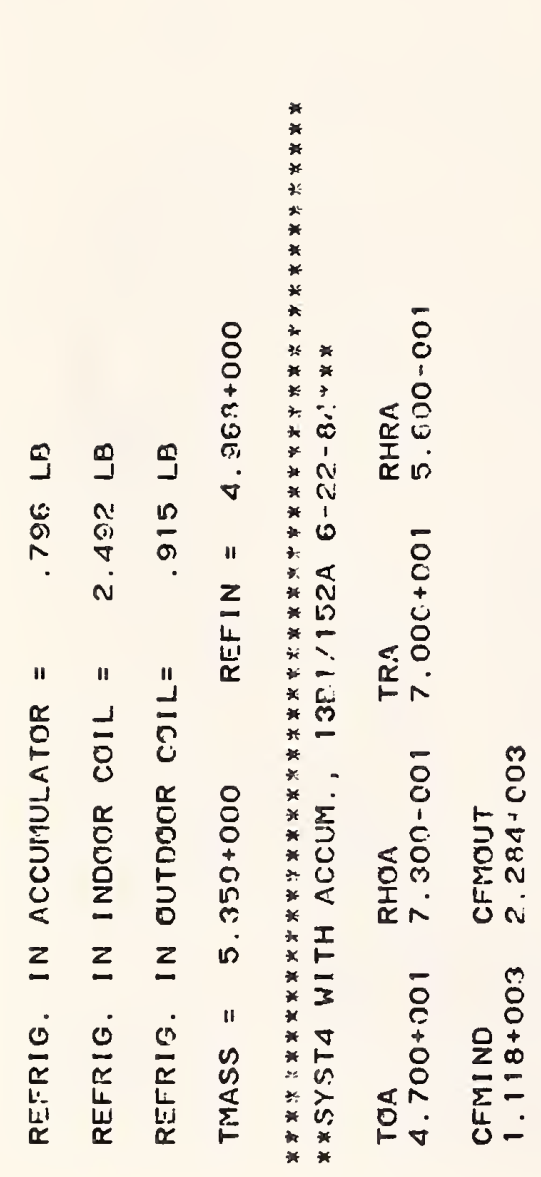

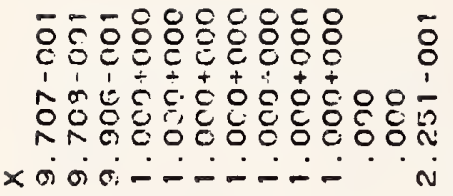

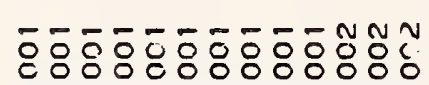
nं 屯心 $n--\infty \sin n-n n \infty$

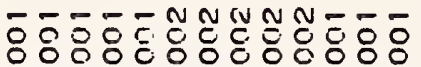

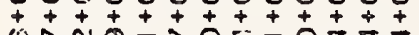
(s) Noñ

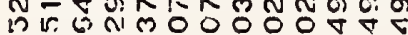

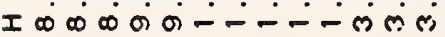

$7+7+t+7+7+1+7+$

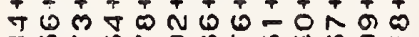

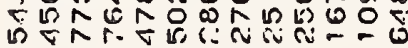
a N

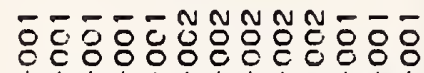

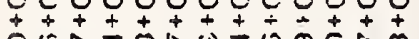

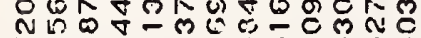

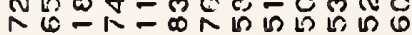

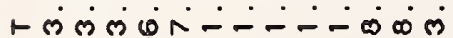

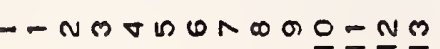

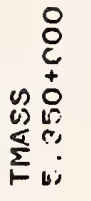

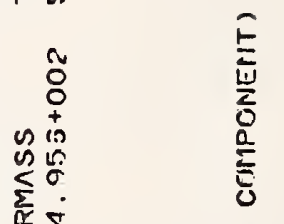

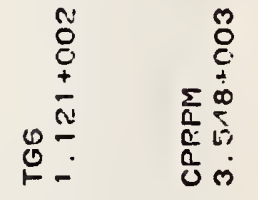

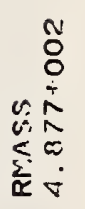

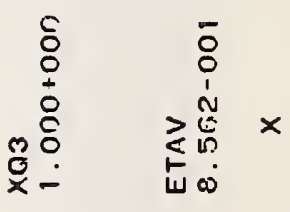

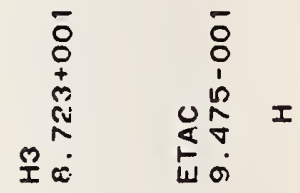

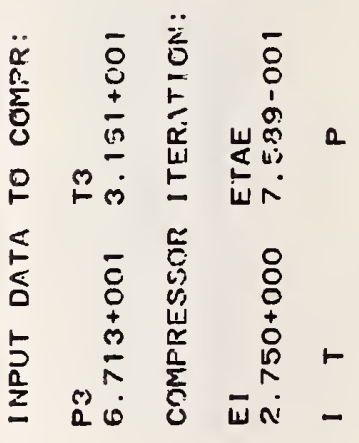

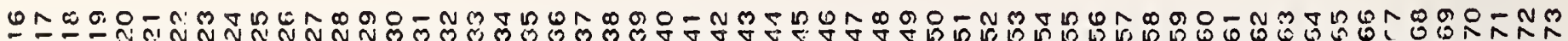

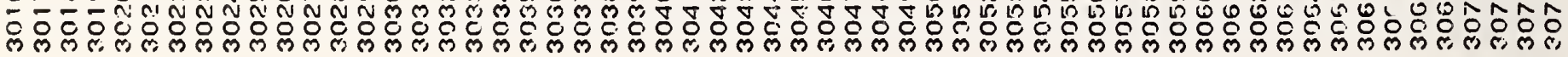




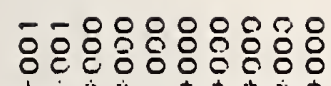

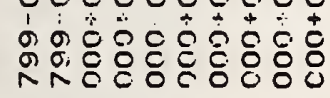
कं $\dot{0}-\div-\div-\div-$

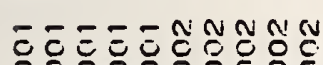

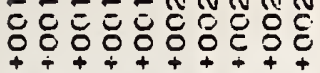

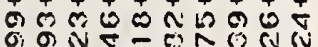

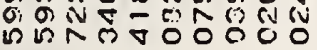

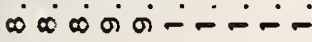

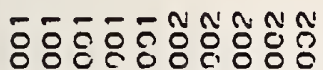
10
0
0

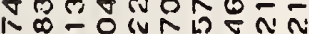

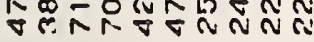
$\sim \wedge \dot{0} \dot{0} \dot{N} \sim \dot{N}$

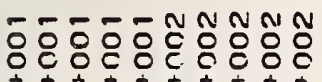

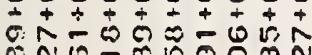

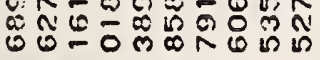

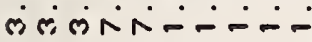

- NM

$\sum_{\substack{0 \\ 0}}^{\substack{0 \\ 0}}$

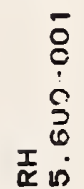

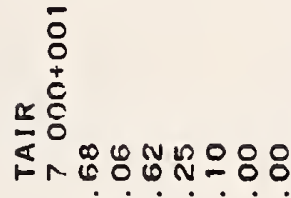

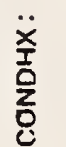

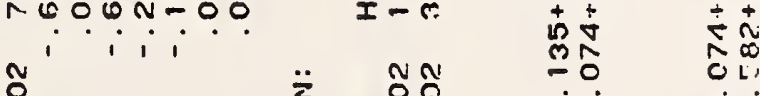
C

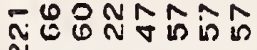
N

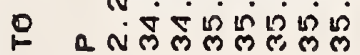

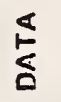

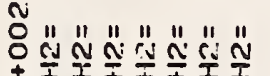

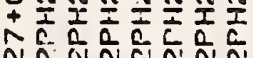

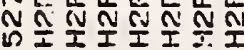

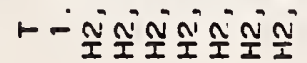

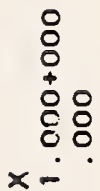

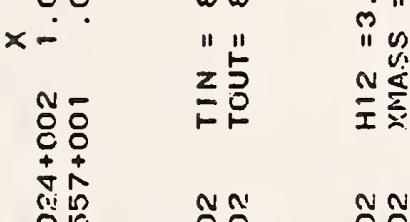

ơ

ลั

a Nं

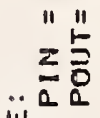

N- $\frac{\mathrm{U}}{\mathrm{J}}$

8

in

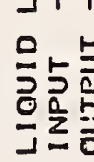

$\overline{8}$
+
1
0
0
i
11
$z$
$z$

5
0
0
0
0
0
0
1
1

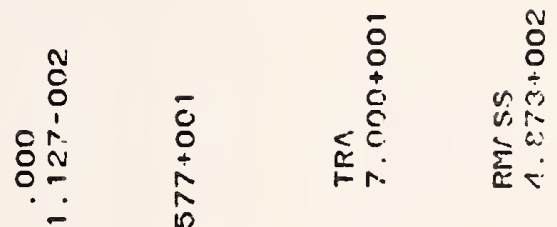

z

-

雚

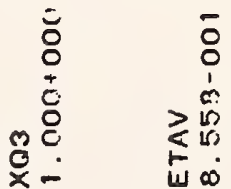

흥융ㅇㅇㅇㅇㅇㅇ융

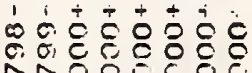
xoci-‥-

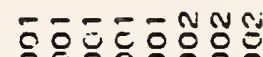

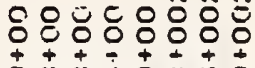

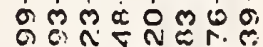

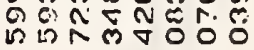

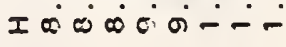

c.

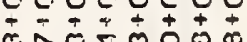
‥$$
\text { द. }
$$

पू añoण

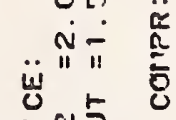

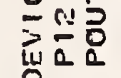

$\begin{array}{ll}z & 1 \\ 0 & 1 \\ 0 & 5 \\ 25 & 5 \\ 0 & 0 \\ 0 & 0 \\ \vdots & 0\end{array}$

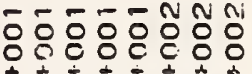

$+7+T=1$

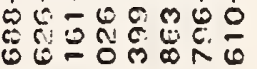

मलंखिता-:-

$\bar{\omega} \stackrel{\wedge}{N}$

- nM

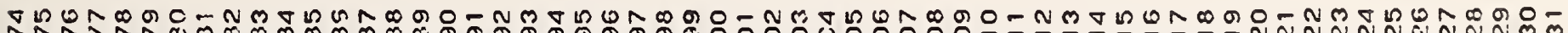

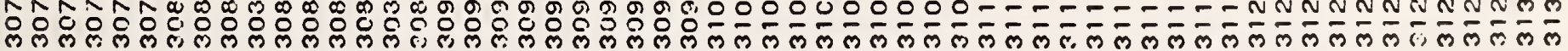




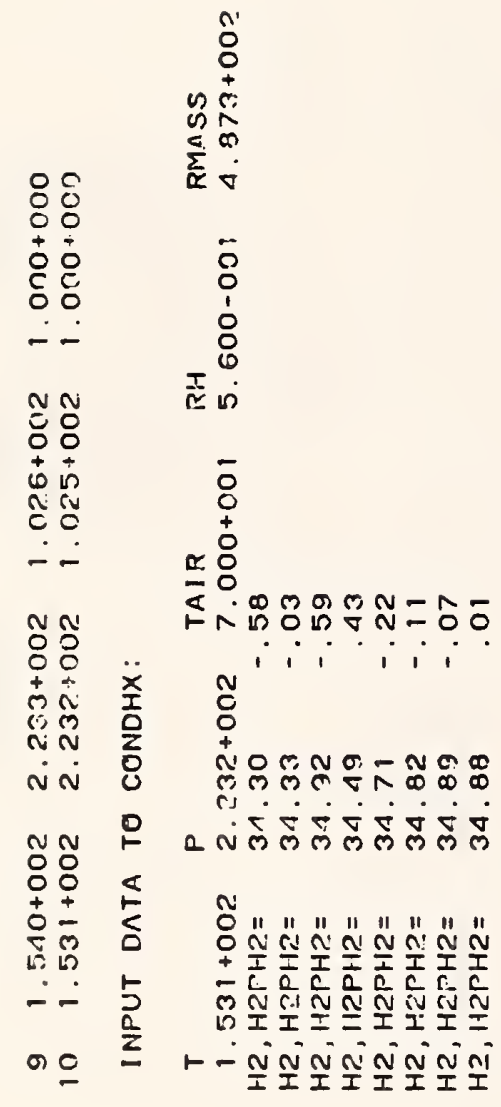

$\overline{8}$
+
0
0
0
8
11
11
2
2

등응융융융융융

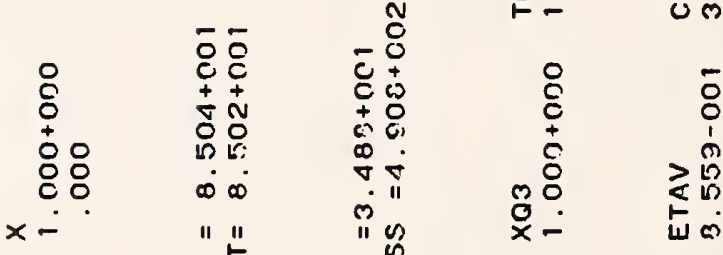

1 1 t t

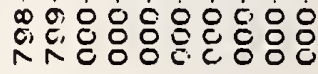

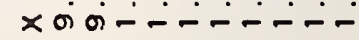

-

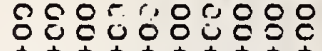

$7++++t+$

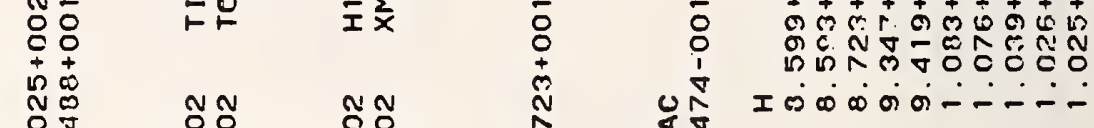

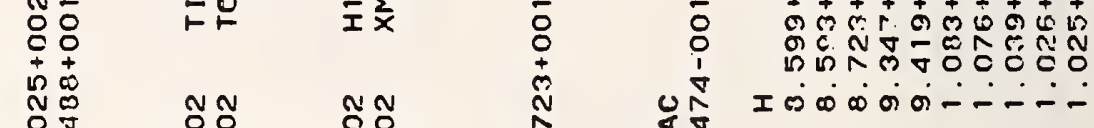

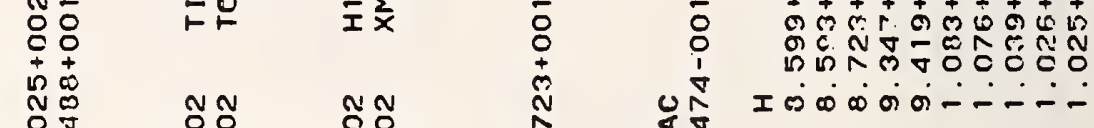

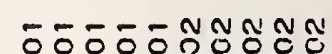

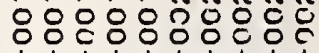

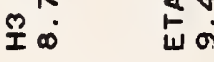

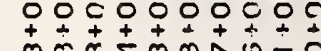

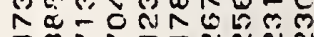

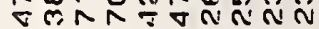

añosoññNán

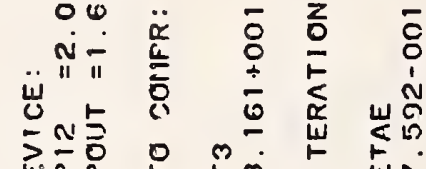

a N

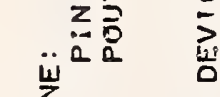

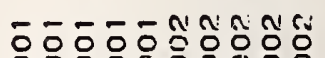

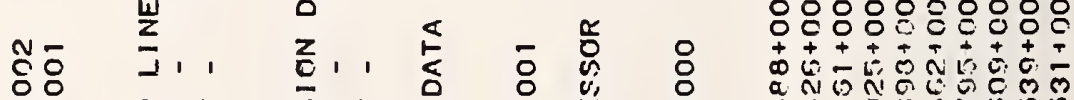

क्षे

紊占

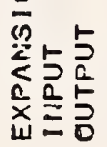

मलंलिin-‥-

色宫

$-\stackrel{n}{n}$

- NMTUWNDO

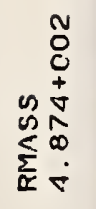

a i

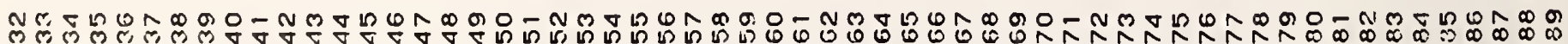

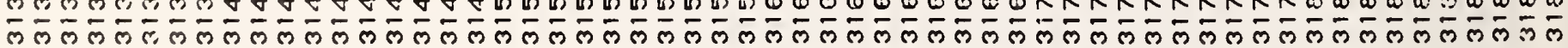




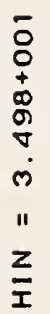

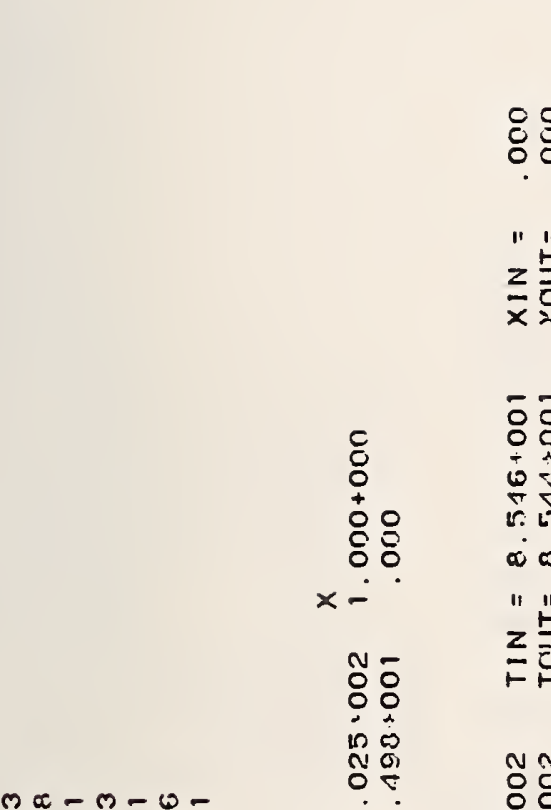

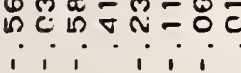

熔

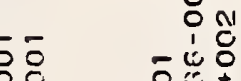

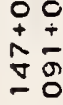

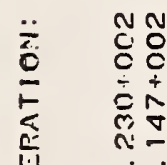

a. तi

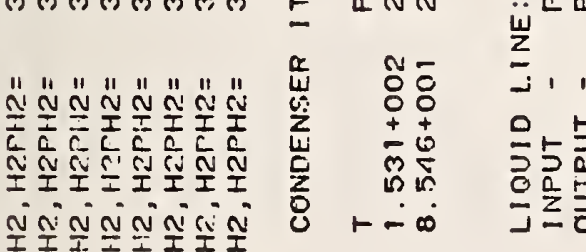

o

L.

I

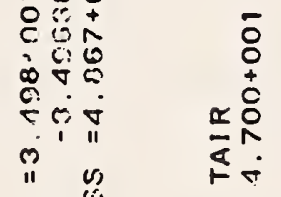

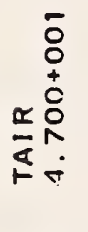

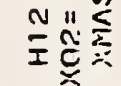

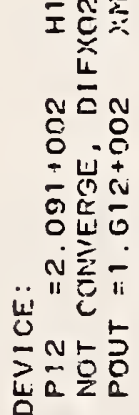

$\overline{0}$
$\vdots$
$\vdots$
$i$
$N$

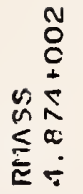

$\overline{8}$
0
$\vdots$
0
0

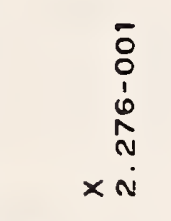

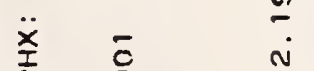

$\begin{array}{ll}\frac{x}{c} & 0 \\ c & 0 \\ \frac{T}{4} & \vdots \\ 0 & i \\ 0 & 0\end{array}$

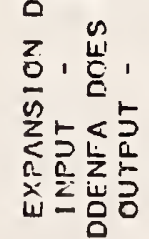

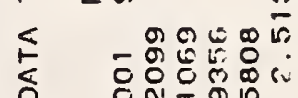

E

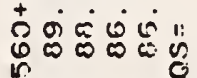

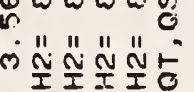

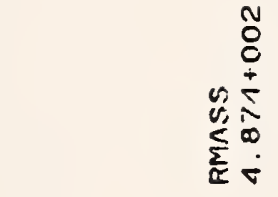

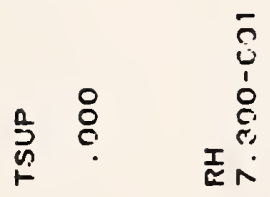

各

路 :

훙

능요

is

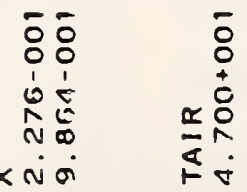

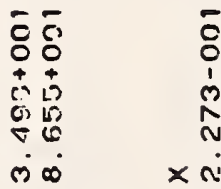

$-e^{2}$

융

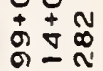

$\operatorname{Tin} \frac{1}{2}$

I $⿻ \circ$

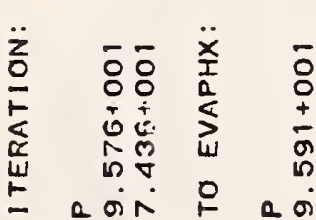

点

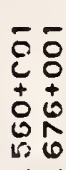

10

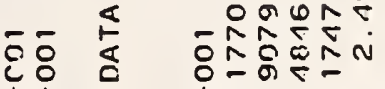

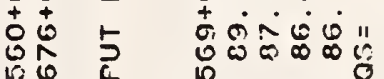

in

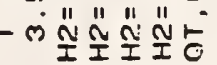

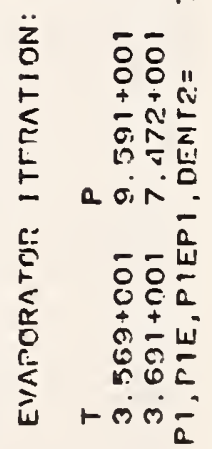

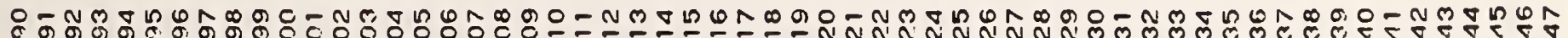
- 


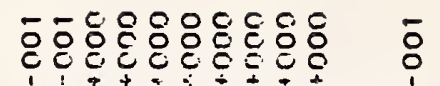
द

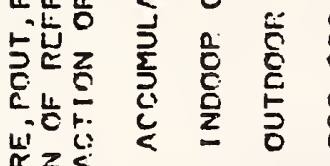

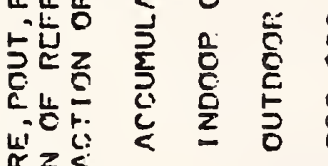

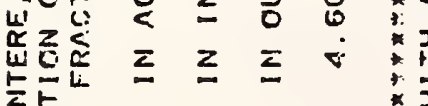

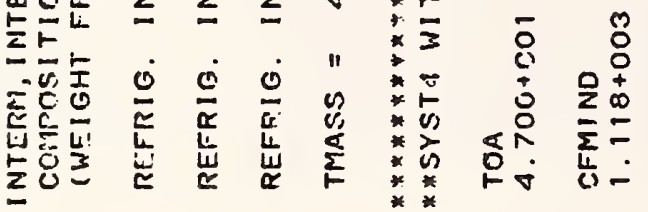

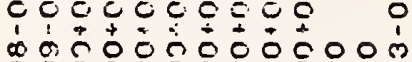
\%

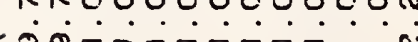

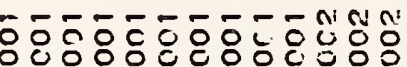

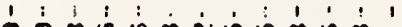

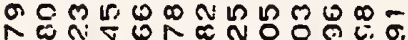

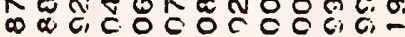
n--incisinginina

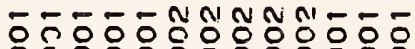
O

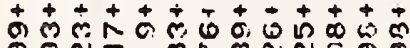

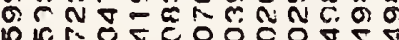

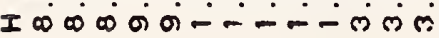

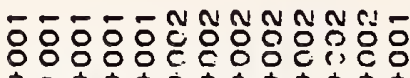

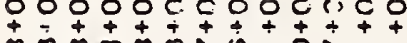

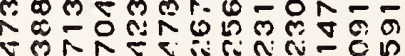

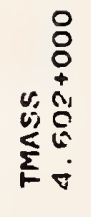

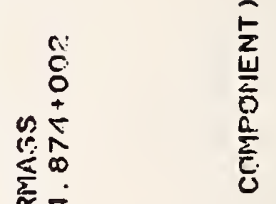

तกம்

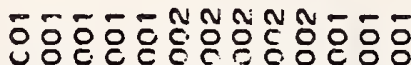

\section{$+7+t i+7+7+7$}

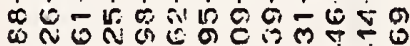

Q

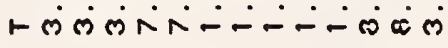

- NMVLUNDOO=NM 


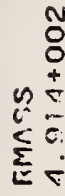

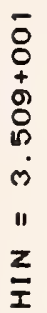

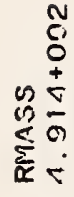

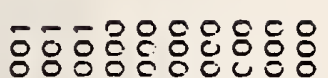

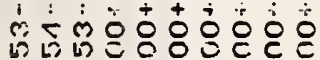

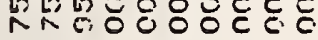
× जं

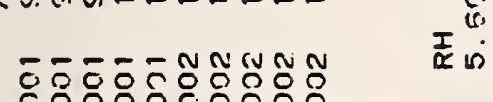

응

$\stackrel{8}{0}$
t.
c.
0

$8:$

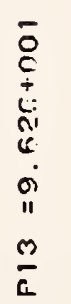

0
8
+
0
0
0
0

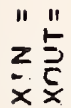

$\begin{array}{r}-\overline{8} \\ \vdots \\ \stackrel{+}{0} \\ \hdashline\end{array}$

I $\infty \infty \infty 0001-\div$

$\bar{c}$

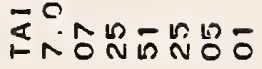

COOCOOLO

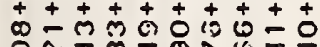

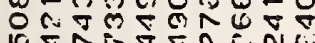

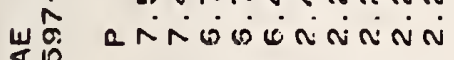

win

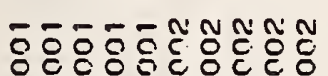

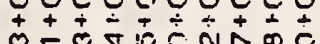

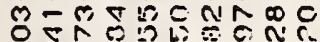

रु-

मलंखिंत-:-:

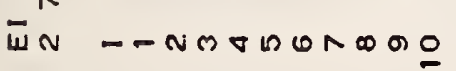

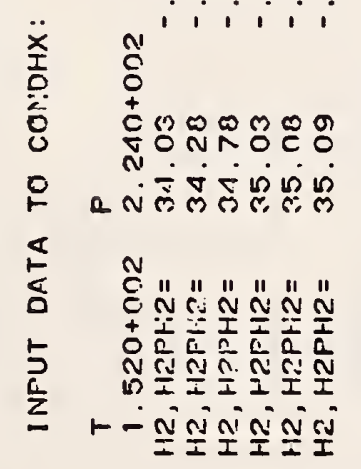

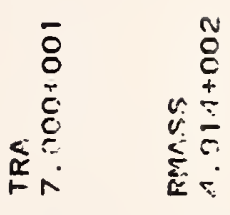

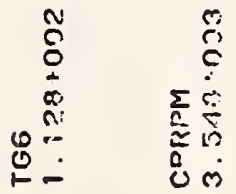

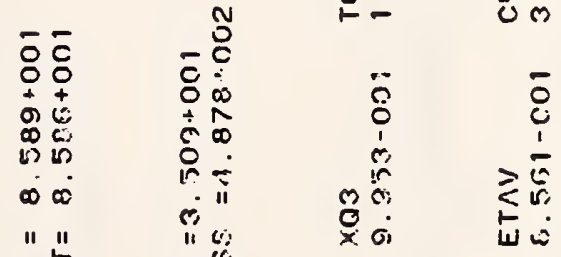

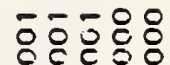

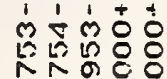

×लंबं:-

$\bar{\delta} \bar{\delta} \overline{\bar{c}} \overline{\bar{c}} \overline{\mathrm{g}}$

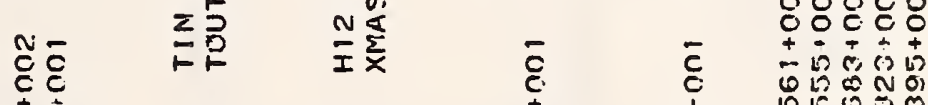

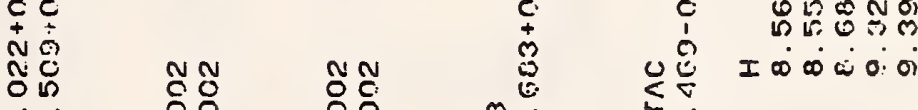

$I-\infty$

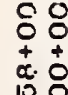

世.

$\bar{c} \bar{c} \overline{0} \overline{0} \overline{0}$

NN

¿ठ

岁"

Ni

a ก

z⿺辶大

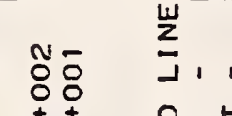

$\sum_{\text {U. }} \cong 5$

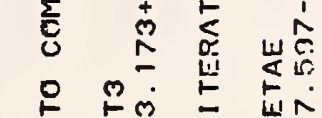

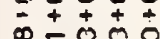

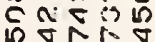

añOOO

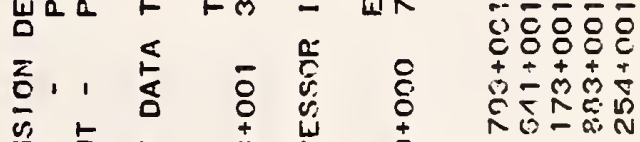

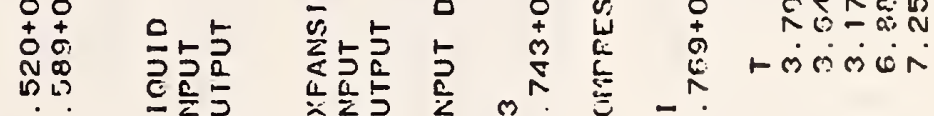

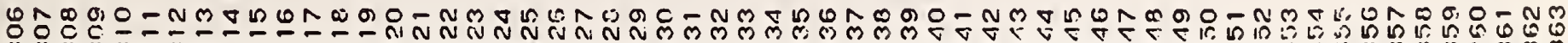

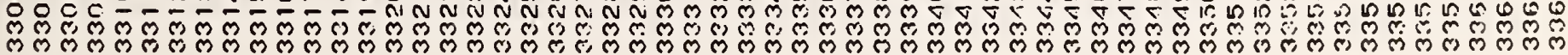




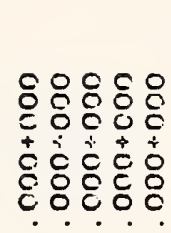

$\ldots$

กำ ำ

ชุต

จะระ

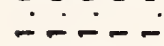

ํํㅇำำำ

$\pm+0+4$

จึ心

जNंNin

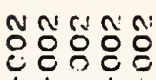

0
+

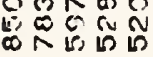

?

-..-

Undo응

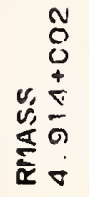

-
0
$\vdots$
0
0

紊的

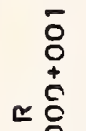

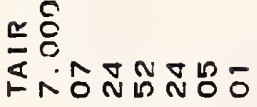

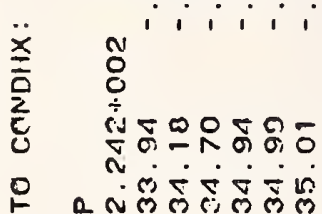

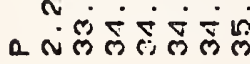

宫

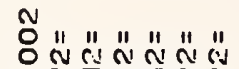

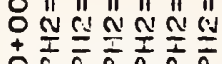

N

bIIIIII

$\vdash-\frac{N}{N} \frac{N}{I N} \frac{N}{N}$

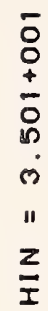

$\frac{2}{3}$

논

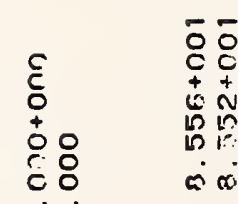

$x-$

I-

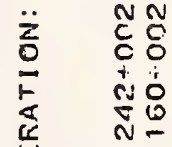

aก

亲 \begin{tabular}{l}
$\overline{8}$ \\
1 \\
$\vdots$ \\
\hline
\end{tabular}

I

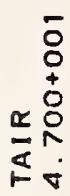

-
8
1
0
0
0
$\times 01$

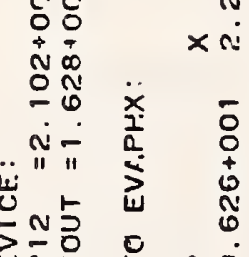

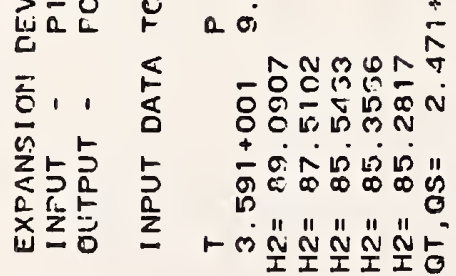

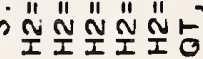

ํํ을

ูํㅇำ

ก N 0
0
0
0
0
0

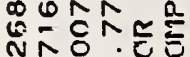
$\times$ त.

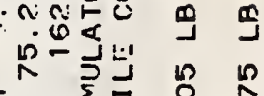
융을

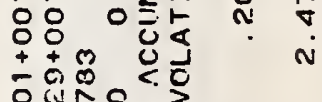
¿

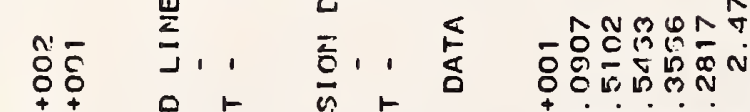

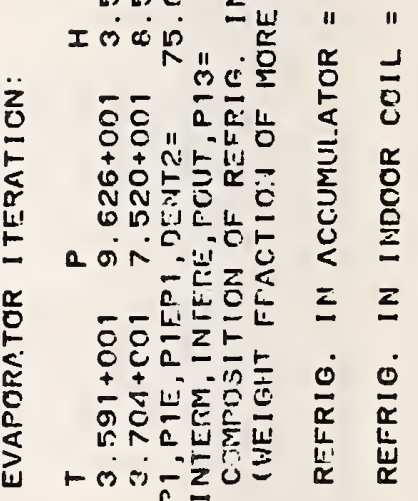

$\bar{\partial} \bar{\partial}$ 요요

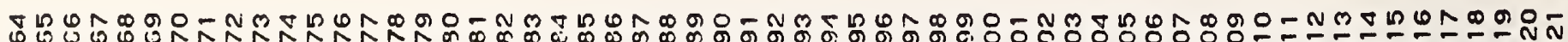

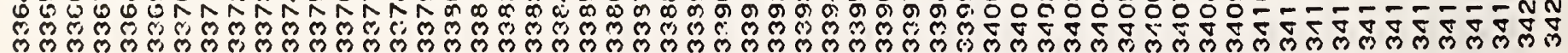




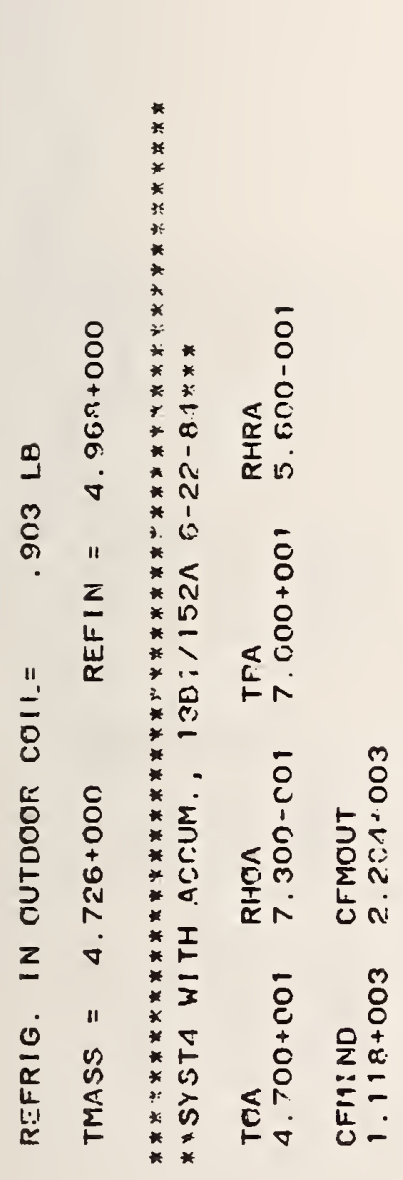

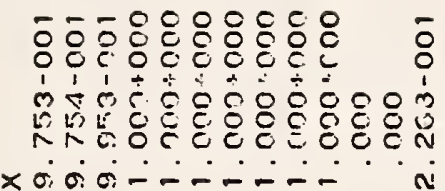

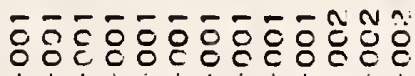

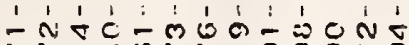

ヘ

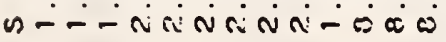

อَ-

o

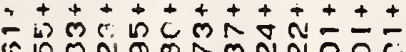

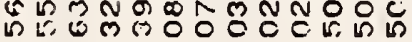

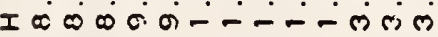

-

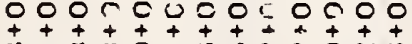

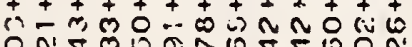

n

- NRம

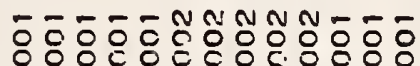

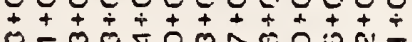

r๐-

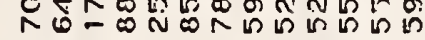

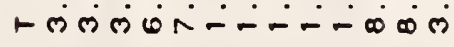

- Nm

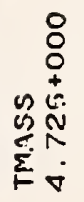

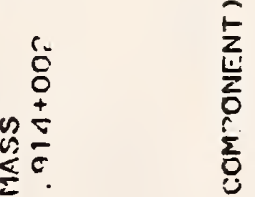

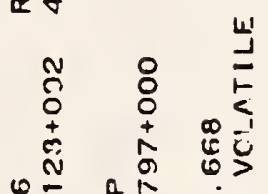

ㄴ: 8

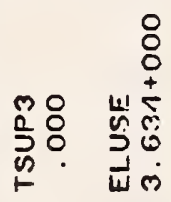

胥

1 론

녕

등다

造这

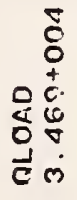

迹恋

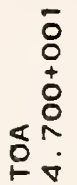

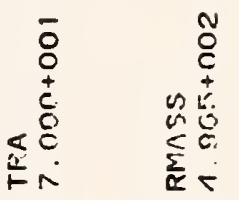

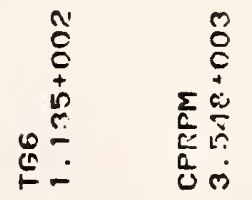

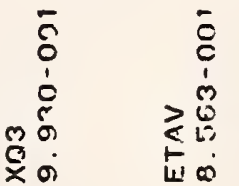

$\bar{c} \bar{c} \overline{0}:$

กิ่

$\times 0.00$ -

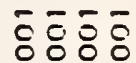

范它的

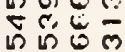

I $\infty \propto \infty$

造兽

흐등

I ${ }^{\infty} \cdot$

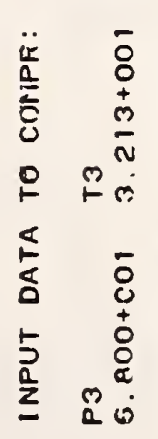

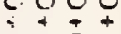

लिएक

anió

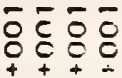

in

रूत्ष

मलंलिं

चथ

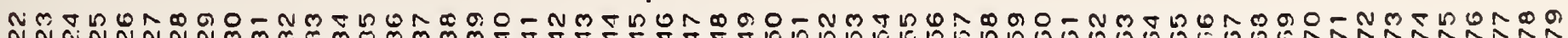

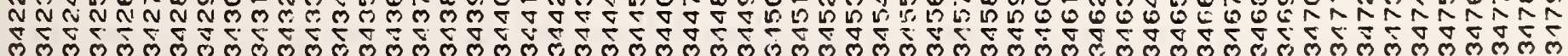




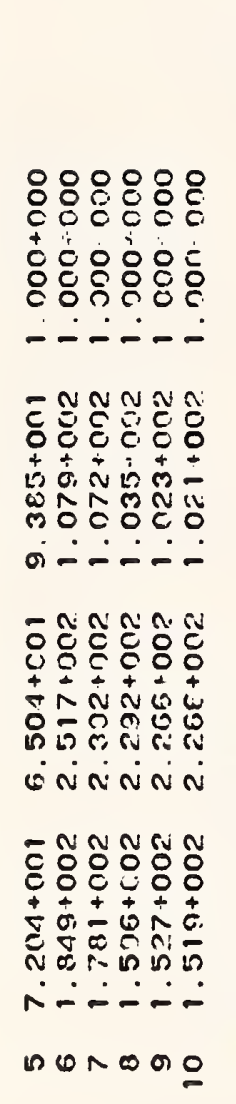

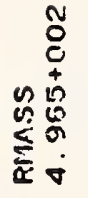

$\ddot{8}$
$\vdots$
$\vdots$
0

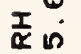

送

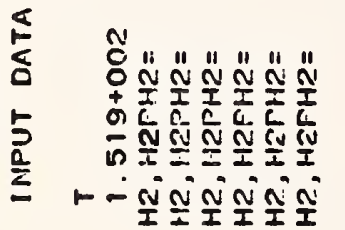

5
$\vdots$
1
5
0
11
$z$
$z$

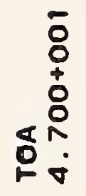

융

$\bar{c}$

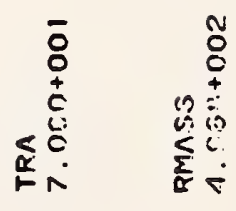

" 2

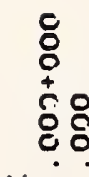

ह-

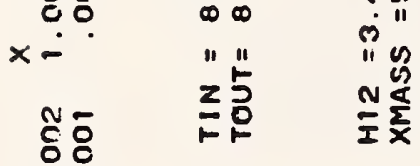

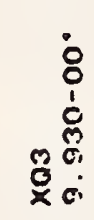

c.

$\gtrless$ 点

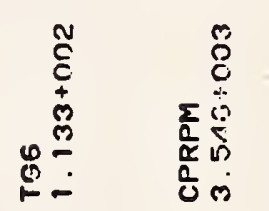

둥융웅영융영

$115+50$

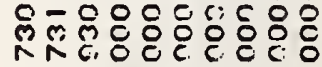

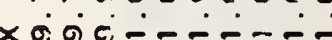

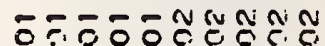

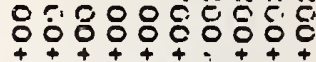

no

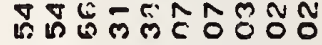

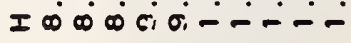

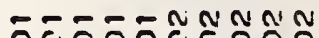

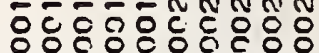

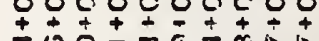

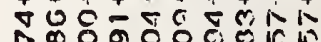

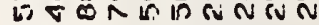

añogoññNa

-

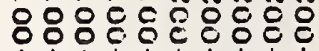

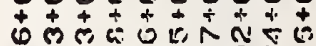

पूल

रुั

मलंलिषन-‥-

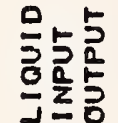

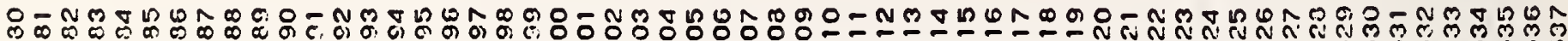

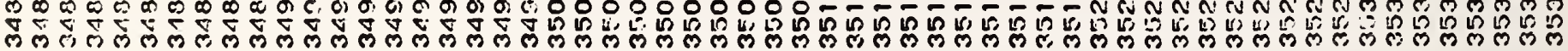


5
8
+
7
0
0
0
11
$z$
$z$

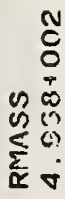

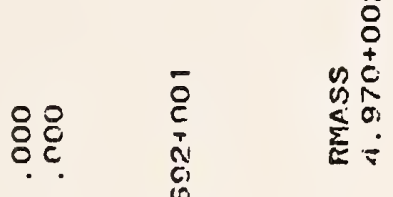

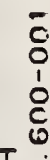

8
8
$\vdots$
$\vdots$
8

$=\frac{11}{2} \frac{5}{x}$

-

$\simeq \stackrel{+}{\circ}$

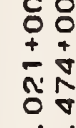

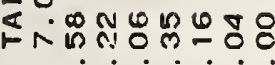

용

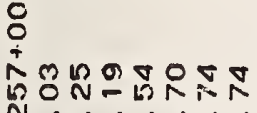

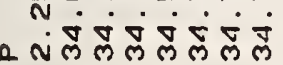

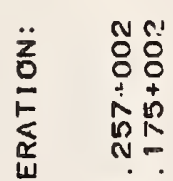

ชิ

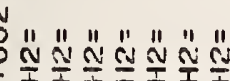

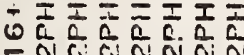

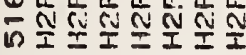

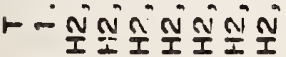

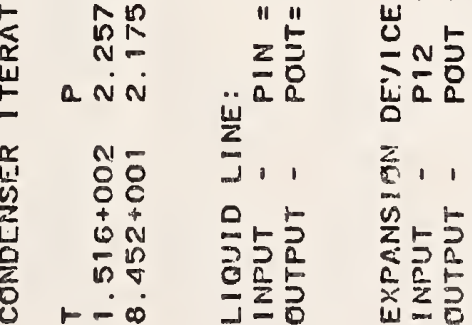

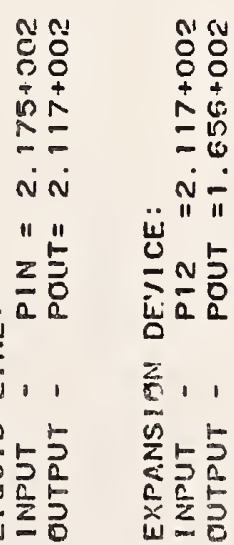

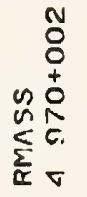

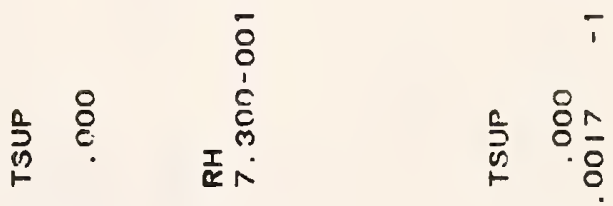

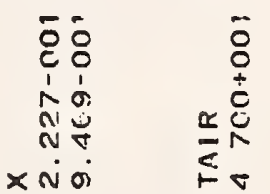

ธํㅇㅇ

a

N $\bar{\sigma}$ x $\cos ^{\circ}$

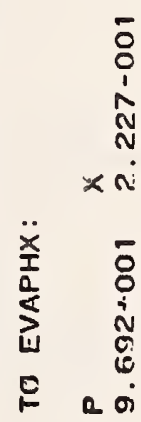

ब -

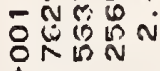

+

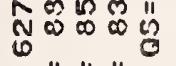

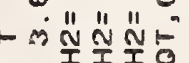

홍요

ס্்

I ल०

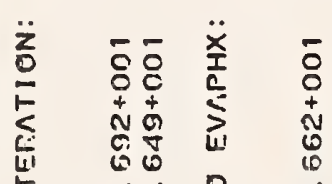

a

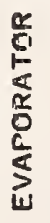

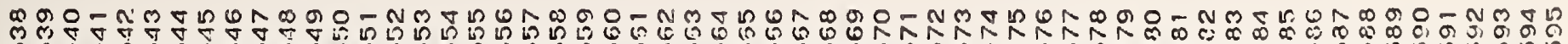

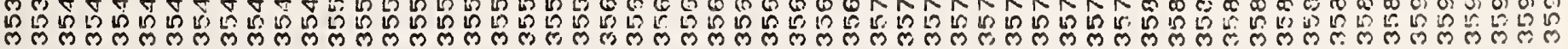




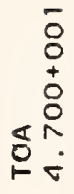

$\overline{8}$
0
$\infty$
0
10
0
$1 "$
$z$
$z$
1

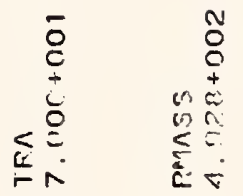

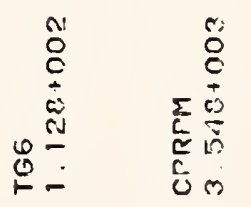

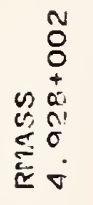

형ㅇㅇㅇㅇㅇㅇㅇㅛ

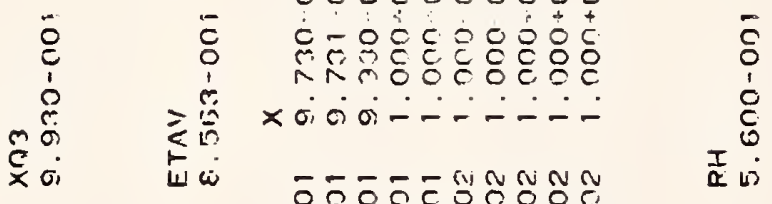

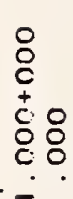



88.858 .082

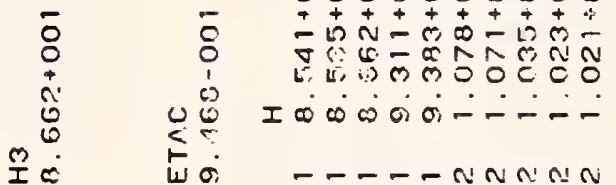

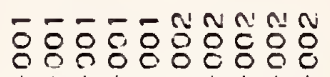

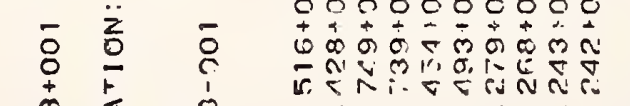

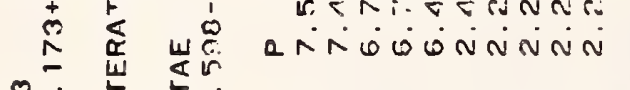

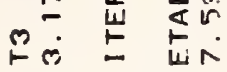

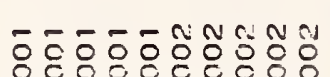

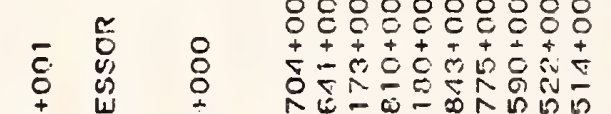

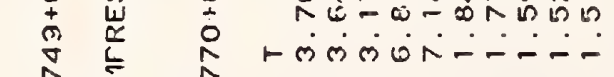

क⿺辶े एँ

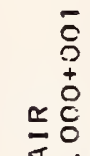

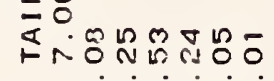

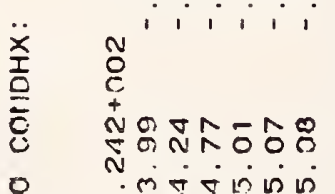

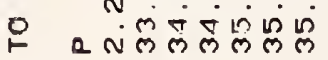

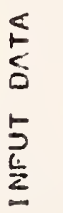

ระุ

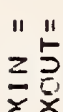

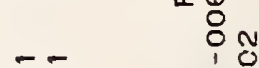

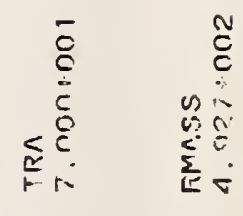

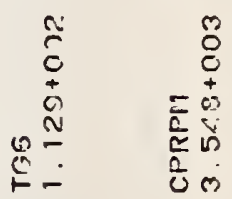

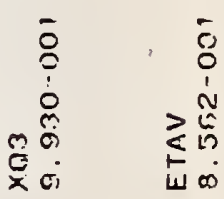

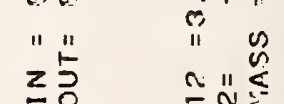

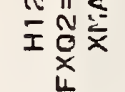

들

$+e^{2}+$

究岕离

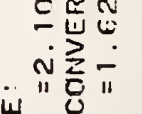

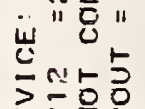

ii ${ }^{-} \mathrm{C}$

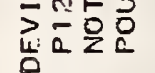

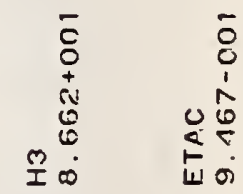

它志

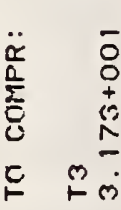

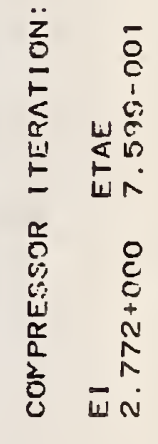

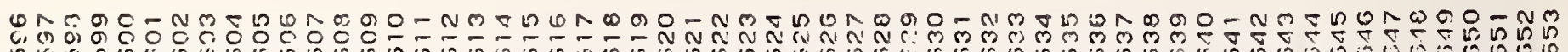

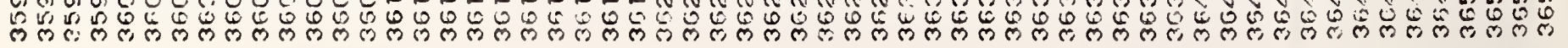




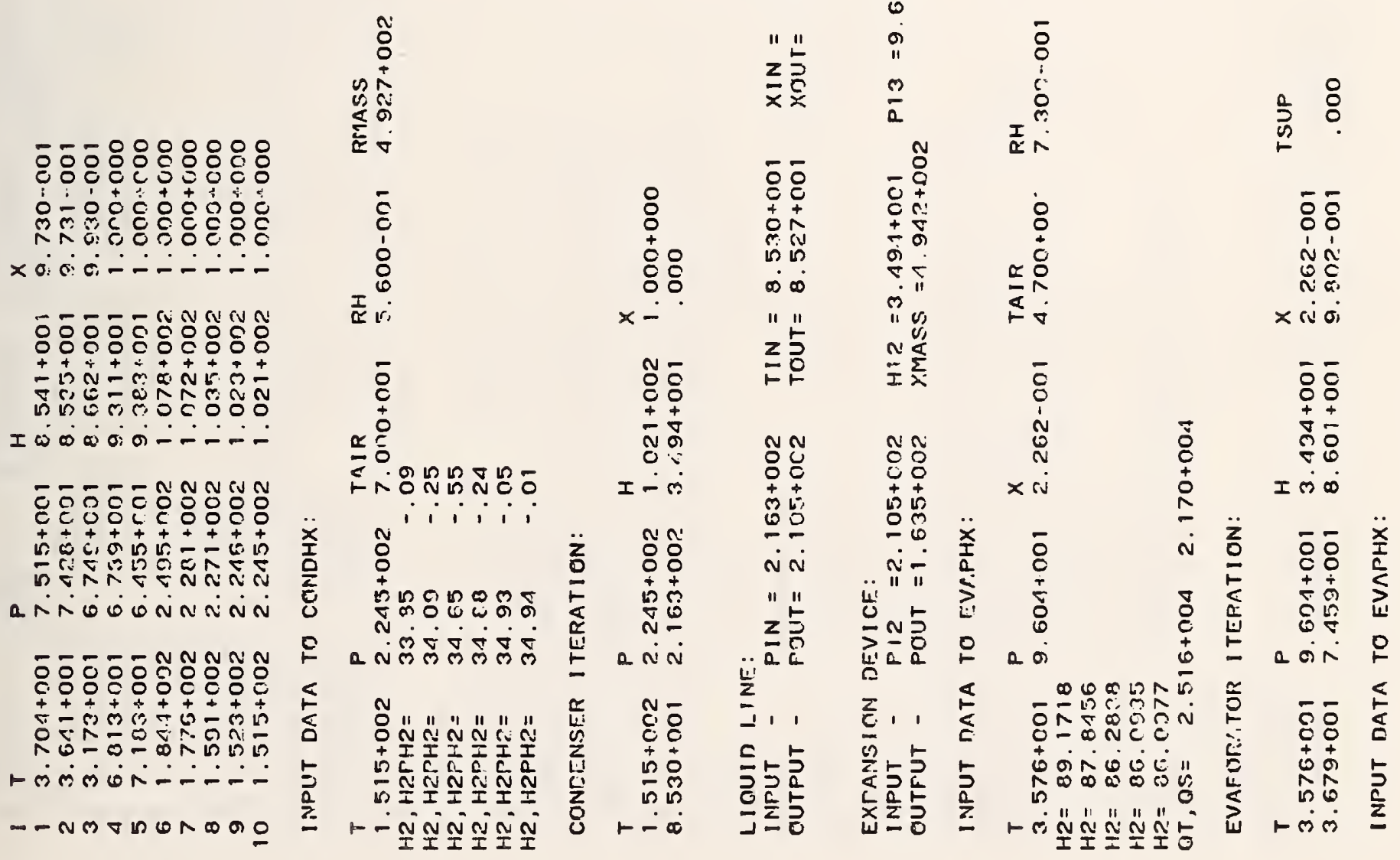

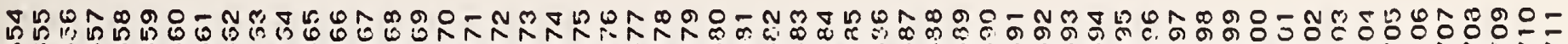

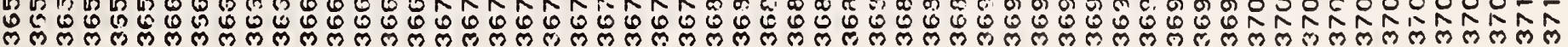




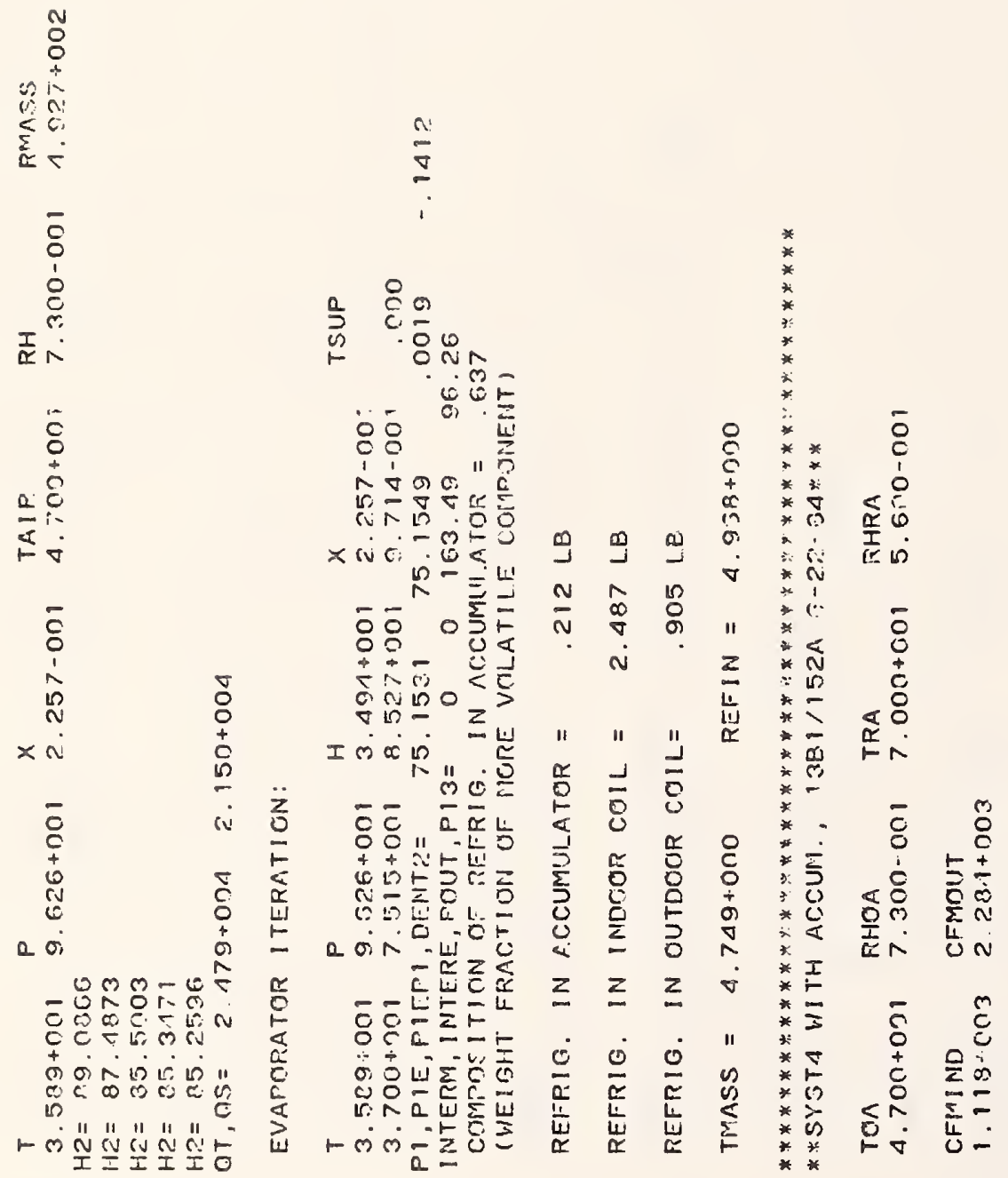

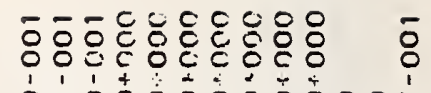

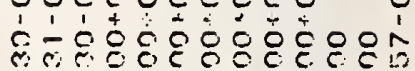
สNG

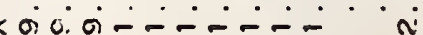

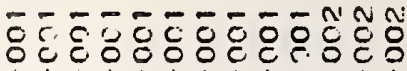

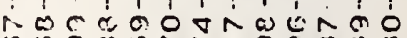
थ n-annanin-mía

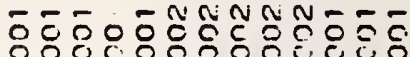
$+\pi+t+4 t+4$ - DU ถึँ I $\infty \omega \theta ल \ldots M M$

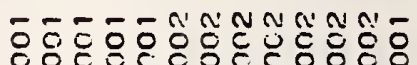
$+\div+\div++\pi=-\div$

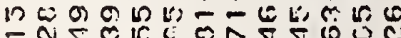

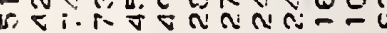

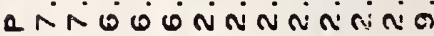

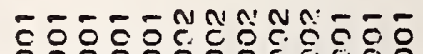

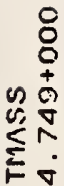

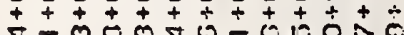

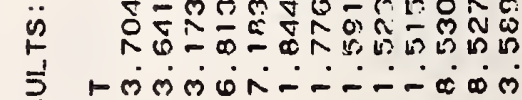
号

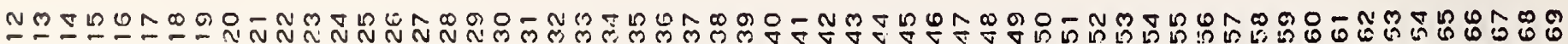

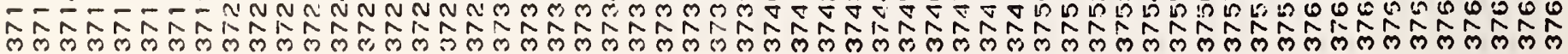


5
8
+
0
0
0
0
10

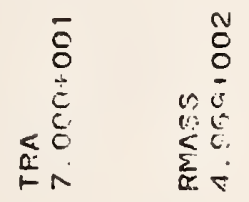

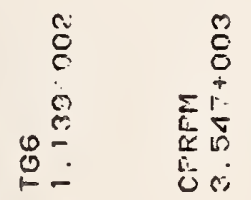

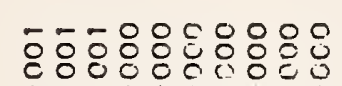

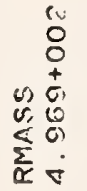

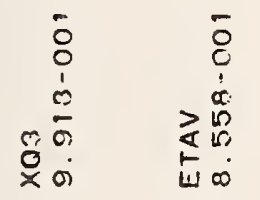

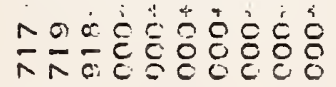
xबंबंध.....

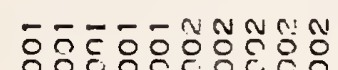

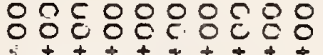

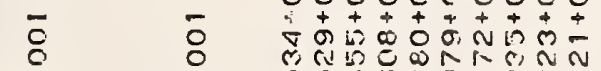

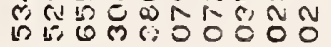

$\begin{array}{ll}+ & \infty \\ 0 & 0 \\ 0 & 0 \\ 0 & 0\end{array}$

I $\omega$ os $\propto$ o 0 o $\ldots \ldots$

Cن

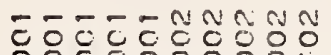

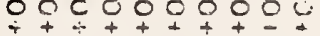

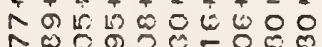

E.

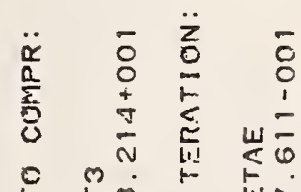

añovoññó

ค म

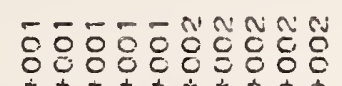

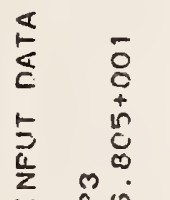

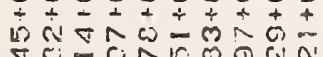

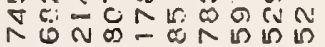

किलिएं-.-

แ)

- nM

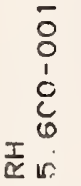

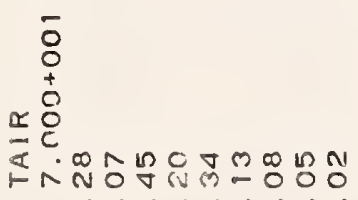

蒙

气ू

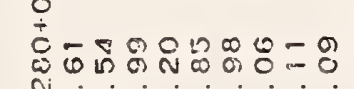

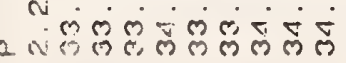

운

$\leqslant$

อี่"

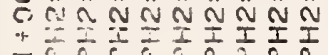

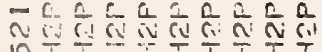

(1)

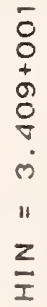

¿

z:

$\overline{0}$
0
+
8
0
$\square$
$\dot{0}$
11
$z$
I

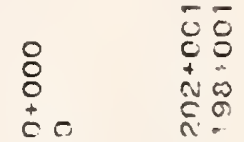

용 क

$x-1$

응

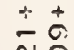

हैं

$I-\dot{~ ल े ~}$

n 2 पूर

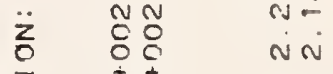

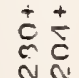

a तं

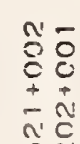

n?

$r-\infty$

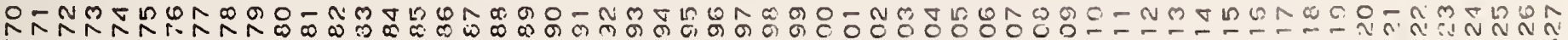

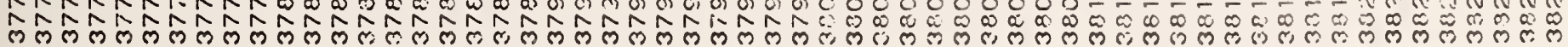




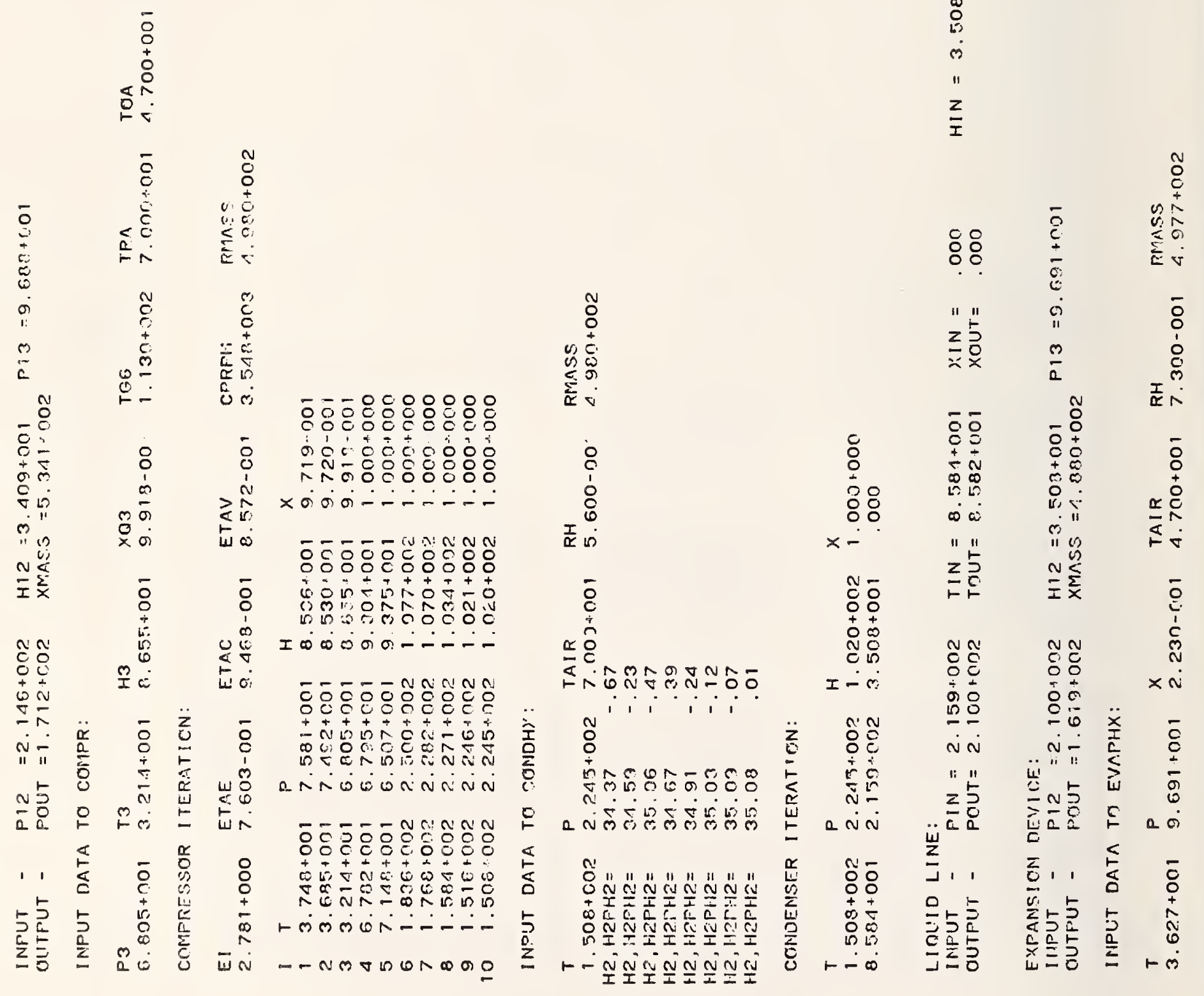

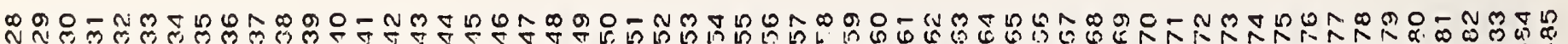
等 


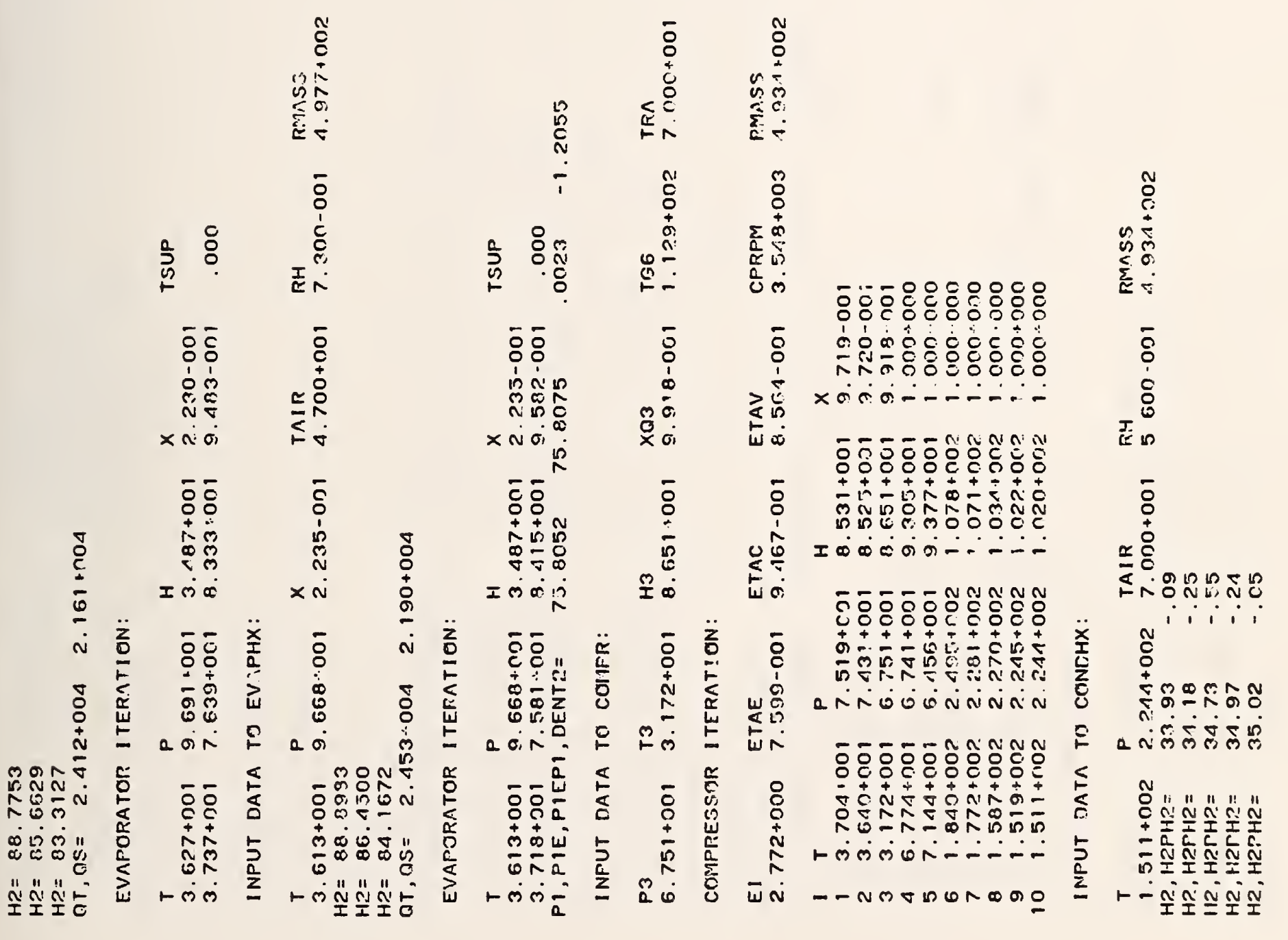

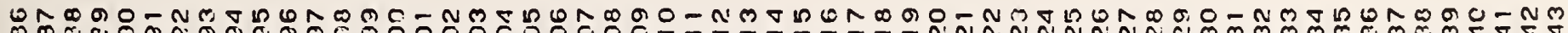
等 


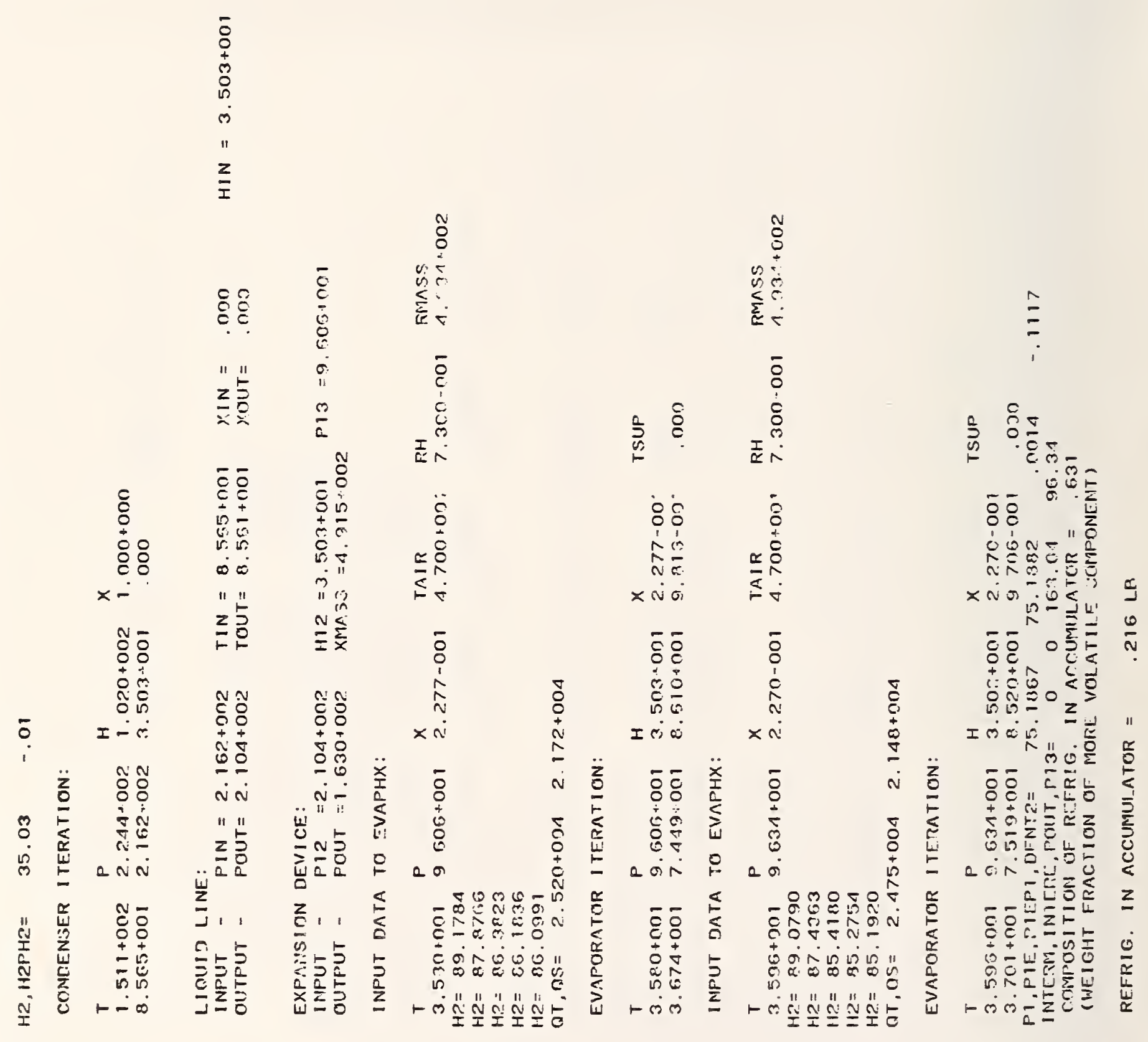

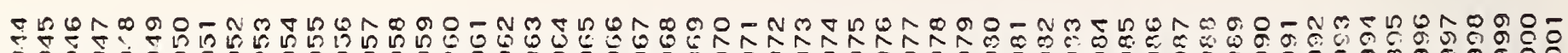

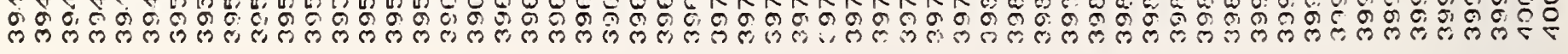




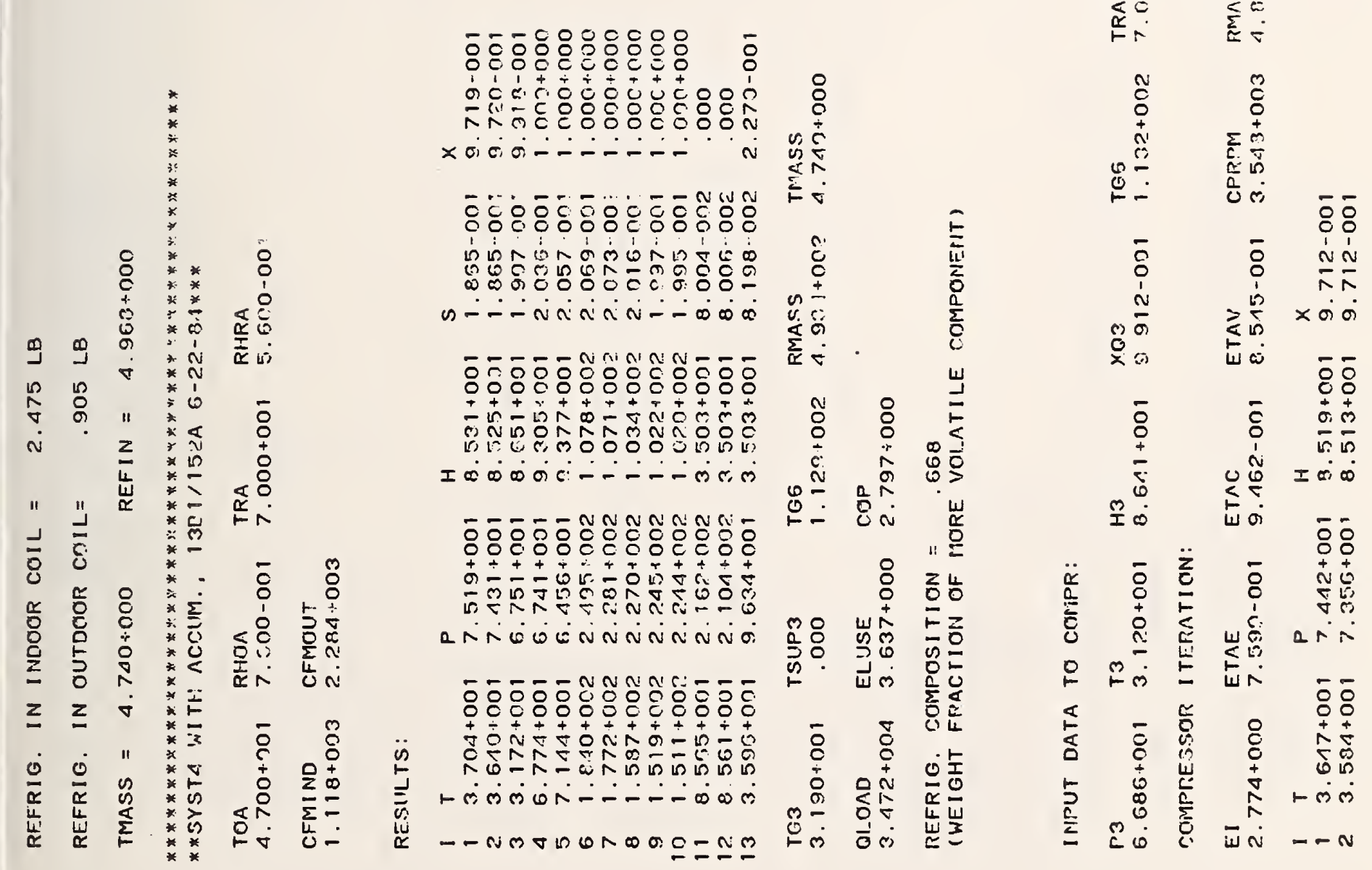

N"

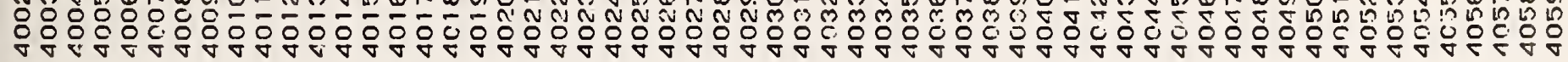




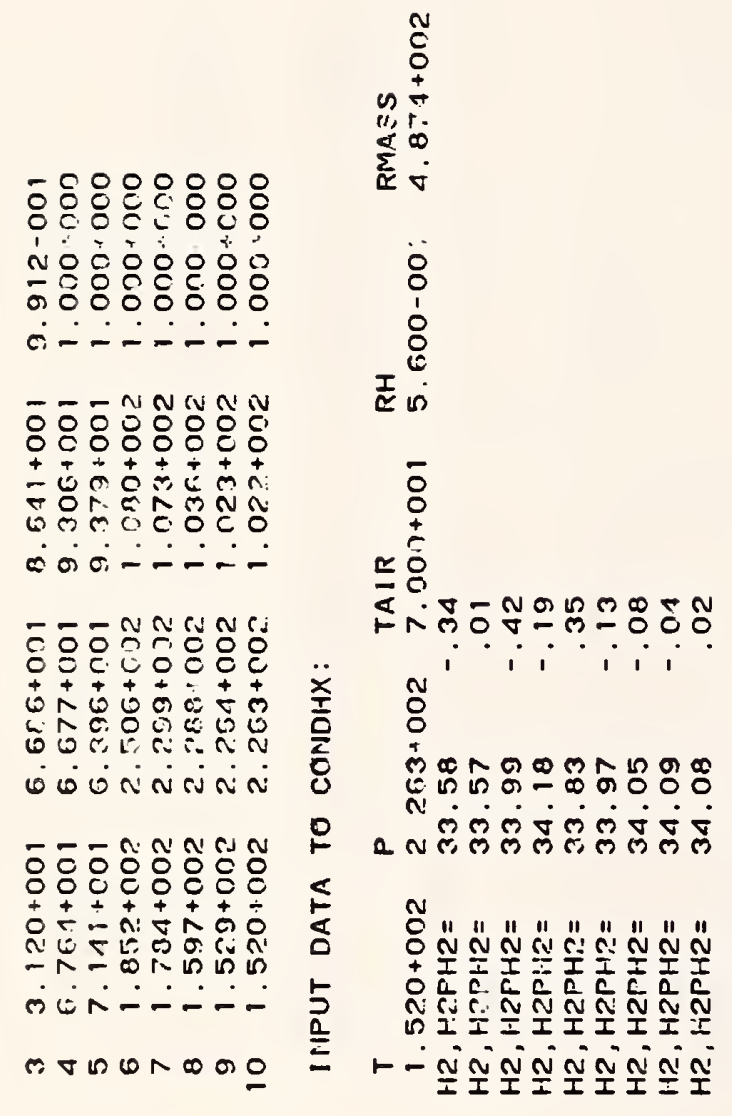

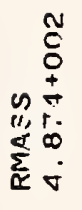

$\because$

$\bar{c}$
0
+
0
0
$⿱ 亠$
$\dot{0}$
11
$z$
$z$
$\bar{I}$

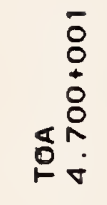

$\frac{2}{x} \frac{5}{x}$

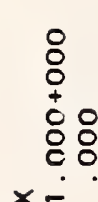

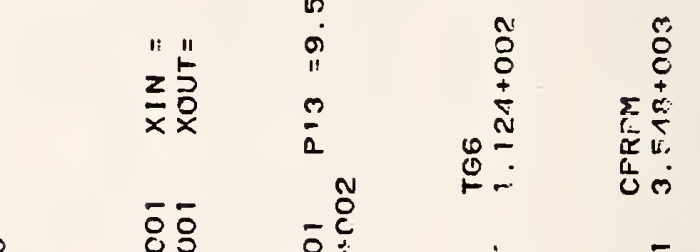

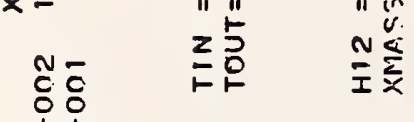

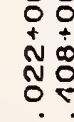

I -

$\cos 2$

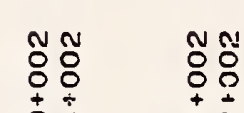

$\overline{8}$

焉

$\begin{array}{lll}1 & 0 \\ 1 & 0 \\ 1 & 1 & 0\end{array}$

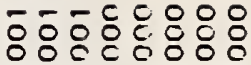

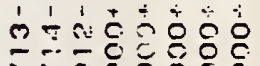
तर xoode.--

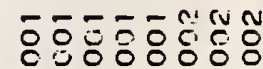

$+7+7+7$

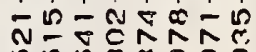
जमूलिल00 I किष 000 \% -

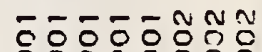

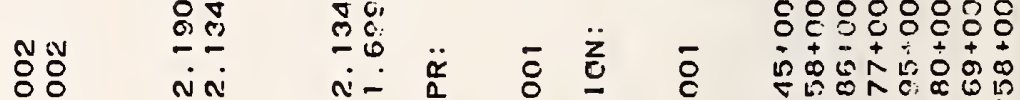

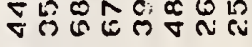
ariogonina

ํํำ 年,

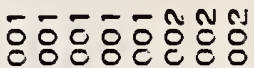
cotocto

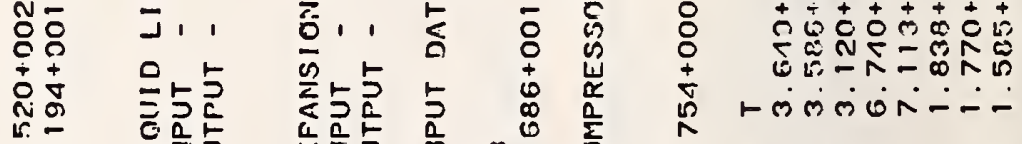

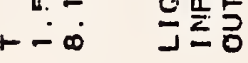

एं

एँ

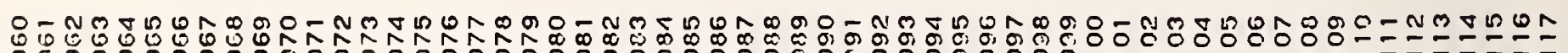

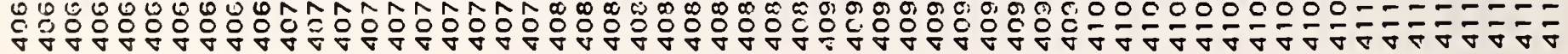





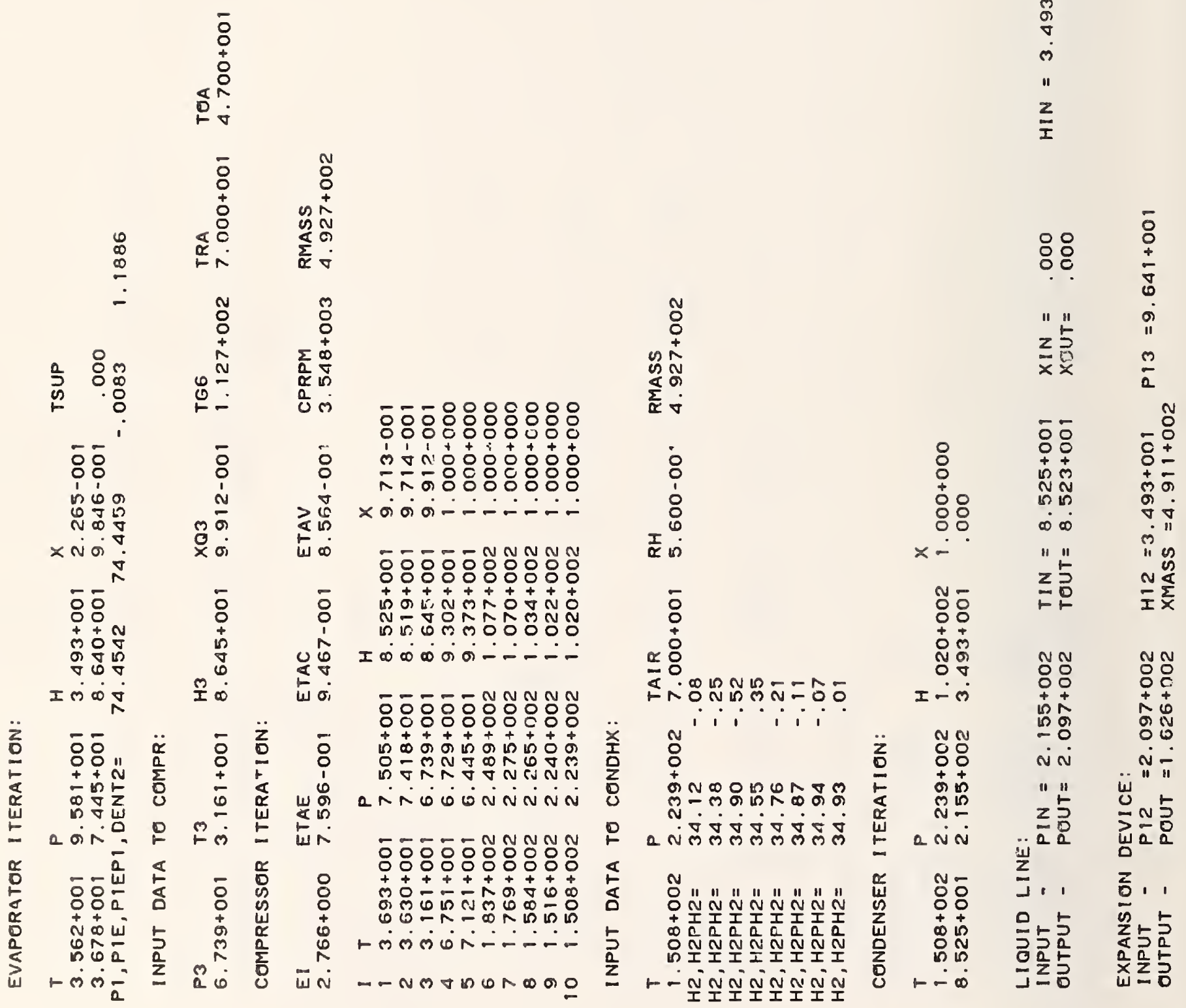

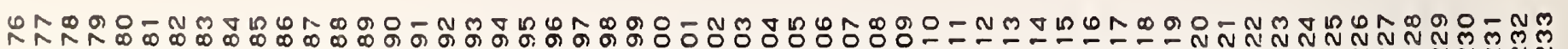

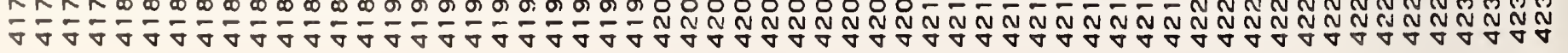




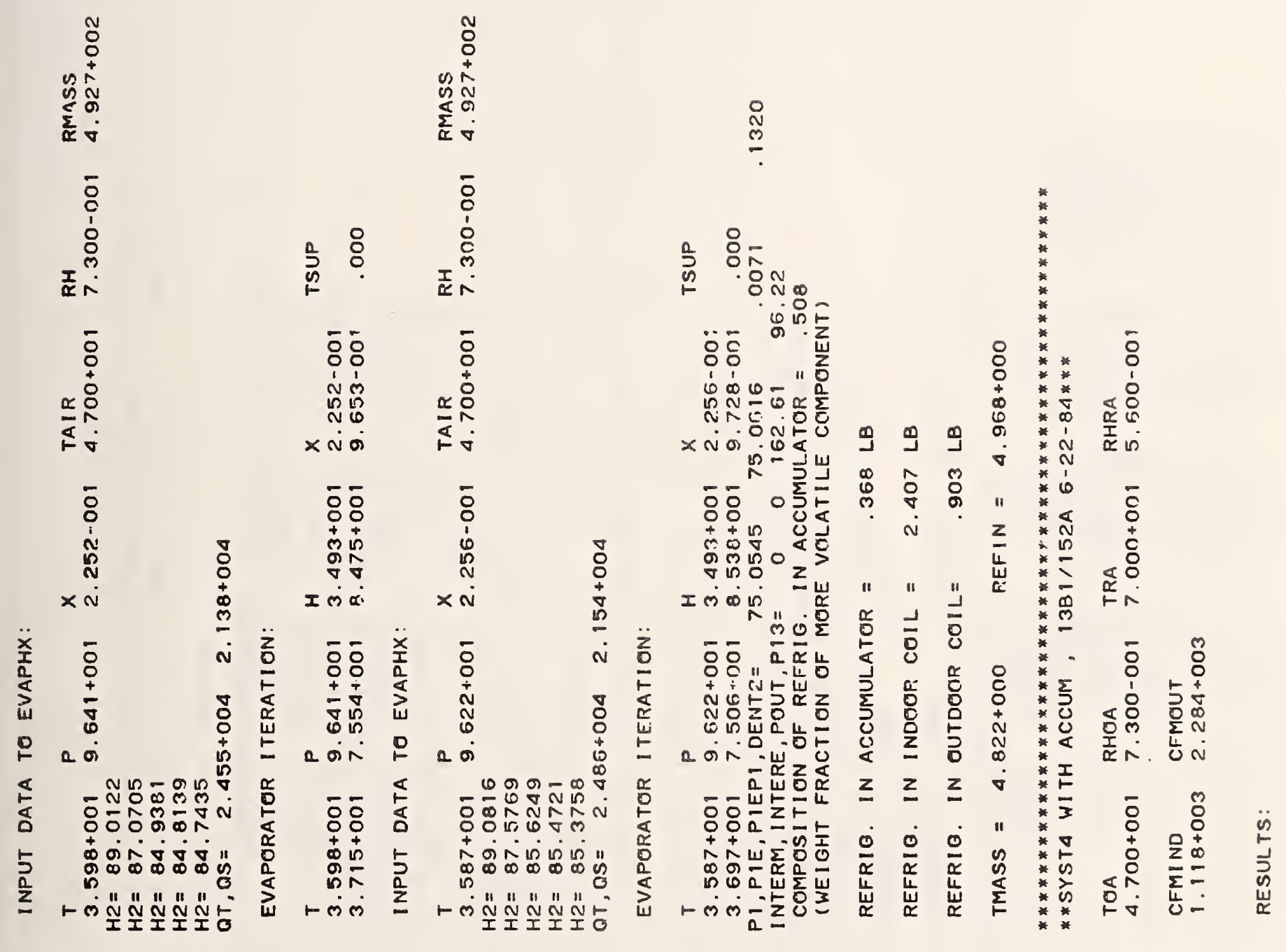

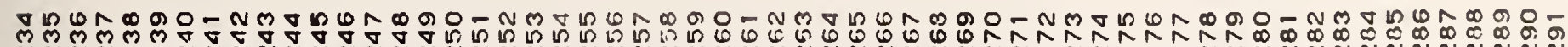

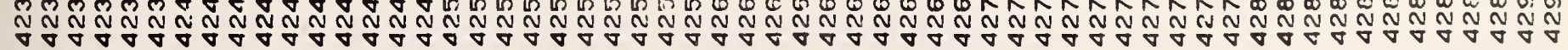




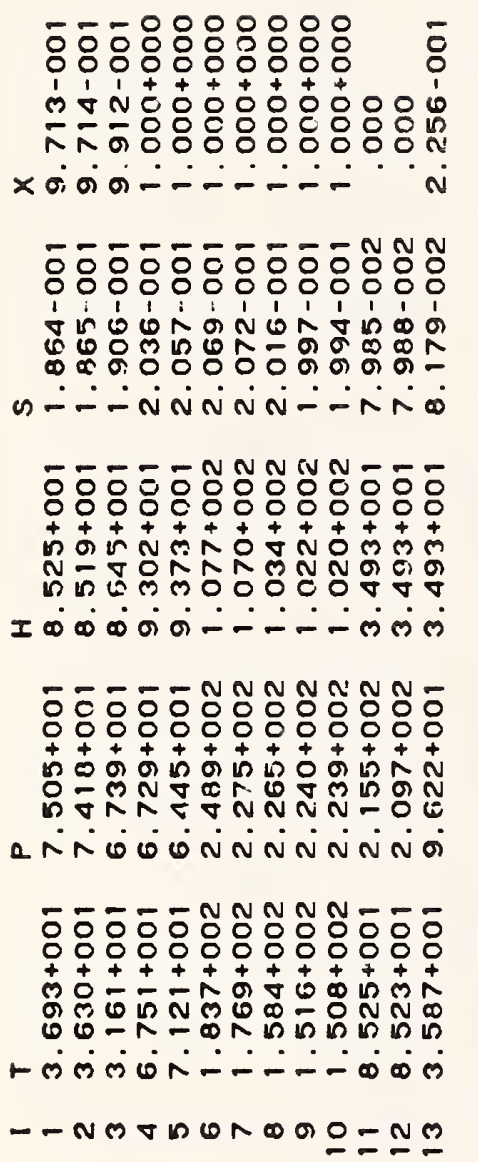

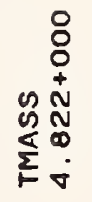

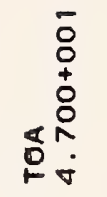

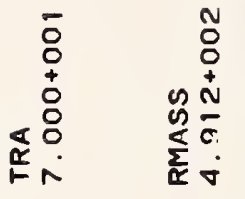

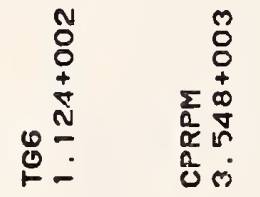

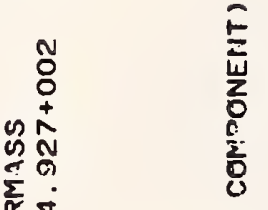

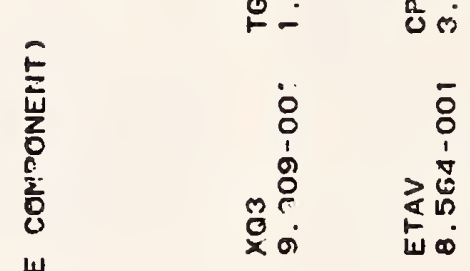

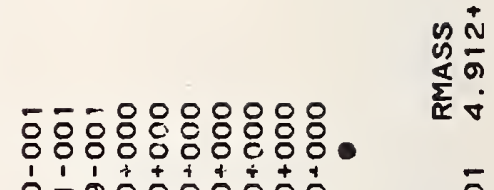

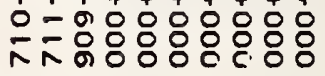

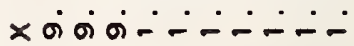

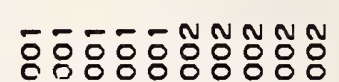

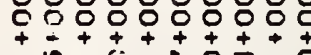

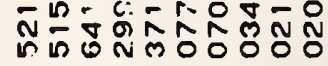
工 $\infty 0^{\circ} \circ$

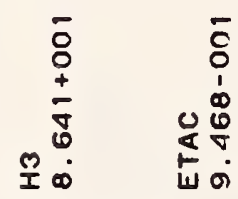

-

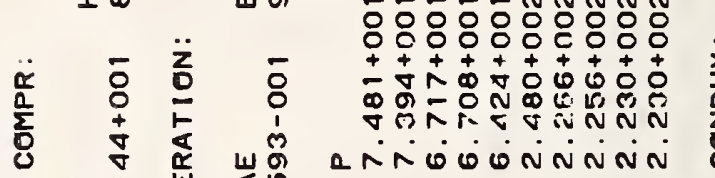

क

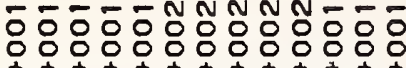

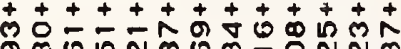

6 6 -

- rimitana

- NM

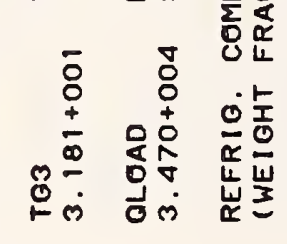

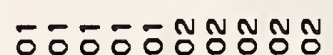

¿००००००००

음 लि

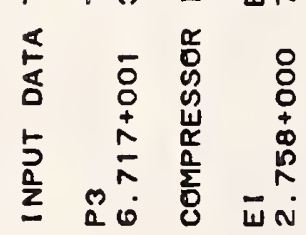

nitis

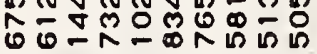

मलंलिष्:-:-

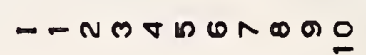

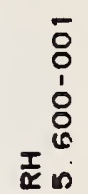

$\overline{8}$
+
$\stackrel{+}{0}$

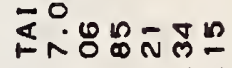
$\therefore i$ 응 +

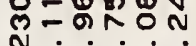

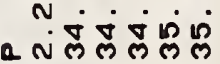

욤

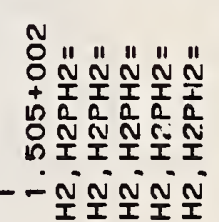

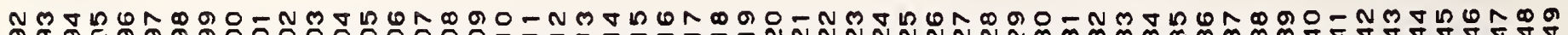

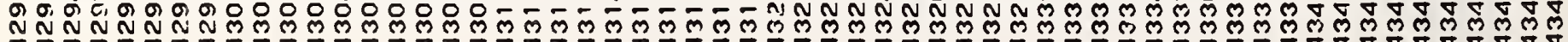
N 
$\overline{8}$
$\frac{1}{30}$
0
in
"1
z

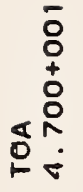

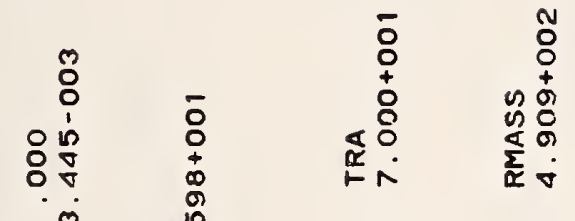

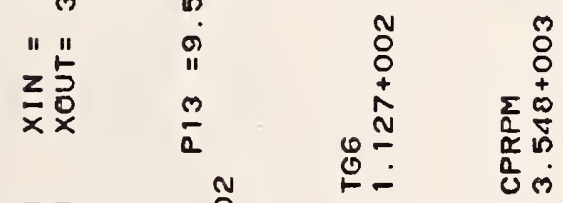

$\stackrel{\infty}{\circ}$

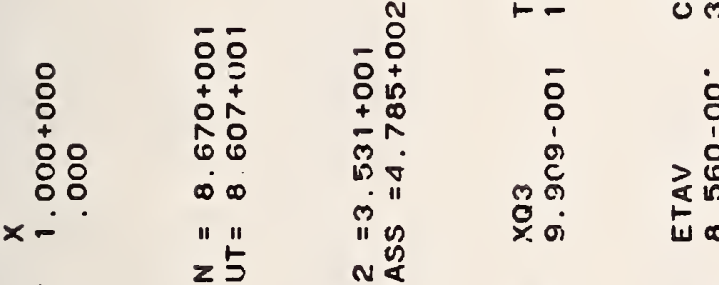

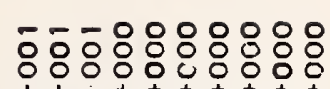

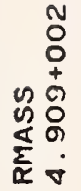

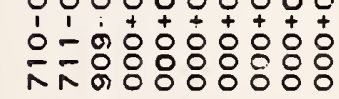

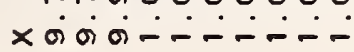

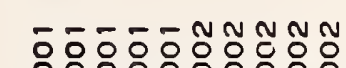

ํํำ

$+$

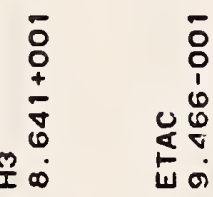

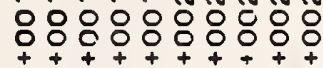

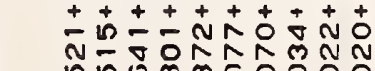

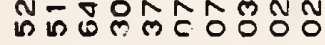

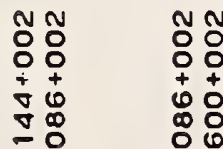

I $\infty \dot{0} 0 \dot{0} 0+\cdots$

-

80000000000

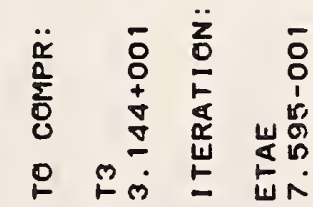

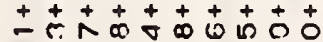

"ü"

" 2 芺

oñognanin

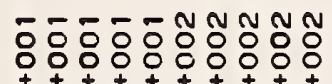

$\ddot{u}^{a}$

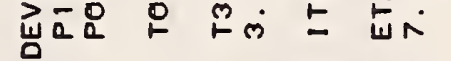

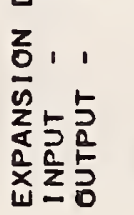

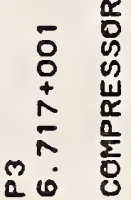

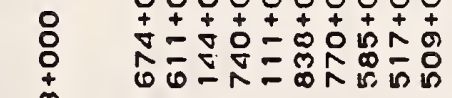

章吉去

मिंलिंत्रि-:

Nิำ

$\vdash-\infty$

$\bar{\omega} \stackrel{\wedge}{N}$

-

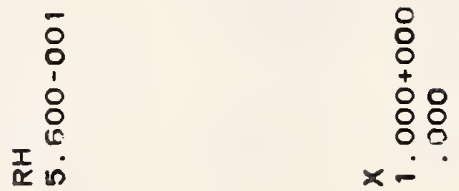

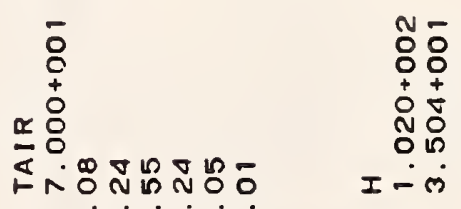

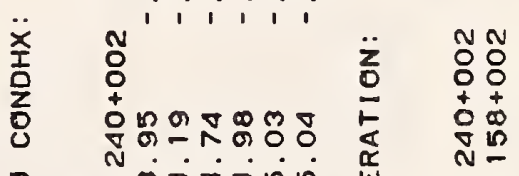

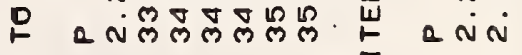

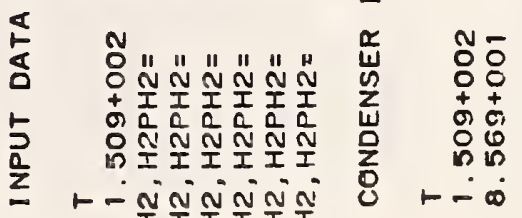

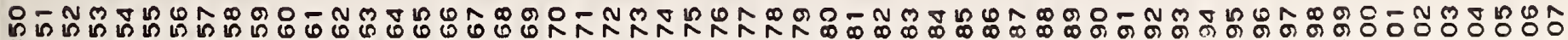

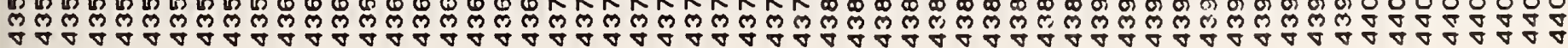


$\overline{8}$
+
8
0
ì
$" 1$
$z$
$z$

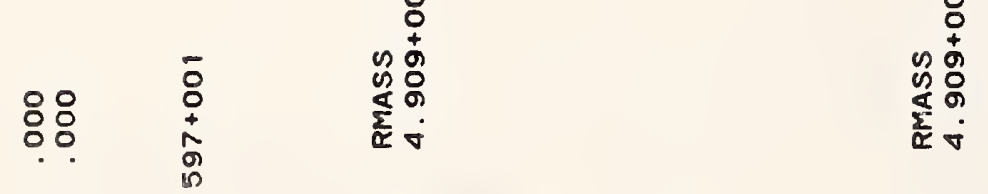

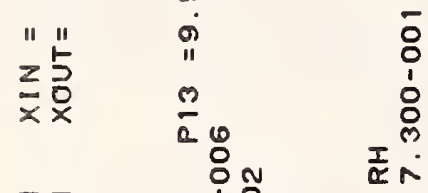

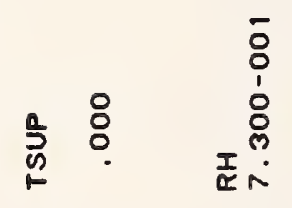

$\frac{0}{0}$

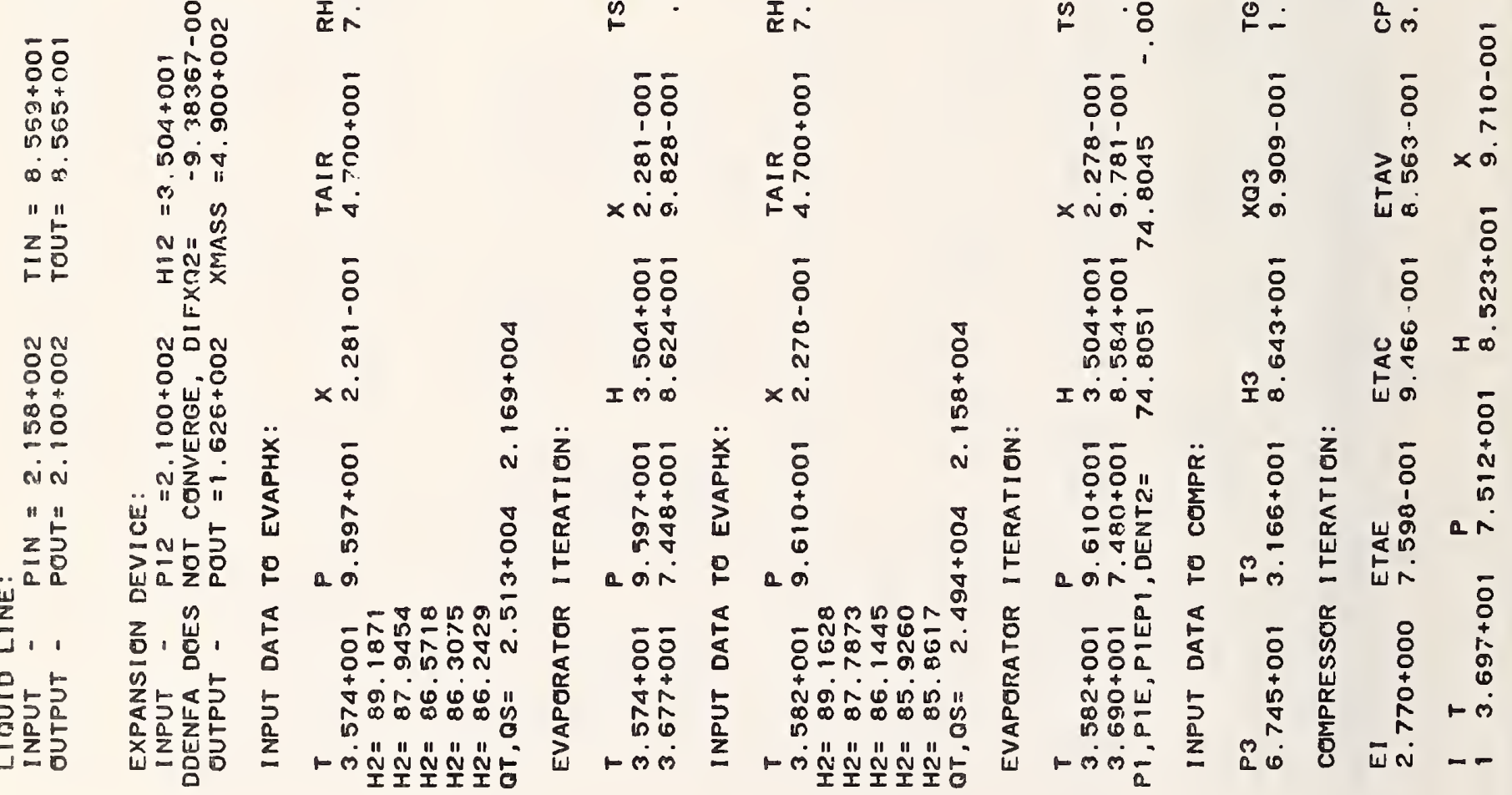

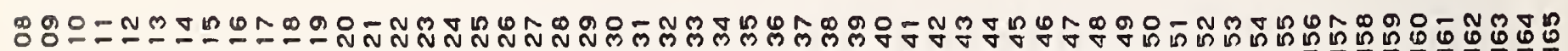

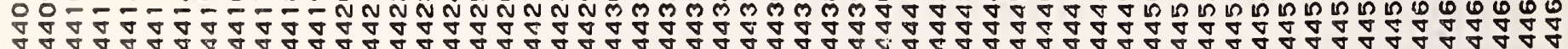


5
8
+
0
15
in
11
2
2

응응응응영영영응

高

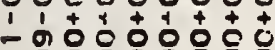

下

कं

-

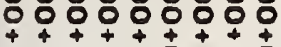

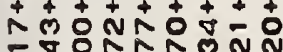

-

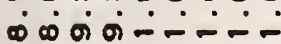

-

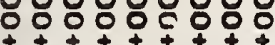

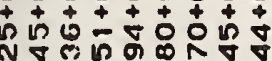

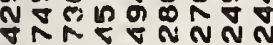

Nंம்

$-5-5 \mathrm{~N}$ N N N

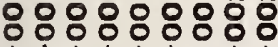

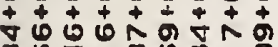

ต 60 -

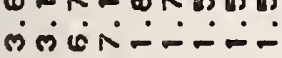

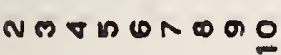

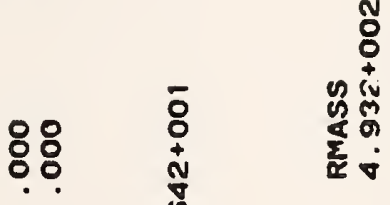

i $\stackrel{+}{N}$

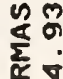

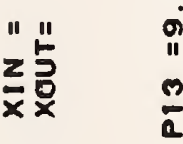

$\begin{array}{r}\overline{0} \\ \vdots \\ \vdots \\ 0 \\ \hdashline\end{array}$

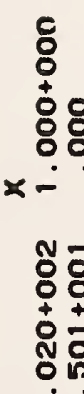

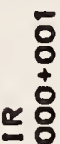

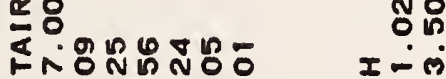

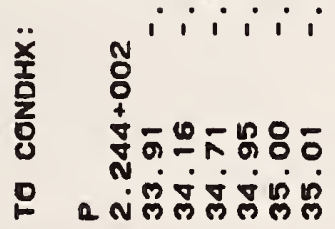

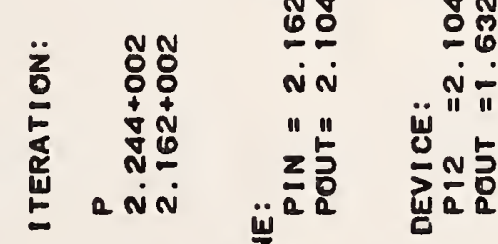

Ơ

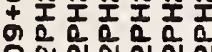

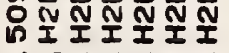

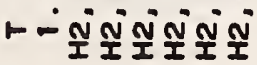

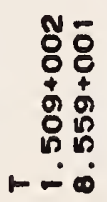

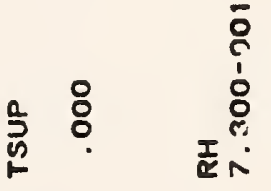

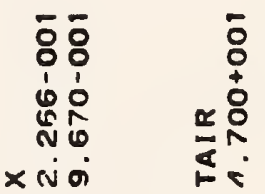

5
0
1
0
6
0

웅

5
8
+
8
8

5

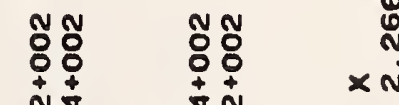

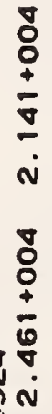

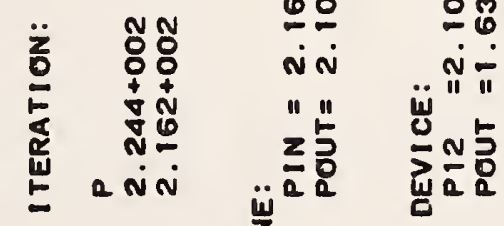

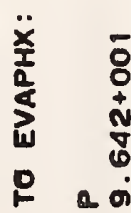

$I \ddot{\circ}$

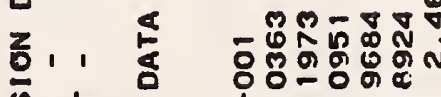

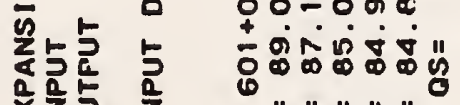

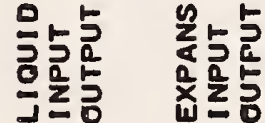

-

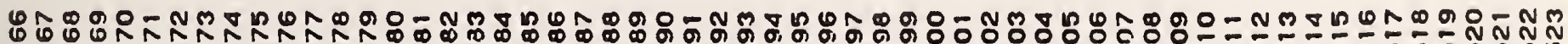

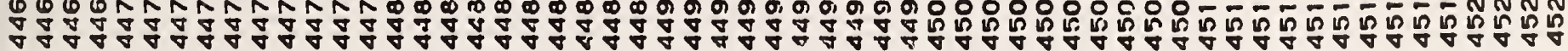




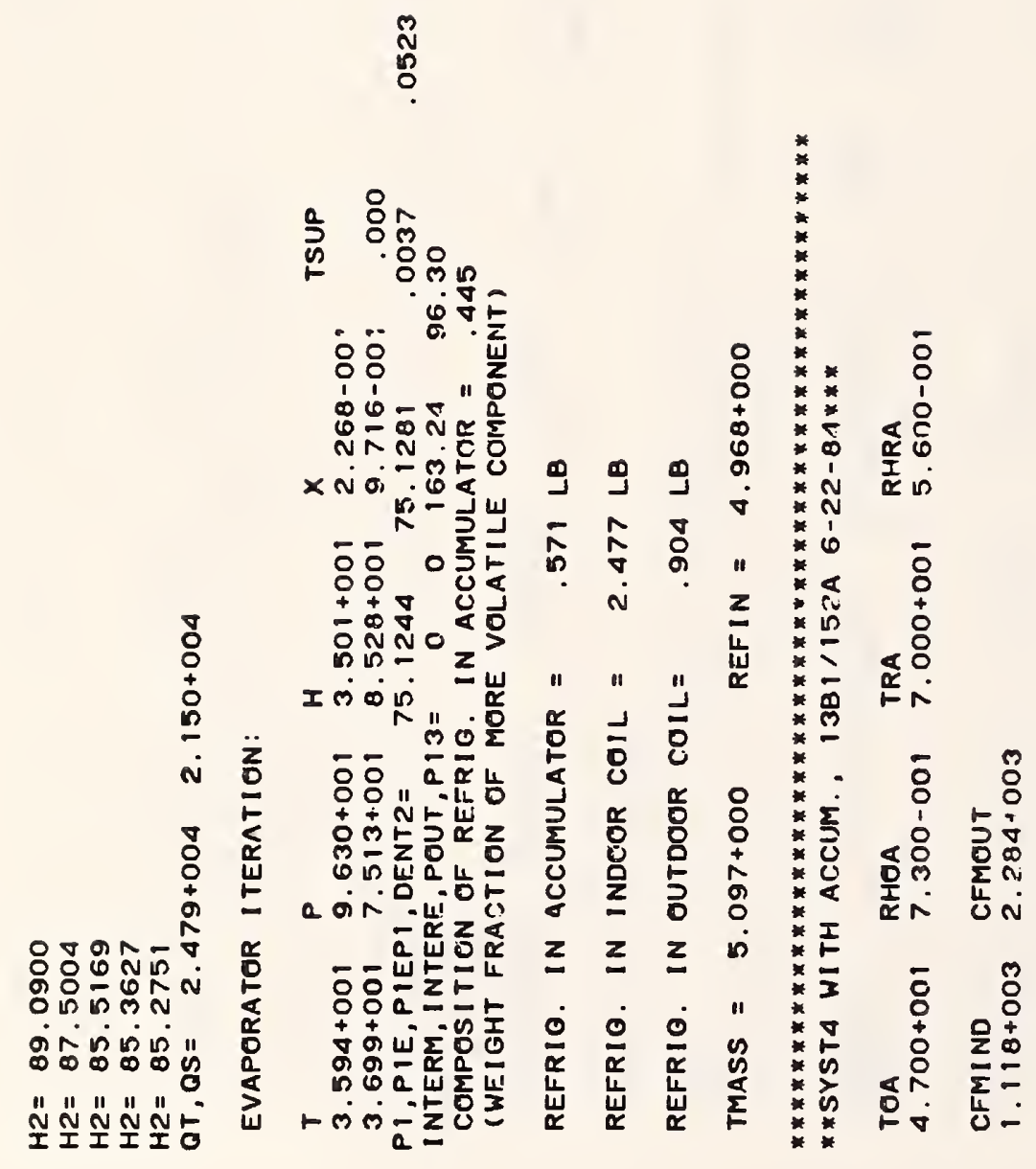

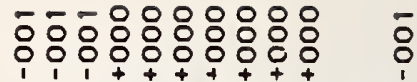
í

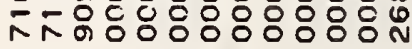
$\times \operatorname{\theta io} \div \therefore \cdots$

-

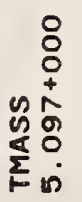

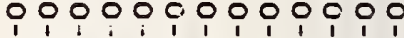

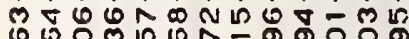

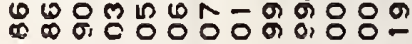

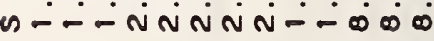
0808000000000

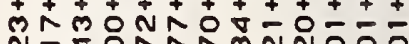

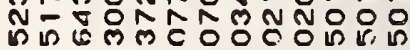

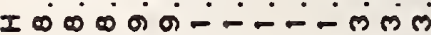

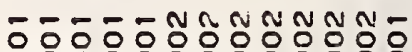
080800000080

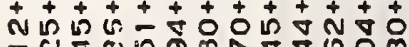
万ก

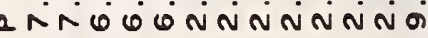
-

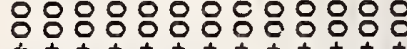

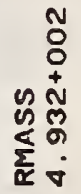

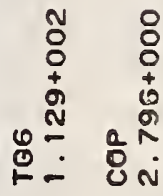

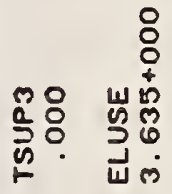

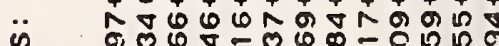
2

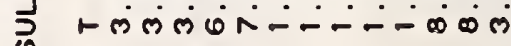
ITIITO

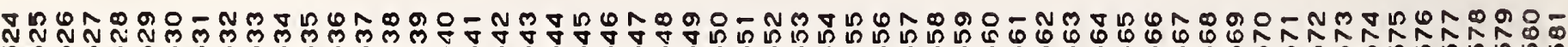

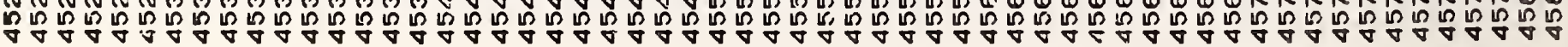


0
0
0
0
0

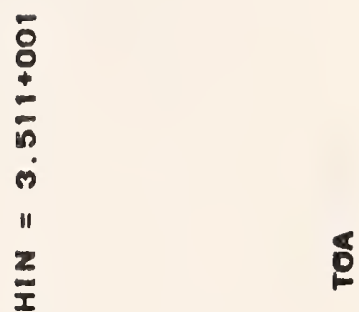

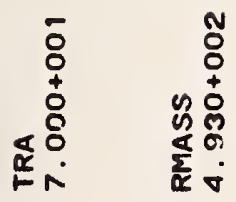

$\because$

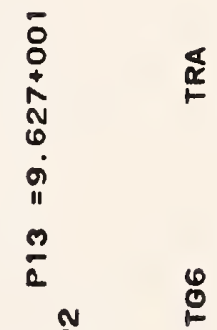

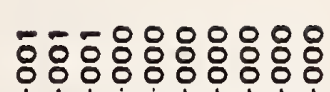

号

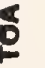

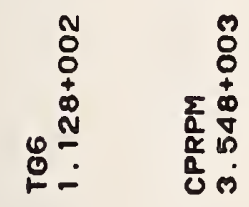

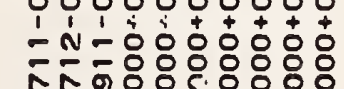

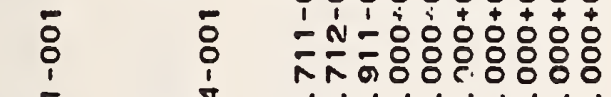

×

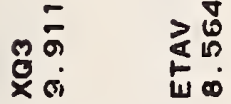

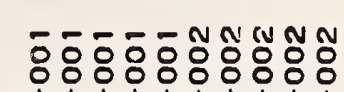

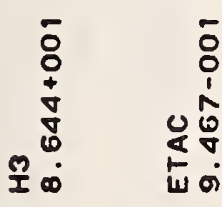

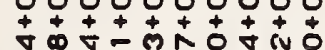

N

ถุด

I $\infty 0_{000}^{\circ} 00^{\circ}-\therefore-$

宸

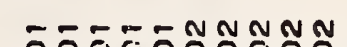

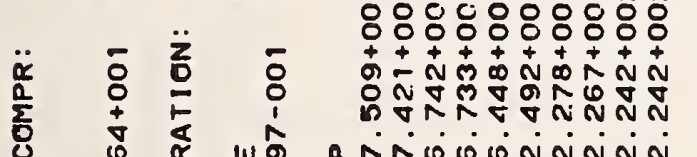

$+7+++7+1$

ํำ

arriogñNañ

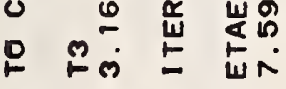

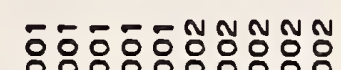

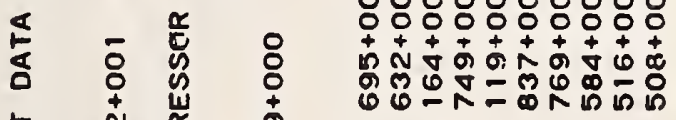

5 N $\frac{\pi}{2}$ 品

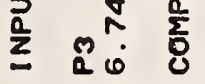

ธี

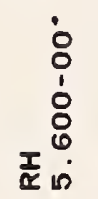

\begin{tabular}{r}
- \\
8 \\
+ \\
\hdashline \\
0
\end{tabular}

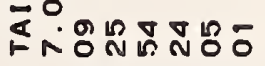

$2 \frac{11}{2}$

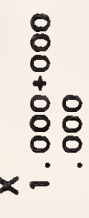

융응

는

ฟั

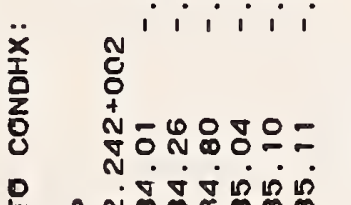

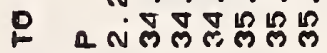
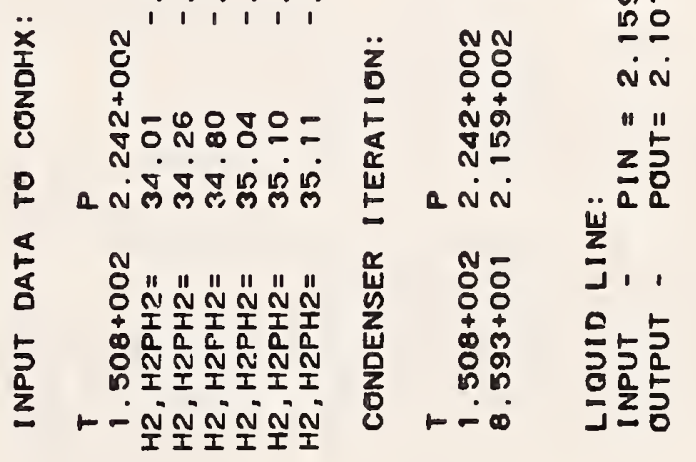

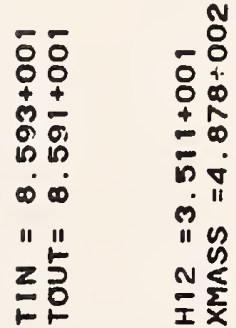

$\stackrel{\circ}{\circ}$

$\stackrel{m}{8}$

$\underline{x}$

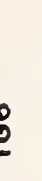




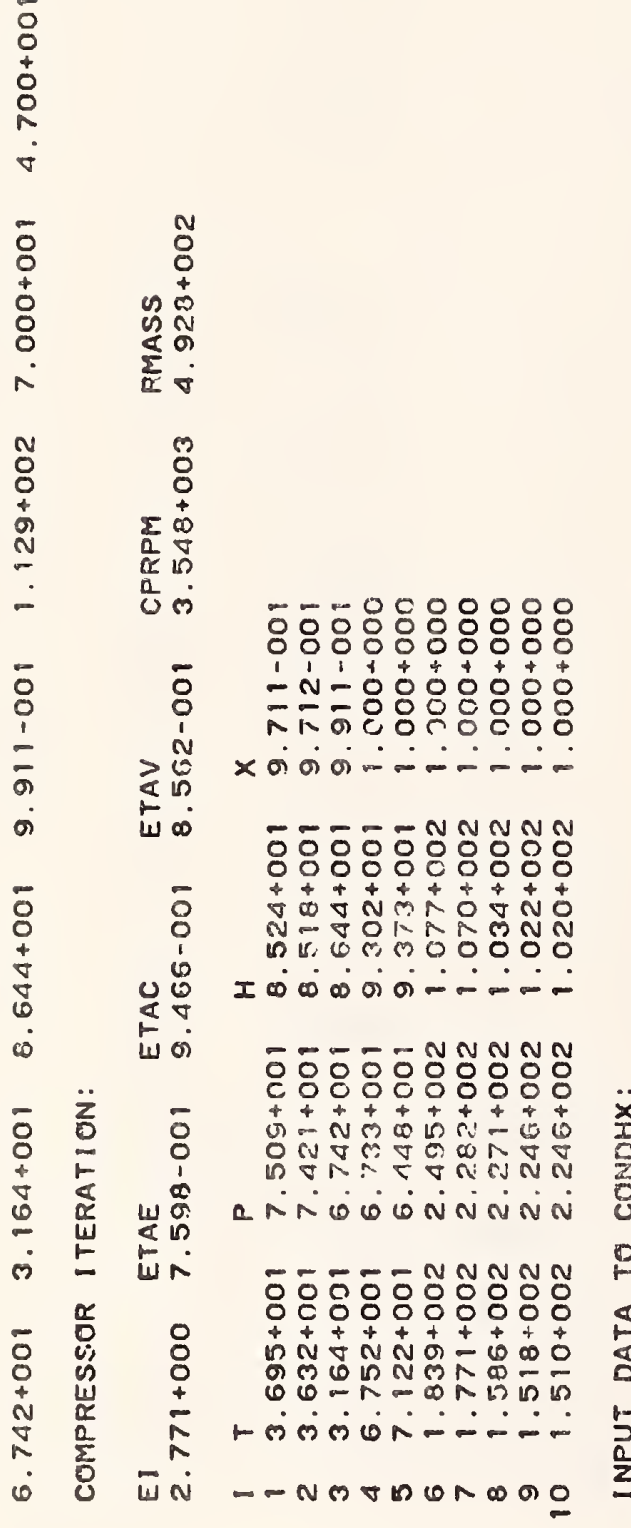

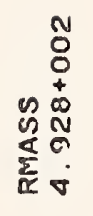

-0
0
1
0
0
0
$\frac{1}{\alpha 2}$

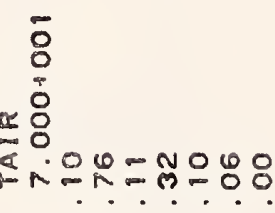

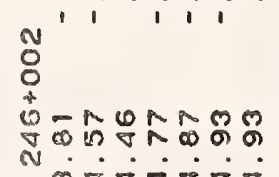

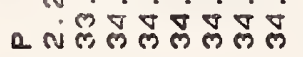

Oั

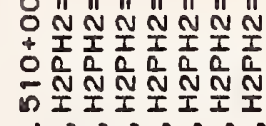

$1-\operatorname{minnNN}$

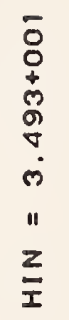

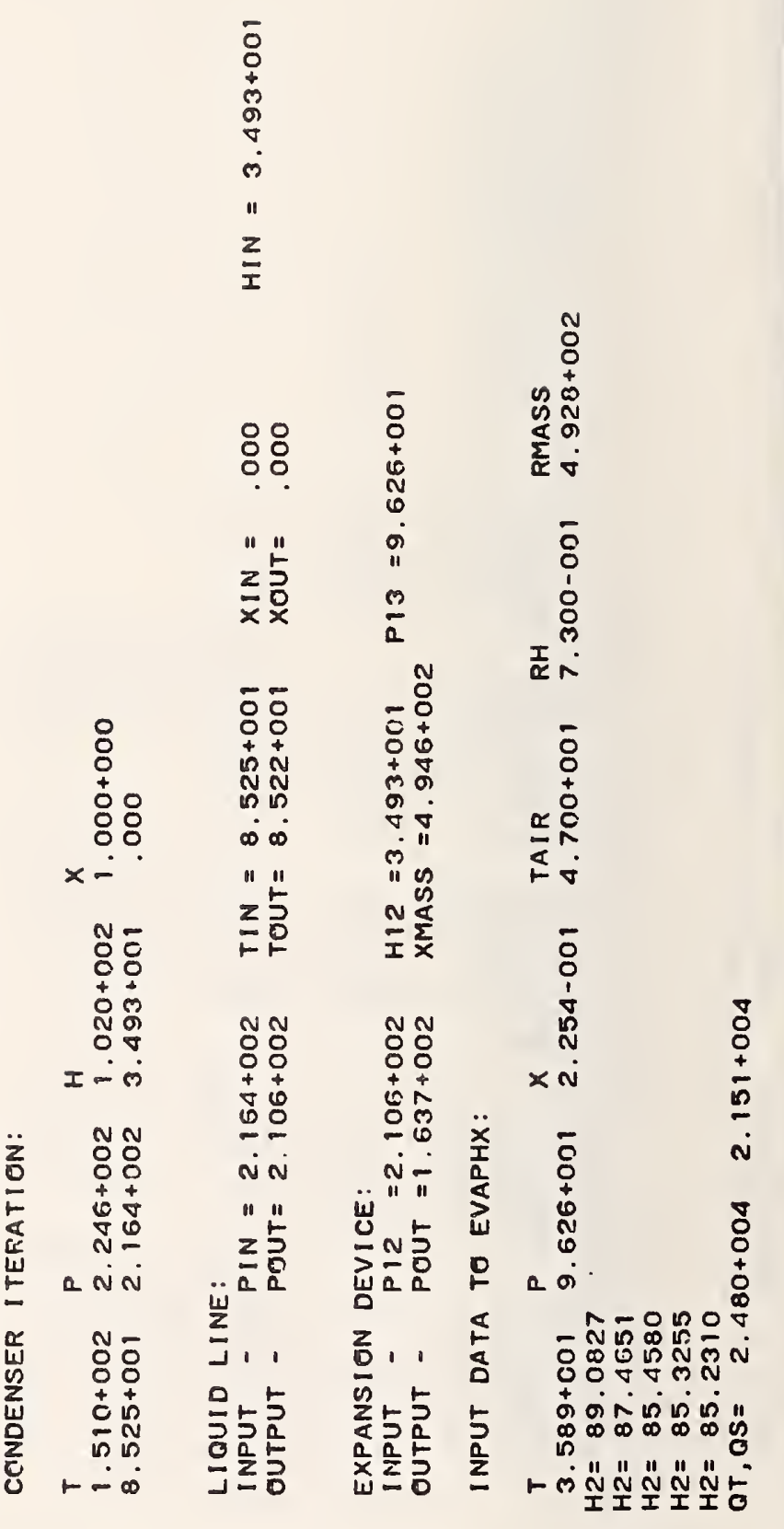

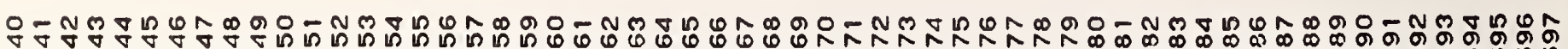

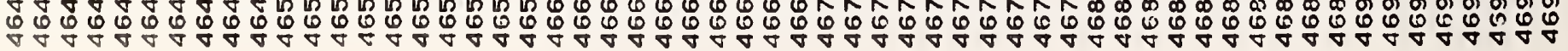




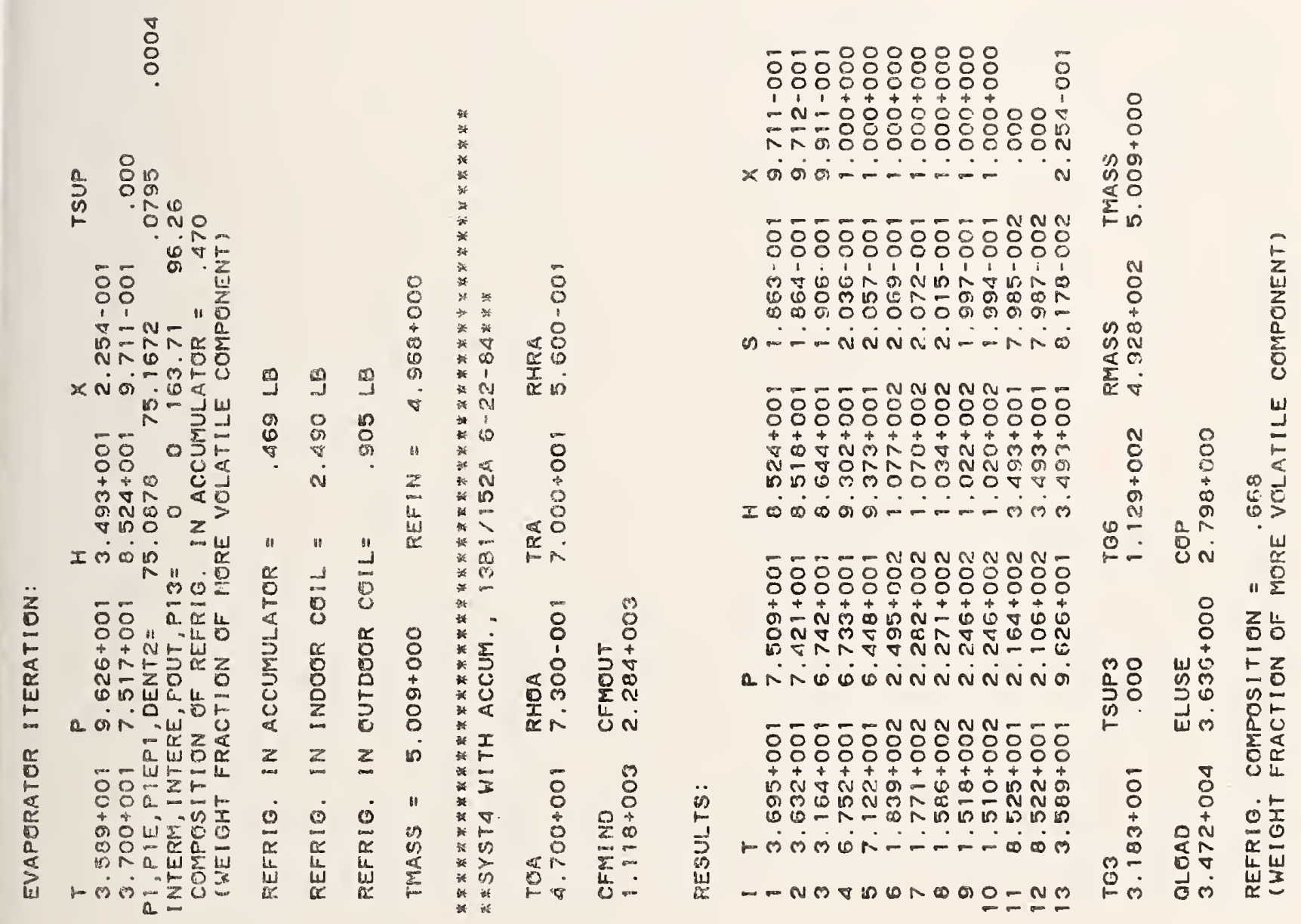

DOO-

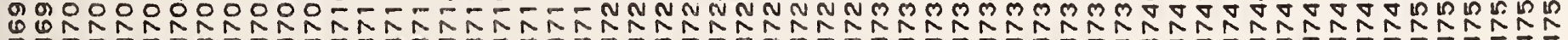
守 


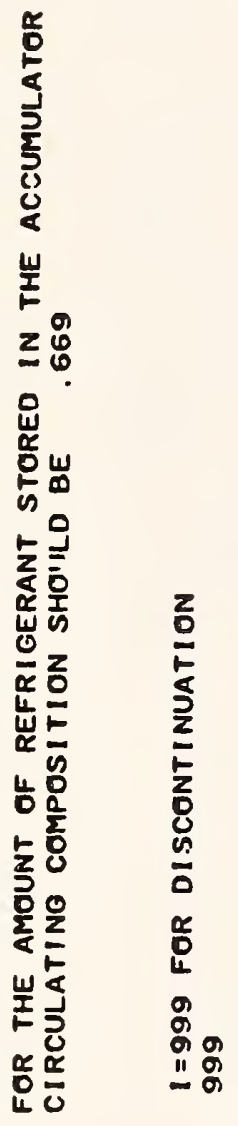

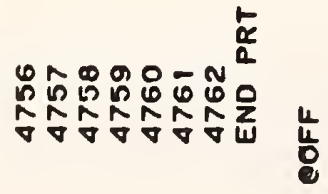


APPENDIX J . LISTING OF THE PROGRAM, HPBI

The following is a complete 1 isting of the program HPBI. All source elements of the program are 1 isted in alphabetical order. For fast reference, the reader can review Tables H1, H2 and H3, which contain name 1 ists of all functions and subroutines with a short statement of their purpose. 


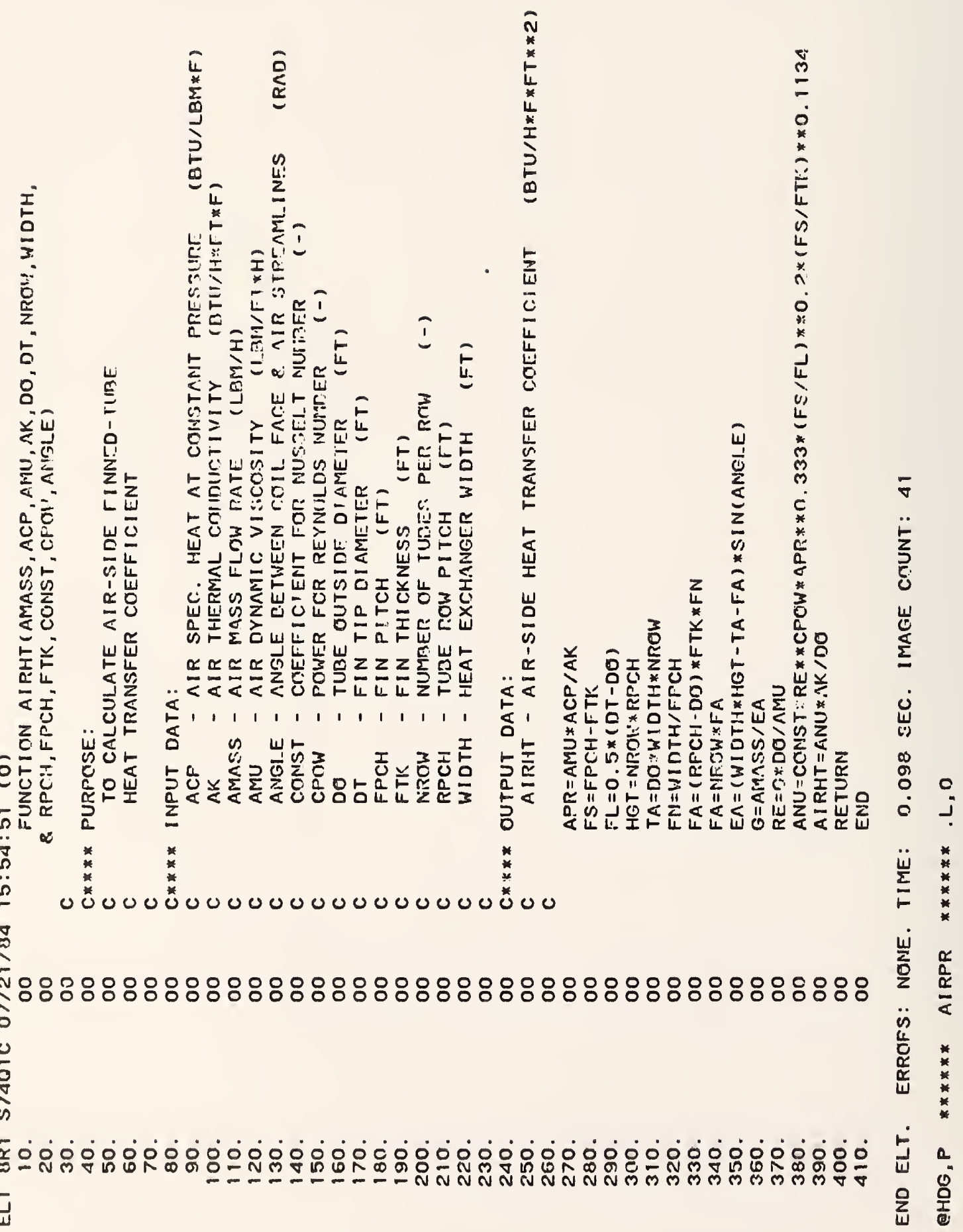




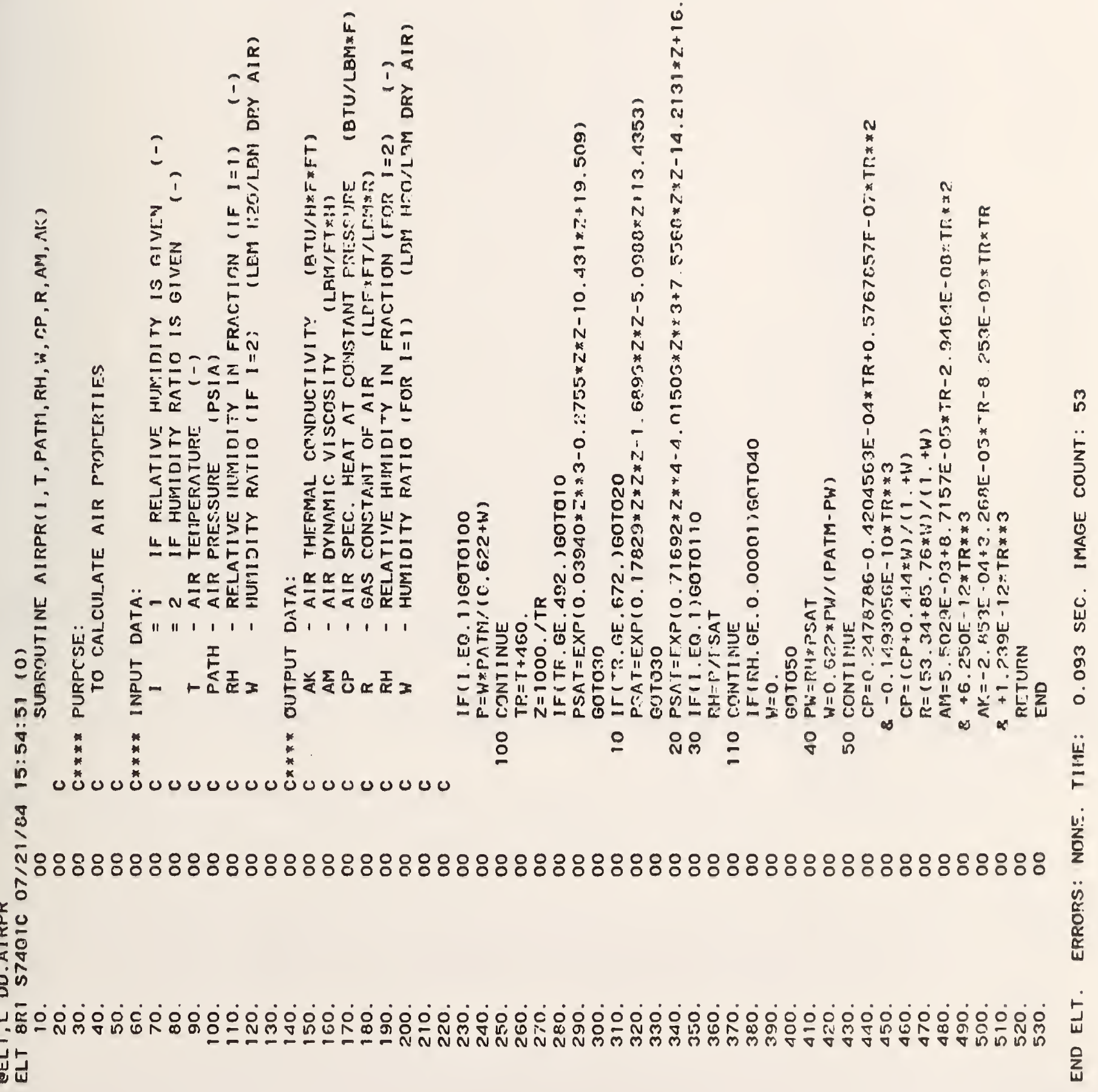



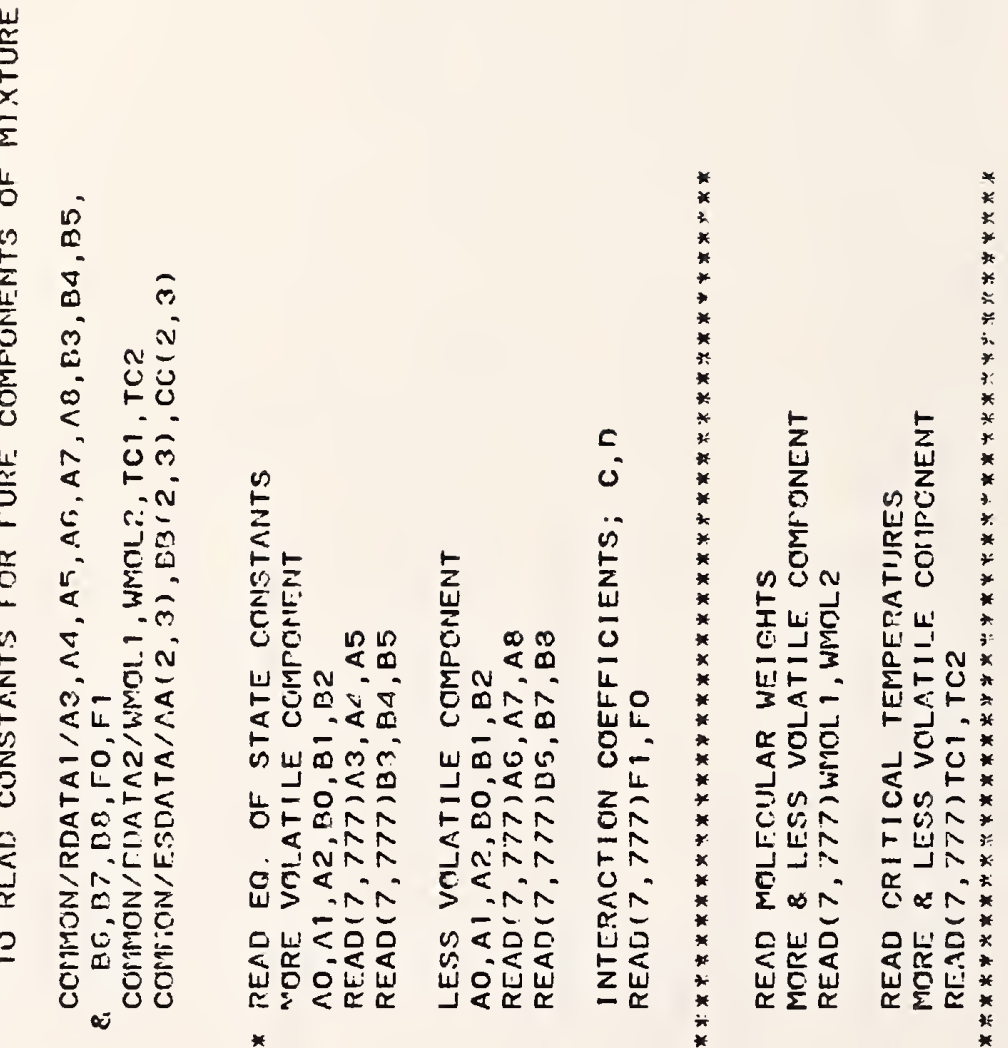

总

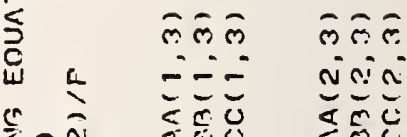

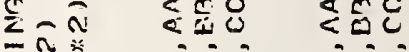

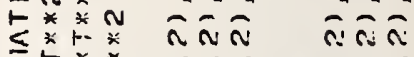

ڤト

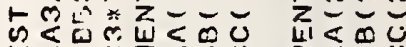

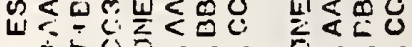

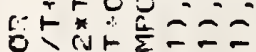

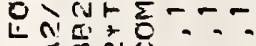

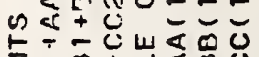

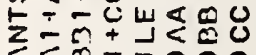

ci二

$\sum_{0}^{2}=-$

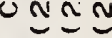

山东苍

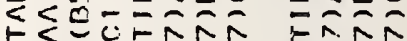

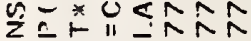

Cô.

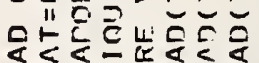

<NÑ

SNANA O

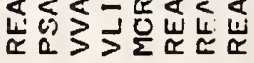

Sniñ

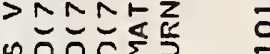
की i⿱ in 00000 $00 \stackrel{*}{*} 00$

000

○

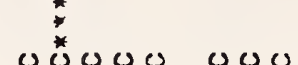

\section{ळ}

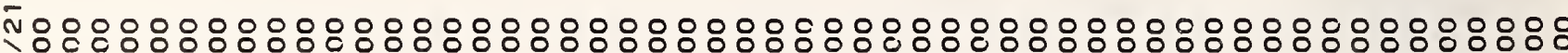

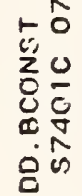

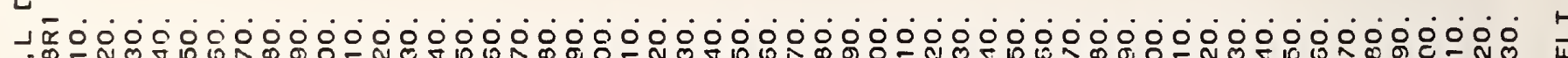
F-NM 


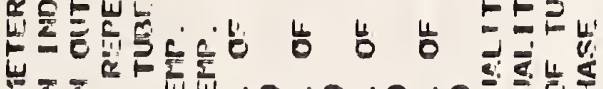

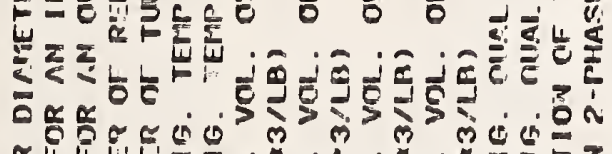

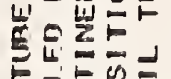

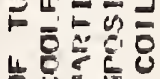
㟔记

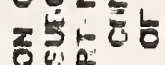
के

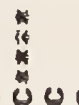

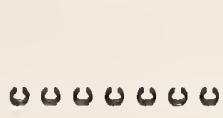

8 00

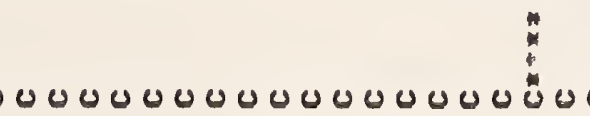

N yo

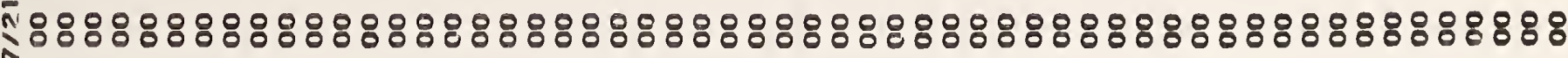

눈

ㅇํㅇ

80

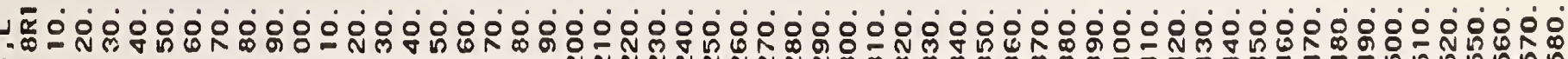
崖 


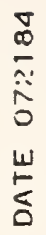
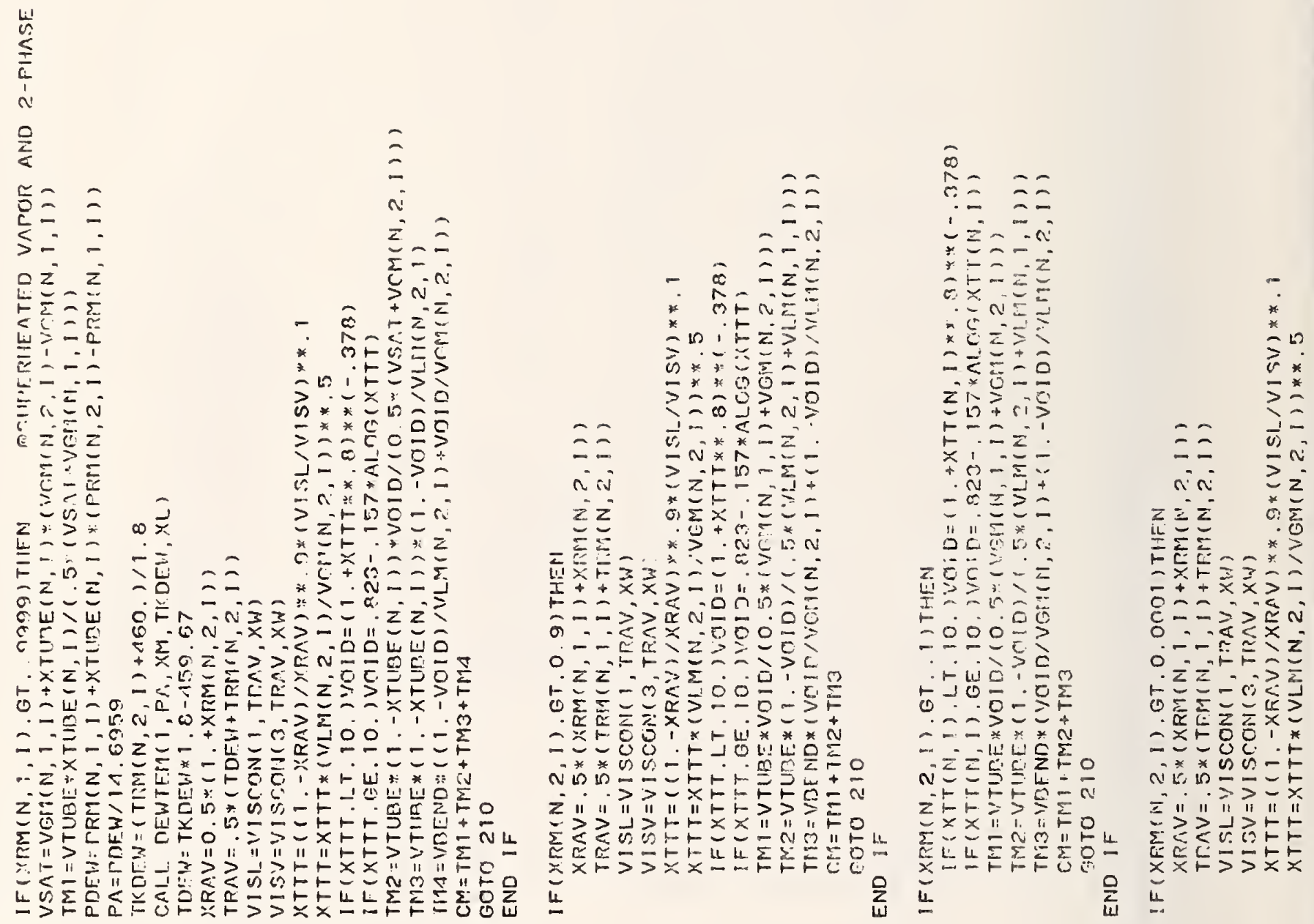

$\circlearrowleft$

U

$\omega$

$\omega$

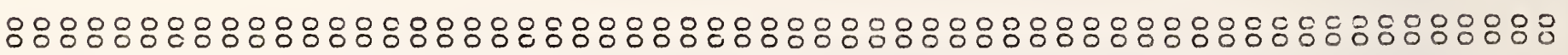
$\frac{10}{0}$

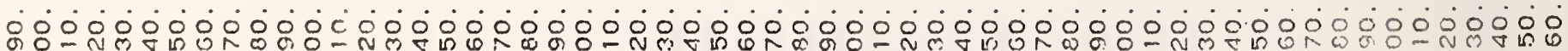

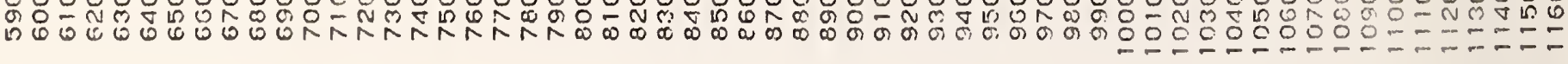




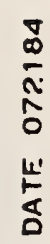

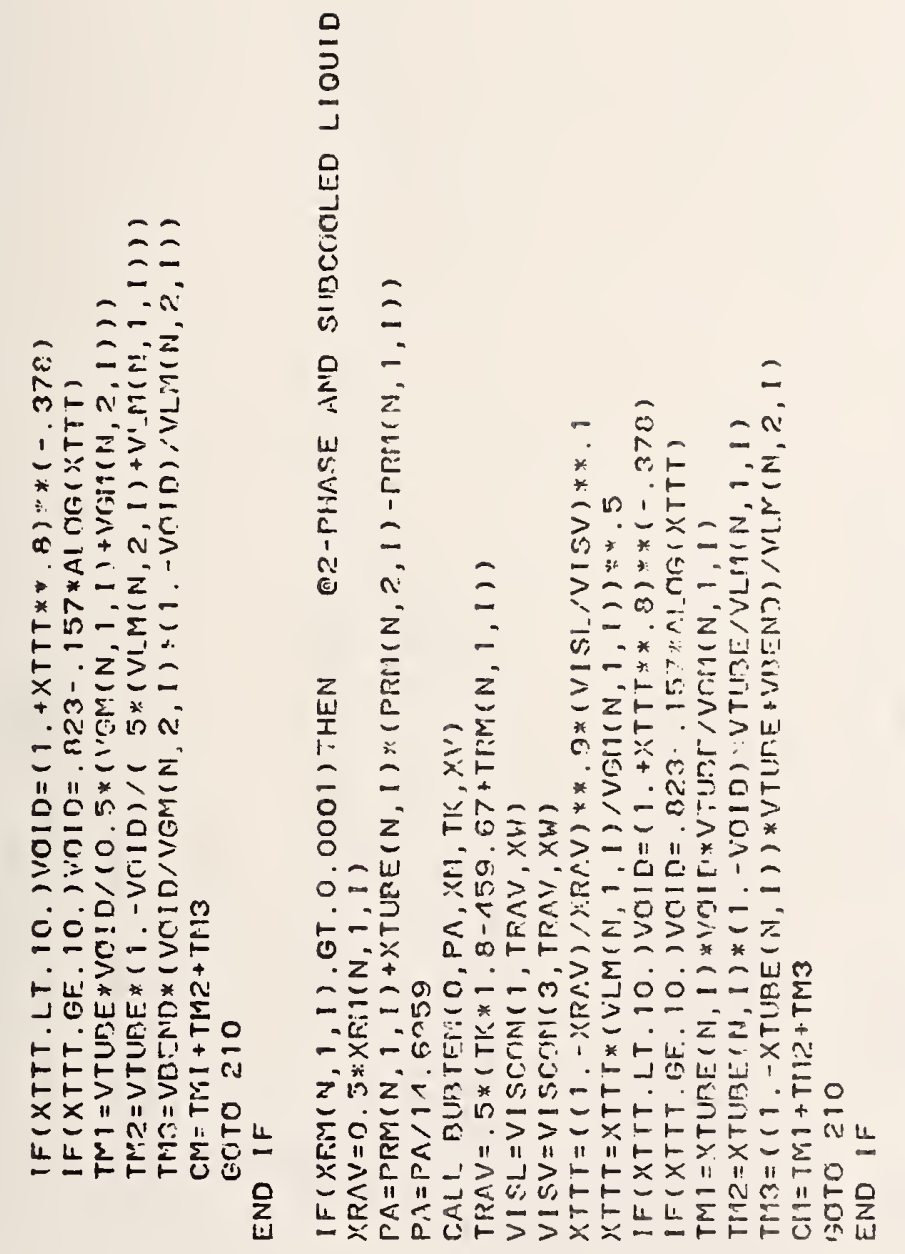

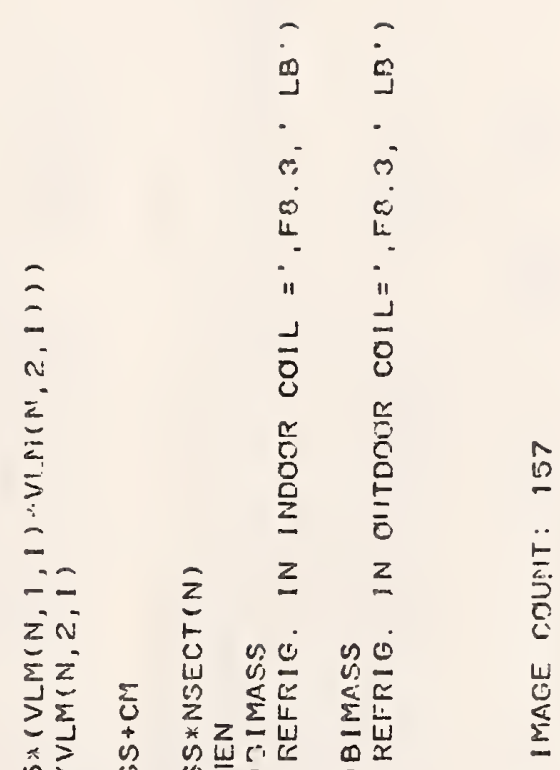

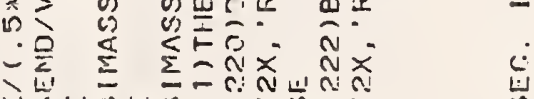

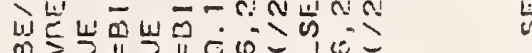

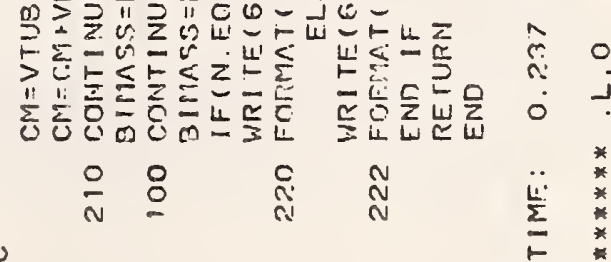

*

0

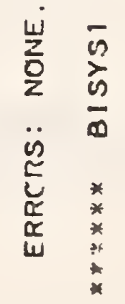

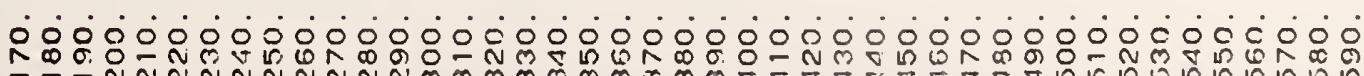

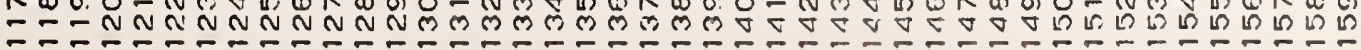




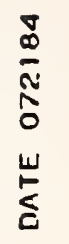
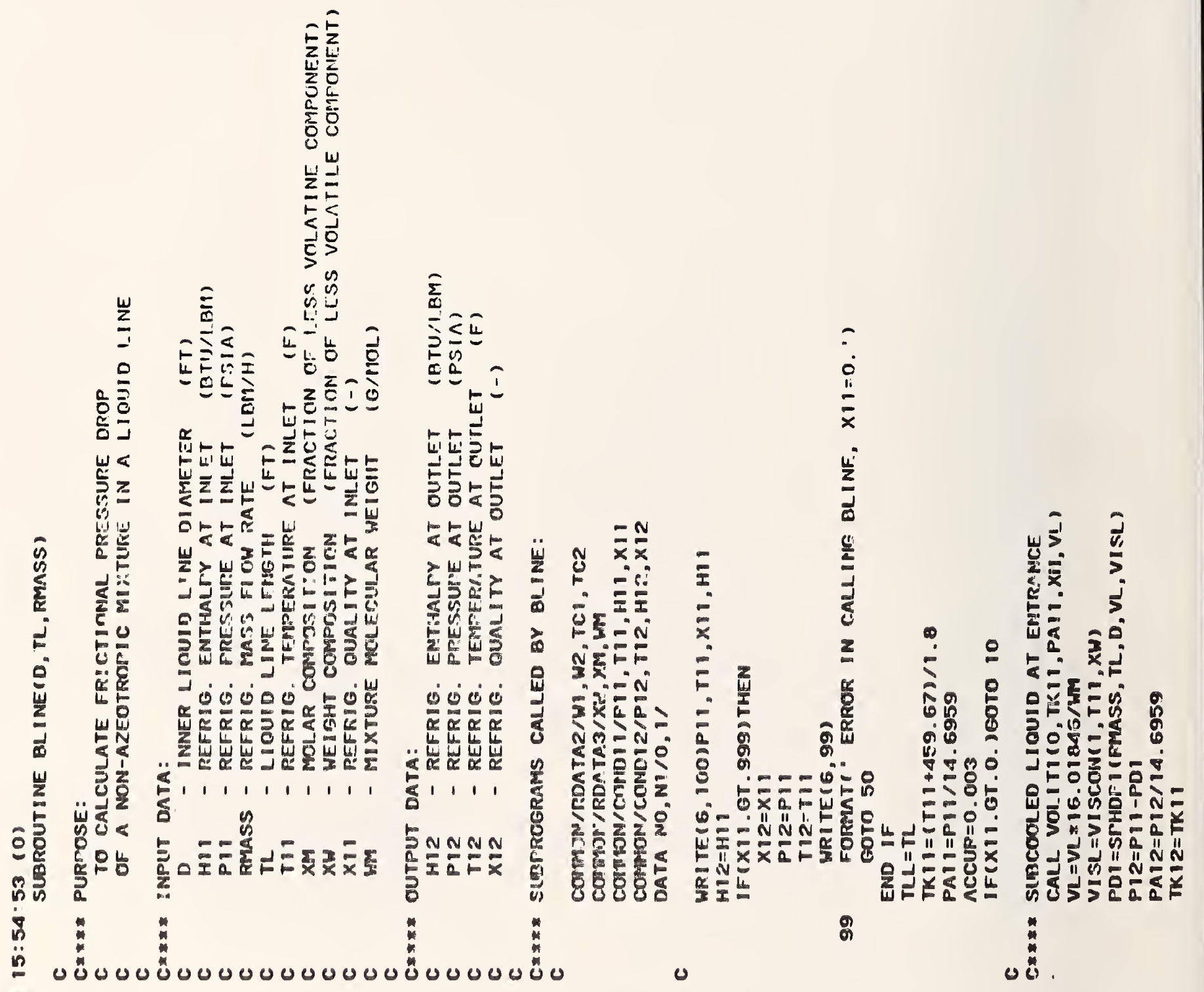

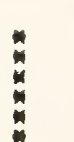
" wo

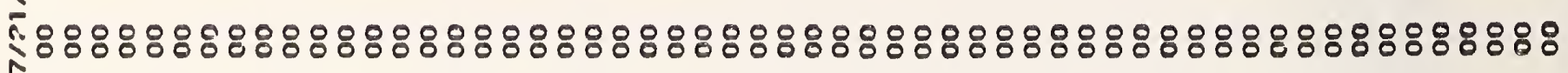

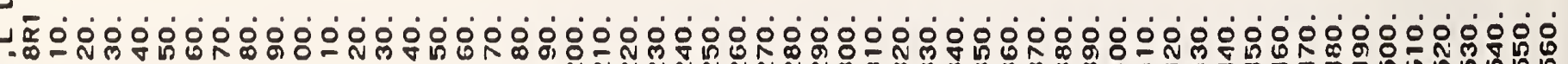
ト 㟧 


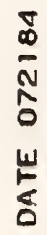

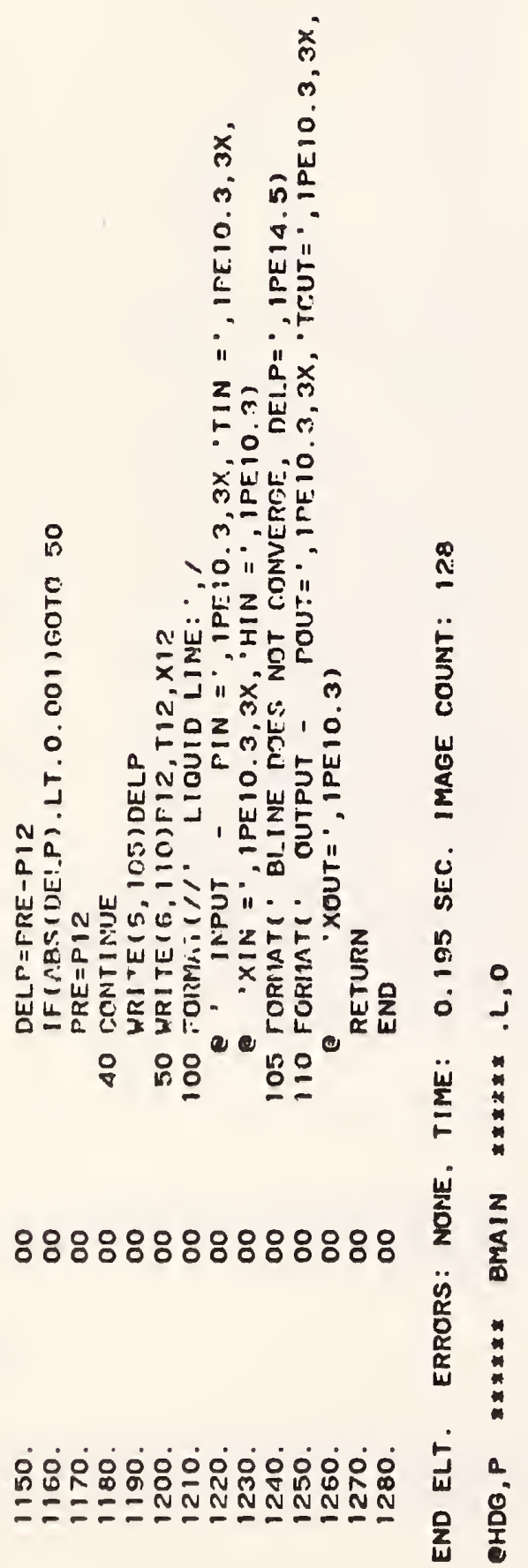



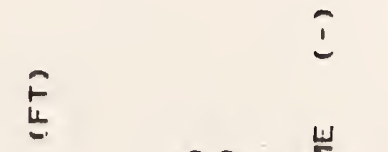

님

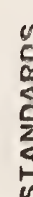

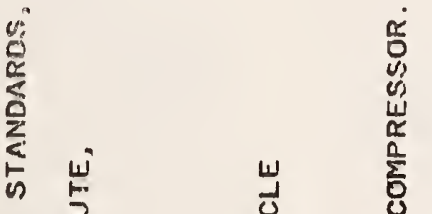

s)

w

c

음

$x$.

㟧

-

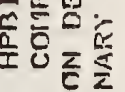

*

位。

o

道

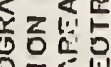

展

行行

z它记

的诰

岂乐这

至品罂玨

n 50

0

电可

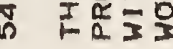

苛 *



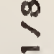

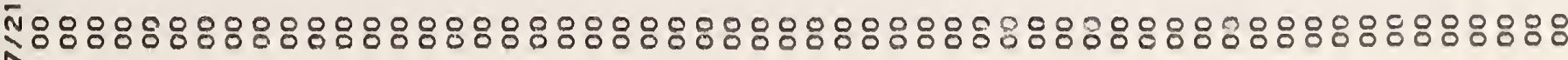
$z^{\circ}$

훙

8 is

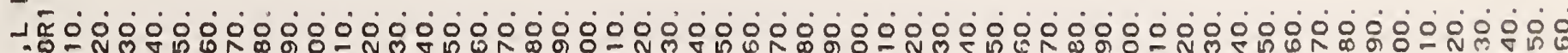
ト 岂吉 


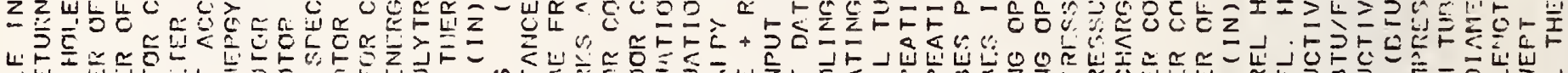

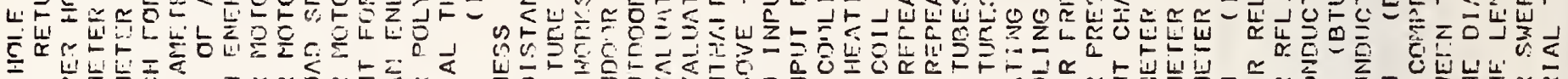

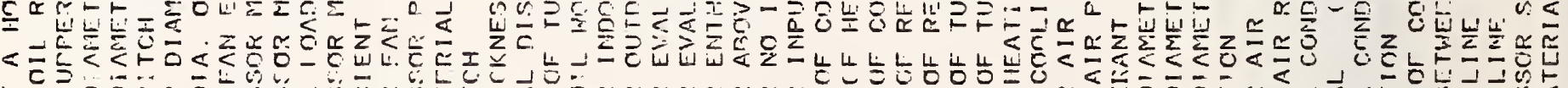

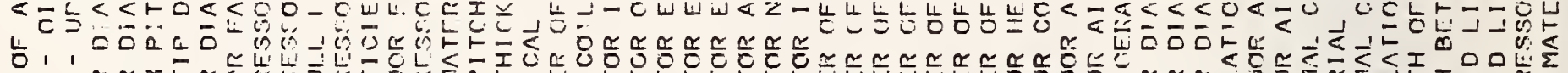

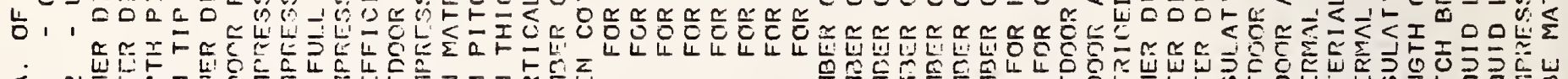

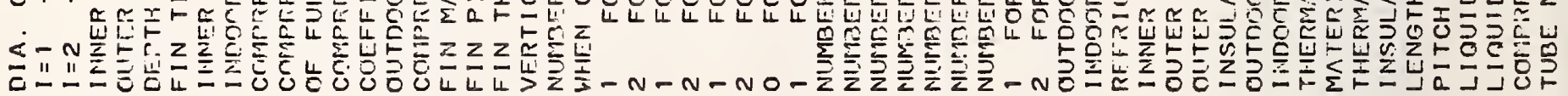

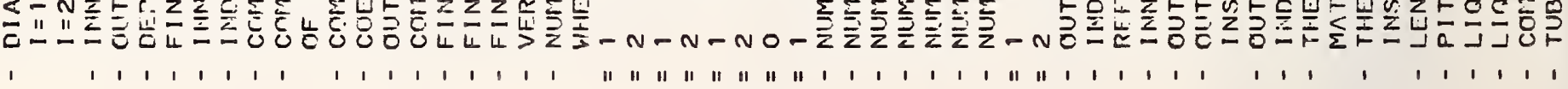

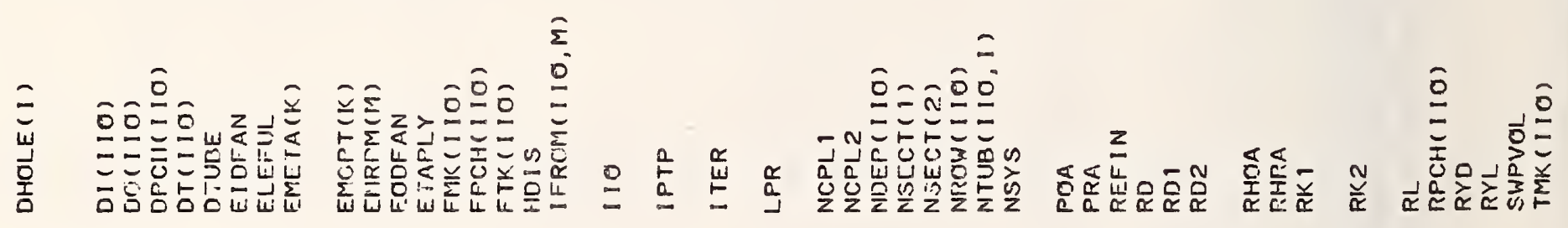

0000000000000000000000000000000000000000000000000000000000

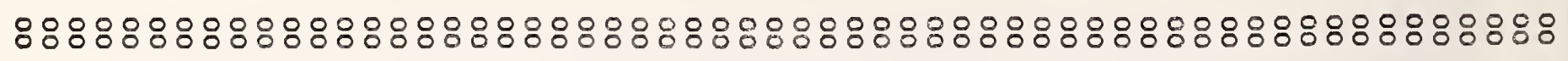

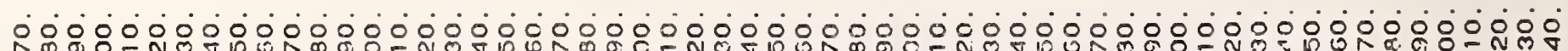

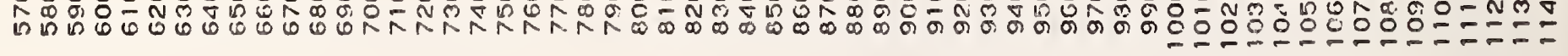




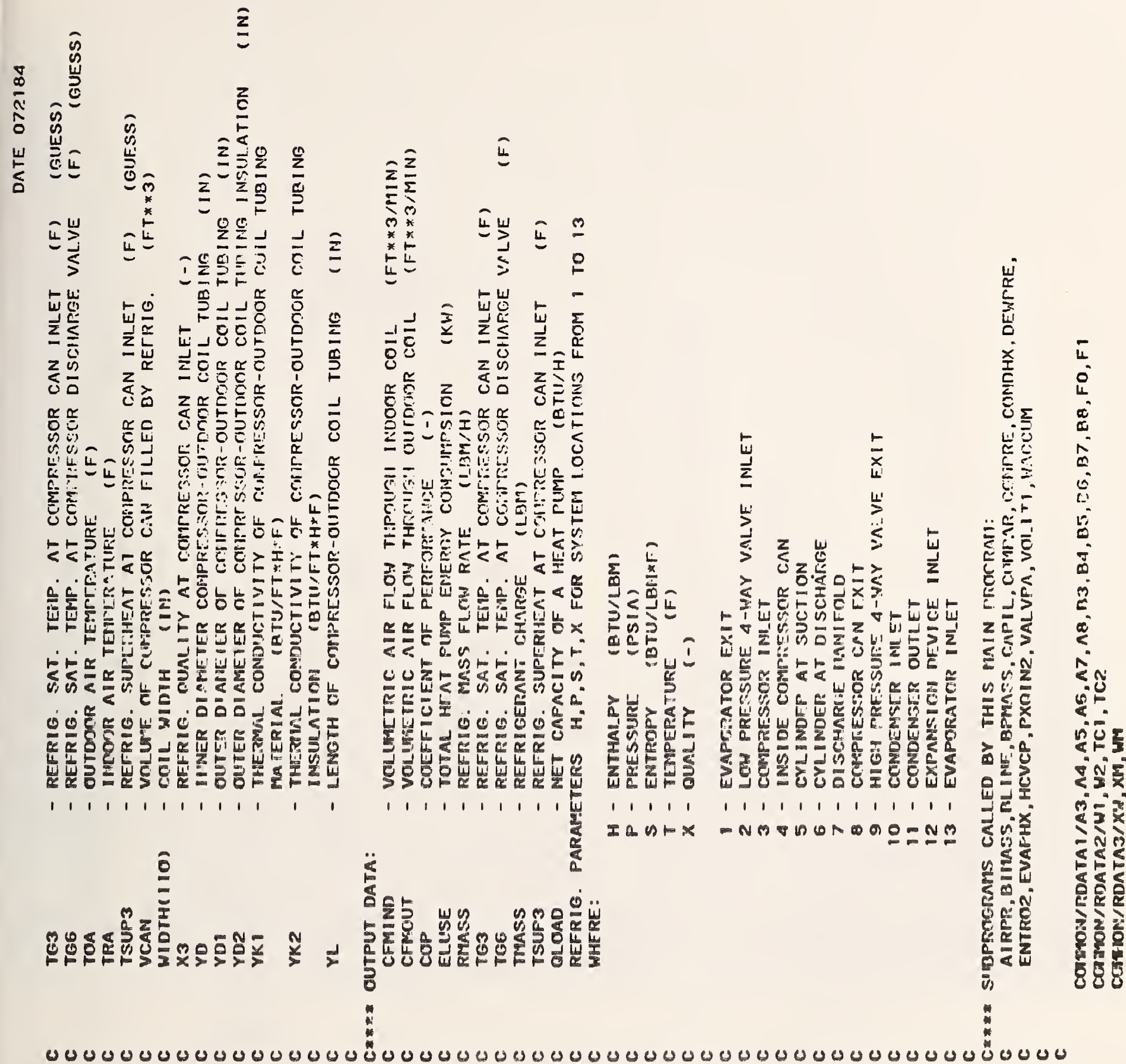

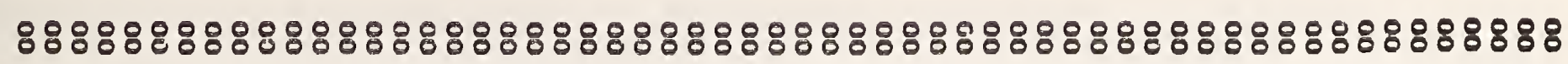

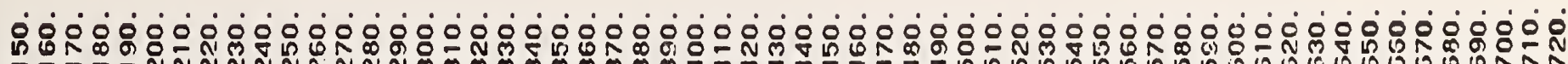
LE: 


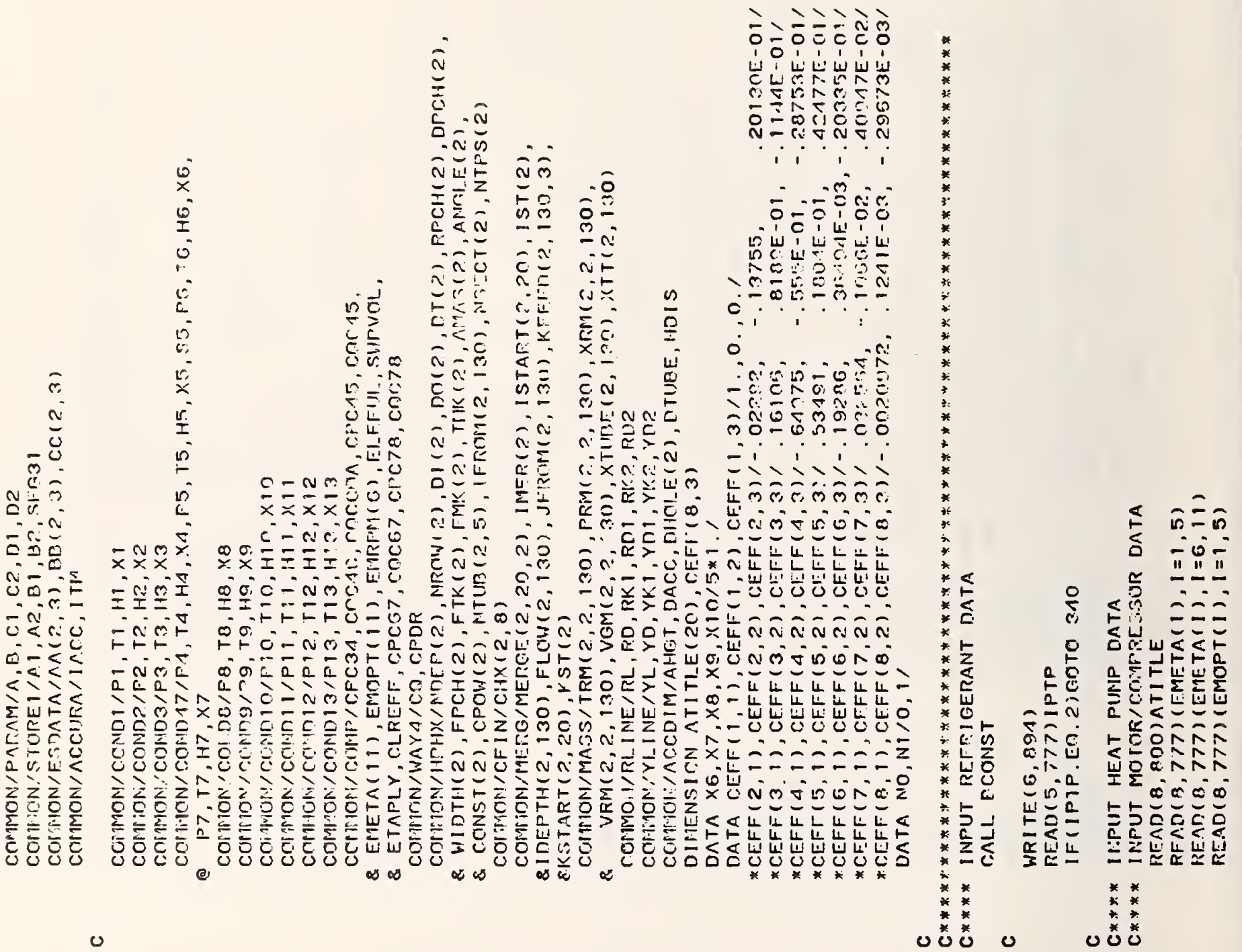

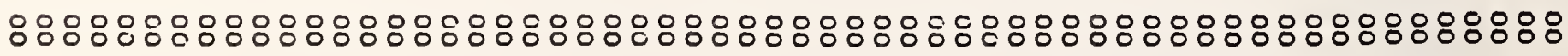

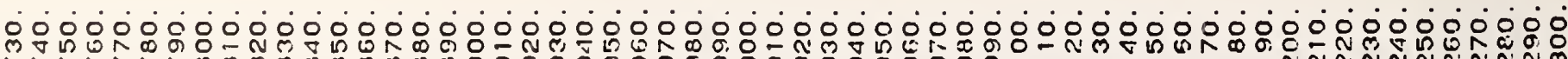

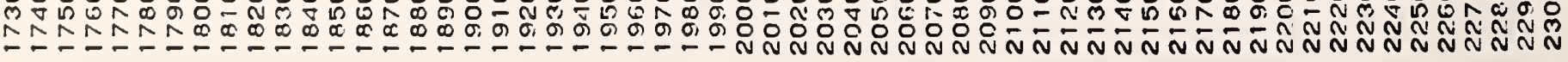




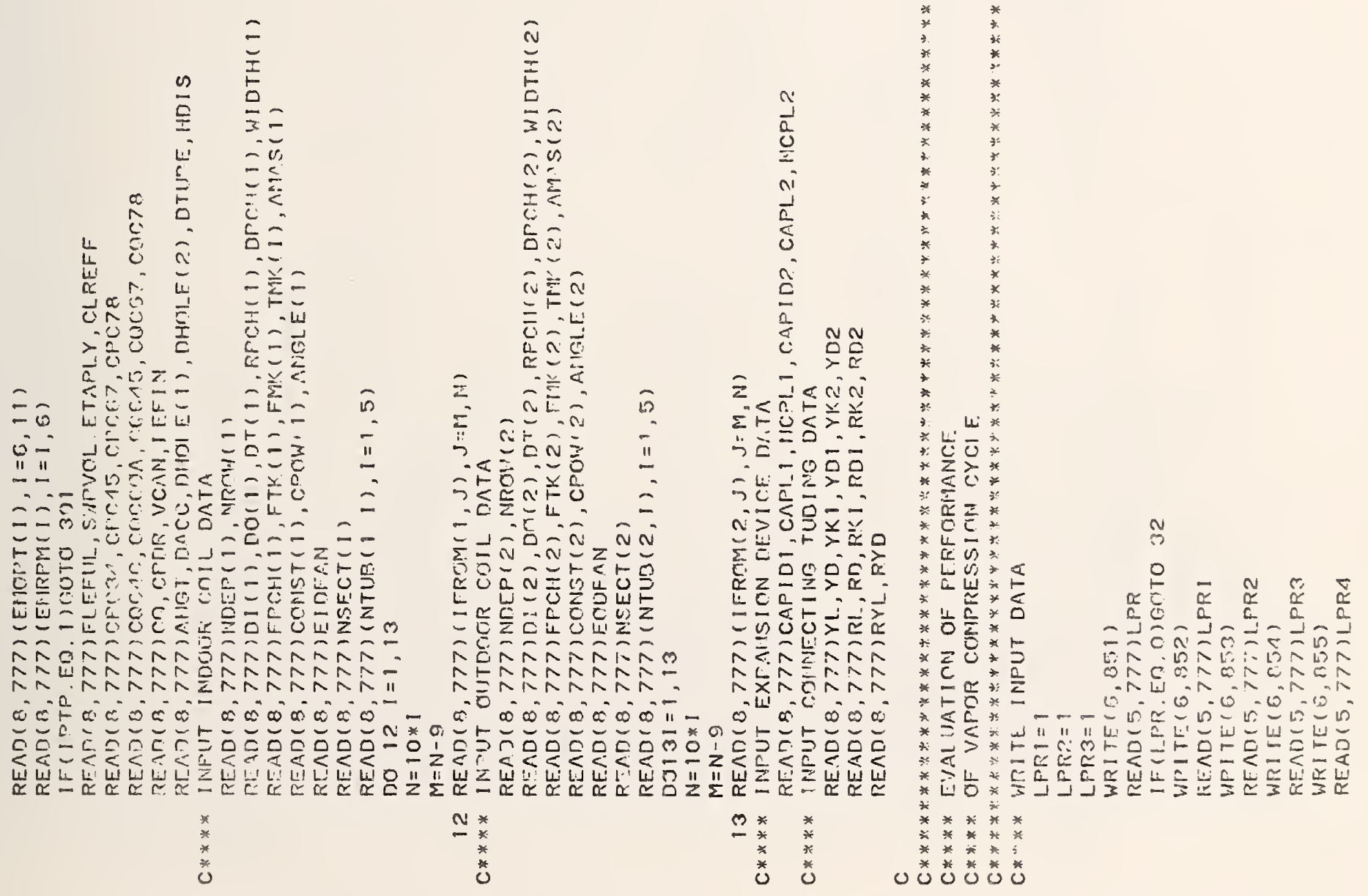

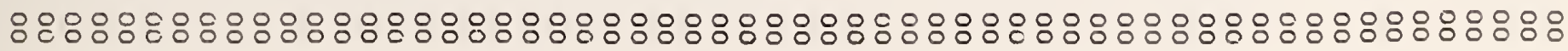

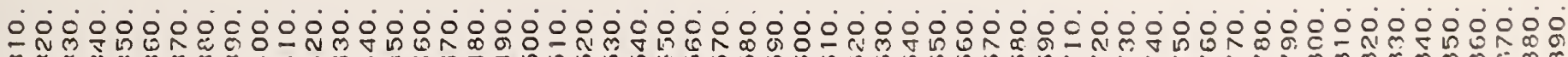

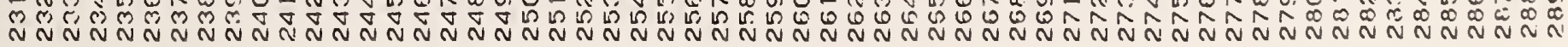




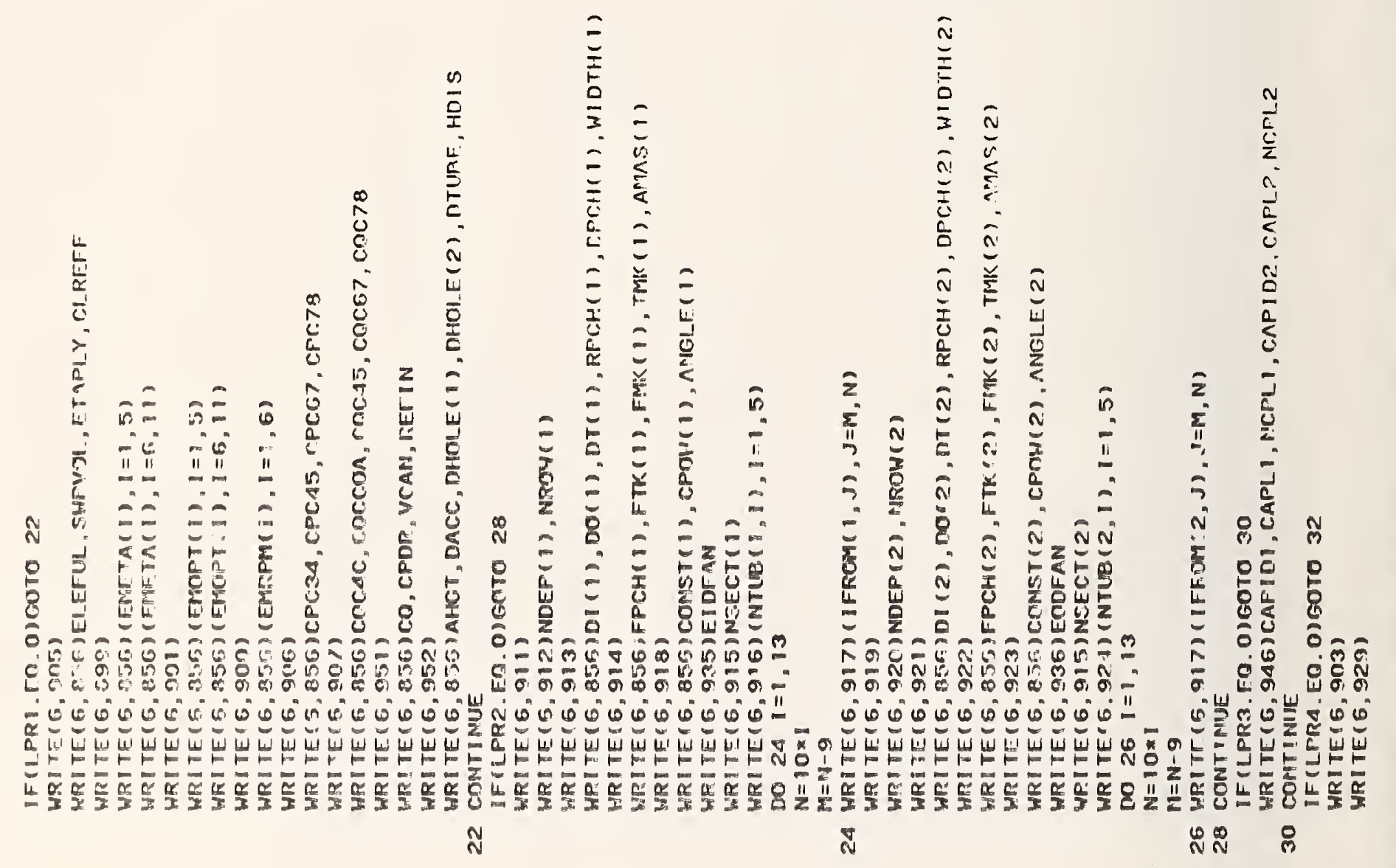

8888888888888888888888888888888888888888888888888888888888

$\frac{2}{5}$

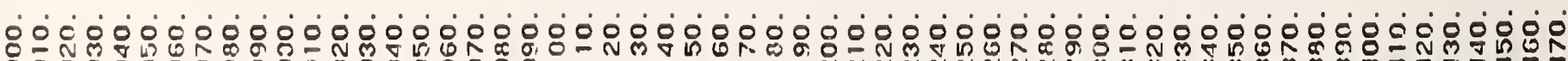

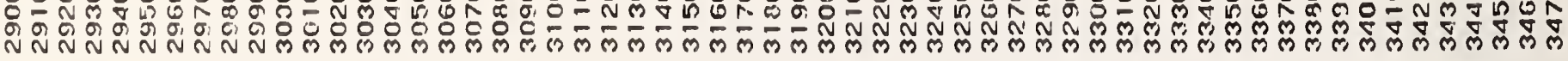




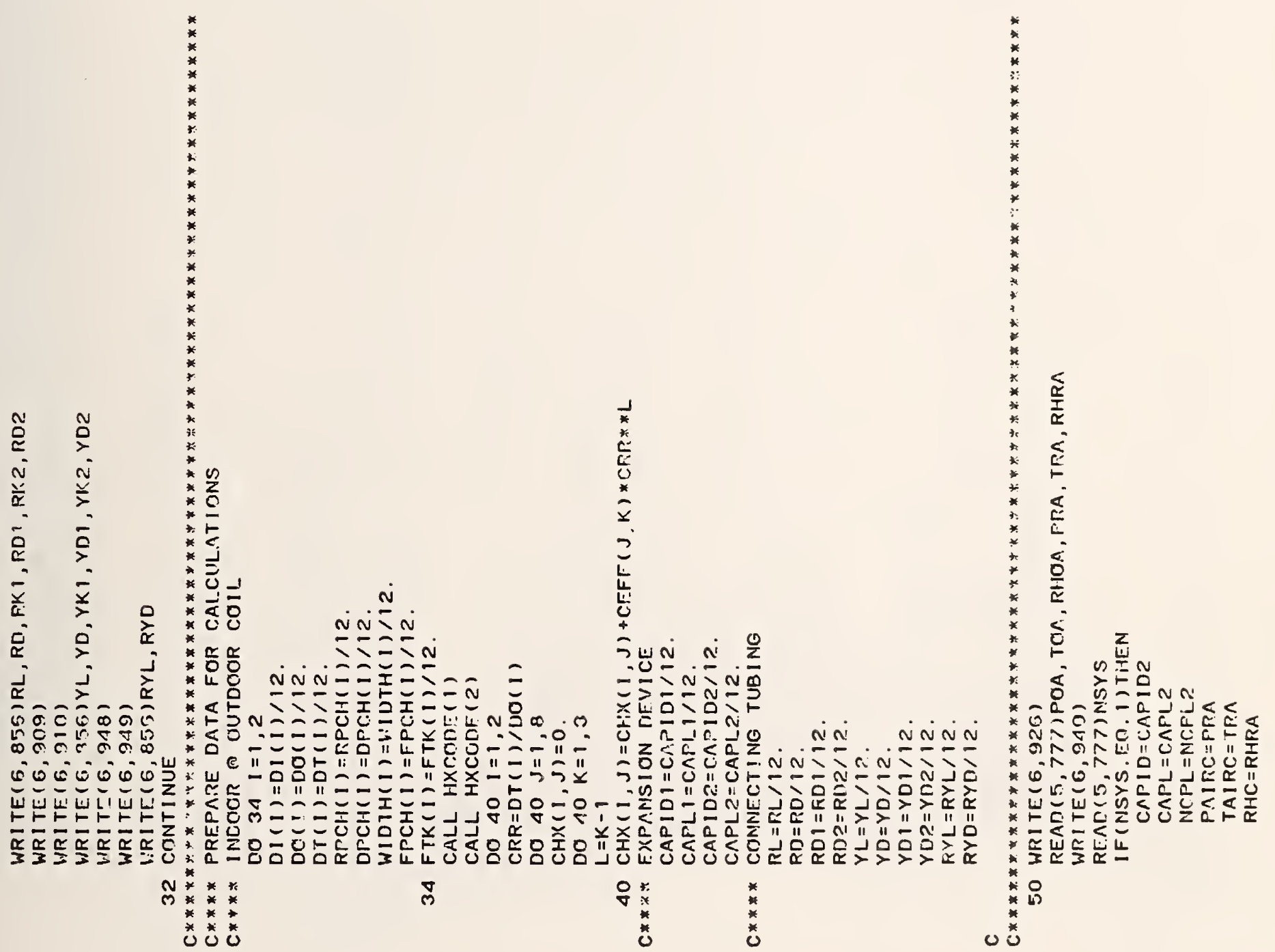

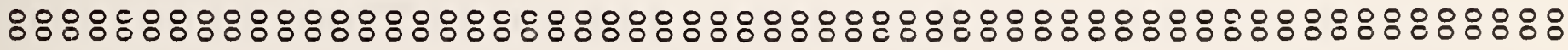

ळó

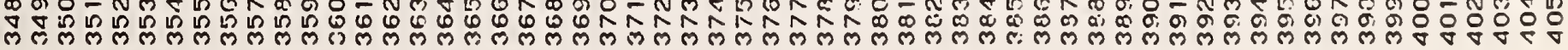




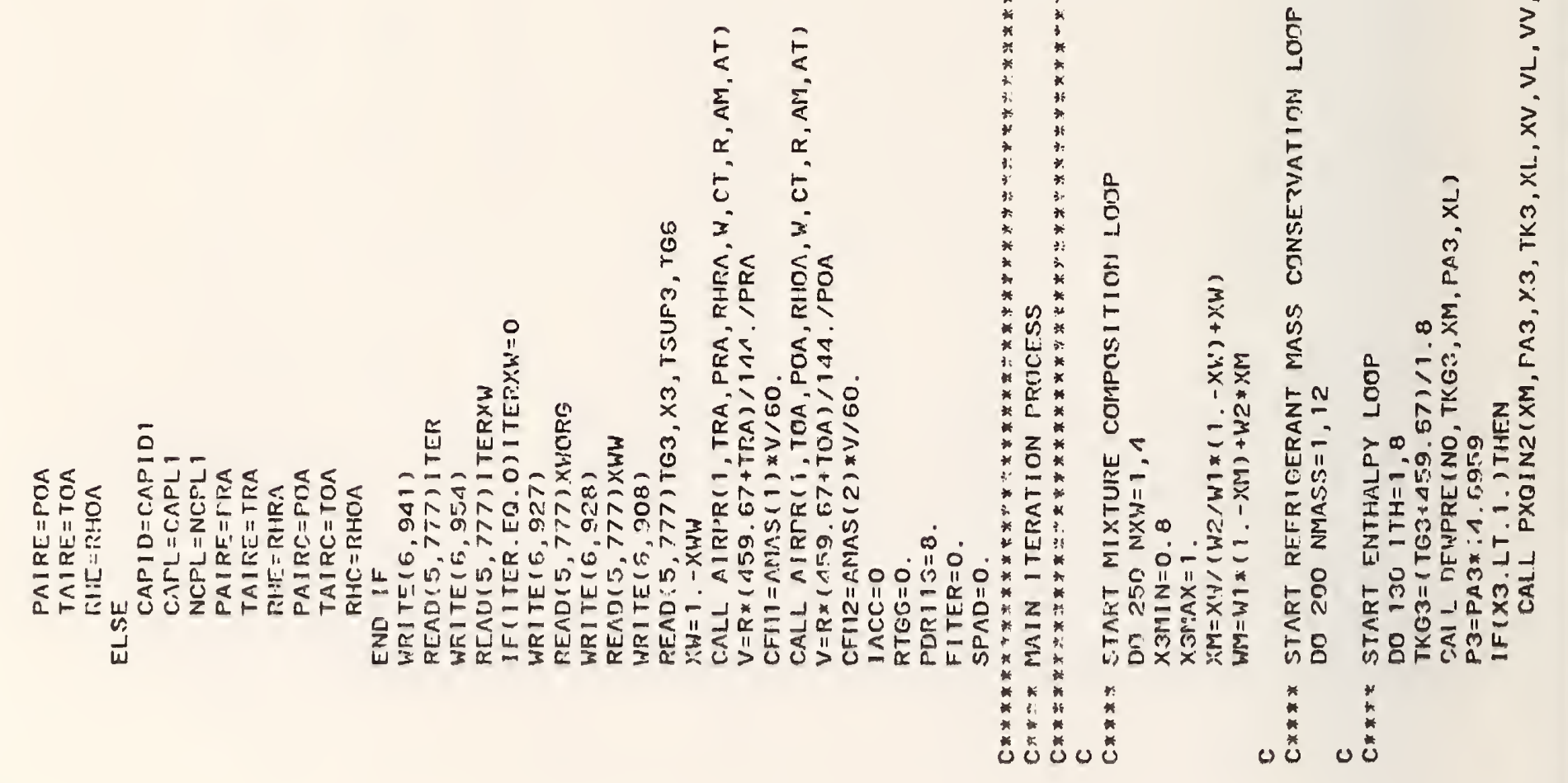

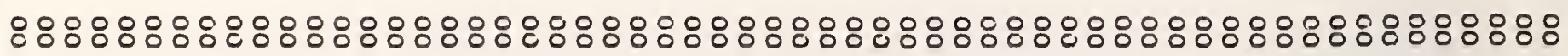

$\frac{z}{\frac{2}{0}}$

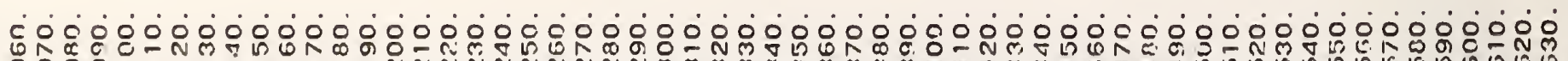

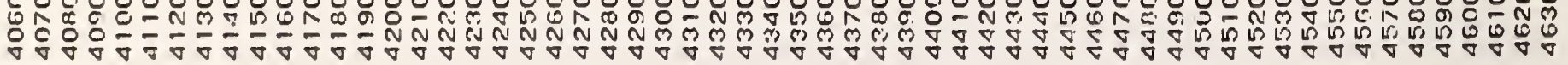




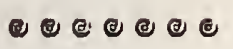

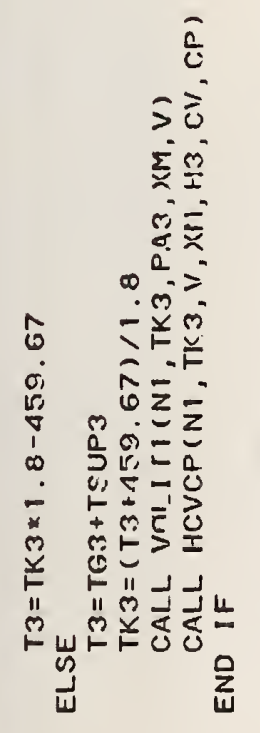

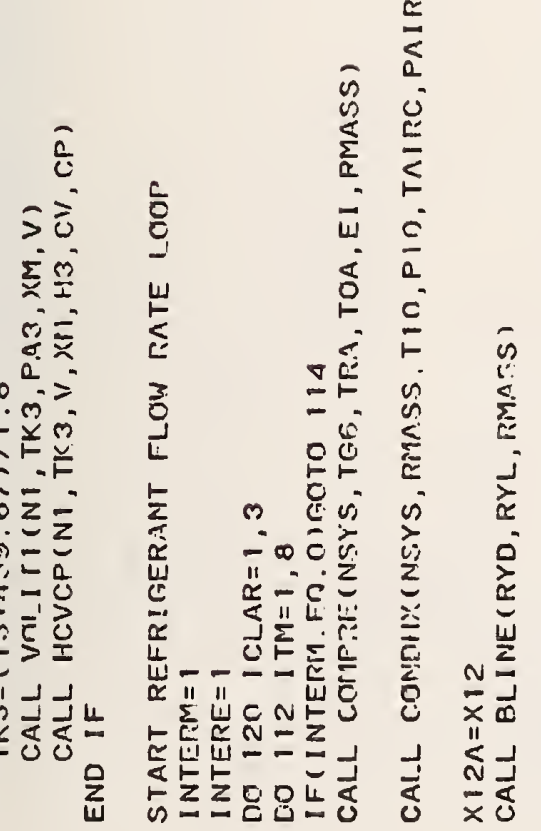

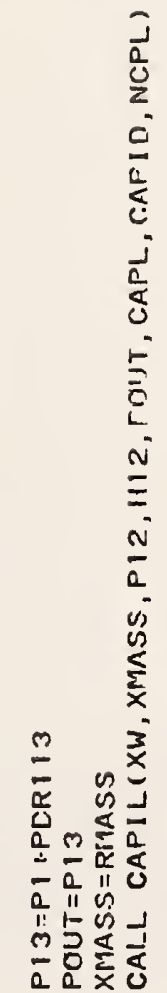

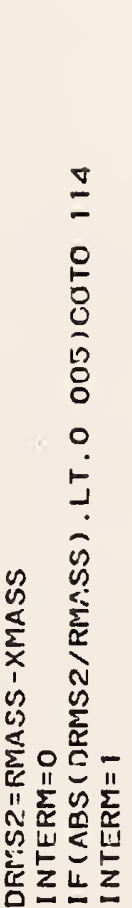

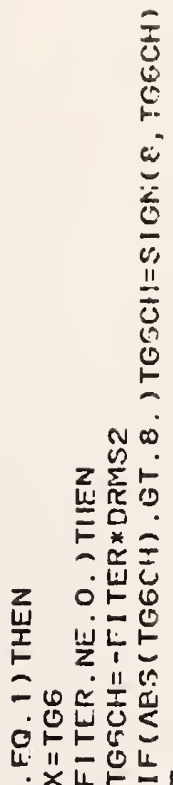

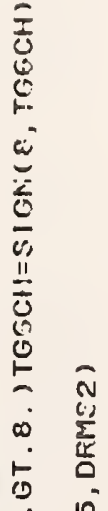

胥

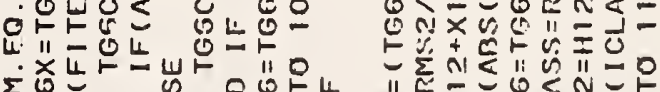

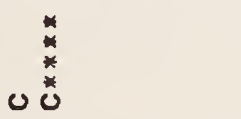

00

0

0

0

0

O

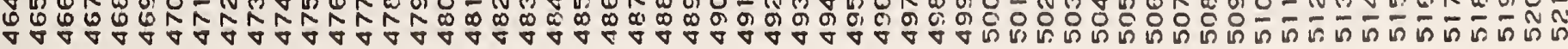




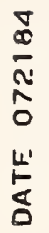

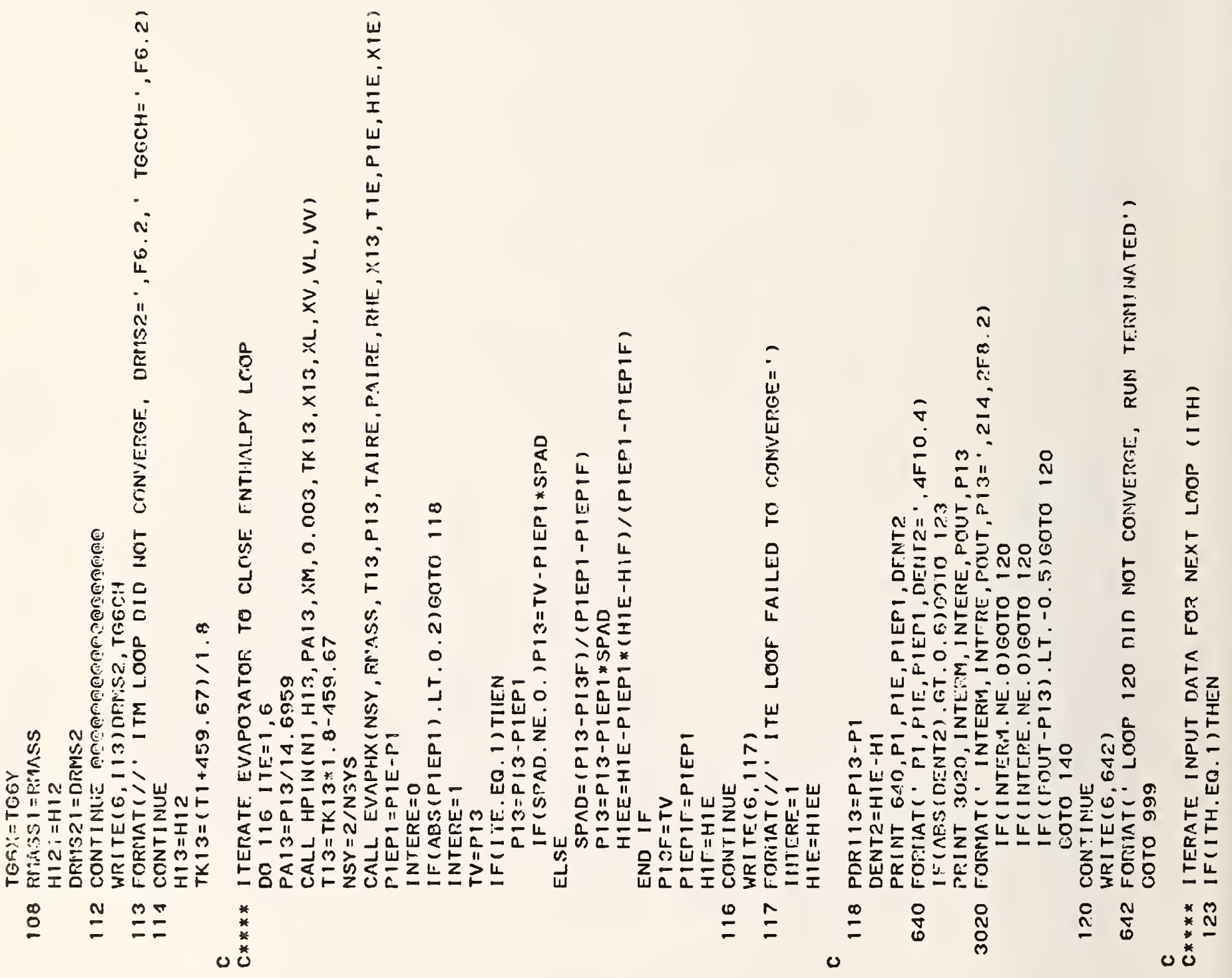

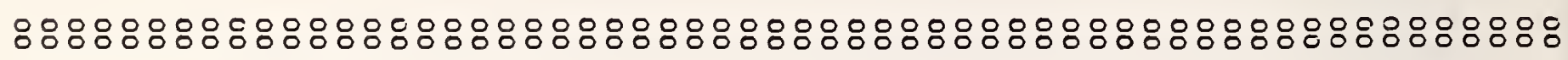

$\frac{z}{\frac{5}{5}}$

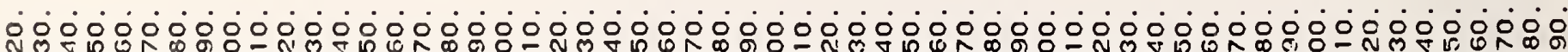

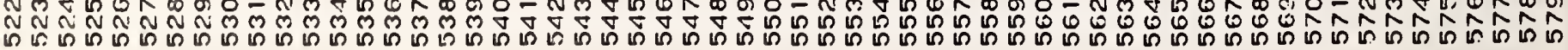



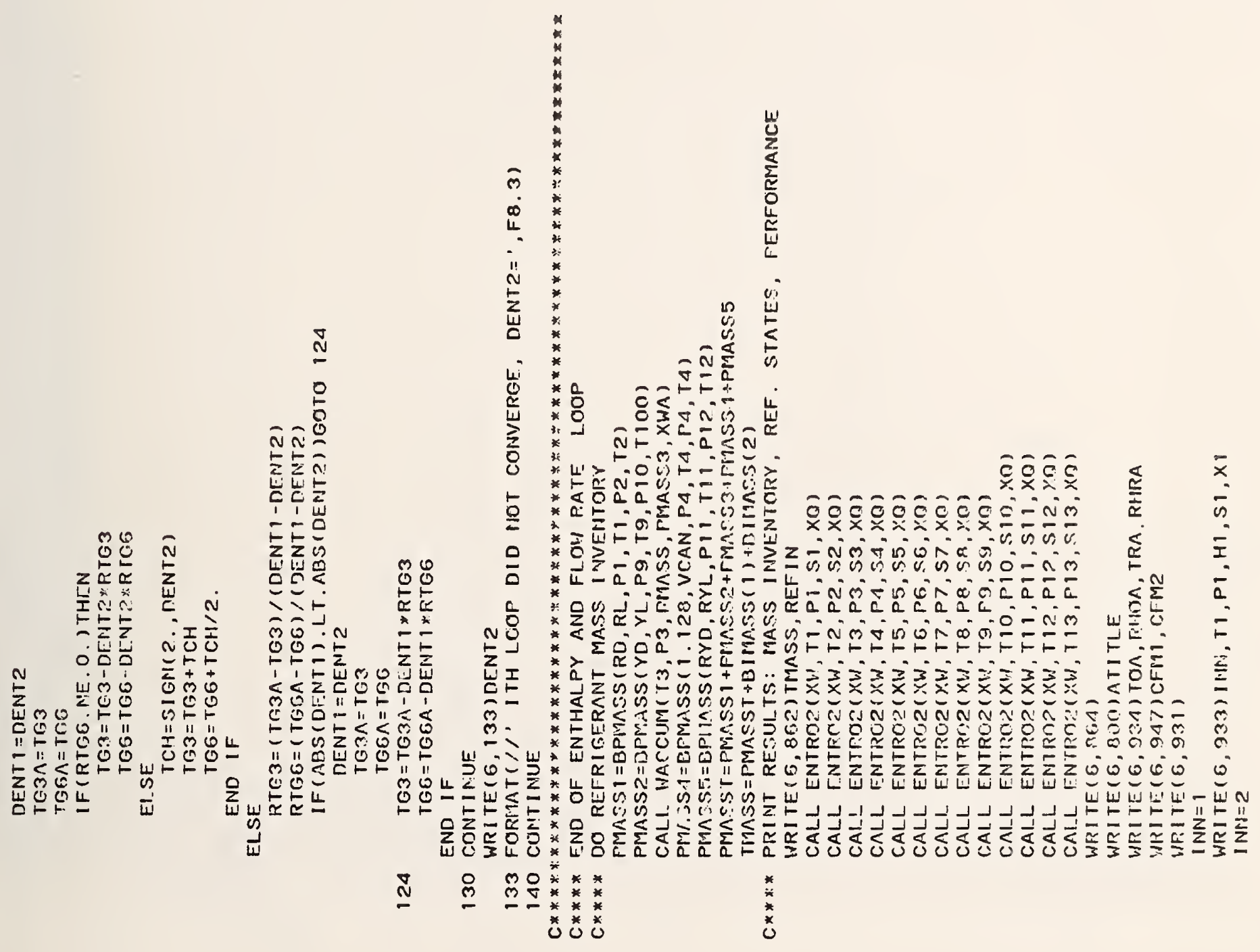

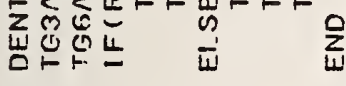

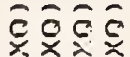

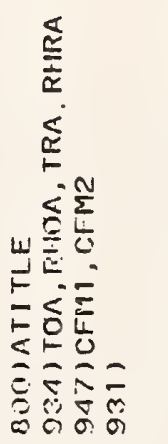

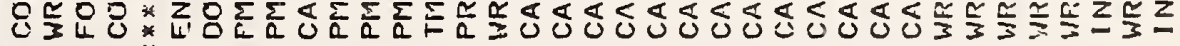

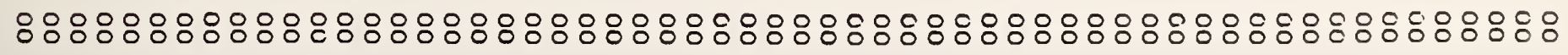

$\frac{z}{\frac{z}{0}}$

O- OO

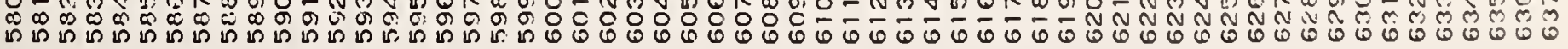




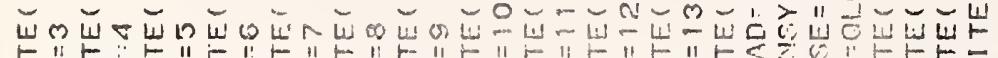

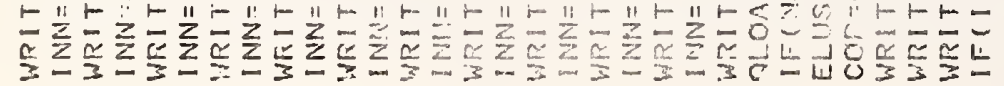
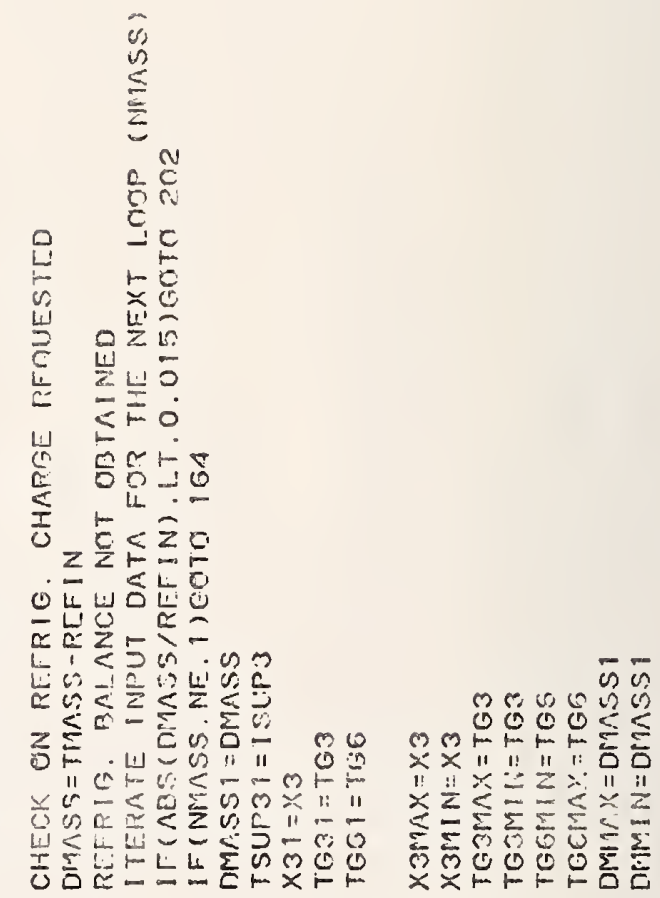

is in

늘

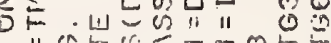

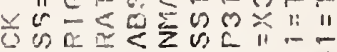
$\leq \frac{1}{2} \sum_{10} \sum \sum$

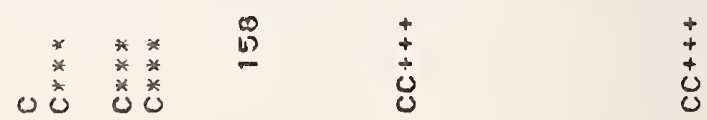

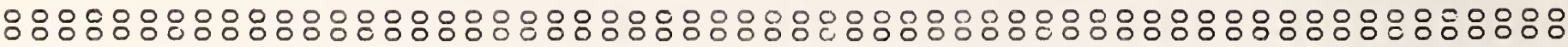

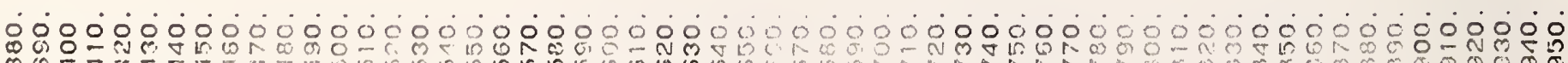

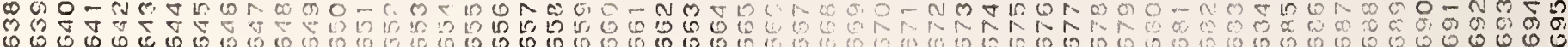


$\frac{1}{0}$

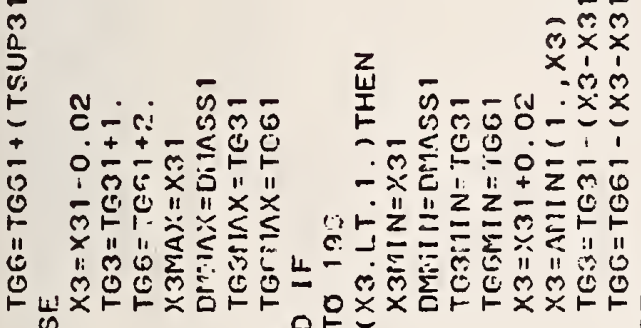

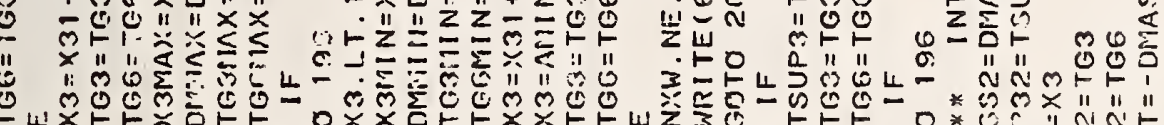

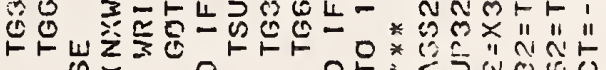
ज़ 员完忘

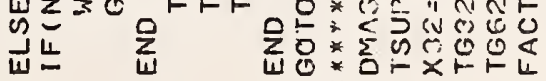
$\stackrel{0}{6}$

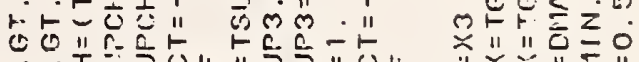
कलं w口

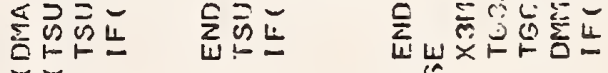
Lut $\underline{4}$

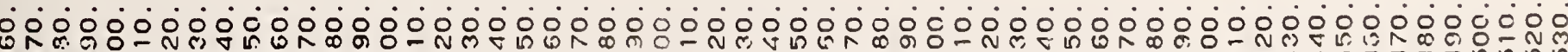
6.6. 


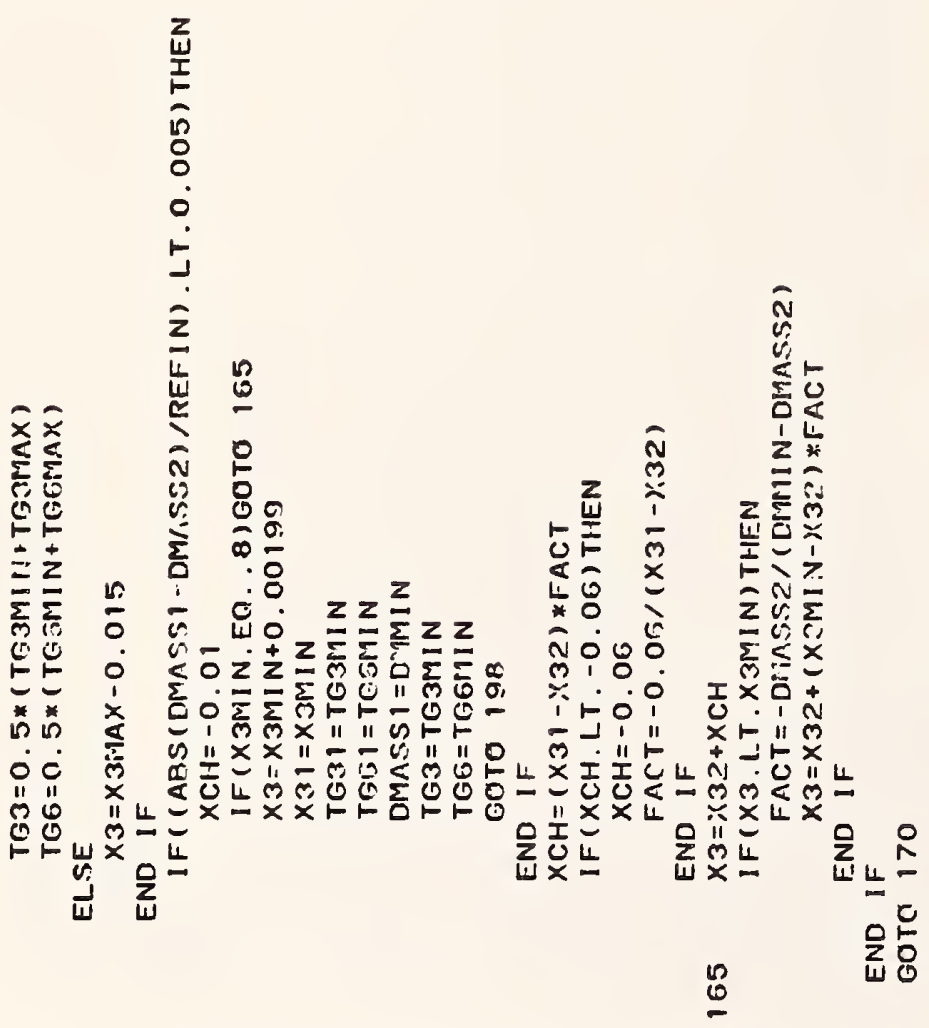

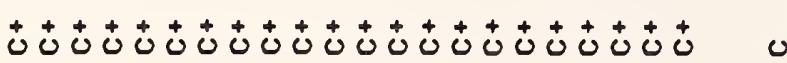

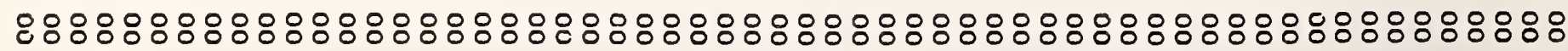

$\frac{2}{5}$

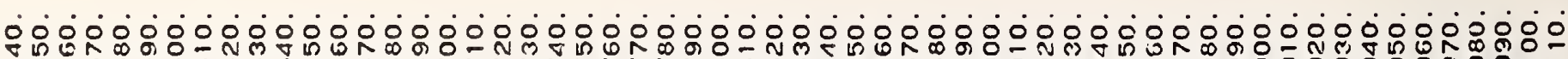

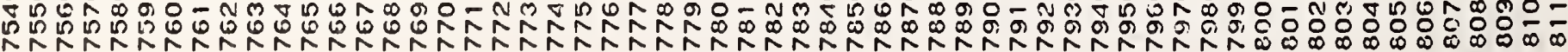




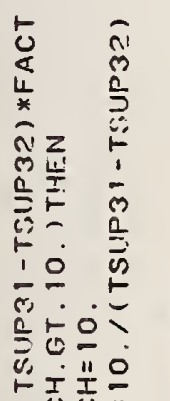
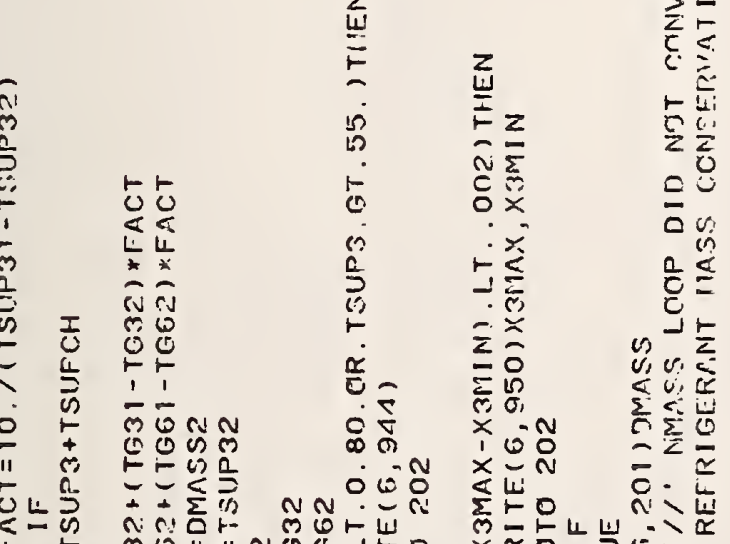

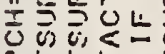

ก⿻上丨

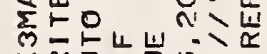

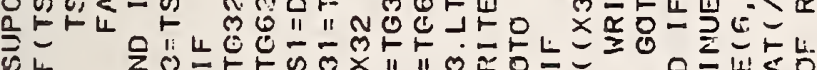

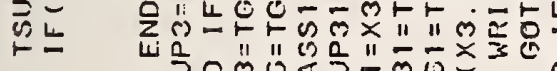

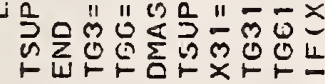

$\stackrel{1}{2}$

w

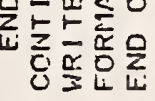

$$
\stackrel{\circ}{9}
$$

高 党*

U.

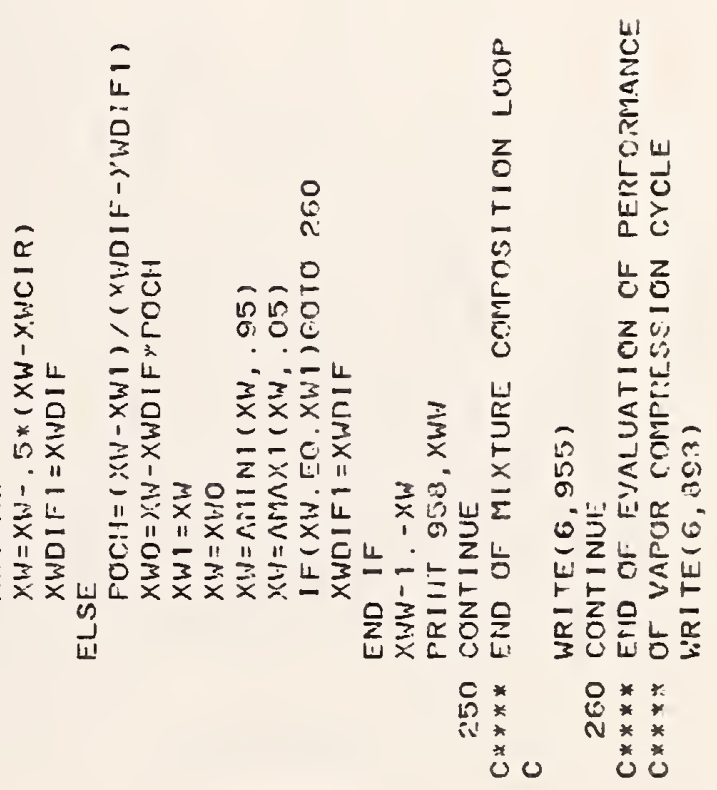

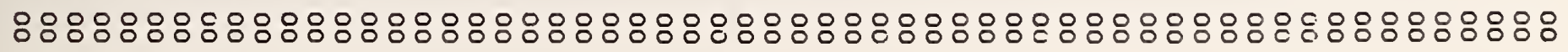

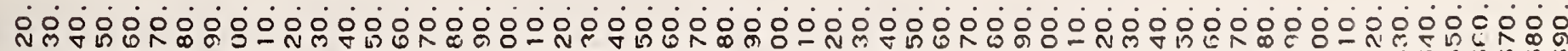

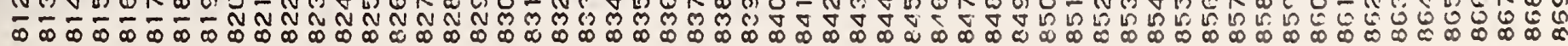




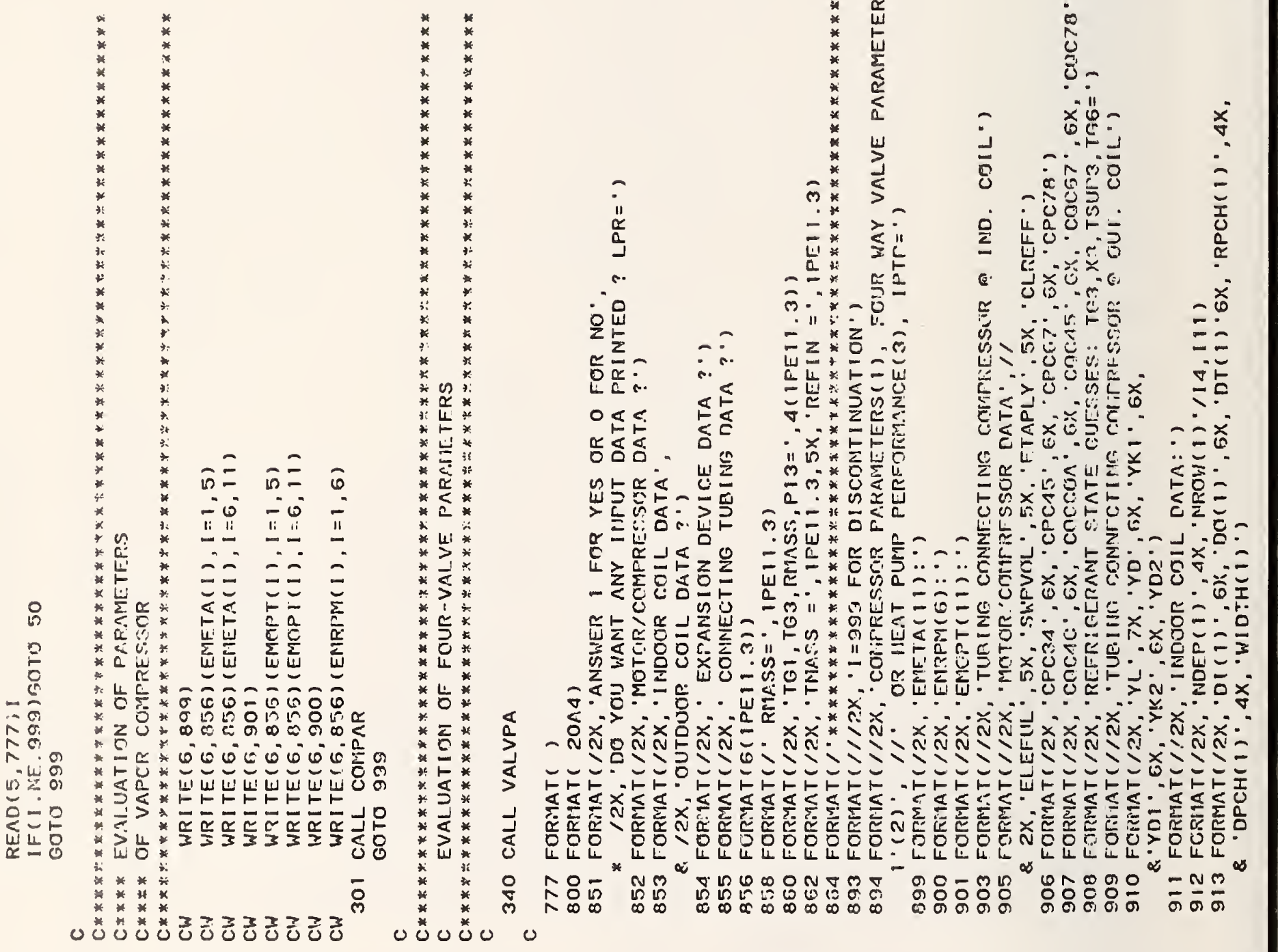

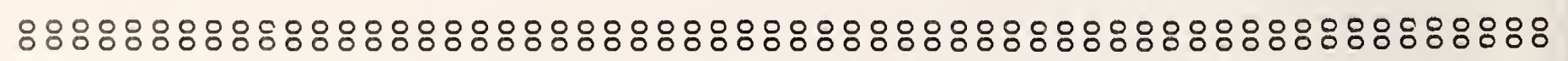

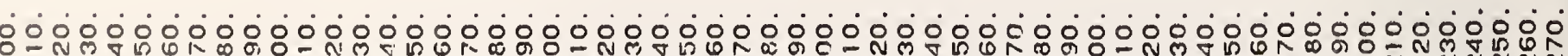
N

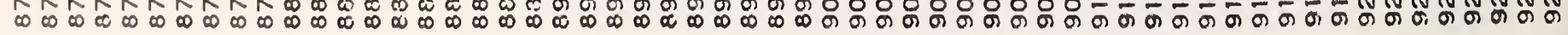




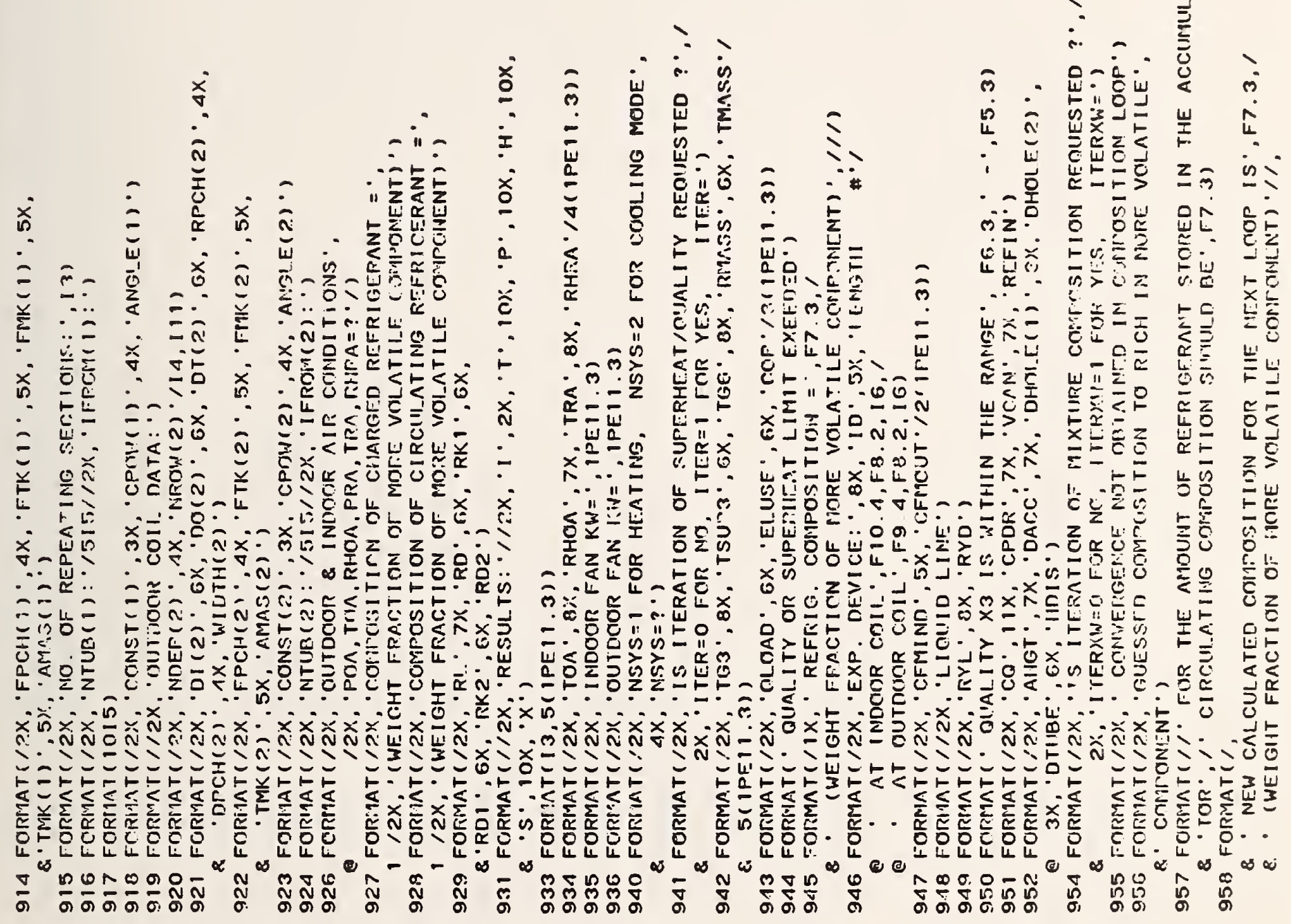

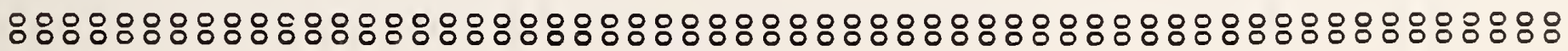

¿́ं

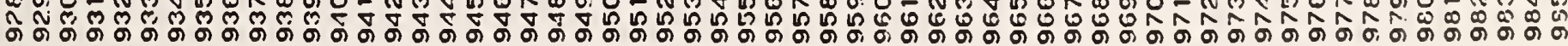




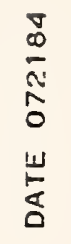

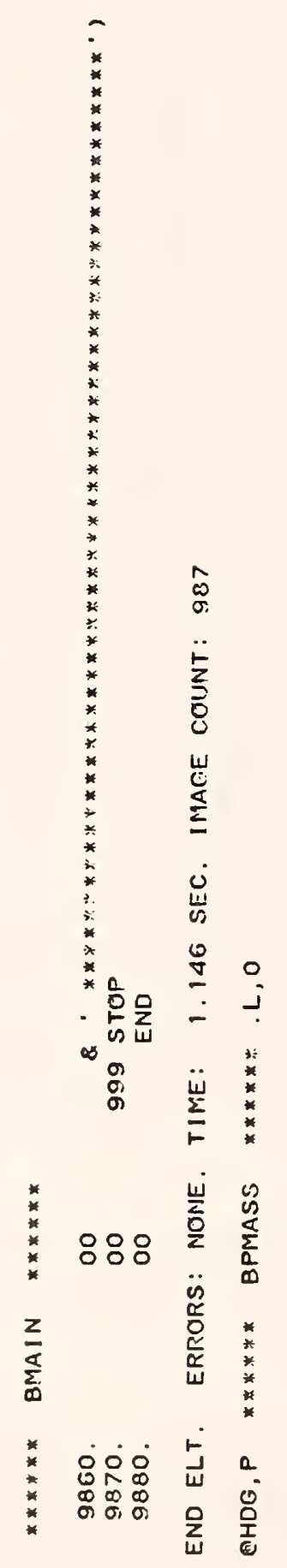




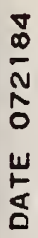

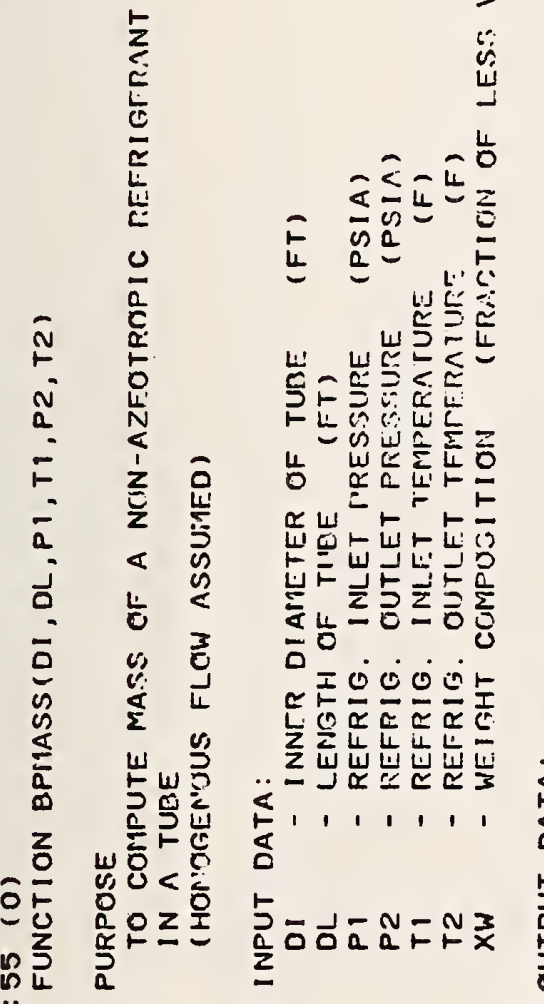

$\stackrel{0}{己}$
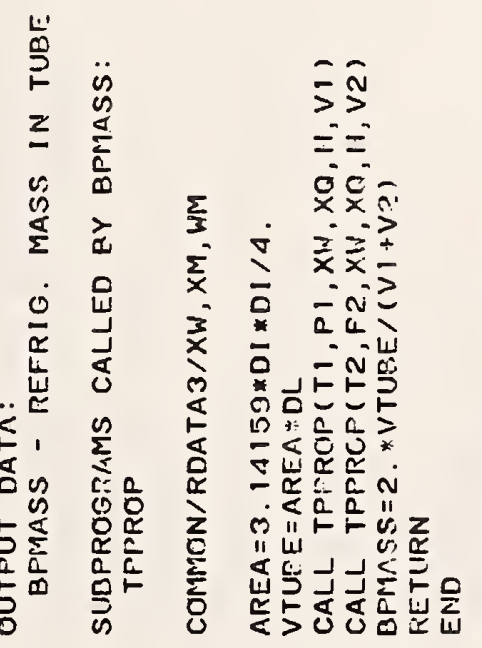

ले

岁

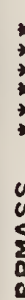
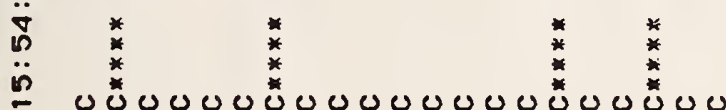

0

岁

$\stackrel{0}{\circ}$
$\dot{0}$
0
0
$\ddot{\omega}$

ळ

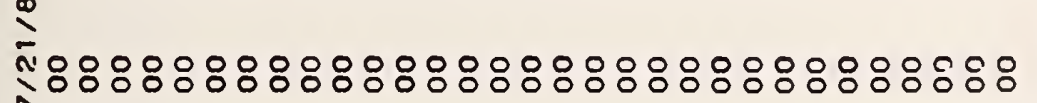
is

$\sum_{0}^{\infty} \frac{0}{0} \frac{0}{0}$

吕

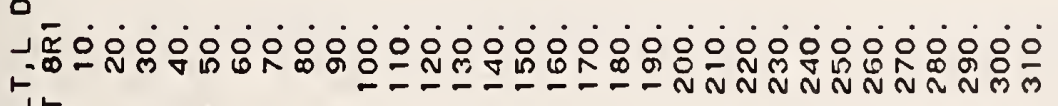

岃志

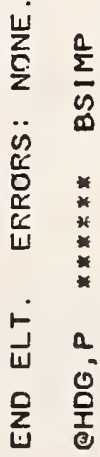




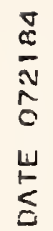

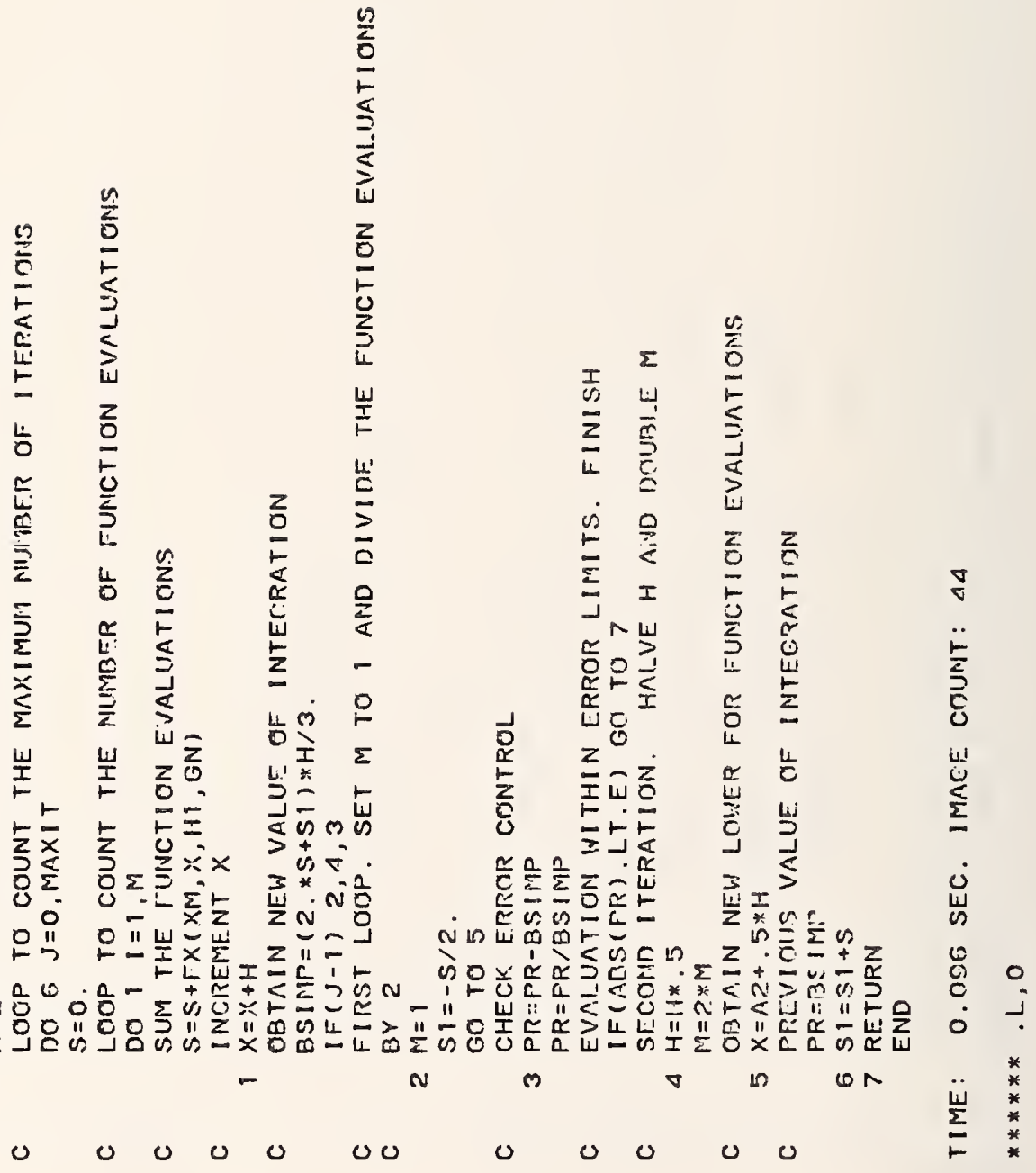

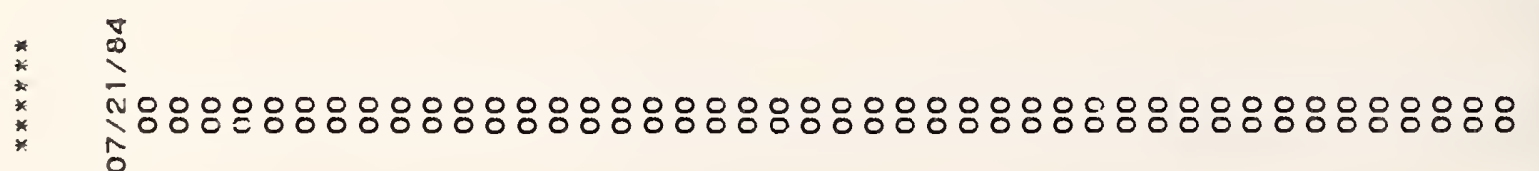

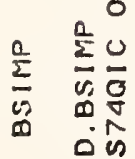

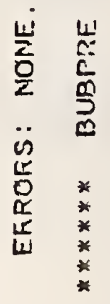

in

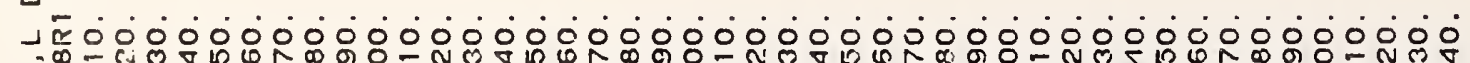

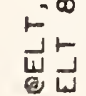

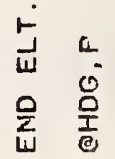




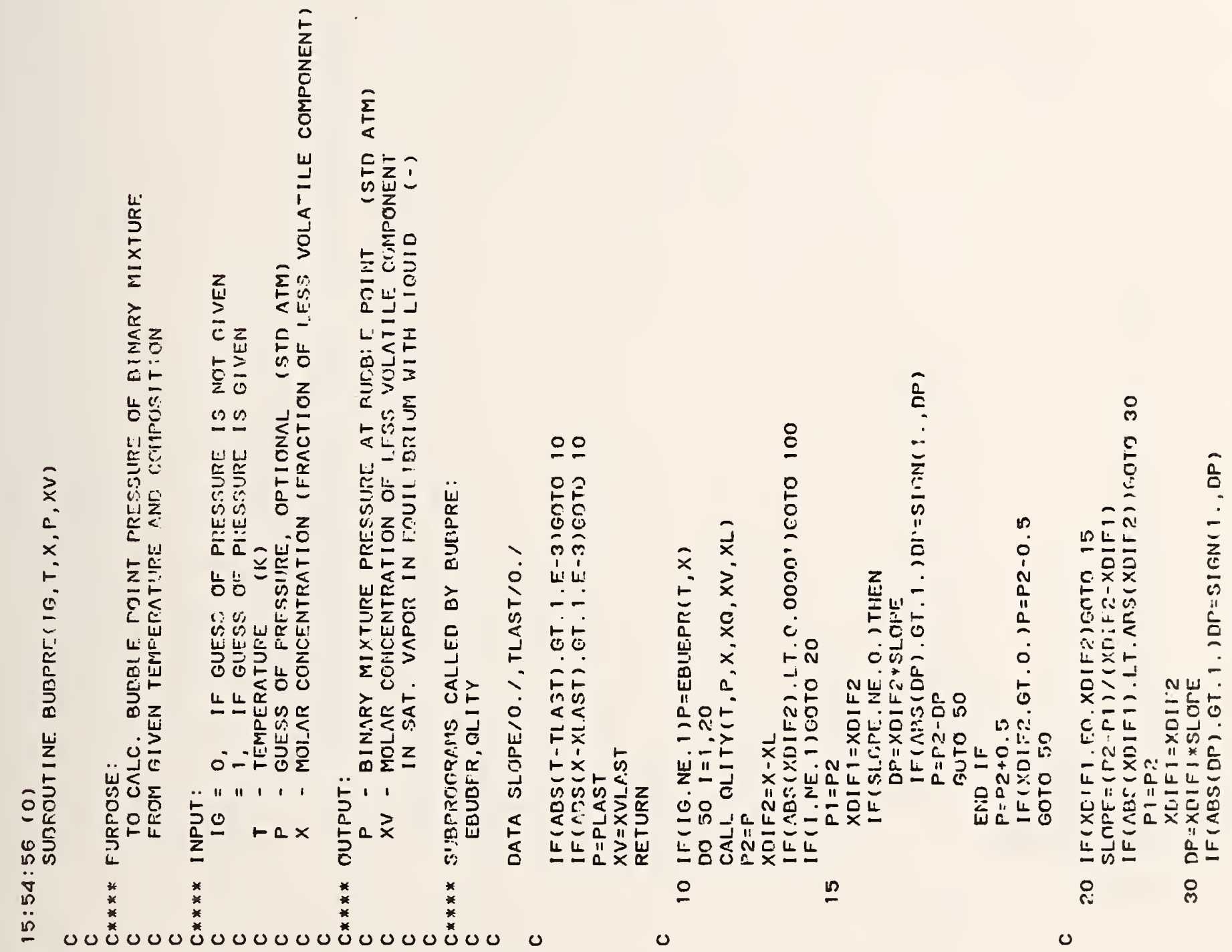
曲

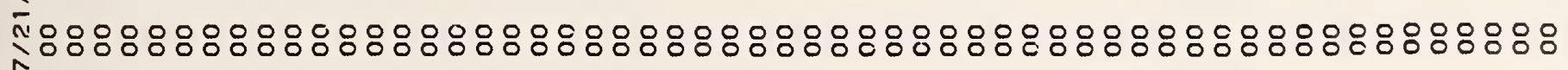

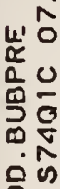

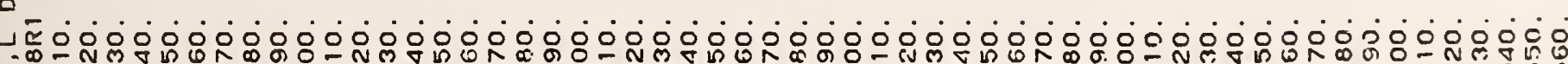
F- - N 这它 


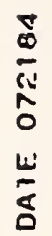

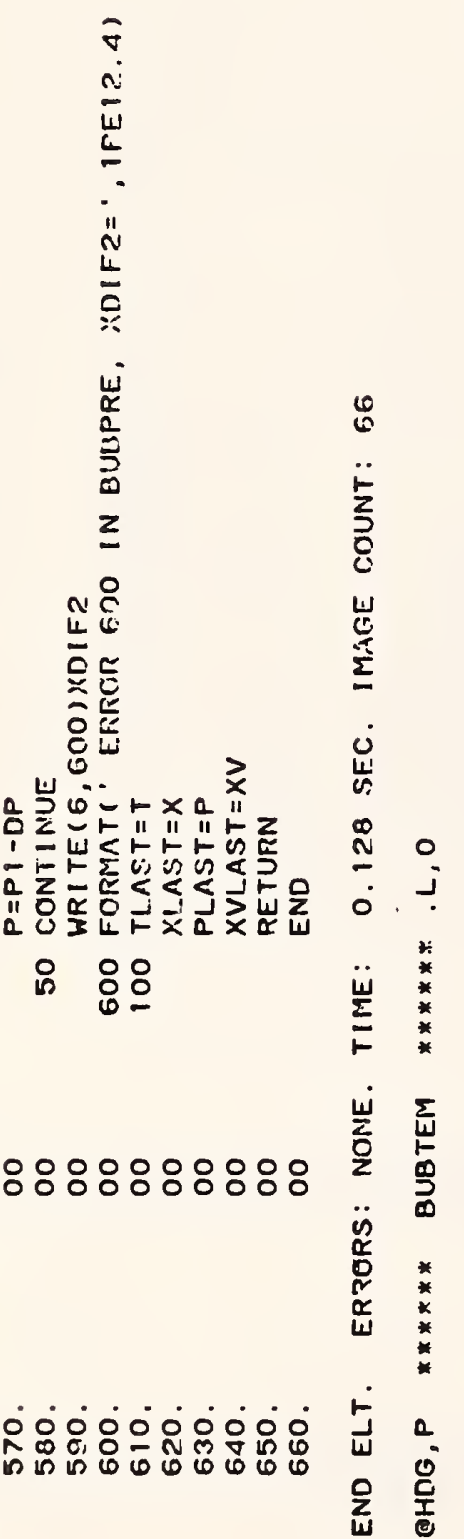




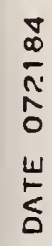

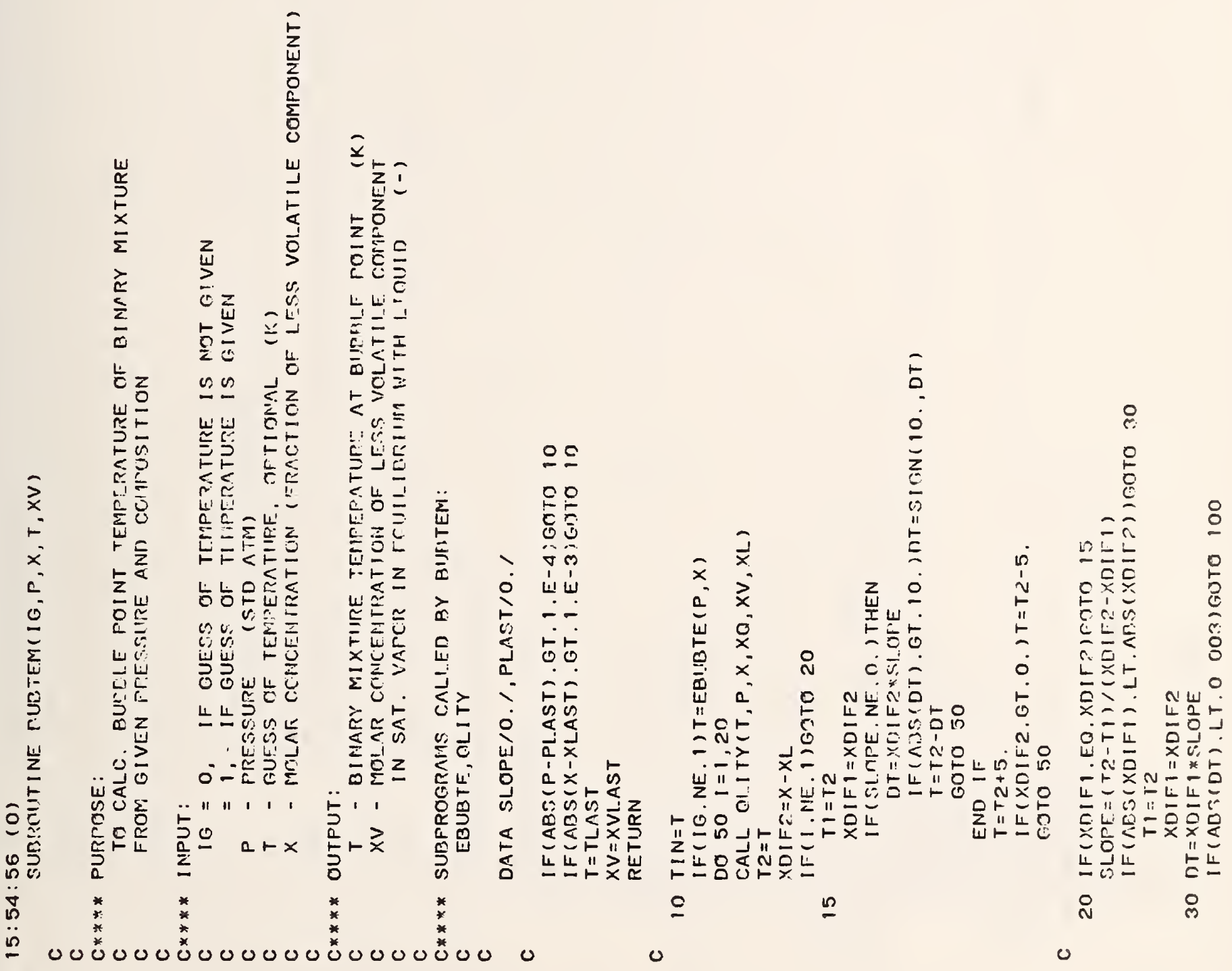

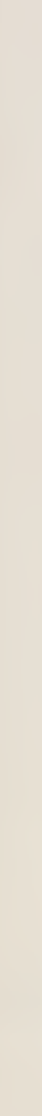




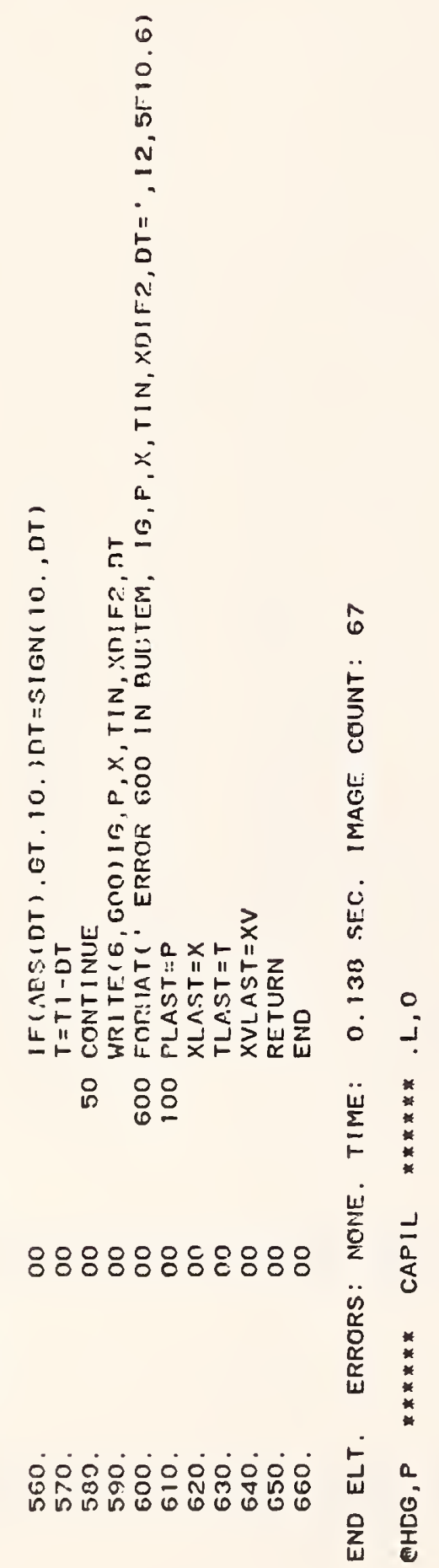




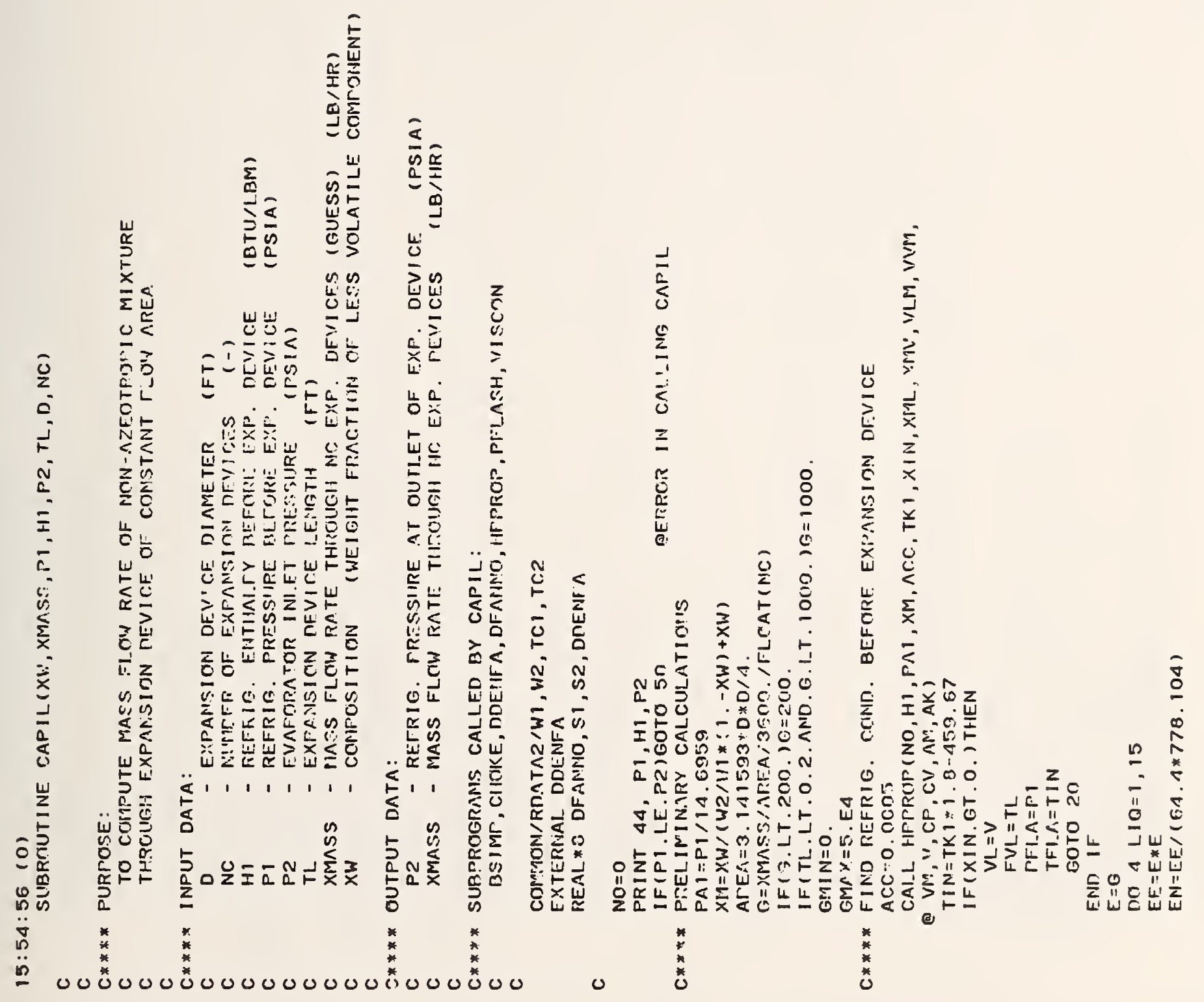




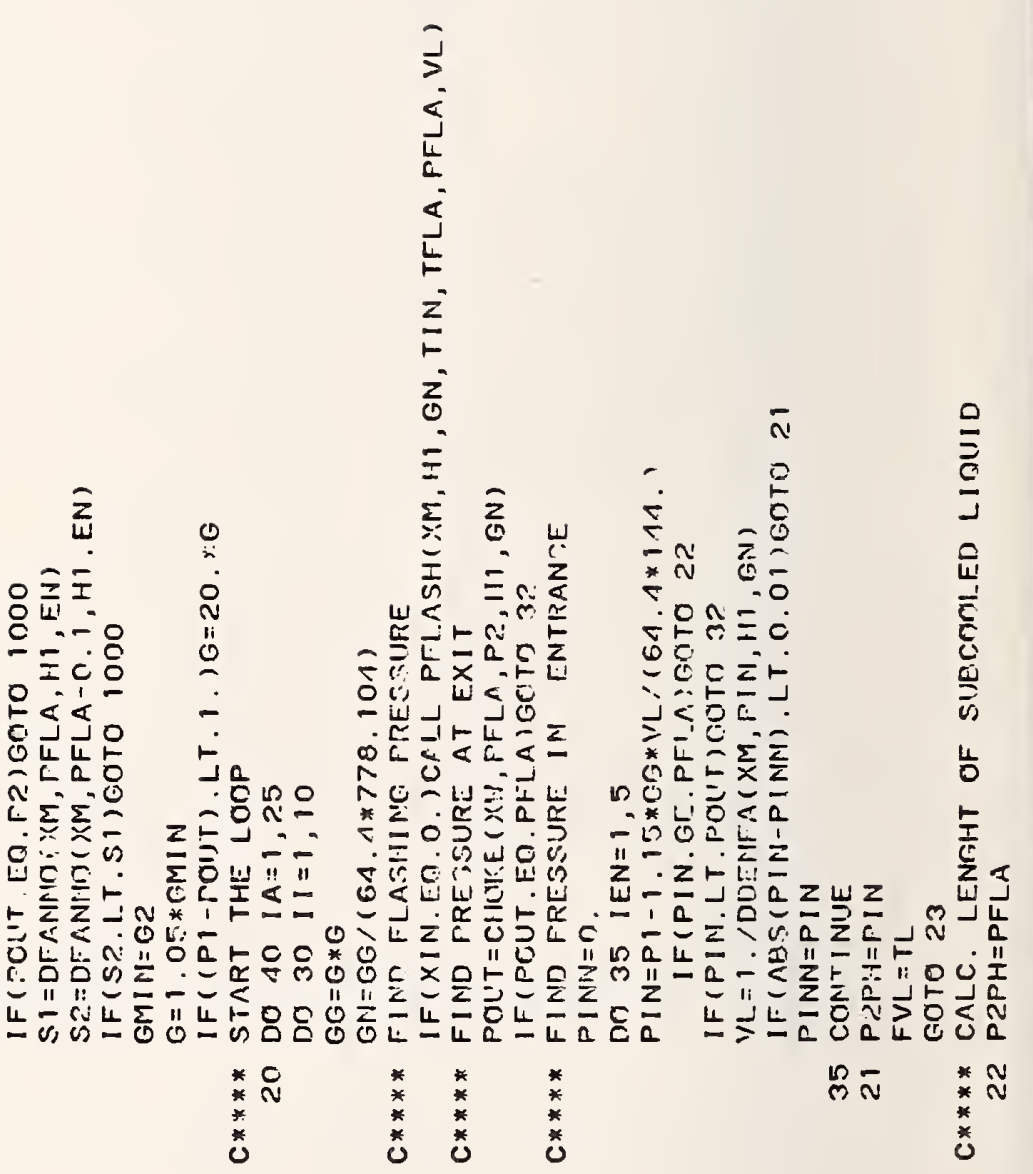

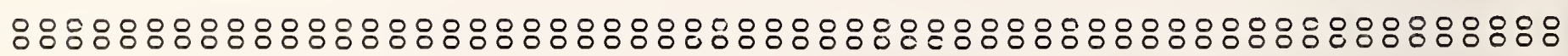

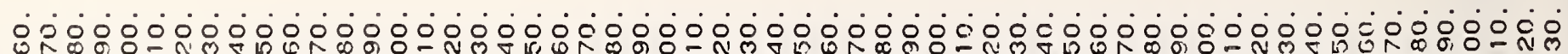
以n 


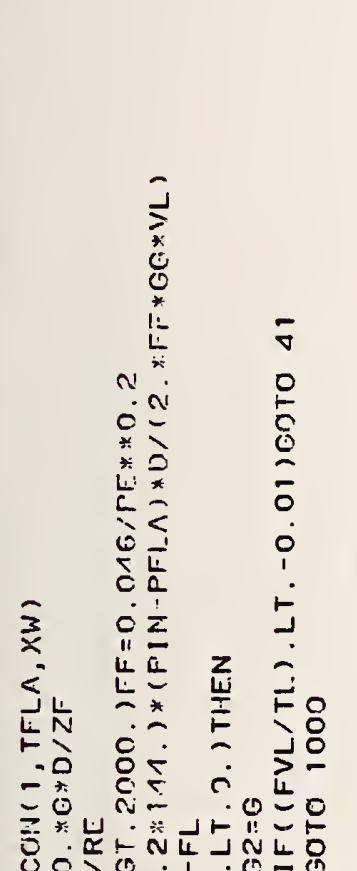

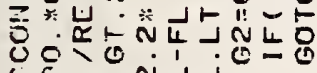

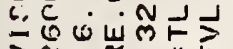
>

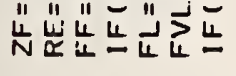
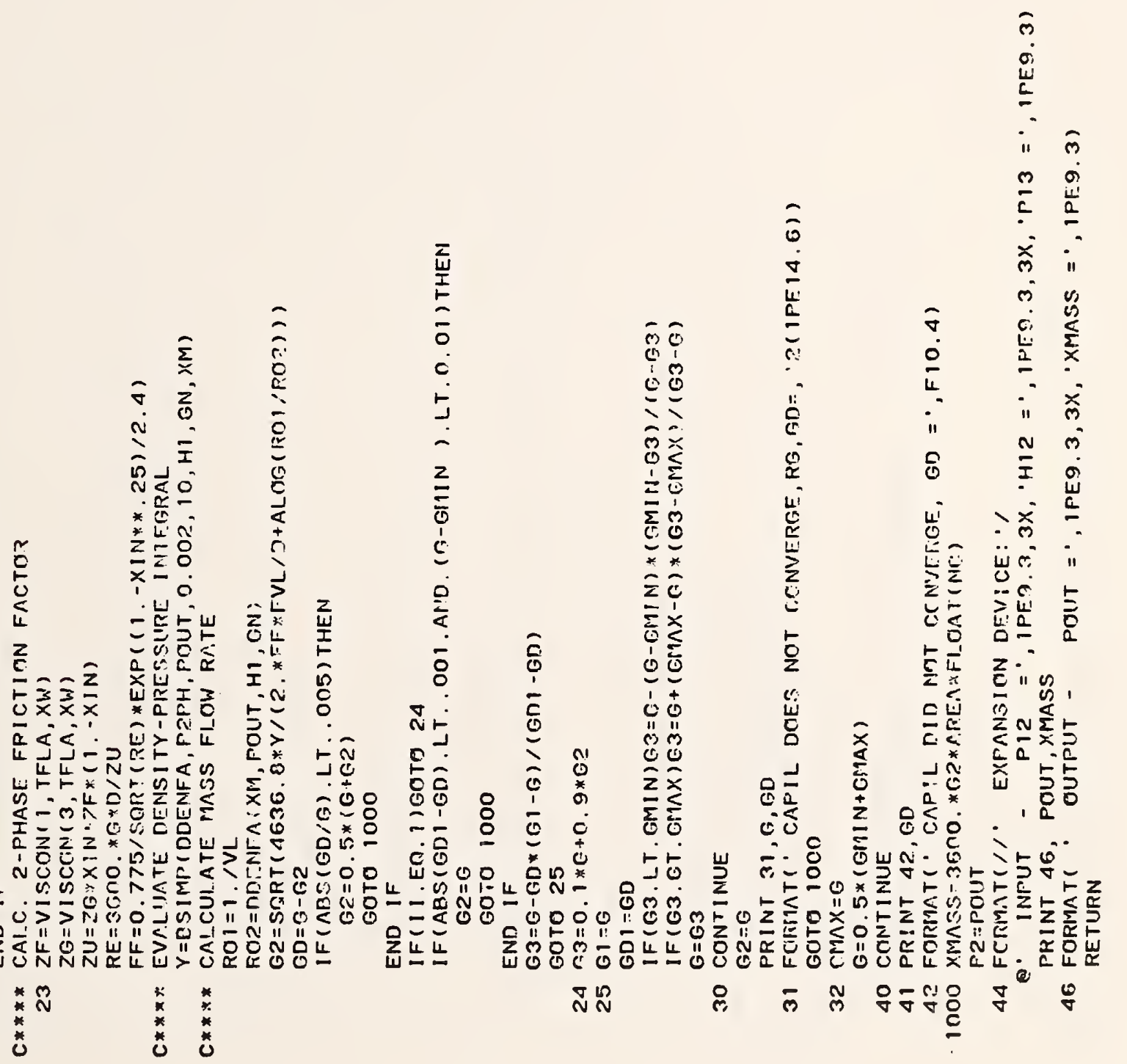

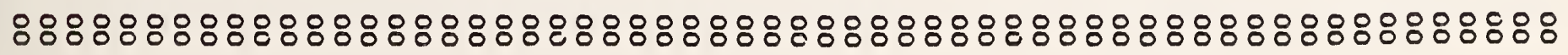

$\frac{1}{d}$

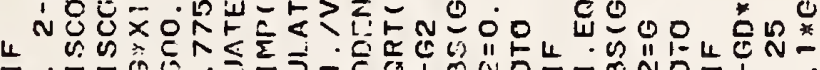

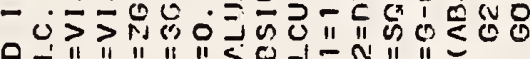

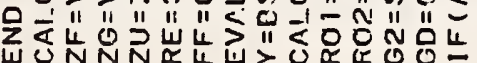

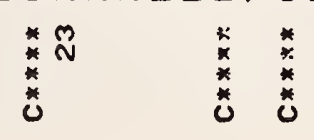

ำ

\%

은ㄷㄴ

10.0

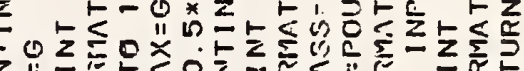

$\bar{m}$

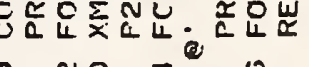

ió

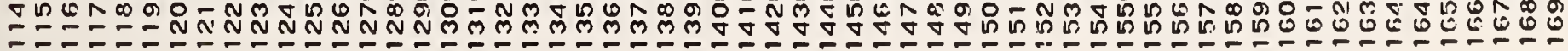




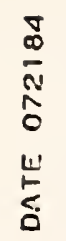

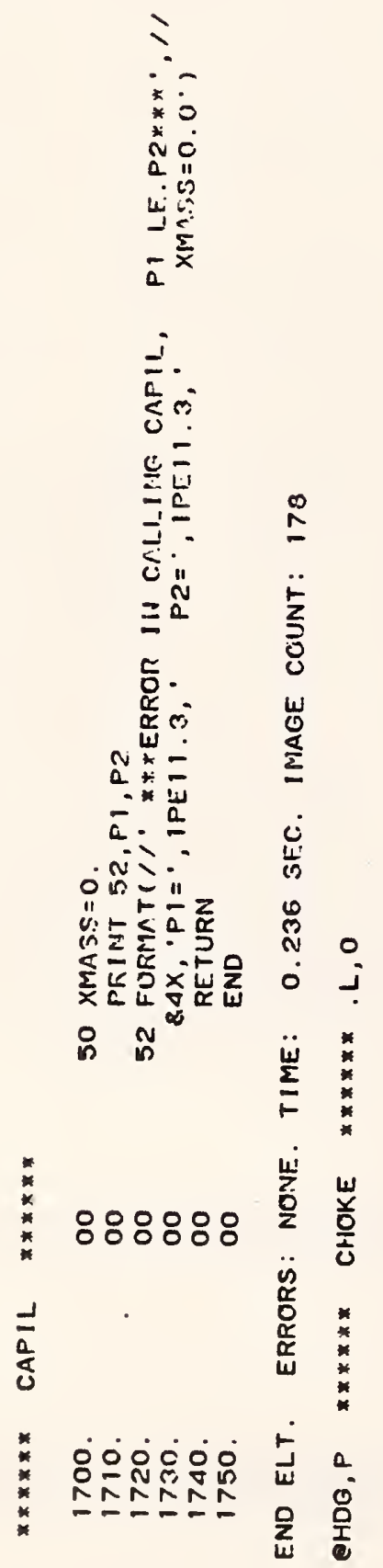




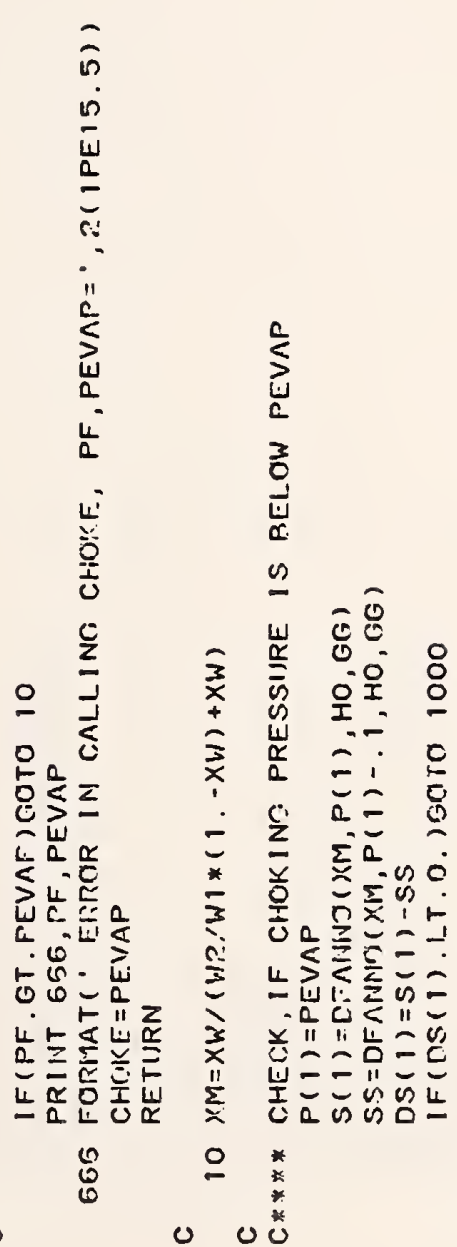

D

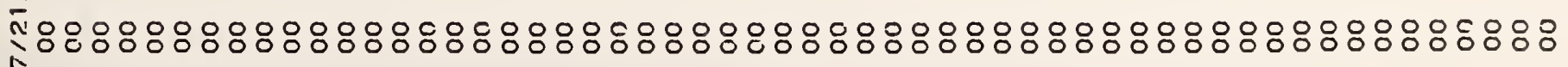

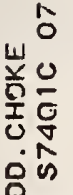

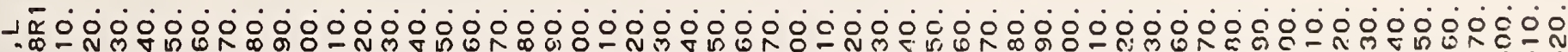
5 峞 


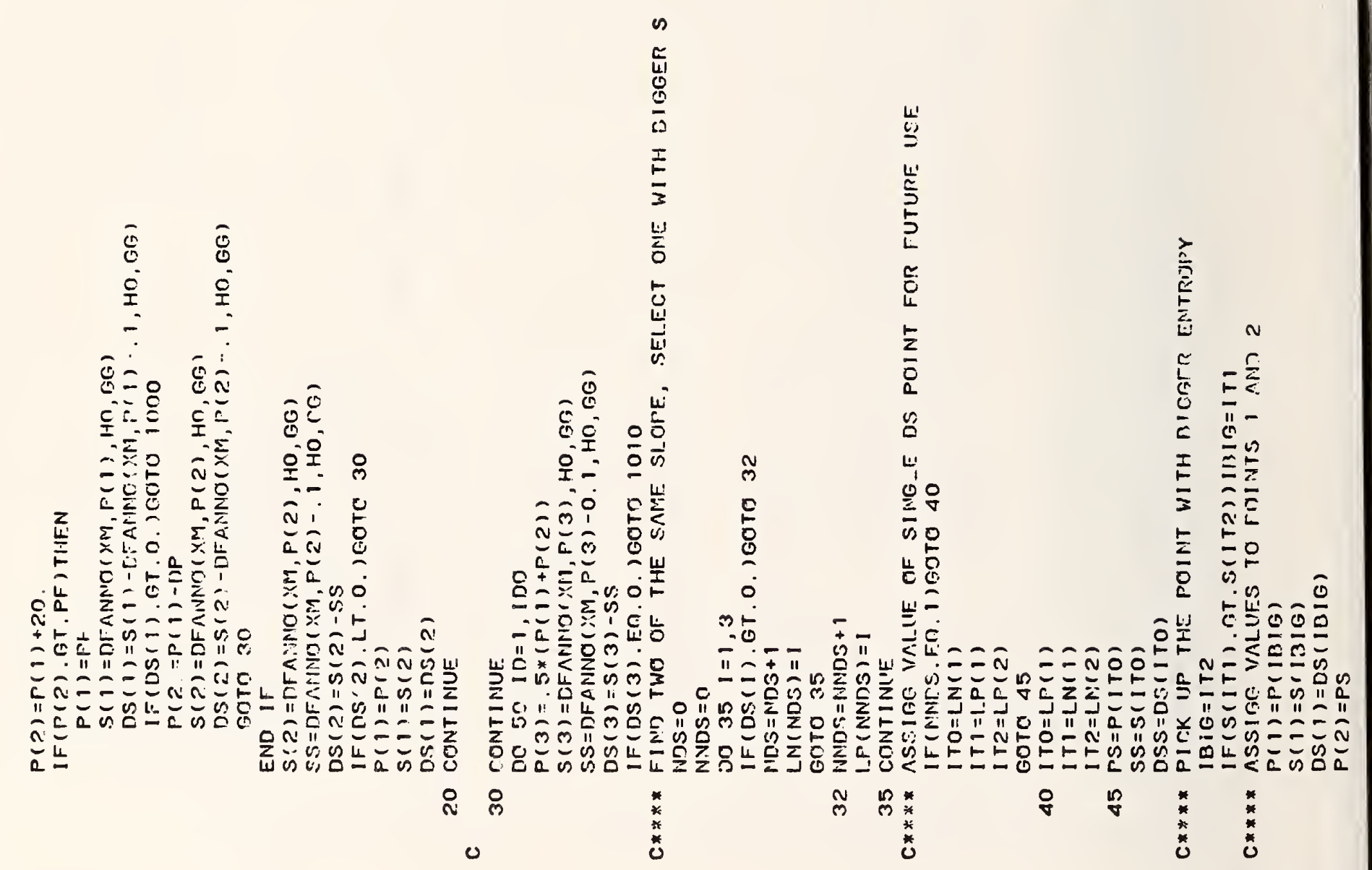



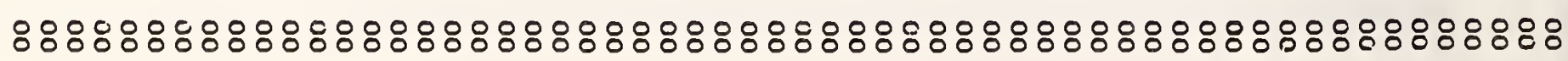

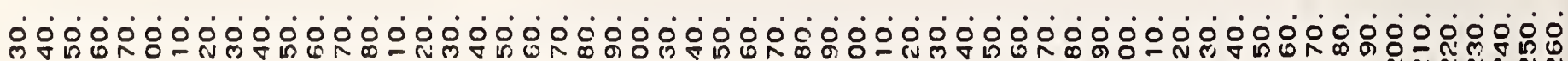

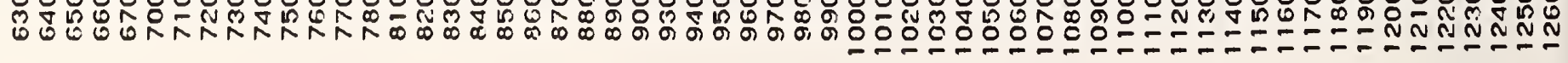




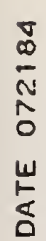

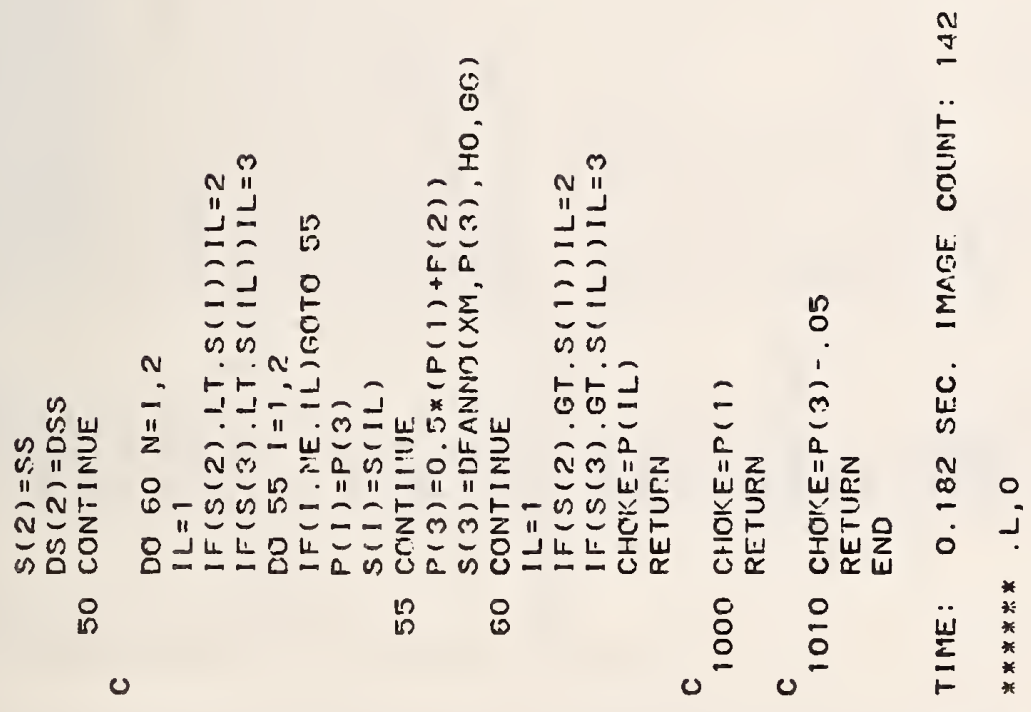

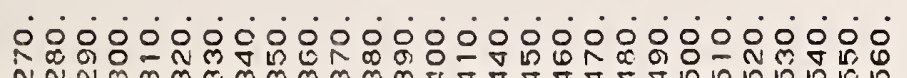

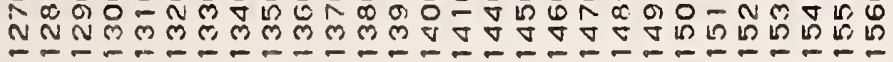

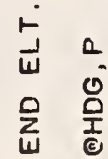




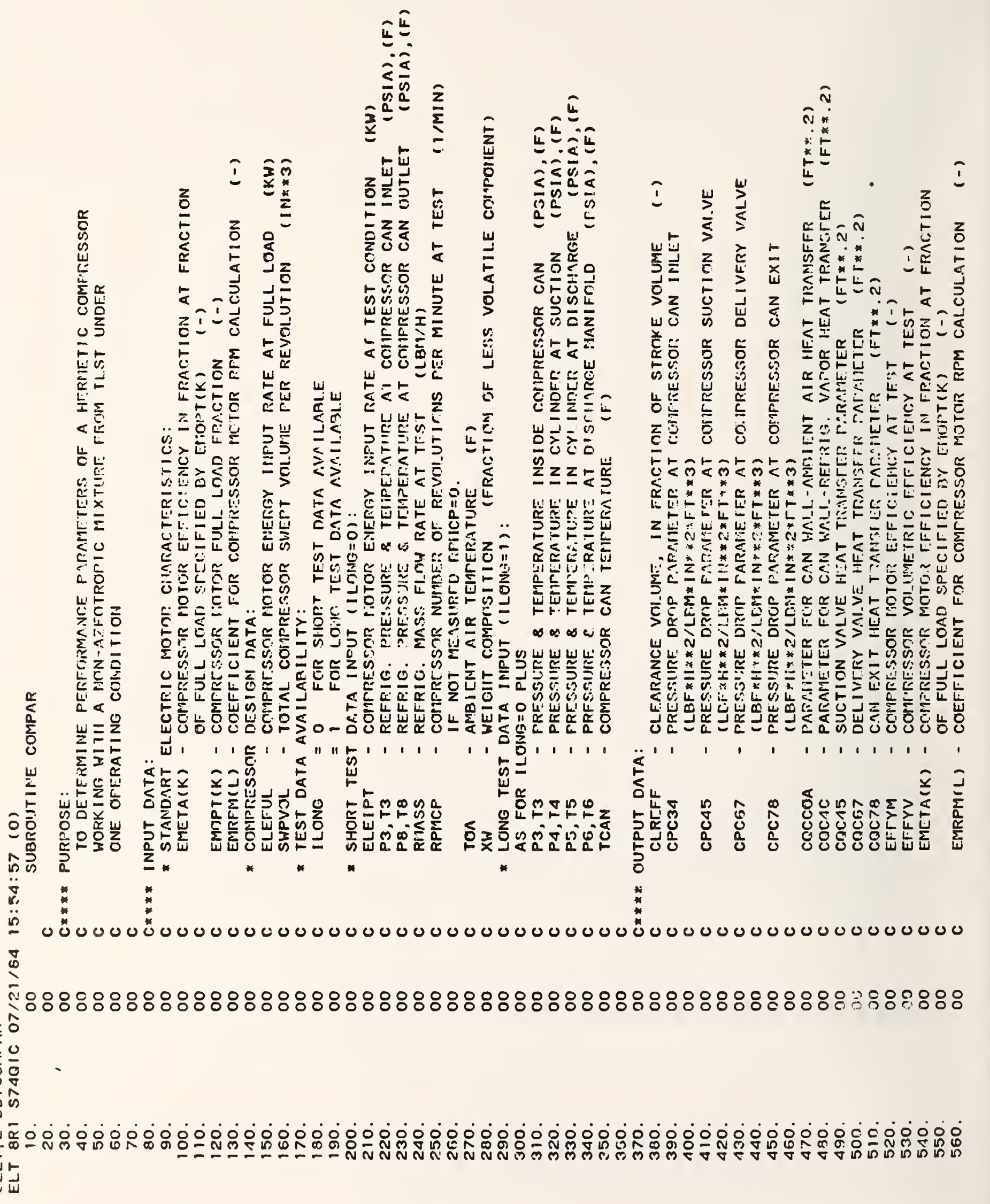




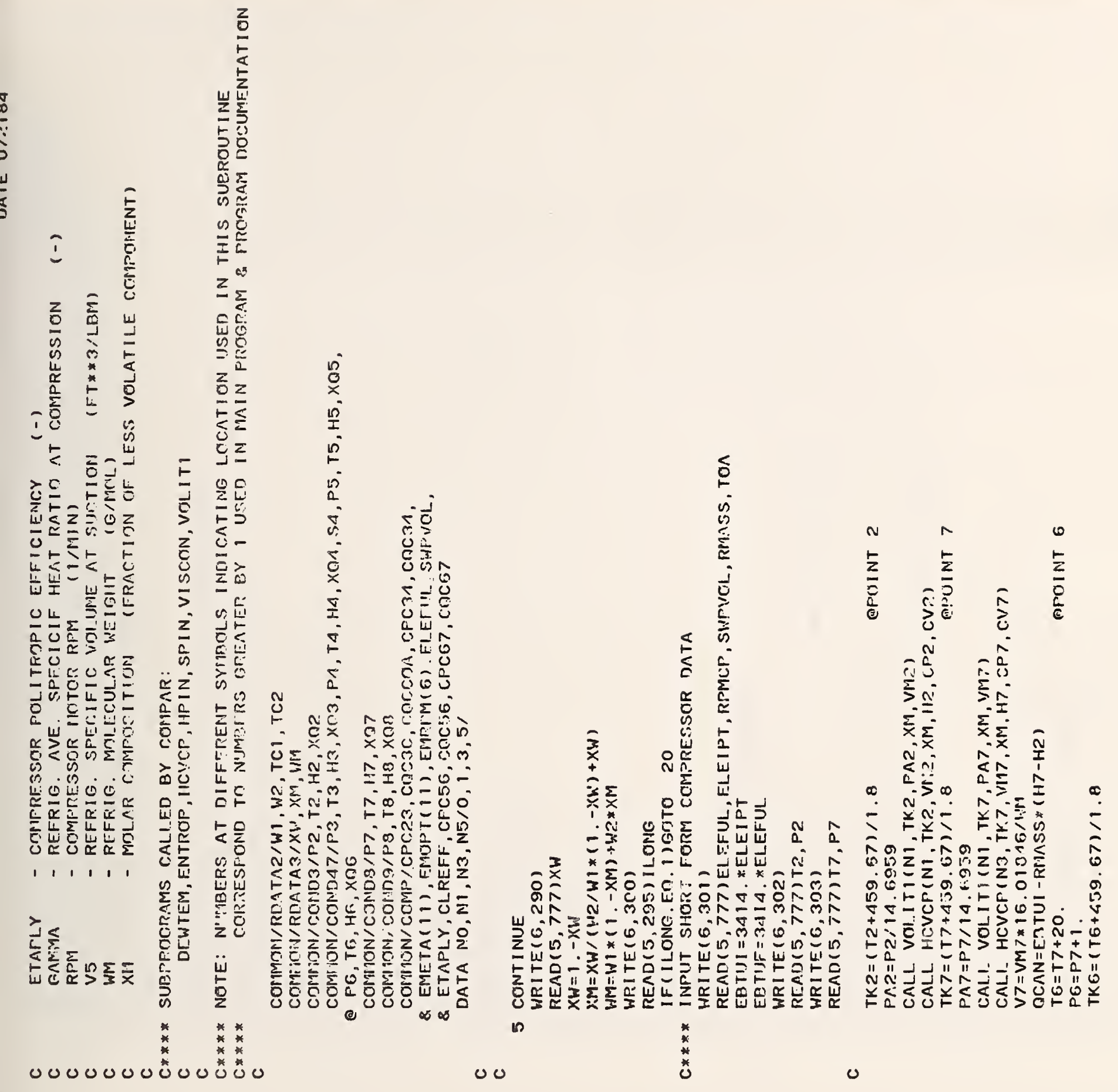

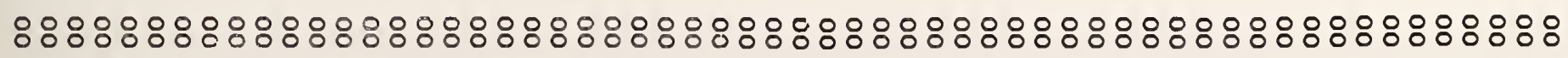

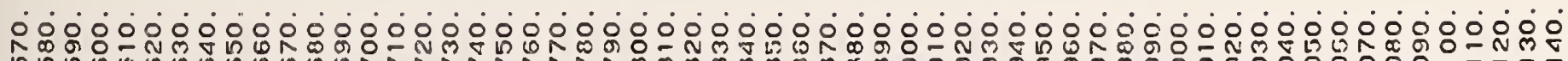

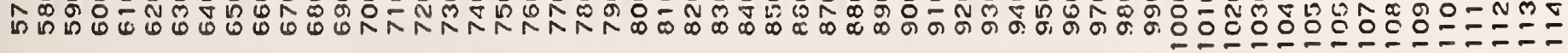




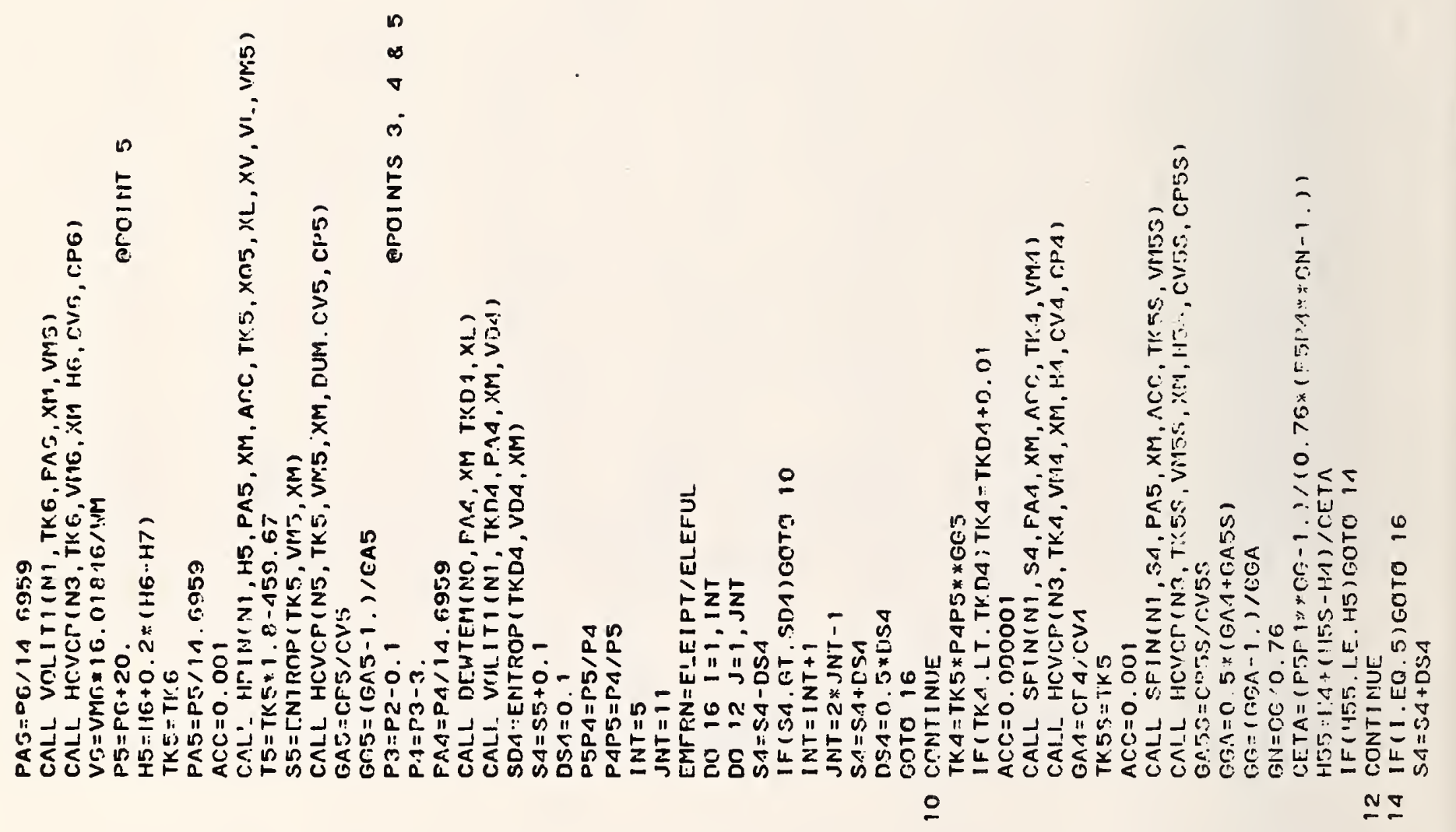

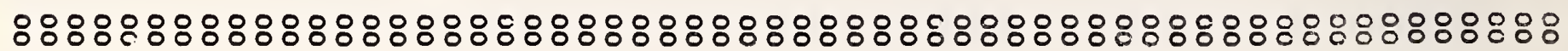
$\frac{\alpha}{\frac{\alpha}{c}}$

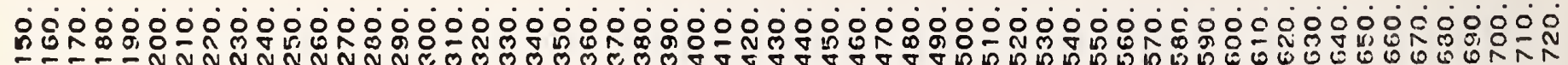

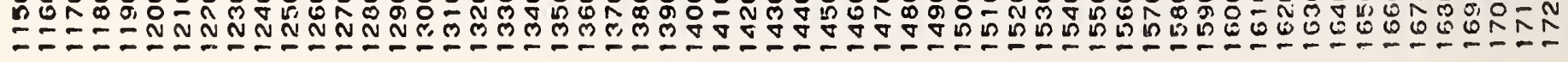




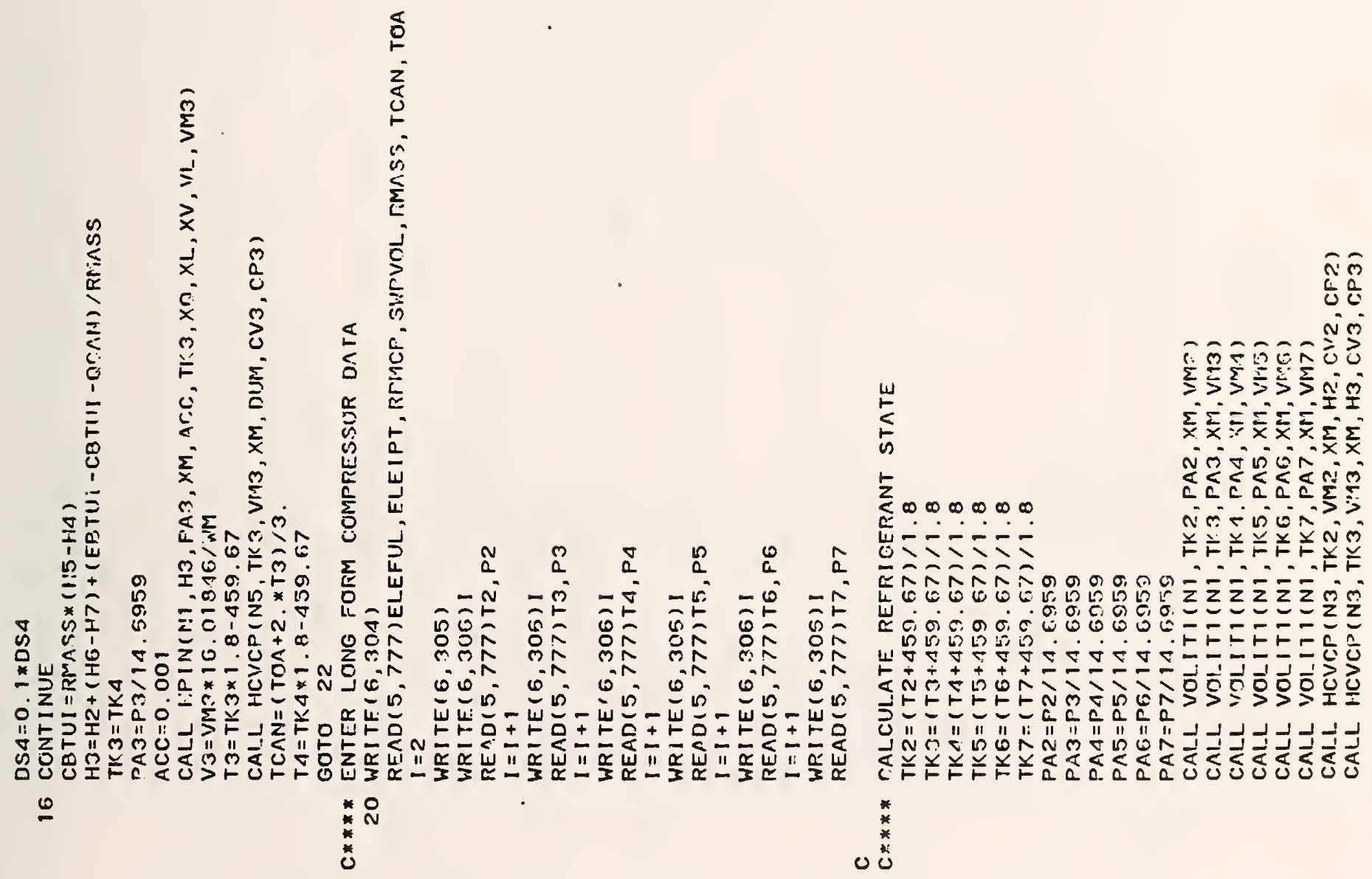

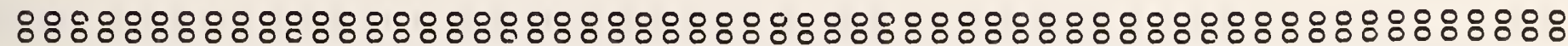
$\frac{\alpha}{\alpha}$

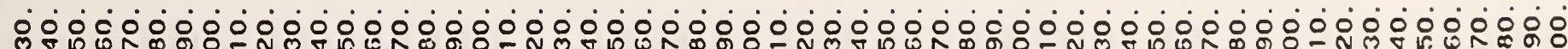

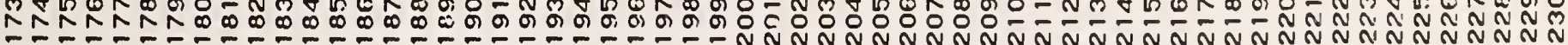




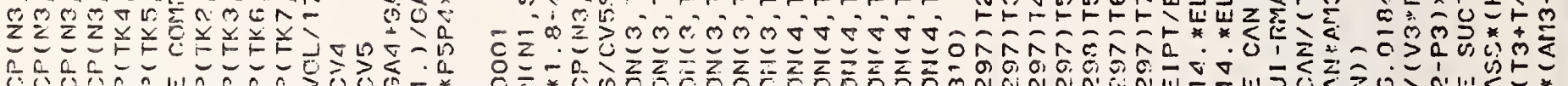

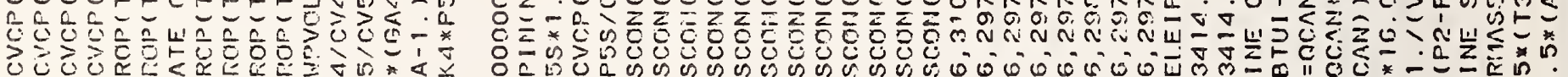

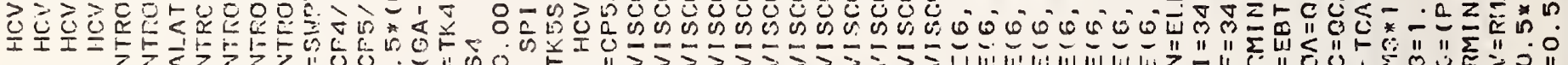

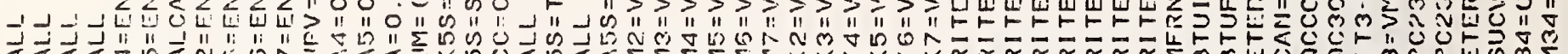

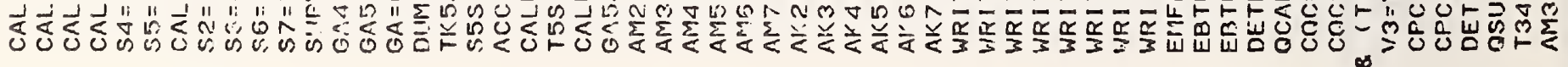

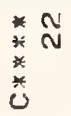

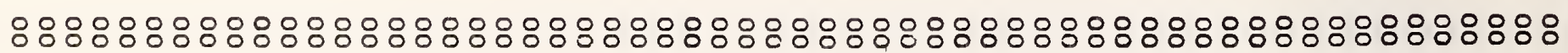

$\frac{\frac{\pi}{c}}{\frac{0}{5}}$

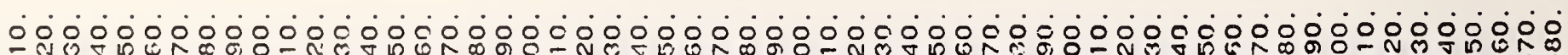
ํํำ 


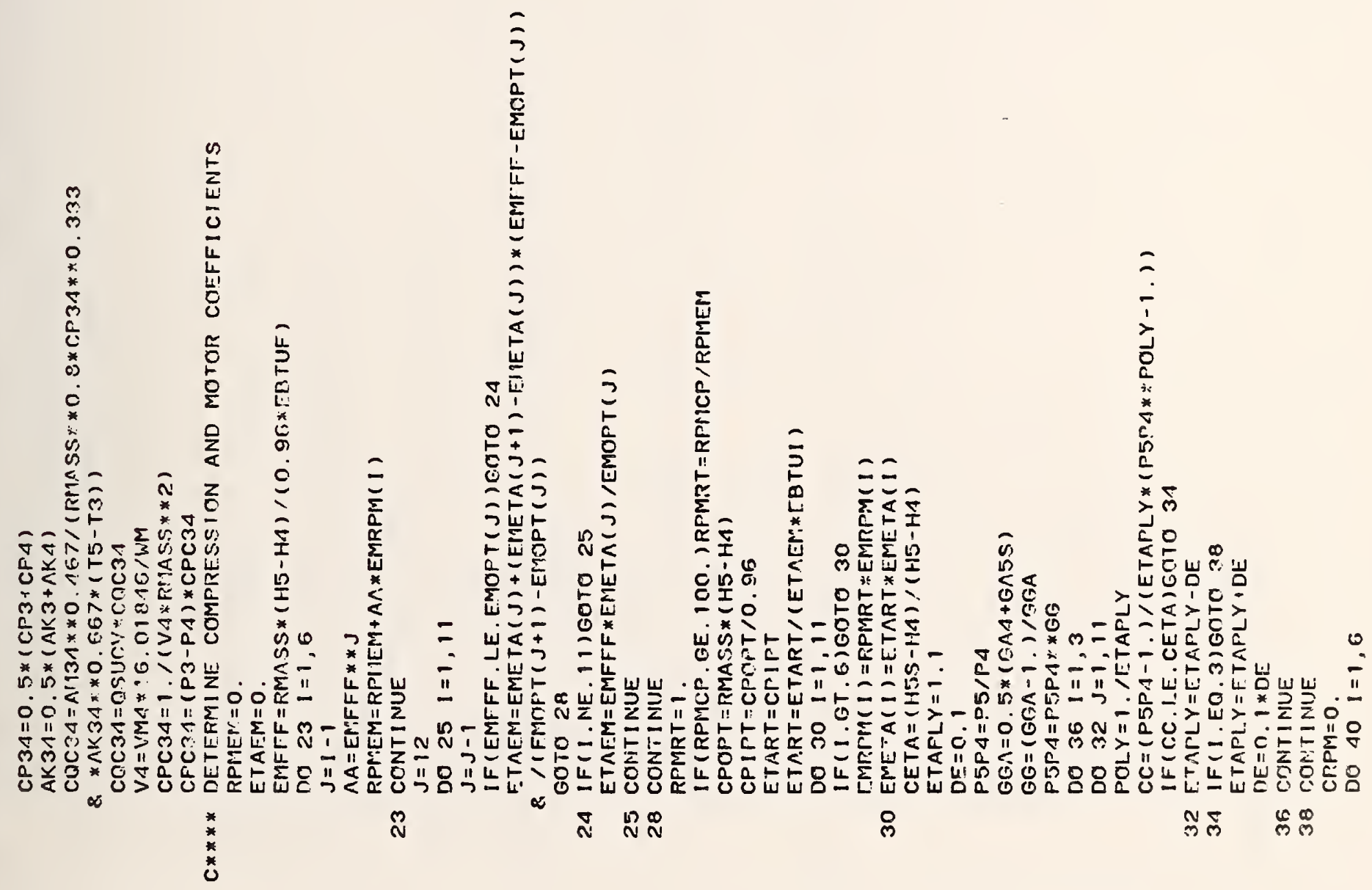

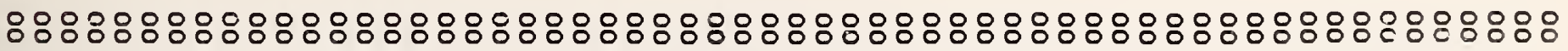

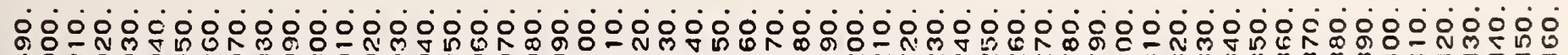

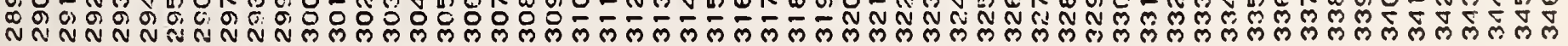




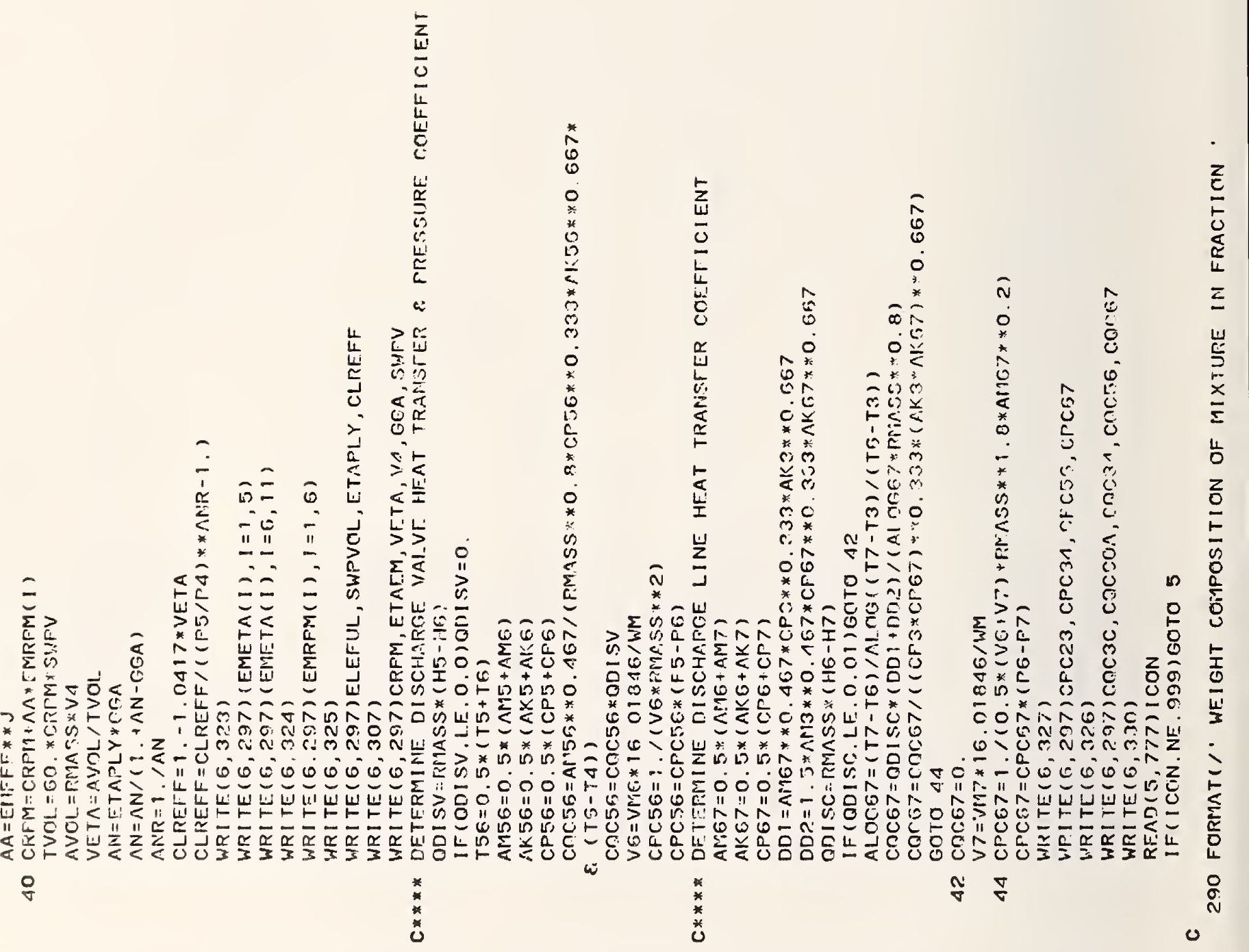

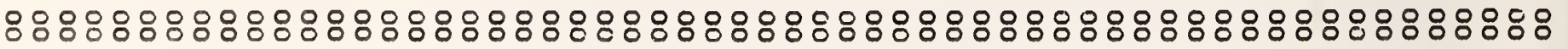

당

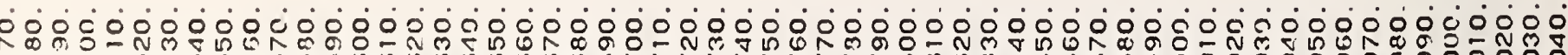

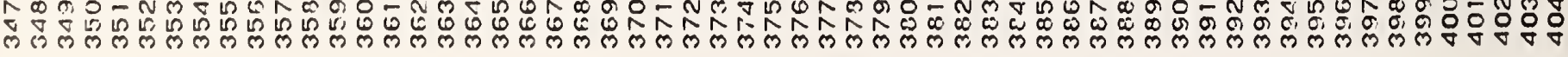




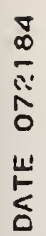

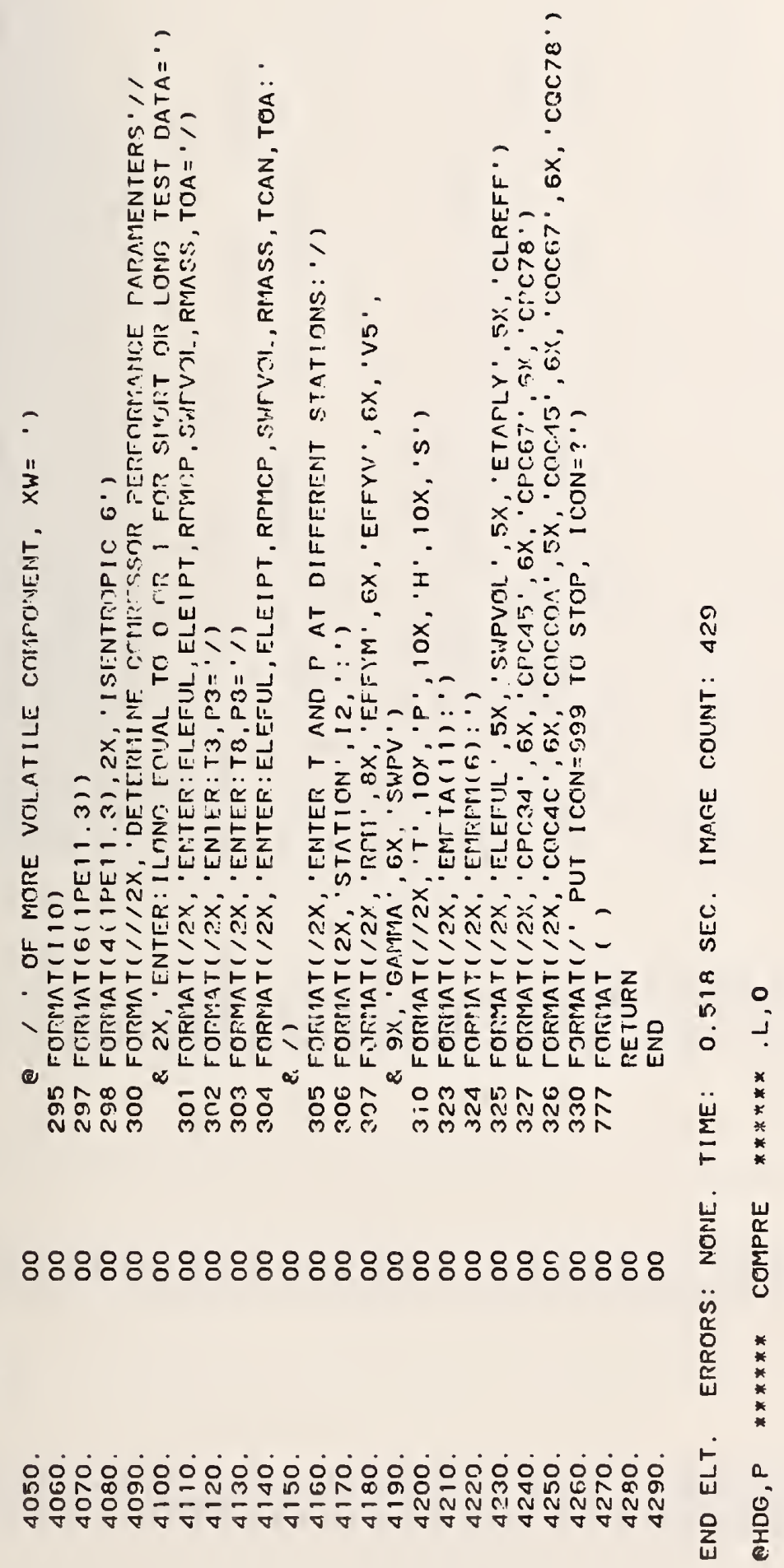



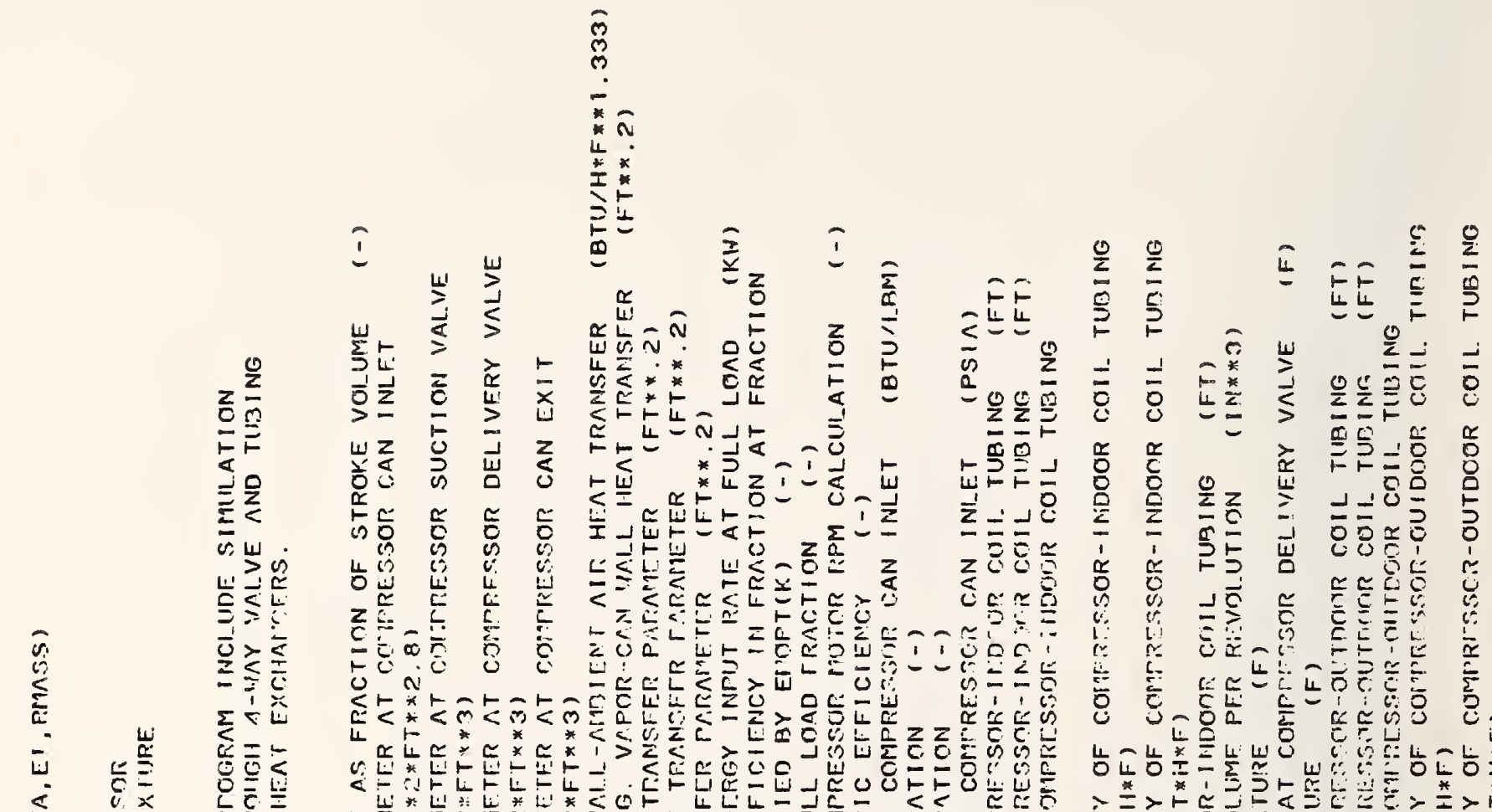

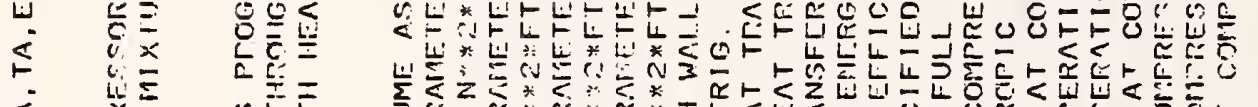

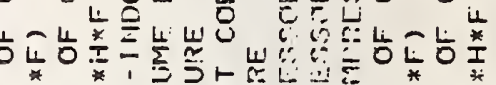

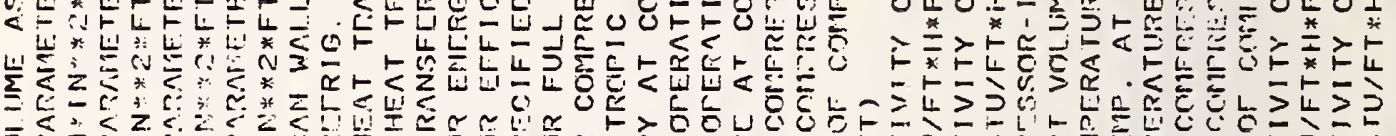

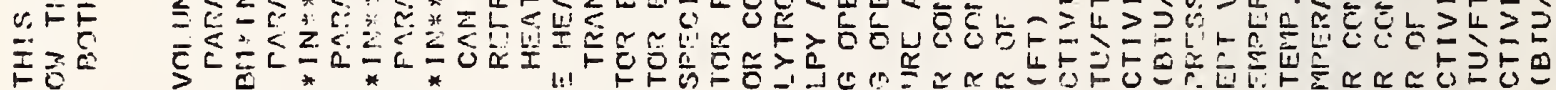

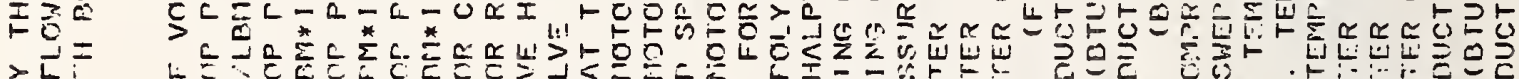

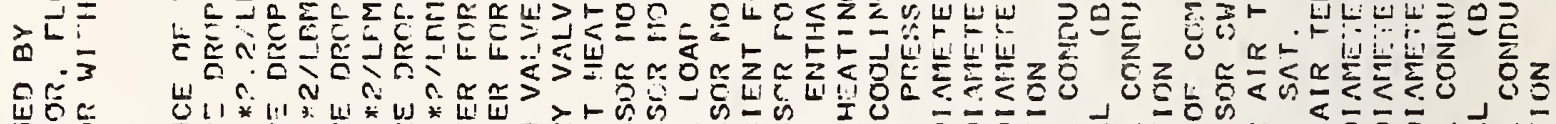

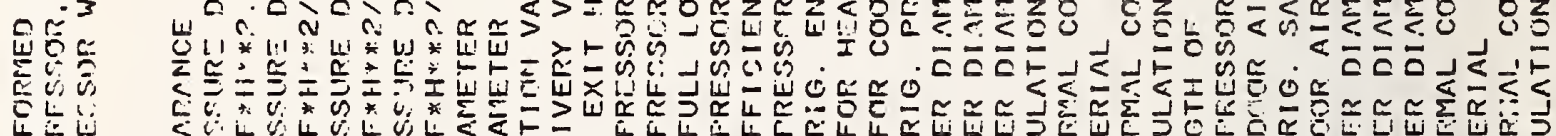

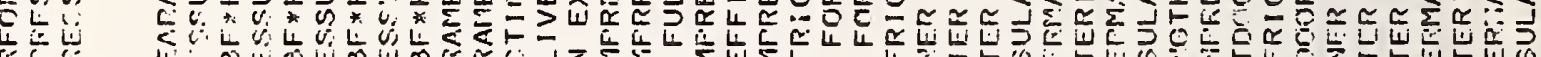

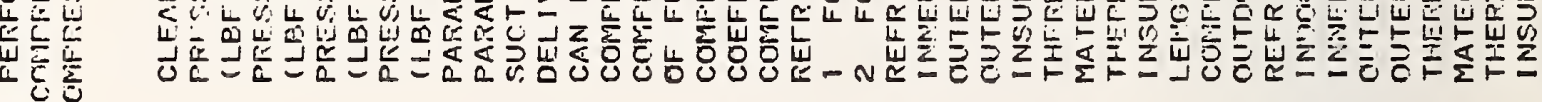
$\varangle$

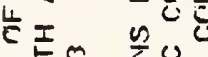

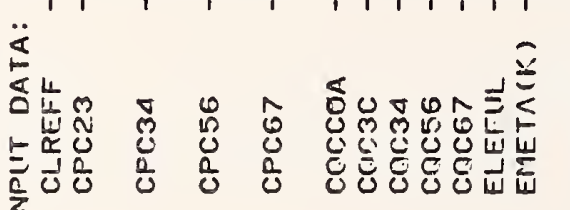

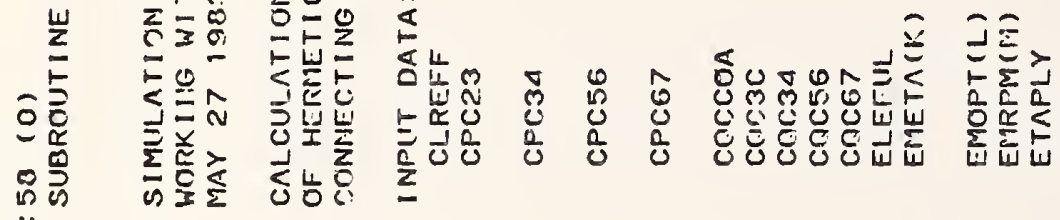

苟 * * * * * *

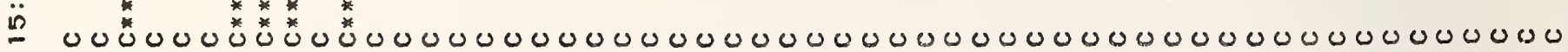

$\stackrel{\infty}{\infty}$

స̃ w

㟶 O

定号

in

* $\rightarrow \frac{\pi}{\infty}$

*

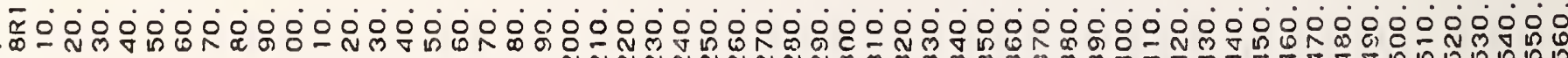
岁吉 


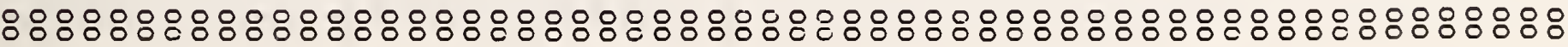

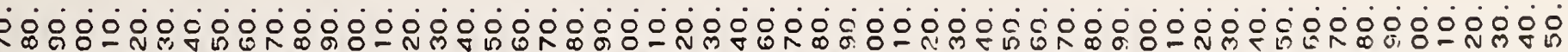

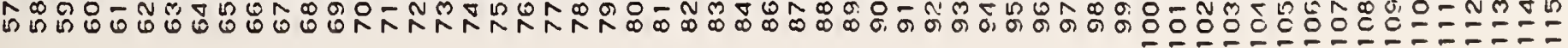




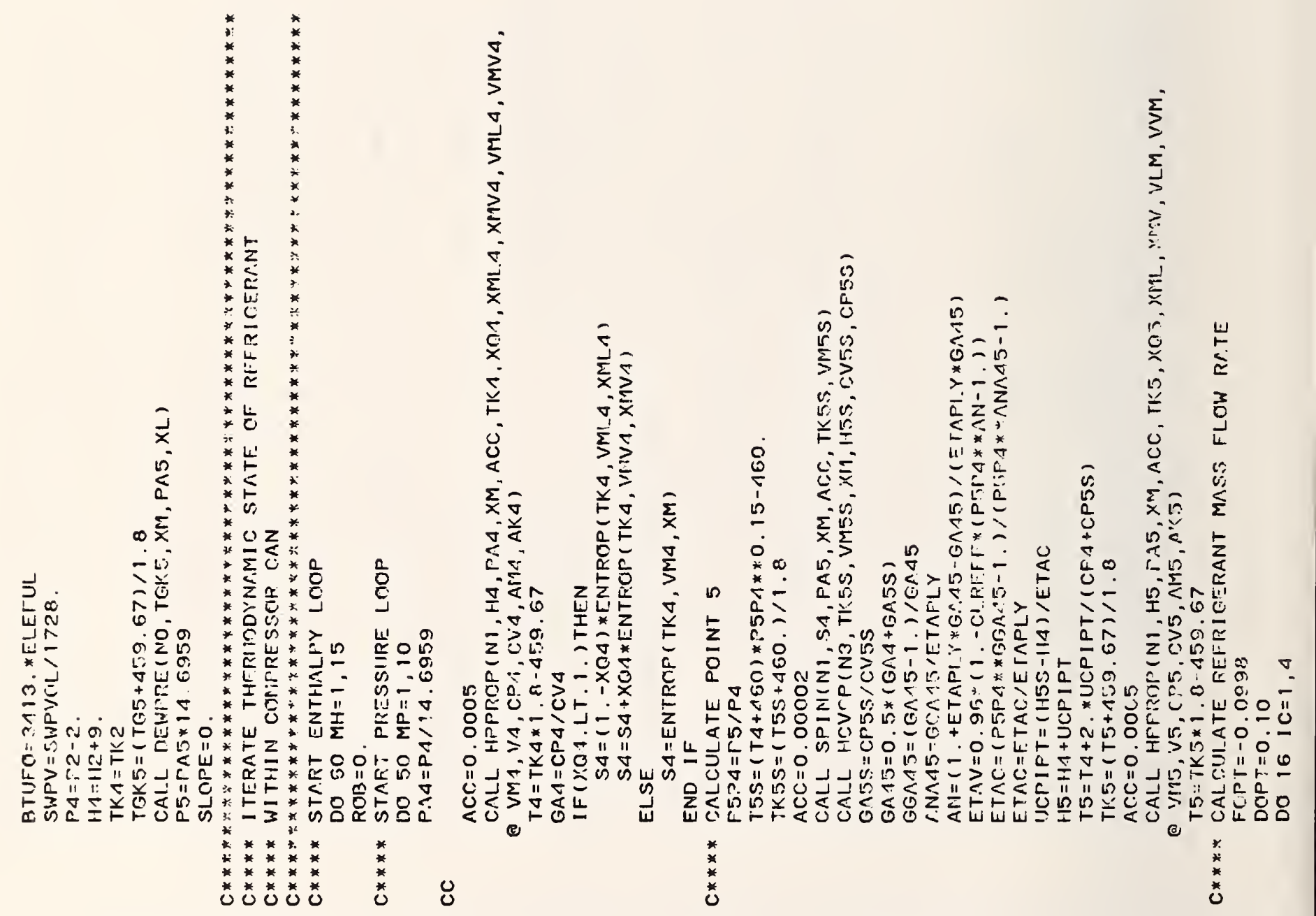

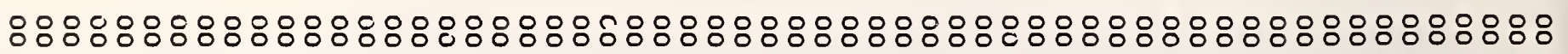
$\frac{\substack{\alpha \\ \alpha}}{\sum_{0}^{0}}$

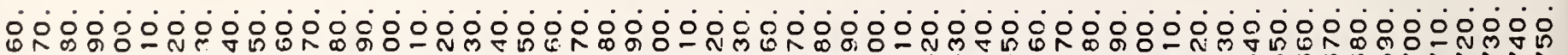

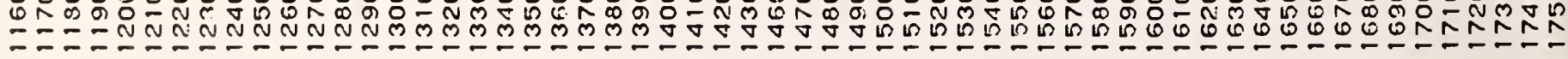




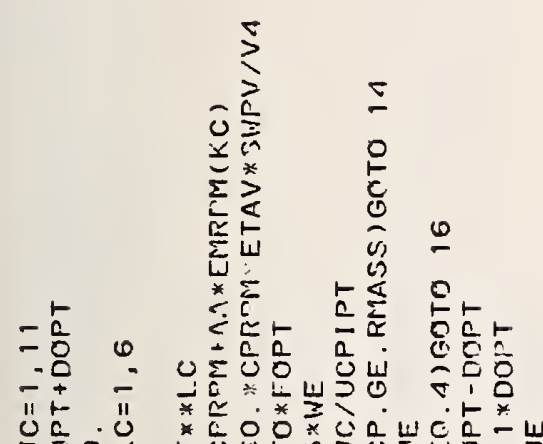

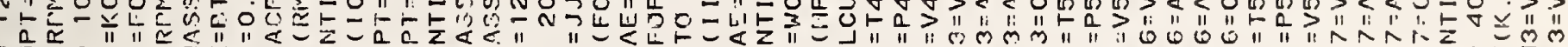

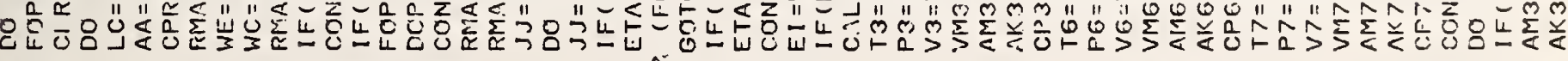

$$
\because \quad \cong \quad \underline{\square}
$$

$\stackrel{\infty}{N} \stackrel{N}{\sim}$ 


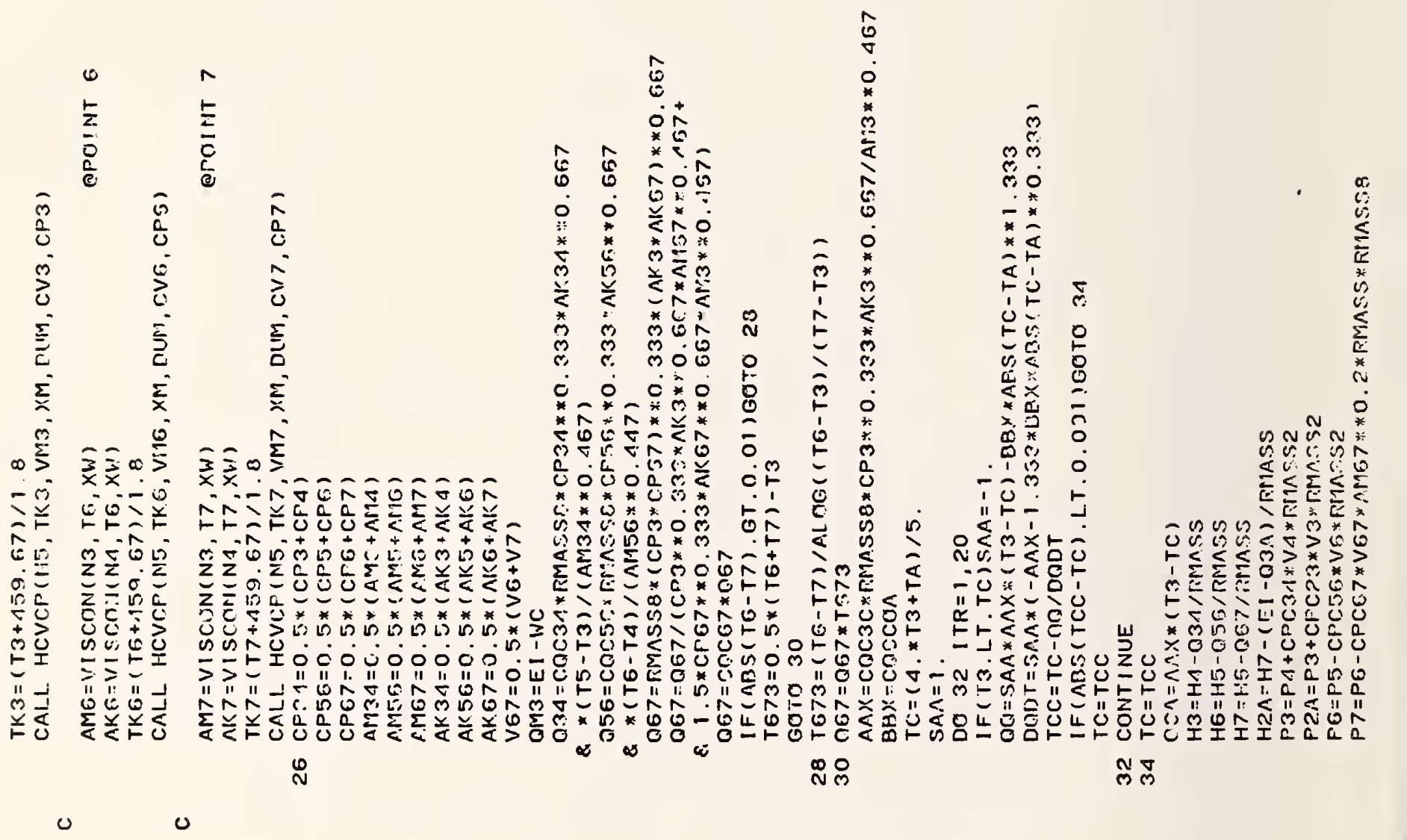

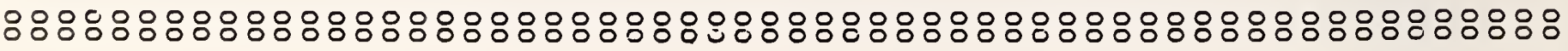

崩

은

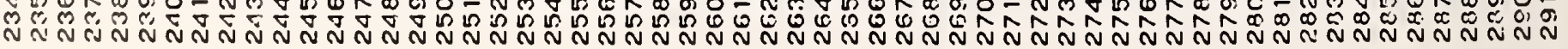




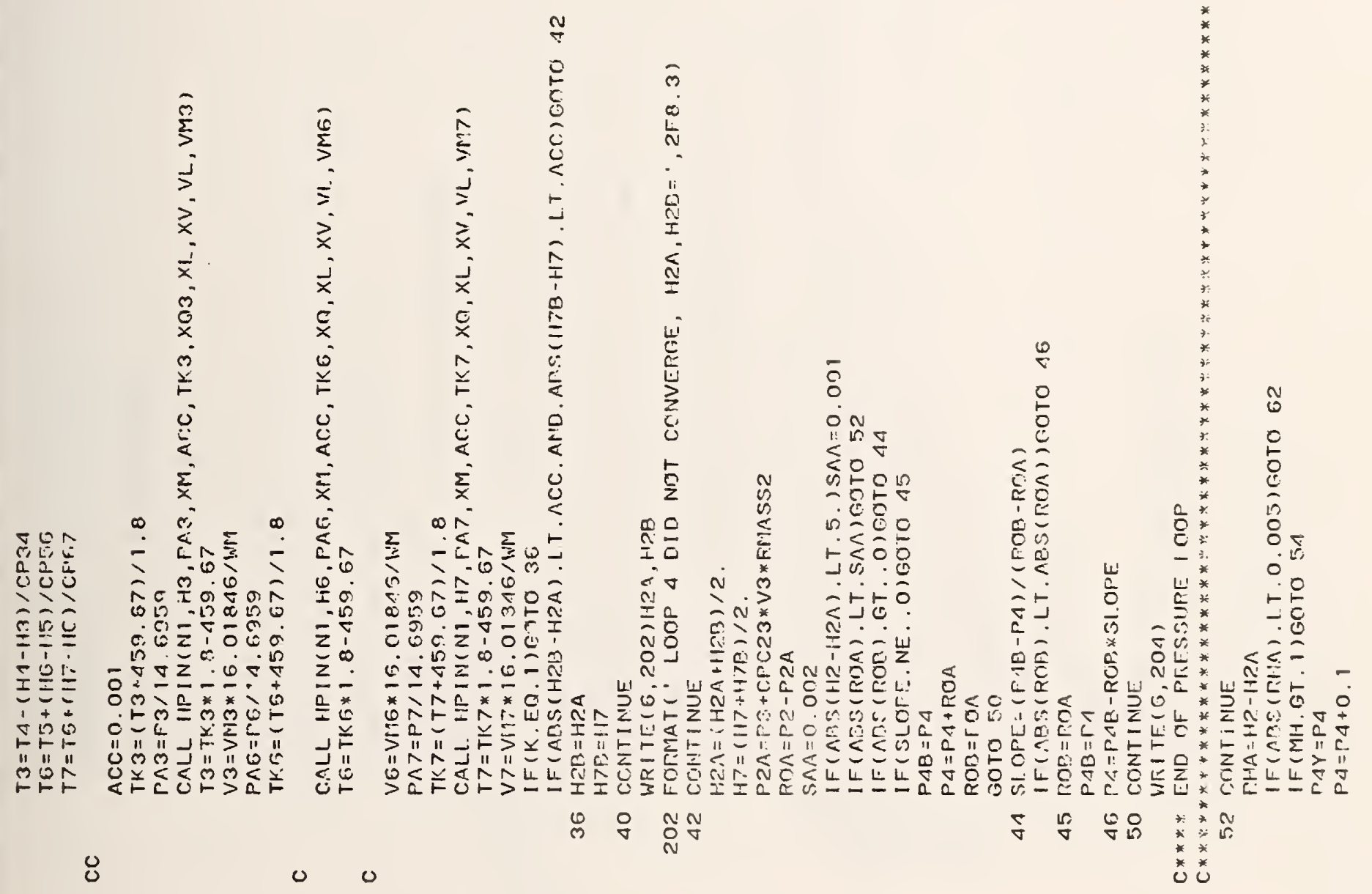

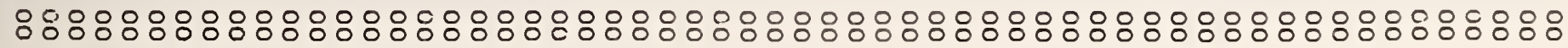

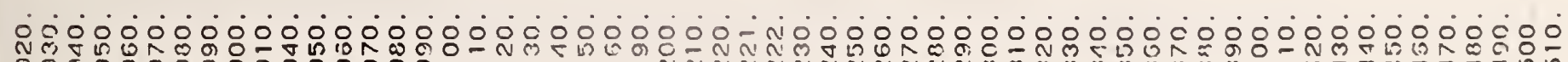
N 

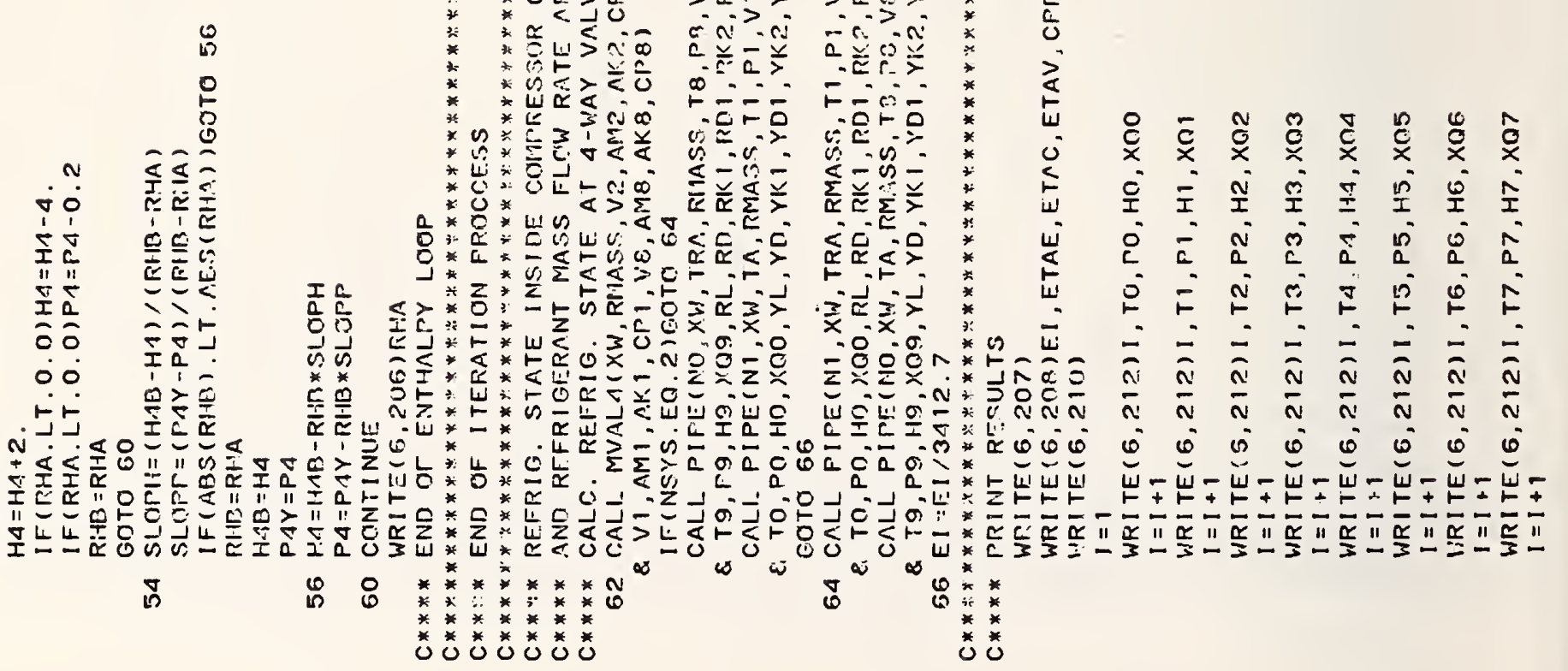

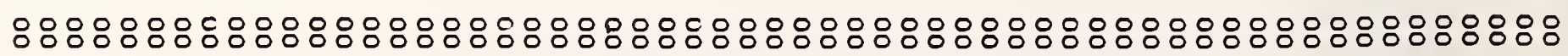

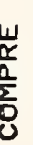

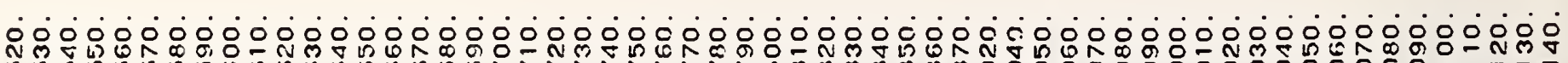

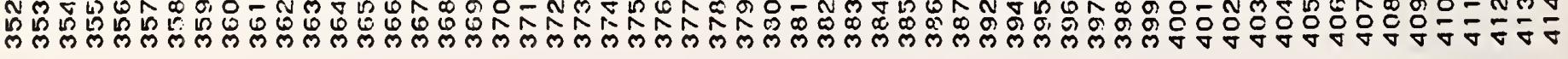




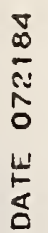

is

$\underline{-}$

ส․

$\stackrel{m}{=}=\frac{x}{x}$

$x^{-m}=\dot{0} ; \bar{x}$

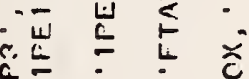

I

$x=-\frac{1}{2}$

ㄷㄷㄷ.:

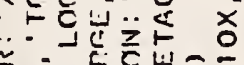

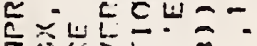

o - $3<x$

$\infty$ o

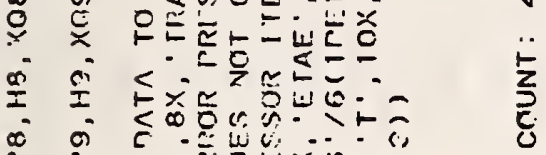

a $a a^{\infty}$ a

o

$== \pm$

ก $\frac{1}{n}$ n.

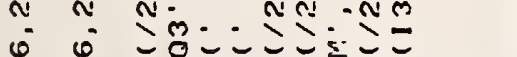

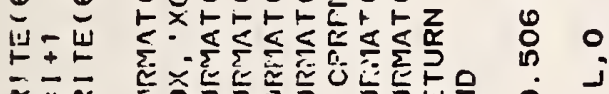

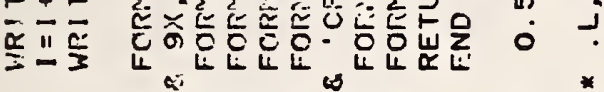

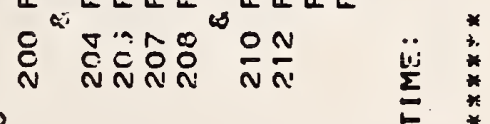

0

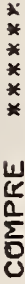

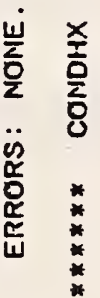

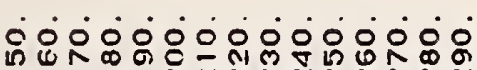

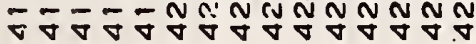

$\begin{array}{ll}\stackrel{5}{w} & a \\ \sum_{w}^{0} & \stackrel{0}{0}\end{array}$ 


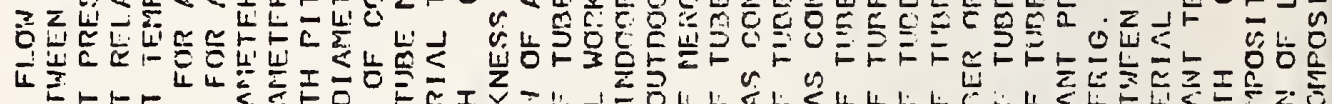
U.

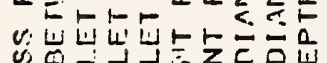

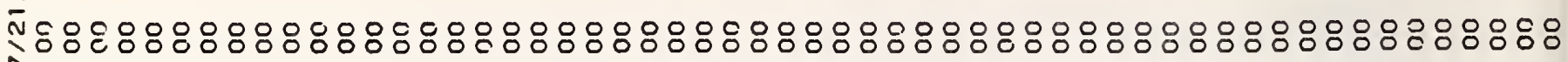

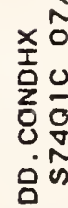

य 岃 
跑

1

䉚

=

는

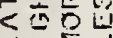

茫落

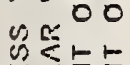

记罗

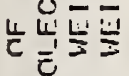

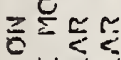

崖崖

论品焉

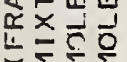

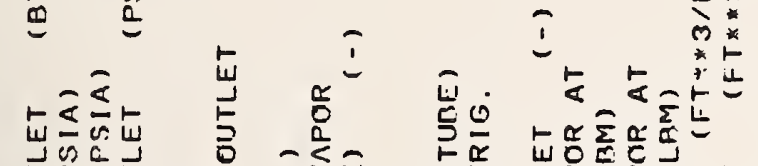

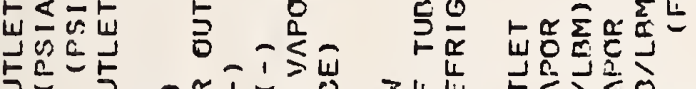

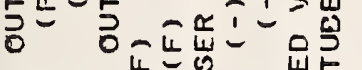

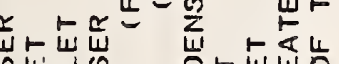

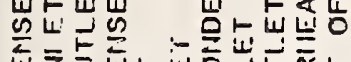

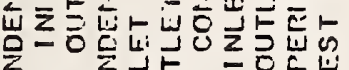

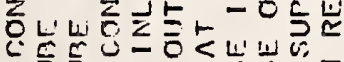

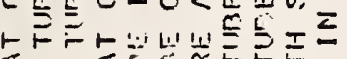

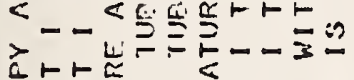

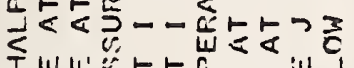

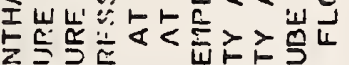

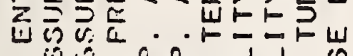

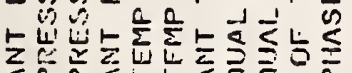

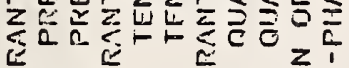

亭岂岩

宁等方出

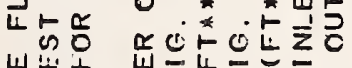

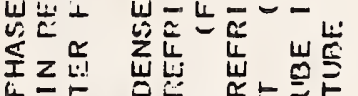

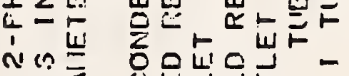

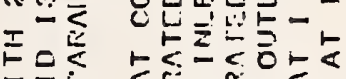

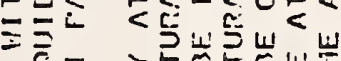

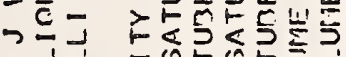

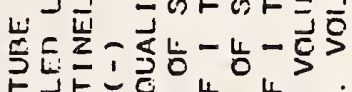

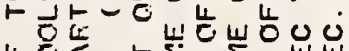

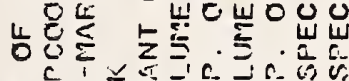

z

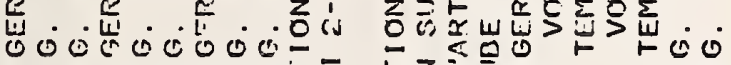

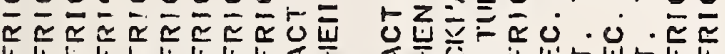

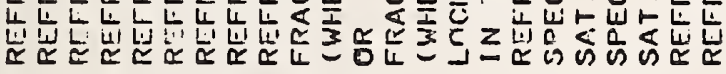

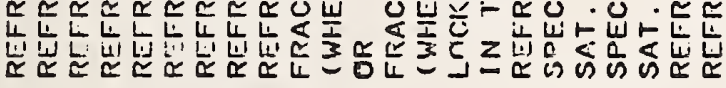

111

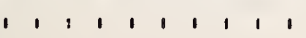

$$
1111
$$

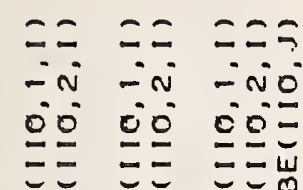

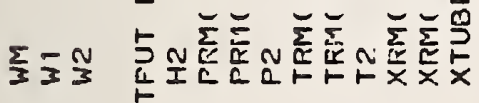
ठ

0000000000000000000000000000000000

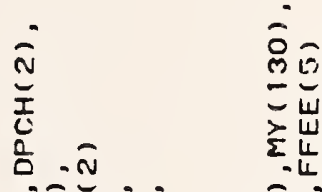

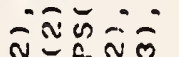

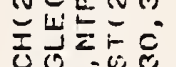

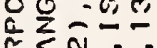

$\sum \leq n-i$

añ

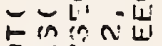

$0 \leq \angle-5=$

i< $<\frac{0}{<} \div$

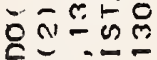

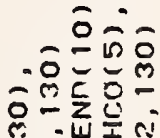

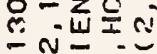

nit:- $=0$

กำ

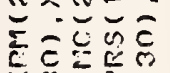

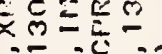

- ñón

cocios

完宫

吕市

爻它

을

岂岂

少

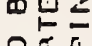

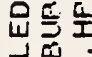

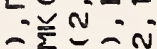

กิำลำ

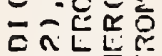

ง กิ

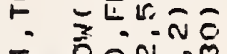

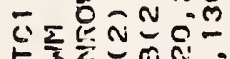

i-

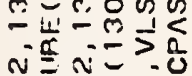

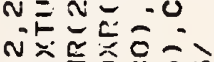

늠ำ

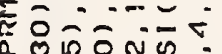

-

ON级

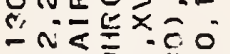

ลñ

-

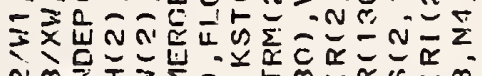

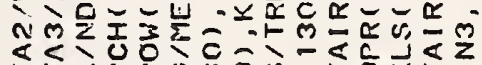

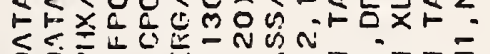

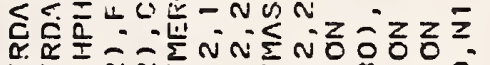

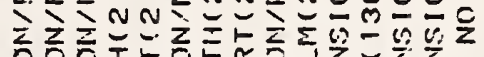

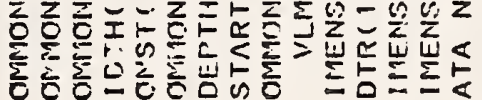

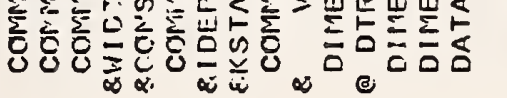

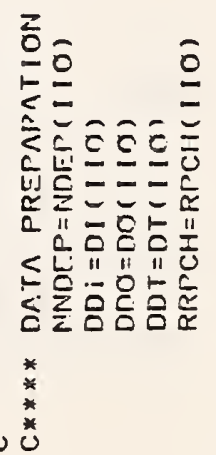

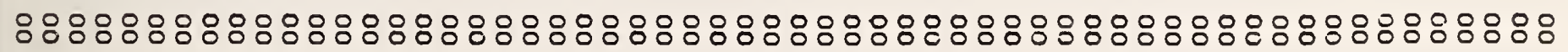

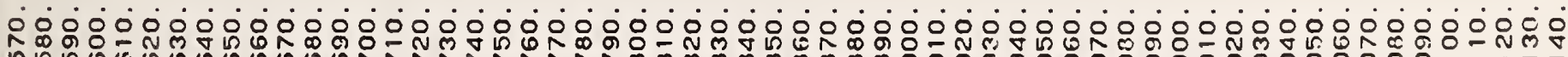
เด 


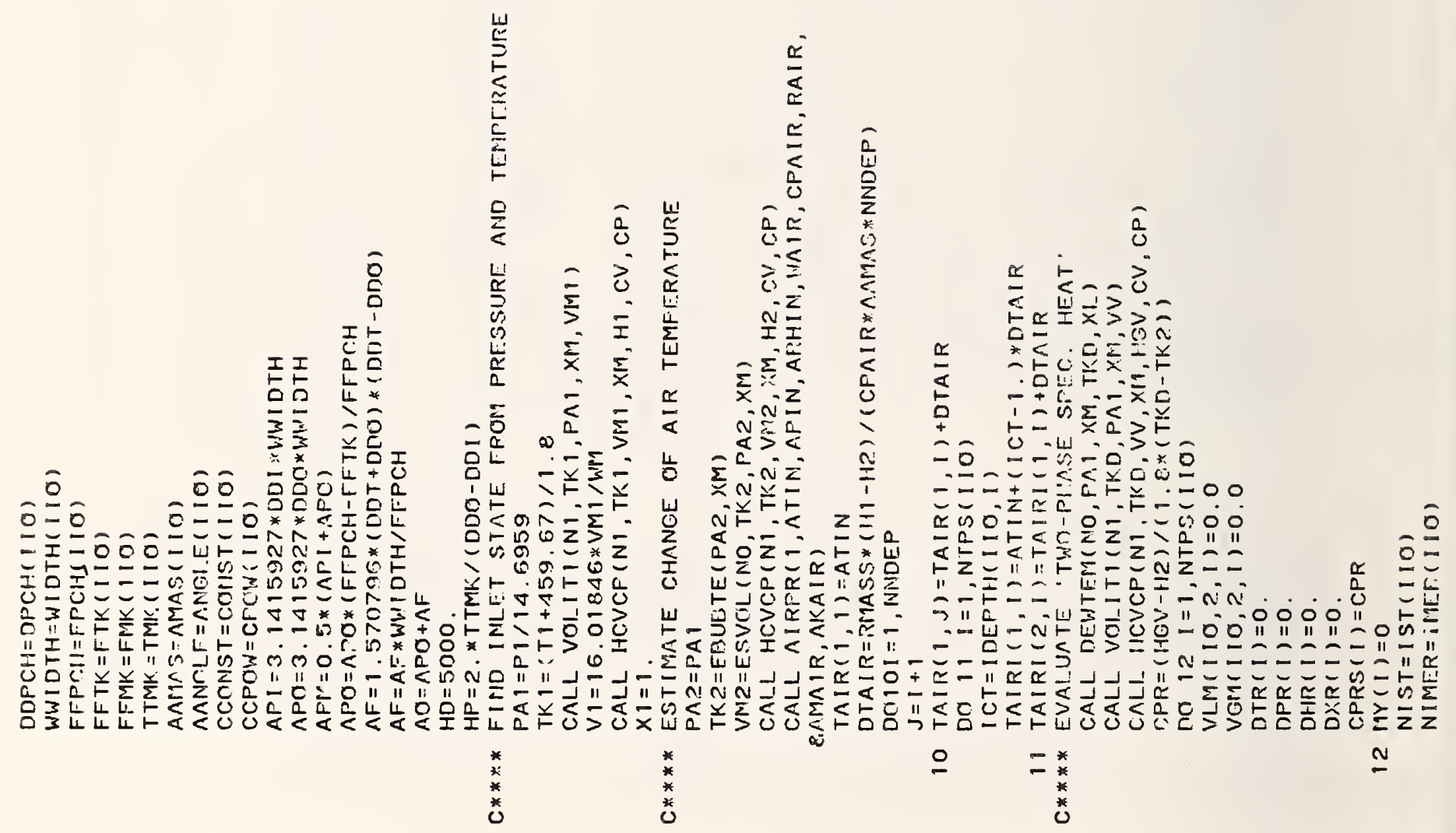

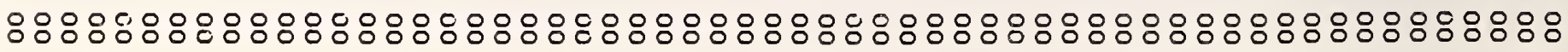




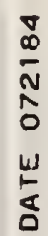

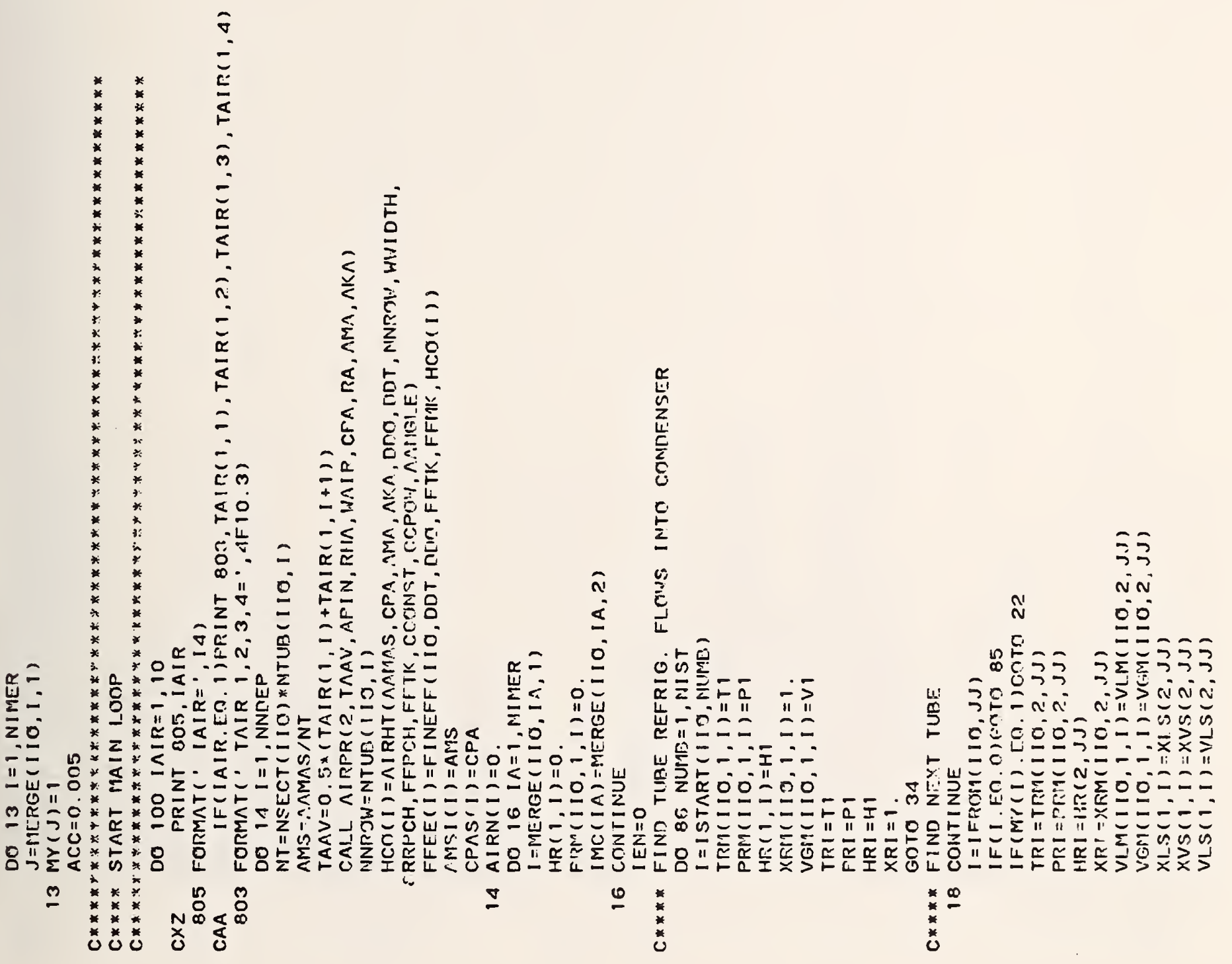

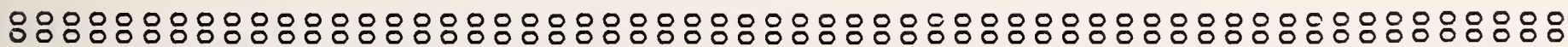
좀

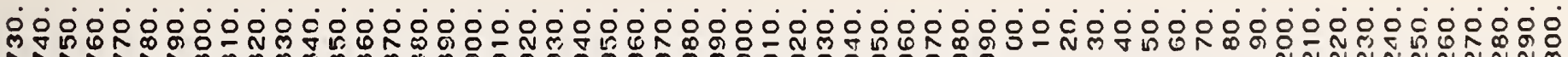

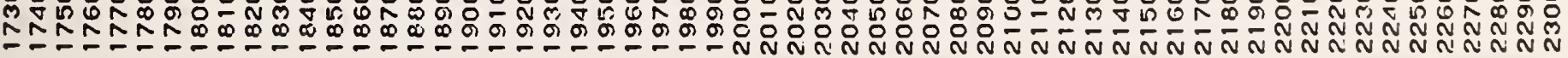




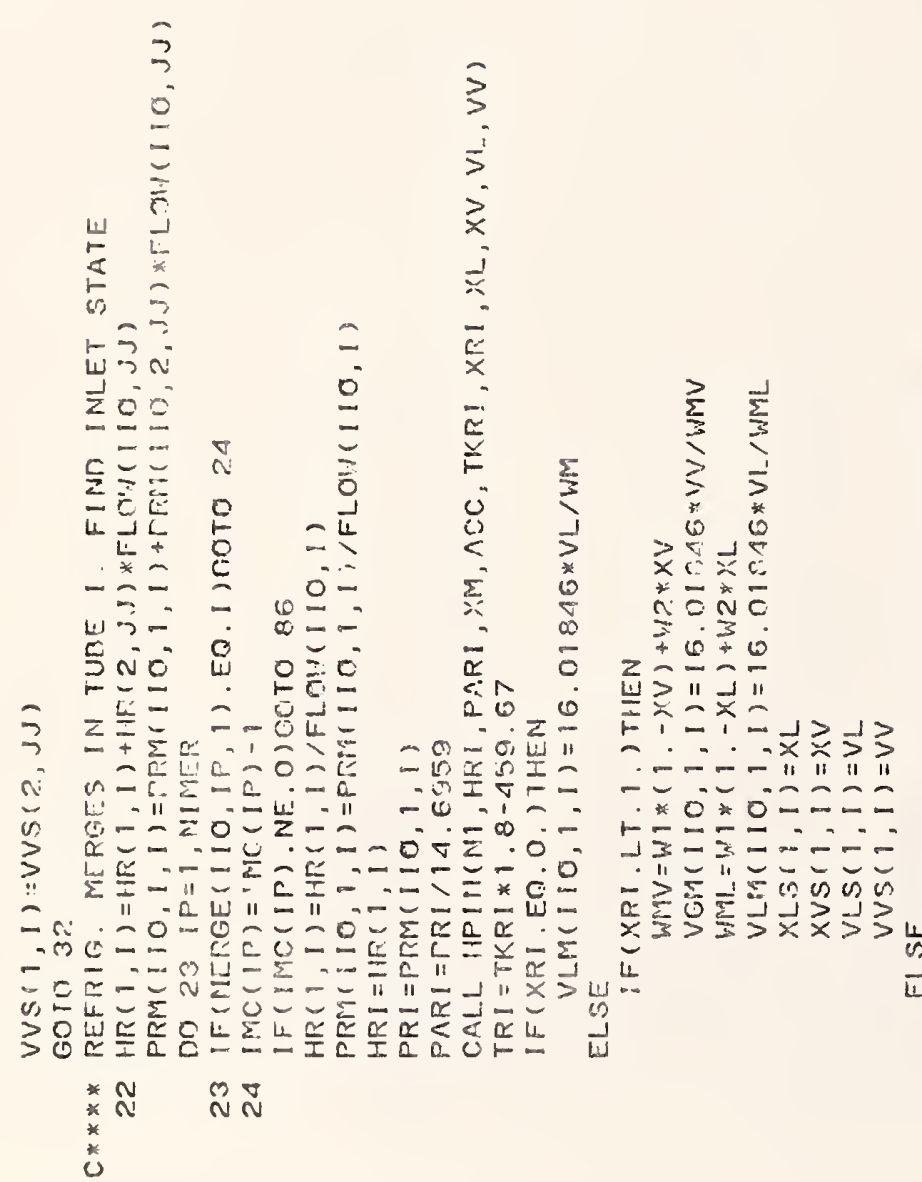

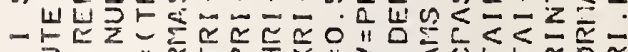
-55

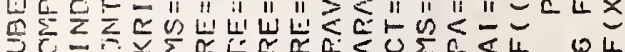

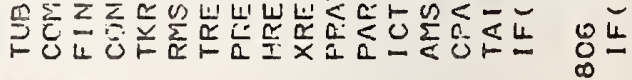

$\begin{array}{lll}* * * & * \\ * & * & *\end{array}$

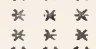

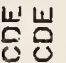

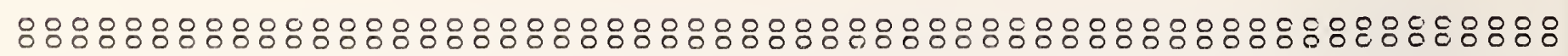

중

N 


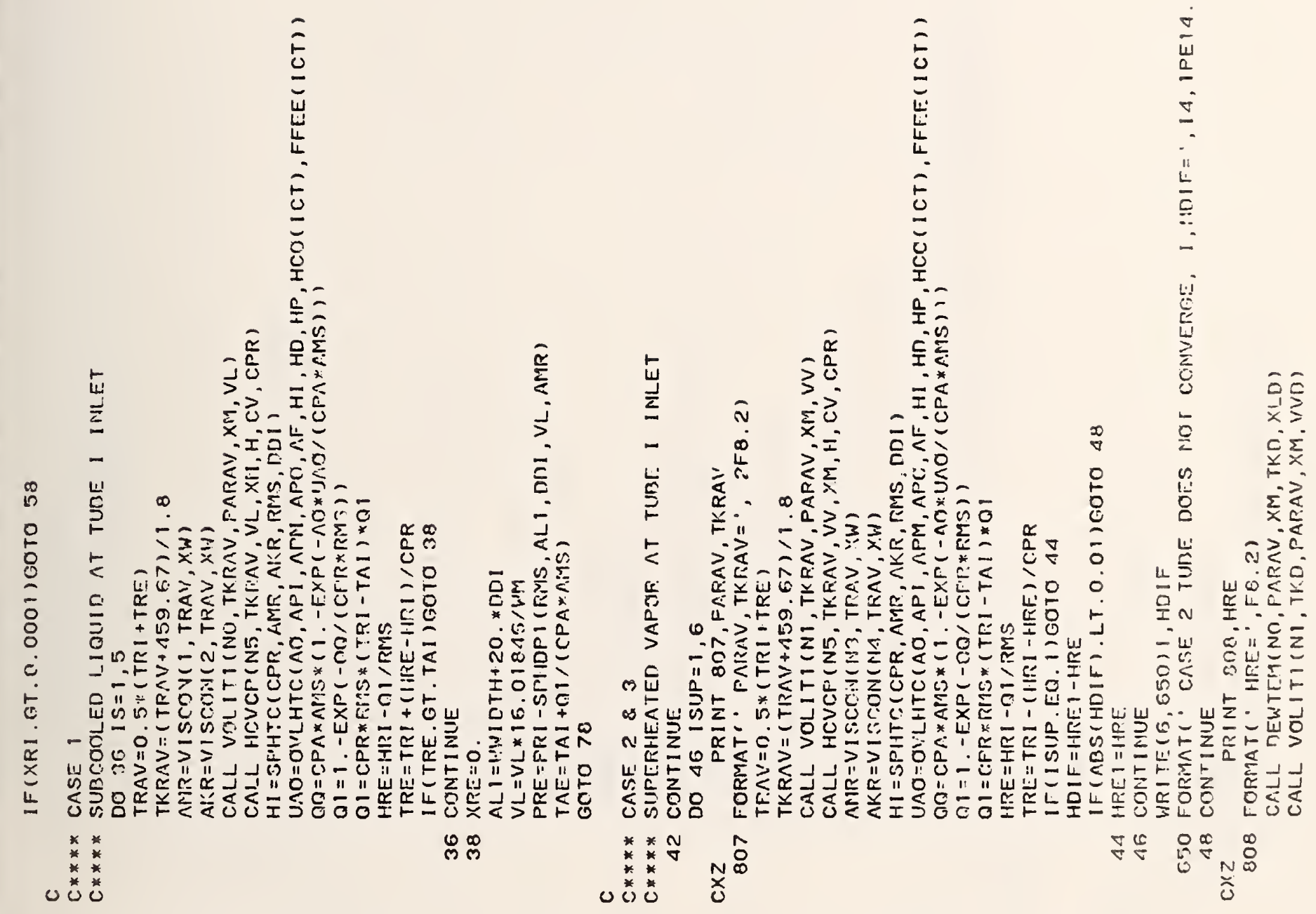

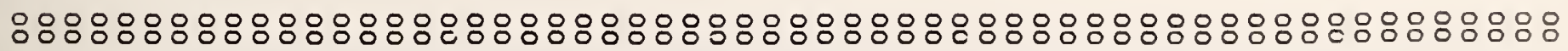

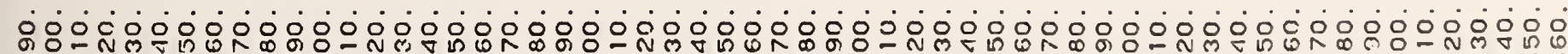

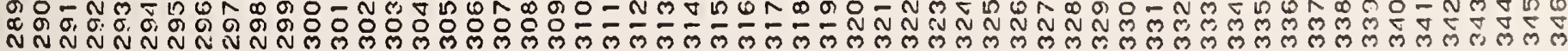




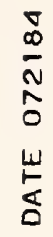

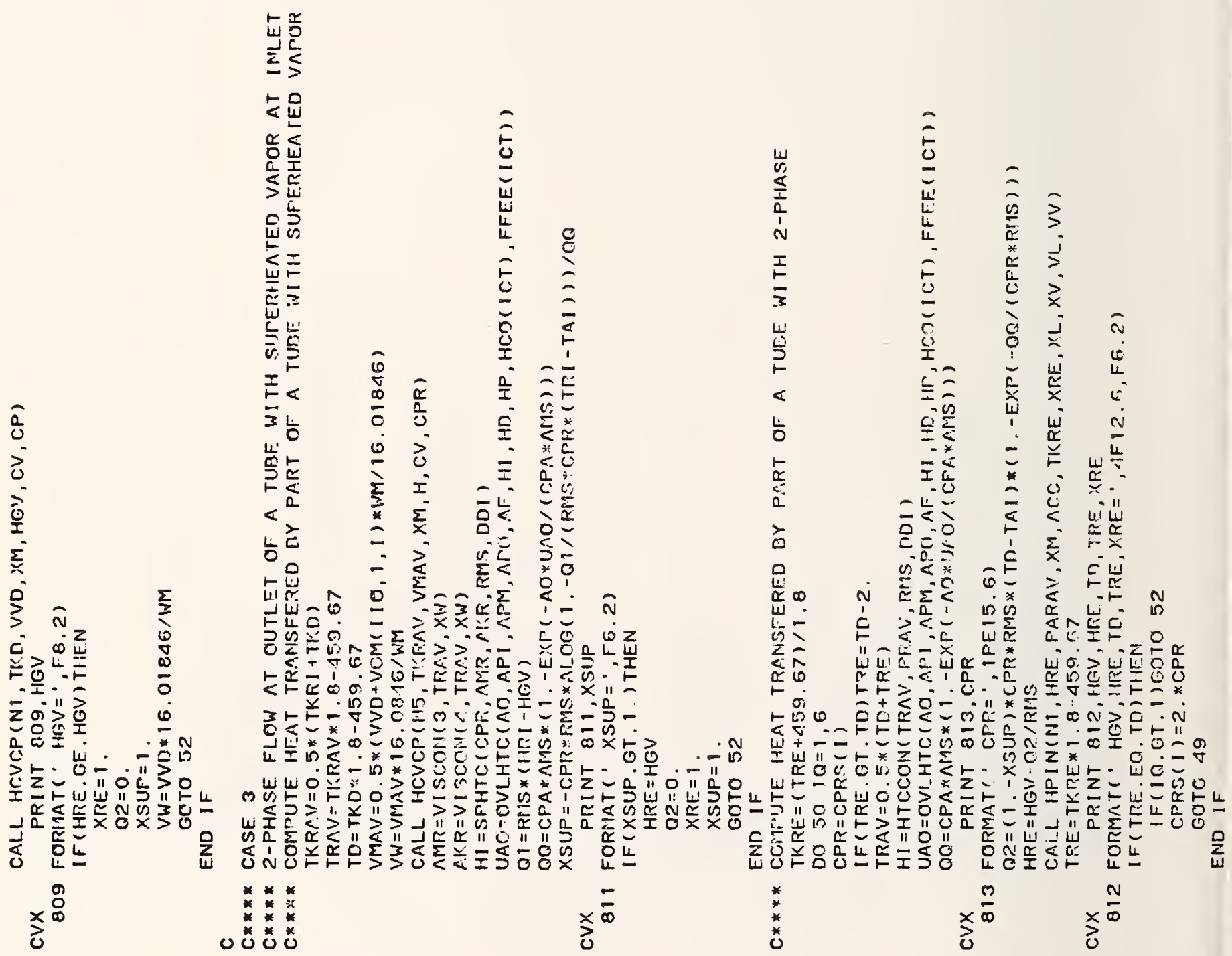

৪৪৪৪৪৪৪৪৪৪৪৪৪৪৪৪৪৪৪৪৪৪৪৪৪৪৪৪৪৪৪৪৪৪৪৪৪৪৪৪৪৪৪৪৪৪৪৪৪৪৪৪৪৪৪৪৪৪

종

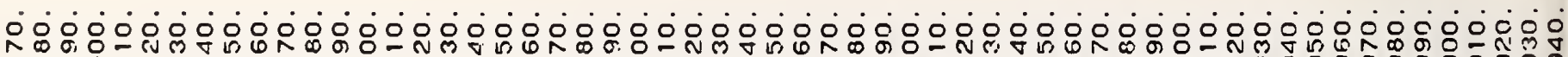
लेलिए户 


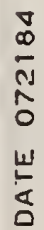

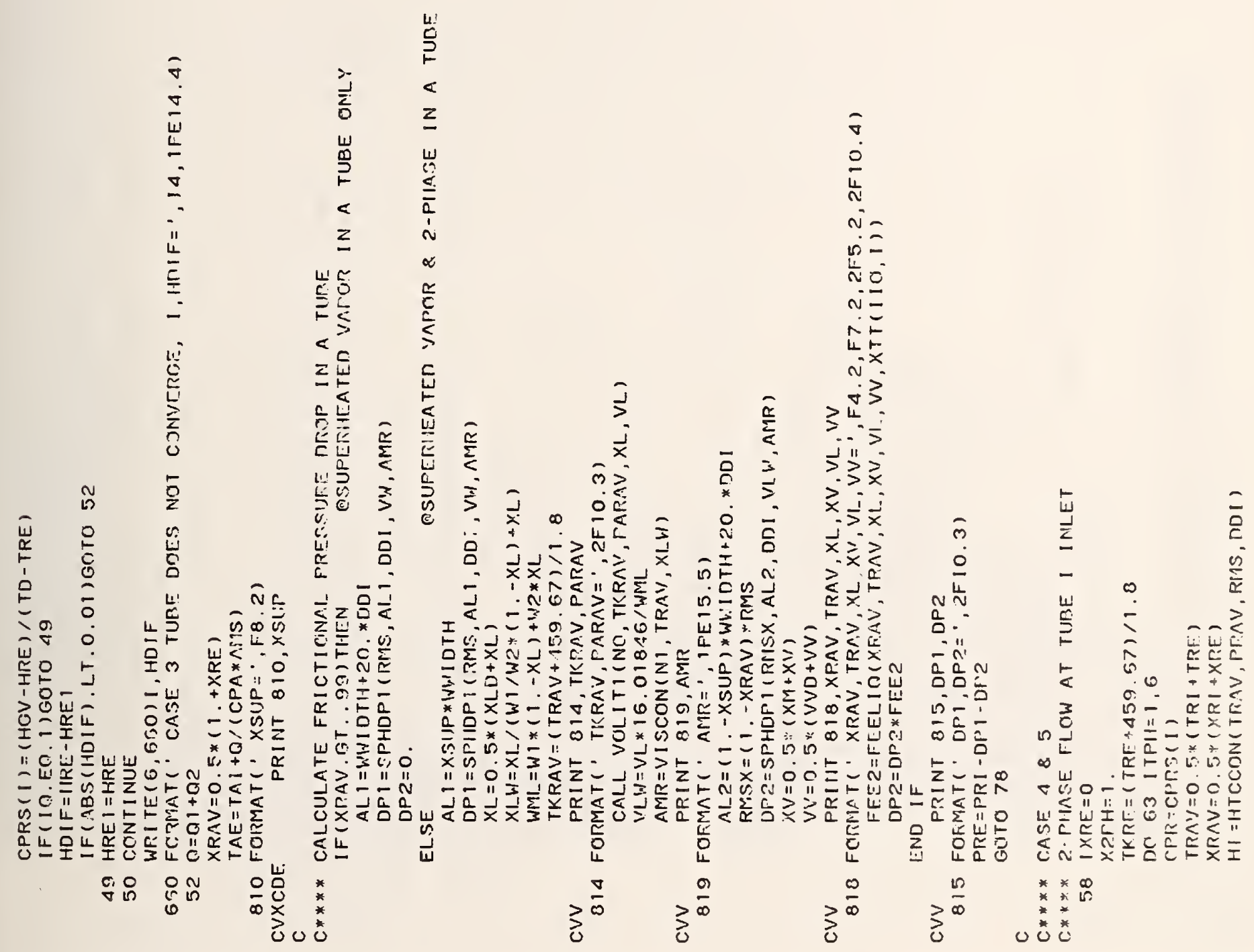

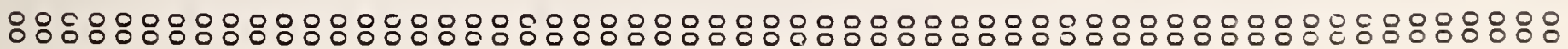

줄

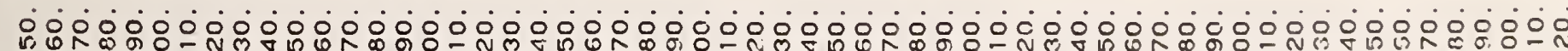

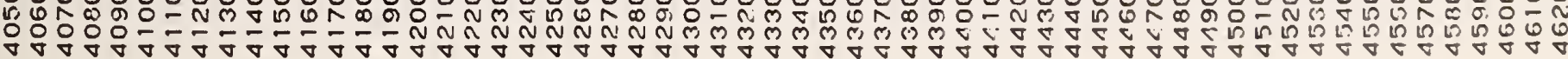



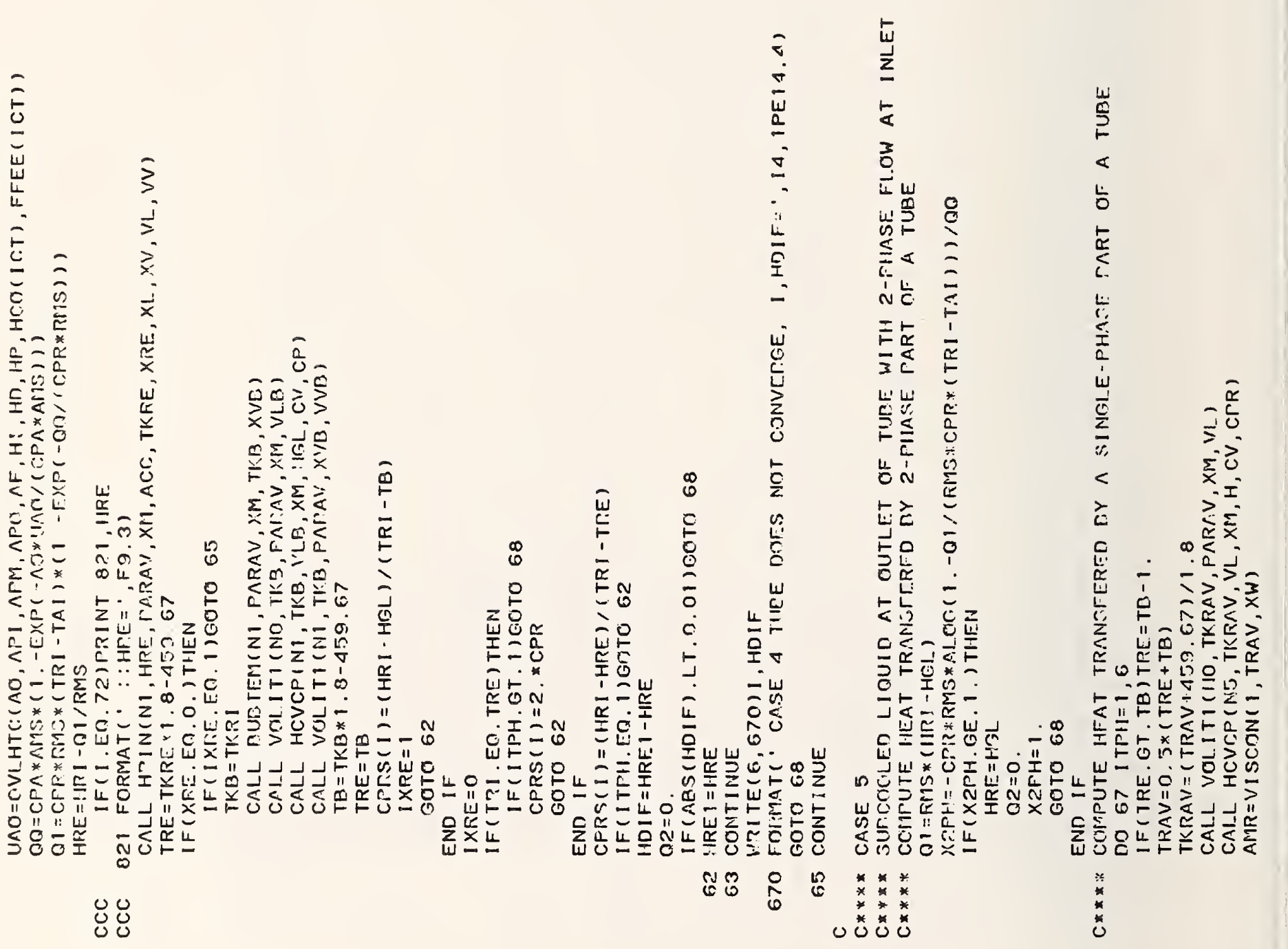

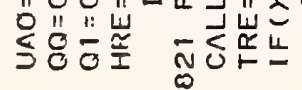

导导

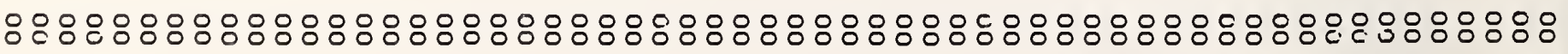

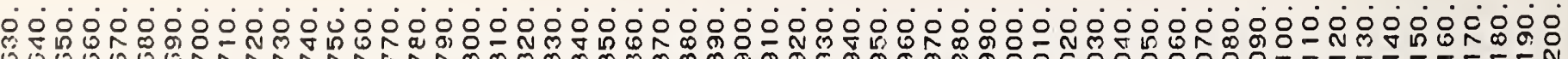

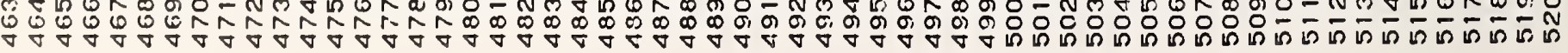




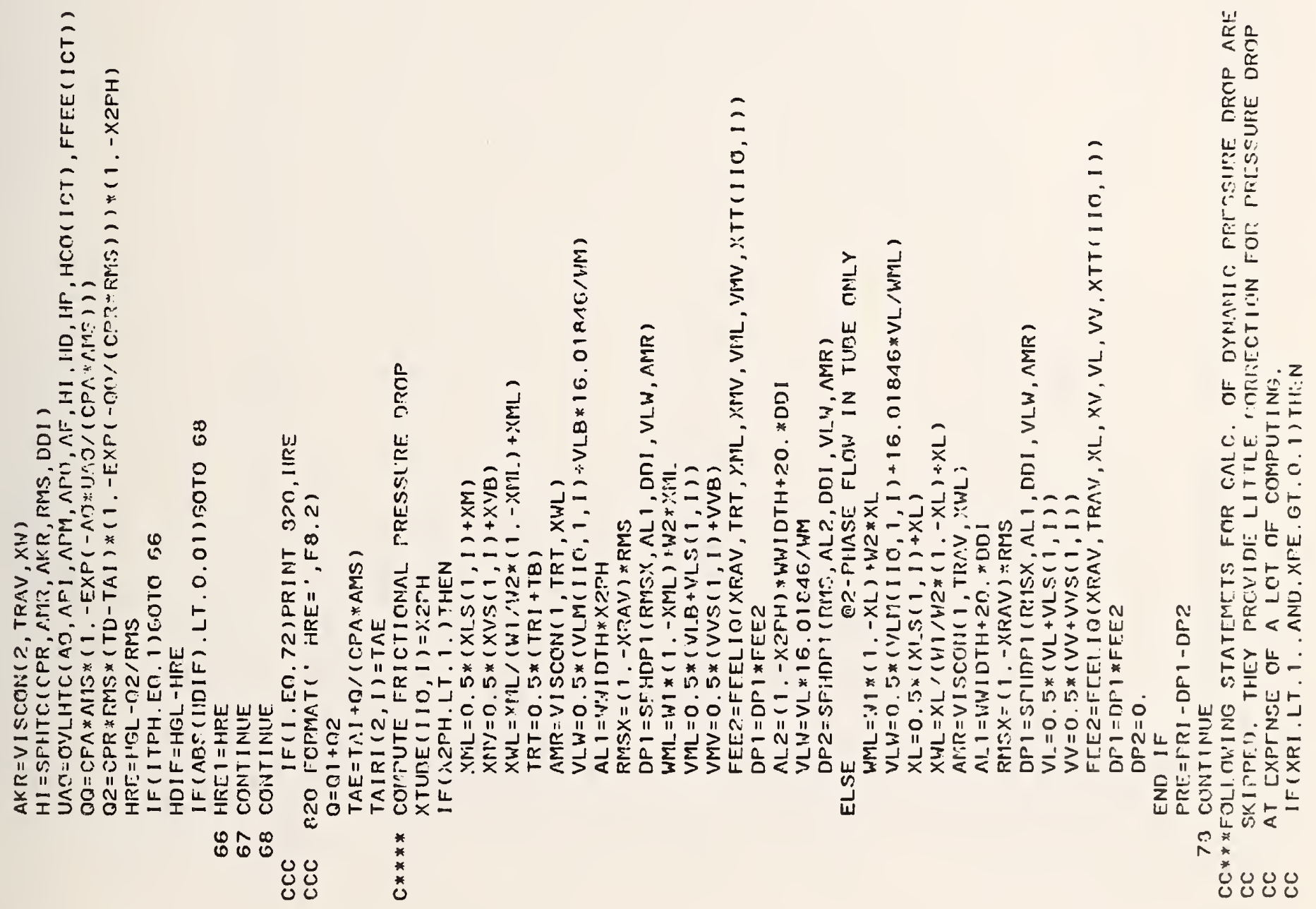

৪৪৪৪৪৪৪৪৪৪৪৪৪৪৪৪৪৪৪৪৪৪৪৪৪৪৪৪৪৪৪৪৪৪৪৪৪৪৪৪৪৪৪৪৪৪৪৪৪৪৪৪৪৪৪৪৪৪

좀

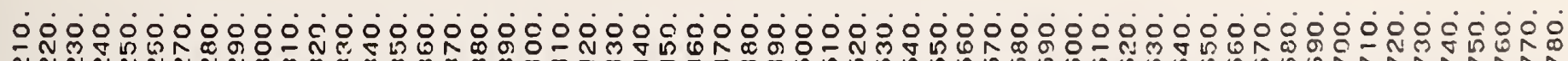

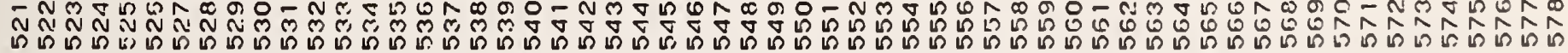




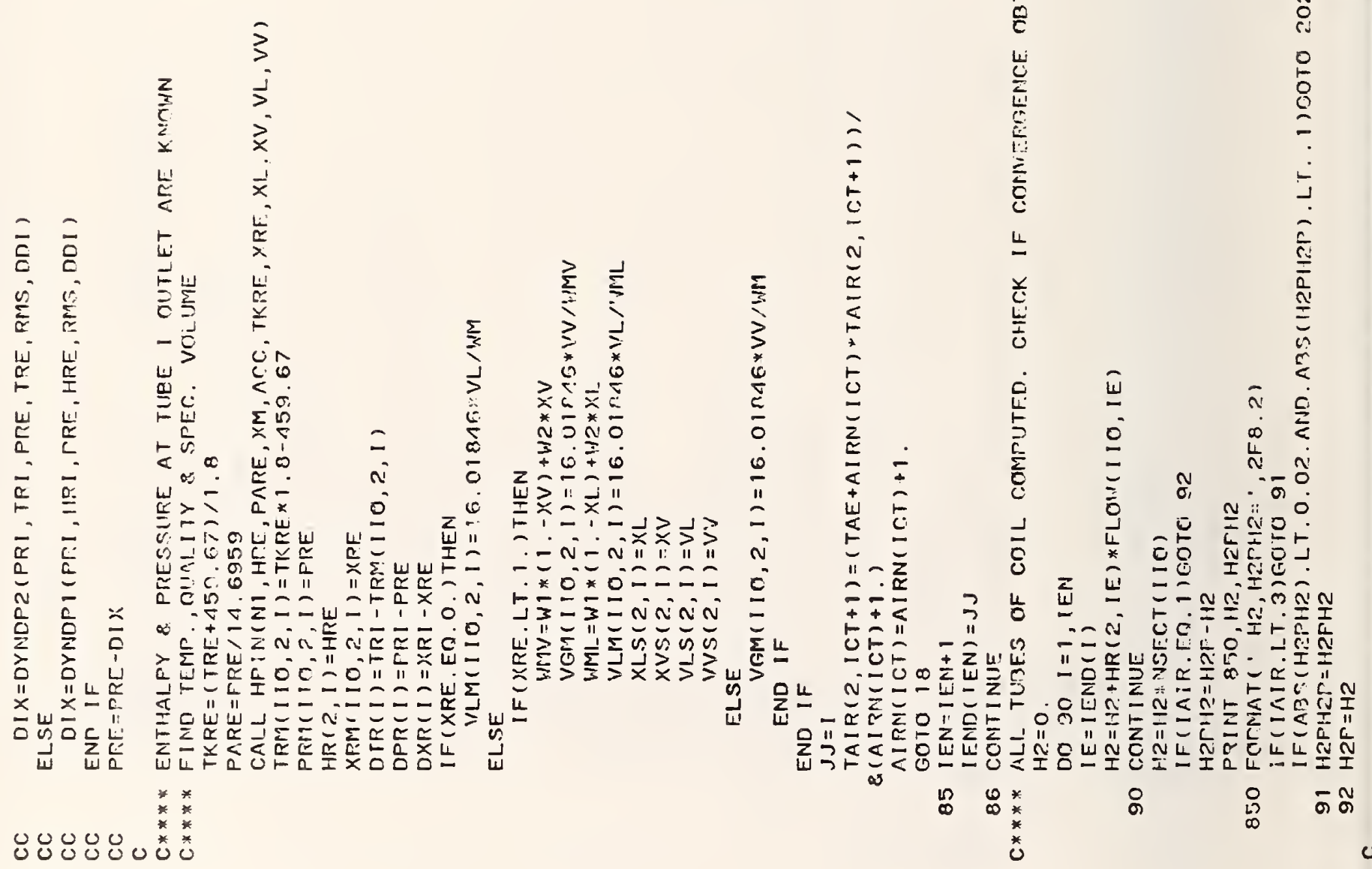

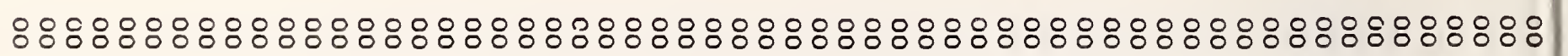

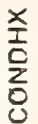

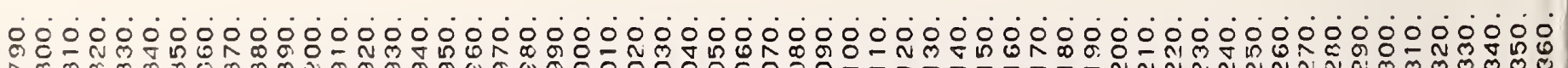

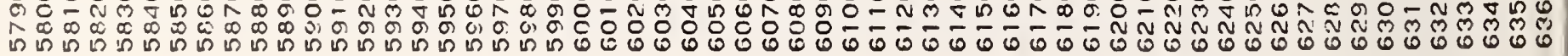




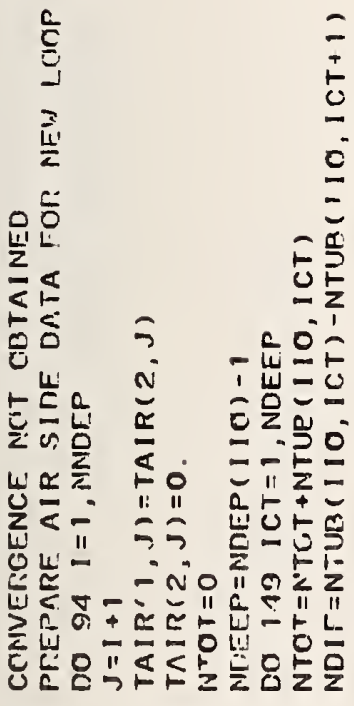

जั

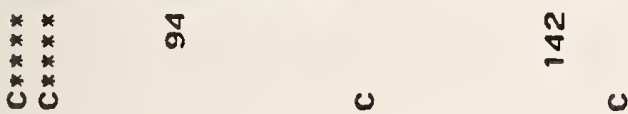

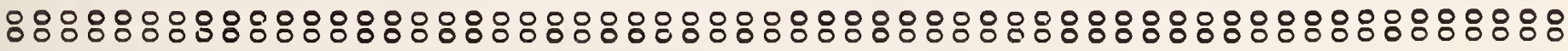

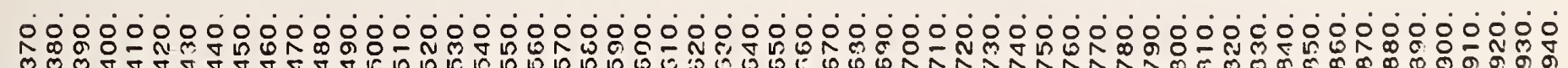

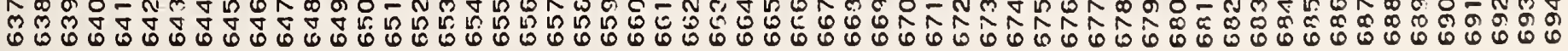




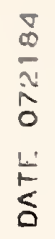

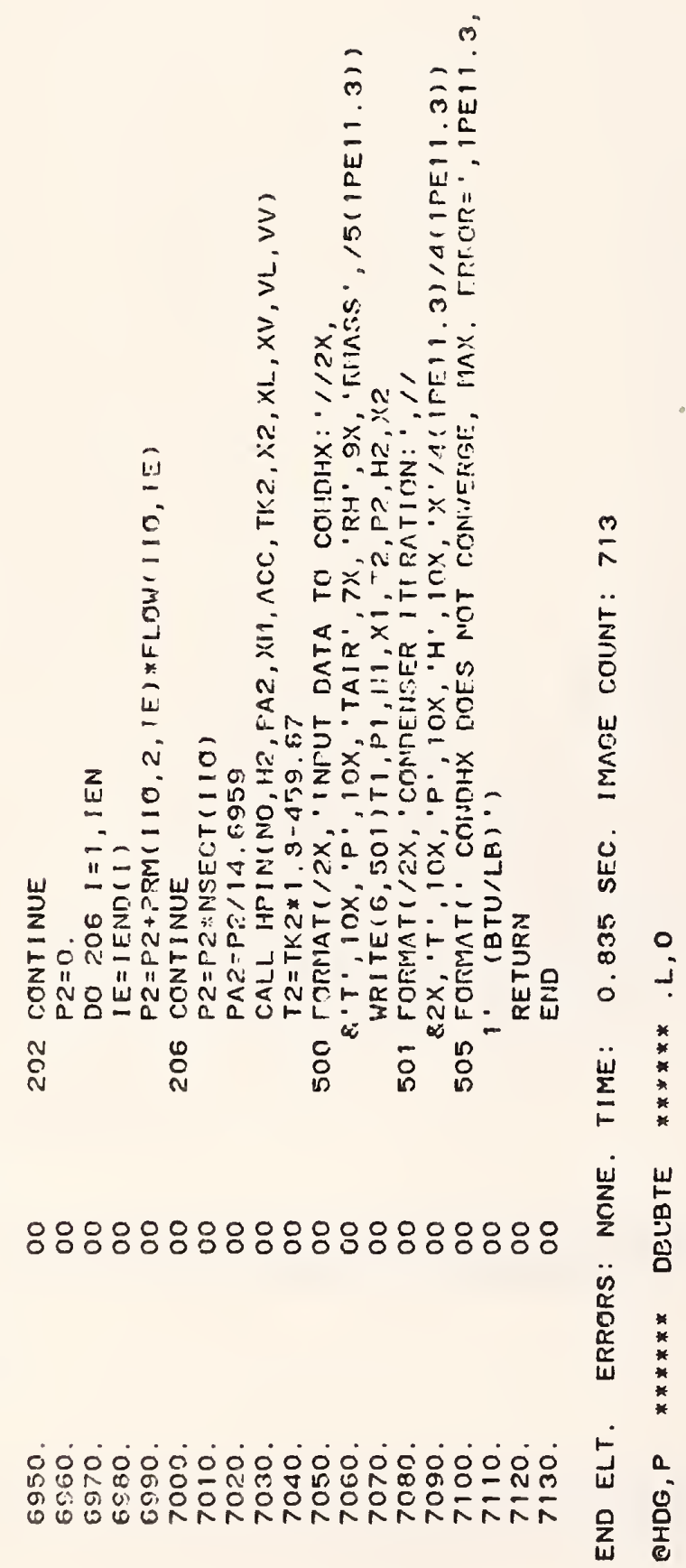




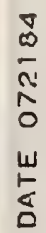

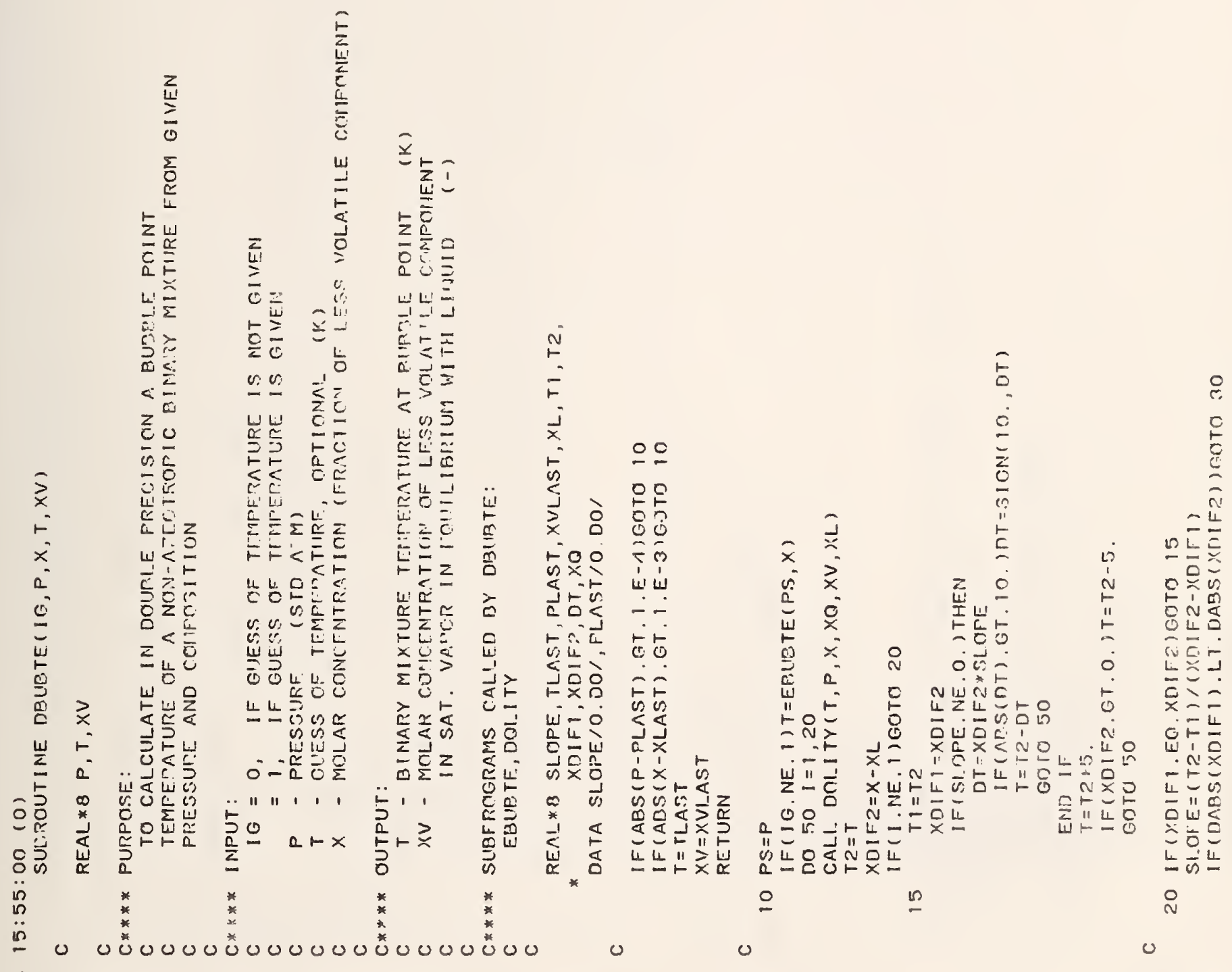




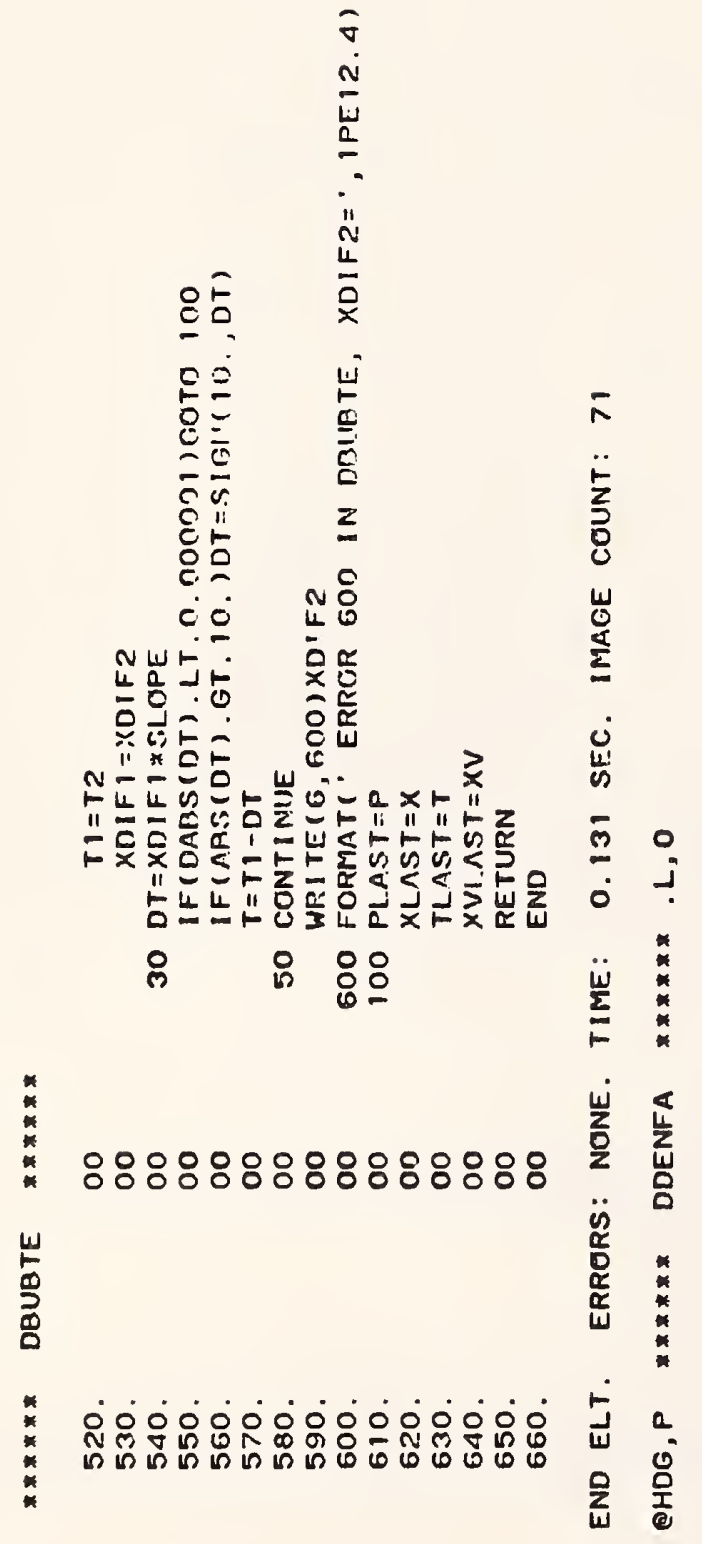




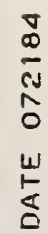

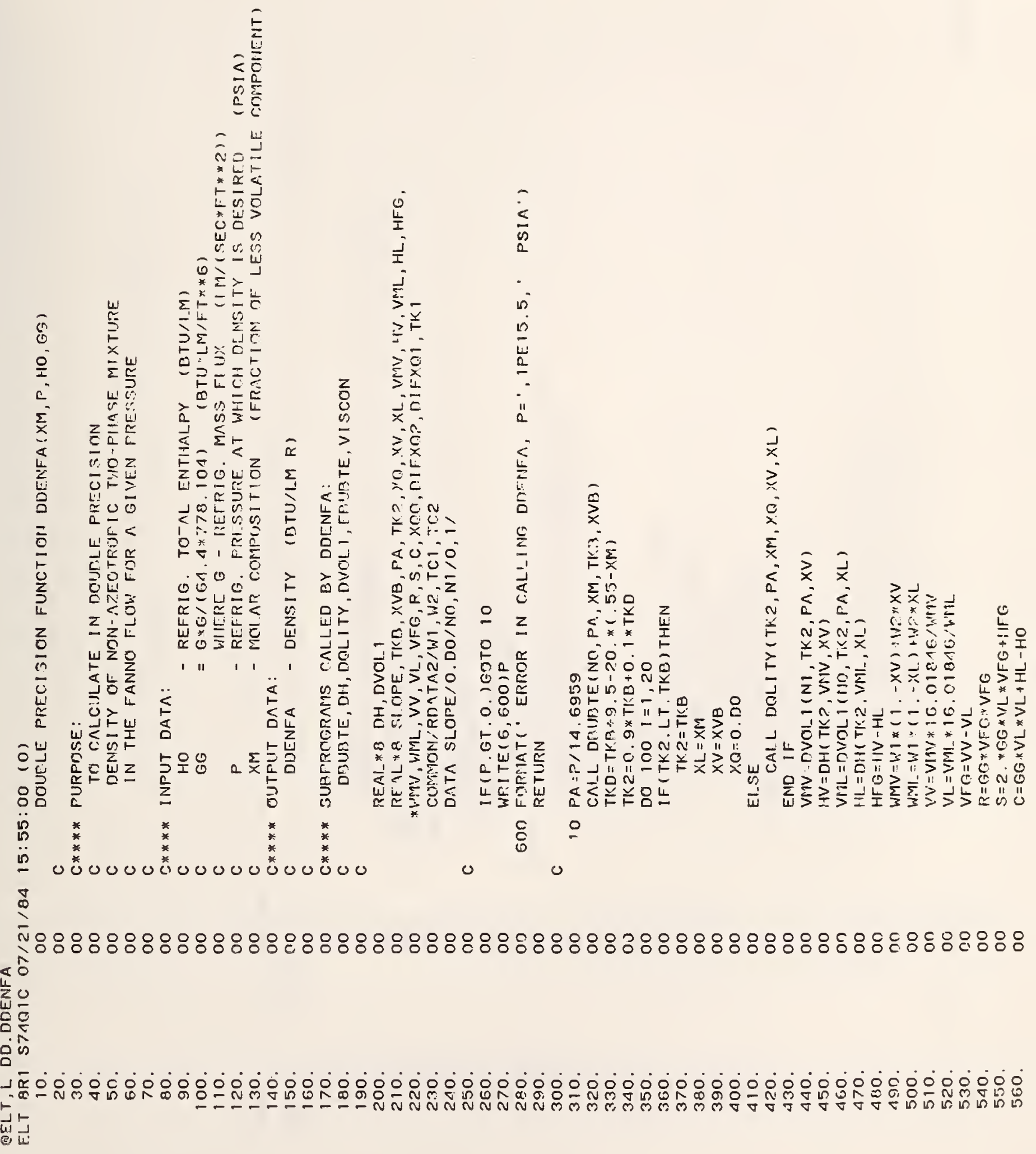



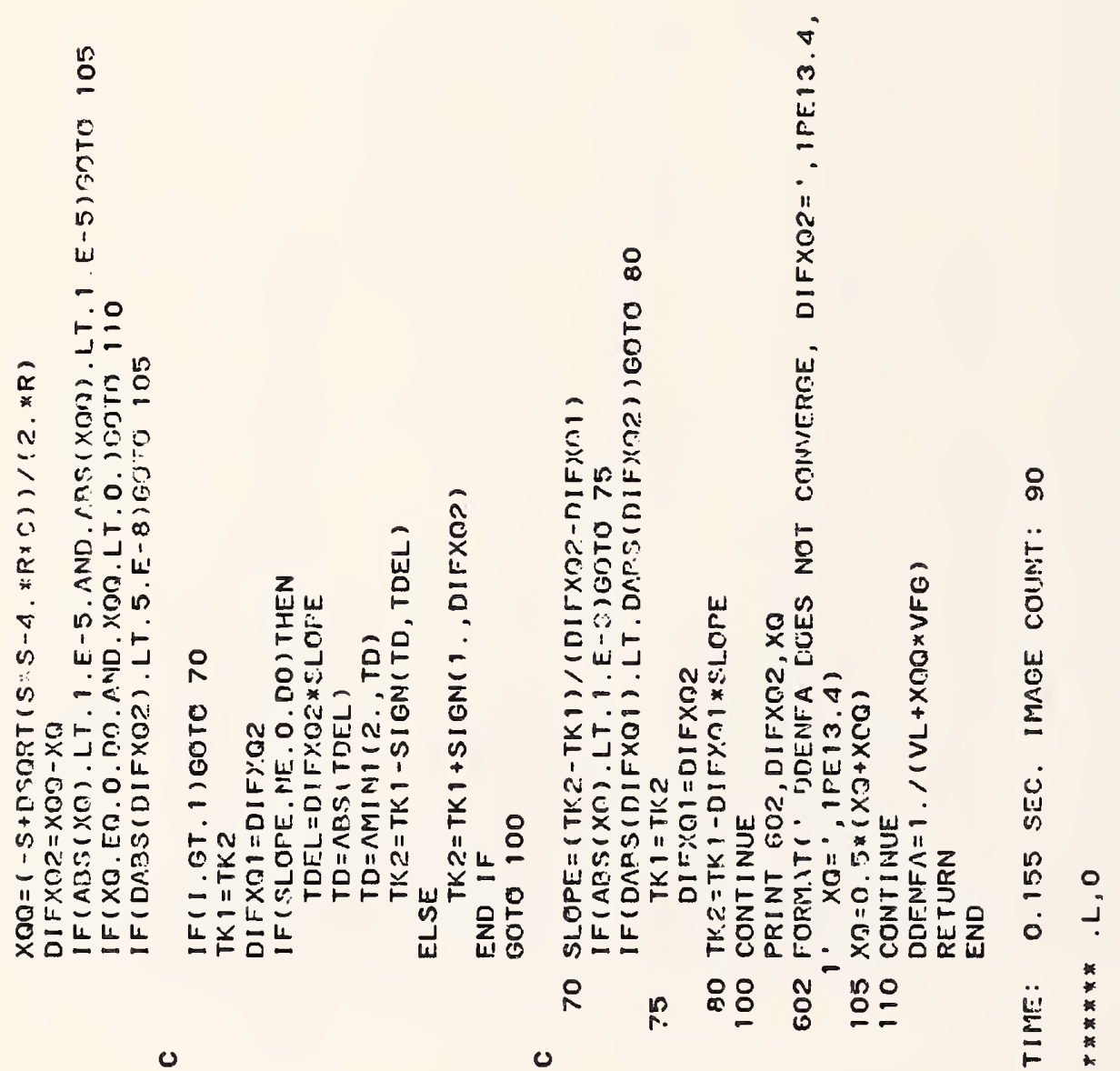

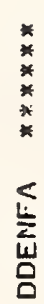

৪৪৪৪৪৪৪৪৪৪৪৪৪৪৪৪৪৪৪৪৪৪৪৪৪৪৪৪৪৪৪৪৪৪

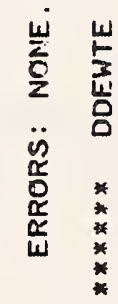

ن

虫 
$\frac{D}{\infty}$
$\frac{N}{N}$
$\omega$
$\frac{5}{\sigma}$

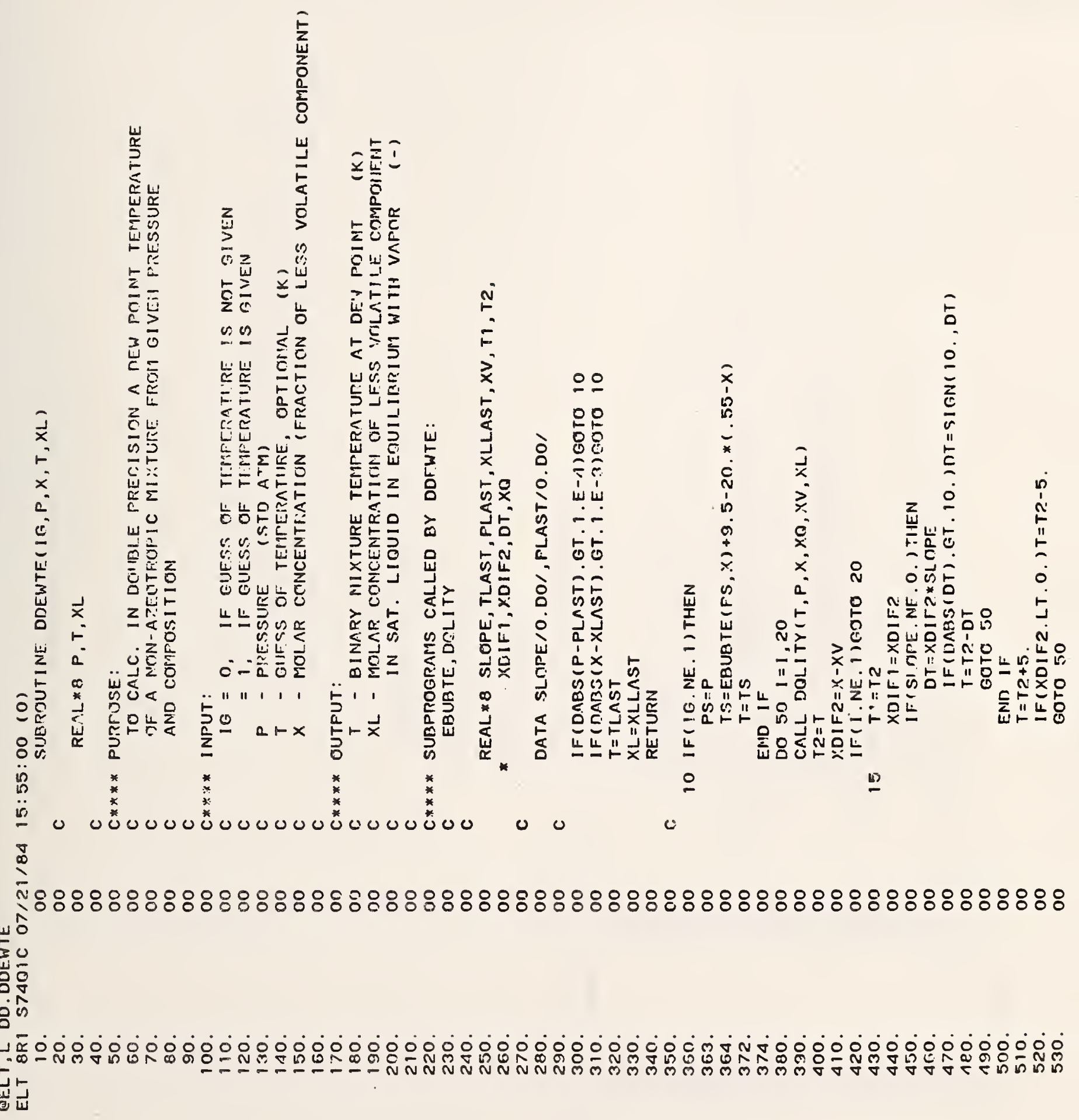




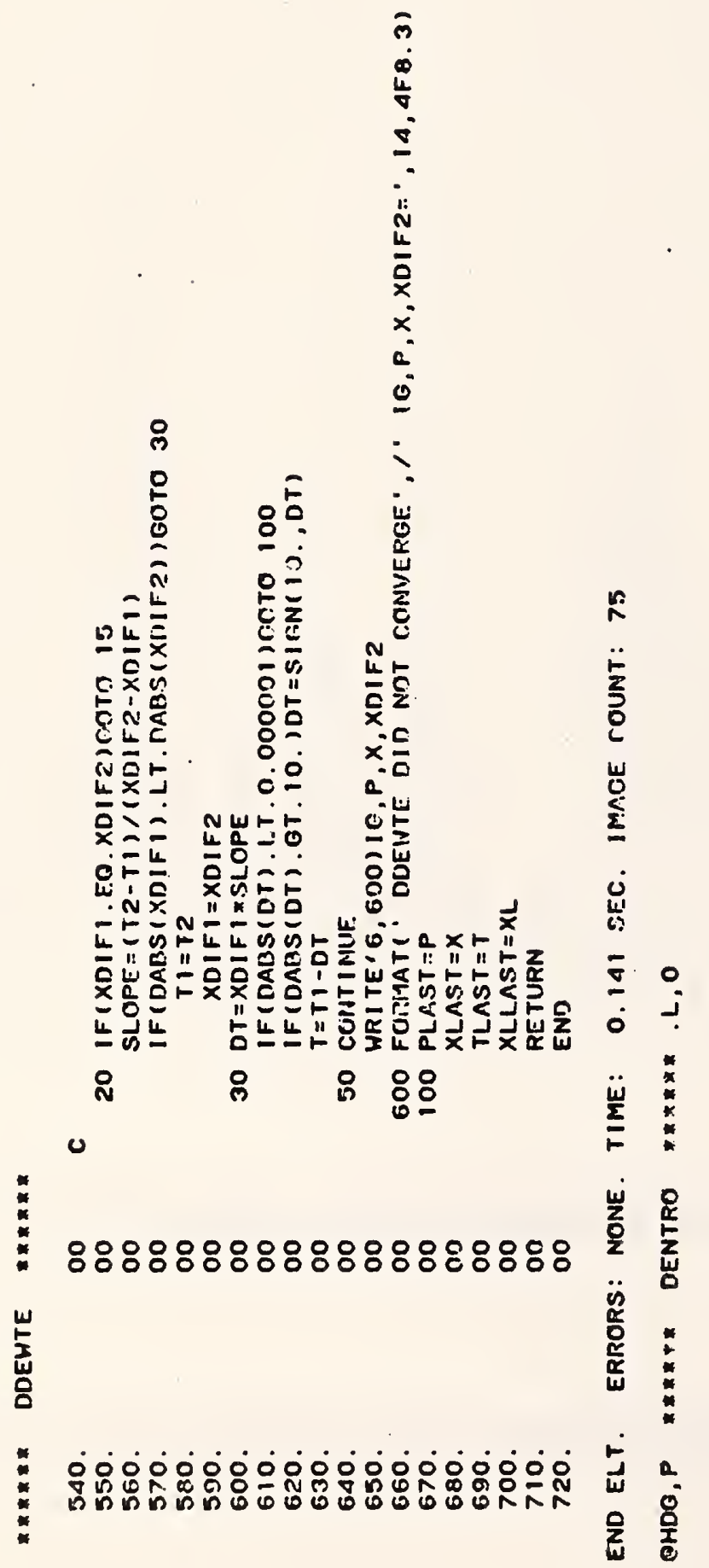




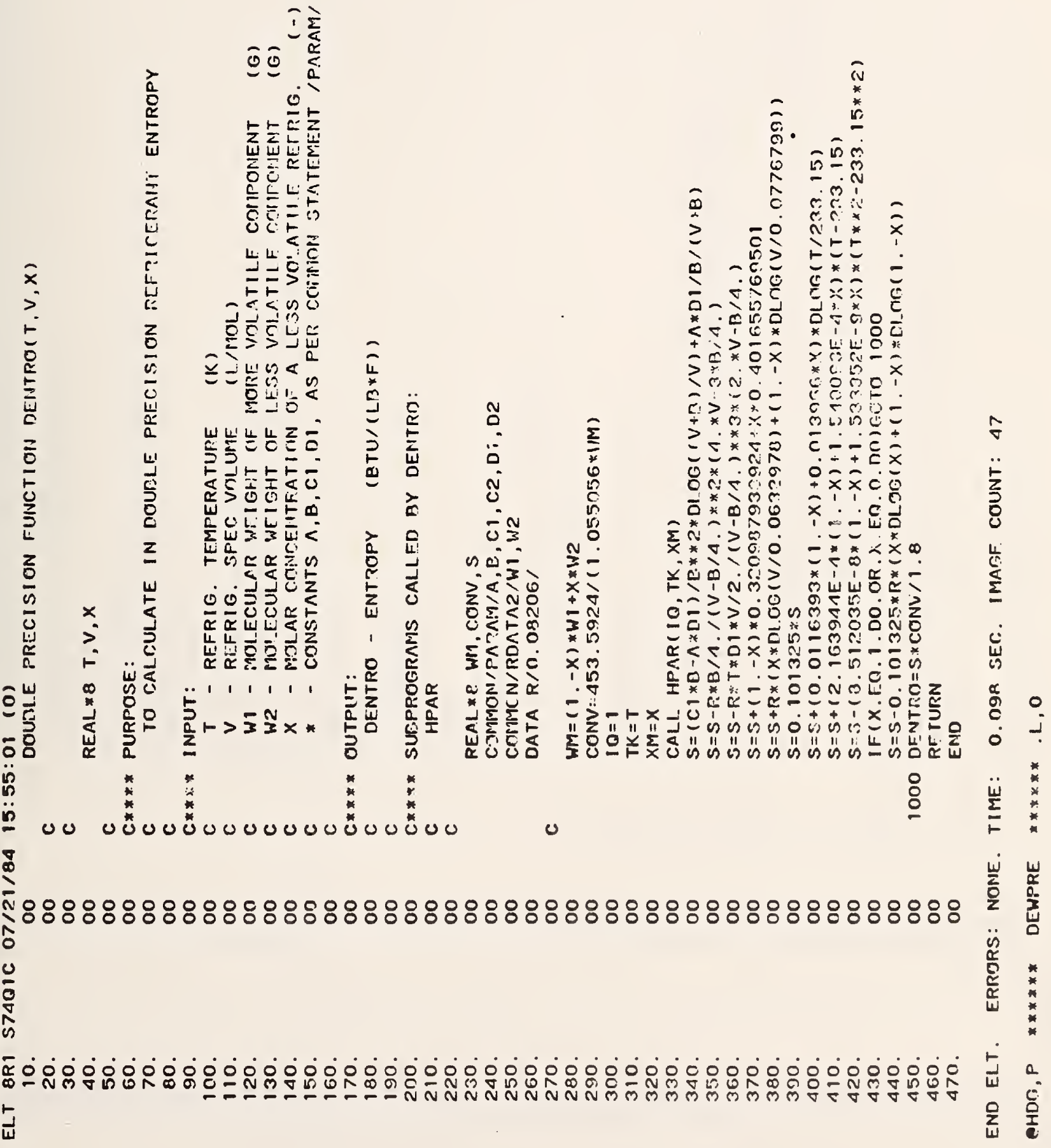




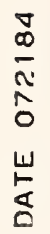

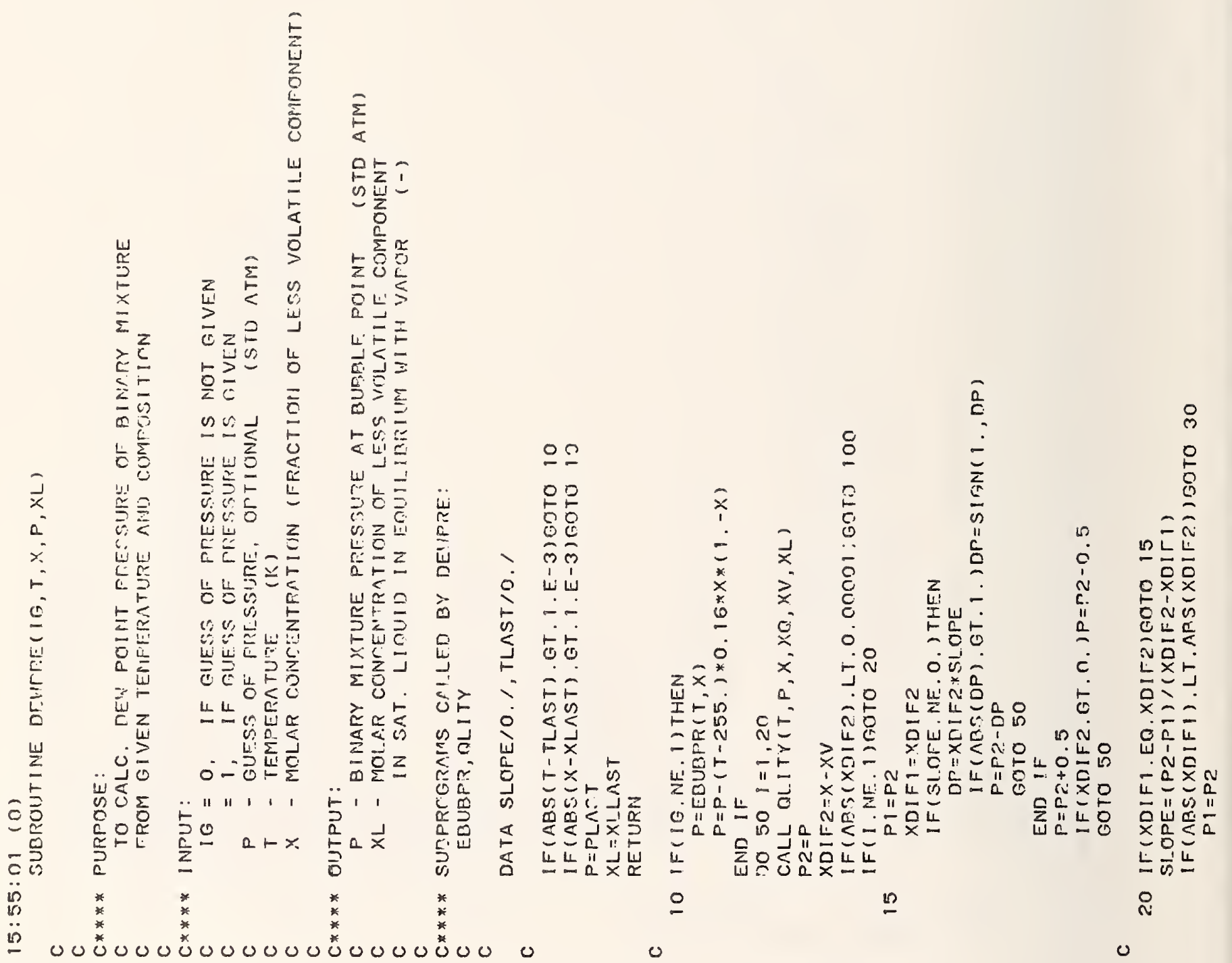

$\stackrel{\square}{\check{1}}$

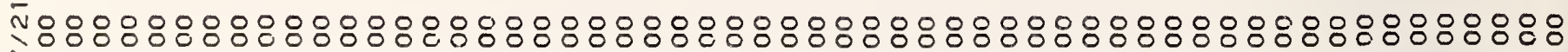
wo

西

$\frac{2}{3} \frac{0}{0}$

㟧

on

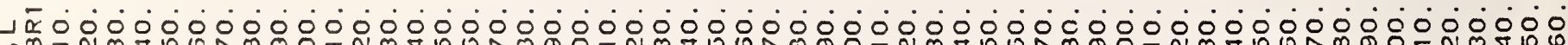
- -2 荘 


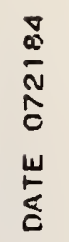

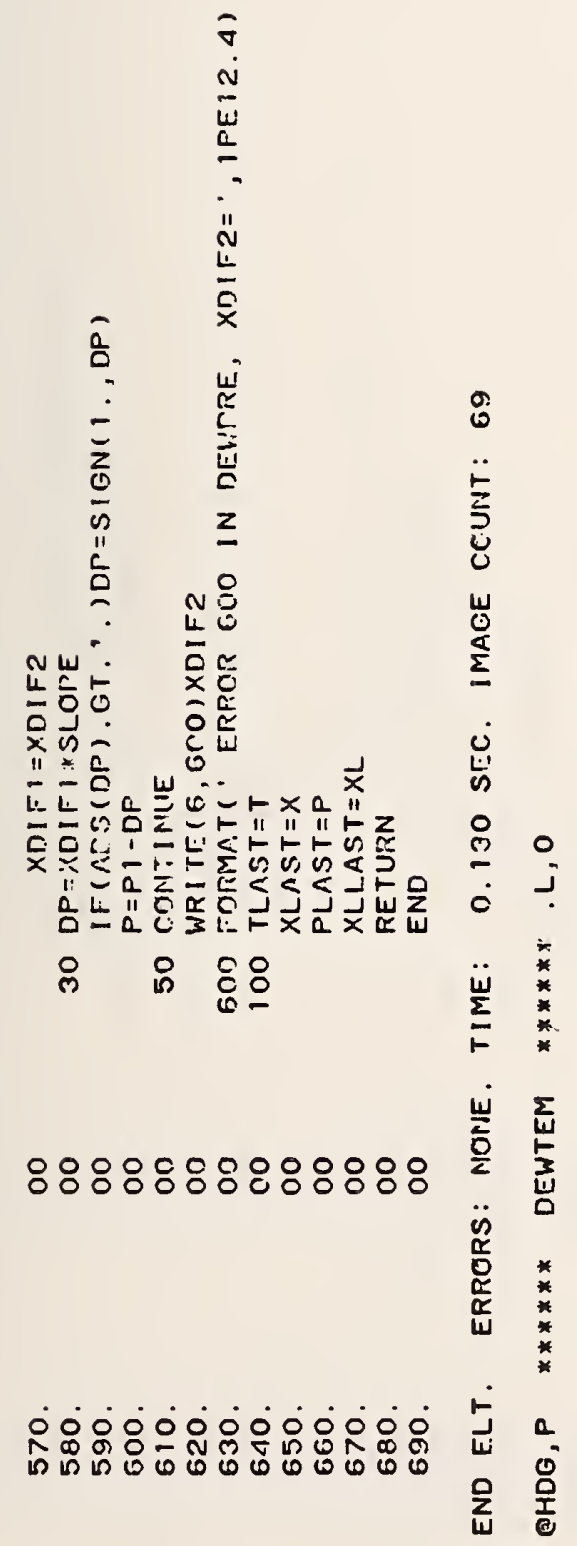




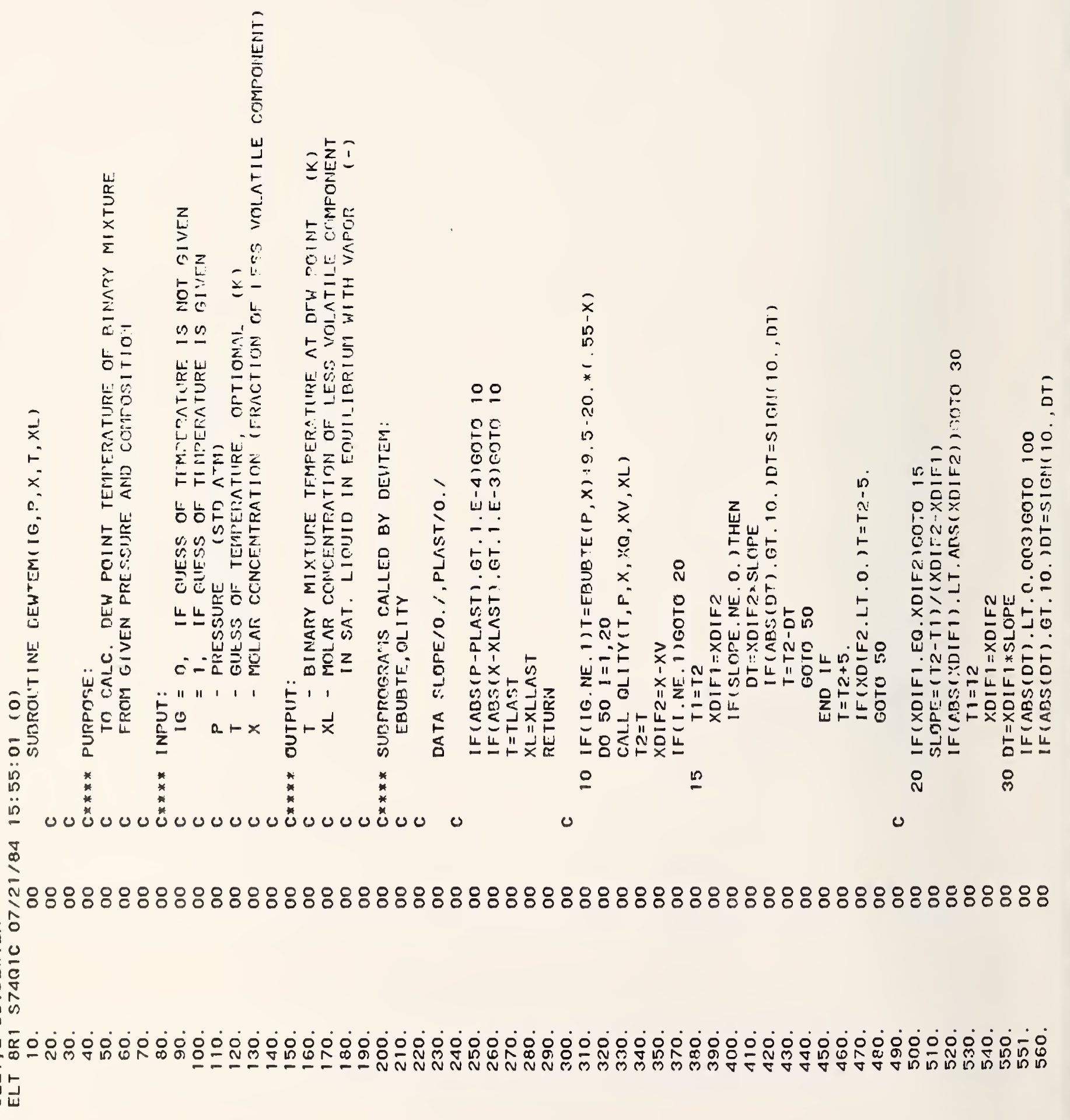




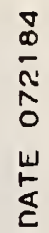

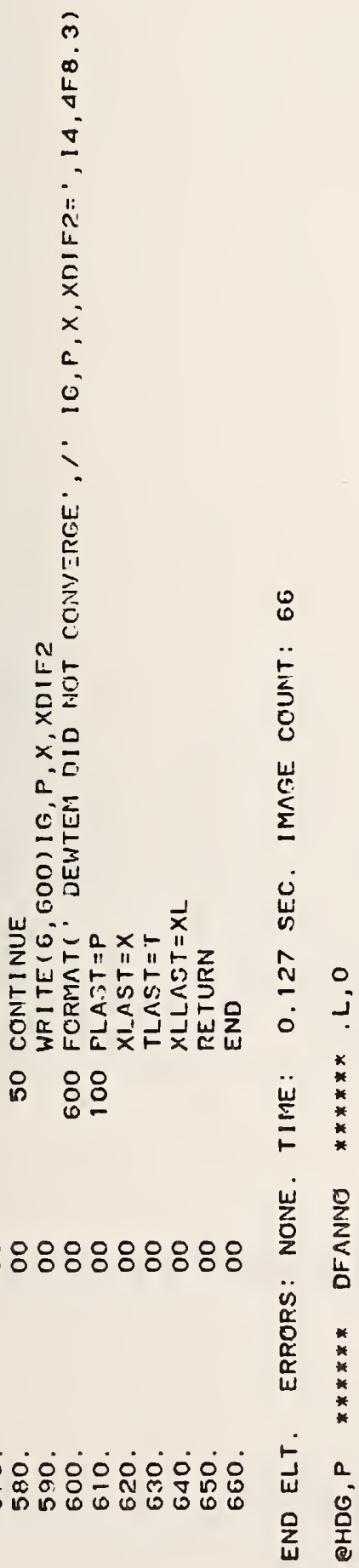


$\sum_{x}^{0}$

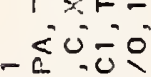

- 00

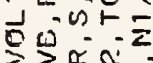

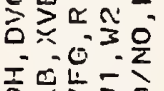

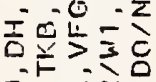

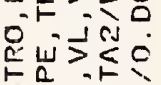

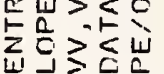

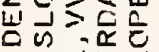

* $\%$ 专育的

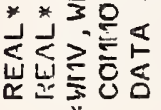

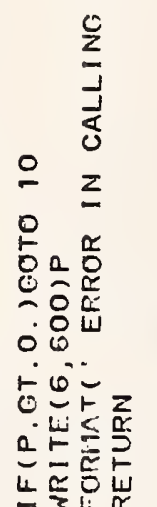

运焉岩崖

商朗

空。

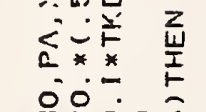

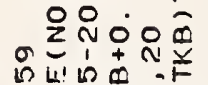

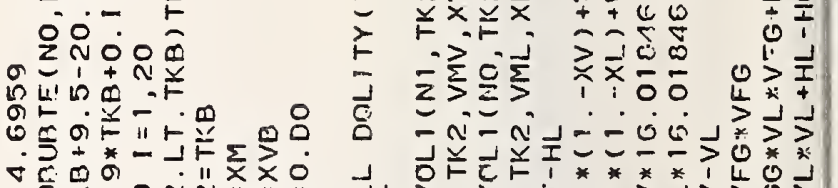

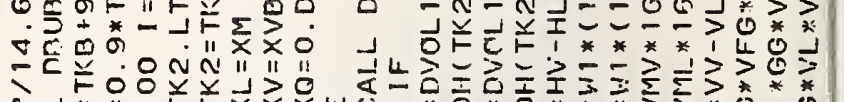
-4

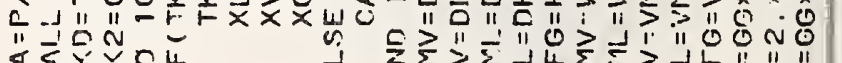

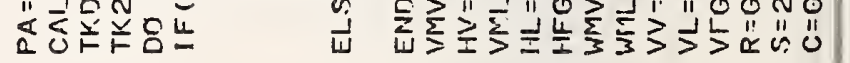
은

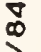

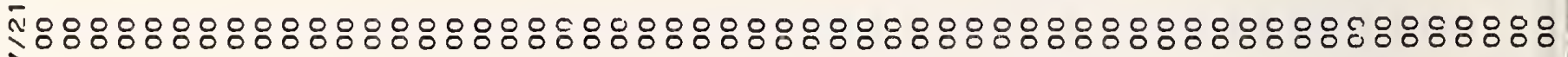
ồ $\sum_{<} \frac{1}{0}$

$\frac{\pi}{4} \frac{1}{0}$ $\dot{0}$ s

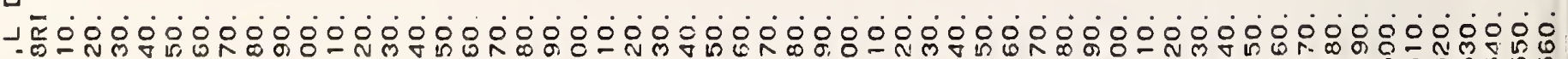
㟧上 


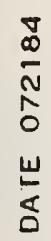

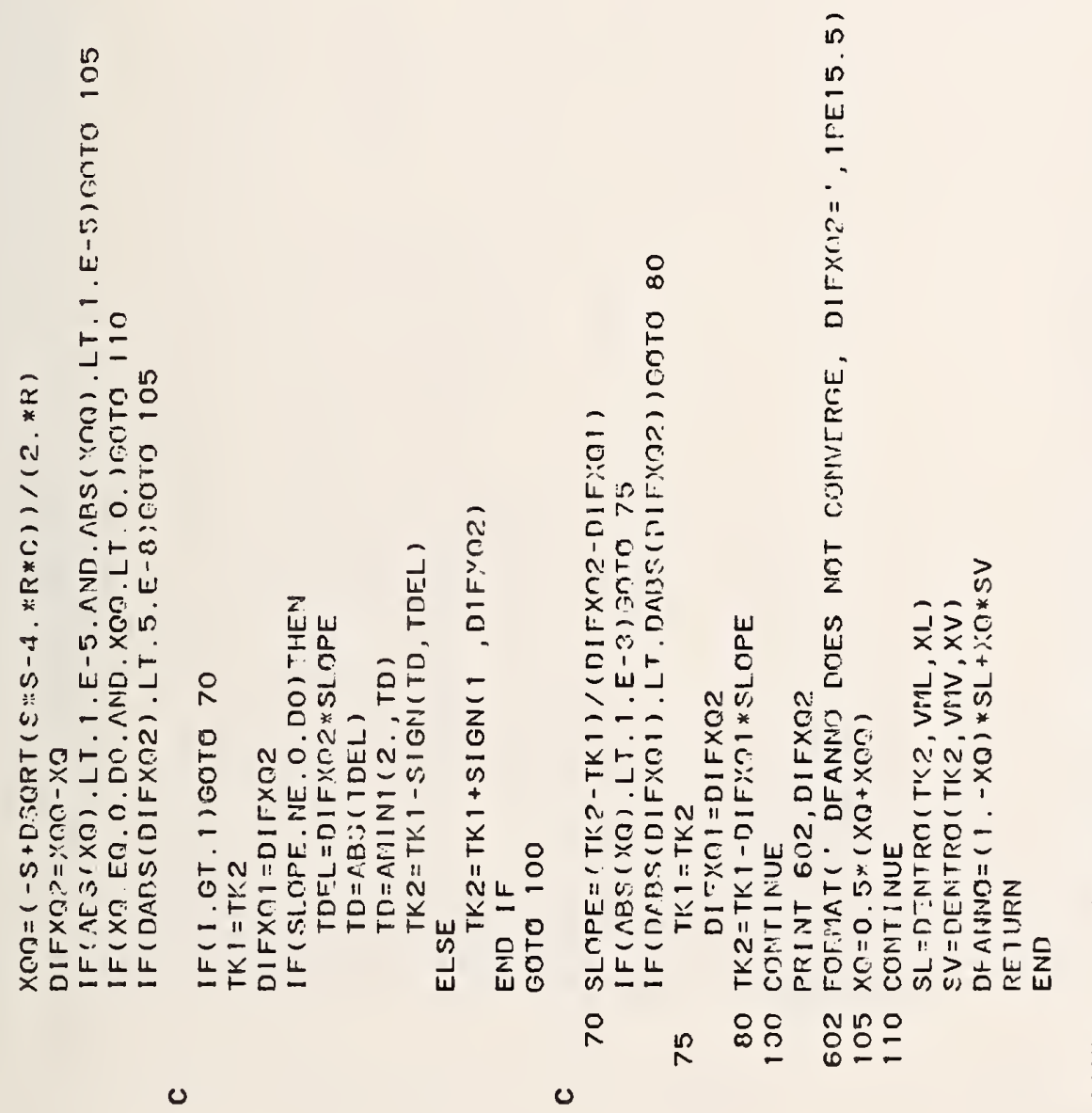

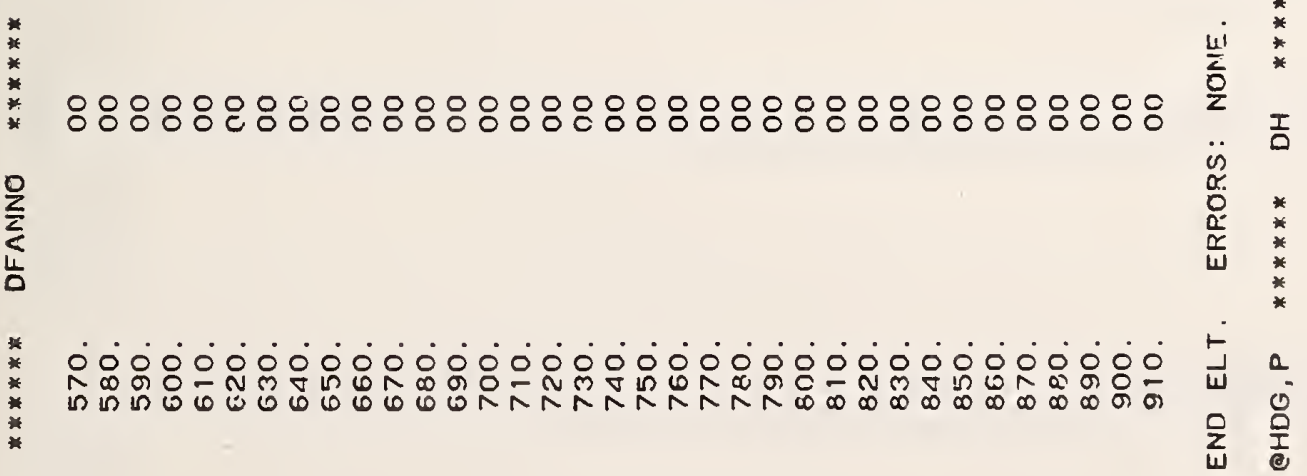


$\frac{5}{N}$
$\frac{N}{0}$
0
$\frac{5}{0}$

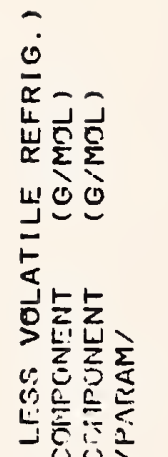

这荌

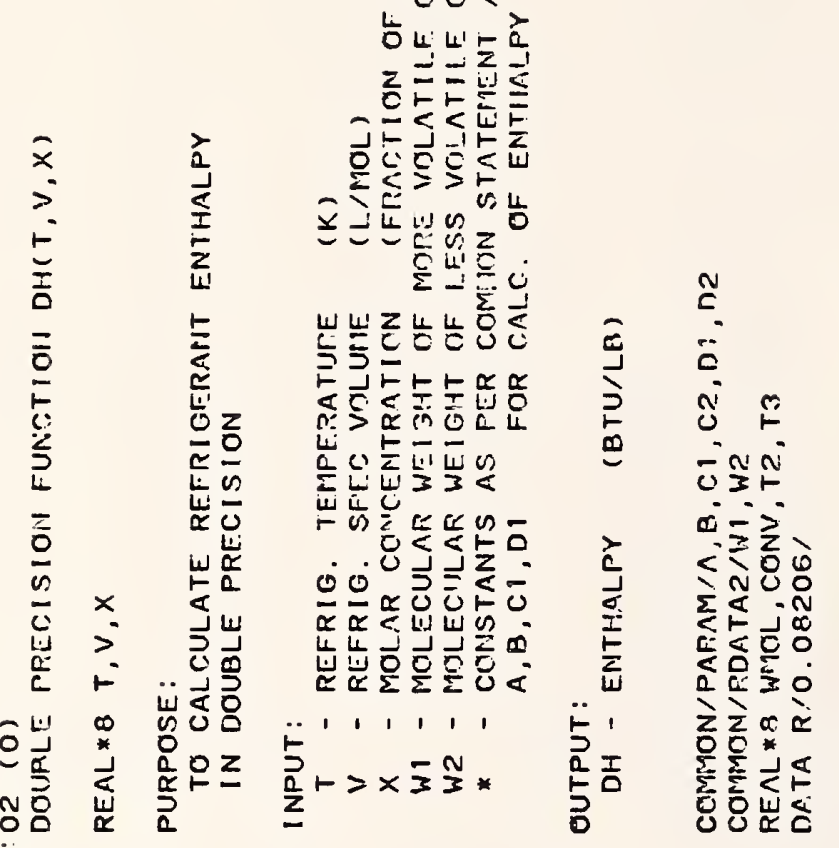

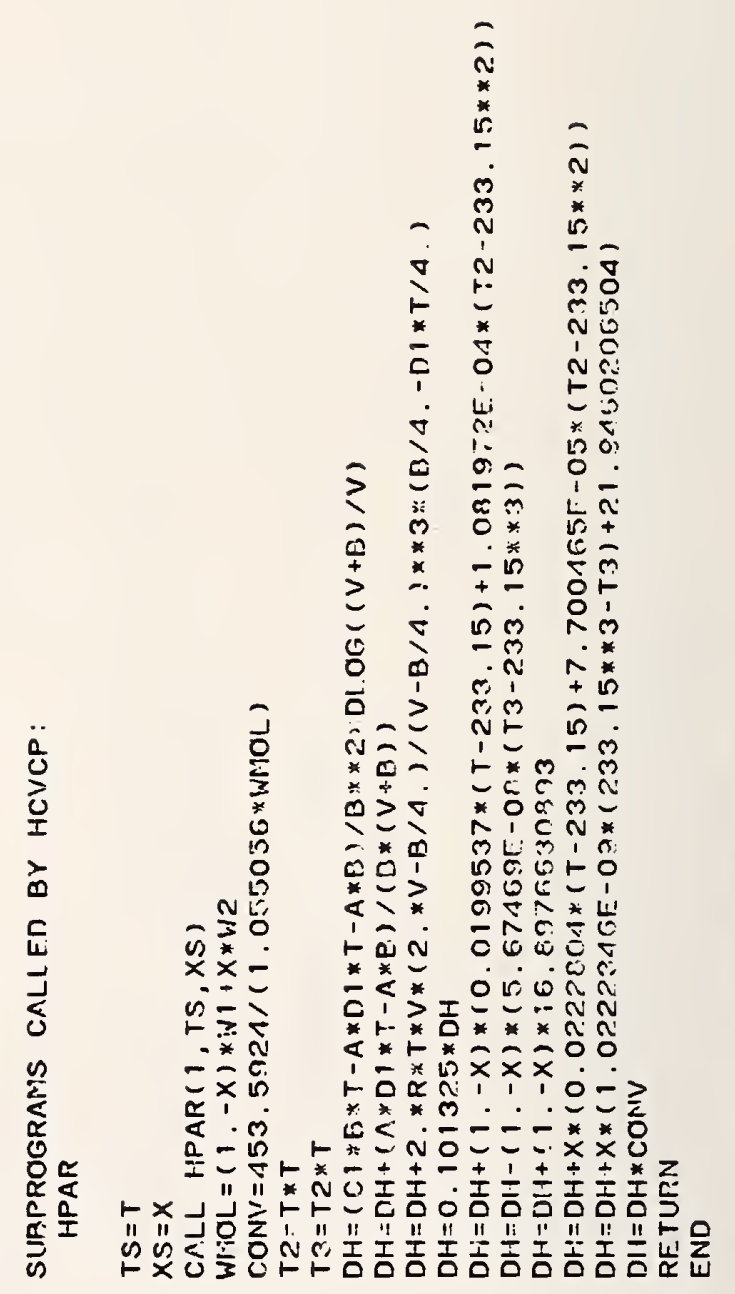

$\stackrel{\infty}{\stackrel{\infty}{Z}}$

岁

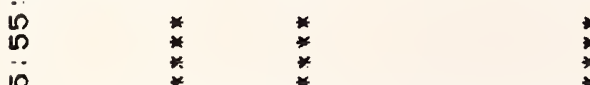

I 0000000000000000000

0

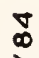
ิㅗㅇ

告市

पx ó 岂 
$\frac{D}{\infty}$
N
0
W
$\stackrel{5}{0}$ 


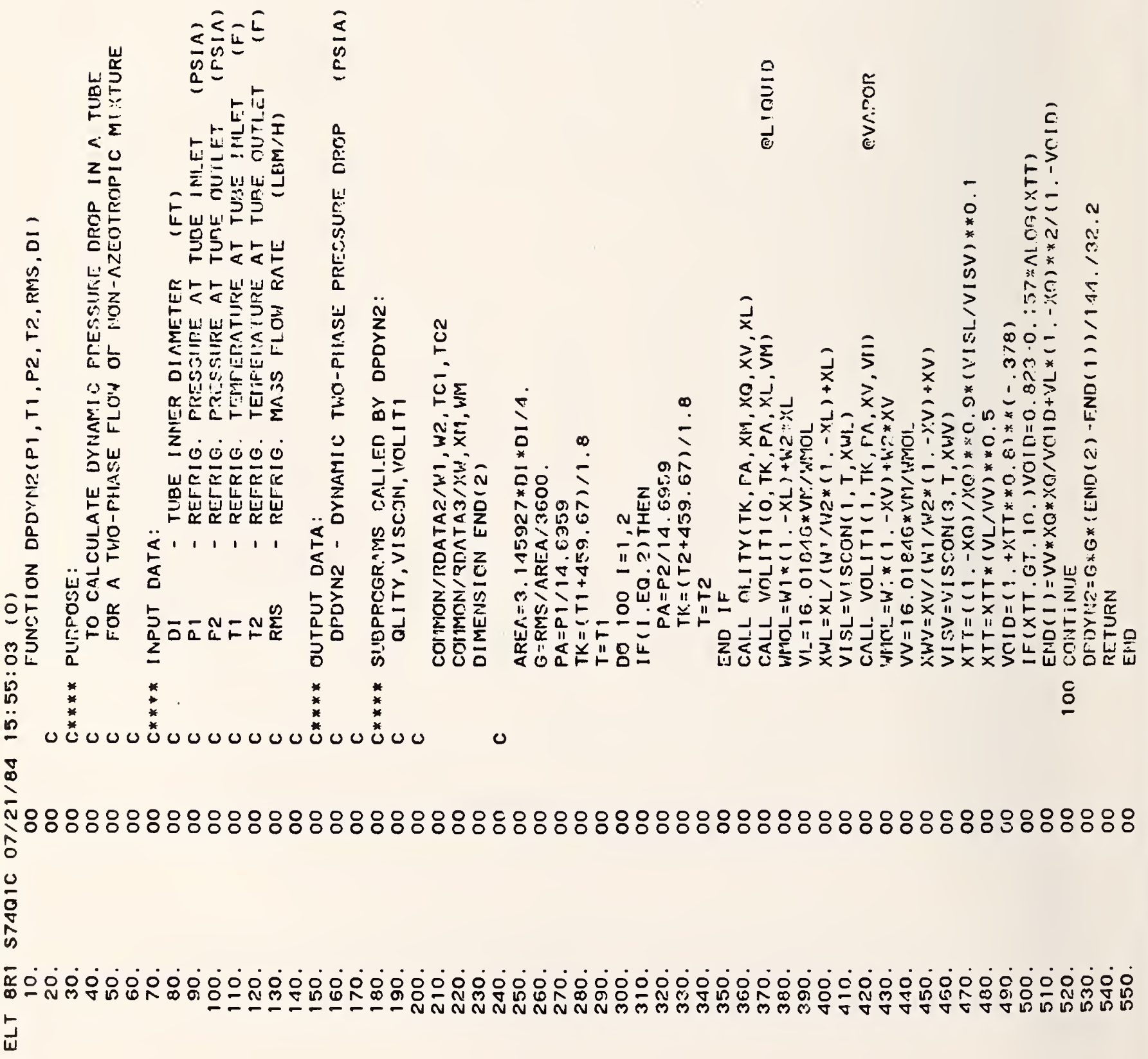




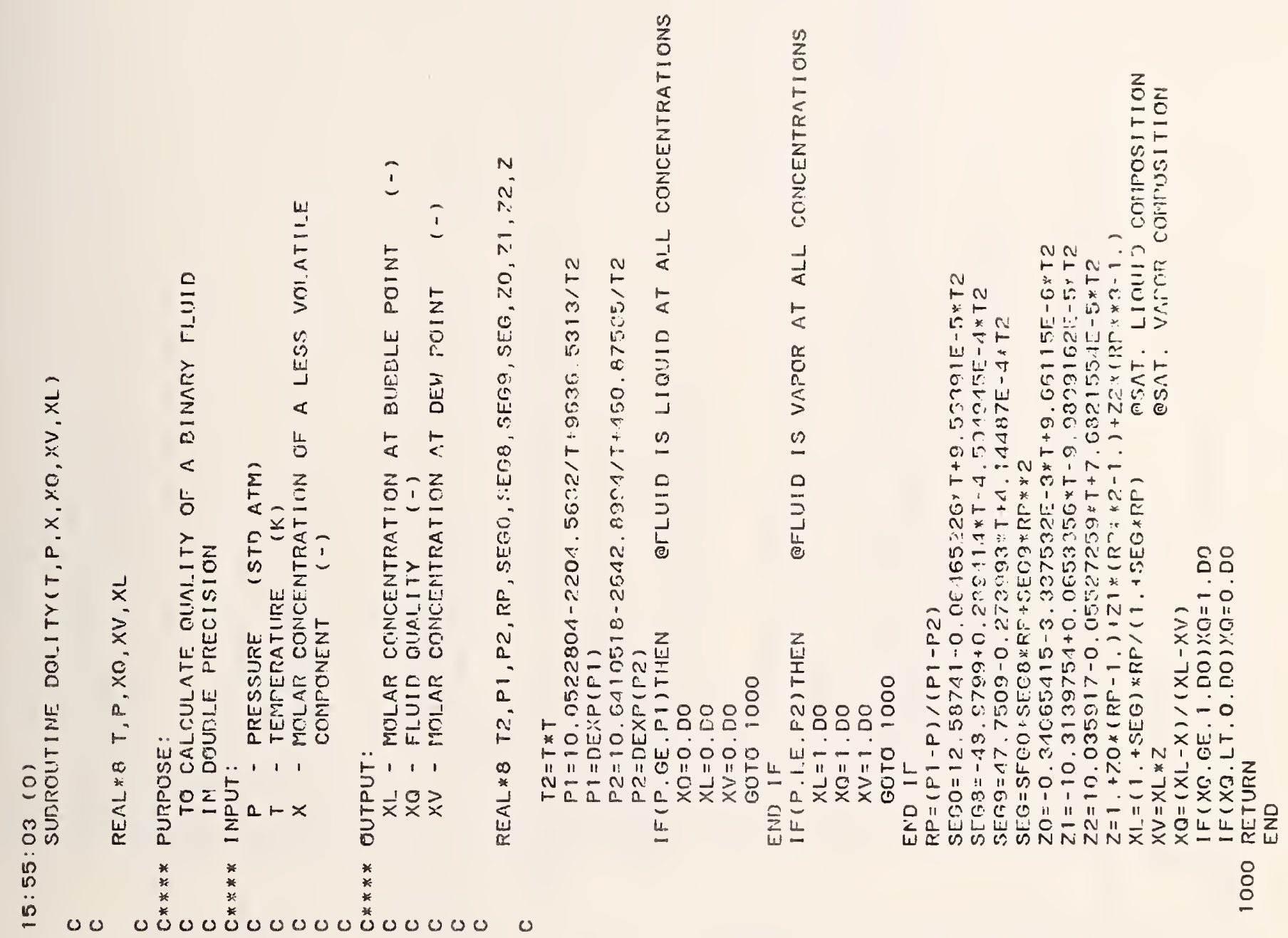

क

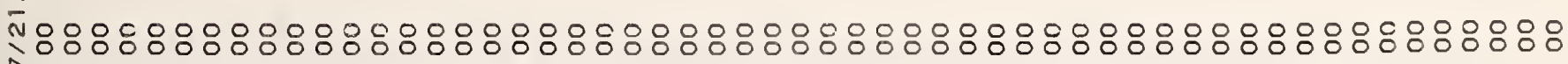
$2 \hat{0}$

$\overline{0} \frac{0}{0}$

in

नज़ 


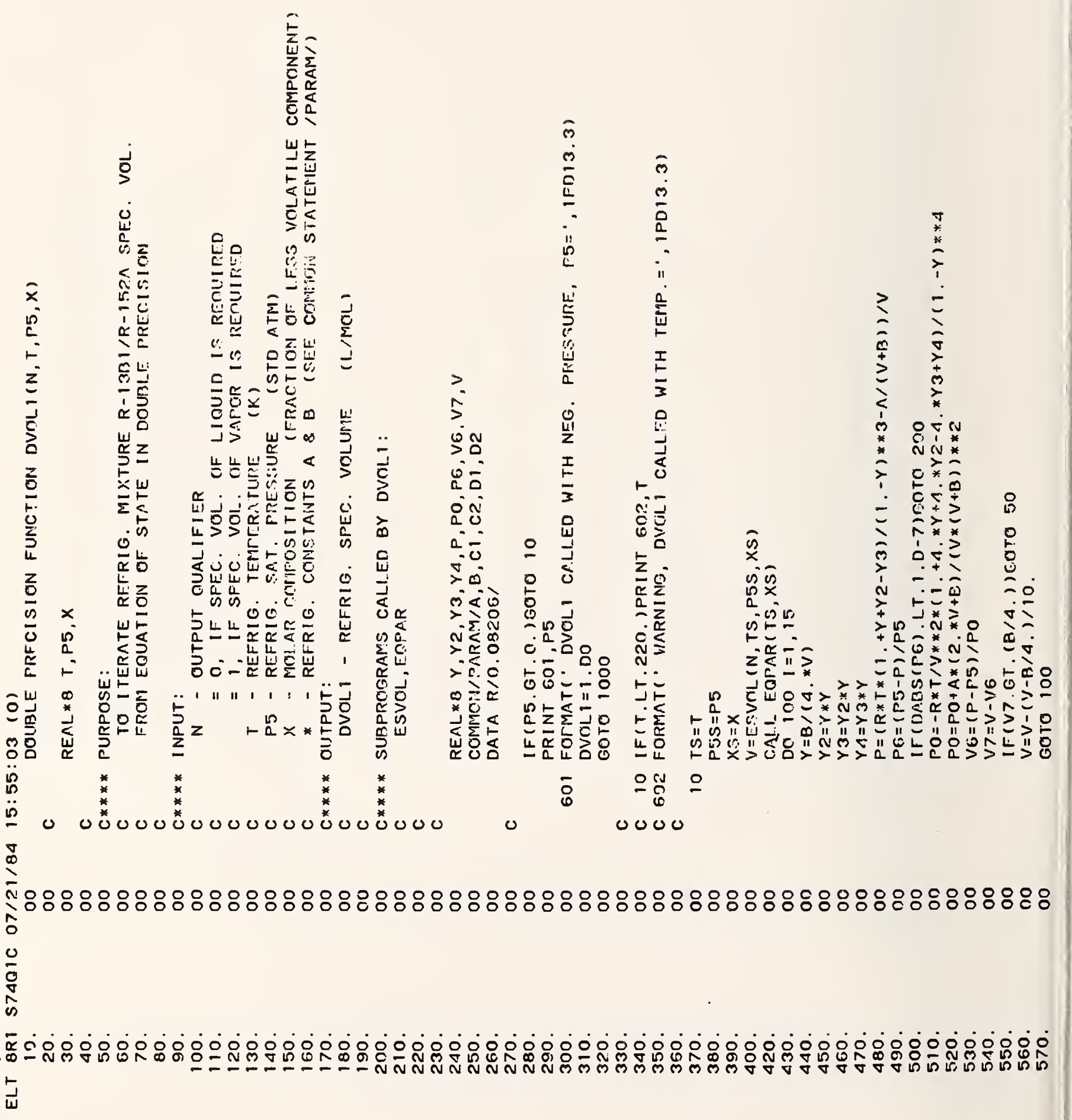




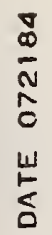

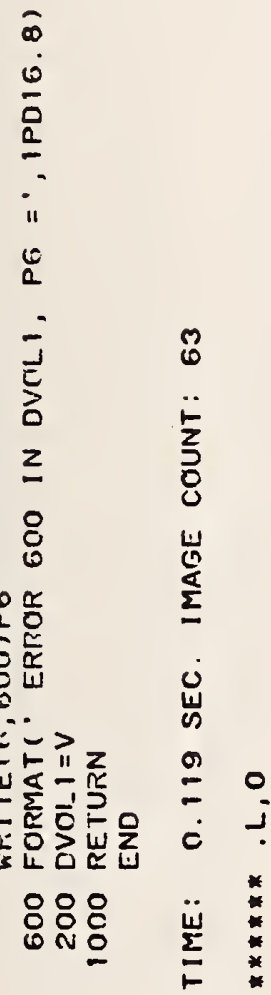

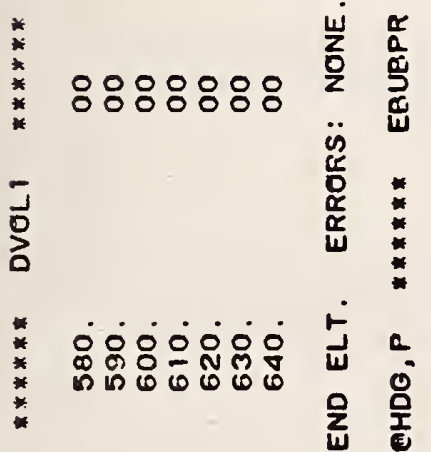




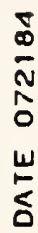

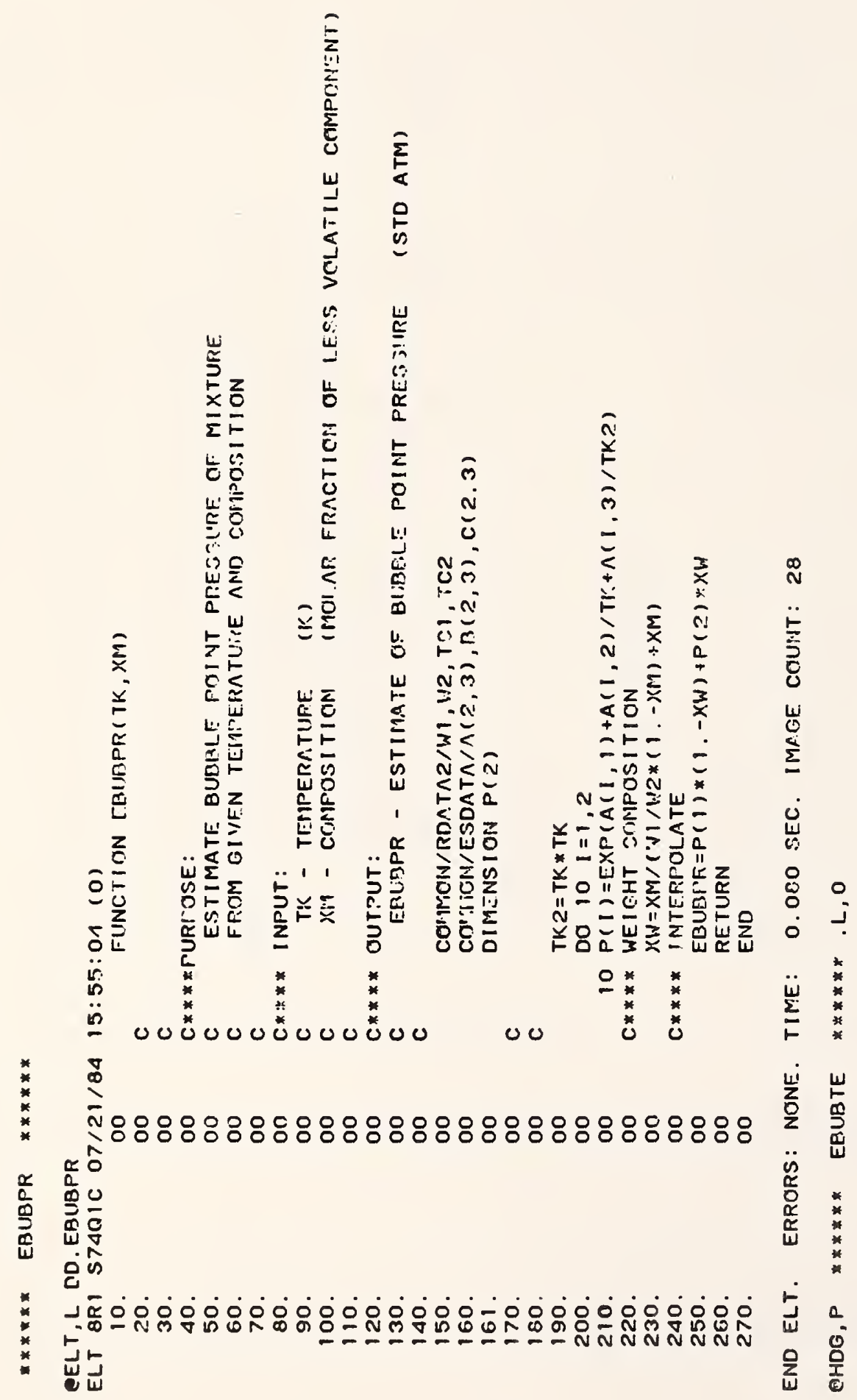




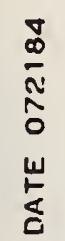

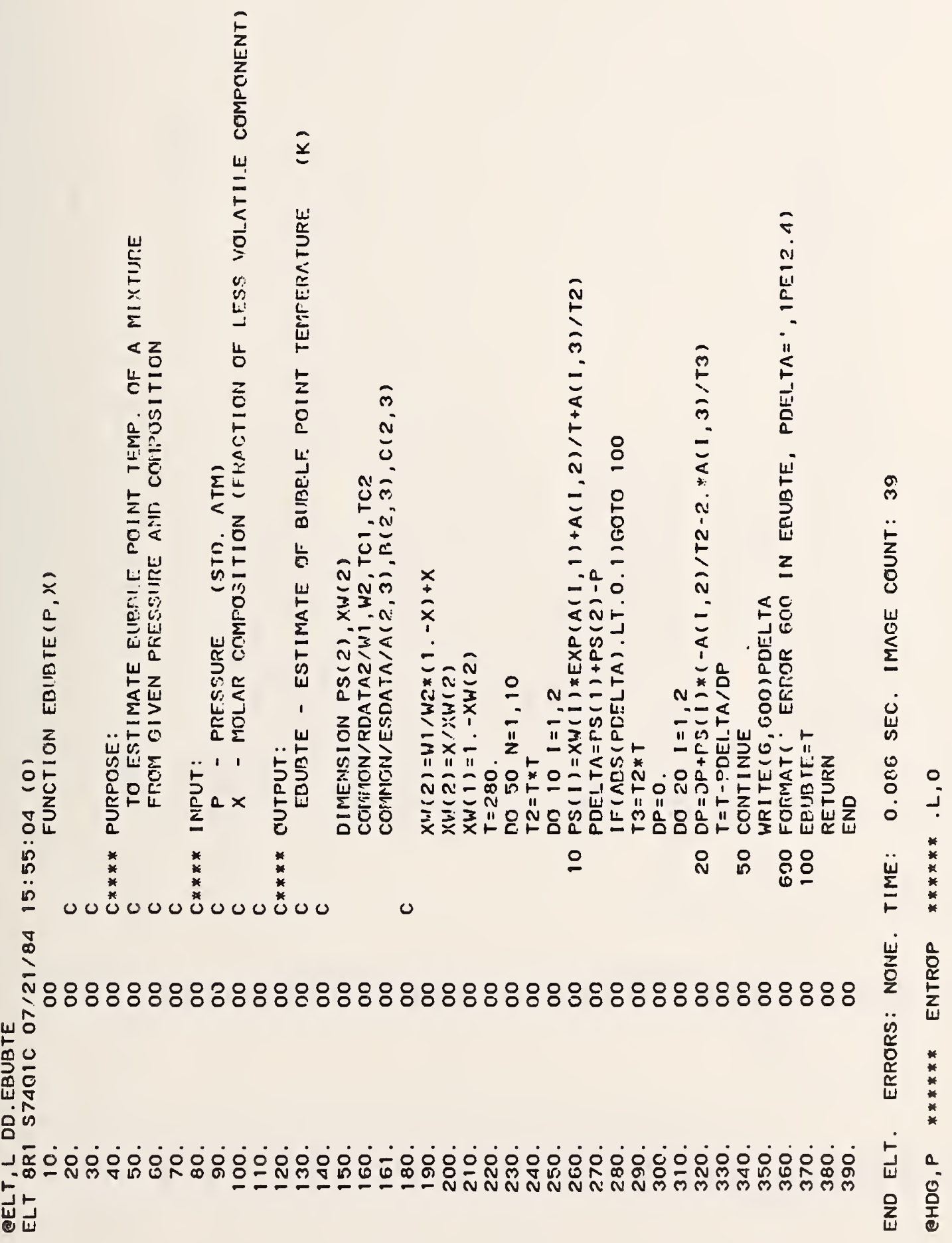




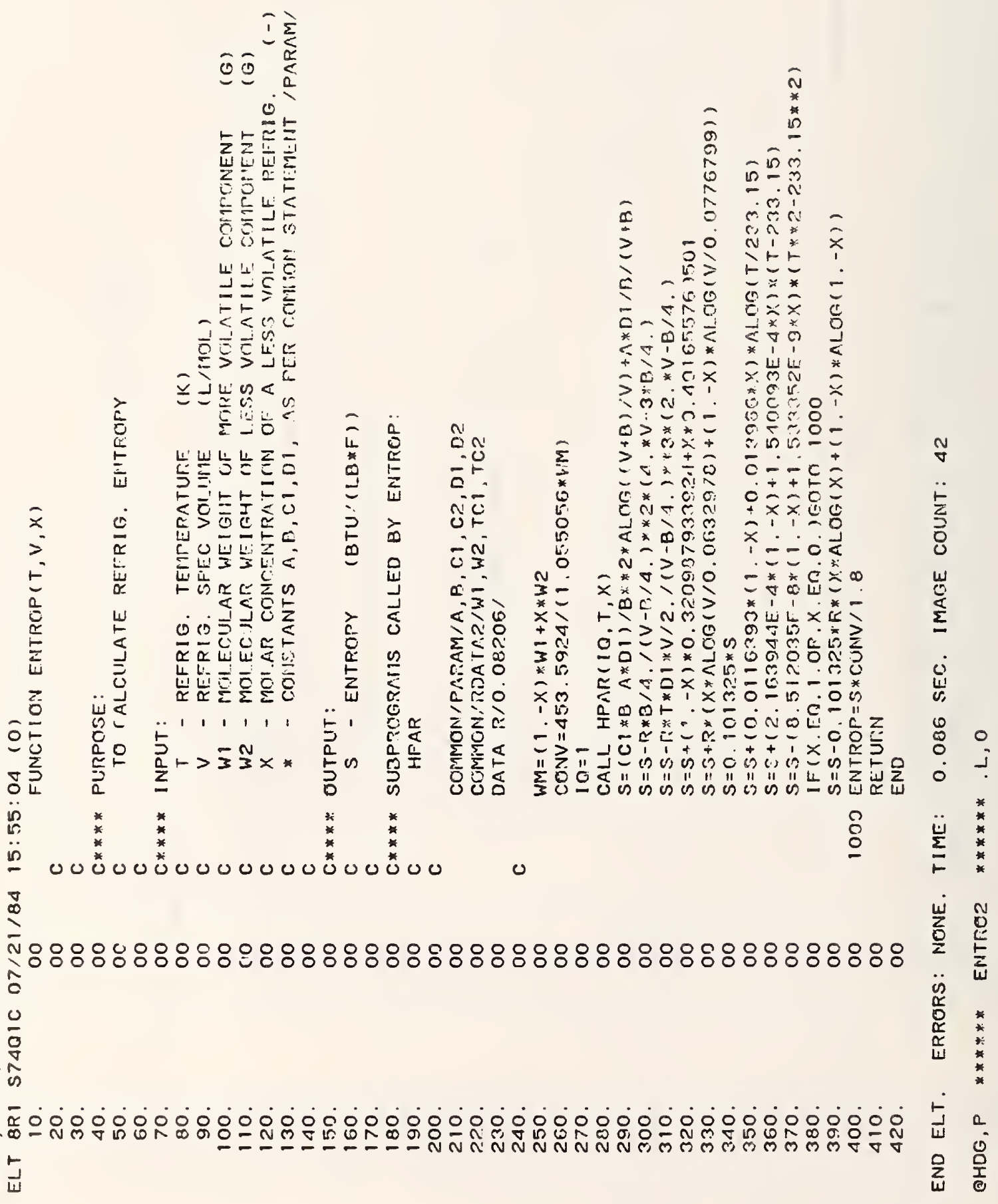




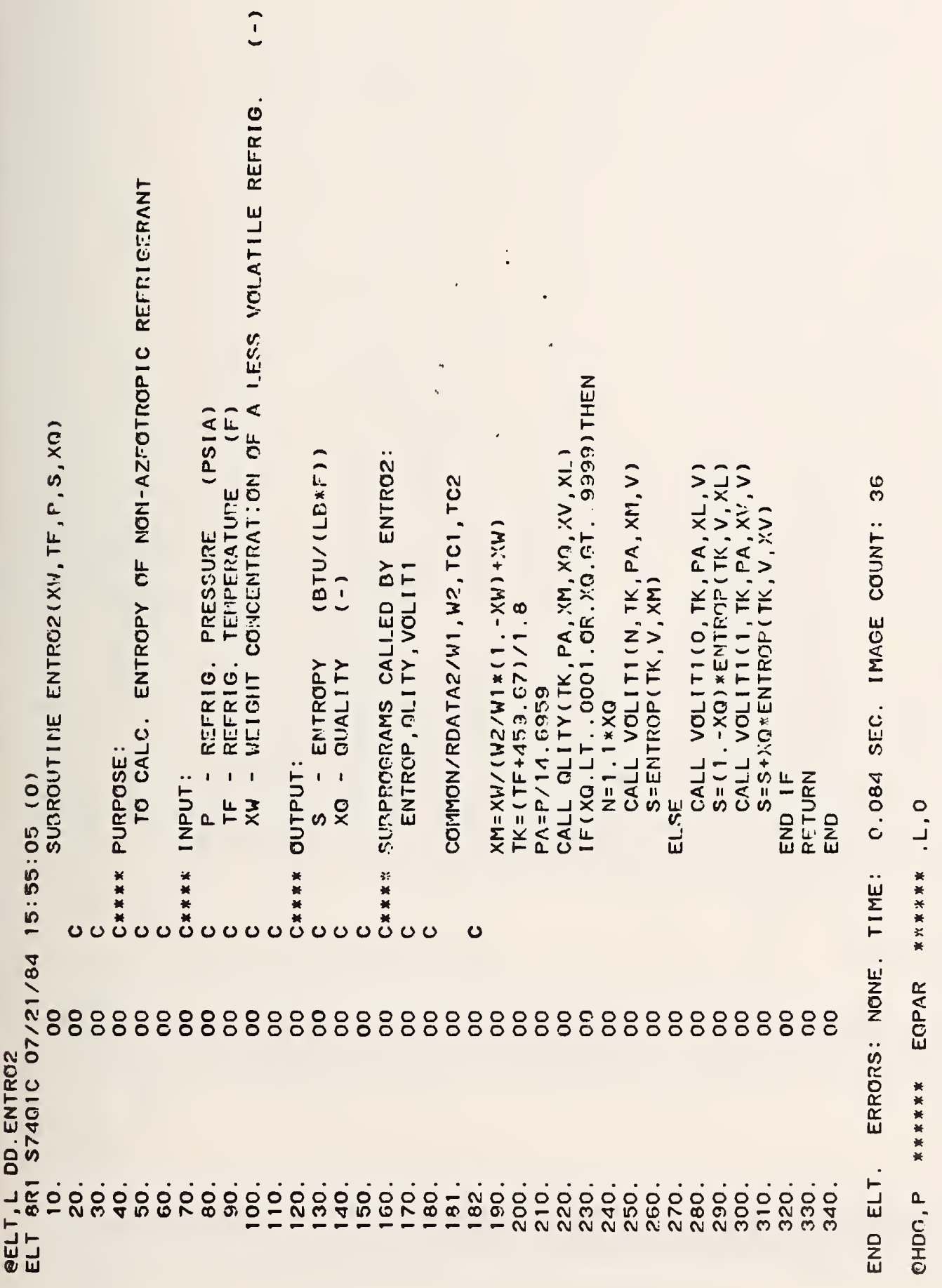




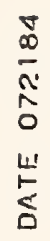

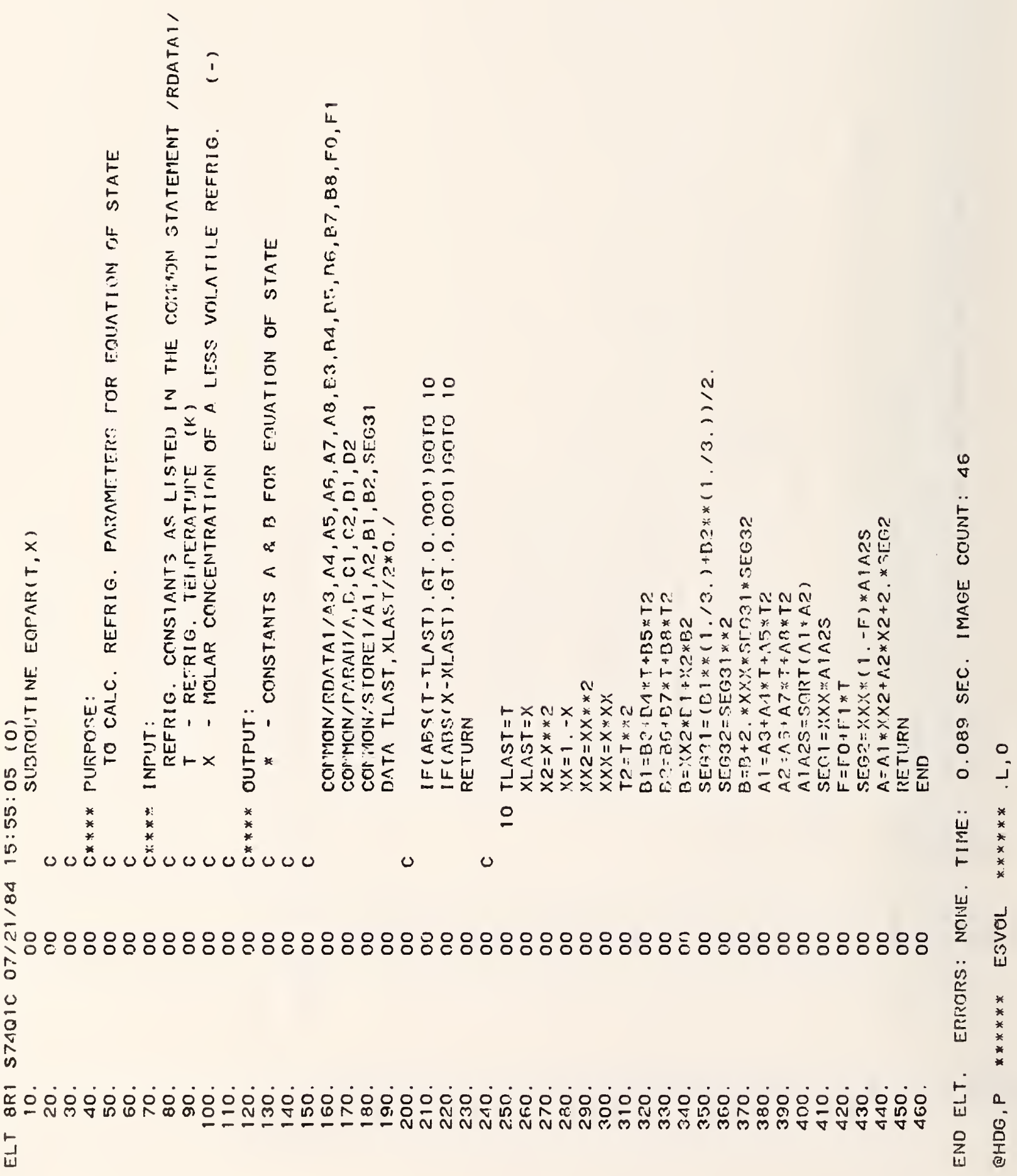




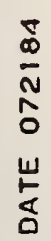

를

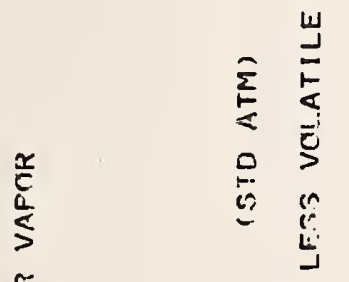

长宸

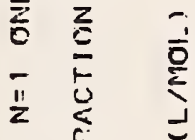

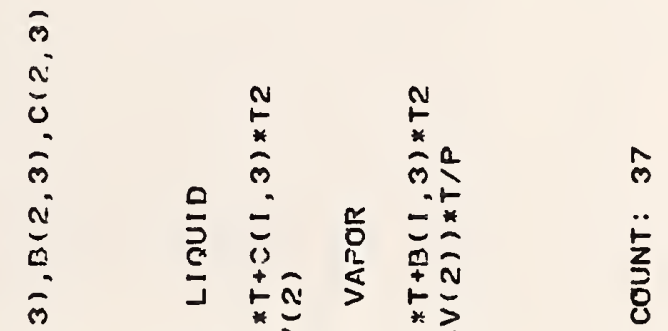

뜬응

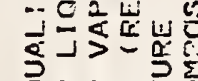

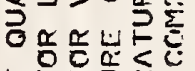

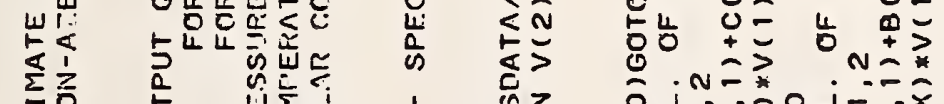

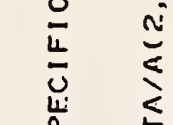

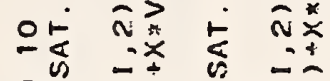

品

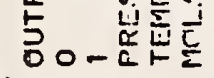

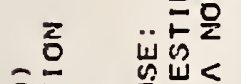

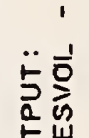

造密

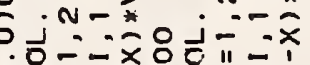

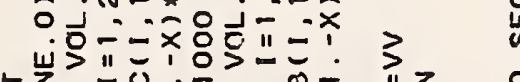

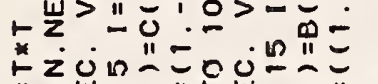

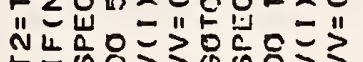

$\because 4 \quad z \quad$ a

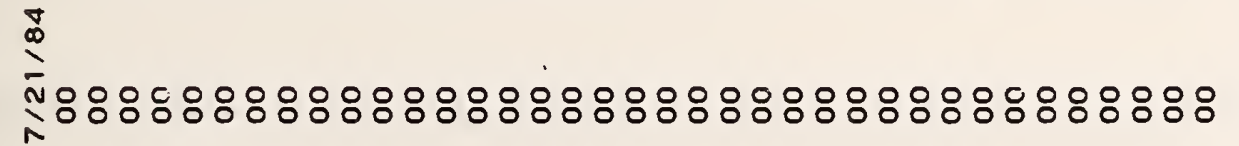

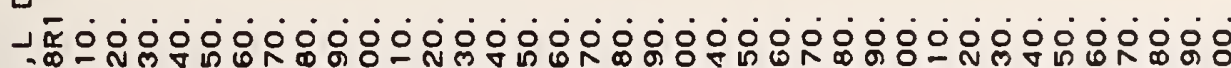
峞 


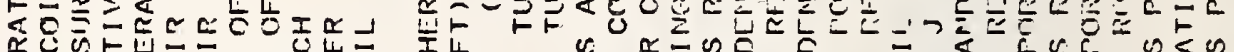

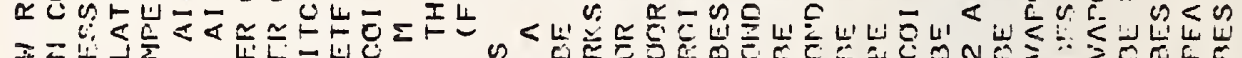

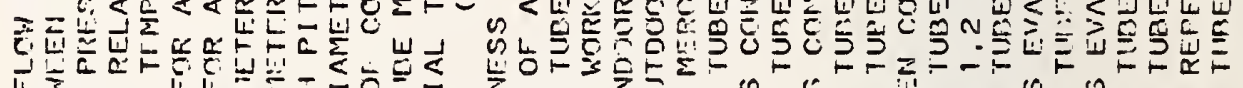
$\begin{array}{lll} & \\ 4 & \end{array}$

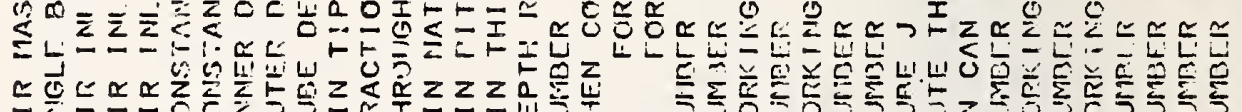

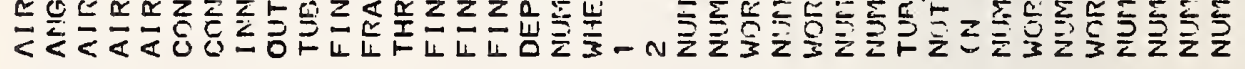

000000000000000000000000000000000000000000000000000000 $\times$

焉

$\sum_{\frac{1}{3}}^{\frac{1}{4}} \frac{0}{0}$

它

峞至 


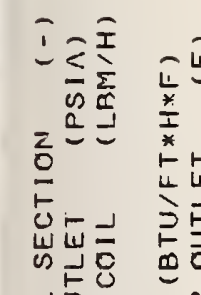

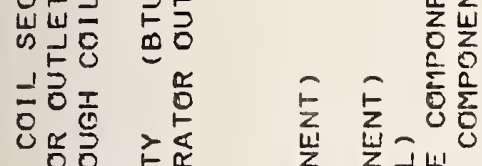

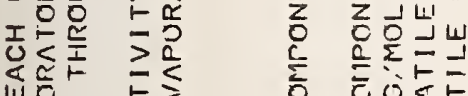

㔺它

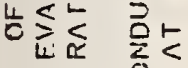

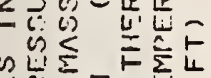

出

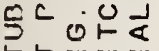

ト上

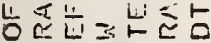

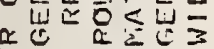

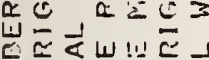

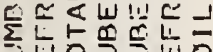

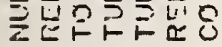

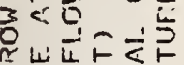

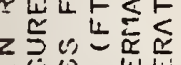

-

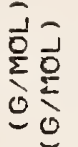

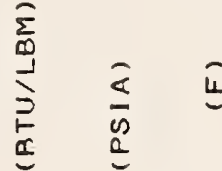

解度

山墕

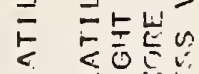

$\overline{\mathrm{s}} \mathrm{C}$

zo

는

-

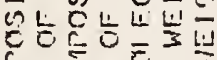

$c=\bar{c}$ 800 o ○上下嵓引

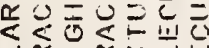

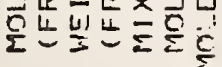

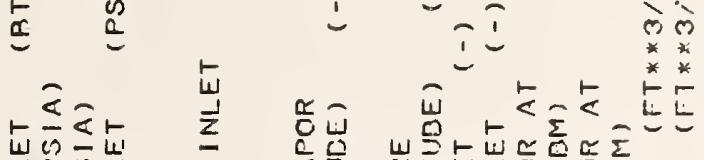

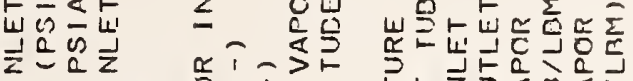
-

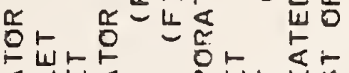

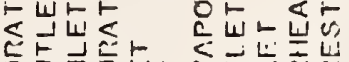
a a

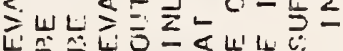

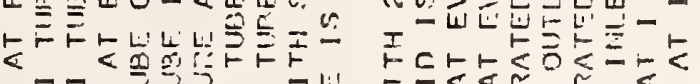

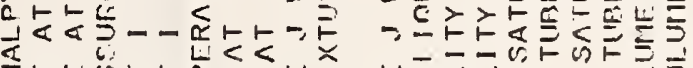

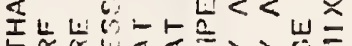

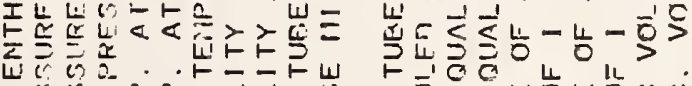
以,

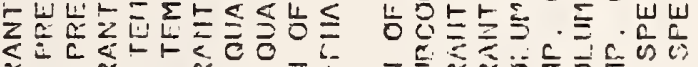
र

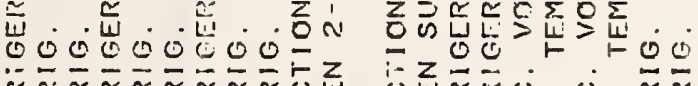

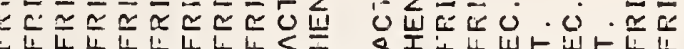

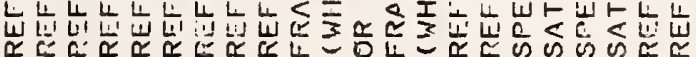
告

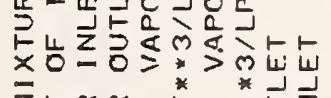

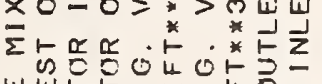

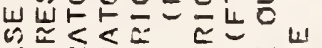

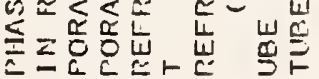

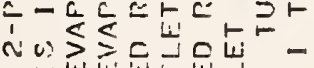

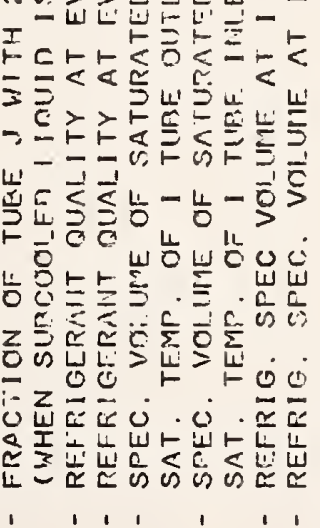

$1,1,1$,

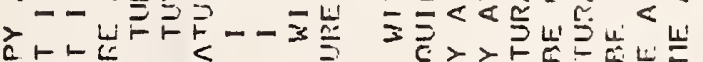

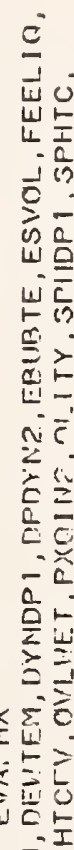

应芴三

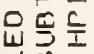

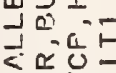

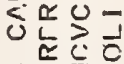

os

$-2-n-n i n$

Nin Nin Niñ

N

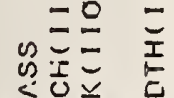

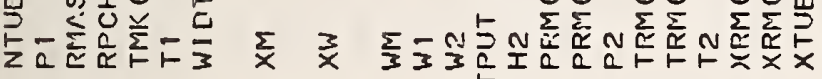

$$
\text { 今 }
$$


$\frac{J}{\infty}$
$\frac{N}{0}$
$\frac{1}{5}$
$\frac{5}{0}$

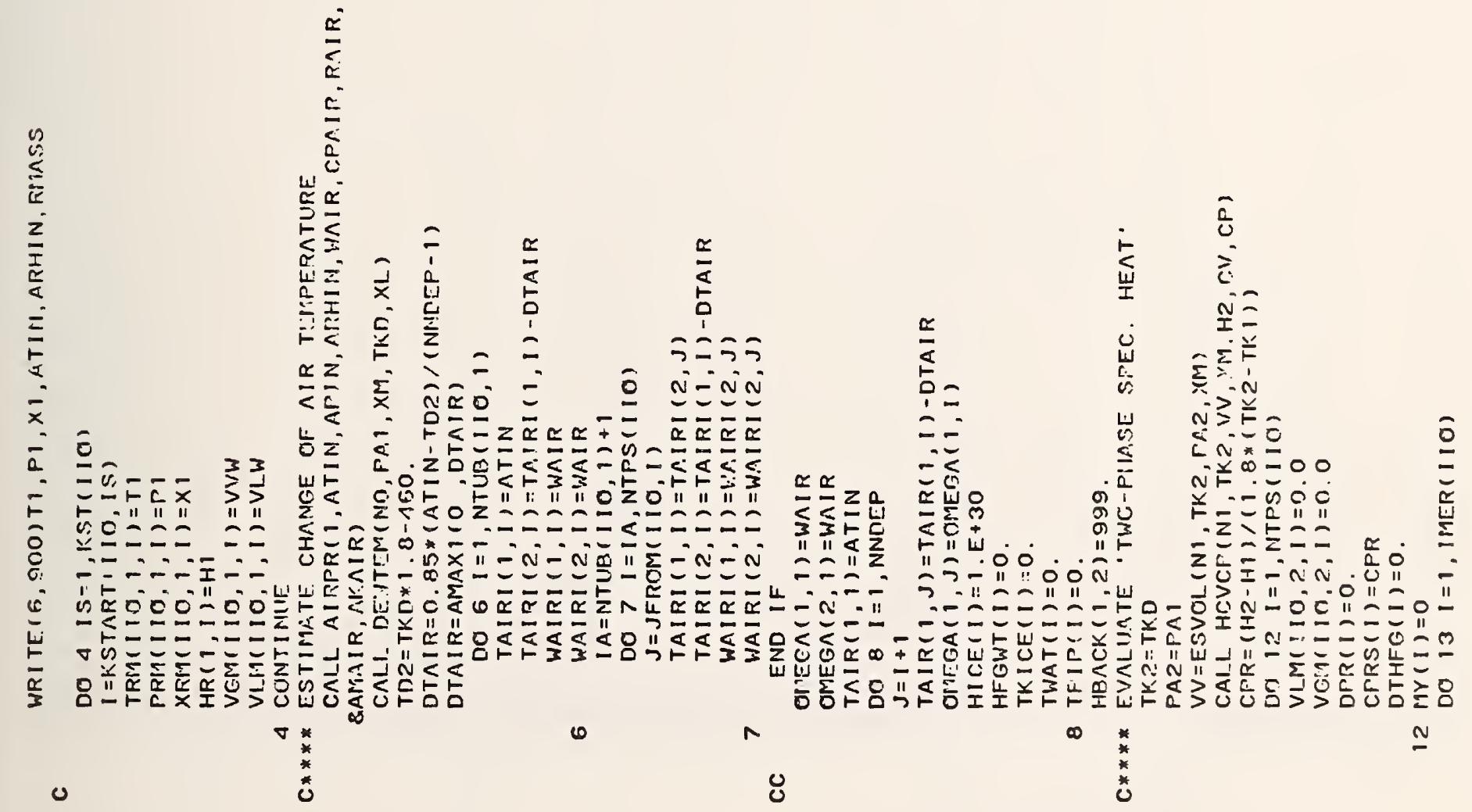

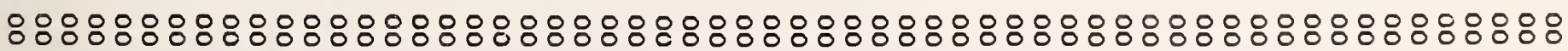

OOO

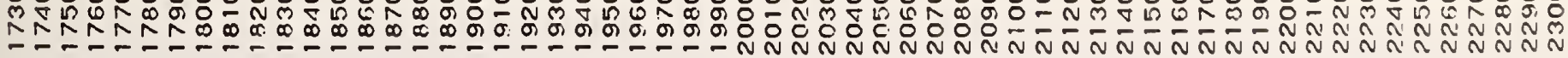




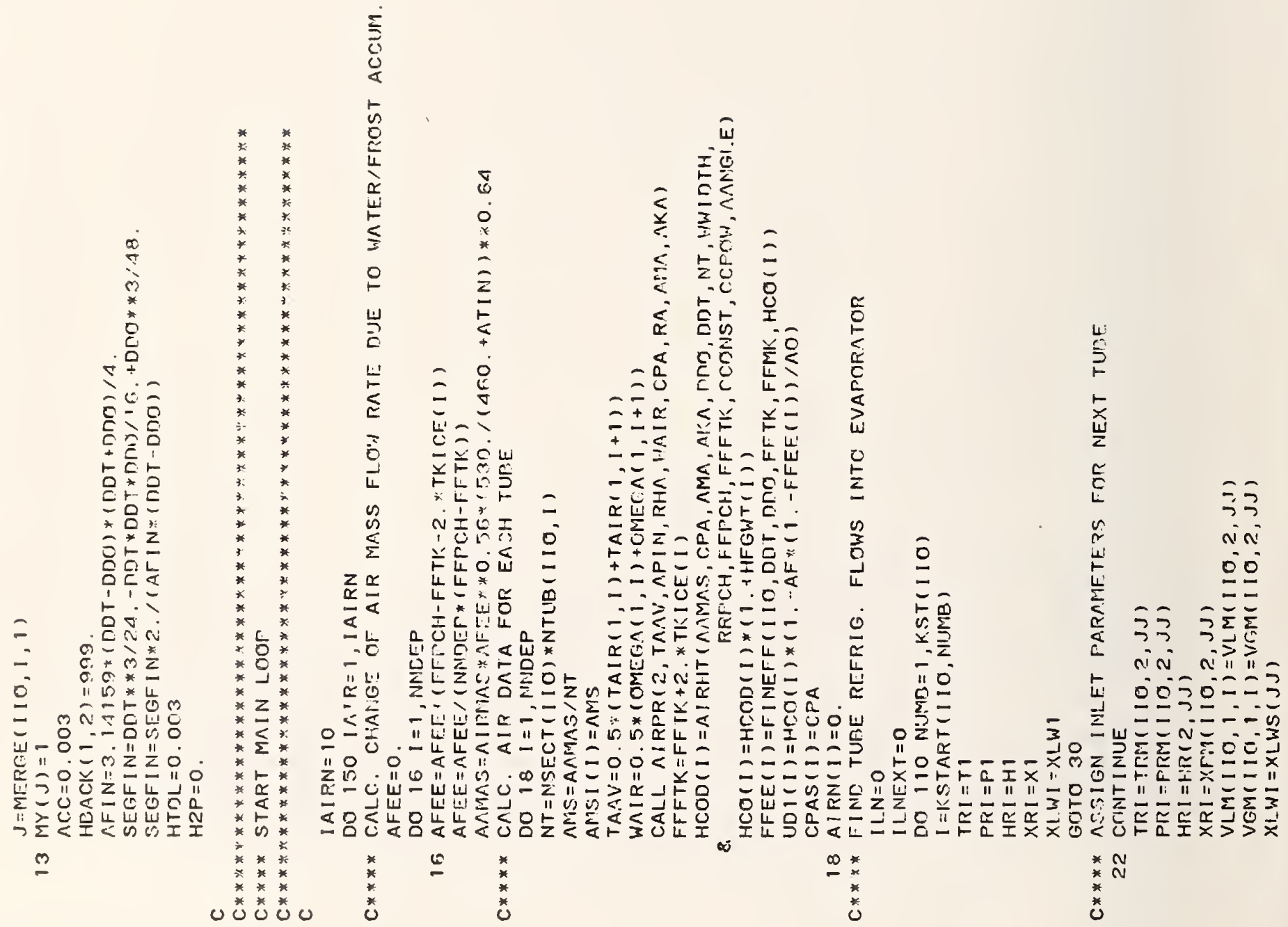

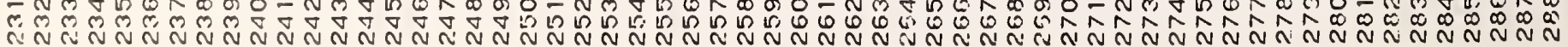


它怘を

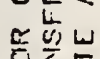

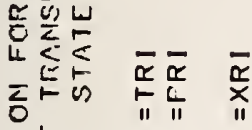

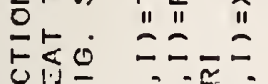

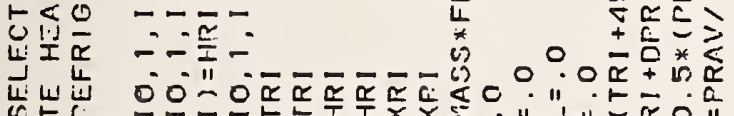

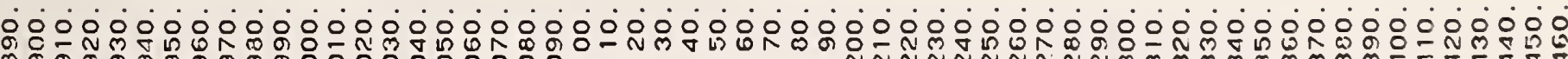

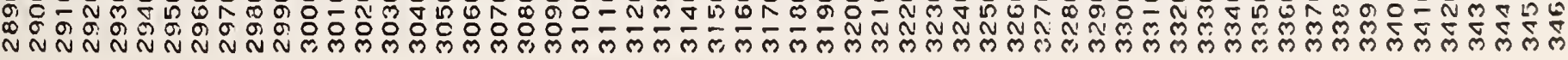




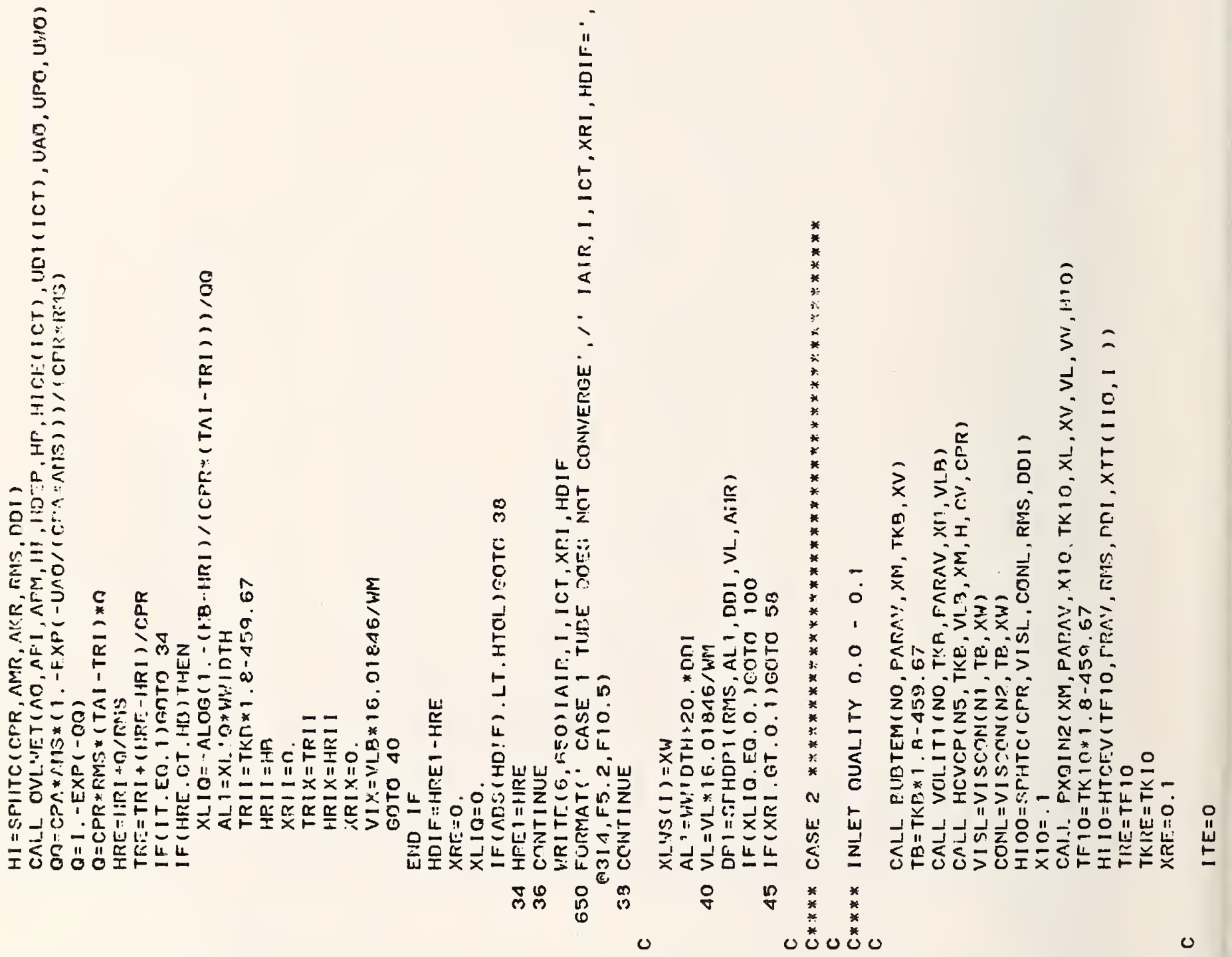

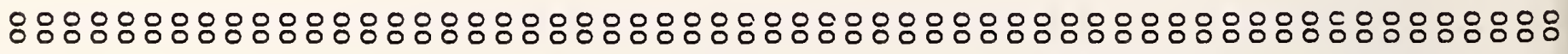

$\frac{x}{\frac{x}{2}}$

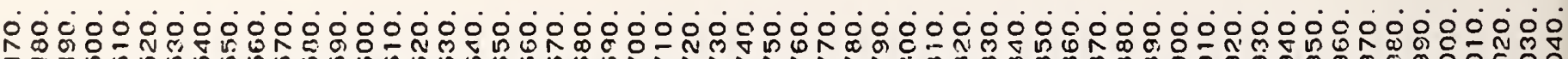

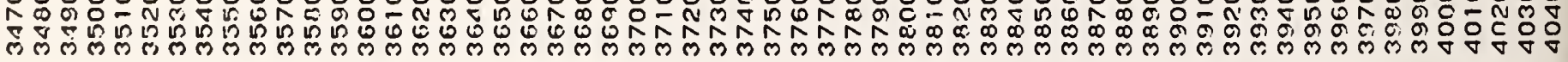


5
0
5
$\vdots$
0
$w$
5
$\vdots$
0

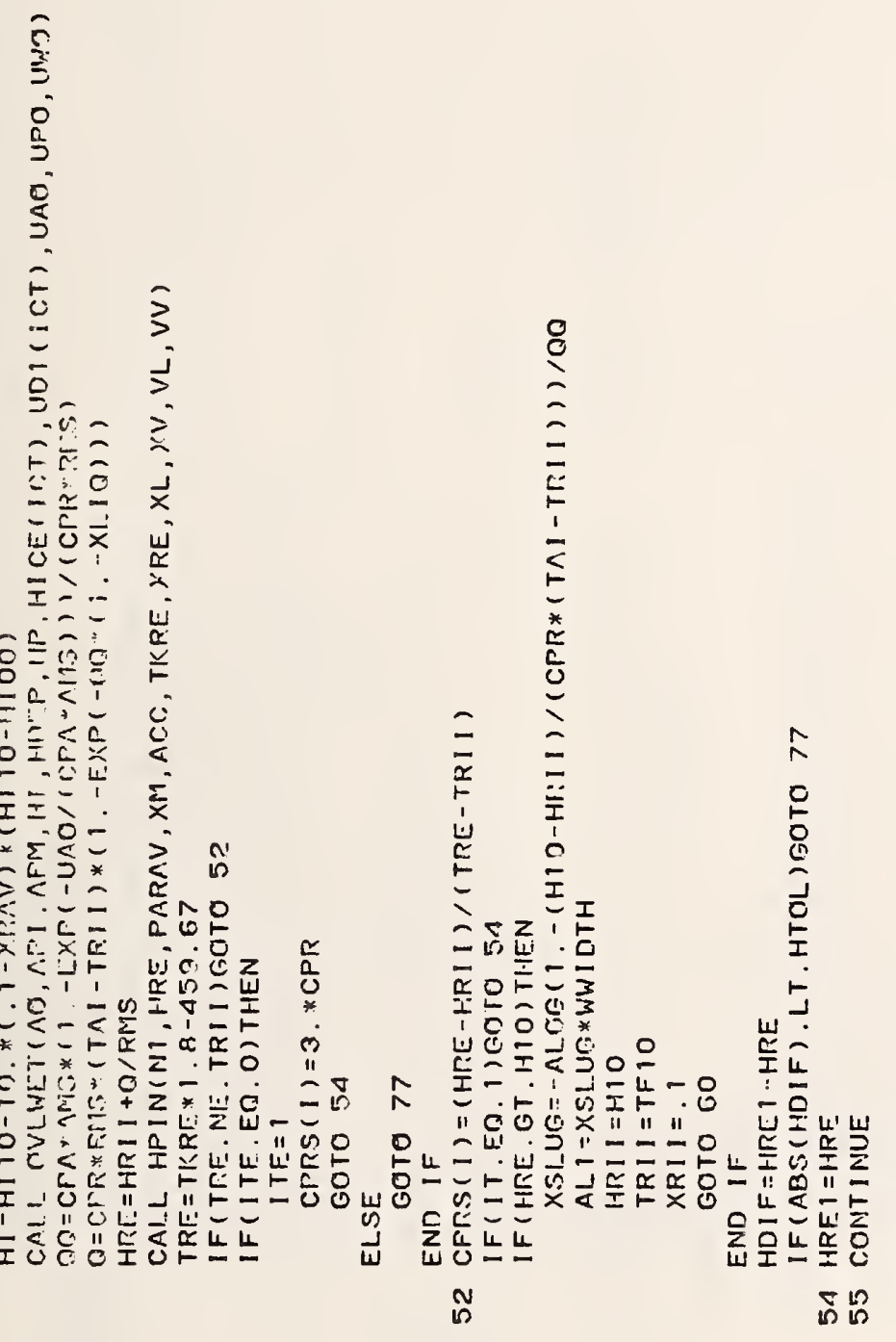

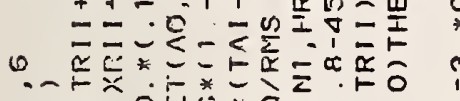

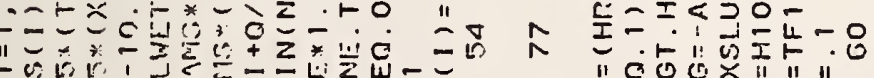

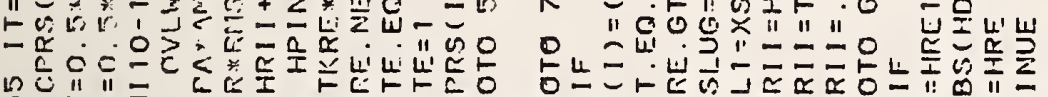

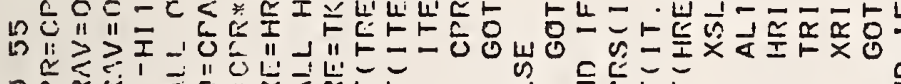

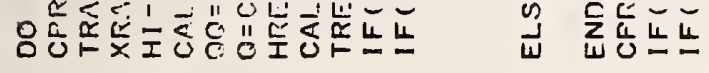

N

in

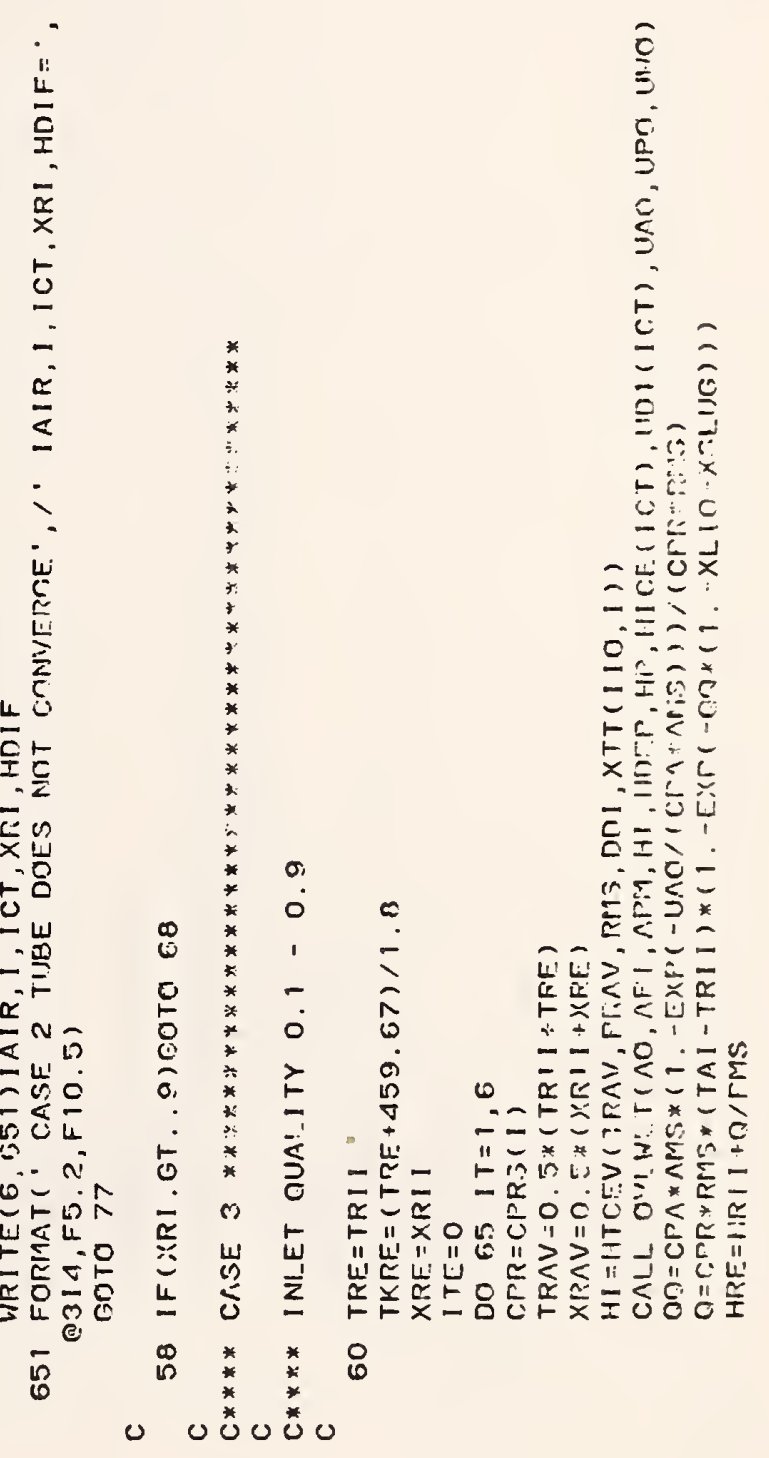

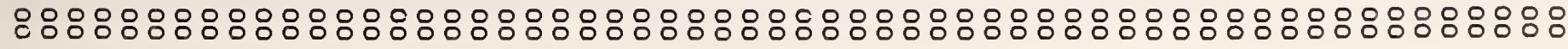

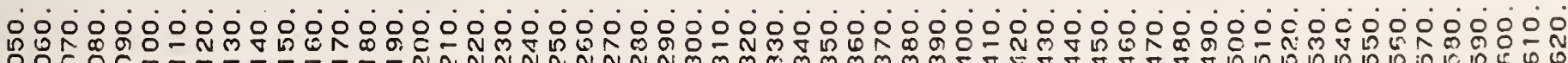

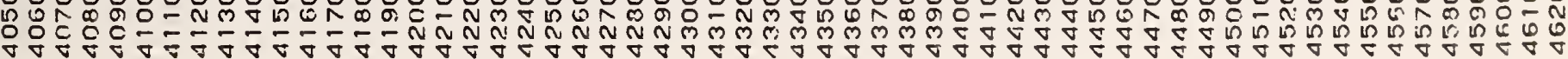




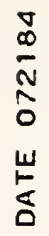

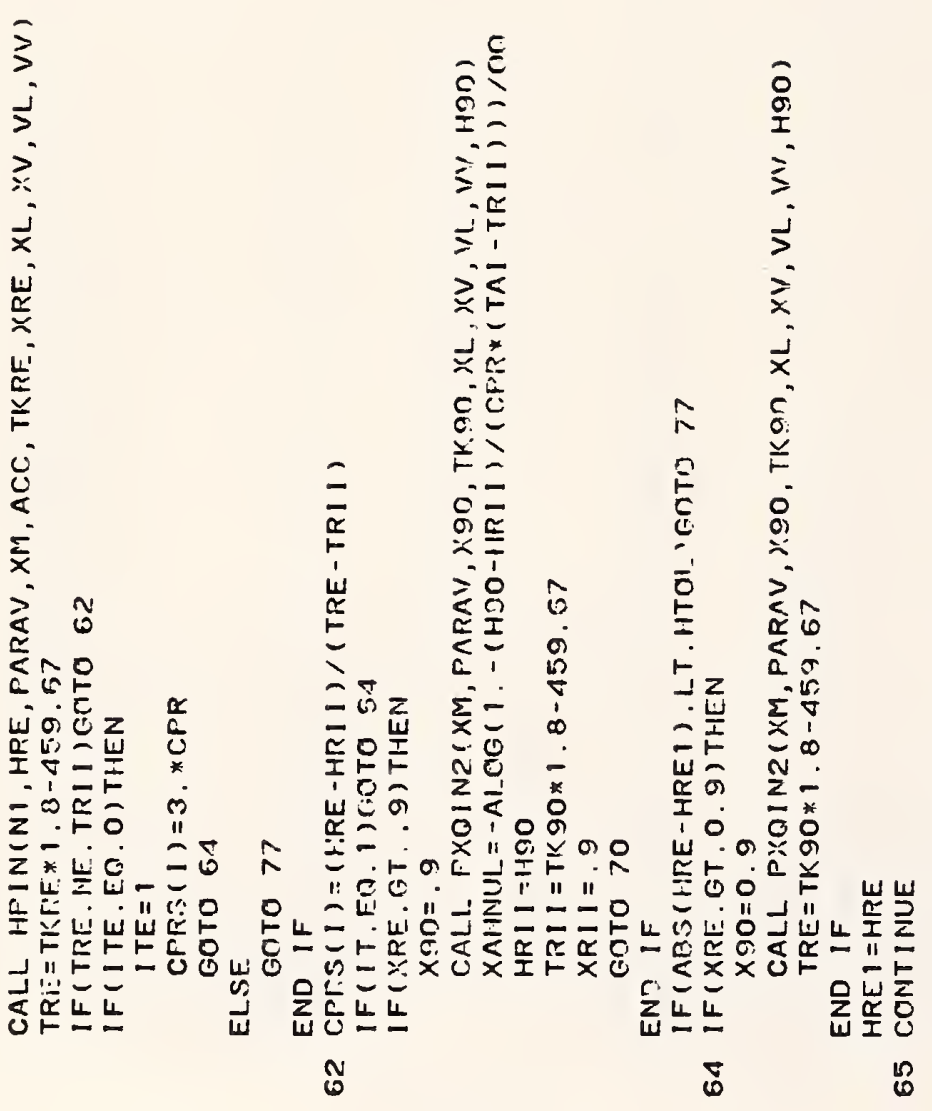

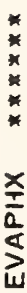

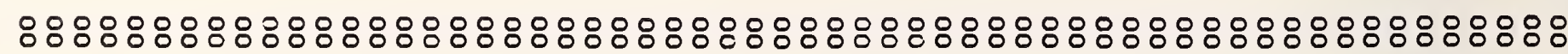

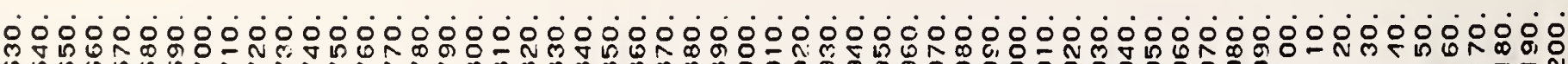

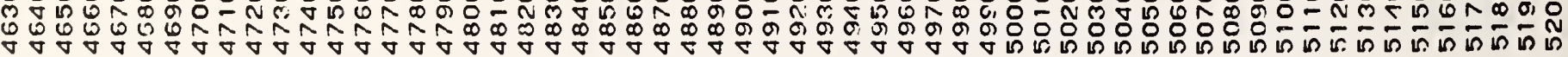




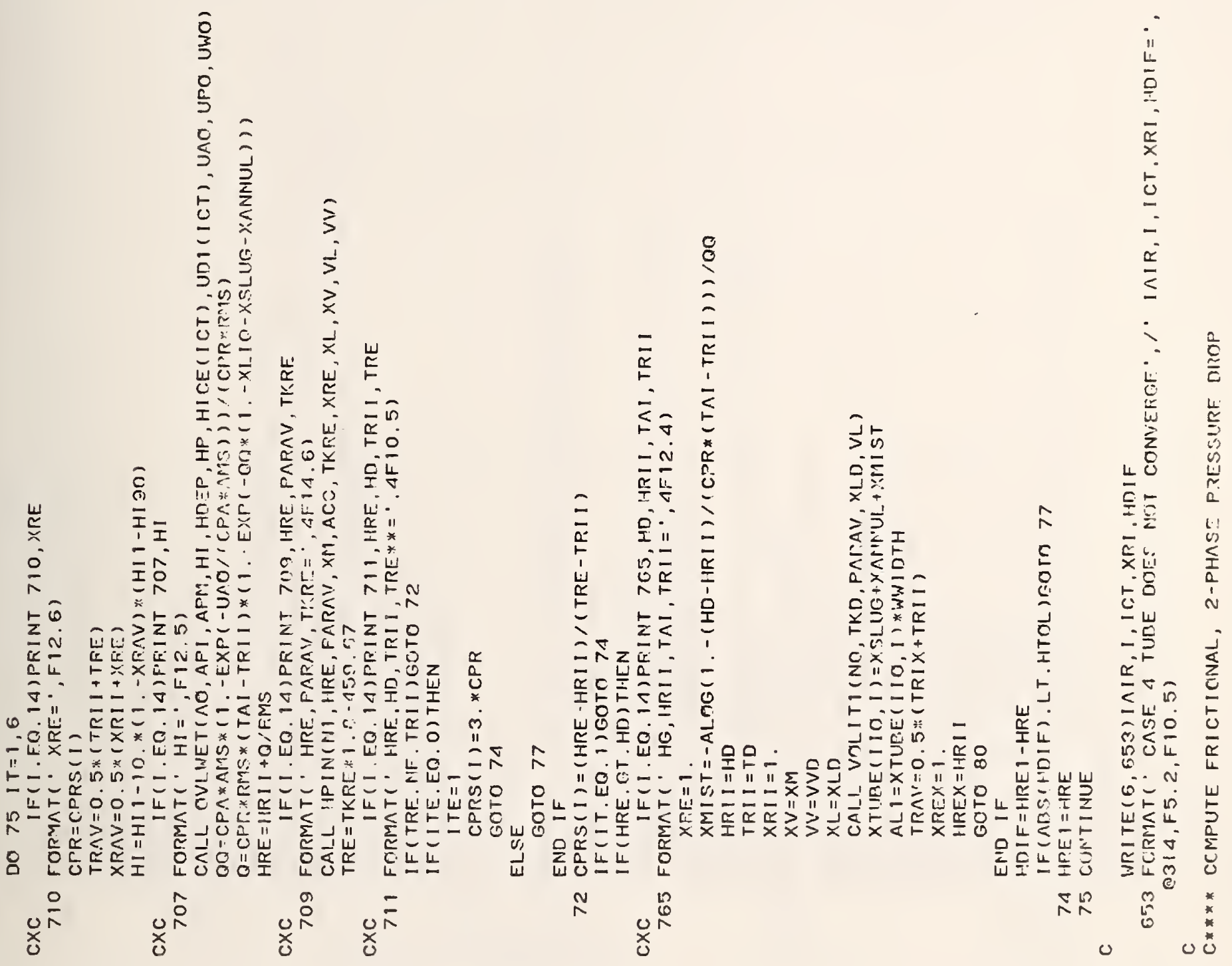

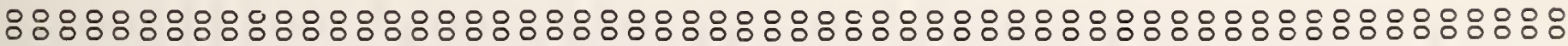

O응은

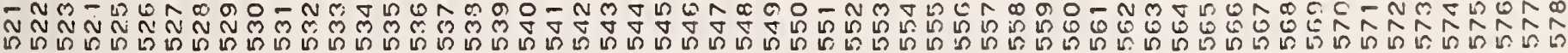




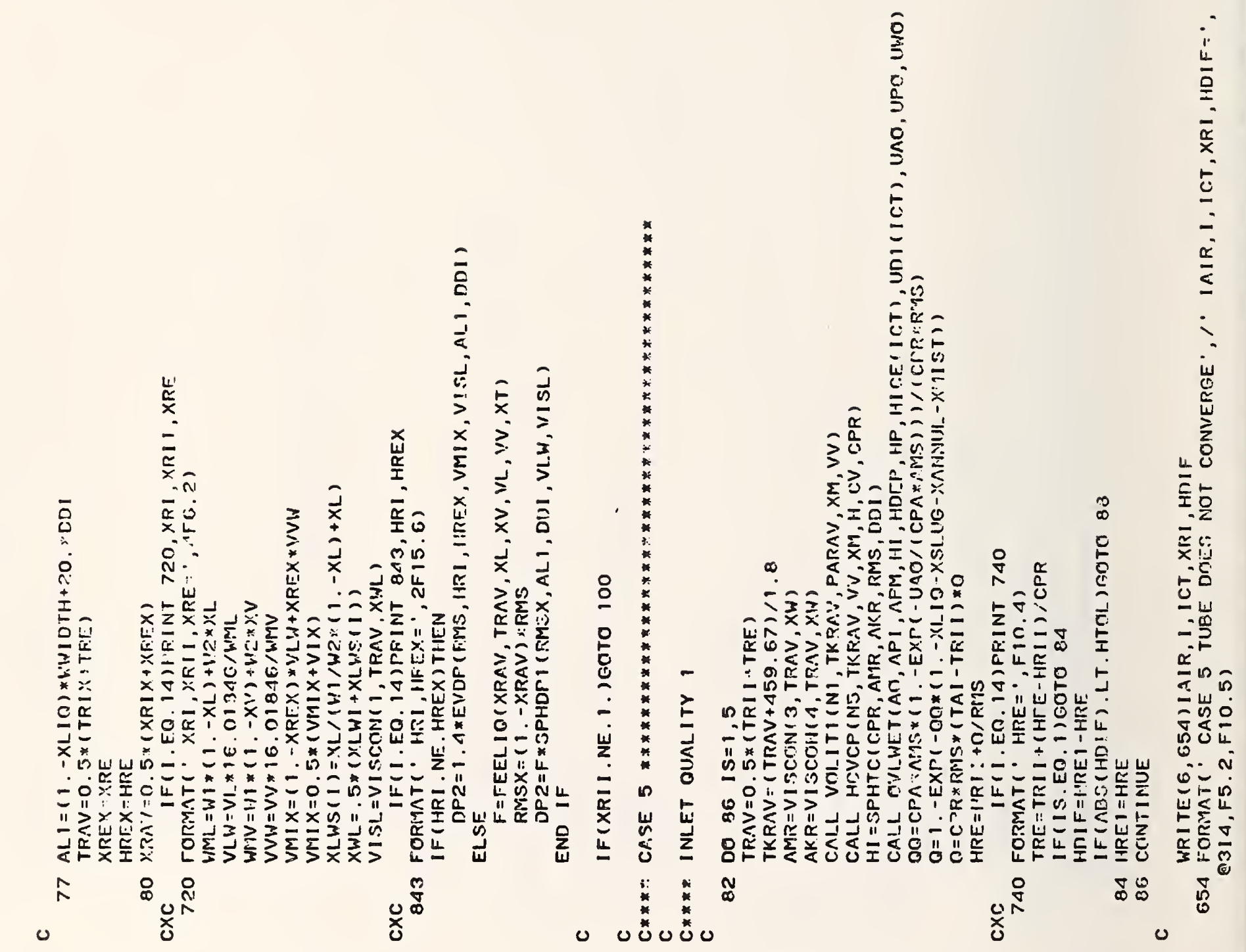


$\frac{7}{N}$
$\frac{N}{0}$
$\frac{1}{5}$

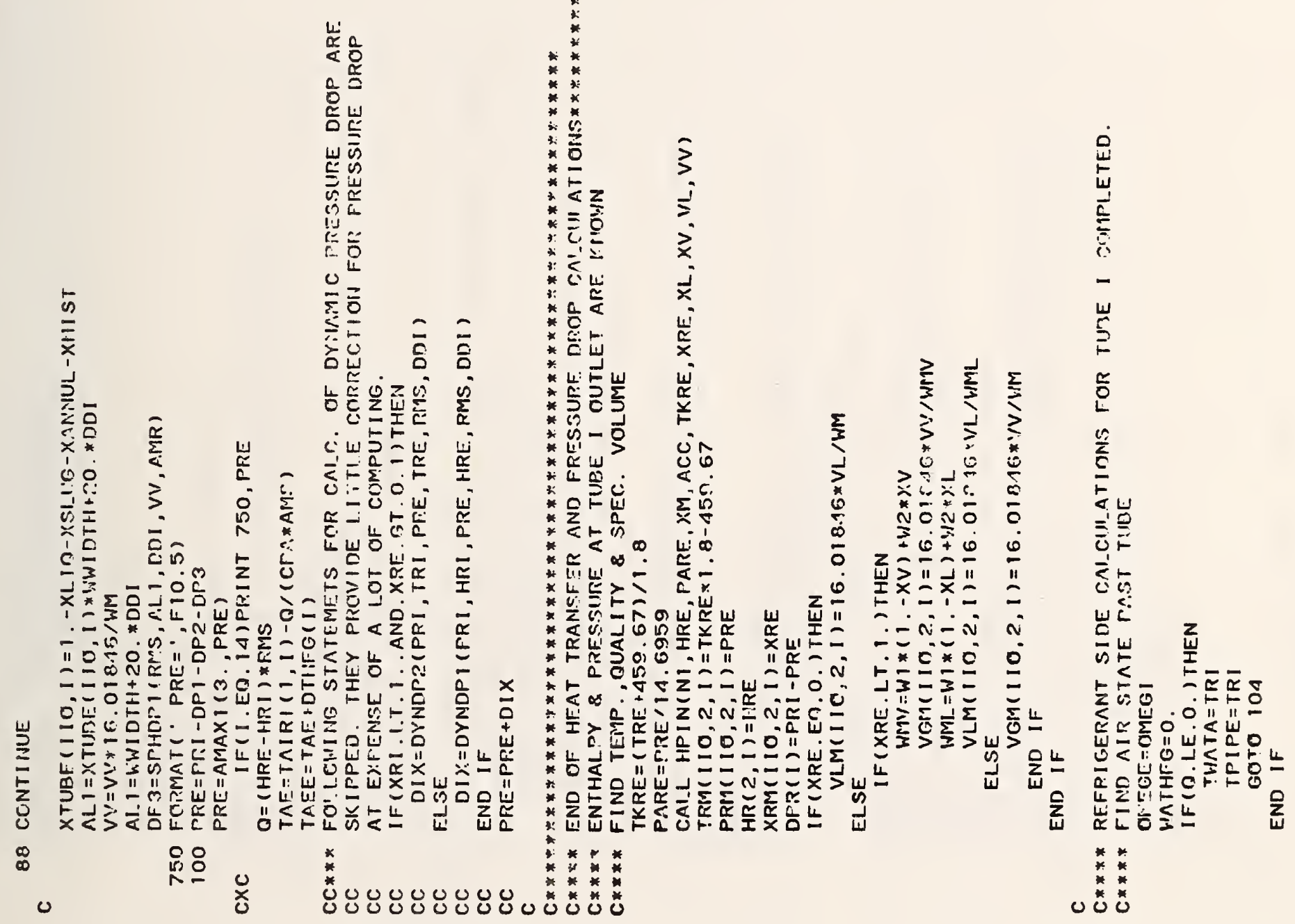

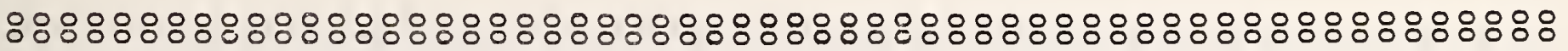
$\frac{x}{\frac{x}{a}}$

வல்

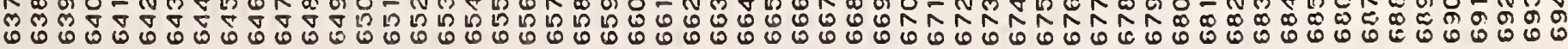




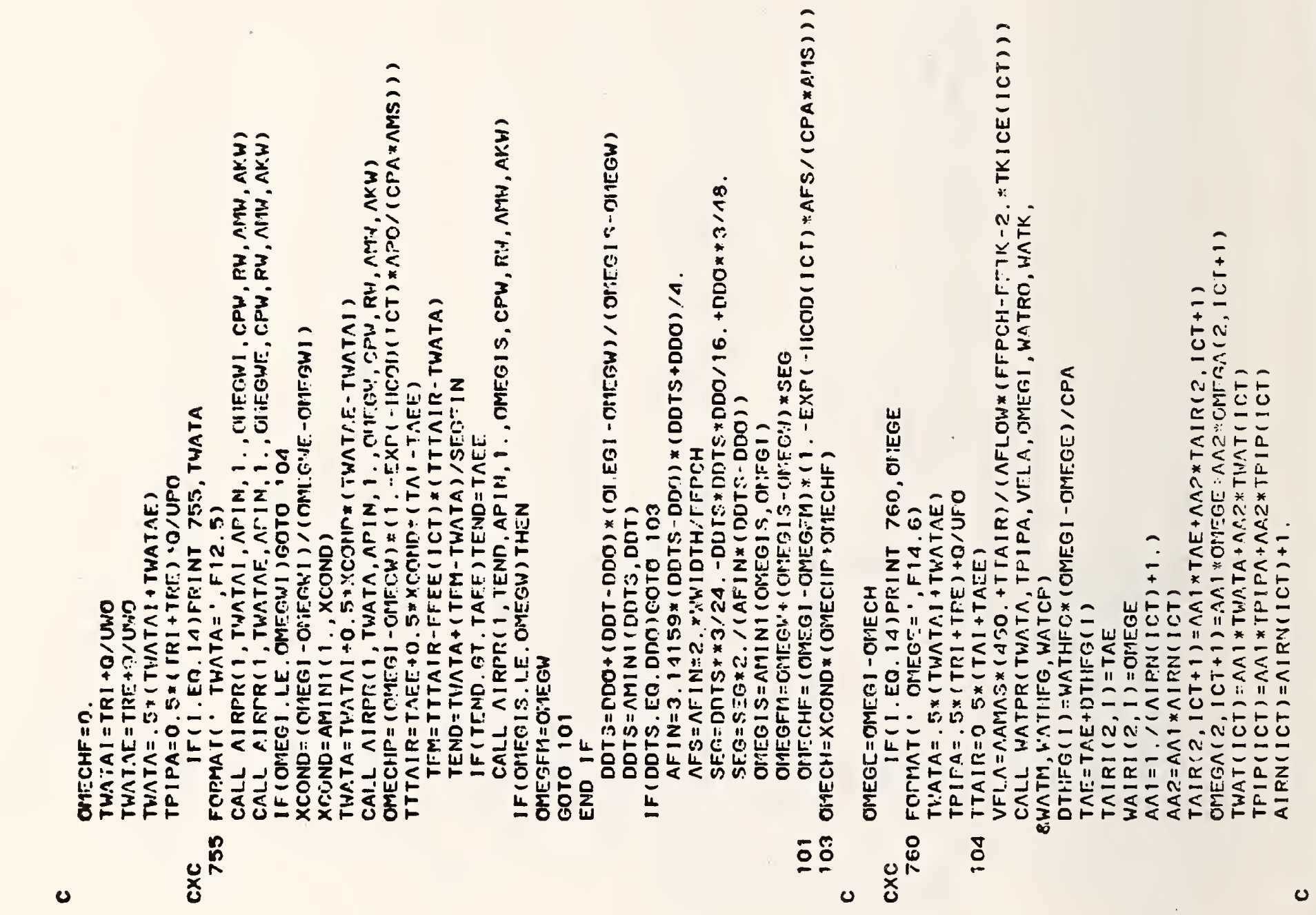

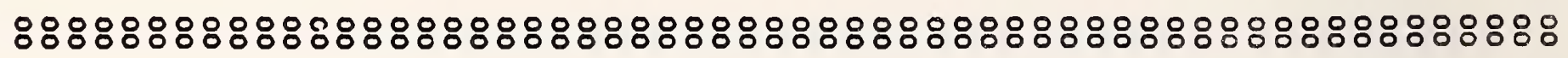

$\frac{x}{2}$

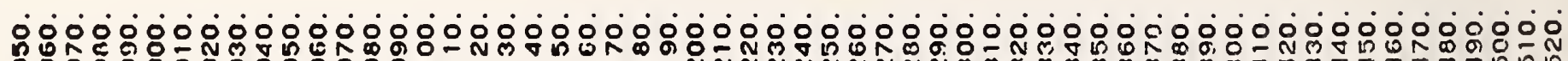

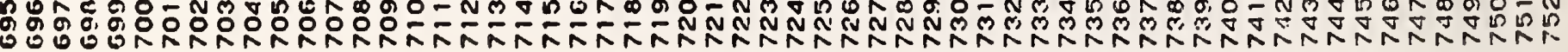




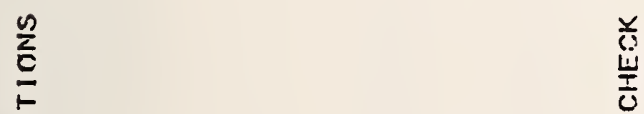

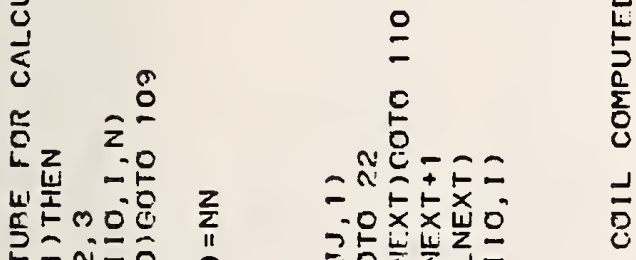

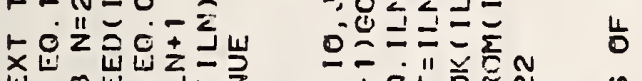

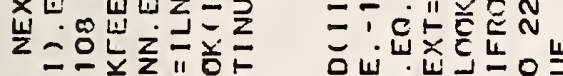

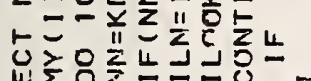

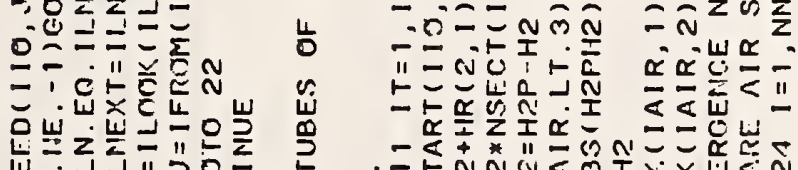

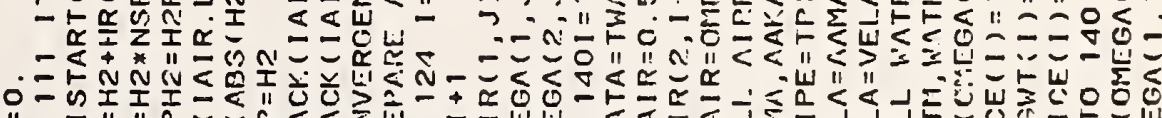

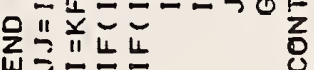

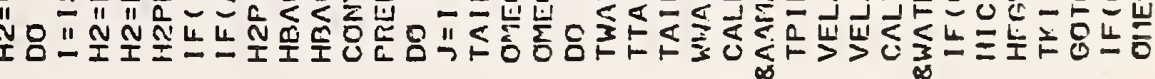

药

$\stackrel{\circ}{\circ}$

$\stackrel{\circ}{=}$

$=\quad \cong \Xi$

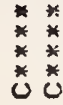

$\stackrel{\mathfrak{N}}{\sim}$

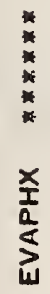

৪৪৪৪৪৪৪৪৪৪৪৪৪৪৪৪৪৪৪৪৪৪৪৪৪৪৪৪৪৪৪৪৪৪৪৪ঃ৪৪৪৪৪৪৪৪৪৪৪৪৪৪৪৪৪৪৪৪৪

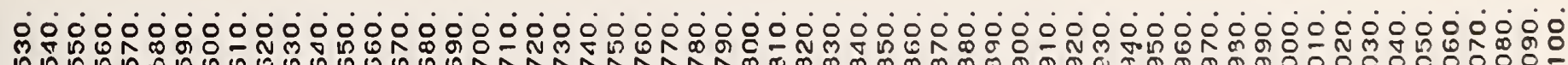

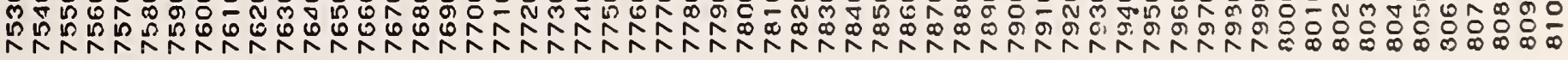



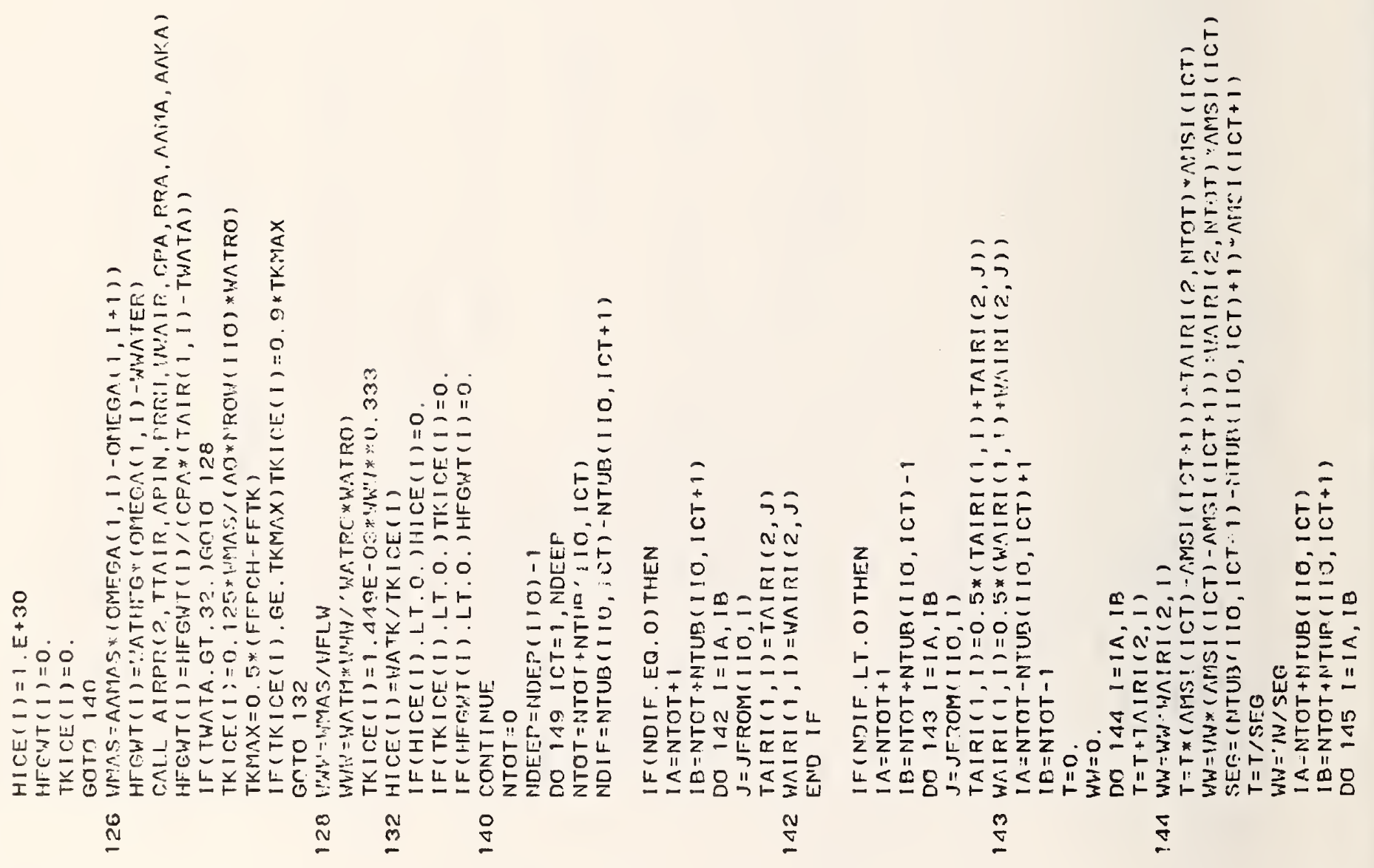

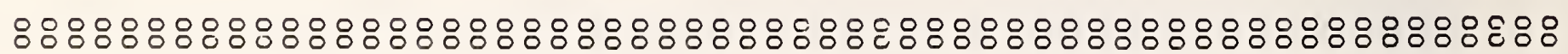

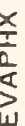

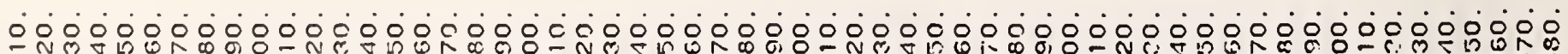

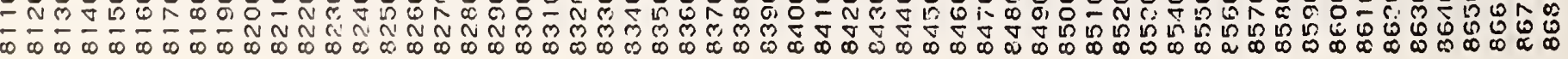




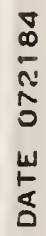

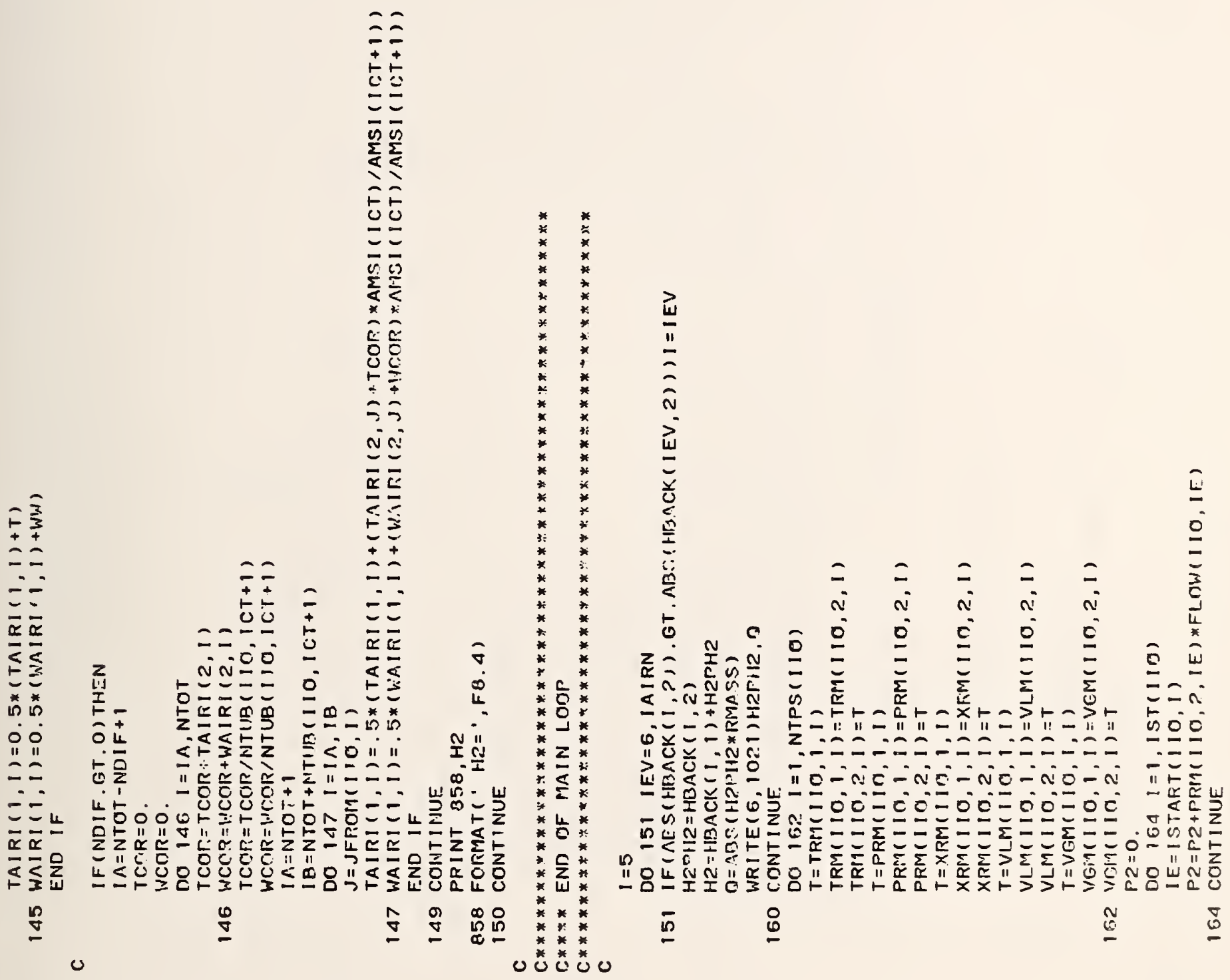

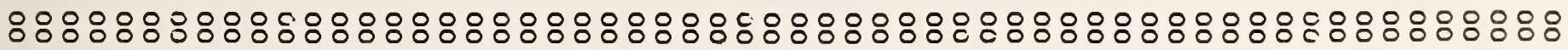

$\frac{x}{1}$

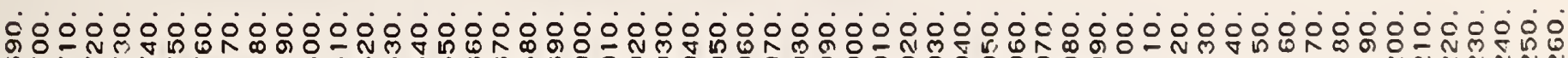

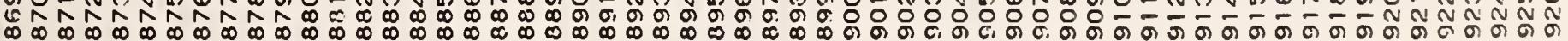




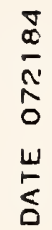

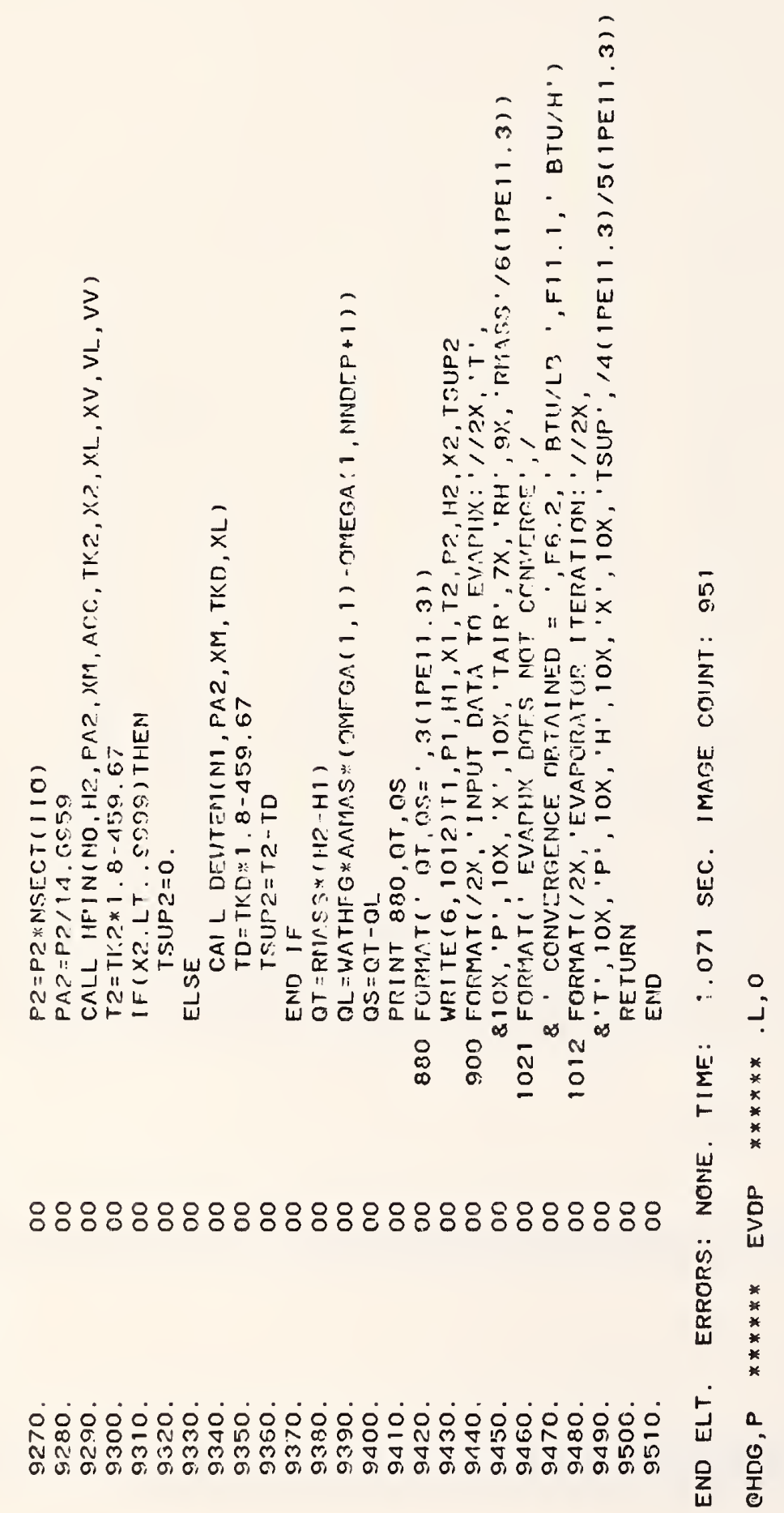




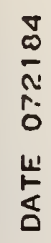

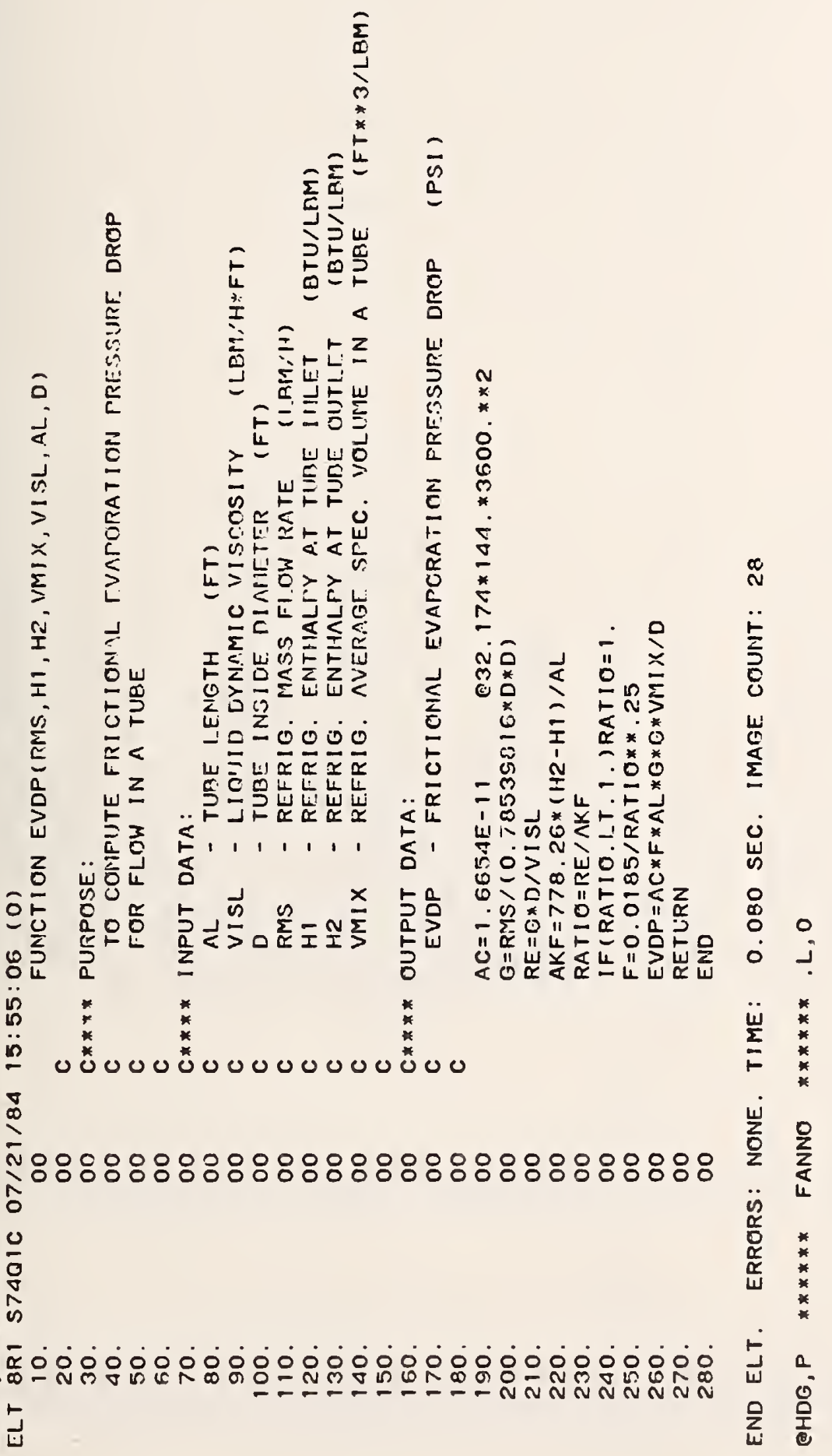




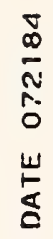

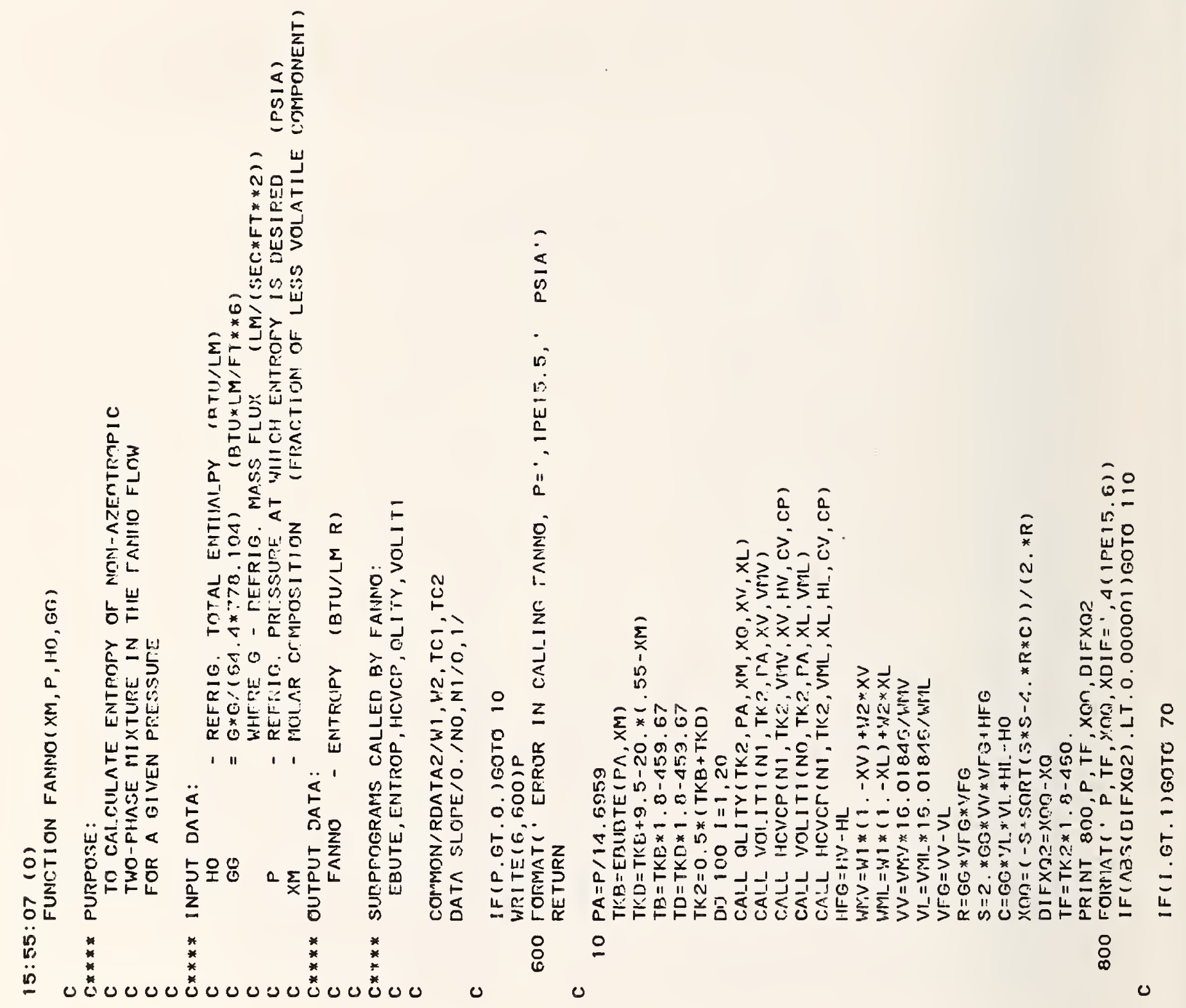

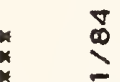

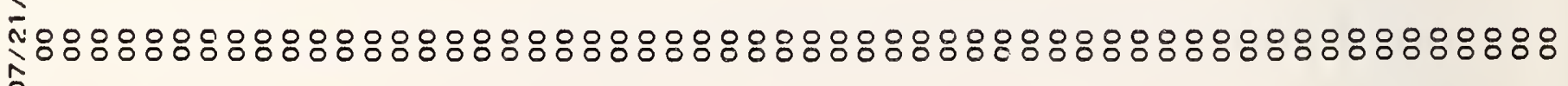

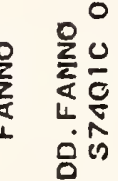

L

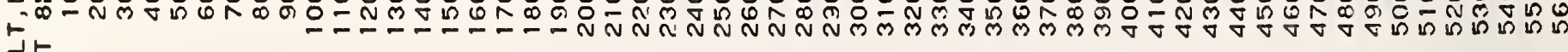
造岕 


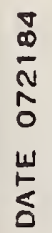

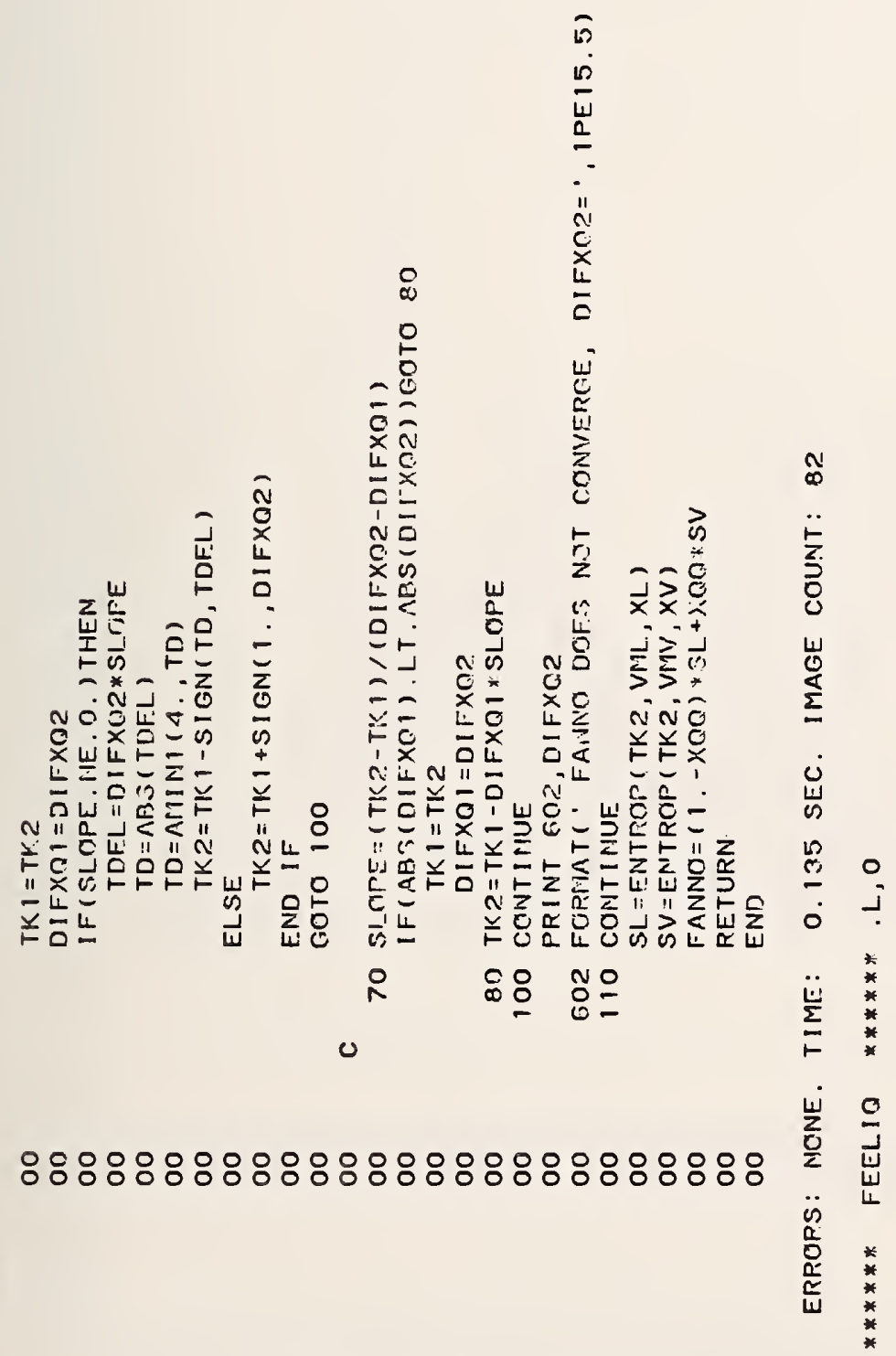

का 


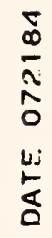
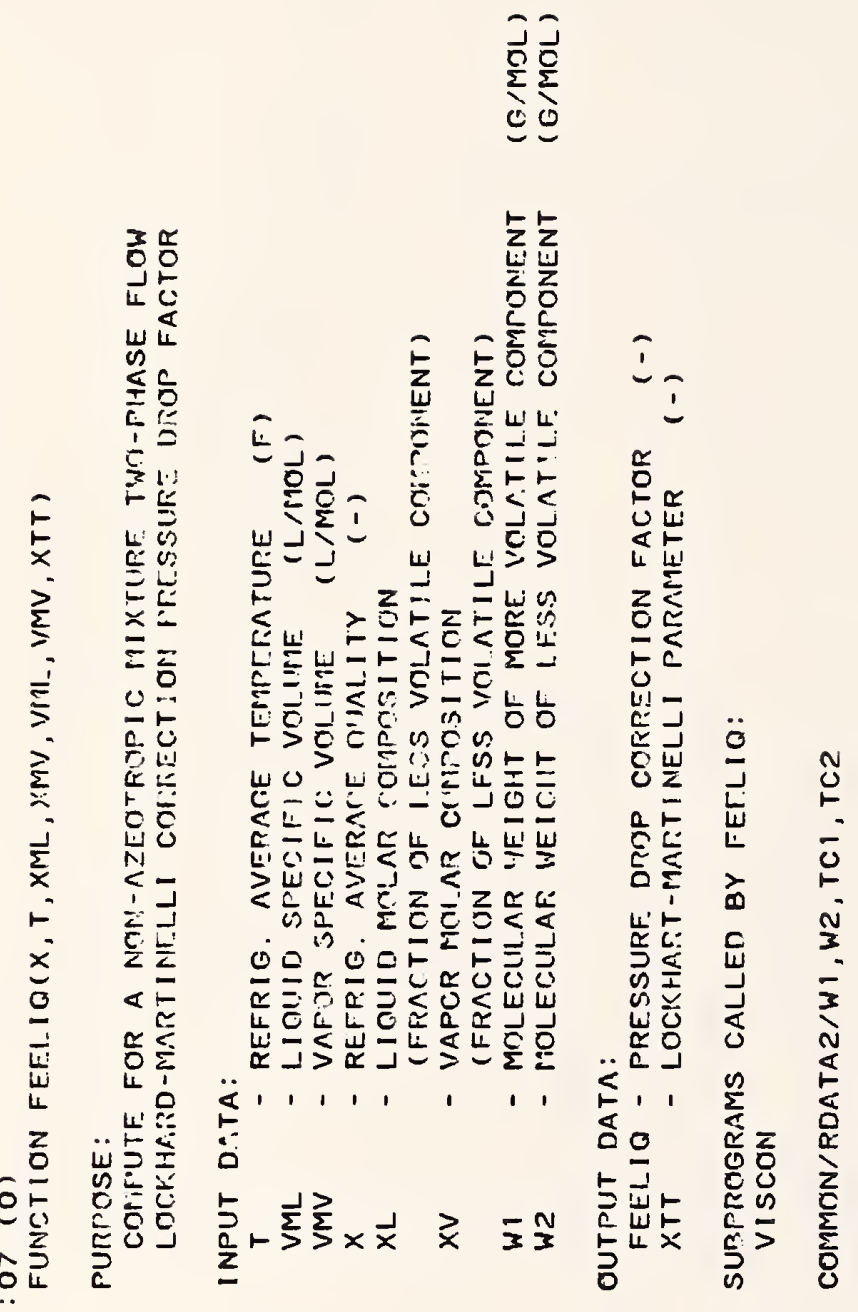
in os

는

$\underset{4}{w} \mathrm{v}$

เ上 


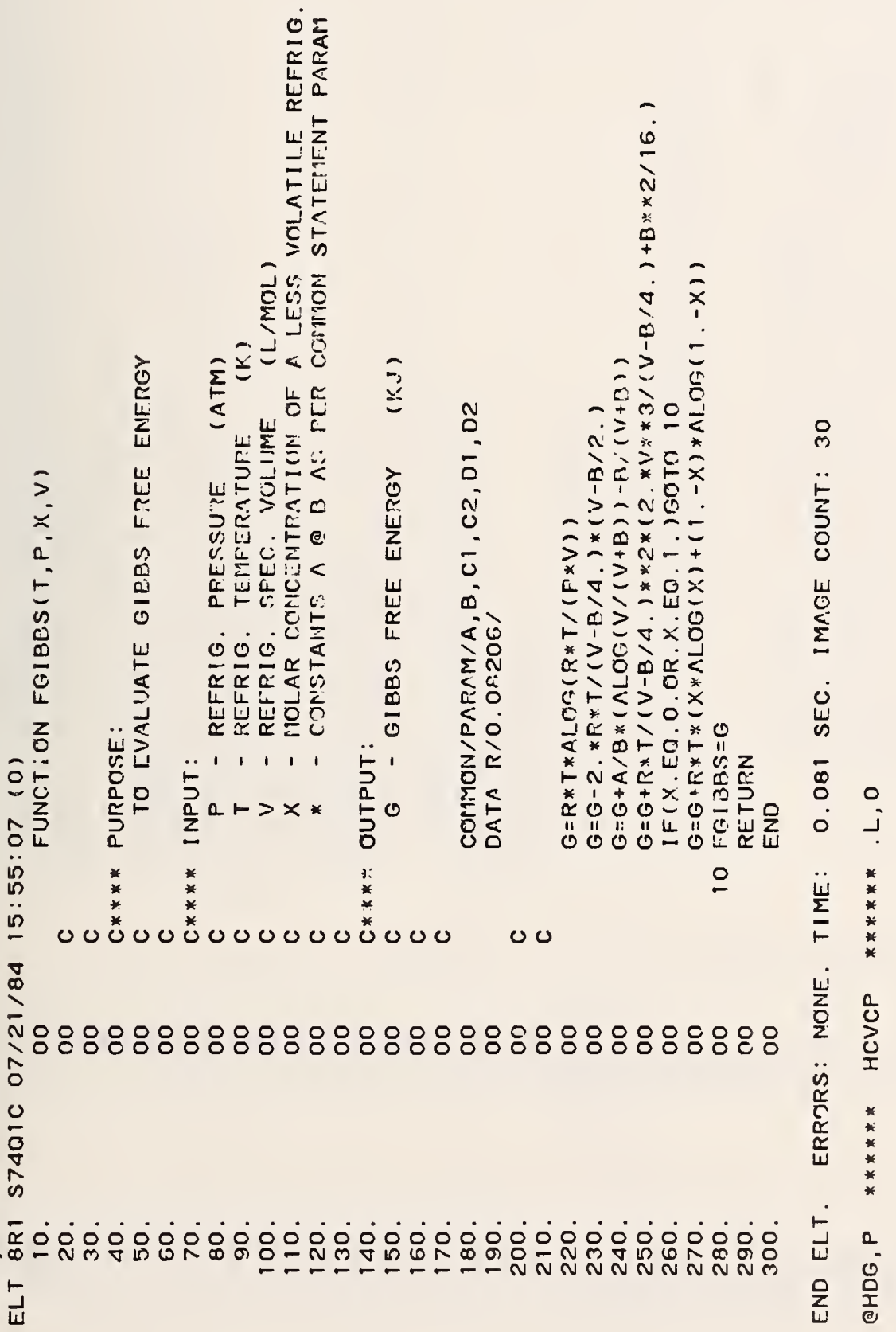




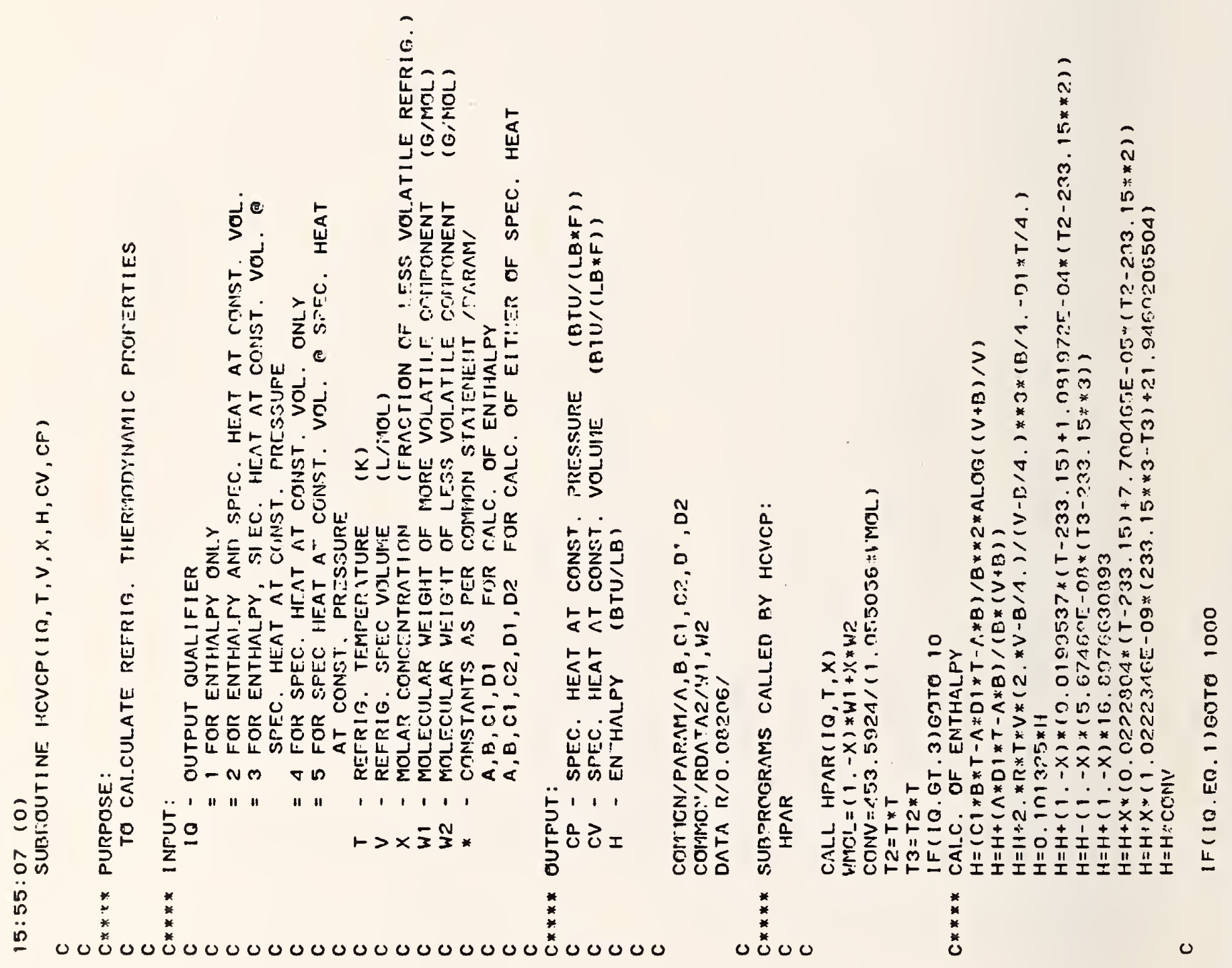

*

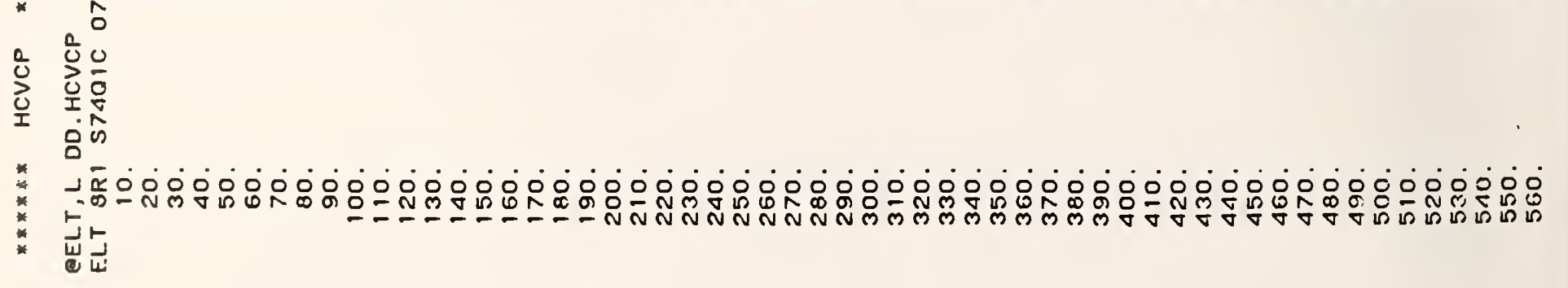


$x^{2}+x^{2}$

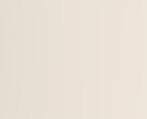

$2 \pi$

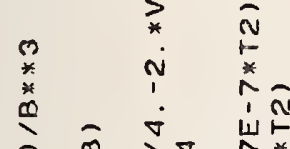

ह

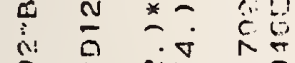

* *

1
$\infty$
$\infty$

$\rightarrow$ i

北

₹

एँ

*

$+\quad \therefore \frac{0}{2}$

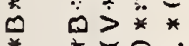

त ले

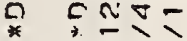

कर्ण

पर

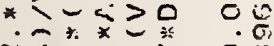

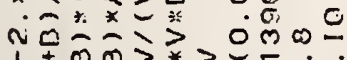

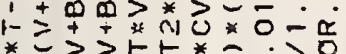

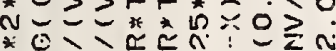

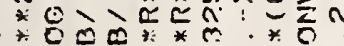

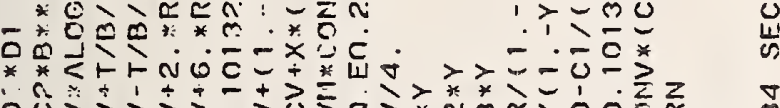

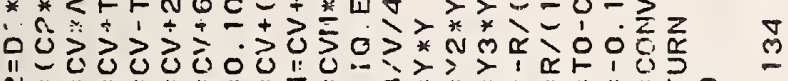

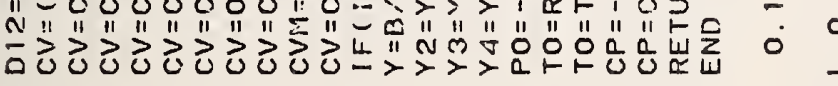
$\therefore$

인

:

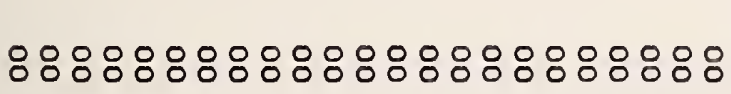

$\frac{a}{0}$

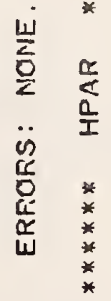

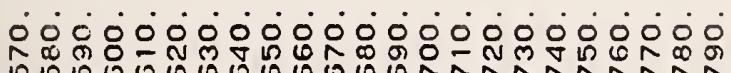

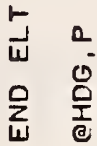




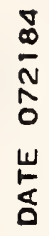

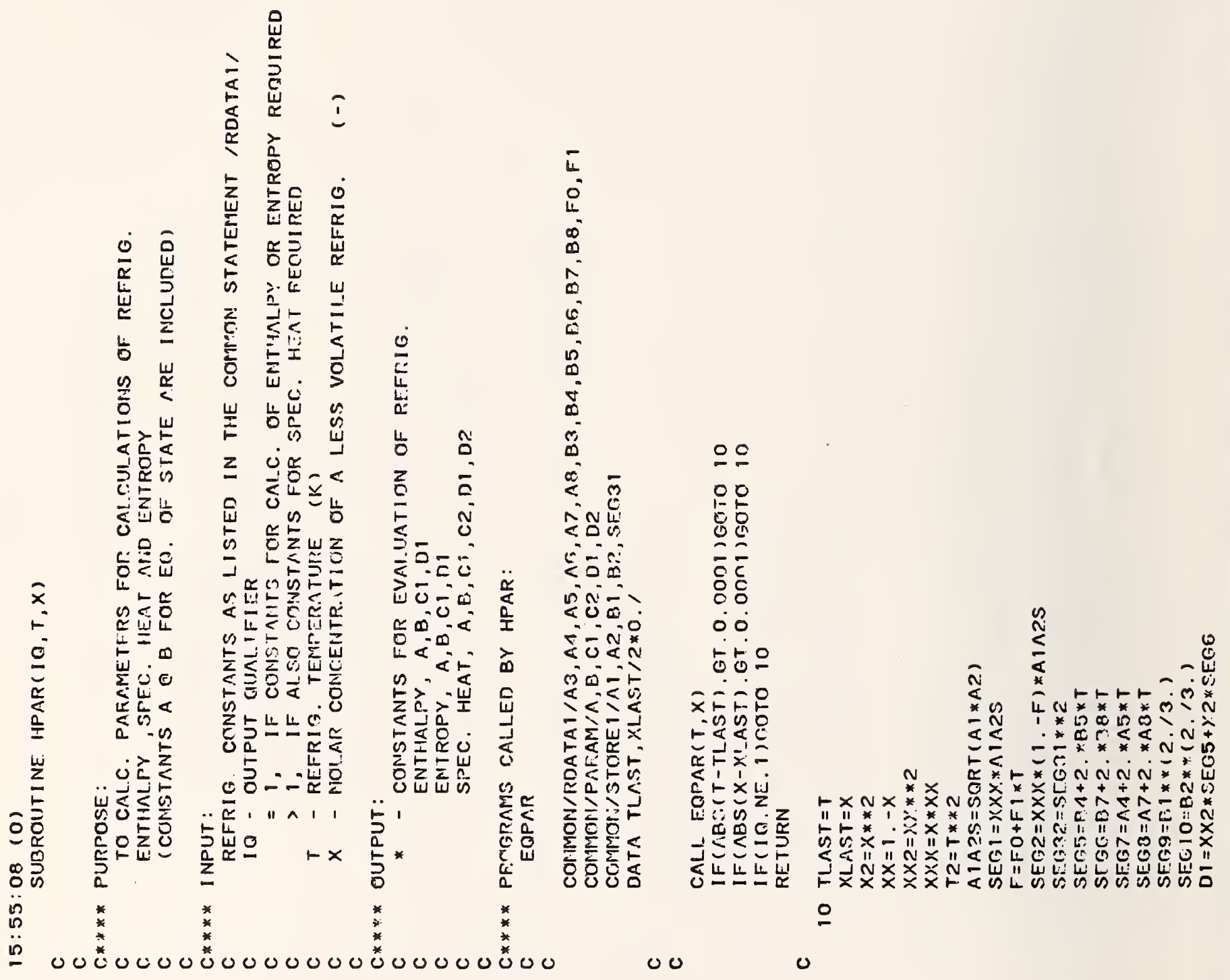

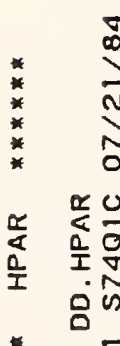

.




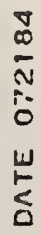

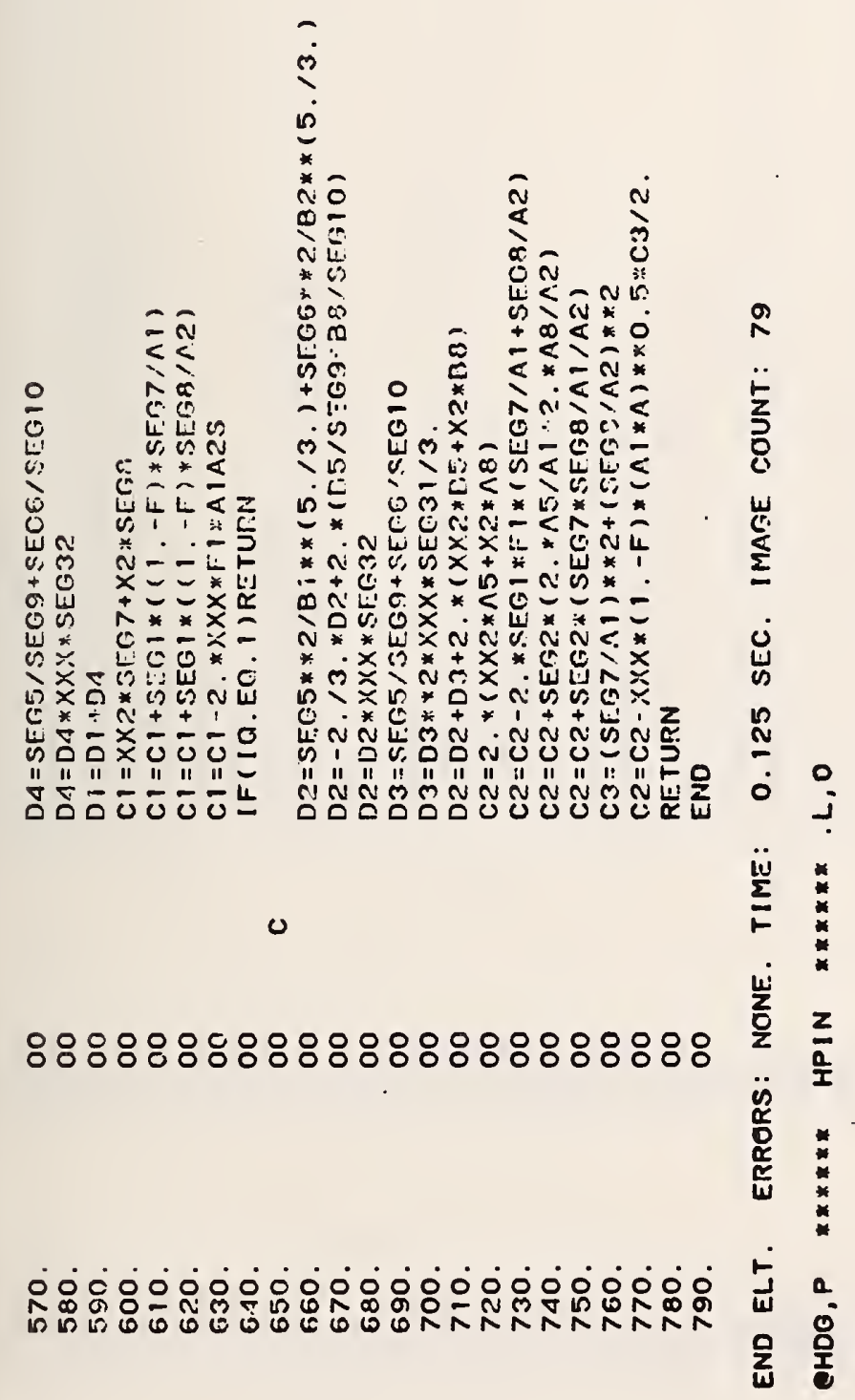




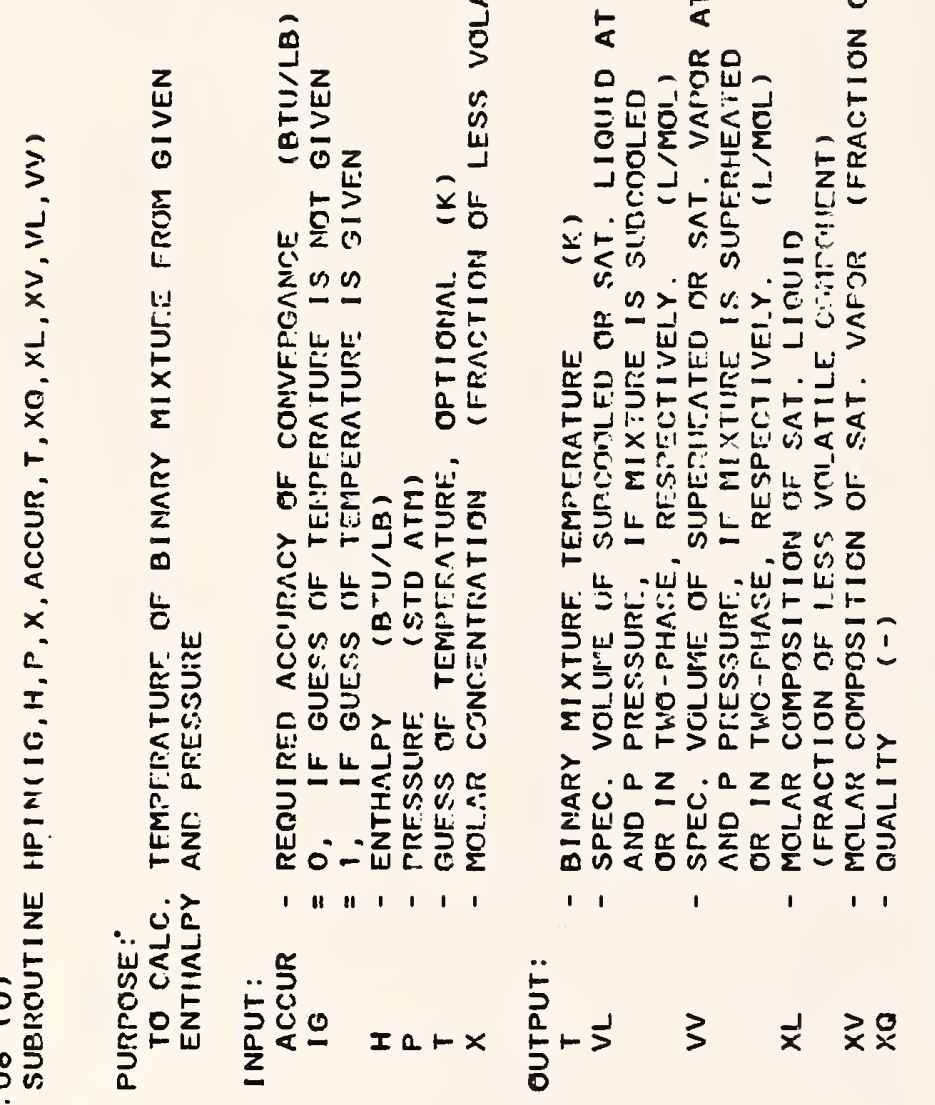

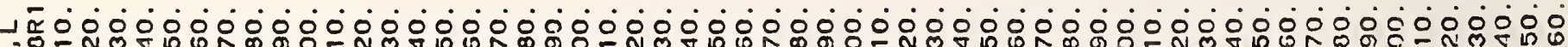
击 㟒 


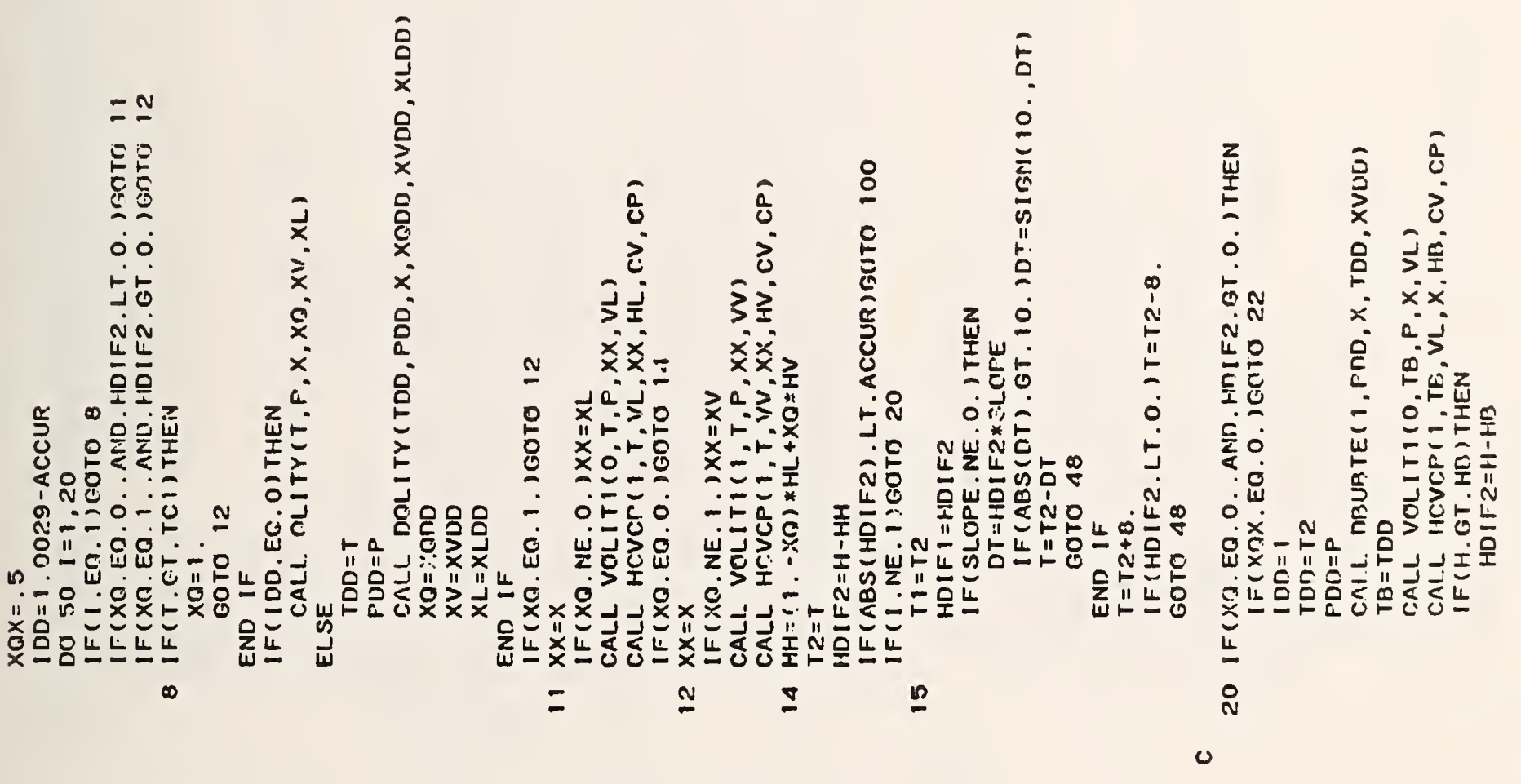

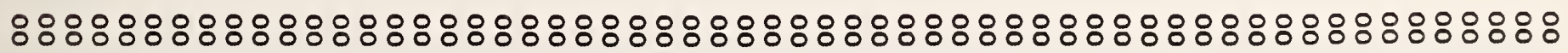

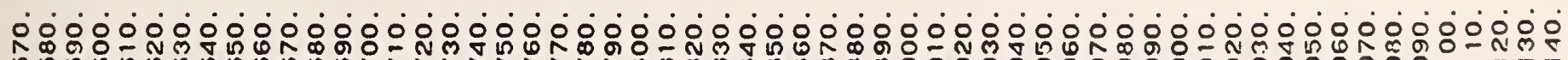

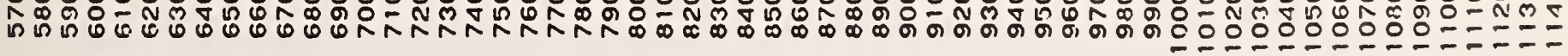



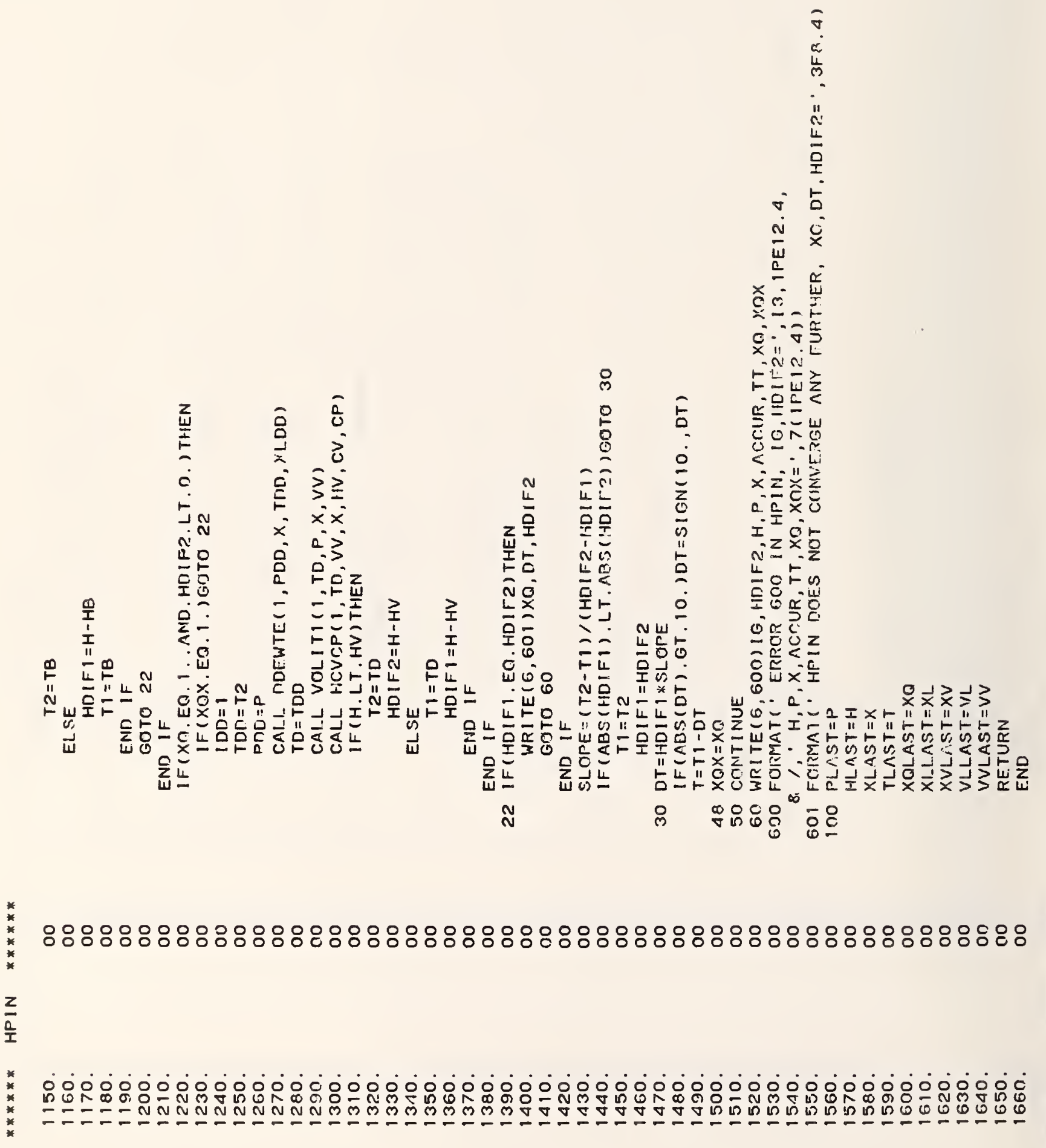


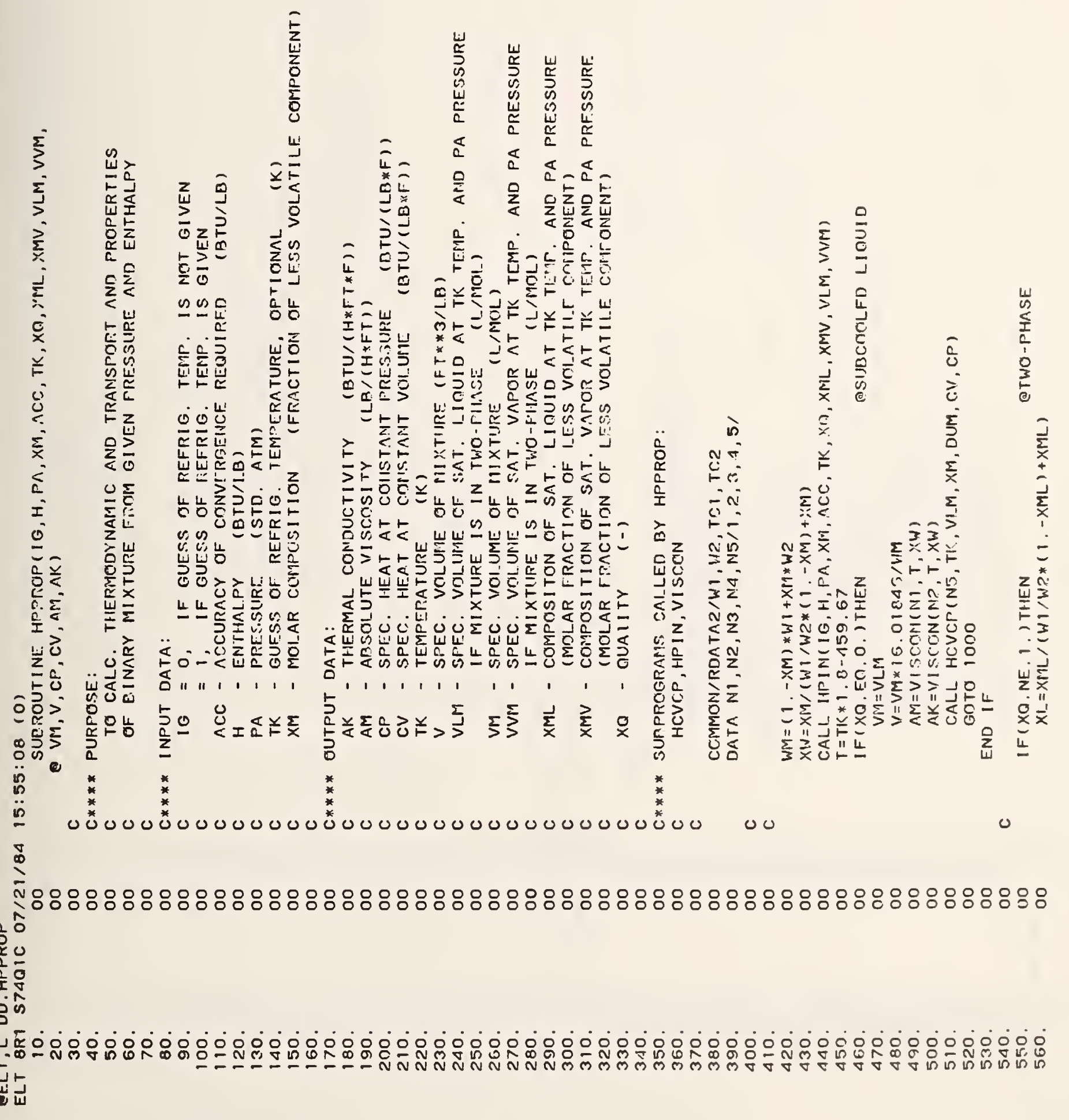




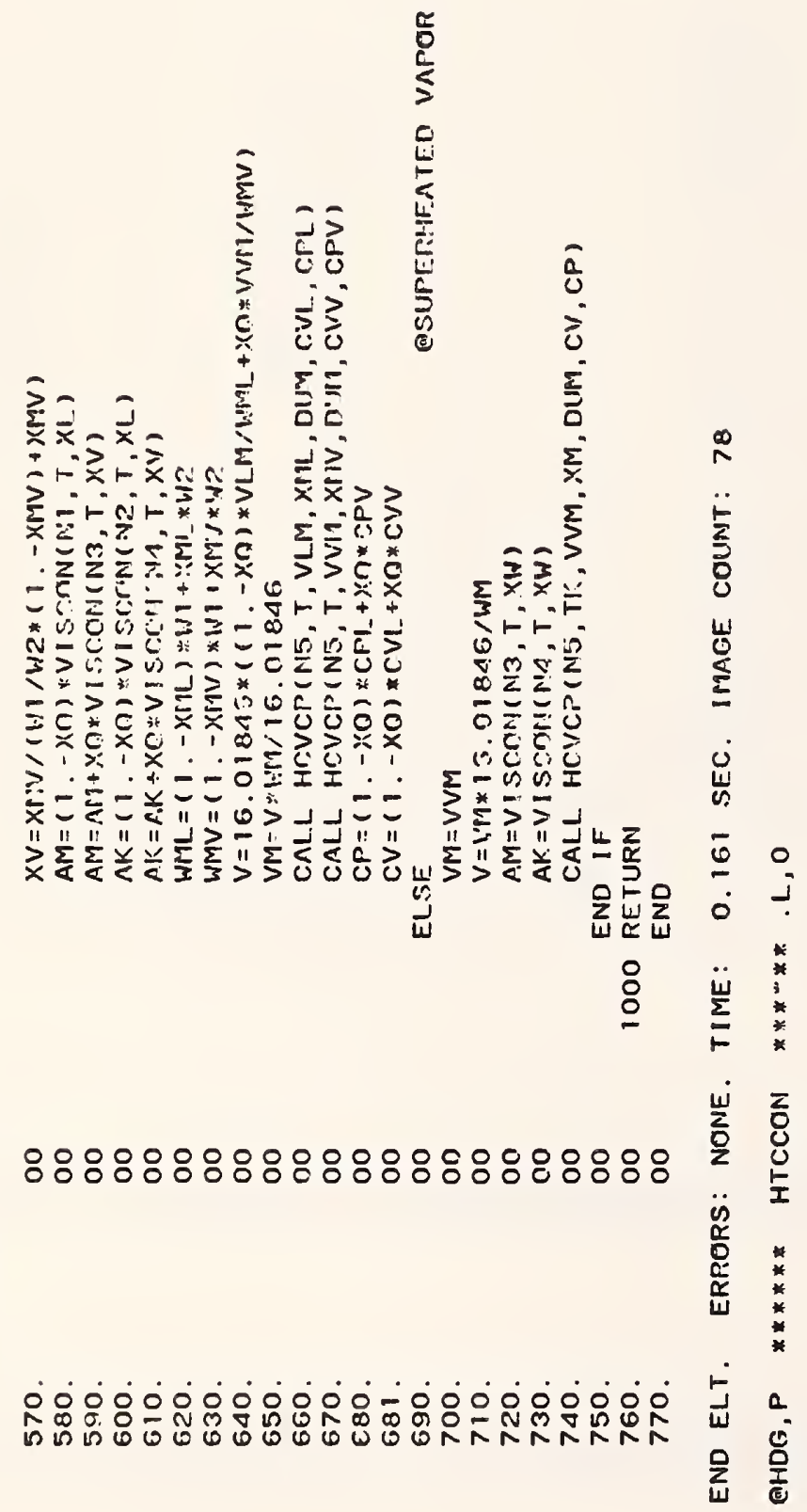




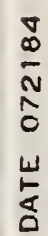

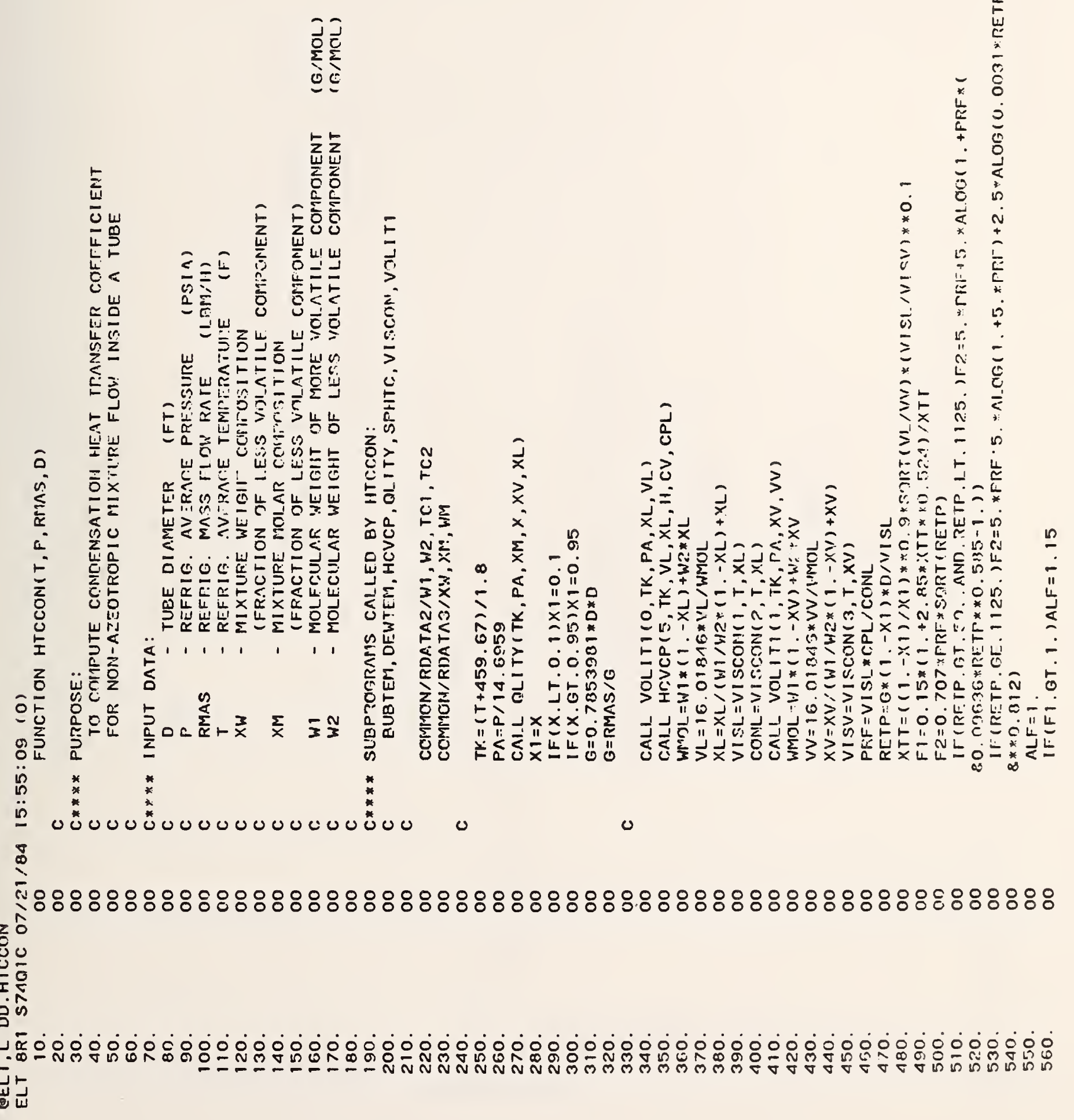




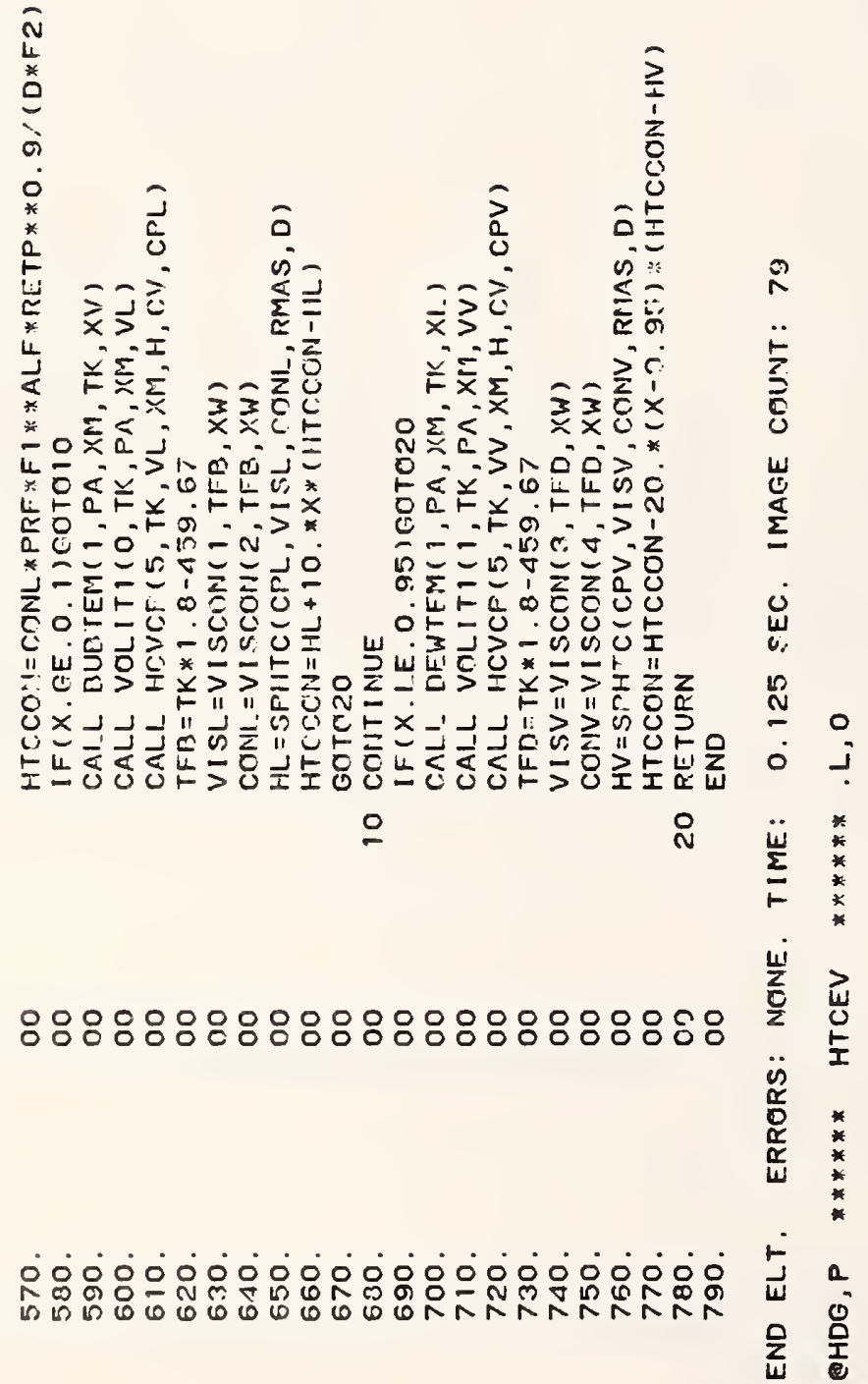




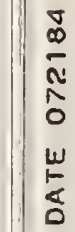

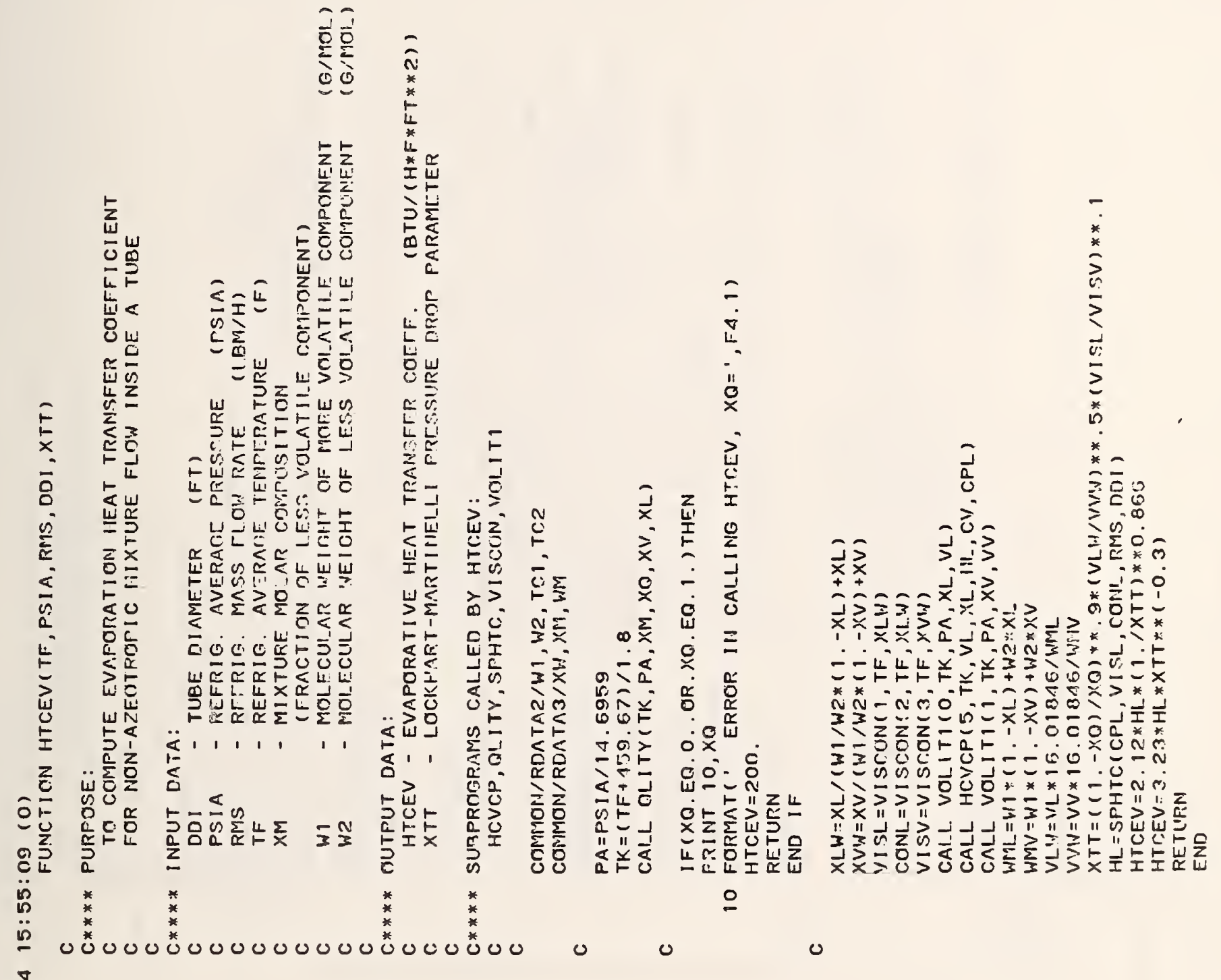
.

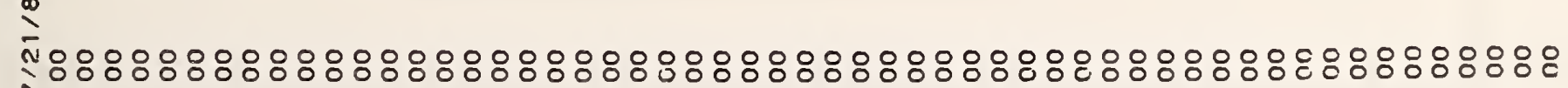
* 岁热

L F- N 峞步 


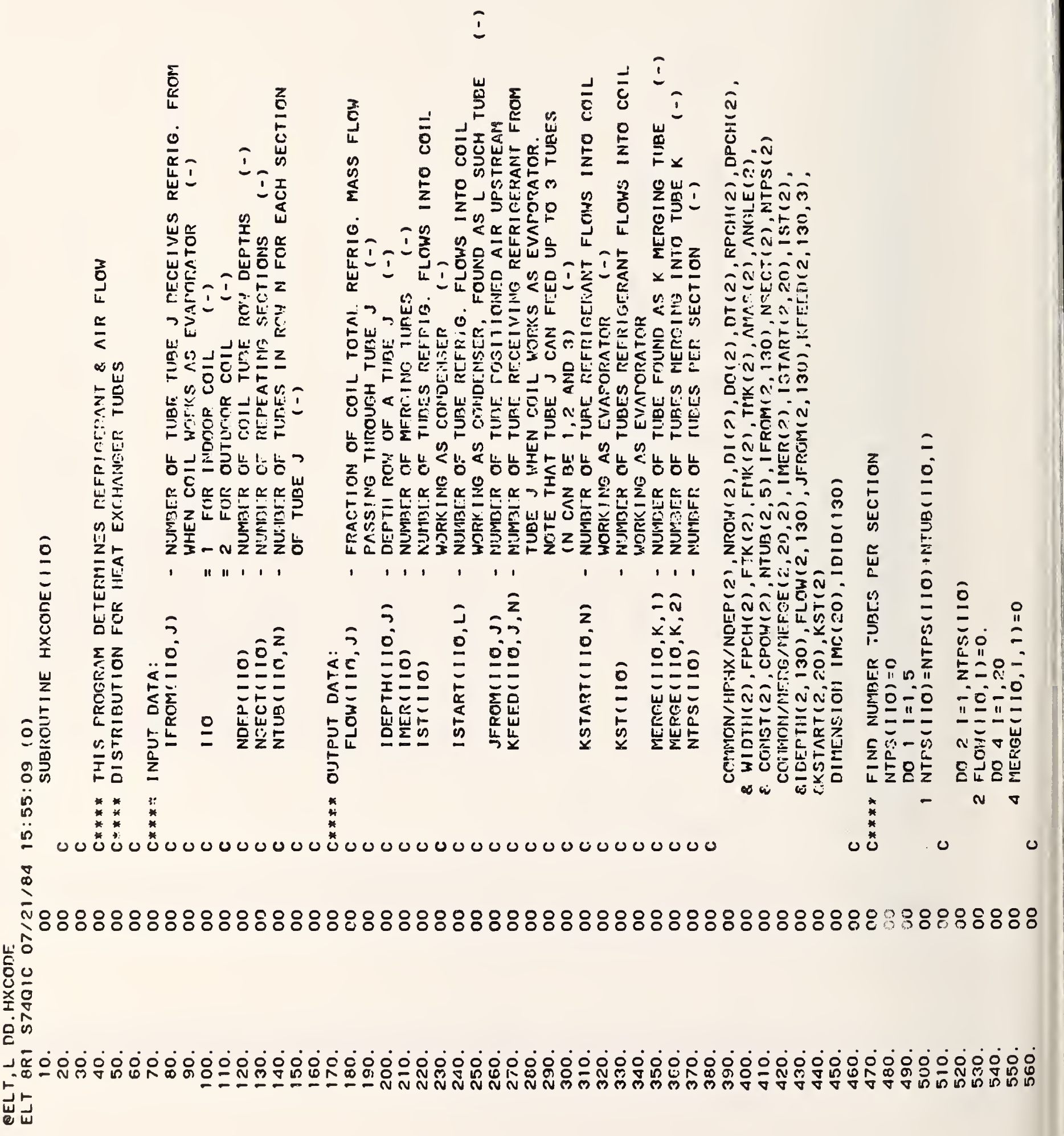


Wh

嵌它

$\sum$

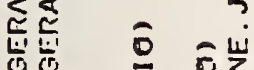

过岕崖

r

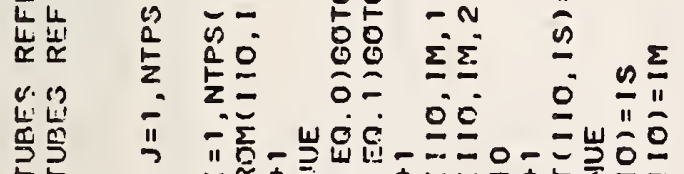

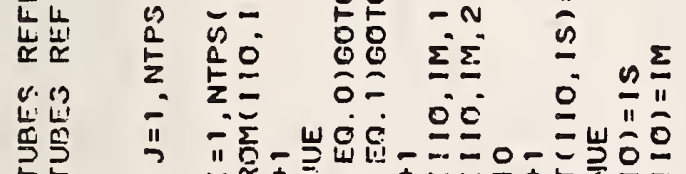

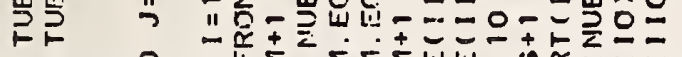

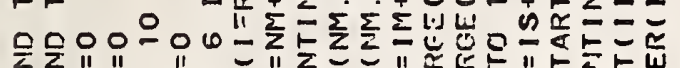

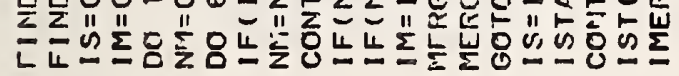<smiles>[18O-]</smiles>

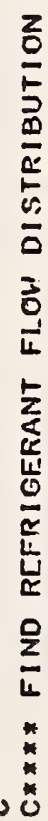

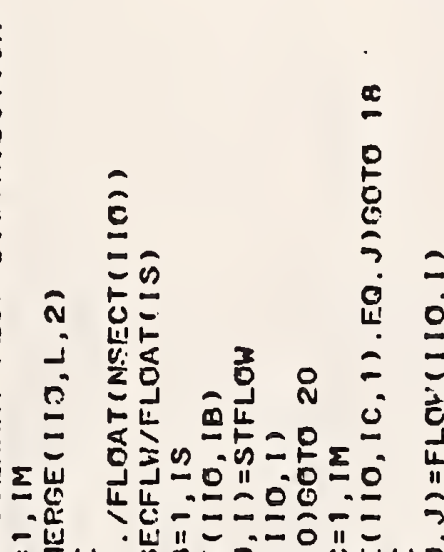

$=$

㟧"

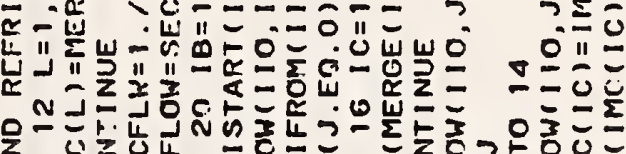

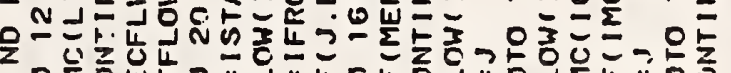

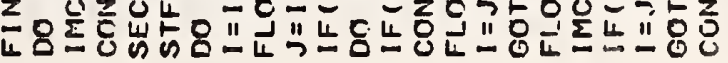

嵌

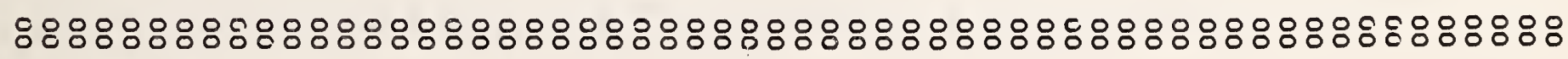

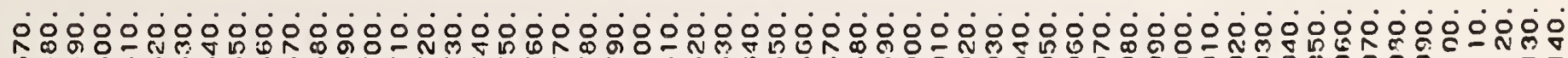

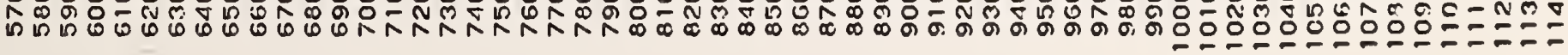




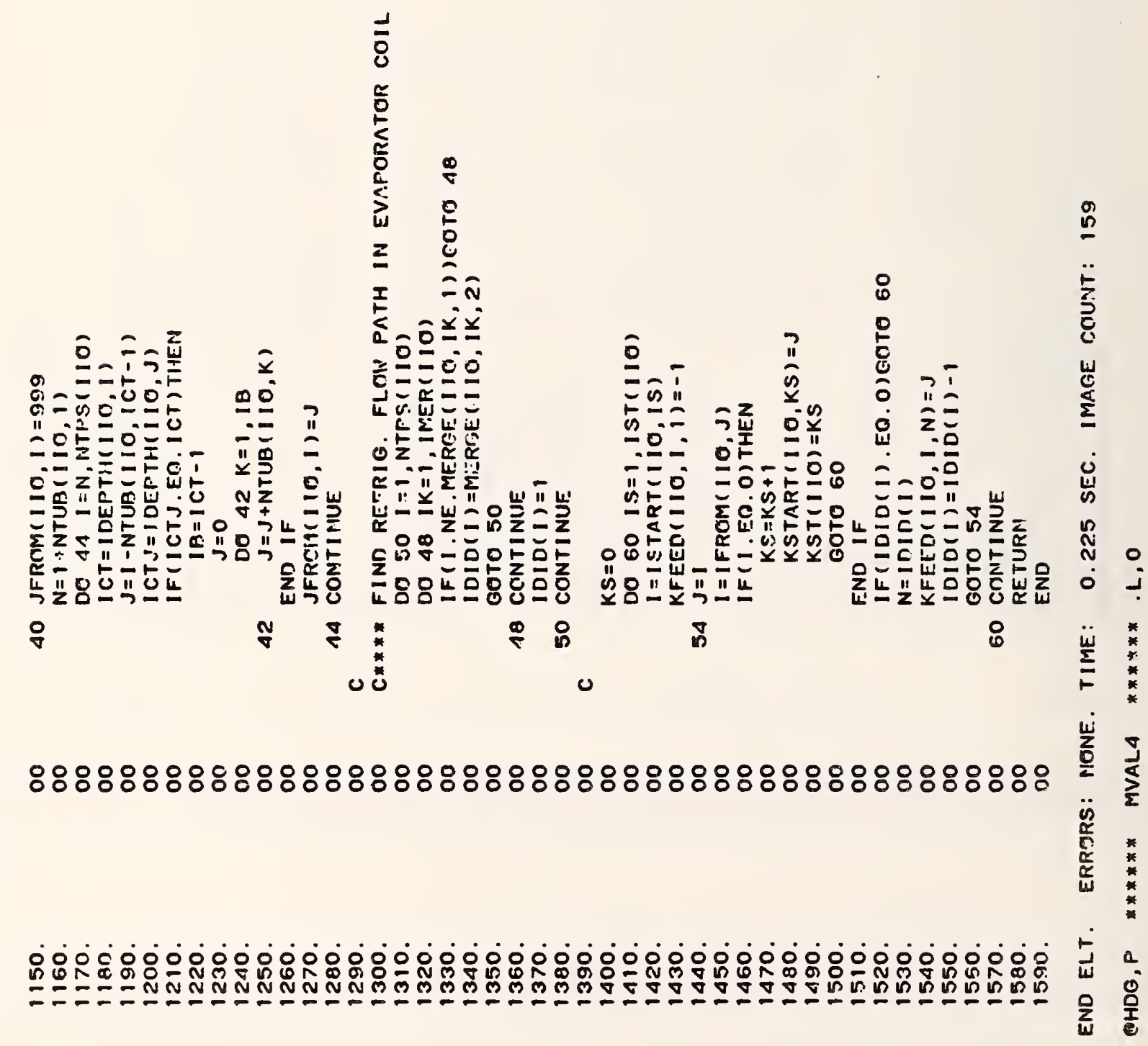




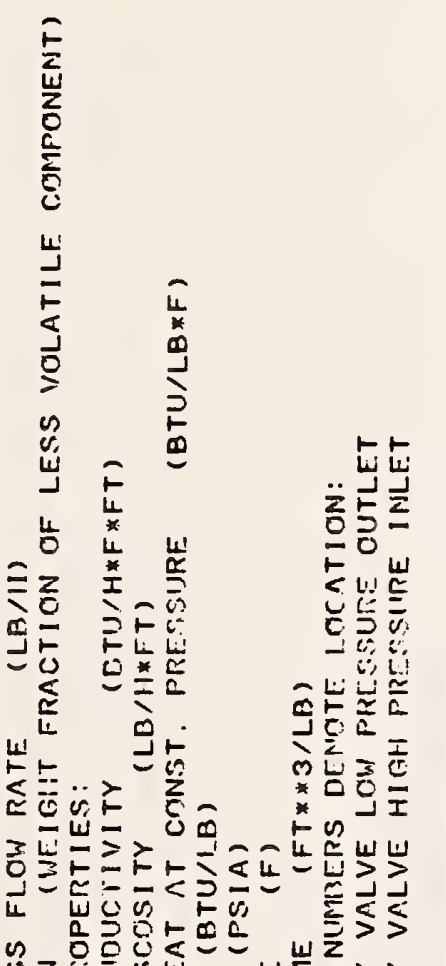

崖势

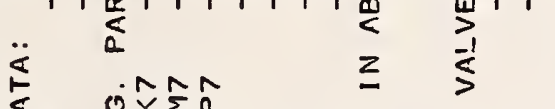

岁' '

공

E

-

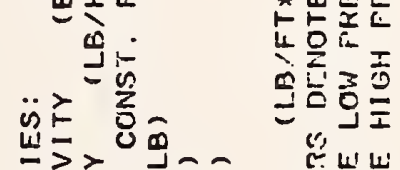

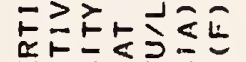

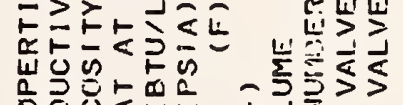

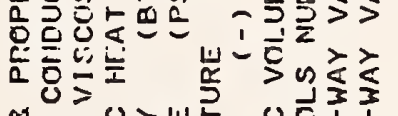

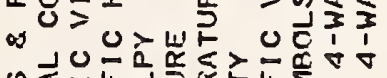

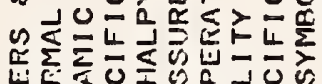

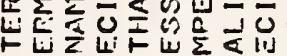

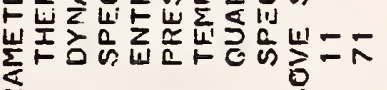

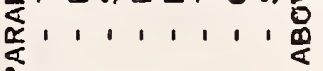

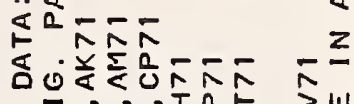

文空。

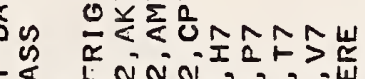

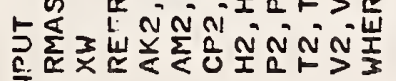

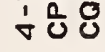

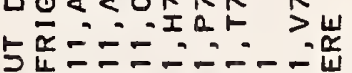

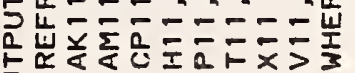
है

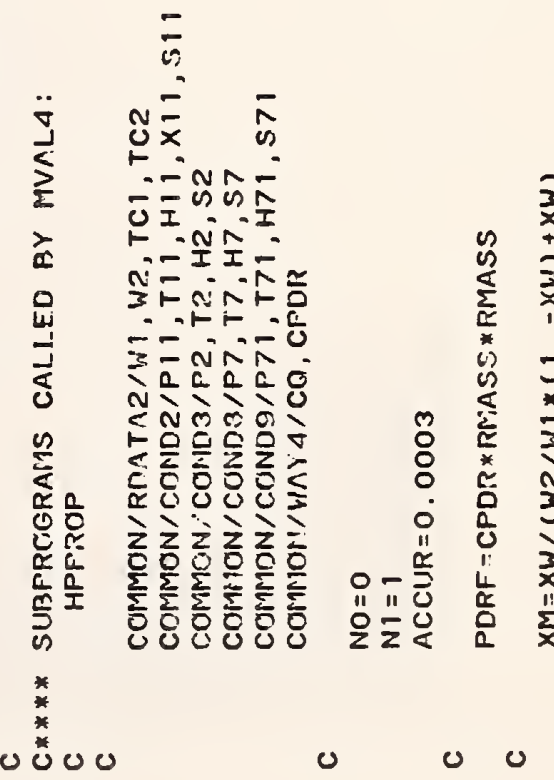

ฐ

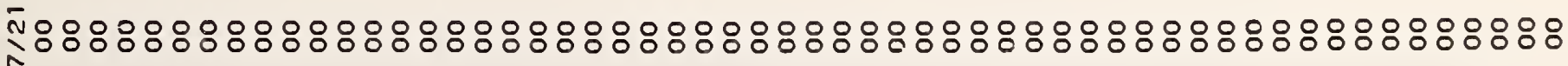

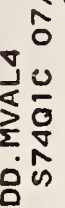

土-

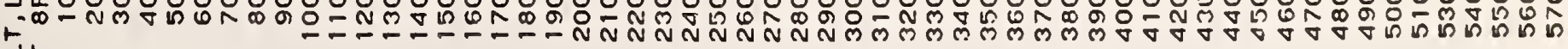
这志 


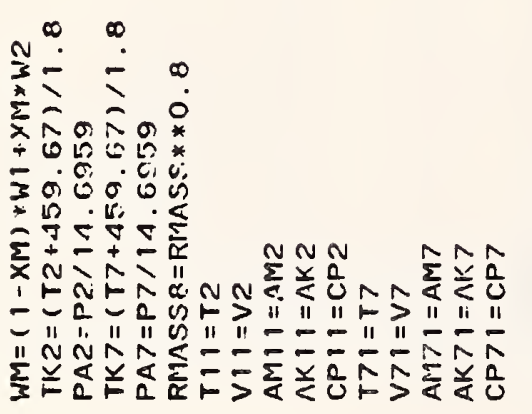

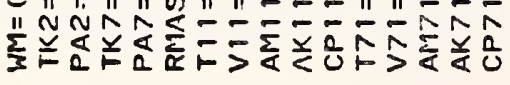

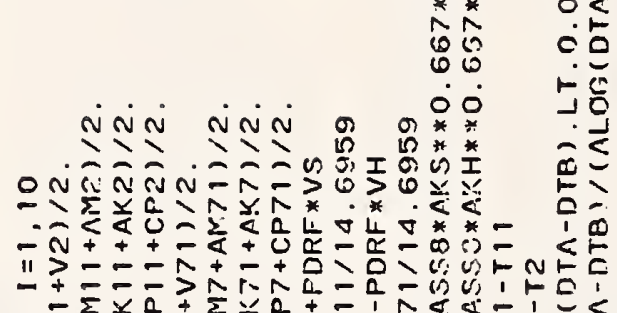

$8=\sum_{0}=0$

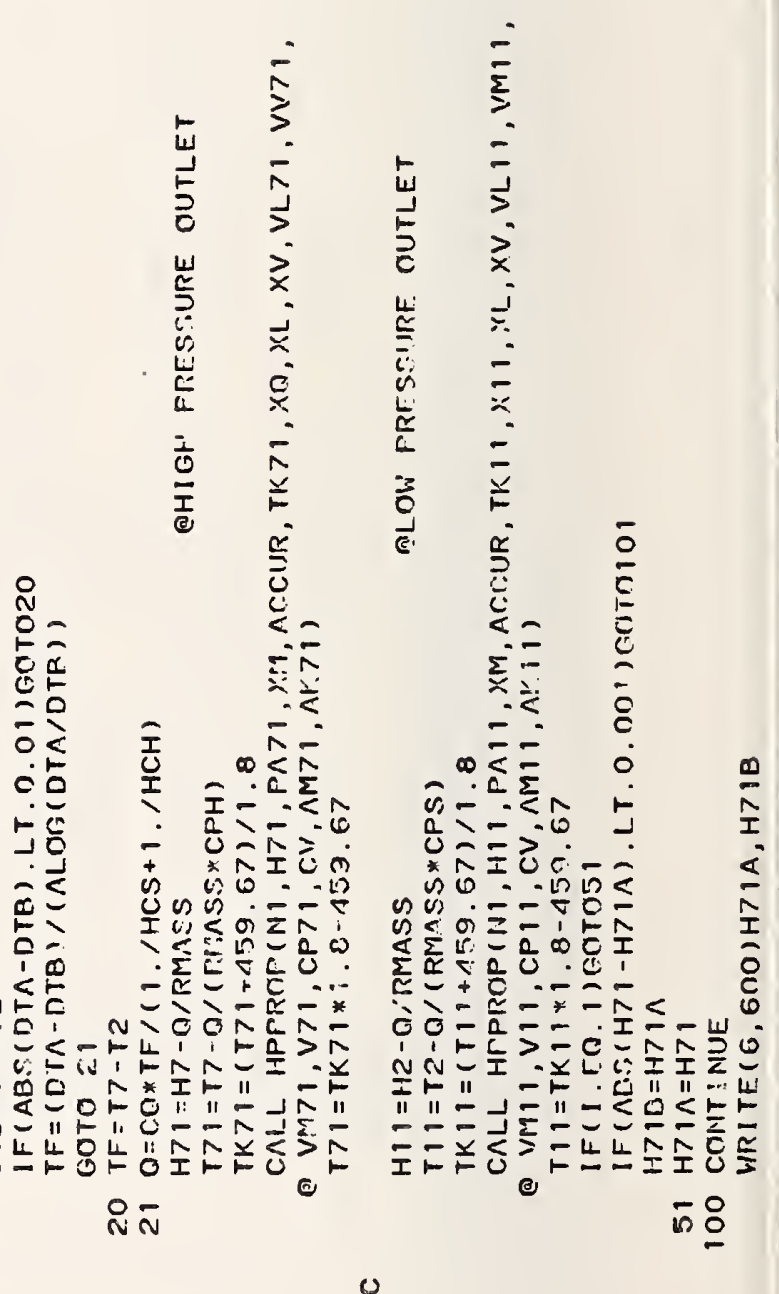

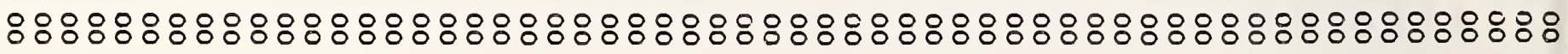

$\frac{\nabla}{5}$

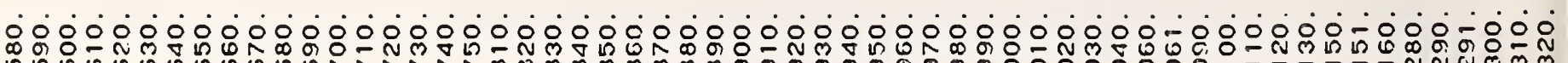

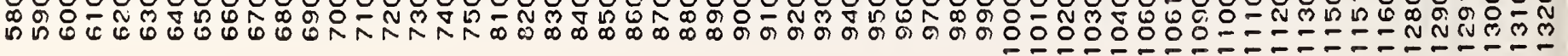




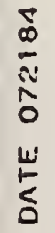

อิ

6

$\frac{2}{20}$

$\frac{11}{2}$

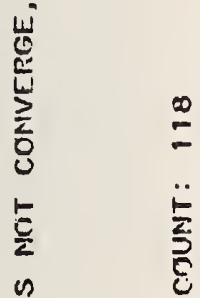

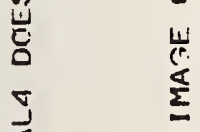

$\sum_{-\infty}^{J}$

ن岁 の

起突致是

LU氏U

응음

$\stackrel{\text { iuj }}{\ddot{u}^{*}}$

*

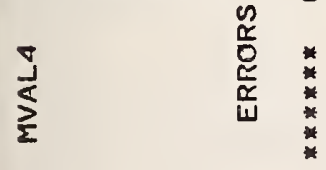

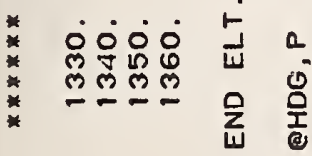




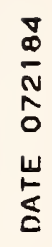
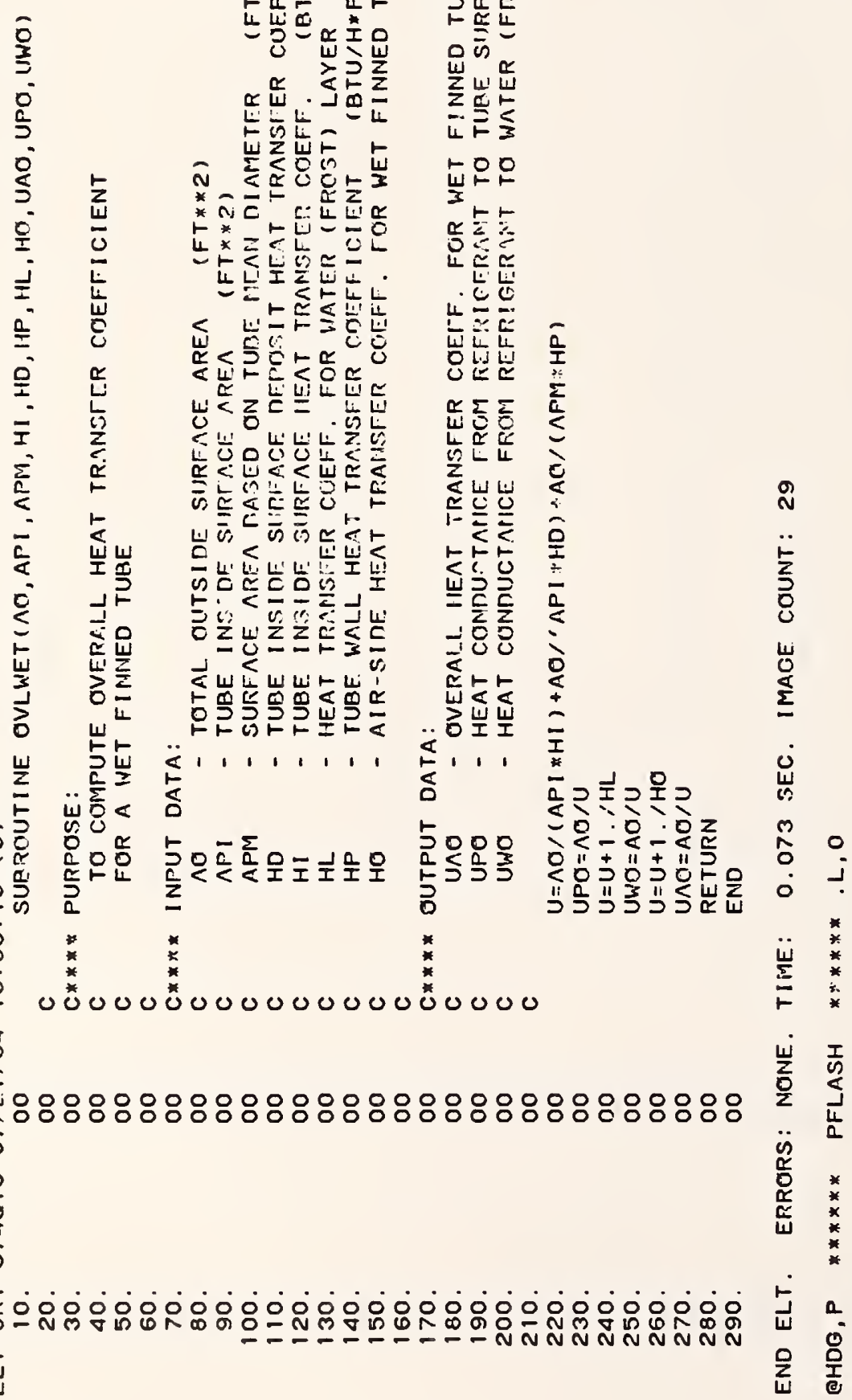


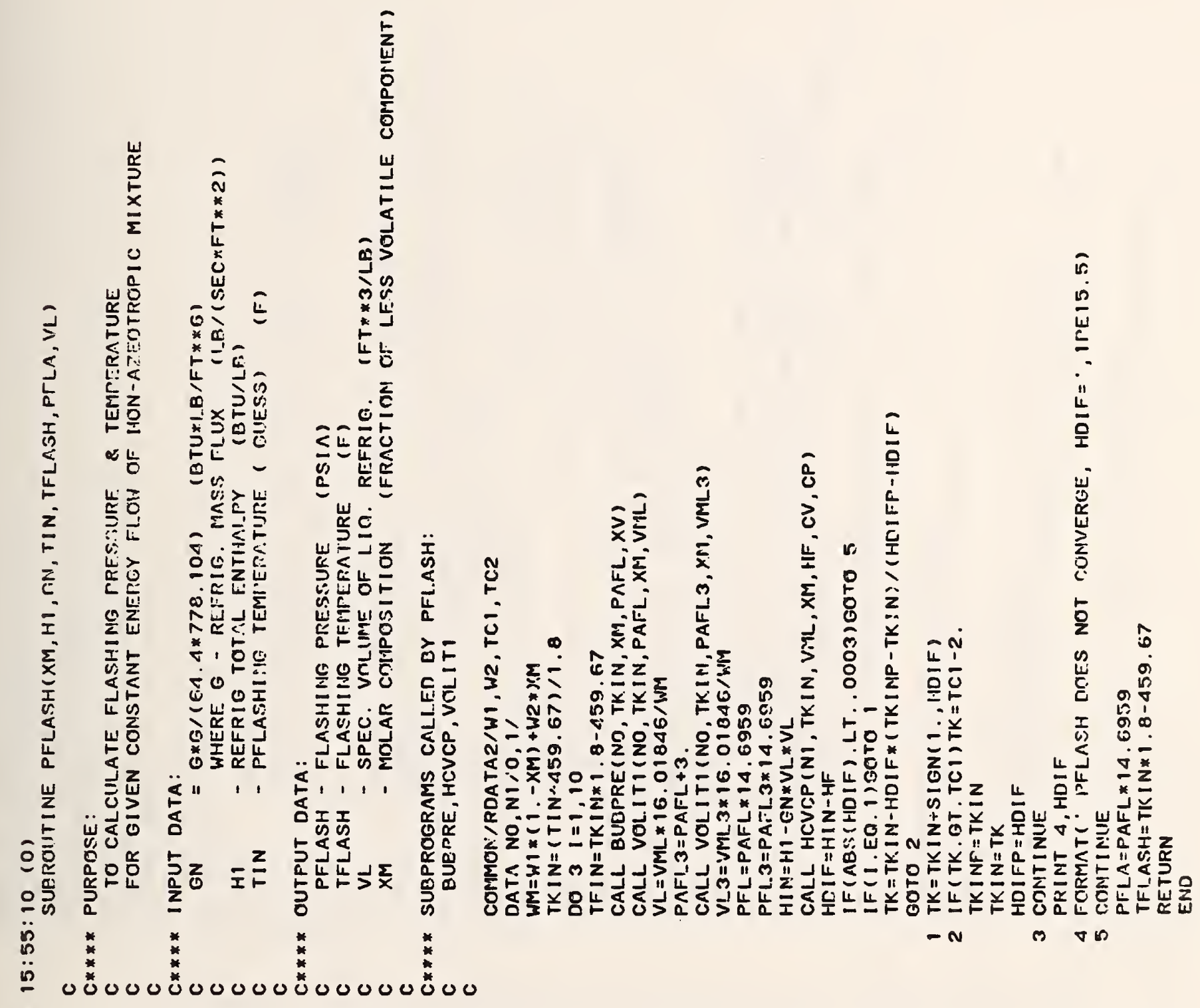

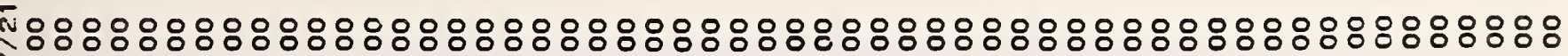
I숭 क्ष

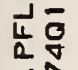

os

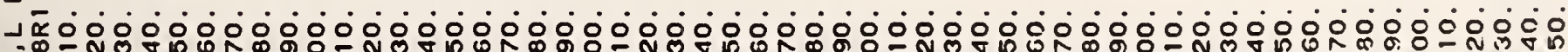
F- N 过 


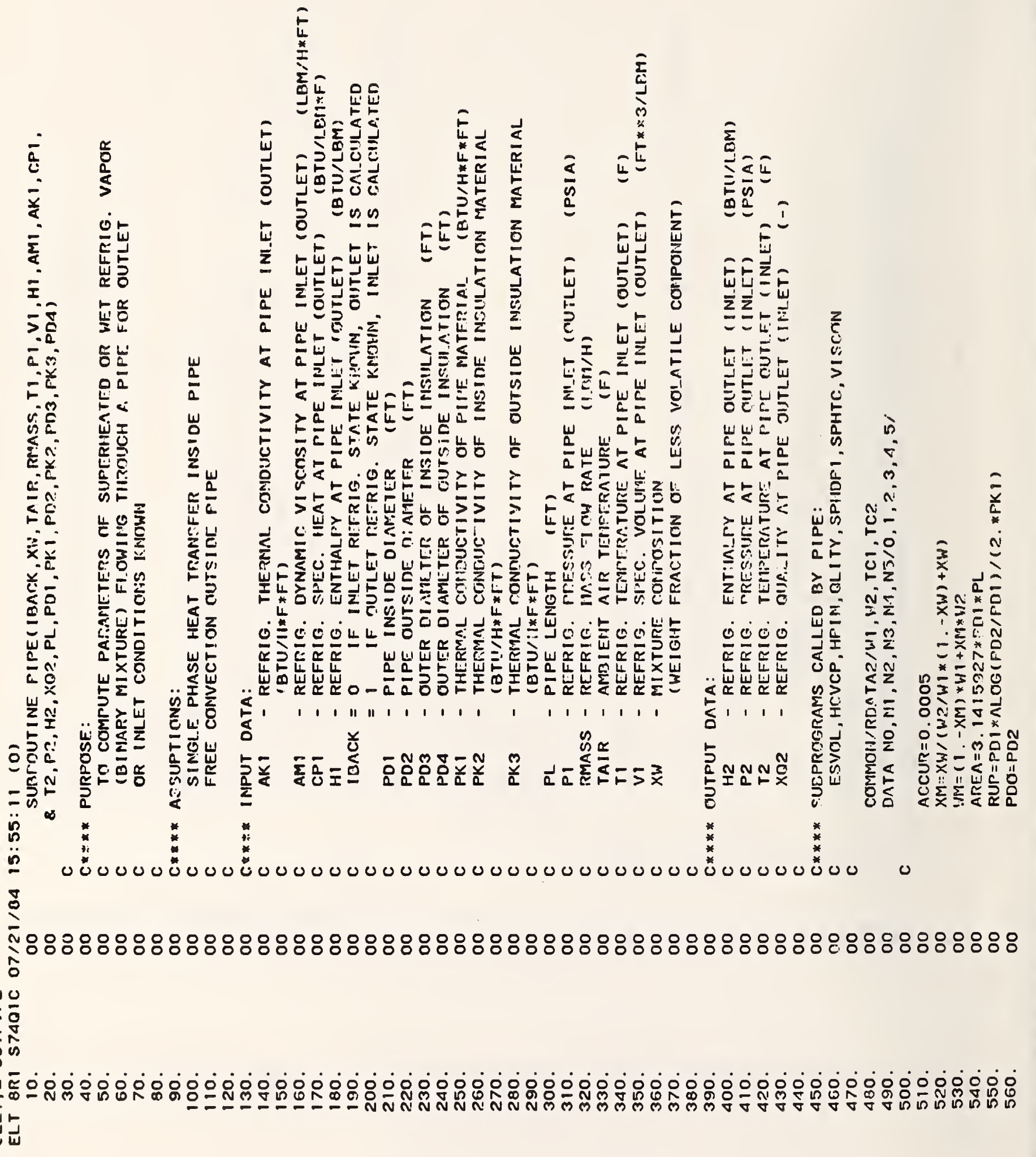




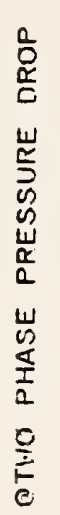




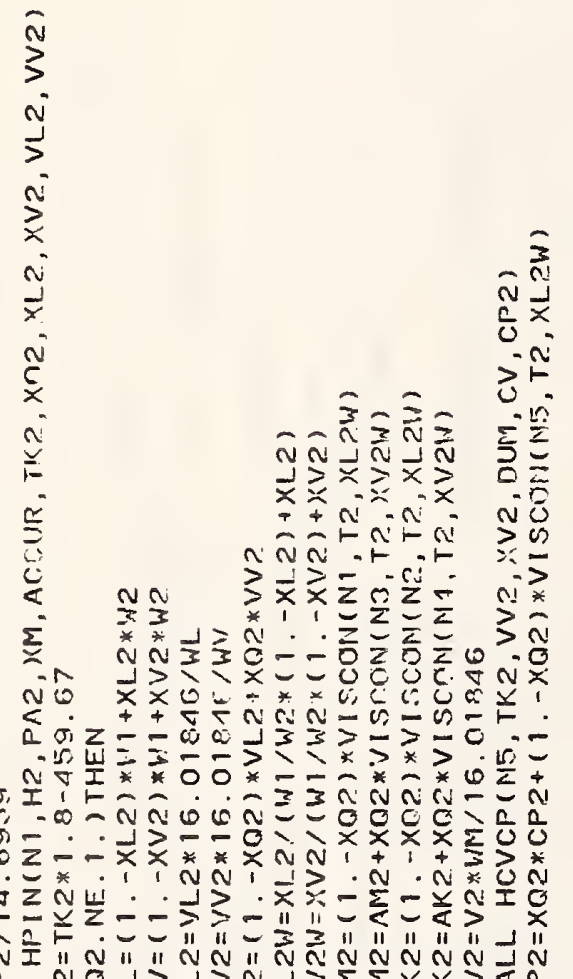

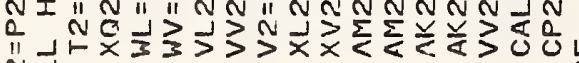

造专台

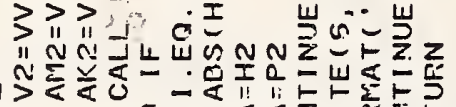


巍

$\frac{D}{\infty}$
$\frac{N}{0}$
0
$\frac{1}{0}$

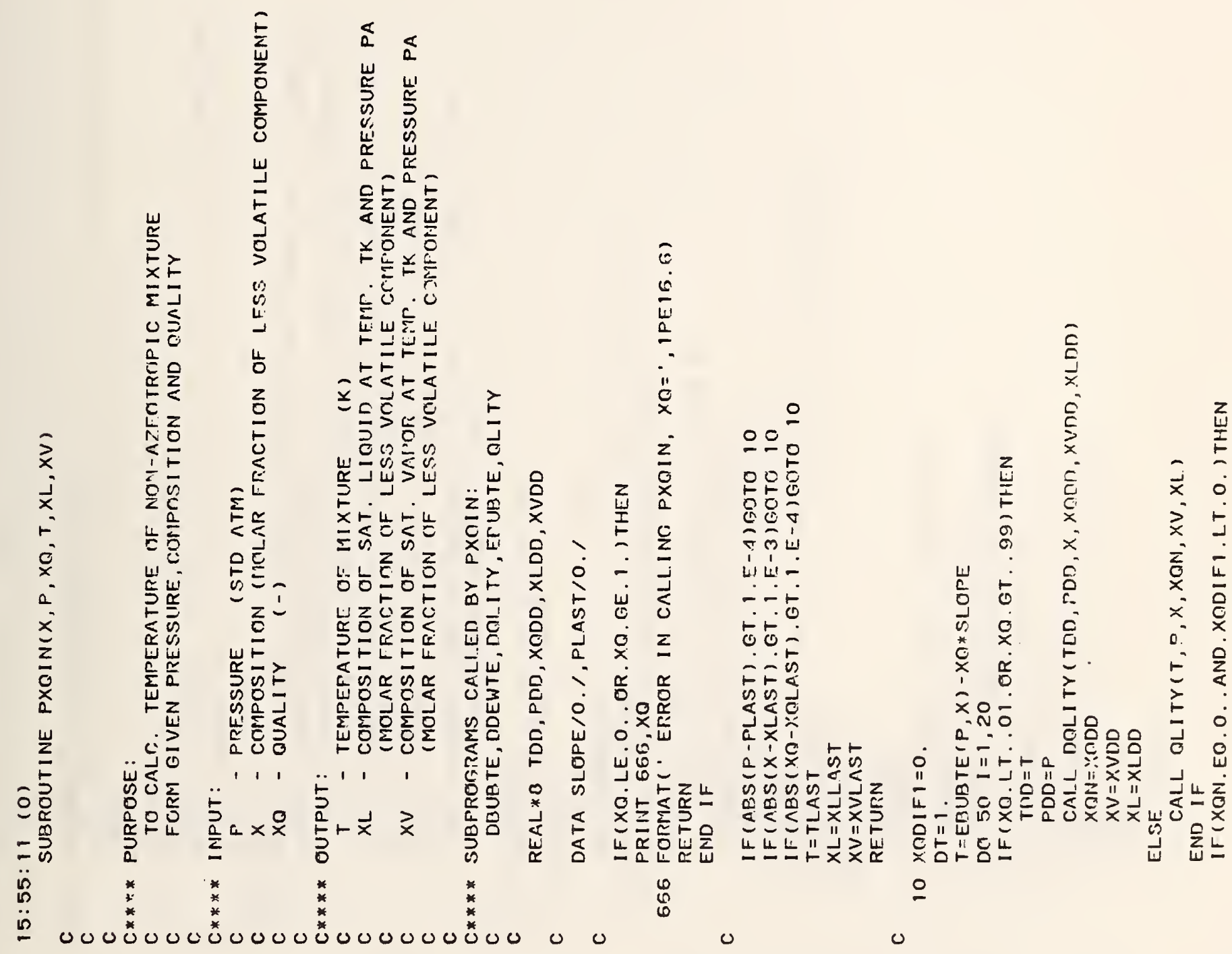

D

N̄ঃ৪৪৪৪৪৪৪৪৪৪৪৪৪৪৪৪৪৪৪৪৪৪৪৪৪৪৪৪৪৪৪৪৪৪৪৪৪৪৪৪৪৪৪৪৪৪৪৪৪৪৪৪৪৪৪

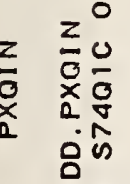

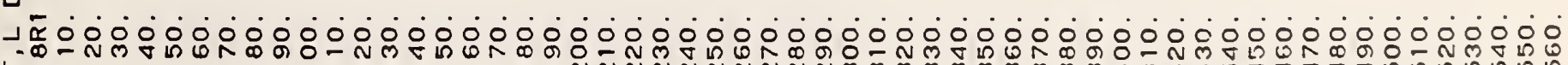
岃上 


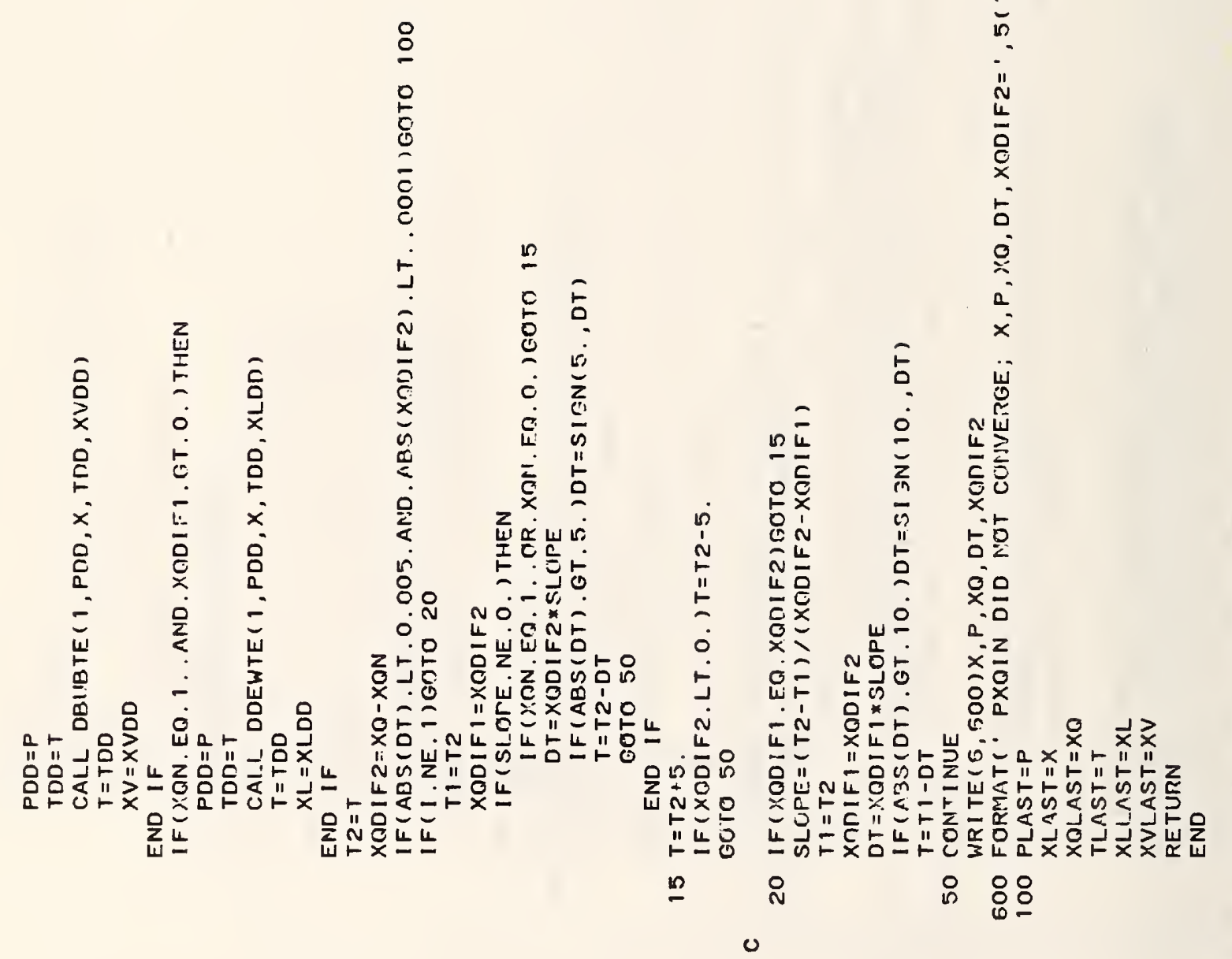




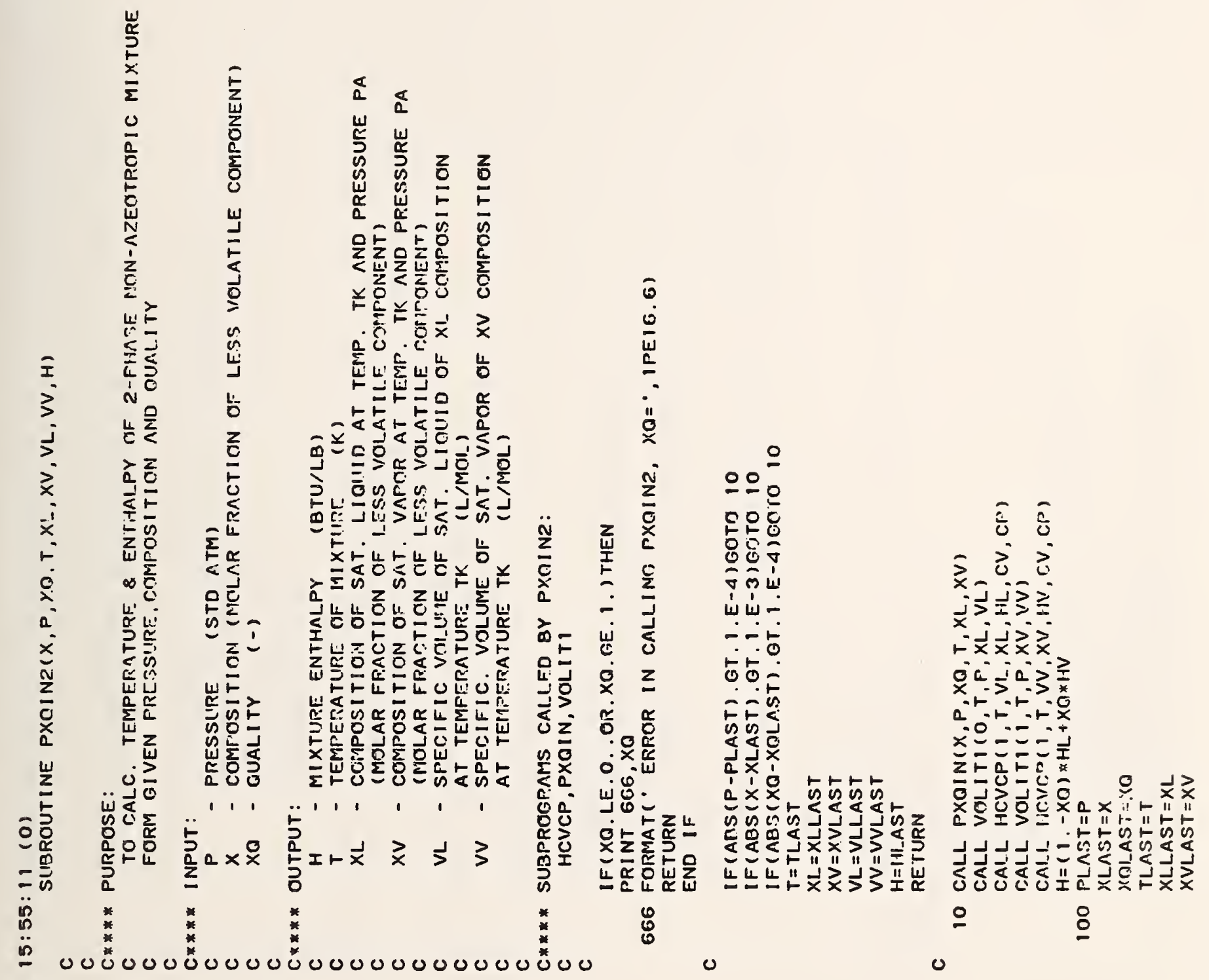

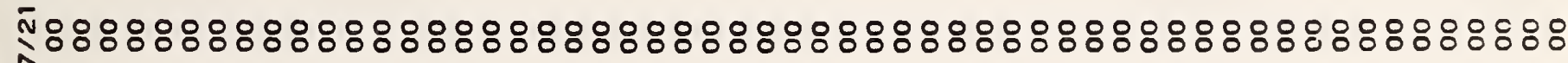
농

$\times \frac{0}{2}$

的

L

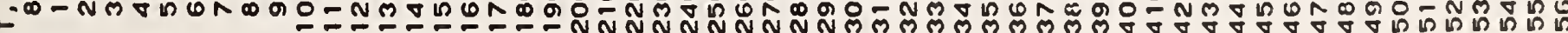
岁古 


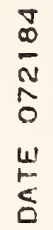

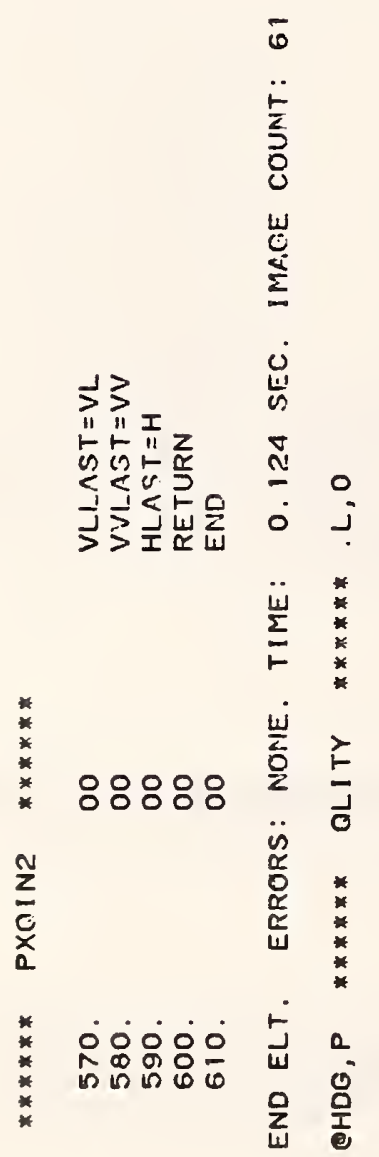




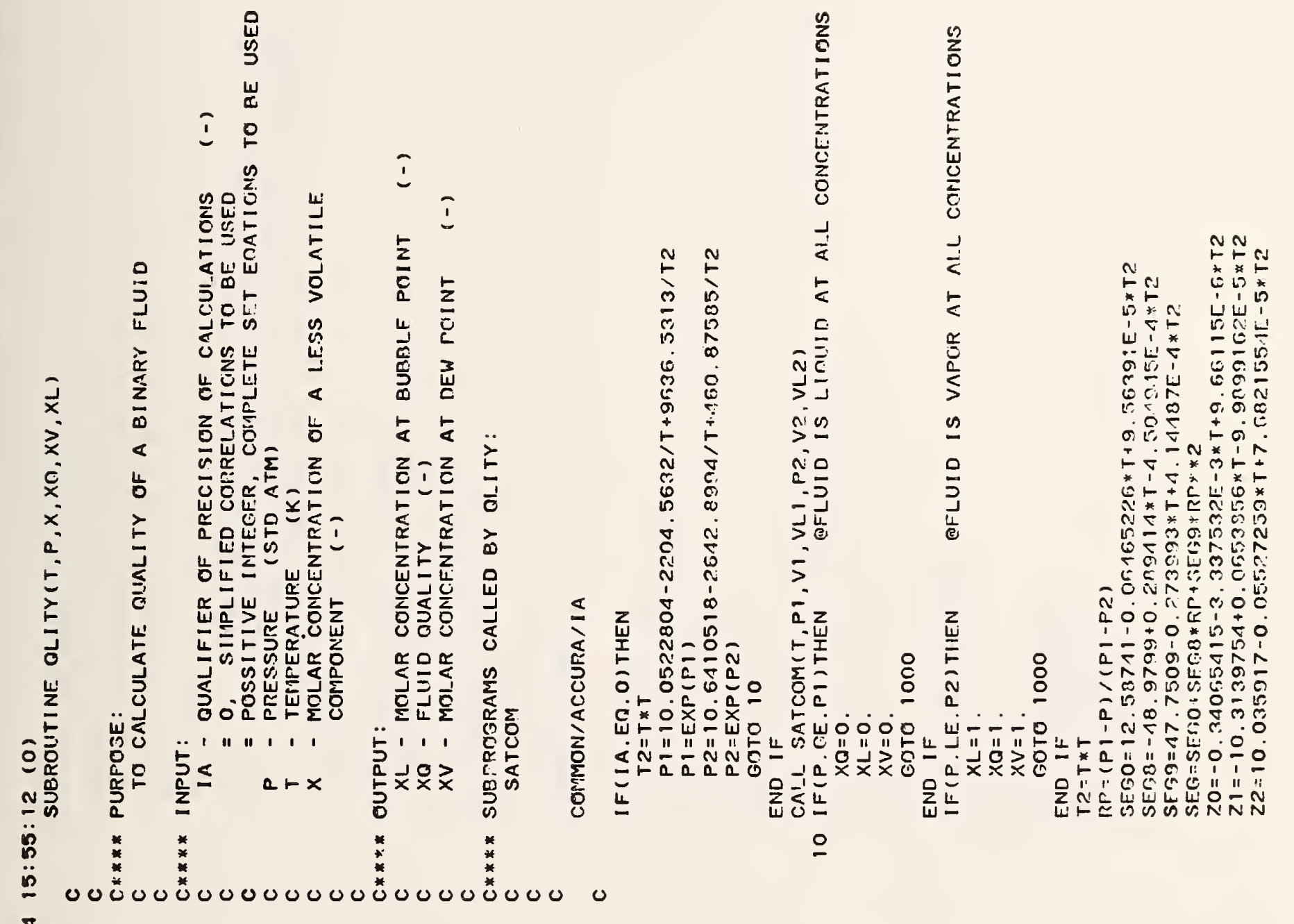
I

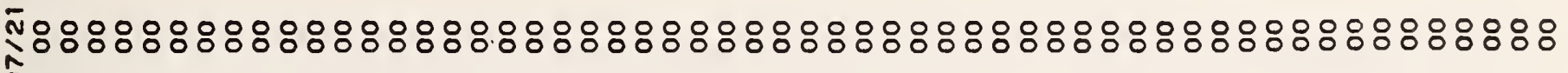
之o

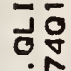

8n

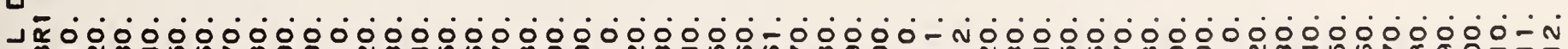
ᄂ 岂 


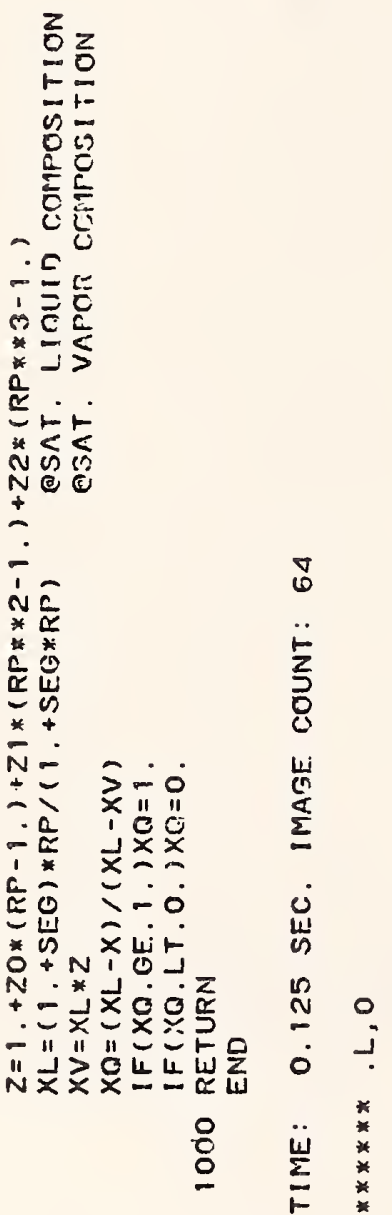

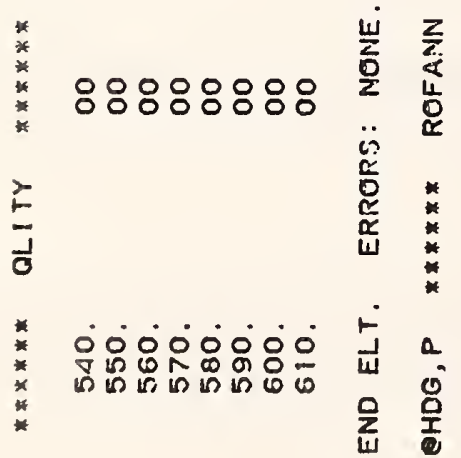




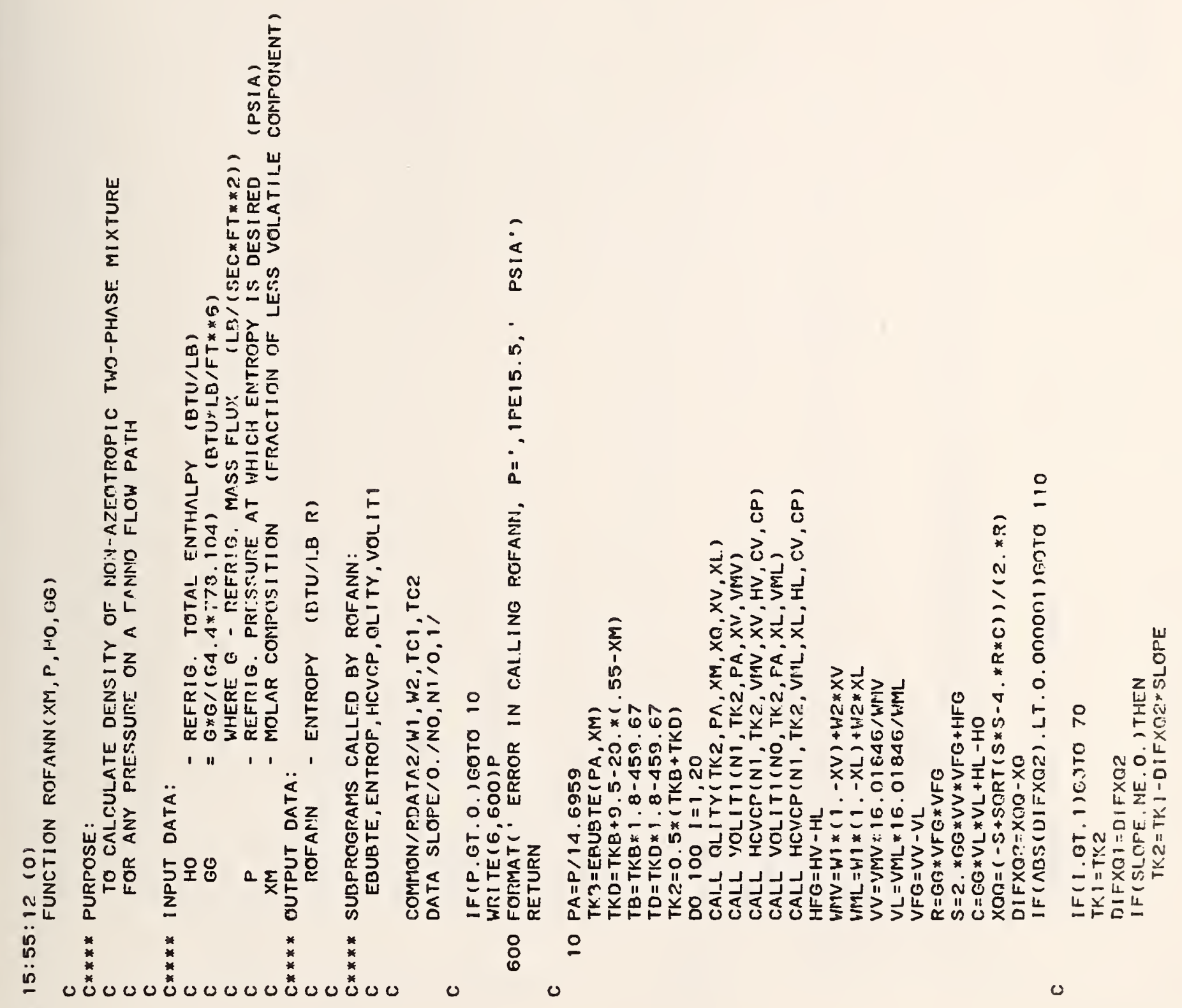

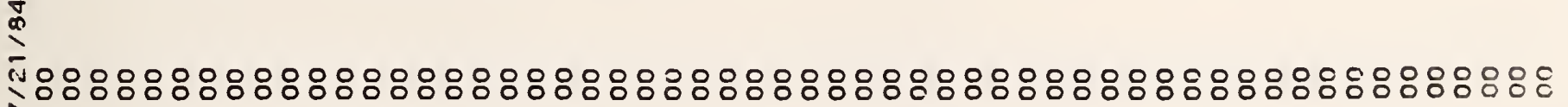

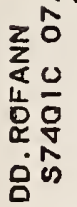

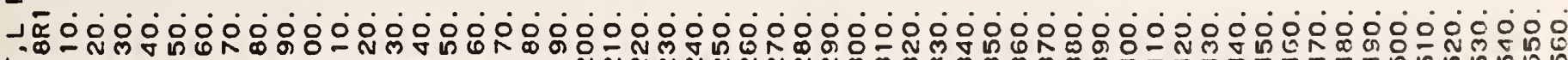
岂志 


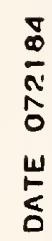

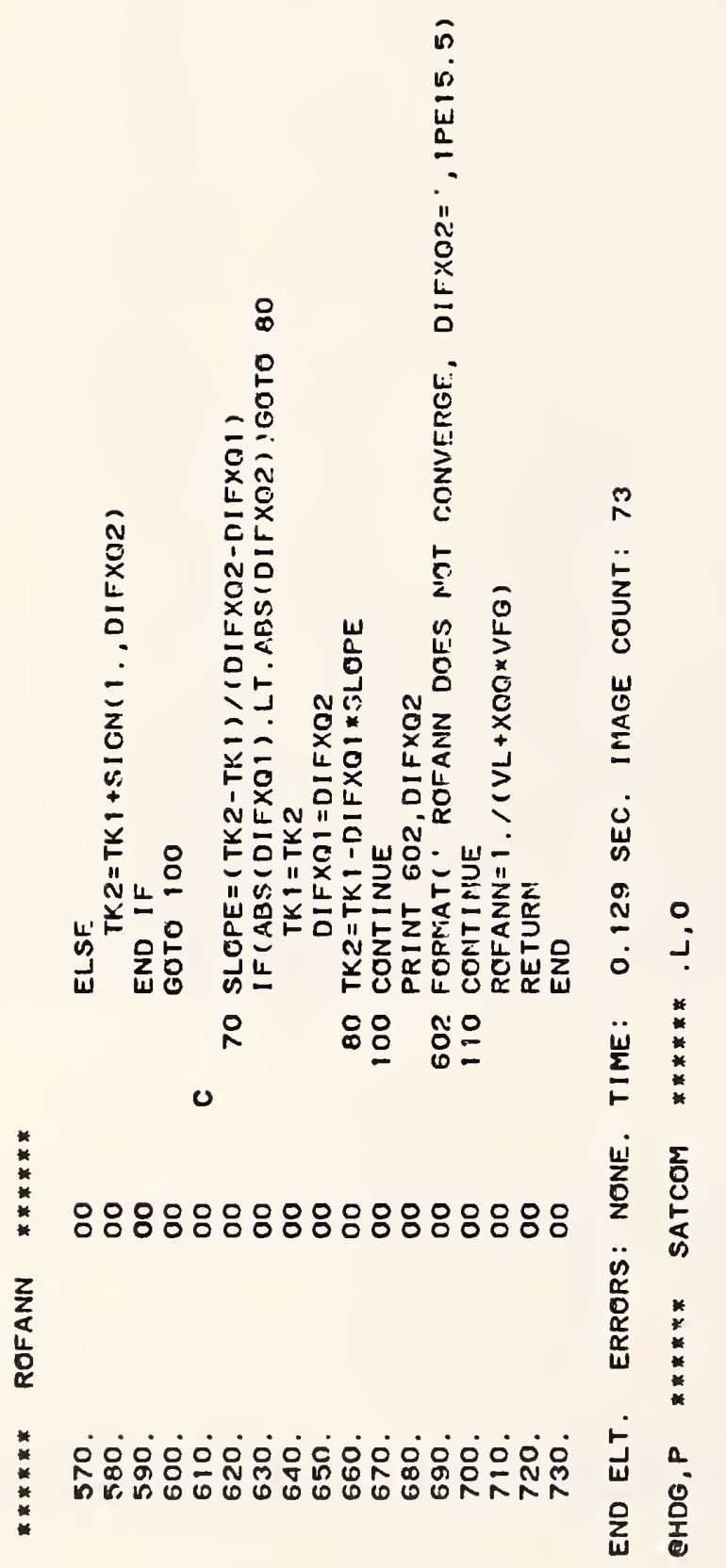




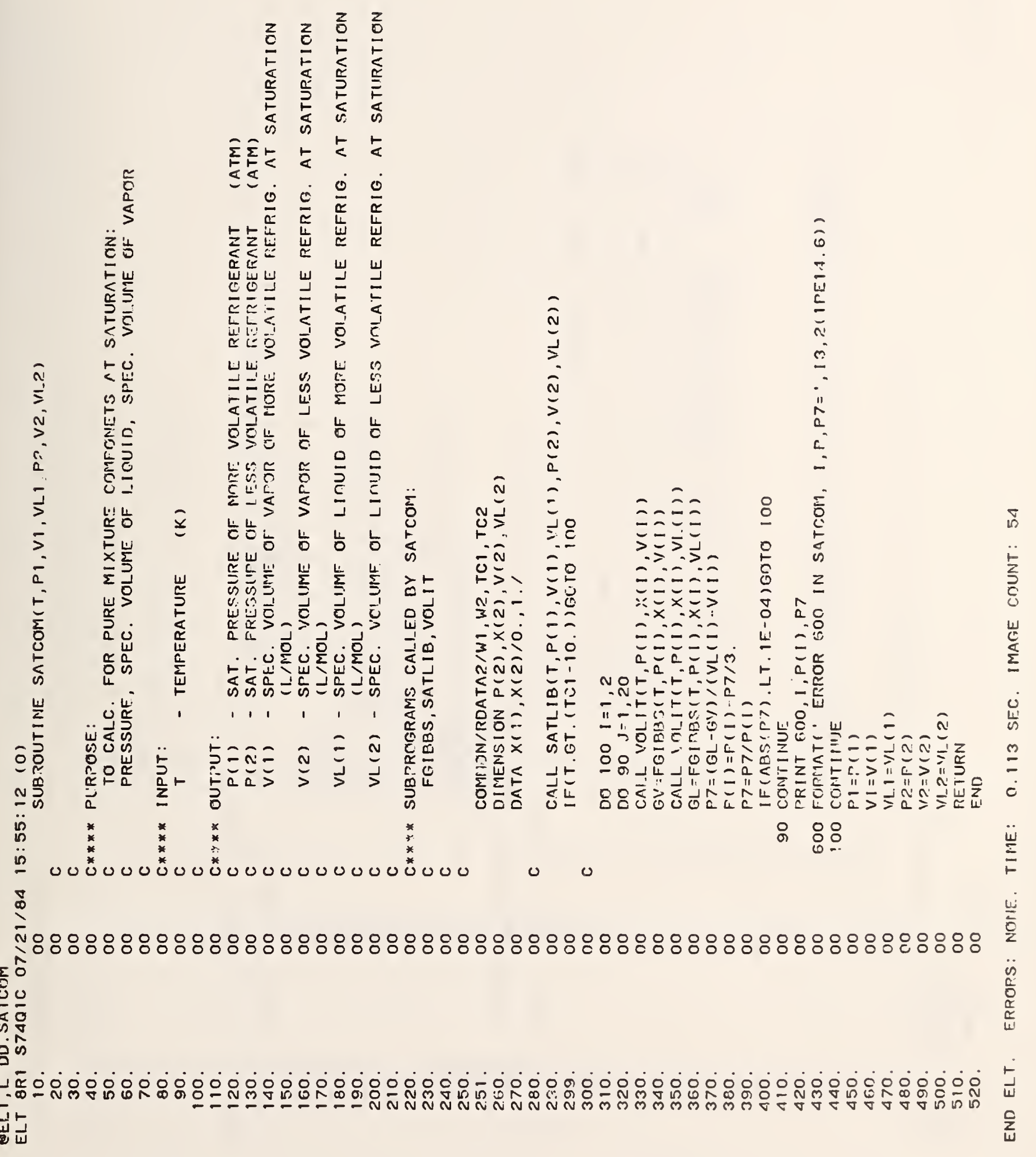




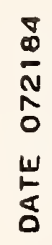

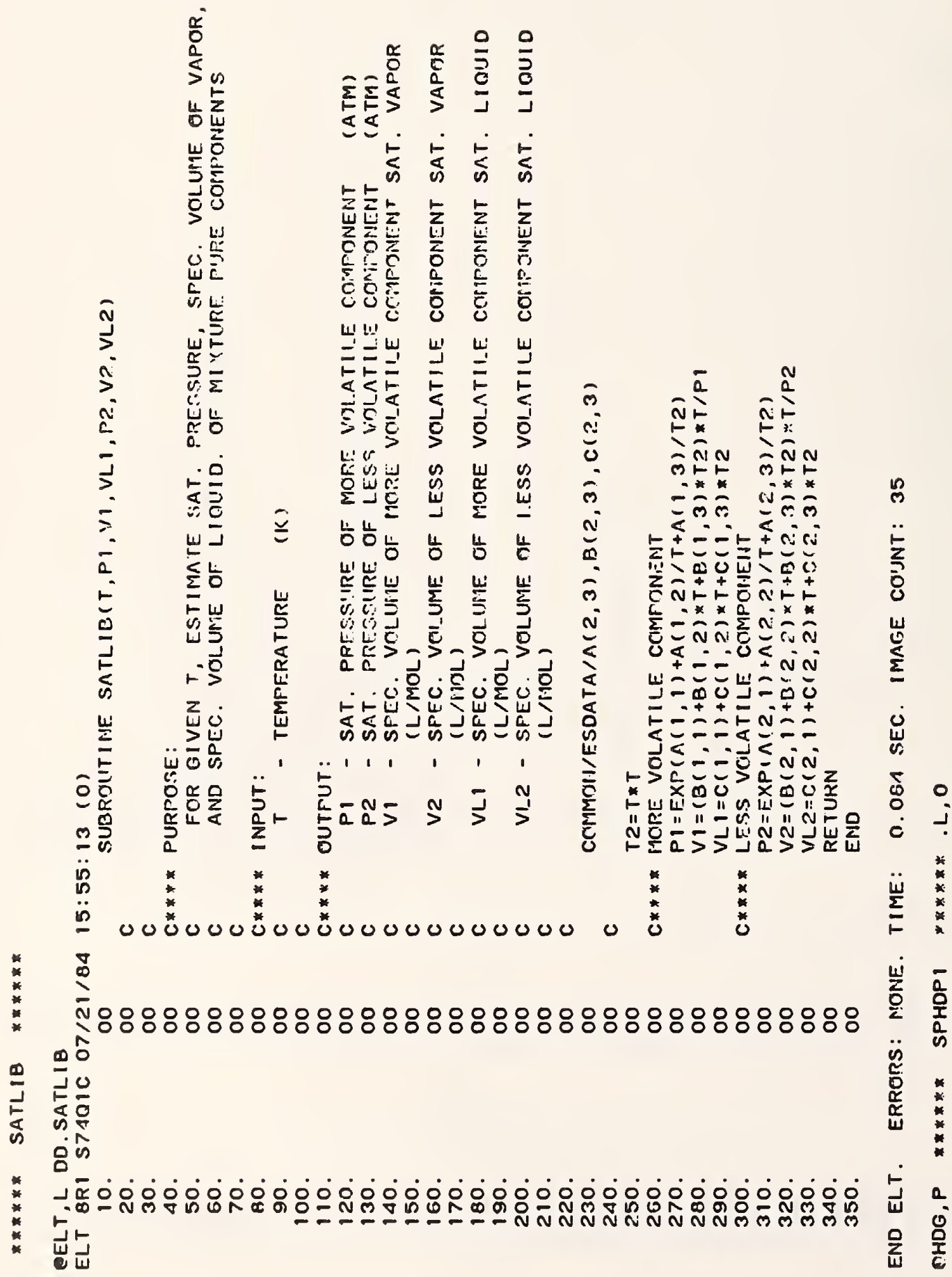




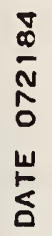

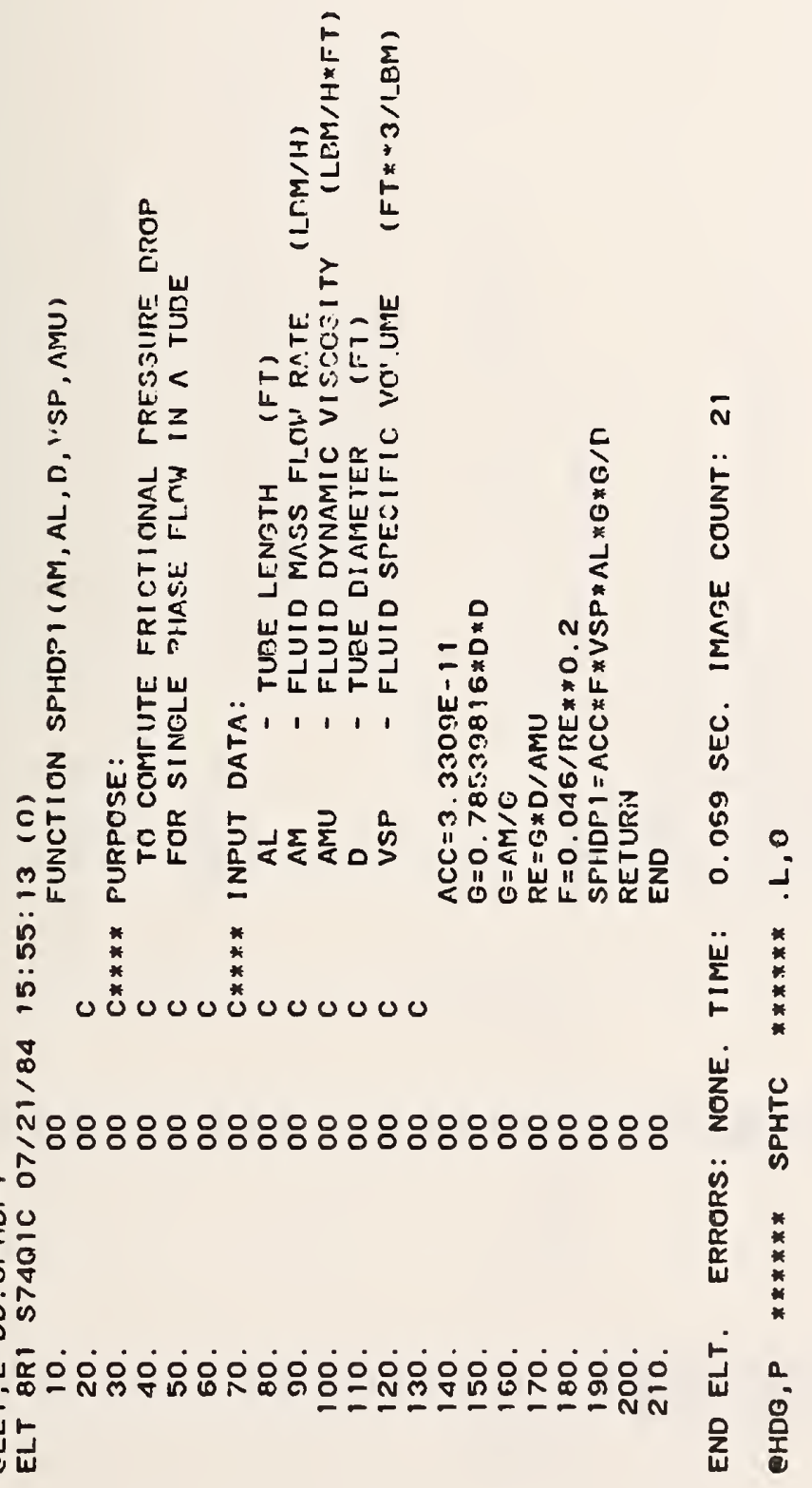




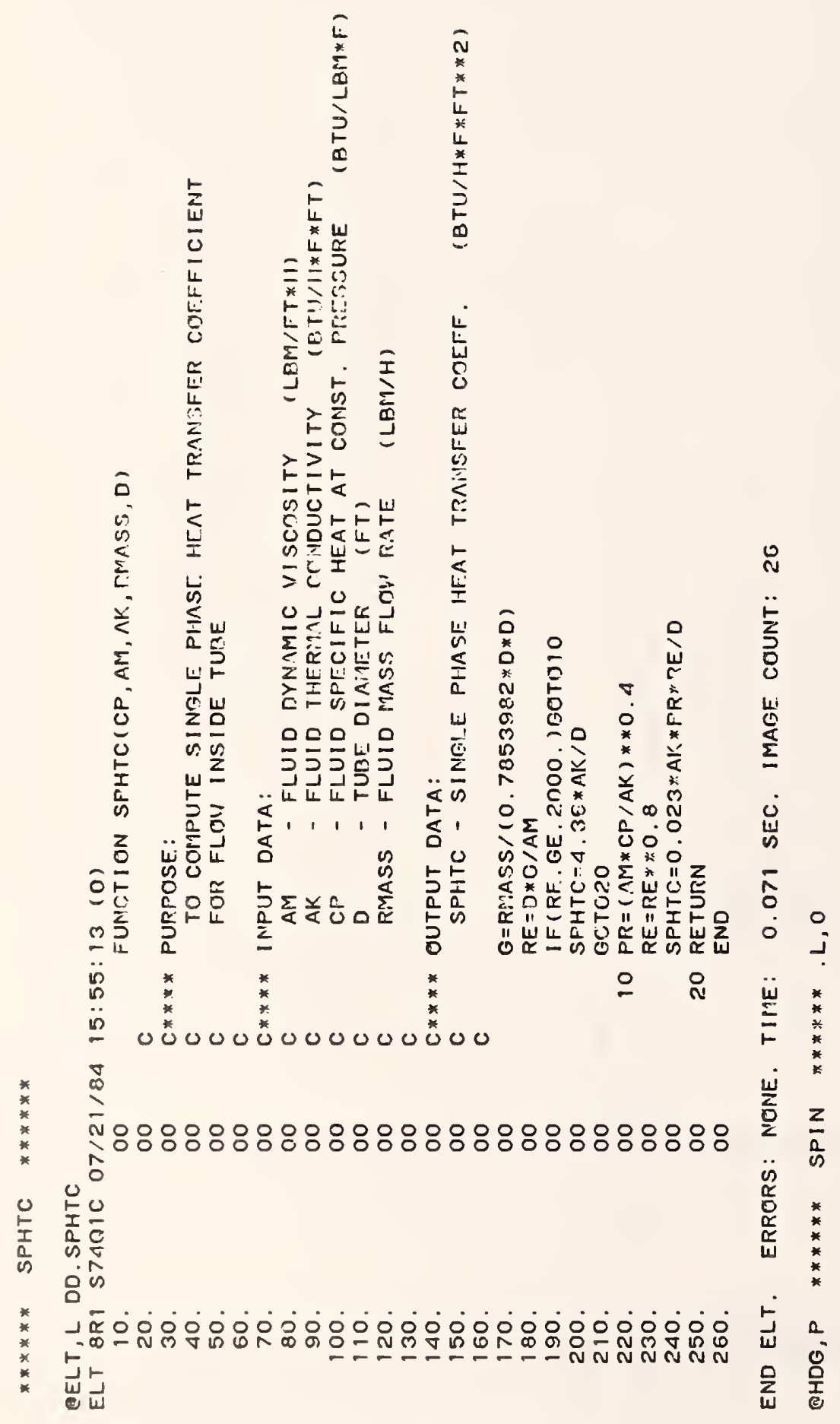




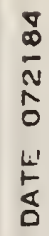

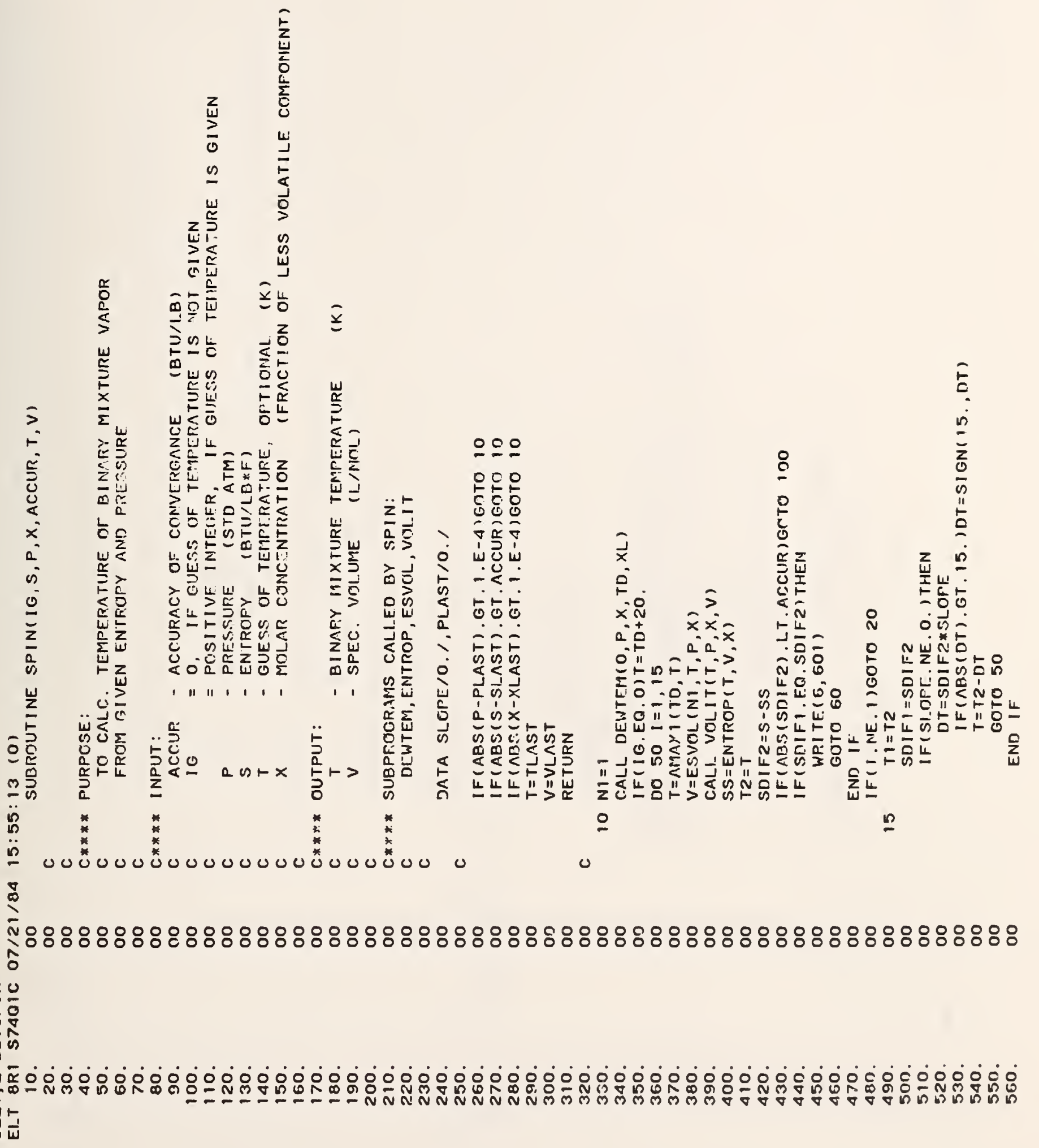




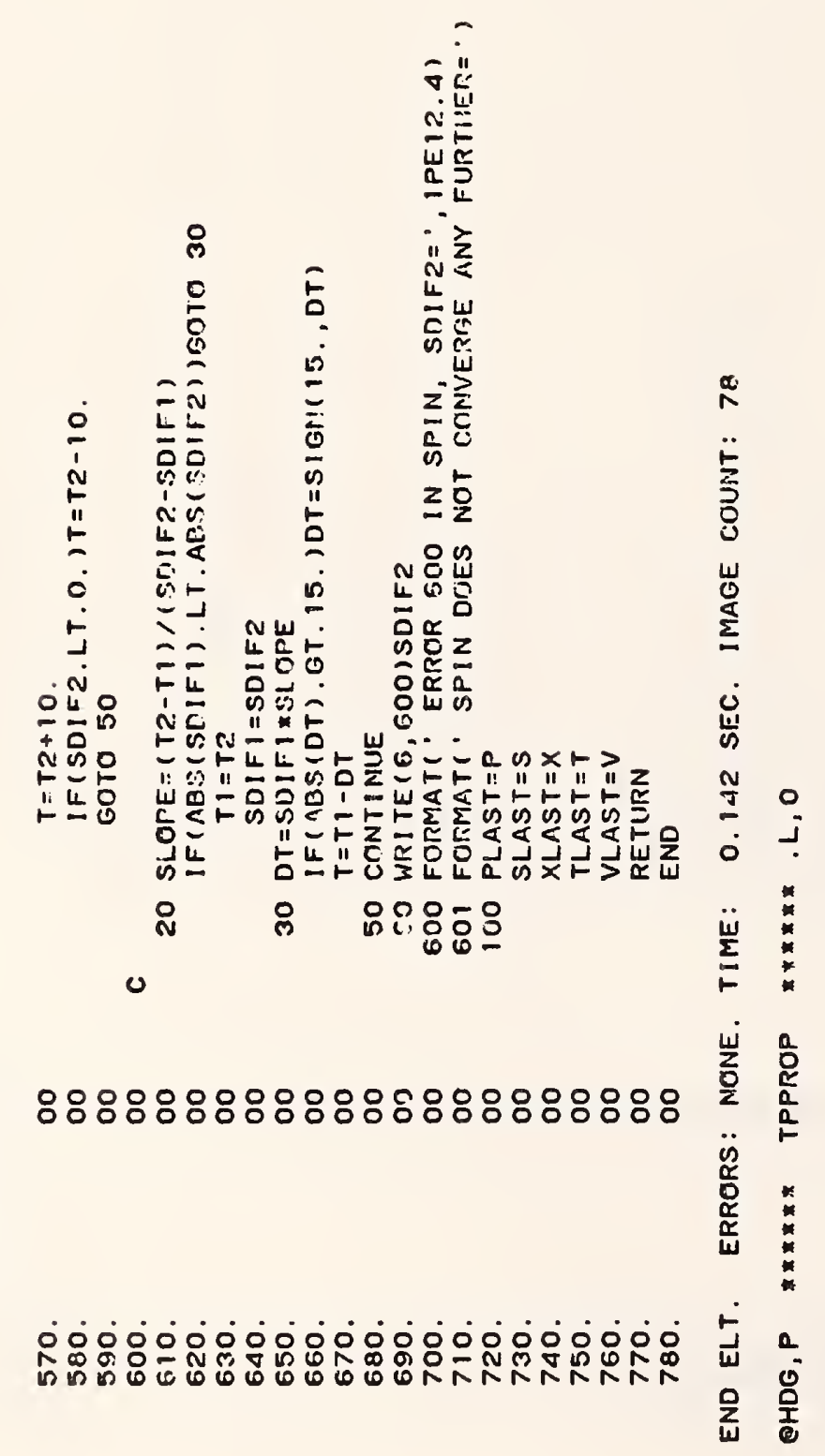




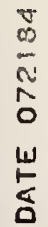

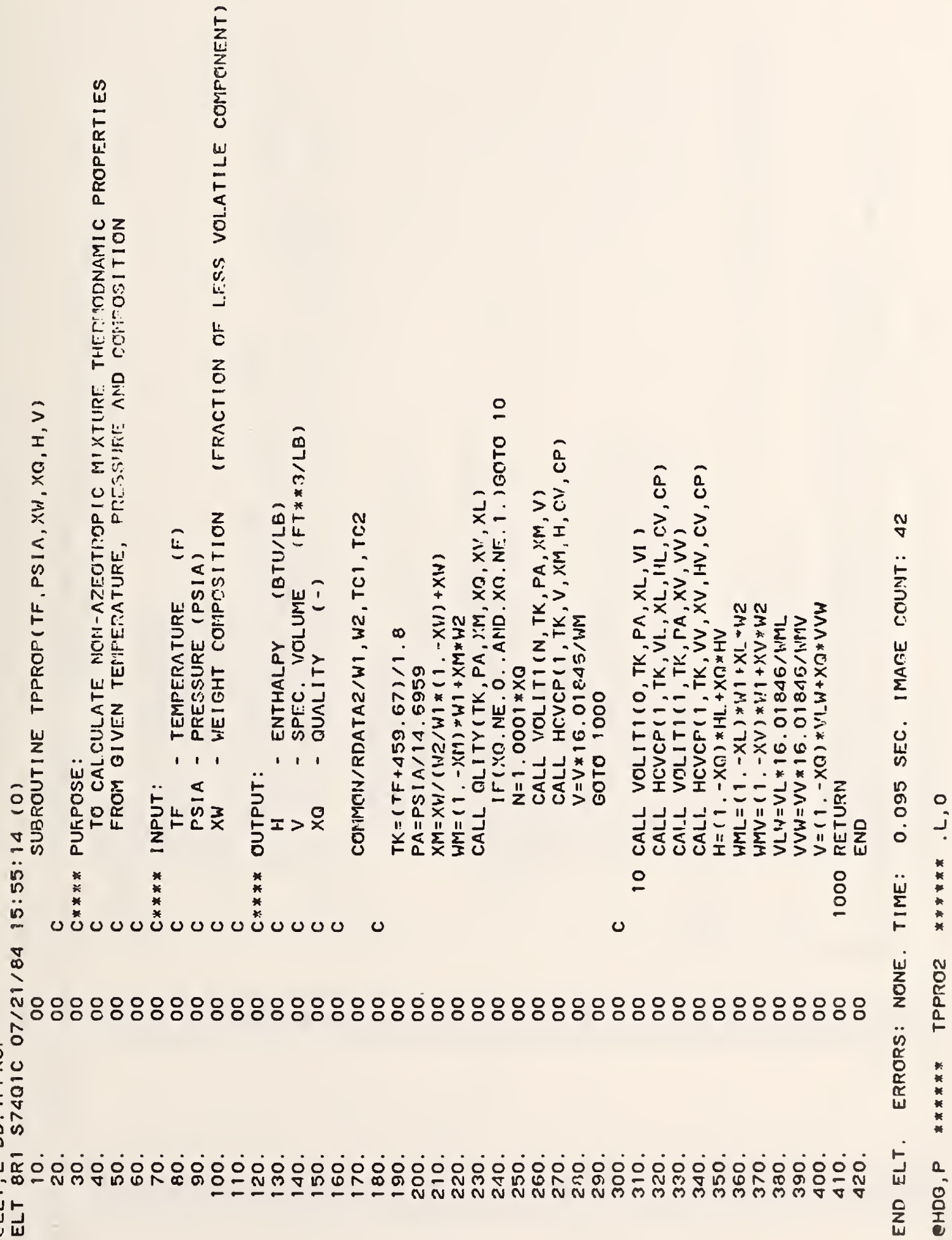




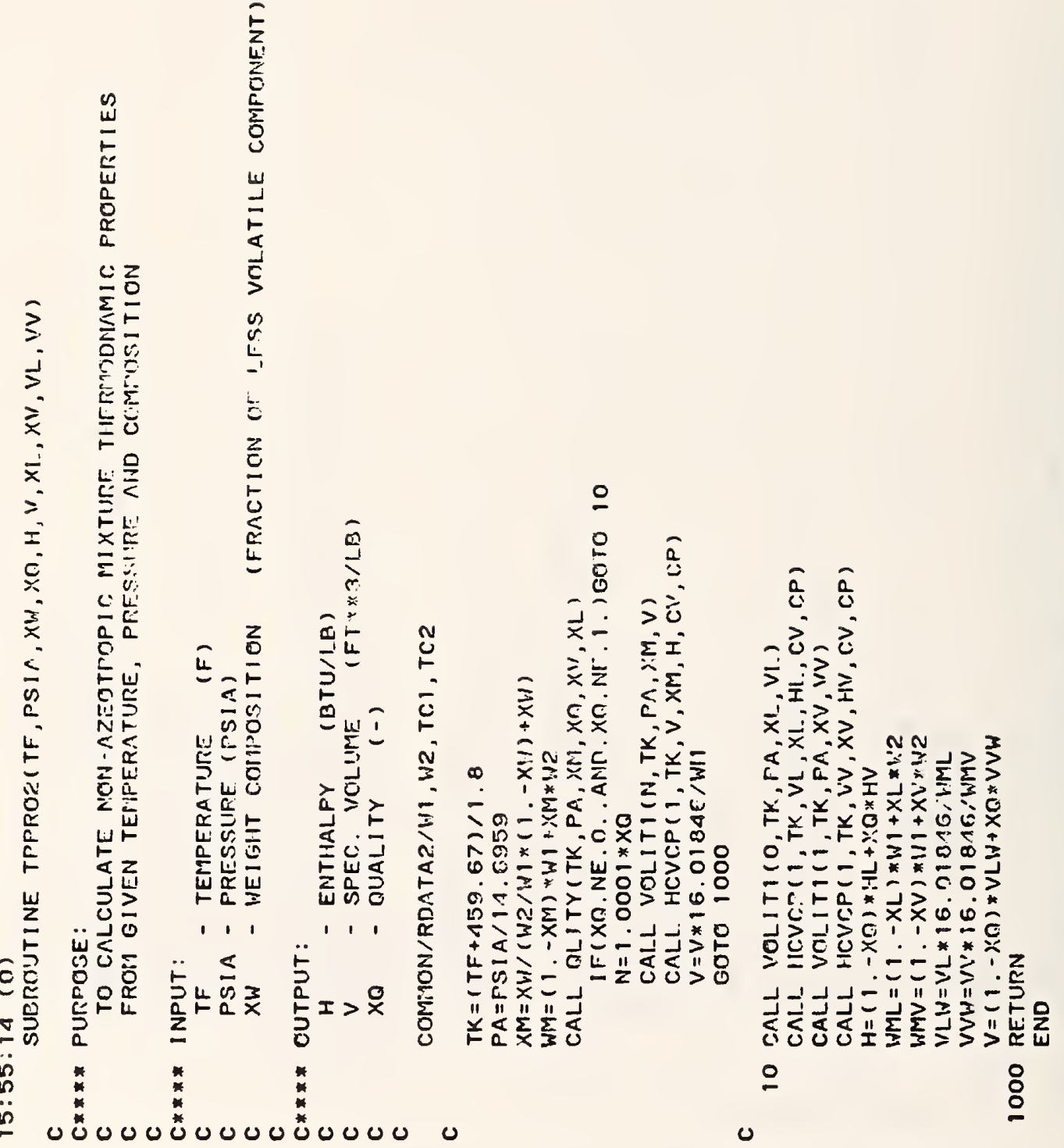

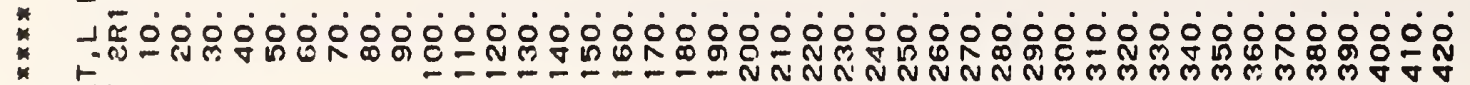
w与 


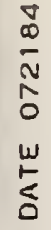

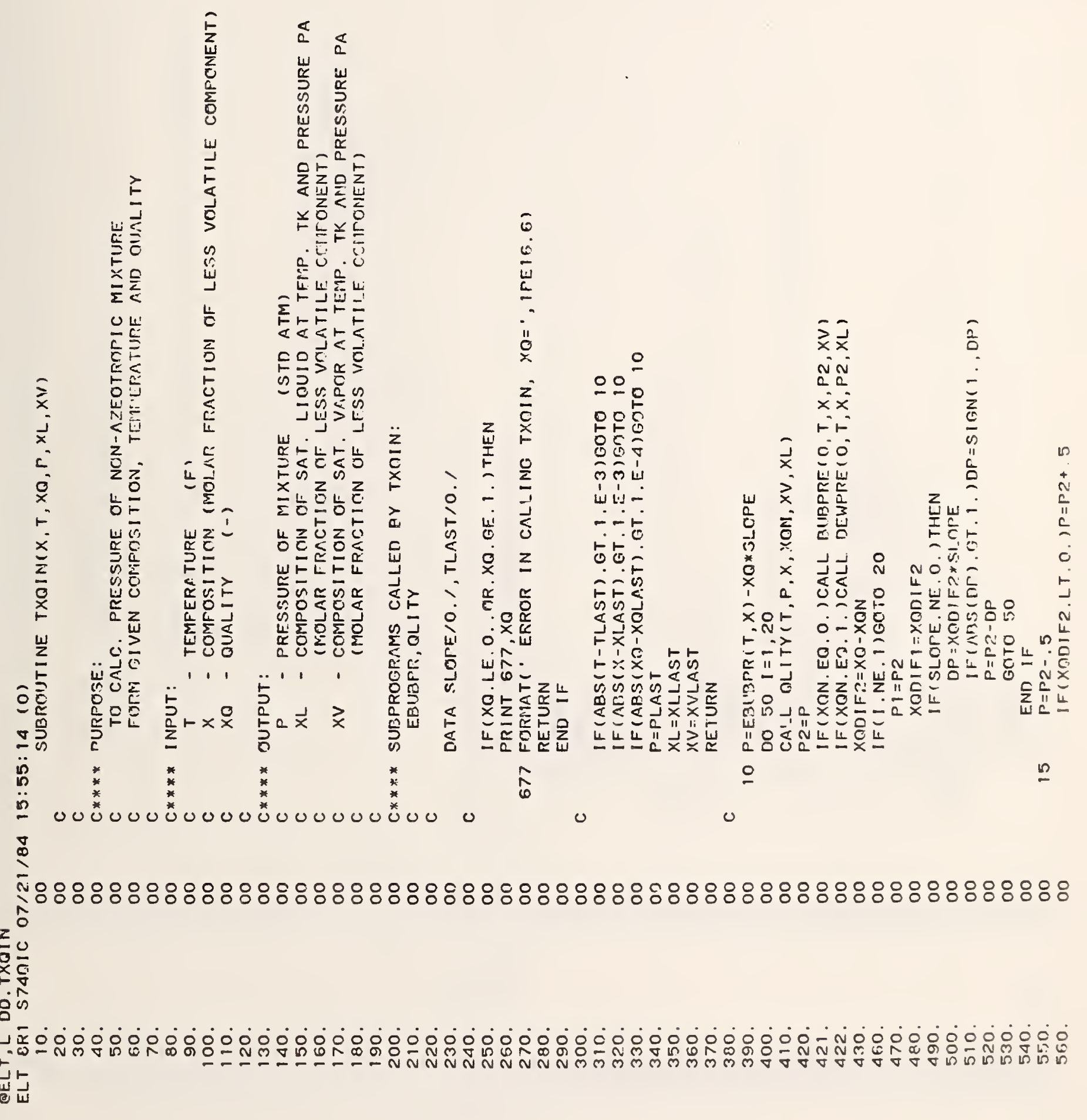


$\frac{7}{\infty}$
$\frac{N}{N}$
0
$\omega$
$\frac{\delta}{\delta}$
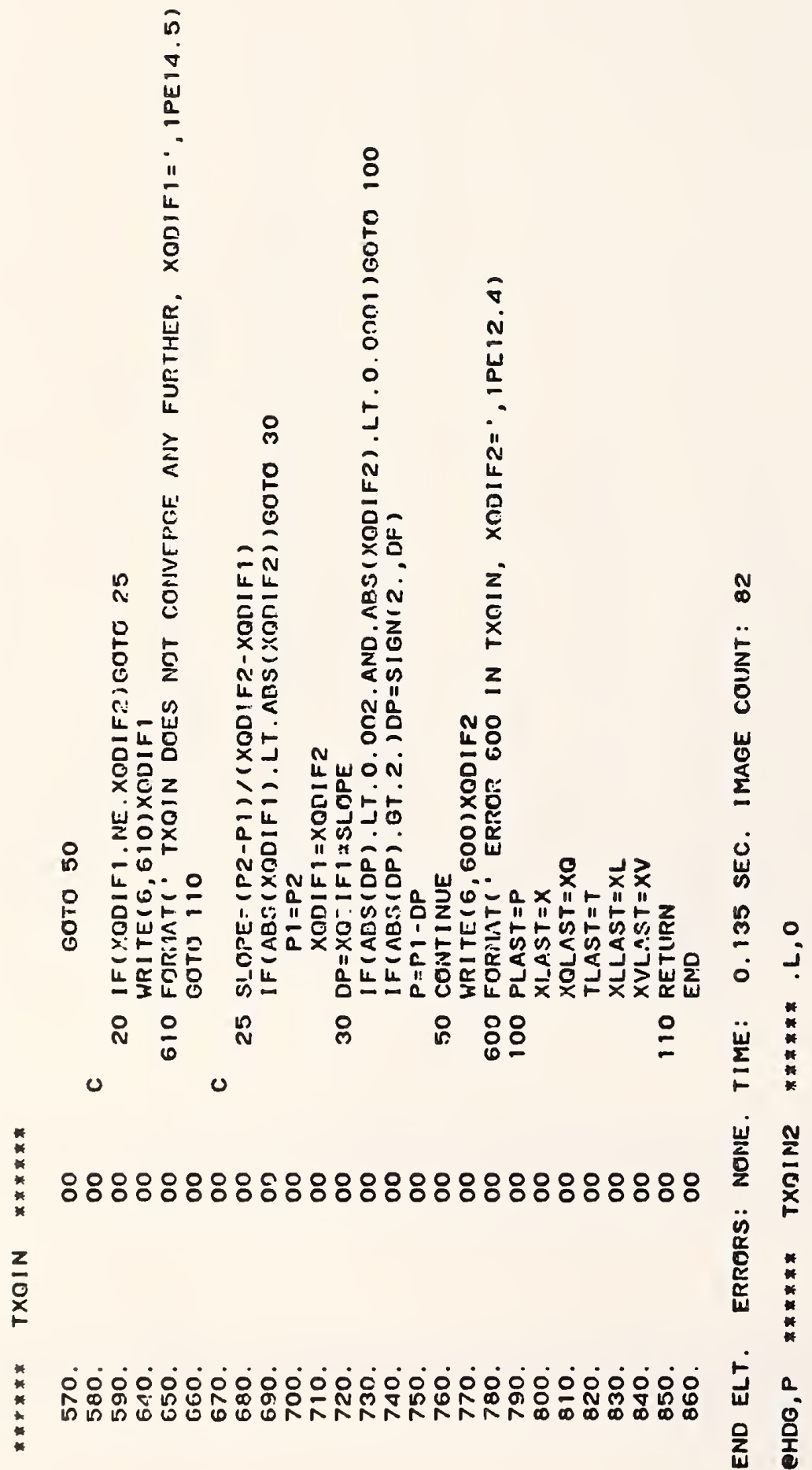


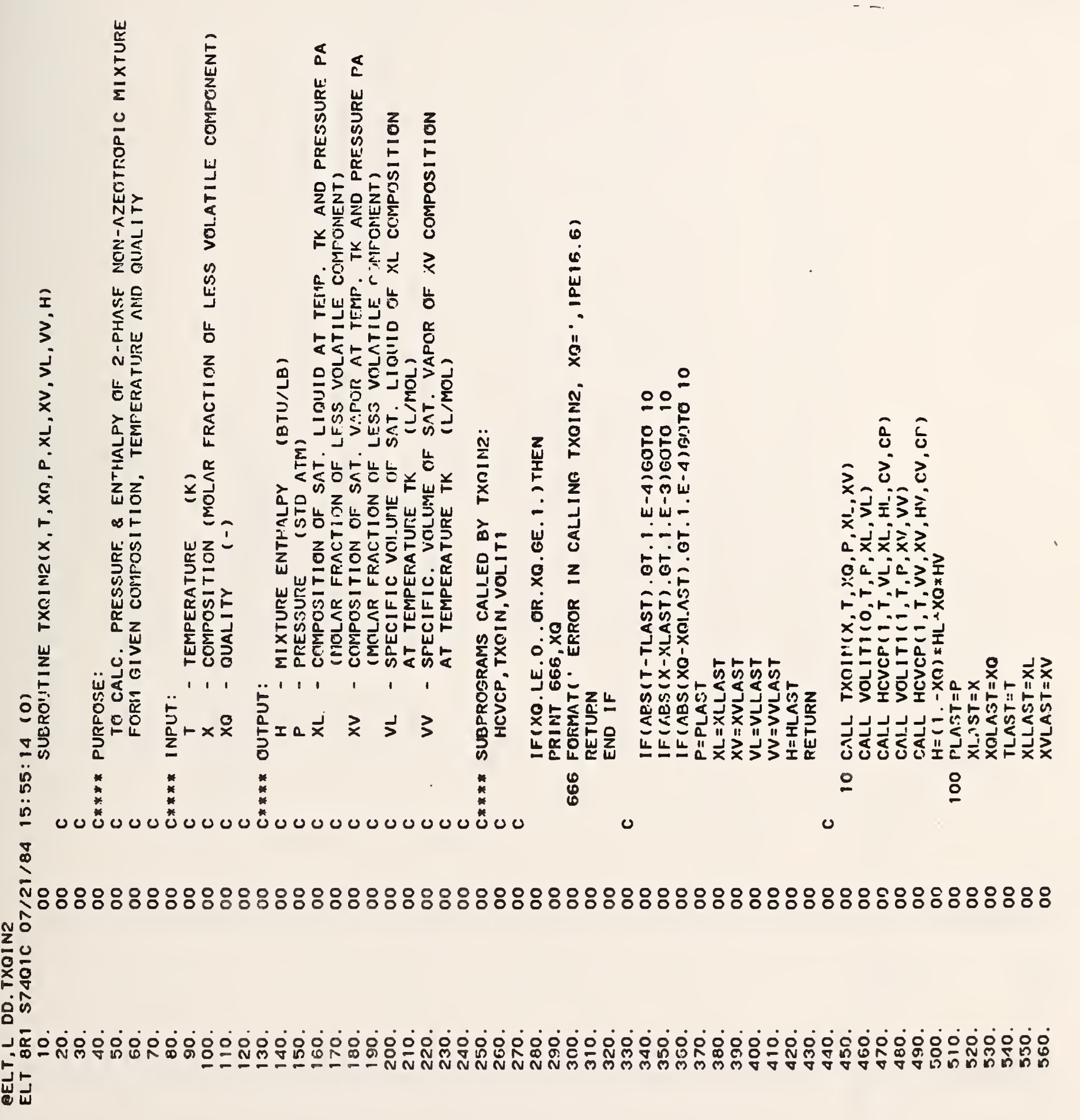




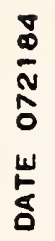

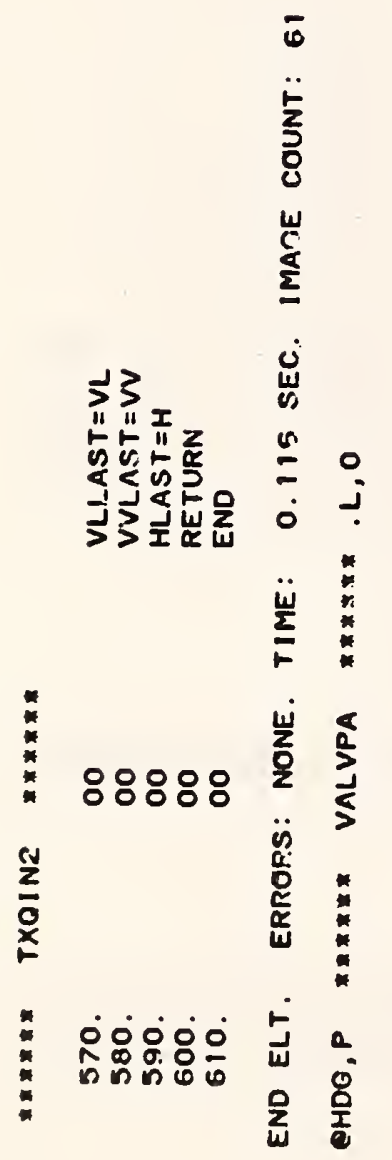




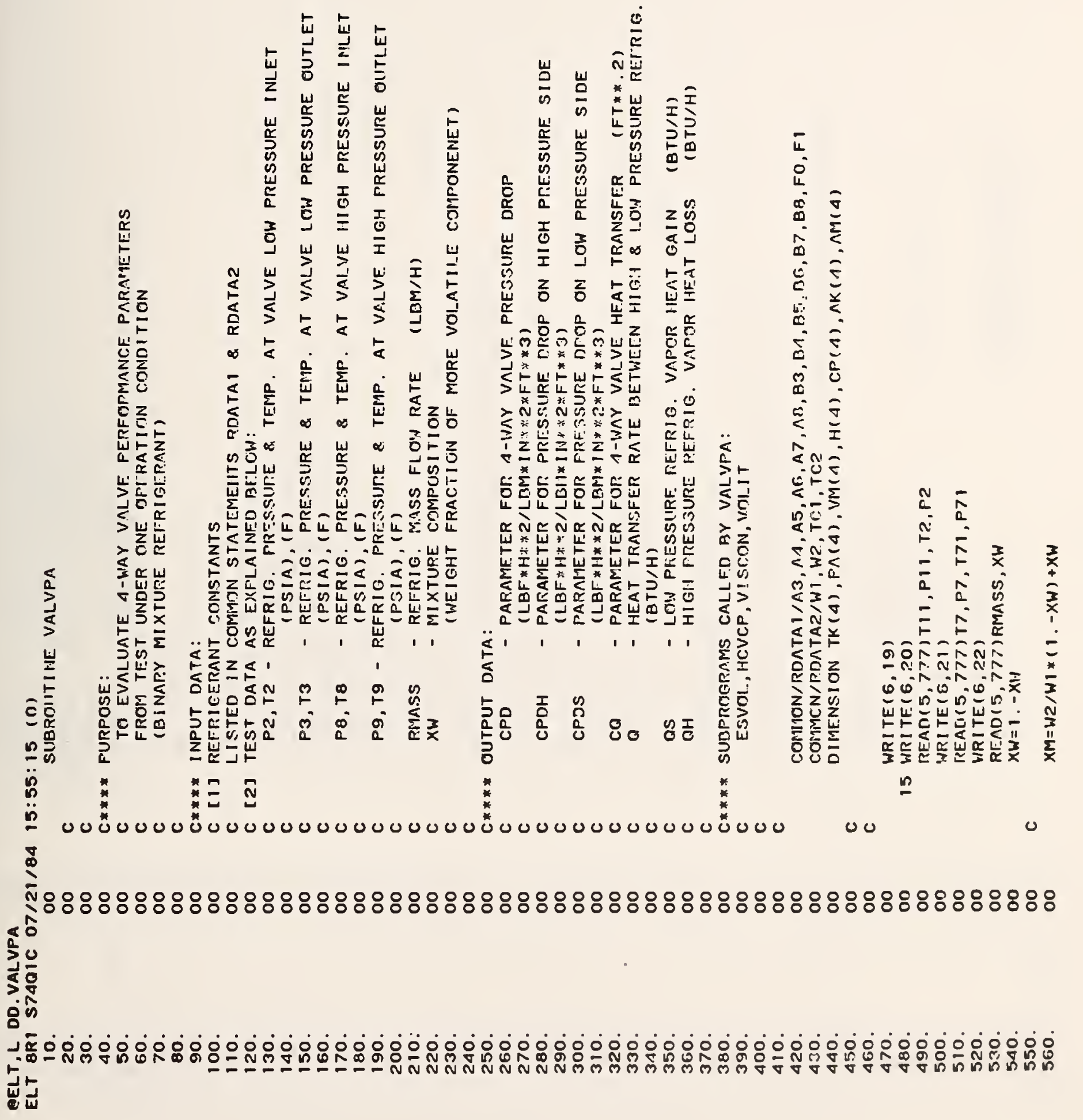




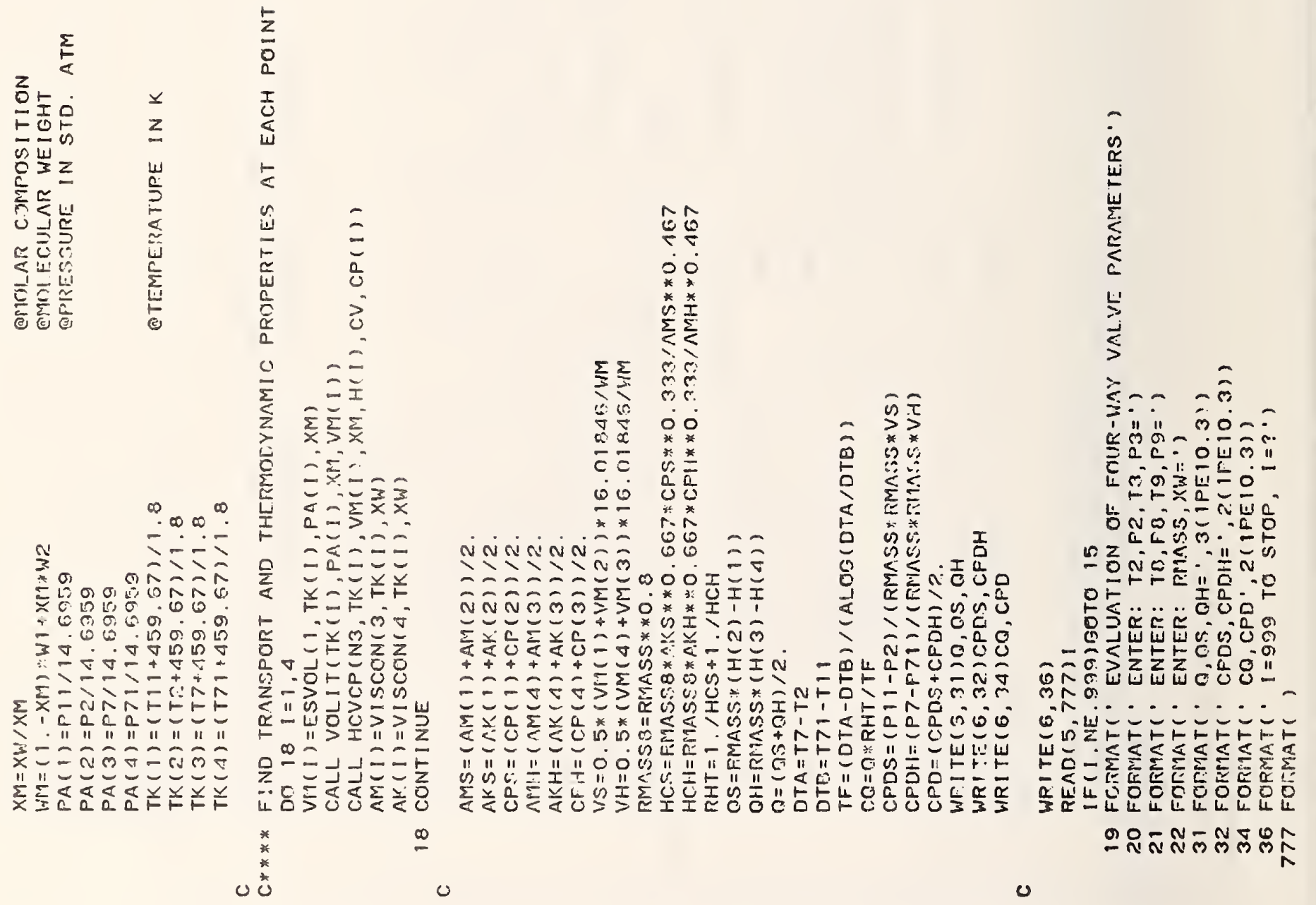

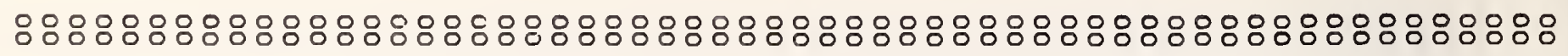

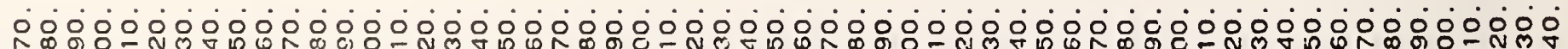
ద 


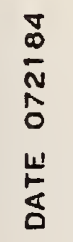

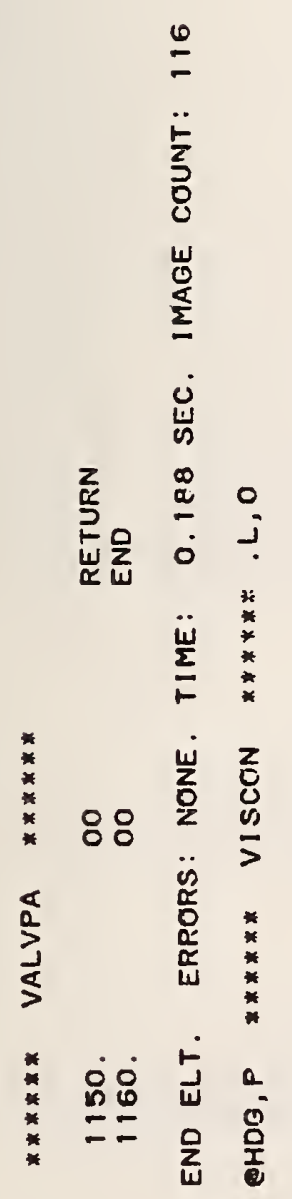




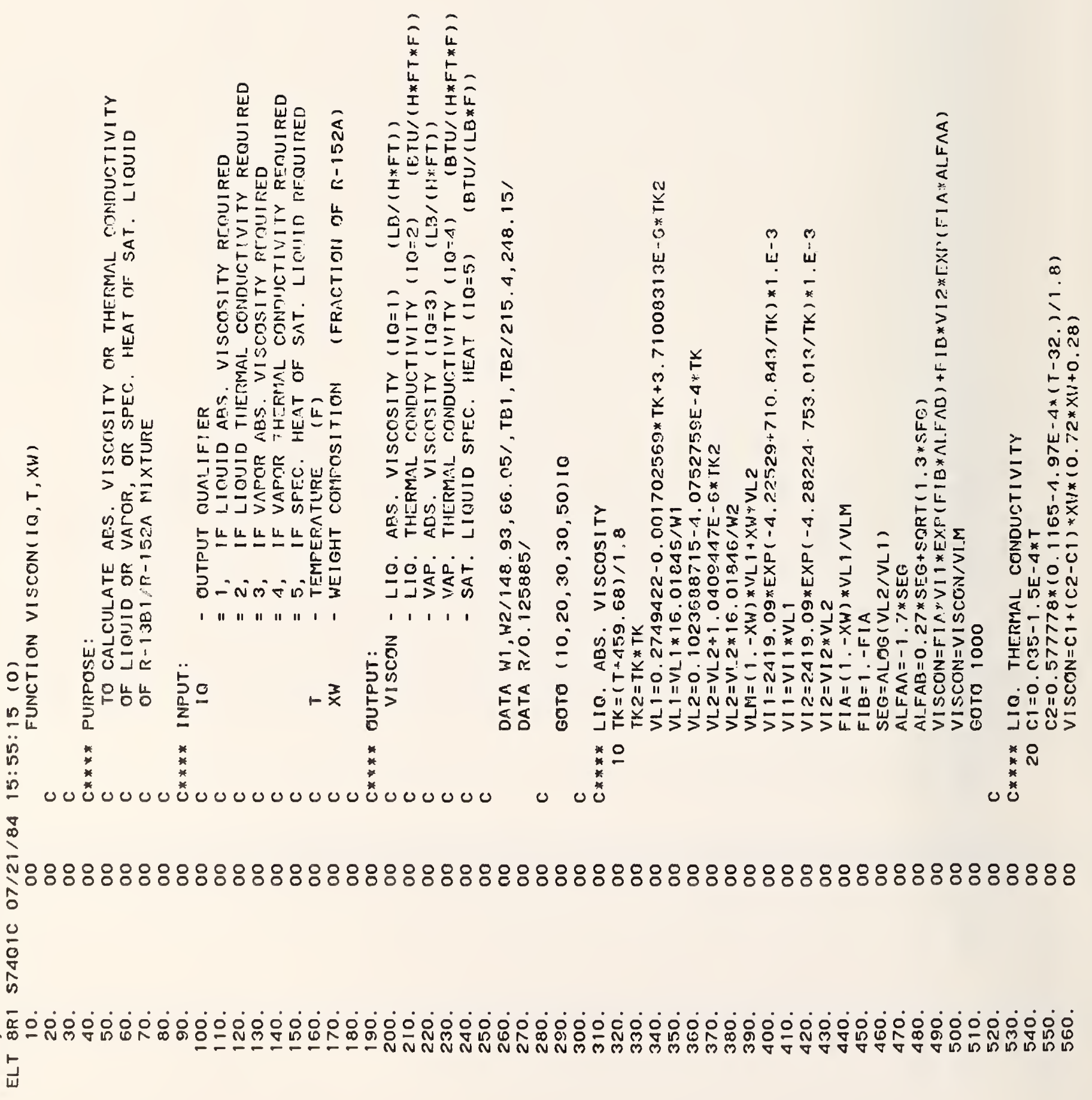




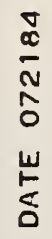

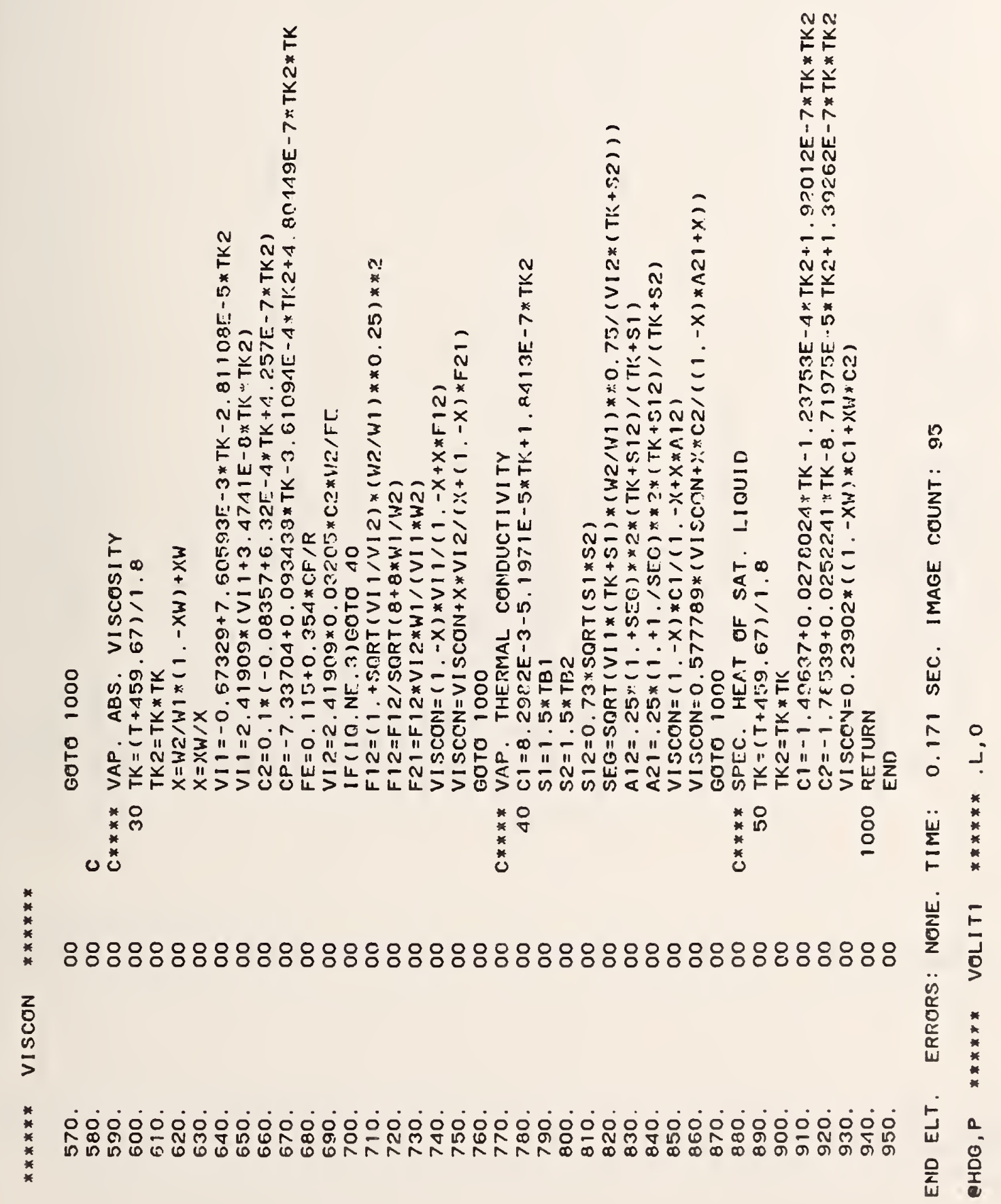




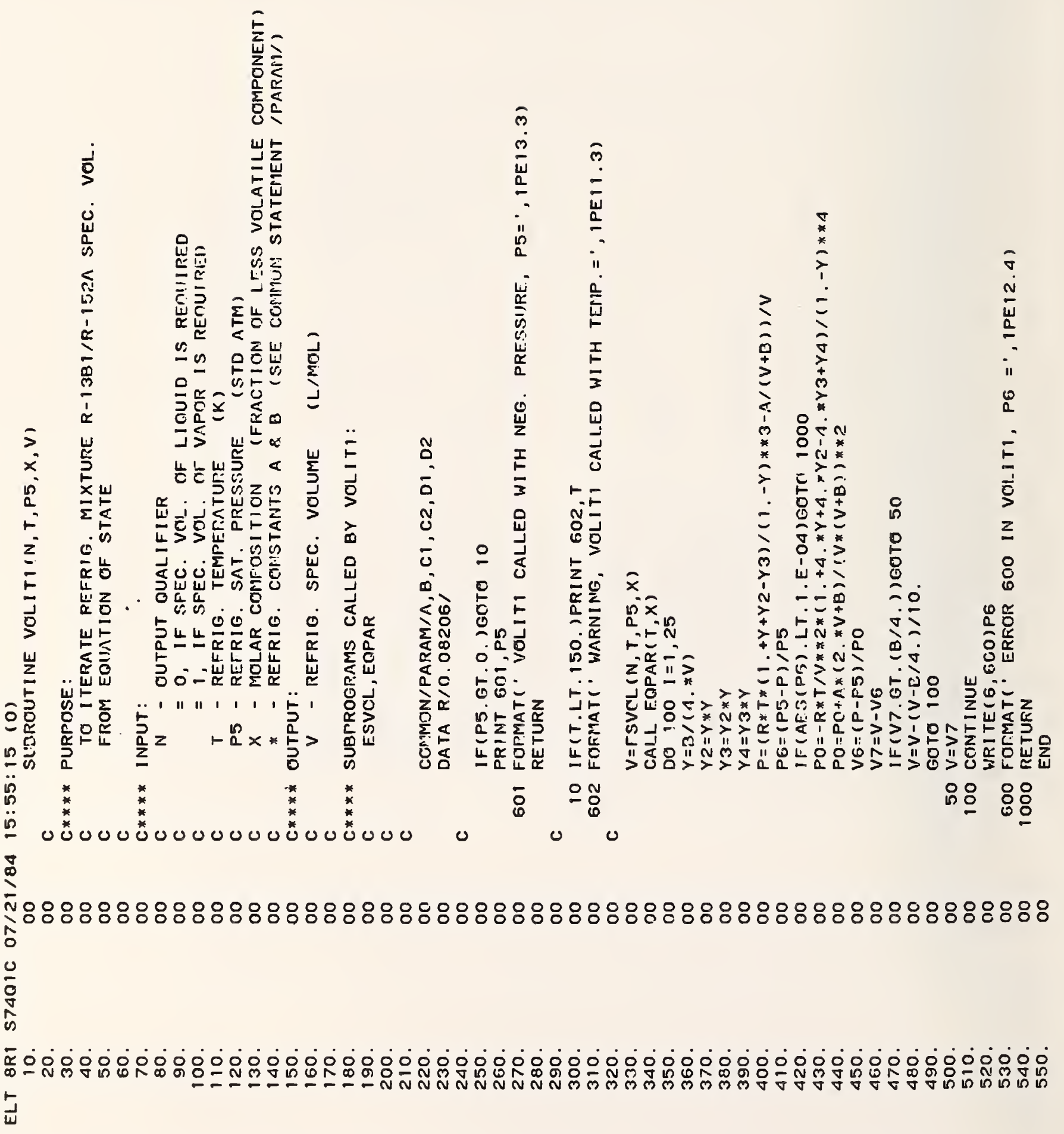


\begin{tabular}{ll}
$\bar{N}$ & 0 \\
\hline & $j$
\end{tabular}

岂

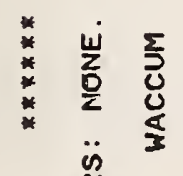

Е

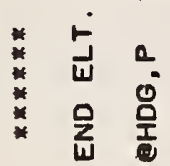




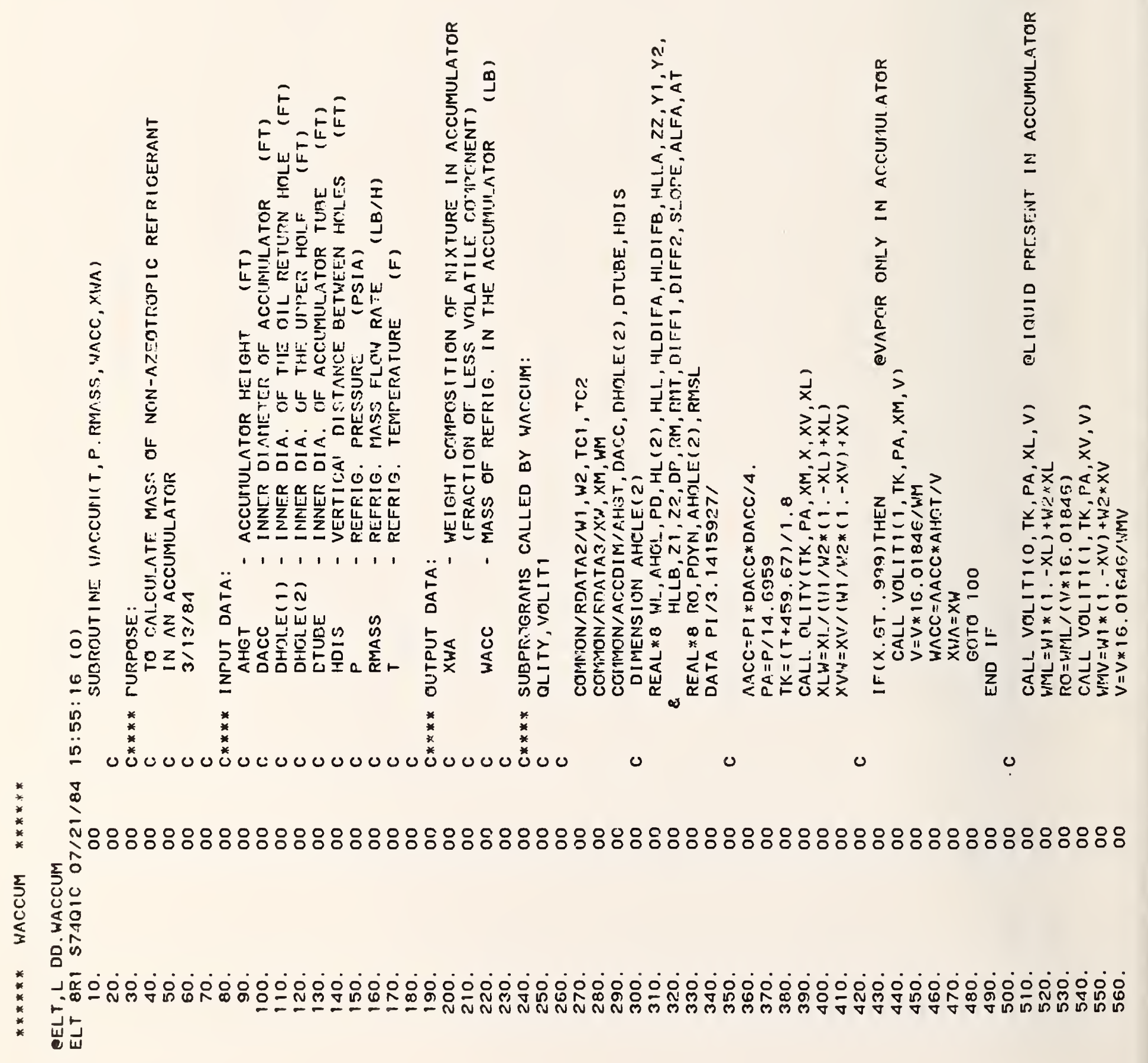




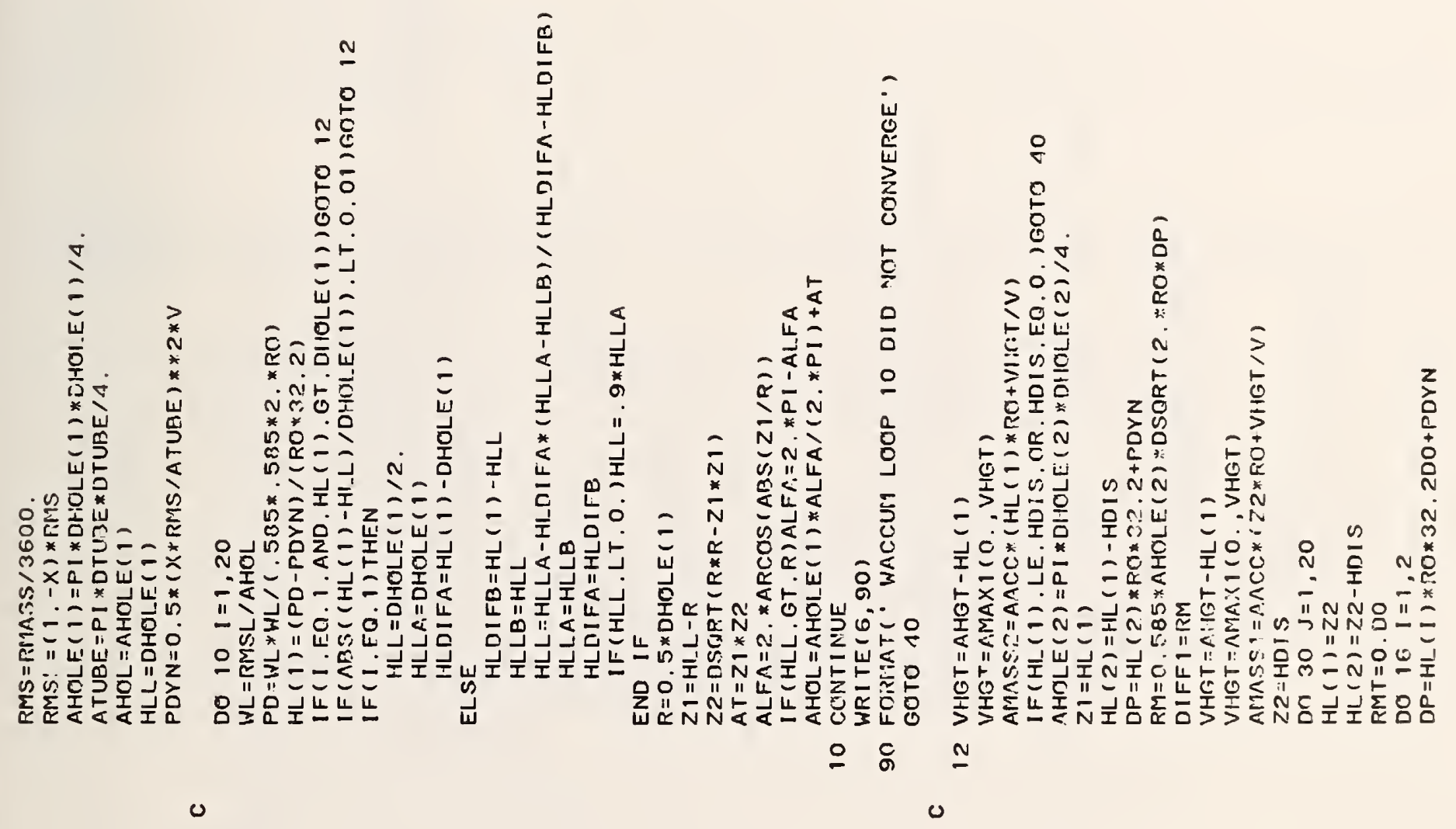

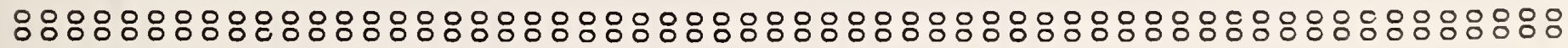

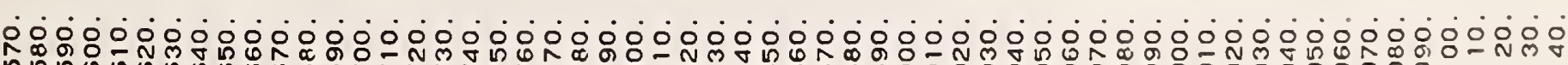
దํ. 


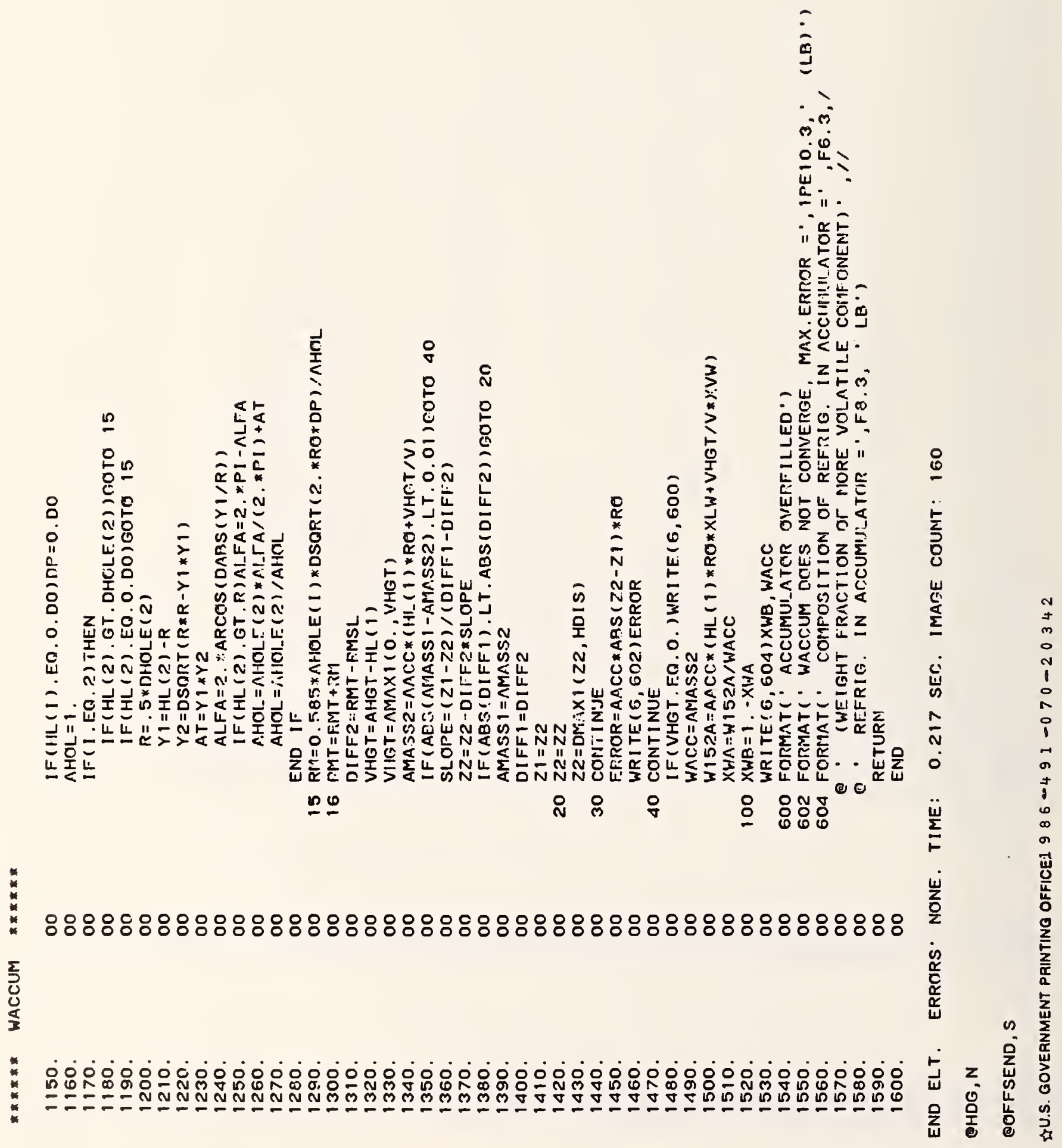


NBS-114A (REV. 2-8C)

\begin{tabular}{|c|c|c|c|}
\hline $\begin{array}{r}\text { NBS-114A (REV. 2-8C) } \\
\text { BIBLIOGRAPHIC DATA } \\
\text { SHEET (See instructions) }\end{array}$ & $\begin{array}{l}\text { 1. PUBLICATION OR } \\
\text { REPORT NO. } \\
\text { NBS/TN-1218 }\end{array}$ & 2. Performing Organ. Report No. Publication Date & 3. \\
\hline
\end{tabular}

4. TITLE AND SUBTITLE

\section{MODELING OF A HEAT PUMP CHARGED WITH A NON-AZEOTROPIC REFRIGERANT MIXTURE}

\section{AUTHOR(S)}

Piotr Domanski

6. PERFORMING ORGANIZATION (If joint or other than NBS, see instructions)

7. Contract/Grant No.

National Bureau of Standards

Department of Commerce

Gaithersburg, MD 20899

Finat

\section{SPONSORING ORGANIZATION NAME AND COMPLETE ADDRESS (Street, City, Stote, ZIP)}

Electric Power Research Institute

3412 Hillview Avenue

P.0. Box 10412

Palo Alto, California 94303

10. SUPPLEMENTARY NOTES

Document describes a computer program; SF-185, FIPS Software Summary, is attached.

11. ABSTRACT (A 200-word or less factual summary of most significant information. If document includes a significant bibliogrophy or literature survey, mention it here)

An analysis of the vapor compression cycle and the main components of an airto-air heat pump charged with a binary non-azeotropic mixture has been performed for steady-state operation. The general heat pump simulation model HPBI has been fomlulated which is based on independent, analytical models of system components and the logic linking them together. The logic of the program requires an iterative solution of refrigerant pressure and enthalpy balances, and refrigerant mixture and individual mixture component mass inventories.

The modeling effort emphasis was on the local thermodynanic phenanena which were described by fundamental heat transfer equations and equation of state relationships among material properties. In the campressor model several refrigerant locations were identified and the processes taking place between these locations accounted for all significant heat and pressure losses. Evaporator and condenser models were developed on a tube-by-tube basis where performance of each coil tube is camputed separately by considering the cross-flow heat transfer with the external air strean and the appropriate heat and mass transfer relationships. A capillary tube model was fonnulated with the aid of Fanno flow theory. Equation of state for mixtures is described and equation constants for RI3BI/R152a mixture are given.

The developed heat pump model was validated by checking computer results against laboratory tests data of one heat pump at two cooling and two heating rating points.

Program HPBI can be used to evaluate potentials of non-azeotropic mixtures working in a heat fump. User's Manual and listing of the progrant is included in the report.

12. KEY WORDS (Six to twelve entries: alphabetical order; capitalize only proper names; and seporate key words by semicolons air conditioner; capiliary tube; coil; compressor; condenser; expansion device; heat pump; modeling; mixture; non-azeotropic refrigerant; vapor modeling cycle

13. AVAILABILITY

X] Unlimited

7 For Official Distribution. Do Not Release to NTIS

[X] Order From Superintendent of Documents, U.S. Government Printing Office, Washington. D.C. 20402.

Order From National Technical Information Service (NTIS), Springfield, VA. 22161
14. NO. OF PRINTED PAGES

391

15. Price 




\section{Periodical}

Journal of Research-The Journal of Research of the National Bureau of Standards reports NBS research ana development in those disciplines of the physical and engineering sciences in which the Bureau is active. These include physics, chemistry, engineering, mathematics, and computer sciences. Papers cover a broad range of subjects, with major emphasis on measurement methodology and the basic technology underlying standardization. Also included from time to time are survey articles on topics closely related to the Bureau's technical and scientific programs. Issued six times a year.

\section{Nonperiodicals}

Monographs-Major contributions to the technical literature on various subjects related to the Bureau's scientific and technical activities.

Handbooks-Recommended codes of engineering and industrial practice (including safety codes) developed in cooperation with interested industries, professional organizations, and regulatory bodies.

Special Publications - Include proceedings of conferences sponsored by NBS, NBS annual reports, and other special publications appropriate to this grouping such as wall charts, pocket cards, and bibliographies.

Applied Mathematics Series-Mathematical tables, manuals, and studies of special interest to physicists, engineers, chemists, biologists, mathematicians, computer programmers, and others engaged in scientific and technical work.

National Standard Reference Data Series-Provides quantitative data on the physical and chemical properties of materials, compiled from the world's literature and critically evaluated. Developed under a worldwide program coordinated by NBS under the authority of the National Standard Data Act (Public Law 90-396).

NOTE: The Journal of Physical and Chemical Reference Data (JPCRD) is published quarterly for NBS by the American Chemical Society (ACS) and the American Institute of Physics (AIP). Subscriptions, reprints, and supplements are available from ACS, 1155 Sixteenth St., NW, Washington, DC 20056.

Building Science Series-Disseminates technical information developed at the Bureau on building materials, components, systems, and whole structures. The series presents research results, test methods, and performance criteria related to the structural and environmental functions and the durability and safety characteristics of building elements and systems.

Technical Notes-Studies or reports which are complete in themselves but restrictive in their treatment of a subject. Analogous to monographs but not so comprehensive in scope or definitive in treatment of the subject area. Often serve as a vehicle for final reports of work performed at NBS under the sponsorship of other government agencies.

Voluntary Product Standards-Developed under procedures published by the Department of Commerce in Part 10, Title 15, of the Code of Federal Regulations. The standards establish nationally recognized requirements for products, and provide all concerned interests with a basis for common understanding of the characteristics of the products. NBS administers this program as a supplement to the activities of the private sector standardizing organizations.

Consumer Information Series-Practical information, based on NBS research and experience, covering areas of interest to the consumer. Easily understandable language and illustrations provide useful background knowledge for shopping in today's technological marketplace.

Order the above NBS publications from: Superintendent of Documents, Governinent Printing Office, Washington, DC 20402.

Order the following NBS publications-FIPS and NBSIR's-from the National Technical Information Service, Springfield, VA 22161.

Federal Information Processing Standards Publications (FIPS PUB)-Publications in this series collectively constitute the Federal Information Processing Standards Register. The Register serves as the official source of information in the Federal Government regarding standards issued by NBS pursuant to the Federal Property and Administrative Services Act of 1949 as amended, Public Law 89-306 (79 Stat. 1127), and as implemented by Executive Order 11717 (38 FR 12315, dated May 11, 1973) and Part 6 of Title 15 CFR (Code of Federal Regulations).

NBS Interagency Reports (NBSIR)-A special series of interim or final reports on work performed by NBS for outside sponsors (both government and non-government). In general, initial distribution is handled by the sponsor; public distribution is by the National Technical Information Service, Springfield, VA 22161, in paper copy or microfiche form. 
U.S. Department of Commerce

National Bureau of Standards

Gaithersburg, MD 20899

Official Business

Penalty for Private Use $\$ 300$ 